\title{
SELECTIVE METAL COORDINATION IN ANTIFOULING COATINGS
}

BY

HANNAH ROBINSON

\begin{abstract}
A thesis
submitted to the Victoria University of Wellington

in fulfilment of the requirements for the degree of

Doctor of Philosophy in Chemistry
\end{abstract}

Victoria University of Wellington 



\section{AbSTRACT}

Marine biofouling is the accumulation of biological material (e.g. microorganisms, soft- and hardfouling organisms) on the surface of an object submerged in seawater, and it remains a worldwide problem for shipping industries. The fouling of ship hulls results in a reduction of speed and manoeuvrability due to frictional drag, as well as increased fuel consumption and accelerated corrosion, and the exorbitant expenses and losses of efficiency attributed to biofouling have prompted the development of antifouling coatings. Current antifouling paints use copper as a biocidal agent, but copper-based paints are increasingly being banned due to environmental concerns about the nontarget effects of leached copper. This project aims to circumvent these concerns and tightening regulations via a revolutionary concept: the development of marine antifouling paints that incorporate $\mathrm{Cu}(\mathrm{II})$-selective ligands to draw the biocidal ingredient (i.e. $\mathrm{CU}(\mathrm{II})$ ) from seawater. A multistage strategy emerged for the development of this technology. First, criteria were established for the project's ideal ligand, and ligands were synthesised or selected based on these criteria. Second, the ligands were incorporated in coatings through covalent modification of the paint binder or additives. Third, methodology was developed and implemented to test each coating's ability to coordinate and retain $\mathrm{Cu}(\mathrm{II})$, as well as its subsequent ability to prevent microfouling by marine bacteria.

The suitability of two ligand classes was assessed: acylhydrazones and tetraaza macrocycles, specifically cyclen. Unlike the acylhydrazones, cyclen met the established criteria and was initially evaluated as a curing agent and/or surface-modifier in a two-pack epoxy system with resin Epikote $^{\mathrm{TM}} 235$. However, the $\mathrm{Cu}(\mathrm{II})$-loading by these coatings was relatively low, being at most $\sim 0.05 \% \mathrm{w} / \mathrm{w}$, and the modification of silica, a common paint additive, with cyclen was explored as an alternative formulation route. The method for the functionalisation of silica with cyclen was optimised, and the maximum $\mathrm{Cu}(I I)$-loading achieved by the product was $2.60 \% \mathrm{w} / \mathrm{w}$. The cyclenfunctionalised silica was incorporated on the surface of an epoxy coating, and a bacterial adherence assay was developed to assess the cellular attachment of marine bacterium Vibrio harveyi to this coating, which was found to be undeterred. Yet, the development of the strategy and testing methodology by which the project's goals may be achieved provides a solid foundation for future work. 


\section{ACKNOWLEDGMENTS}

I would like to express my deepest gratitude to those who have provided me with support during my $\mathrm{PhD}$ candidature. First and foremost, I would like to thank my supervisors, Associate Professor Simon Hinkley and Professor Bradley Williams, for giving me this opportunity to gain skills and experience in new areas and become an independent research scientist. Simon, I truly appreciate your direction, reliability, encouragement, assistance (both personal and professional), and unwavering positivity. We have literally come through fire to make it to this point. Yet, I consider myself fortunate and privileged to work under your tutelage, and I recognise all of the time and effort you have put into this endeavour: thank you. Bradley, I have greatly valued your advice and gained much insight from our discussions: your knowledge and perspective have helped to advance this work.

Mark Glenny, I am so grateful for your collaboration and provision of materials and counsel to a paint newbie.

Darren Day, please accept my deepest thanks for allowing me access to your laboratory facilities, resources, equipment, and guidance. Jen Soundy, thank you for showing me the ropes and providing your input in regard to the microbiology. Thank you to all others who supported the microbiological work in my thesis: Shuguang Zhang, Rebecca Edgar, Rashad Syed, and Indira Rasiah.

Janet Pitman, I am sincerely grateful for the desk space in TTR you provided for me after the Ferrier fire.

Bruce Charlier, I greatly appreciate your ICP-MS analysis of my many samples. Herbert Wong, Andrew Lewis, and lan Vorster, thank you for the numerous hours of support in NMR analysis. Yinrong Lu and Sarah Spencer, thank you for conducting the MS and SEM-EDS analyses, respectively. Thank you, Donal Krouse, for sharing your expert opinion on the statistical analyses.

I am indebted to the staff and students at the Ferrier Research Institute who have welcomed me into the group and made this such a positive experience. Special thanks to Rosannah, Jono, my cohort (Liz, Roselis, Rinu, and Dan), and my A block buddies, especially Amira, Alison, Norman, and Keith.

Finally, thank you to my parents, family, and friends for their unwavering support, love, and encouragement. Mom and dad, you have always been my biggest cheerleaders, and I am blessed to have you as my parents. Anna and Courtney, thank you for your faithful correspondence and steadfast friendship. Andrew, more thank-yous than could fill a thesis - you were the best part. 


\section{DEClARATION AND COPYRIGHT}

The copyright of this thesis resides with the author. No quotation from it should be published without prior consent, and the information derived from it should be acknowledged.

The work in this thesis was completed by the author at the Victoria University of Wellington, New Zealand. It has not been submitted before, in part or in whole, for a higher degree at this university or any other.

Some of the work described in this thesis has been reported in the following poster and publication:

1. Robinson, H., Burr, D., Daines, A. M., Glenny, M., Hinkley, S. F. R., \& Williams, D. B. G. Assessing the antimicrobial activity of copper-coordinating coatings. in ASM Microbe Conference (2018), SCANZ Convention (2018), \& Microbes \& Molecules NZMS Annual Conference (2018).

2. Daines, A. M., Robinson, H., Glenny, M., Williams, D. B. G. \& Hinkley, S. F. R. Linear and macrocyclic water soluble polyacylhydrazones and their utilisation in coatings. Prog. Org. Coatings 121, 38-44 (2018).

The author wrote Sections 2.4 (paragraphs 1-2, 4) and $\mathbf{3 . 2}$ (paragraphs 1-2, 4) in the research article above, and these sections are included in Chapters 2 (Section 2.3.2) and 6 (Section 6.2.2.2) of this thesis, as permitted by Elsevier and the Victoria University of Wellington Faculty of Graduate Research. 


\section{TABle OF Contents}

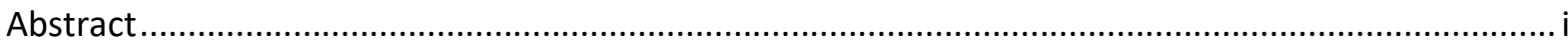

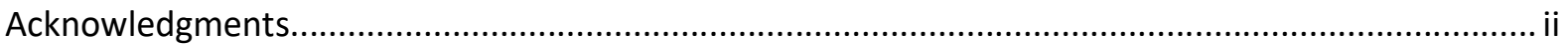

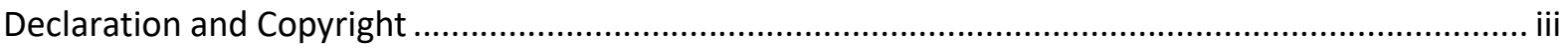

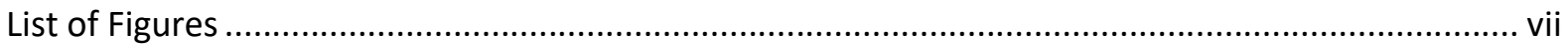

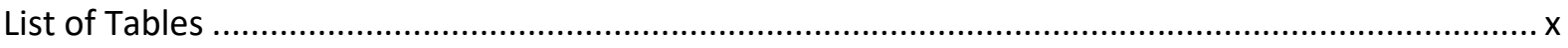

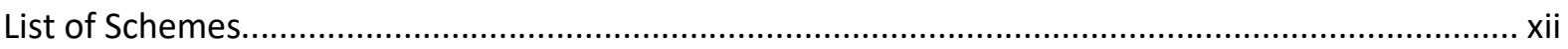

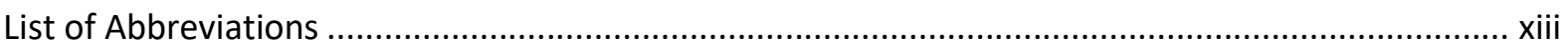

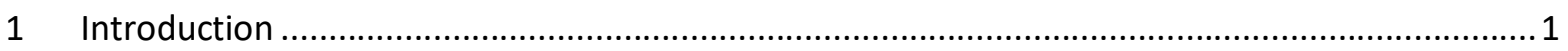

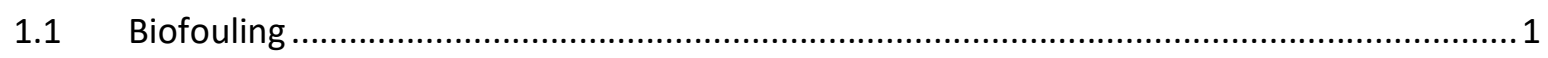

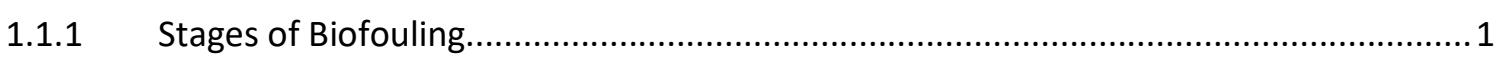

1.1.2 Negative Consequences of Biofouling .............................................................. 4

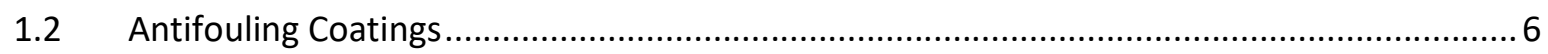

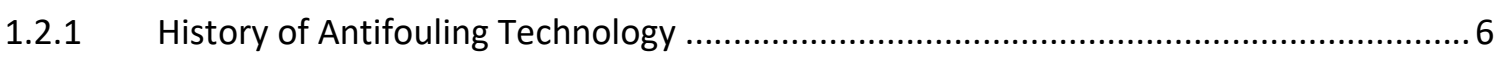

1.2.2 Biocide-Based Antifouling Coatings ............................................................... 10

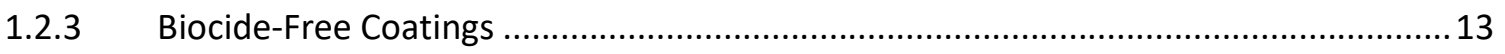

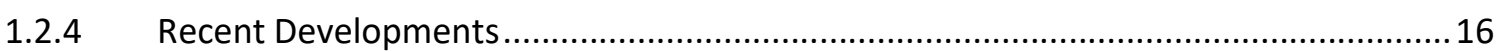

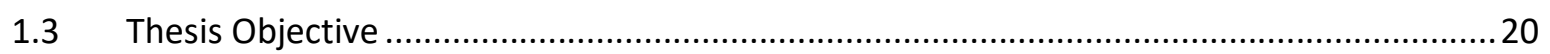

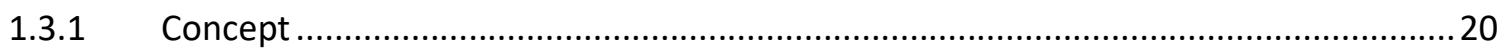

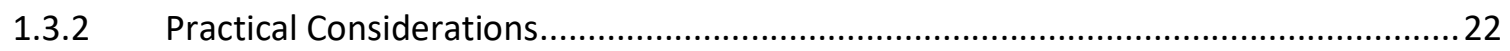

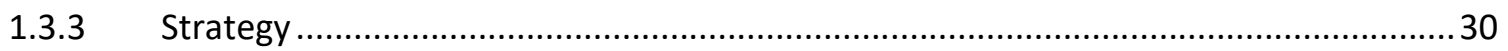

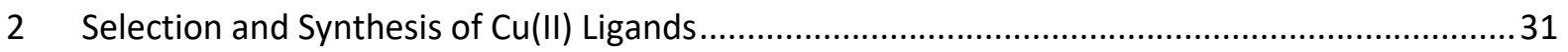

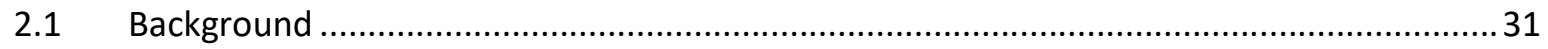

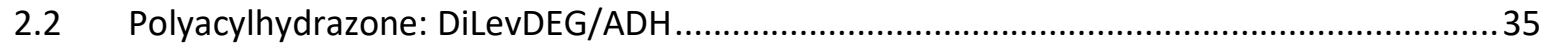

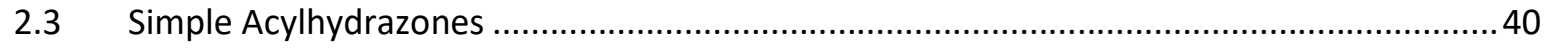

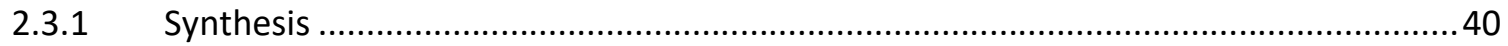

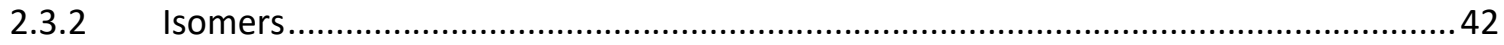

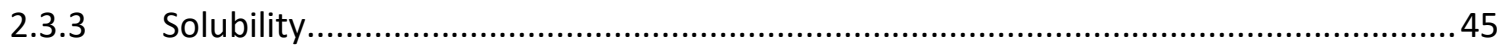

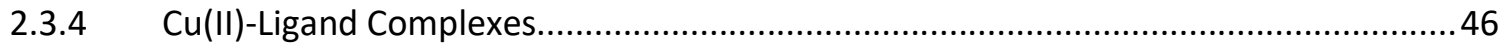

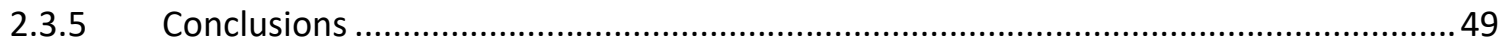

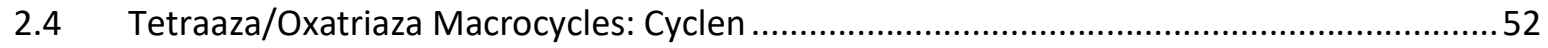

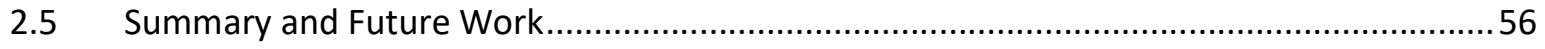

3 Incorporation of Cu(II)-Ligand Complexes in Coating Binders ...........................................58

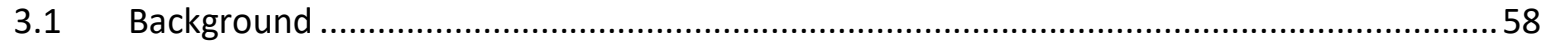


3.2 Polyacylhydrazone DiLevDEG/ADH-Substituted Resin in a Commercial Acrylic Paint.

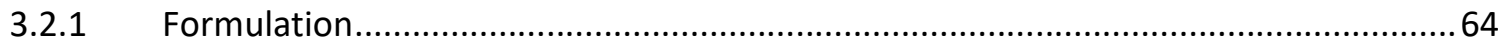

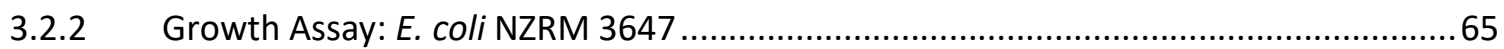

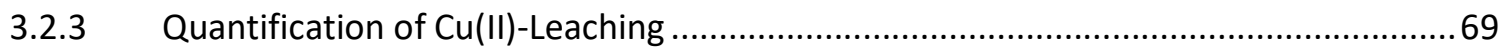

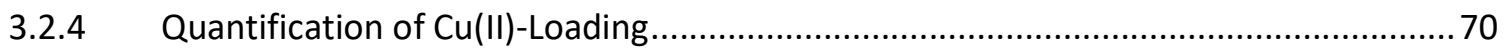

3.2.5 DiLevDEG/ADH Polyacylhydrazone-Leaching ......................................................... 73

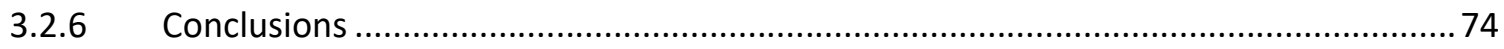

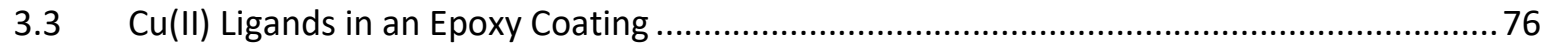

3.3.1 Modifications of the Hardener Component.................................................................. 80

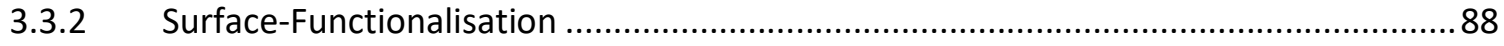

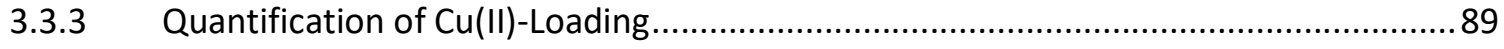

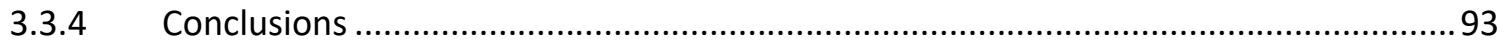

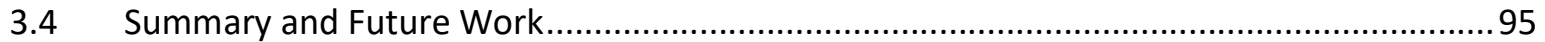

4 Incorporation of $\mathrm{Cu}(\mathrm{II})$-Ligand Complexes in Coating Additives ................................................97

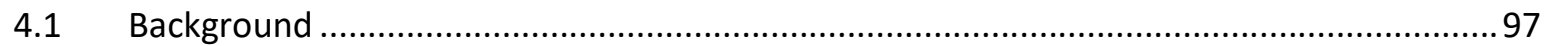

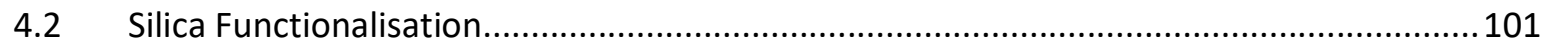

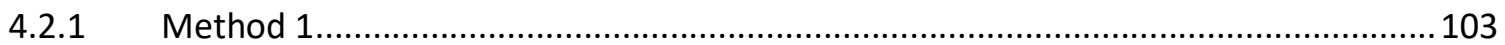

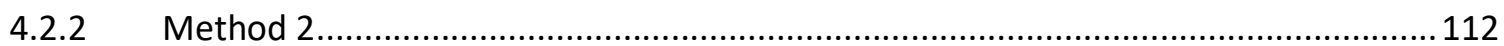

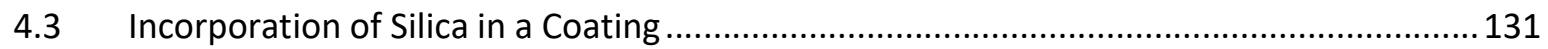

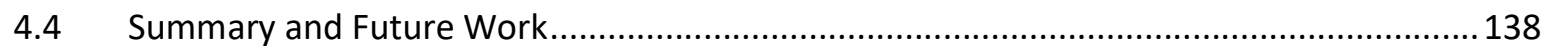

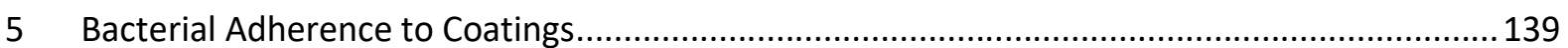

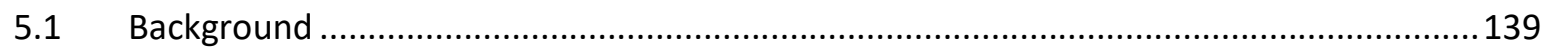

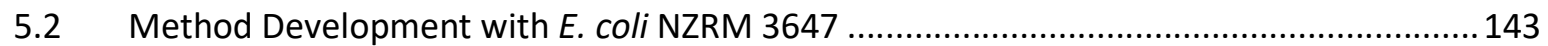

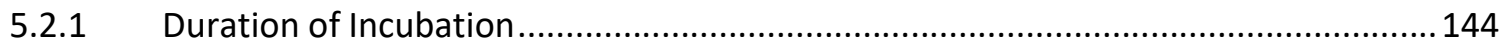

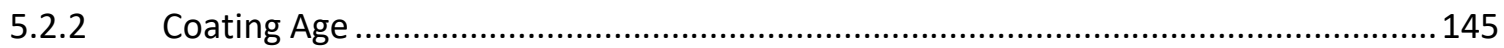

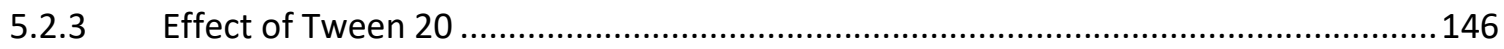

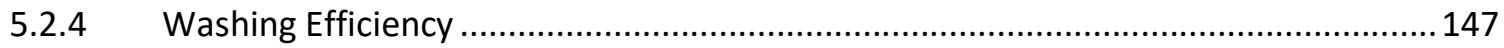

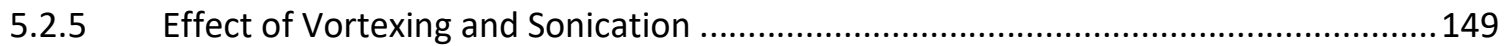

5.2.6 Efficiency of Vortexing and Sonication ( \pm Tween 20) ...........................................149

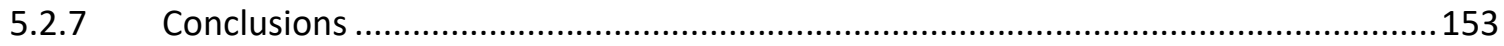

5.3 Adherence of E. coli NZRM 3647 to $\mathrm{SiO}_{2}$ /epx-Type Coatings .......................................... 154

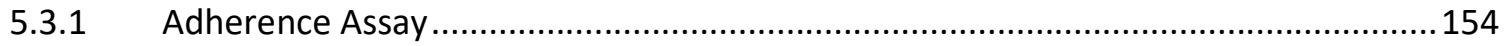

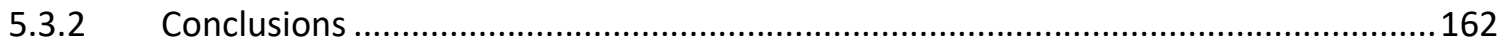

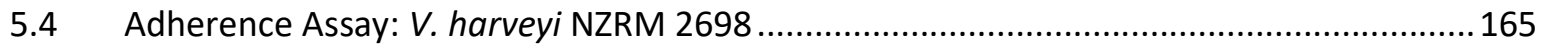

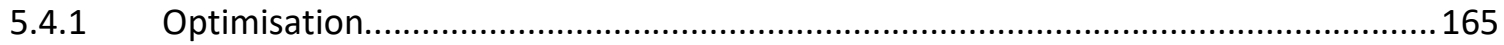

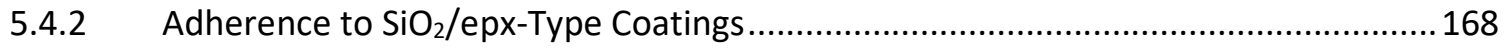




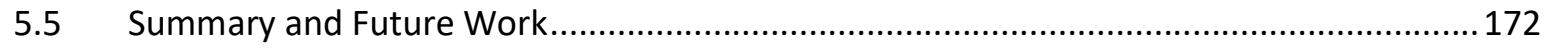

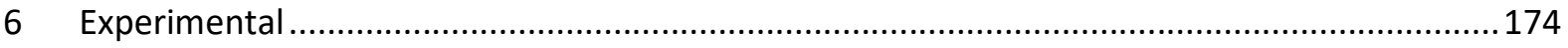

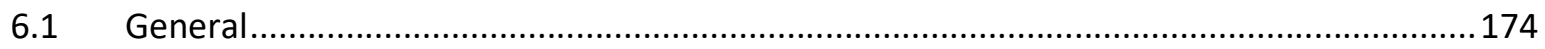

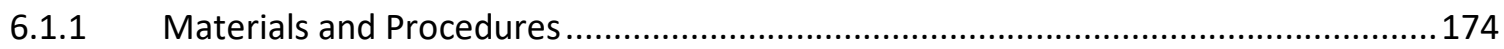

6.1.2 Instrumentation, Equipment, and Analysis ....................................................... 174

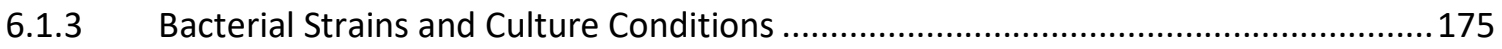

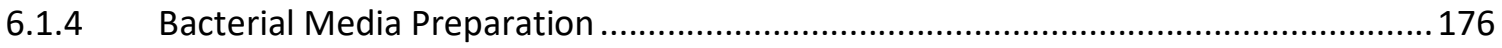

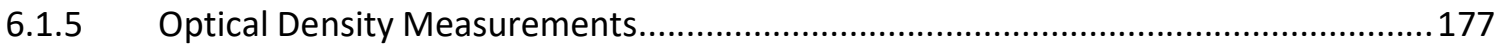

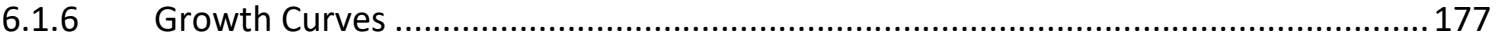

6.1.7 Enumeration of Cells by the Drop Plate Method ....................................................... 178

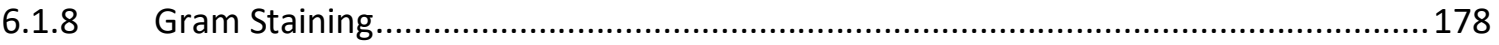

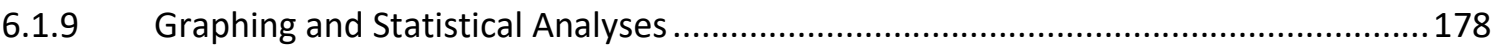

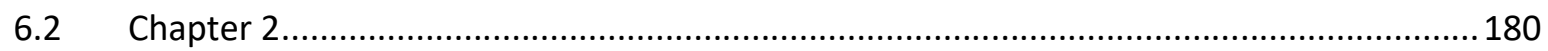

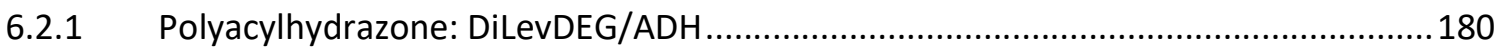

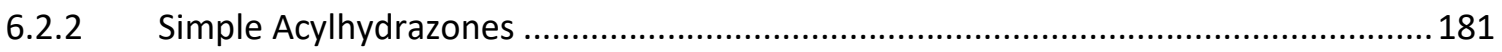

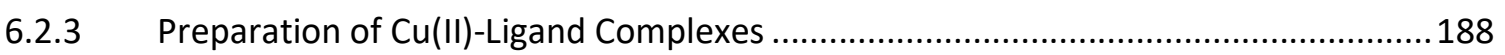

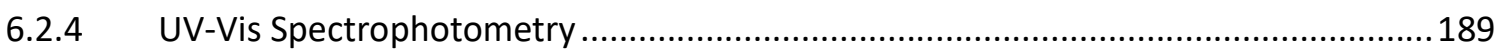

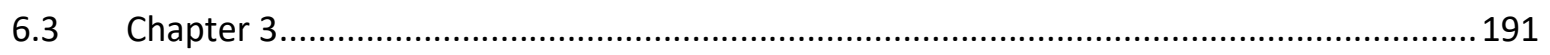

6.3.1 DiLevDEG/ADH-Substituted Resin in a Commercial Acrylic Paint................................191

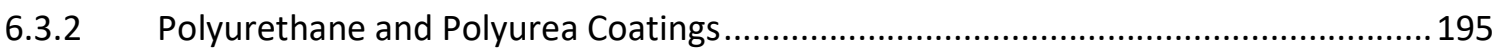

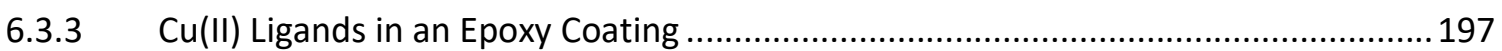

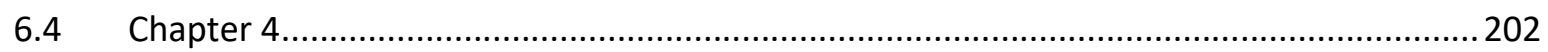

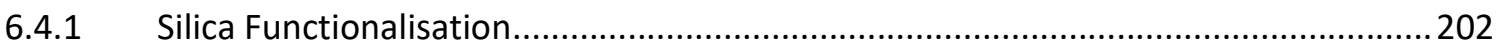

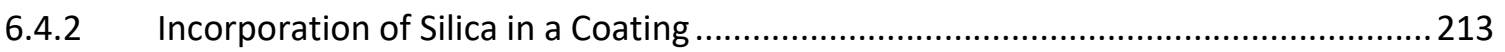

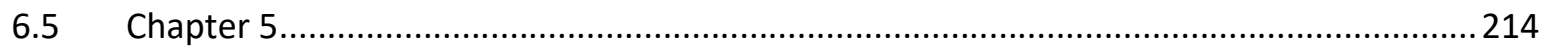

6.5.1 Method Development with E. coli NZRM 3647 .....................................................214

6.5.2 Adherence of E. coli NZRM 3647 to $\mathrm{SiO}_{2}$ /epx-Type Coatings ....................................223

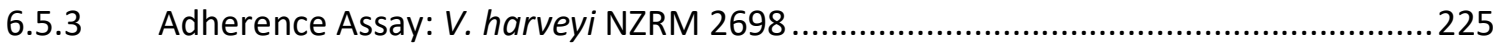

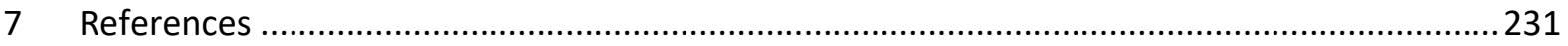

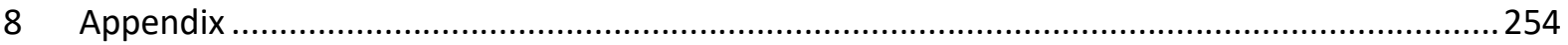

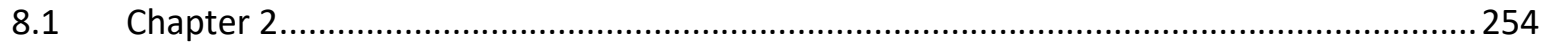

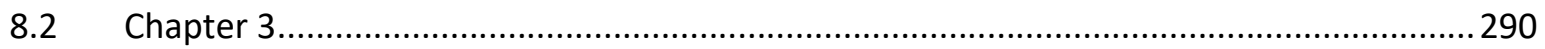

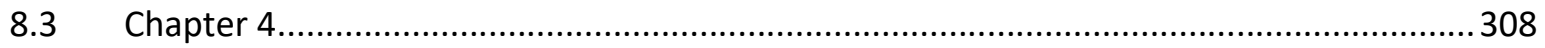

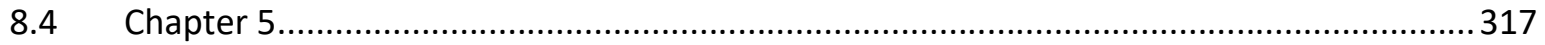




\section{LIST OF FIGURES}

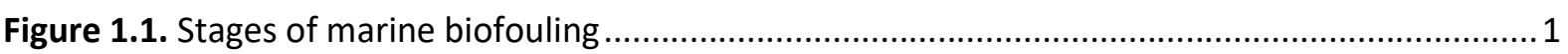

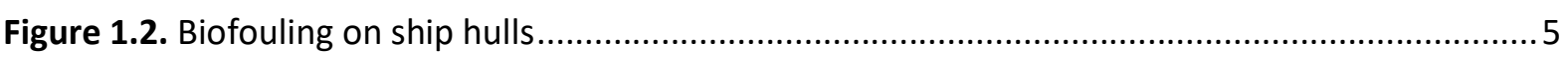

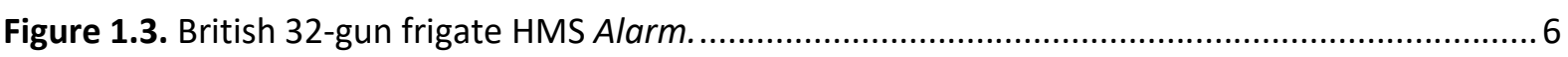

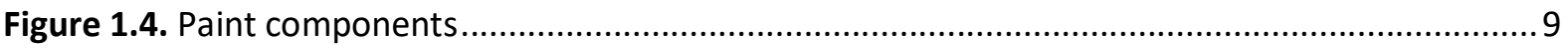

Figure 1.5. Classifications of biocide-based antifouling paints........................................................ 10

Figure 1.6. Hydrophilic antifouling coating with PEG brushes ....................................................... 14

Figure 1.7. Detachment of marine foulers from a PDMSe-based fouling-release coating ..................15

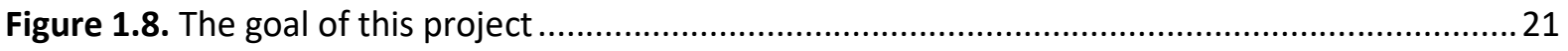

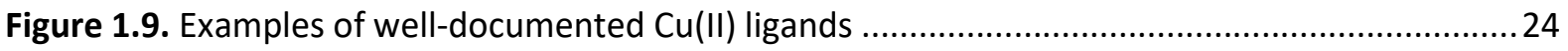

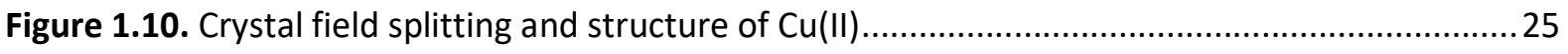

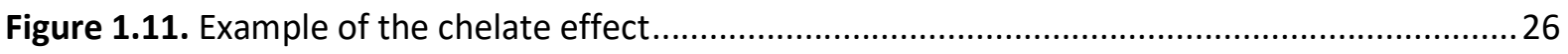

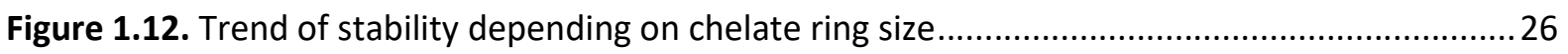

Figure 1.13. Example Job plot of a metal complex with $1: 1$ metal:ligand stoichiometry ....................27

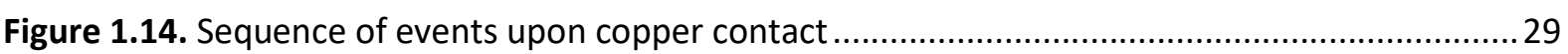

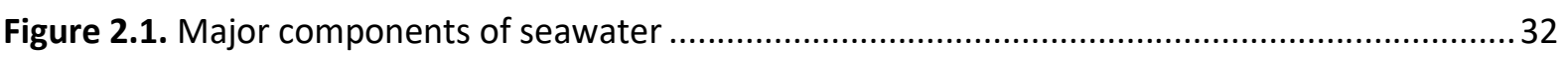

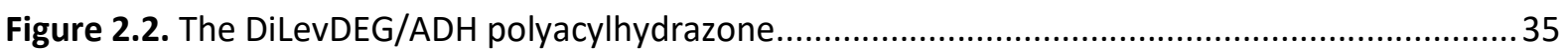

Figure 2.3. Proposed $\mathrm{Cu}(\mathrm{II})$-chelation by the acylhydrazone functionality in DiLevDEG/ADH .............36

Figure 2.4. ${ }^{13} \mathrm{C}$ NMR spectrum of the DiLevDEG/ADH polyacylhydrazone ...........................................37

Figure 2.5. Relationship between the colour of $\mathrm{Cu}(\mathrm{II})$ complexes and the electron configuration of

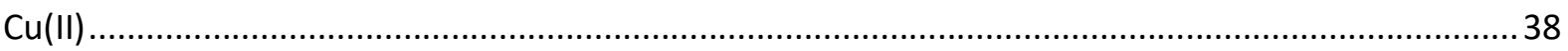

Figure 2.6. UV-Vis spectra of DiLevDEG/ADH, $\mathrm{Cu}\left(\mathrm{NO}_{3}\right)_{2}$, and $\mathrm{Cu}(I I)-\mathrm{DiLevDEG} / \mathrm{ADH} \ldots \ldots \ldots \ldots \ldots \ldots \ldots \ldots . . . . . . . . . . . . .39$

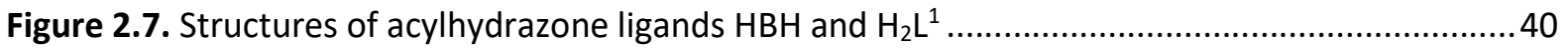

Figure 2.8. Possible geometric ( $E / Z)$-isomers and $\left(E^{\prime} / Z^{\prime}\right)$-rotamers of acylhydrazones .......................42

Figure 2.9. Signals attributed to rotamers in a ${ }^{13} \mathrm{C}$ NMR spectrum (5i: C1) .................................... 43

Figure 2.10. Examples of intramolecular hydrogen bonding in monoacylhydrazone 1a ....................43

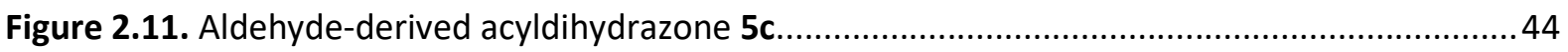

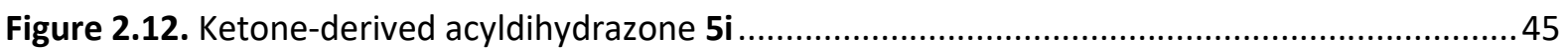

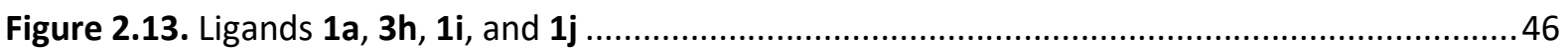

Figure 2.14. Job plot obtained by varying the molar ratio of ligand 1a to copper(II) nitrate. ............47

Figure 2.15. Absorption spectra of $0.100 \mathrm{mM}$ solutions of $1 \mathrm{a}$ and increasing concentrations of copper(II) nitrate

Figure 2.16. Expanded views of the regions in Figure 2.15 containing isosbestic points ...................49 
Figure 2.17. Example of different constitutional states in an acylhydrazone .50

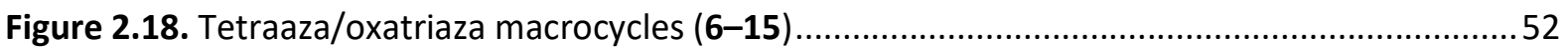

Figure 2.19. $\mathrm{Cu}(\mathrm{II})$-chelation by cyclen in the trans-I conformation ...................................................55

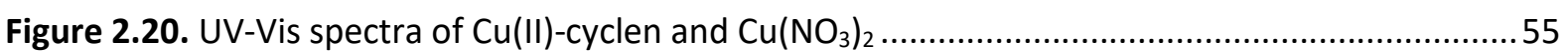

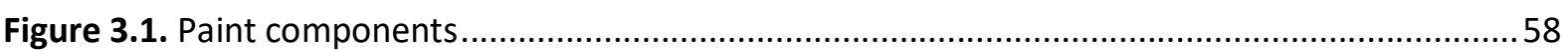

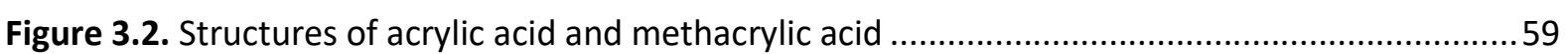

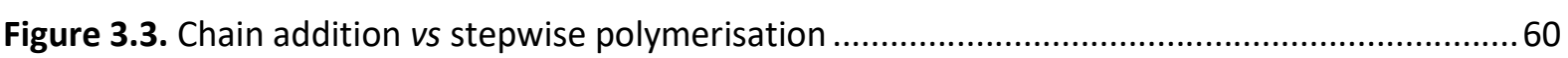

Figure 3.4. Methodology for assessing the $\mathrm{Cu}(\mathrm{II})$-chelating ability of the acrylic and DAp/acr paints 63

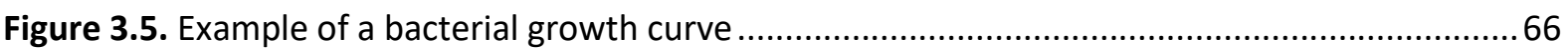

Figure 3.6. Cu(II)-loading process for acrylic and DAp/acr paints ...................................................67

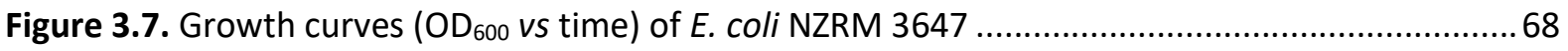

Figure 3.8. ${ }^{13} \mathrm{C}$ NMR spectra of the leachate and DiLevDEG/ADH polyacylhydrazone .......................... 74

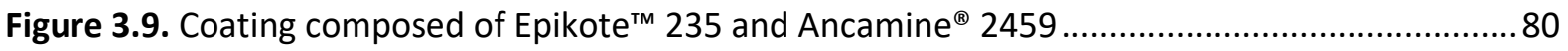

Figure 3.10. FT-MIR and NIR spectra of overcured and undercured Epikote ${ }^{\mathrm{TM}}$ 235/Ancamine ${ }^{\circledR} 2459$ coatings

Figure 3.11. Example of monitoring the $\mathrm{Cu}(\mathrm{II})$-addition to under/overcured Ancamine ${ }^{\circledR}$ 2459/

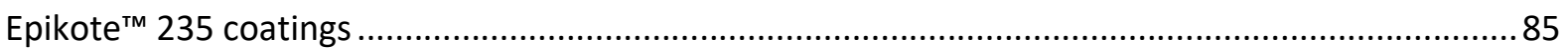

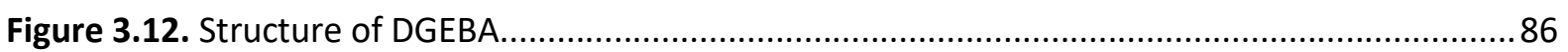

Figure 3.13. Monitoring the $\mathrm{Cu}(\mathrm{II})$-addition to coatings prepared from Epikote ${ }^{\mathrm{TM}} 235$ and DETA.......87

Figure 3.14. $\mathrm{Cu}(\mathrm{II})$-addition to coatings prepared from Epikote ${ }^{\mathrm{TM}} 235$ and cyclen.............................91

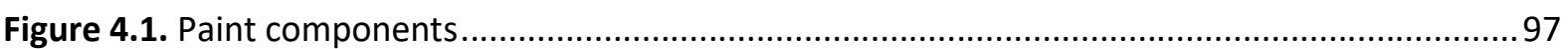

Figure 4.2. Structures of macrocycles cyclen and cyclam ............................................................99

Figure 4.3. The organic and inorganic components of silane coupling agent GLYMO .......................102

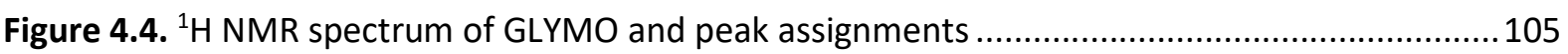

Figure 4.5. ${ }^{1} \mathrm{H}$ NMR spectra of the filtrates from Procedures 1-4 compared to GLYMO.................... 106

Figure 4.6. IR spectra of silica, GLYMO starting materials, and purported $\mathrm{GmSiO}_{2}$ products from

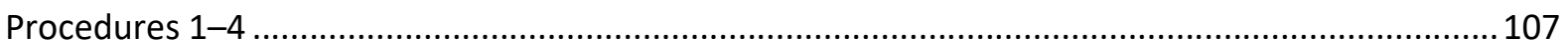

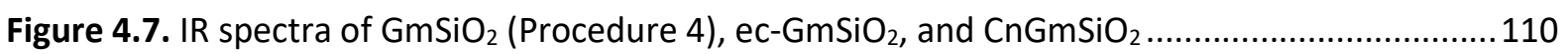

Figure 4.8. Potential structure of self-polymerised GLYMO ..........................................................114

Figure 4.9. Proposed mechanism of nucleophilic attack on the less hindered carbon of the metal-

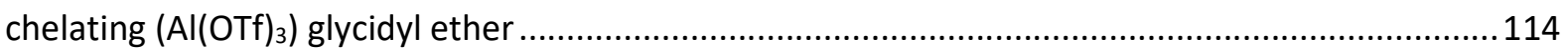

Figure 4.10. ${ }^{1} \mathrm{H}$ NMR spectra of cyclen, GLYMO, and samples at 2 and $69 \mathrm{~h}$ from the $\mathrm{Cn}(\mathrm{Gm})_{\mathrm{n}}$ Procedure 5 reaction mixture 115 
Figure 4.11. ${ }^{1} \mathrm{H}$ NMR spectra of GLYMO and the samples at 2 and $69 \mathrm{~h}$ from the $\mathrm{Cn}(\mathrm{Gm})_{\mathrm{n}}$ Procedure 5 reaction mixture

Figure 4.12. ${ }^{1} \mathrm{H}$ NMR spectra of GLYMO and the samples at $3,5,6$, and 10 days from the $\mathrm{Cn}(\mathrm{Gm})_{\mathrm{n}}$ Procedure 6 reaction mixture

Figure 4.13. 2D HSQC NMR spectrum of $\mathrm{Cn}(\mathrm{Gm})_{n}$ from Procedure 6 with peak assignments. 118

Figure 4.14. $2 \mathrm{D}\left({ }^{1} \mathrm{H},{ }^{13} \mathrm{C}\right)$ - $\mathrm{HMBC}$ NMR spectrum of $\mathrm{Cn}(\mathrm{Gm})_{\mathrm{n}}$ from Procedure 6 with peak assignments

Figure 4.15. $\pm \mathrm{Cu}(\mathrm{II})-\mathrm{CnGmSiO}$ products from Batches 1-4

Figure 4.16. ${ }^{1} \mathrm{H}$ NMR spectra of GLYMO, following methoxy group hydrolysis over time 124

Figure 4.17. GLYMO hydrolysis over time under the sets of conditions reported in Table 4.8 125

Figure 4.18. SEM secondary electron images of the $\mathrm{SiO}_{2} /$ epx-type squares 133

Figure 4.19. Example EDS spectrum (counts vs keV) 135

Figure 4.20. Interactions between the sample and the incident electron ( $\left.\mathrm{e}^{-}\right)$beam . 136

Figure 4.21. SEM backscatter electron images of the marine paint. 137

Figure 5.1. Factors that affect bacterial surface attachment 140

Figure 5.2. Enumeration of E. coli NZRM 3647 cells via the drop plate method. 142

Figure 5.3. Steps of the bacterial adherence assay and areas to be addressed for method development 143

Figure 5.4. Testing the effect of duration of incubation on bacterial adherence ( $24 \mathrm{~h}$ vs $48 \mathrm{~h}$ )........144

Figure 5.5. Testing the effect of coating age on bacterial adherence .............................................146

Figure 5.6. Testing the effect of Tween 20 in the bacterial adherence assay ................................147

Figure 5.7. Enumeration of bacterial counts in the saline washes.................................................. 148

Figure 5.8. Testing the combined effect of vortexing and sonication on planktonic bacteria...........149

Figure 5.9. Testing the efficiency of vortexing and sonication with Tween 20 in the removal of adherent bacteria from uncoated squares 150

Figure 5.10. Testing the efficiency of vortexing and sonication with or without Tween 20 in the removal of adherent bacteria from uncoated squares. 151

Figure 5.11. Testing the efficiency of vortexing and sonication with or without Tween 20 in the removal of adherent bacteria from $\mathrm{SiO}_{2} /$ epx-coated squares 152

Figure 5.12. Results of E. coli NZRM $3647 \mathrm{CFU} / \mathrm{cm}^{2}$ on the $\mathrm{SiO}_{2} /$ epx-type coatings .........................155

Figure 5.13. Optimisation of the bacterial adherence assay for $V$. harveyi NZRM 2698 ..................165

Figure 5.14. Gram-staining of a culture of $V$. harveyi NZRM 2698 in Luria marine broth.................. 166

Figure 5.15. Optimisation of the bacterial adherence assay for $V$. harveyi NZRM 2698 (cont.) ........167

Figure 5.16. Results of $V$. harveyi NZRM $2698 \mathrm{CFU} / \mathrm{cm}^{2}$ on the $\mathrm{SiO}_{2} /$ epx-type coatings 169 


\section{LIST OF TABLES}

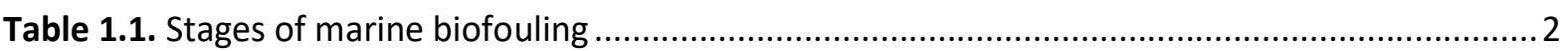

Table 1.2. Antifouling products used prior to the mid-19 ${ }^{\text {th }}$ century ................................................ 7

Table 1.3. Antifouling paints used on steel hulls after the mid-19 ${ }^{\text {th }}$ century ....................................... 8

Table 1.4. Examples of hard, soft, and intermediate ligands and metal ions ....................................23

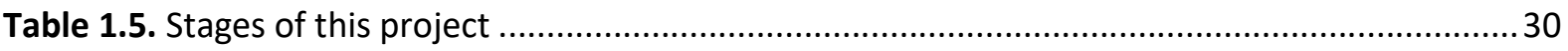

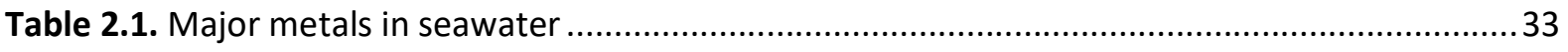

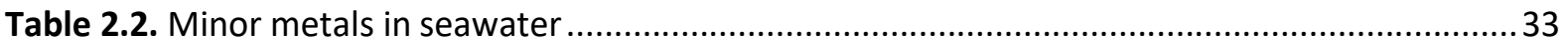

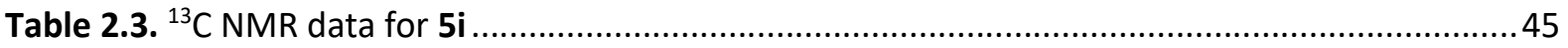

Table 2.4. Stability constants of metal complexes of the macrocyclic compounds ............................53

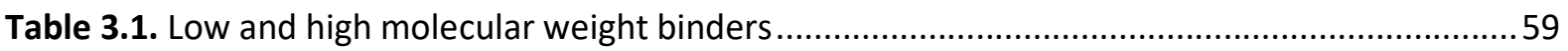

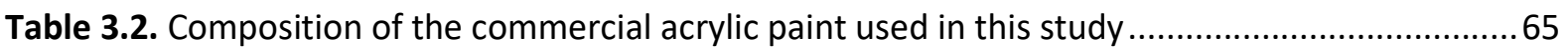

Table 3.3. Amounts of copper leached from the $\mathrm{Cu}(\mathrm{II})$-loaded commercial acrylic and $35 \% \mathrm{w} / \mathrm{w}$

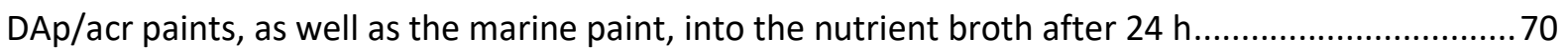

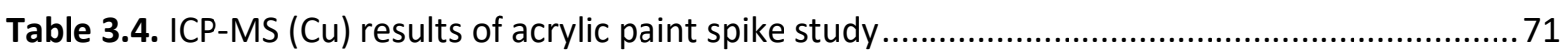

Table 3.5. Amounts of copper retained by different batches of the commercial acrylic and $35 \% \mathrm{w} / \mathrm{w}$

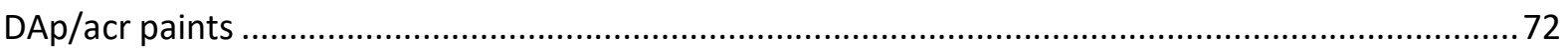

Table 3.6. Descriptions of epoxy resin, hardeners, and surface ligands used in this study .................78

Table 3.7. Epoxy coatings prepared with epoxy resin Epikote ${ }^{\mathrm{TM}} 235$ and the following hardeners:

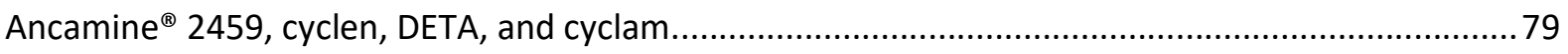

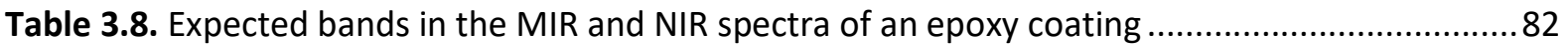

Table 3.9. Properties of epoxy coatings prepared with epoxy resin Epikote ${ }^{\mathrm{TM}} 235$ and hardener

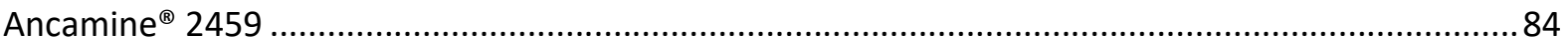

Table 3.10. Epoxy coatings prepared with Epikote ${ }^{\mathrm{TM}} 235$ and $\mathrm{Cu}(\mathrm{II})$ ligands as hardeners ..................86

Table 3.11. Epoxy coatings prepared with Epikote $^{\mathrm{TM}} 235$ and Ancamine ${ }^{\circledR} 2459$ and surface-

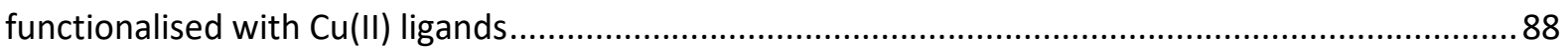

Table 3.12. Epoxy coatings prepared with Epikote ${ }^{\mathrm{TM}} 235$ and to be measured for $\mathrm{Cu}(\mathrm{II})$-loading .......90

Table 3.13. Amount of copper retained by the Ancamine ${ }^{\circledR} 2459 /$ Epikote $^{\mathrm{TM}} 235$ coating prepared in stoichiometric proportions

Table 3.14. Amounts of copper retained by coatings prepared from Epikote ${ }^{\mathrm{TM}} 235$ and cyclen in stoichiometric proportions or under/overcured with cyclen.

Table 3.15. Amounts of copper retained by coatings determined to be high $\mathrm{Cu}(\mathrm{II})$-loading based on colour intensity 
Table 4.1. Reaction conditions and outcomes in Procedures 1-4 for $\mathrm{GmSiO}_{2}$ synthesis 104

Table 4.2. Data and peak assignments from the IR spectra of silica and GLYMO starting materials and purported $\mathrm{GmSiO}_{2}$ products from Procedures 1-4 108

Table 4.3. Data and peak assignments from the IR spectra of $\mathrm{GmSiO}_{2}$ (Procedure 4), ec- $-\mathrm{GSiO}_{2}$, and $\mathrm{CnGmSiO}_{2}$

Table 4.4. Reaction conditions and outcomes in Procedures 1-6 for $\mathrm{Cn}(\mathrm{Gm})_{\mathrm{n}}$ synthesis 113

Table 4.5. Batches of $\pm \mathrm{Cu}(I I)-\mathrm{CnGmSiO}_{2}$ prepared 121

Table 4.6. $\mathrm{CHN}$ elemental analysis and percent functionalisation results for $\mathrm{Batch} 1 \mathrm{Cu}(\mathrm{II})-\mathrm{CnGmSiO}$, as well as the percent error between the expected and measured $\% \mathrm{~N}$

Table 4.7. ICP-MS (Cu) results for Batch $1 \mathrm{Cu}(\mathrm{II})-\mathrm{CnGmSiO}_{2}$

Table 4.8. Conditions for GLYMO hydrolysis experiments

Table 4.9. CHN elemental analysis and percent functionalisation/adsorption results for silica, Batches 1-4 $\pm \mathrm{Cu}(\mathrm{II})-\mathrm{CnGmSiO}_{2}$, and adsorbed $\mathrm{Cu}(\mathrm{II})$-cyclen 127

Table 4.10. Percent error between the expected and measured $\% \mathrm{~N}$ from $\mathrm{CHN}$ results 127

Table 4.11. ICP-MS (Cu) results for silica $\pm \mathrm{Cu}(\mathrm{II})$, Batches $1-4 \pm \mathrm{Cu}(\mathrm{II})-\mathrm{CnGmSiO}_{2}$, and adsorbed $\mathrm{Cu}(\mathrm{II})$-cyclen 128

Table 4.12. Loading of the macrocycle and copper on silica. 130

Table 4.13. EDS results for Batch $2 \mathrm{Cu}(\mathrm{II})-\mathrm{CnGmSiO}_{2} / \mathrm{epx}$, Batch $4 \mathrm{CnGmSiO}_{2} / \mathrm{epx}$, Batch 4 $\mathrm{CnGmSiO}_{2}+\mathrm{Cu}(\mathrm{II}) / \mathrm{epx}$, and $\mathrm{SiO}_{2} / \mathrm{epx}$

Table 4.14. EDS results for the marine paint at the sampled areas in Figure 4.21

Table 5.1. The $\mathrm{OD}_{600}$ of the $E$. coli cultures after $24 \mathrm{~h}$ incubation and the copper content of the test squares, as well as the copper-leachate concentrations after $24 \mathrm{~h}$ 156

Table 5.2. Literature data of $\mathrm{Cu}(\mathrm{II})$ toxicity in cultures of $E$. coli 158

Table 5.3. $\mathrm{OD}_{600}$ of cultures of $E$. coli NZRM 3647 in M63 minimal medium after incubation for $24 \mathrm{~h}$ at $37^{\circ} \mathrm{C}, \pm$ uncoated test squares and \pm shaking 159

Table 5.4. Literature data from copper ion-containing coatings compared to this study 161

Table 5.5. The $\mathrm{OD}_{600}$ of the $V$. harveyi cultures after $24 \mathrm{~h}$ incubation and the copper content of the test squares. 170 


\section{LIST OF SCHEMES}

Scheme 1.1. Hydrolysis of TBT from the acrylic polymer (methyl methacrylate) in a TBT self-polishing

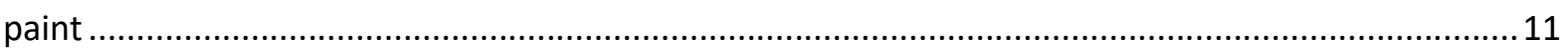

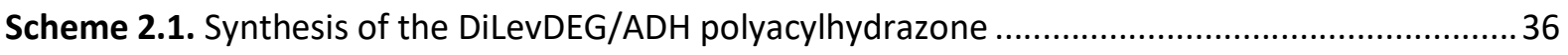

Scheme 2.2. General scheme for the synthesis of acylhydrazones................................................. 41

Scheme 3.1. Reaction of a diisocyanate with a polyol to produce a polyurethane ............................61

Scheme 3.2. Reaction of bisphenol A with epichlorohydrin to produce an epoxy resin .....................61

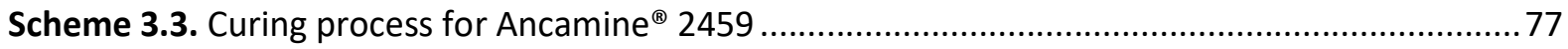

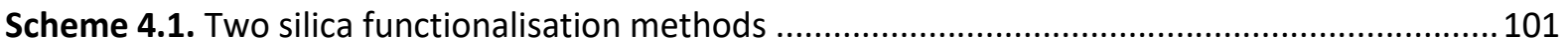

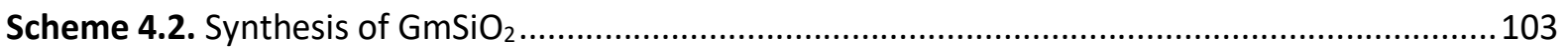

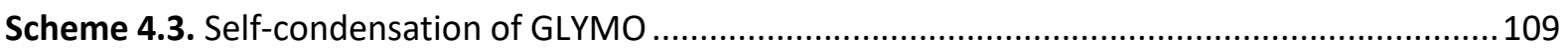

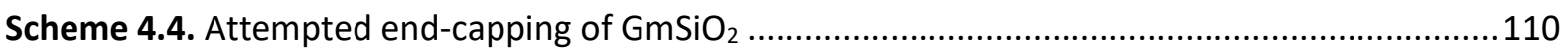

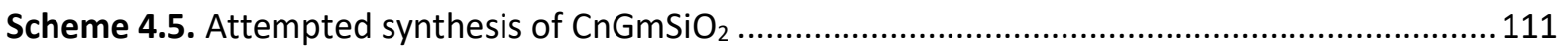

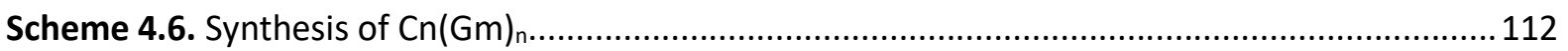

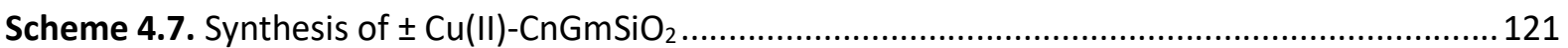

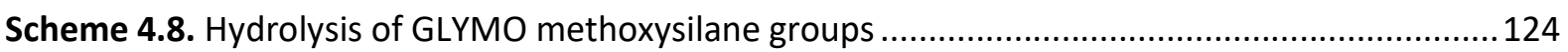




\section{LiST OF ABBREVIATIONS}

\begin{tabular}{|c|c|}
\hline Ac & acetyl group \\
\hline $\mathrm{AcOH}$ & acetic acid \\
\hline $\mathrm{ADH}$ & adipic acid dihydrazide \\
\hline AHEW & amine hydrogen equivalent weight \\
\hline $\mathrm{AHL}$ & $\mathrm{N}$-acyl-homoserine lactone \\
\hline $\mathrm{Al}(\mathrm{OTf})_{3}$ & aluminium triflate (trifluoromethanesulfonate) \\
\hline ANOVA & analysis of variance \\
\hline $\mathrm{aq}$ & aqueous \\
\hline ATR & attenuated total reflectance \\
\hline $\mathrm{AU}$ & absorbance units \\
\hline BET & Brunauer, Emmett, and Teller \\
\hline BPA & bisphenol A \\
\hline BPF & bisphenol F \\
\hline $\mathrm{br}$ & broad (IR, NMR), biological replicate (statistics) \\
\hline BSED & backscattered electron detector \\
\hline $\mathrm{CDCl}_{3}$ & chloroform-d \\
\hline $\mathrm{CD}_{3} \mathrm{OD}$ & methanol- $d_{4}$ \\
\hline CFSE & crystal field stabilisation energy \\
\hline CFT & crystal field theory \\
\hline CFU & colony forming unit \\
\hline $\mathrm{CHX}$ & chlorhexidine \\
\hline $\mathrm{Cn}(\mathrm{Gm})_{\mathrm{n}}$ & cyclen-(GLYMO) $)_{n}$ \\
\hline $\mathrm{CnGmSiO}_{2}$ & cyclen-GLYMO-silica \\
\hline COSY & correlation spectroscopy \\
\hline CP-MAS & cross-polarisation magic angle spinning \\
\hline $\mathrm{Cu}(\mathrm{II})-\mathrm{CnGm}$ & copper(II)-cyclen-GLYMO \\
\hline $\mathrm{Cu}(\mathrm{II})-\mathrm{CnGmSiO}{ }_{2}$ & copper(II)-cyclen-GLYMO-silica \\
\hline DAp/acr & DiLevDEG/ADH polyacylhydrazone/acrylic \\
\hline $\mathrm{DCM}$ & dichloromethane \\
\hline$d d$ & doublet of doublets \\
\hline $\mathrm{ddH}_{2} \mathrm{O}$ & double-distilled water \\
\hline DEG & diethylene glycol \\
\hline
\end{tabular}




\begin{tabular}{|c|c|}
\hline DETA & diethylenetriamine \\
\hline DGEBA & bisphenol A diglycidyl ether \\
\hline DiLevDEG & dilevulinoyl diethylene glycol \\
\hline DLVO & Derjaguin-Landau-Verwey-Overbeek \\
\hline DMF & dimethylformamide \\
\hline DMSO & dimethylsulfoxide \\
\hline DTPA & diethylene triamine pentaacetic acid \\
\hline $\mathrm{e}^{-}$ & electron \\
\hline ec-GmSiO 2 & end-capped GLYMO-silica \\
\hline EDTA & ethylene diamine tetraacetic acid \\
\hline en & ethylenediamine \\
\hline EPA & Environmental Protection Agency \\
\hline EPS & extracellular polymeric substances \\
\hline eq & equivalents \\
\hline ESI & electrospray ionisation \\
\hline Et & ethyl \\
\hline EtAc & ethyl acetate \\
\hline EtOH & ethanol \\
\hline FT-IR & Fourier-transform infrared spectroscopy \\
\hline GA & glutaraldehyde \\
\hline GLYMO & (3-glycidyloxypropyl)trimethoxysilane \\
\hline $\mathrm{GmSiO}_{2}$ & GLYMO-silica \\
\hline $\mathrm{h}$ & hour \\
\hline $\mathrm{HBH}$ & o-hydroxyacetophenone benzoylhydrazone \\
\hline $\mathrm{H}_{2} \mathrm{~L}^{1}$ & 2-thiophenecarbonyl hydrazone of 3-isatin \\
\hline HMBC & heteronuclear multi-bond correlation \\
\hline HRMS & high-resolution mass spectrometry \\
\hline HSAB & Hard Soft Acid Base \\
\hline $\mathrm{HSQC}$ & heteronuclear single quantum correlation \\
\hline HV & accelerating voltage \\
\hline I & ionic strength \\
\hline ICP-MS & inductively coupled plasma mass spectrometry \\
\hline ICP-OES & inductively coupled plasma optical emission spectroscopy \\
\hline IR & infrared \\
\hline
\end{tabular}


International Organisation for Standardisation

JIS

Japanese Industry Standard

$k$

constant

$K_{\mathrm{d}}$

dissociation constant

$\mathrm{kDa}$

kilodalton

$K_{\mathrm{f}}$

formation constant

kph

kilometres per hour

L

ligand

LA

laser ablation

LC-MS

liquid chromatography mass spectrometry

LFD

low vacuum secondary electron detector

LMB

Luria marine broth

LOD

limit of detection

$\log _{10}$

logarithm (base 10)

LPS

lipopolysaccharide

M

metal

m

medium (IR), multiplet (NMR)

Me

methyl

$\mathrm{MeOH}$

methanol

$\min$

minute

MIR

mid infrared

MPG

monopropylene glycol

MRI

magnetic resonance imaging

MS

mass spectrometry

MSN

mesoporous silica nanoparticle

$m / z$

NIR

mass-to-charge

near infrared

NMR

nuclear magnetic resonance

NO

nitric oxide

ns

not significant

NZRM

New Zealand Reference Culture Collection: Medical Section

$\mathrm{OD}_{600}$

optical density (measured at $600 \mathrm{~nm}$ )

$\mathrm{p}$

pentet

PDA

polydopamine 


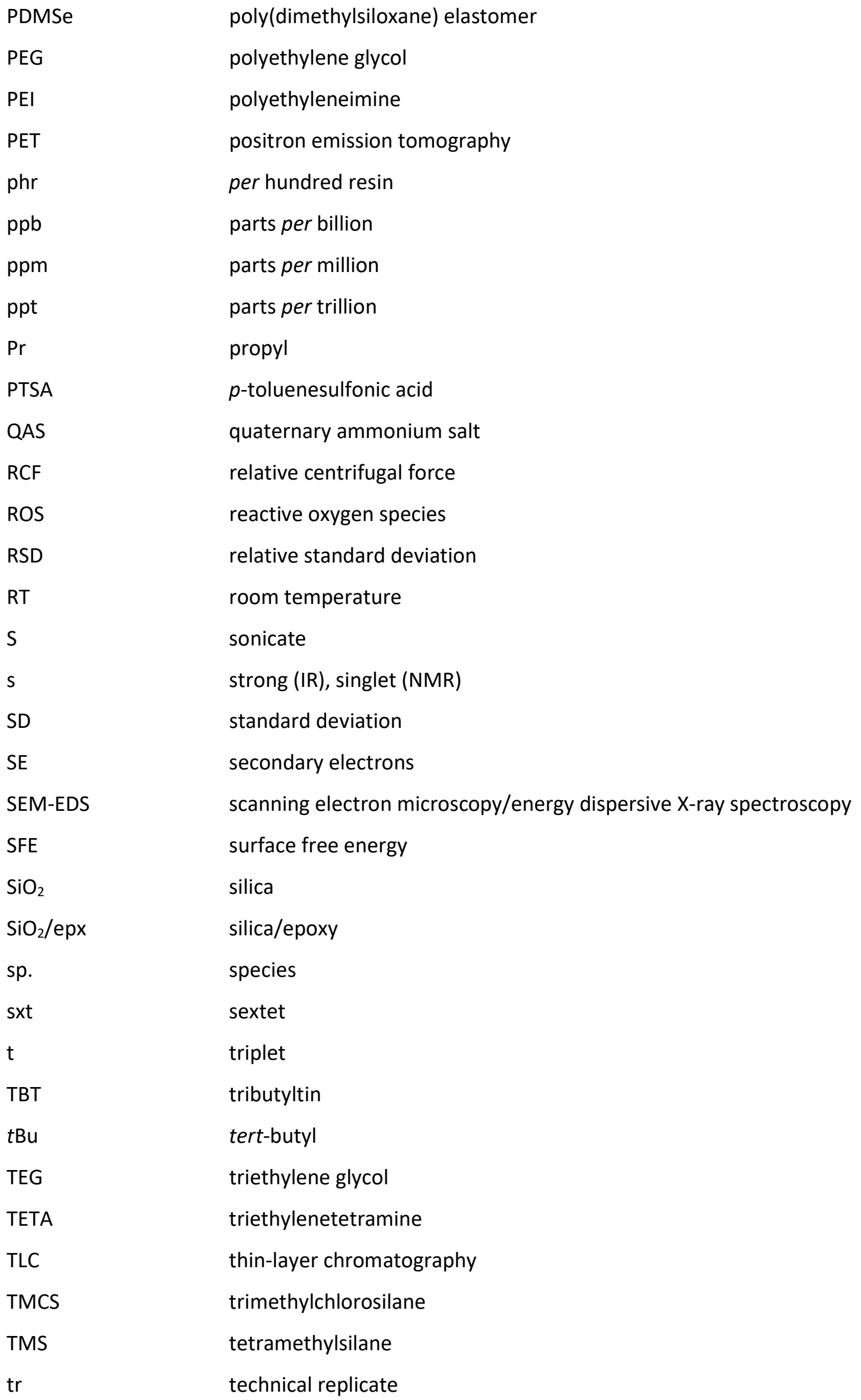


T20

UV-Vis

V

$\mathrm{vbr}$

vs

vw

W

WD

WPE

$\mathrm{XRC}$

XRF
Tween 20

ultraviolet-visible

vortex

very broad

very strong

very weak

weak

working distance

weight per epoxide

X-ray crystallography

X-ray fluorescence 


\section{INTRODUCTION}

\subsection{BIOFOULING}

\subsubsection{Stages of Biofouling}

Following submersion in seawater in the marine environment, an object, artificial or natural, will accumulate biological material on its surface through a process known as biofouling, beginning with the formation of a conditioning layer of adsorbed proteins, polypeptides, and polysaccharides within one minute of immersion (Figure 1.1, Table 1.1). ${ }^{1-4}$ This molecular conditioning film, the first stage of biofouling, is the result of physical forces, such as Brownian motion, electrostatic interactions, and Van der Waals forces. ${ }^{3}$ The succeeding biofouling stages involve colonisation by fouling organisms, which are divided into three groups: microorganisms (e.g. fungi, diatoms, marine bacteria, cyanobacteria, protozoans, microalgae), soft-fouling organisms (e.g. bryozoans, sponges, brown and multicellular algae, soft corals, anemones, tunicates, hydroids), and hard-fouling organisms (e.g. polychaete worms, mussels, barnacles).$^{2-4}$ In common parlance, the resultant fouling from the organisms in these three categories is referred to as slime, weed, and shell, respectively. 5,6

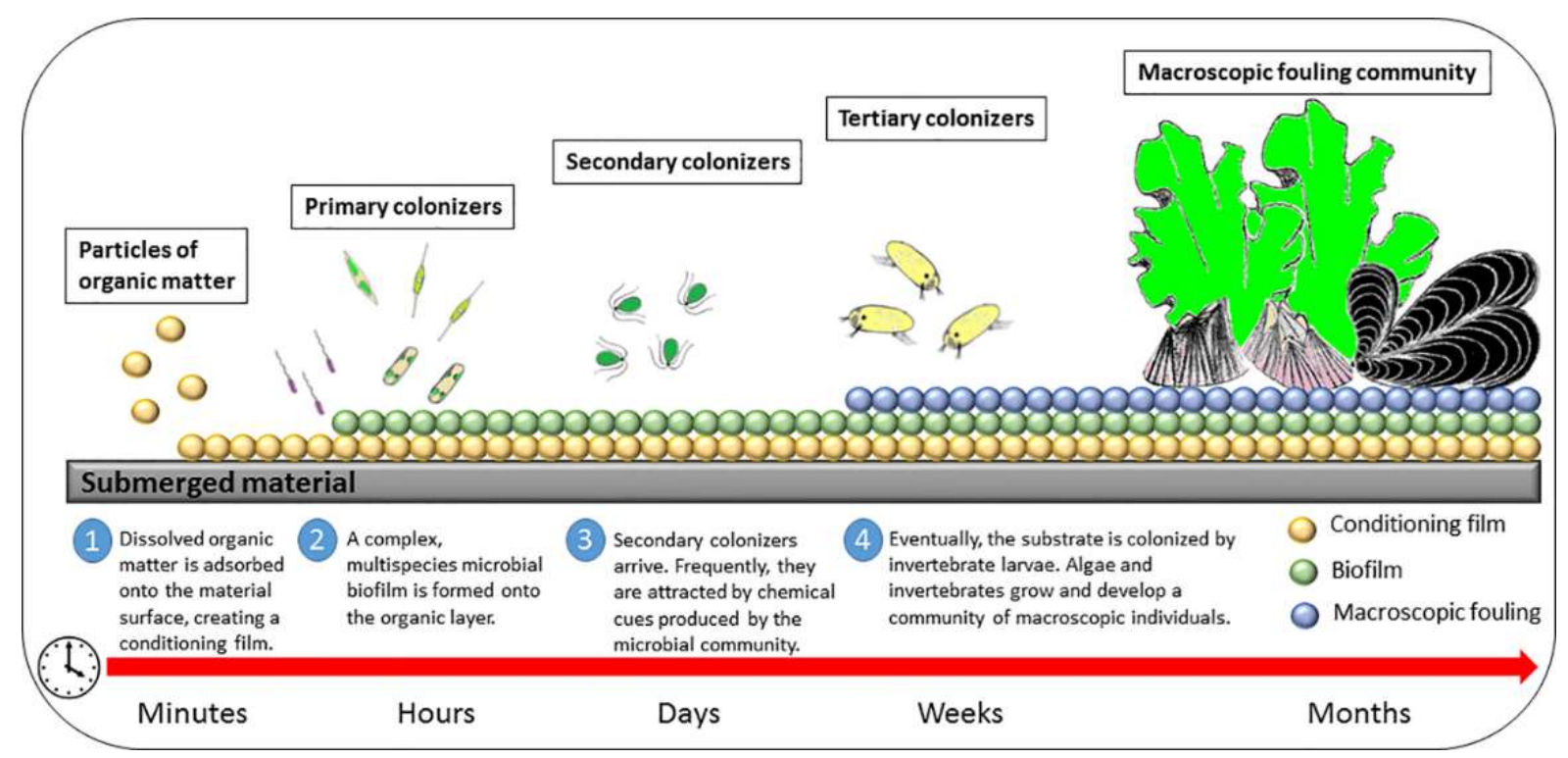

Figure 1.1. Stages of marine biofouling. ${ }^{7}$

"Schematic representation of the biofouling process" (https://journals.plos.org/plosone/article?id=10.1371/journal.pone.0123652) from Martín-Rodríguez et al. 7 , licensed under CC BY 4.0 (https://creativecommons.org/licenses/by/4.0/). 
Table 1.1. Stages of marine biofouling (adapted from Almeida et al. ${ }^{3}$ ).

Processes Involved

STAGE 1: Physical forces

(e.g. electrostatic interactions, Brownian motion, Van der Waals forces)

STAGE 2: Reversible adsorption of organisms by, usually, physical forces, and their subsequent adhesion interacting together with protozoans and rotifers Attached Organisms

Adhesion of organic molecules

(e.g. proteins, polysaccharides,

polypeptides, proteoglycans, some

Conditioning

$1 \min$

inorganic molecules)

\section{Bacteria}

(e.g. Pseudomonas putrefaciens, Vibrio

alginofyticos);

Fungi;

Diatoms - unicellular algae

Microbial biofilm

(e.g. Achnantes brevipes, Amphora

coffeaeformis, Amphiprora paludosa

Nifzschia pusilla, Licmophora abbreviata)

\section{STAGE 3: Arrangement of}

microorganisms with greater protection

from predators, toxicants, and

environmental alterations, making it

easier to obtain the nutrients necessary

for the attachment of other

microorganisms

Spores of microalgae

(e.g. Ulothrix zonata, Enteromorpha

intestinalis):

Protozoans

Biofilm

1 week

(e.g. Vaginicola sp., Zoolhamnium sp., Vorticella sp.)
STAGE 4: Increase in the capture of more particles and organisms (e.g. larvae of marine macroorganisms) as a

consequence of the pre-existence of the biofilm and the roughness created by the irregular microbial colonies that comprise it

\section{Larvae of macroorganisms}

(e.g. Balanus amphitrite (Crustacea), Laomedia flexuosa (Coelenterata), Electra crustulenta (Bryozoa), Spirorbis borealis (Polychaeta), Mytilus edulis (Mollusca), Styela coriacea (Tunicata))
Film consisting of the

attachment and

development of marine 2-3 weeks

invertebrates and growth

of macroalgae (seaweed) 
In the second stage of biofouling, the conditioning layer promotes adhesion by serving as a source of nutrients and site of interaction with organic molecules, and microorganisms, such as bacteria, fungi, and diatoms (i.e. unicellular algae), colonise the conditioned surface within hours of submersion. ${ }^{3,8}$ Among these primary colonisers, bacteria are dominant due to their high concentration in seawater $\left(10^{5}-10^{6}\right.$ cells $\left./ \mathrm{mL}\right){ }^{9}$ Microorganisms are transported to the surface by fluid dynamics, diffusion, gravity, Brownian motion, cell mobility, and electrostatic interactions. Their initial adhesion to the surface is reversible, resulting from weak interactions (e.g. Van der Waals forces, hydrophobic and electrostatic interactions). But, following the production of extracellular polymeric substances (EPS), adhesion becomes irreversible, and a microbial biofilm develops. ${ }^{8}$ This biofilm, sometimes called microfouling, is an aggregation of attached cells embedded in the matrix of self-produced EPS, which are highly hydrated biopolymers (e.g. polysaccharides, lipids, nucleic acids, proteins). ${ }^{4,10,11}$ The matrix keeps the cells in close proximity, allowing for synergistic interactions. Microbial life in a biofilm has numerous advantages compared to planktonic cells living individually: biofilm cells form microconsortia, experience enhanced nutrient acquisition from the environment, retain extracellular enzymes, communicate and cooperate intercellularly, have a large gene pool available for genetic exchanges, recycle nutrients, and display enhanced tolerance to antibiotics and biocides. ${ }^{10,11}$ As antimicrobial substances diffuse through the biofilm, they can bind to or be degraded by EPS in the matrix, rendering them inactive. ${ }^{11}$ These advantages allow microbial communities to thrive in the challenging marine environment in which conditions are changeable and sometimes detrimental and nutrients are often growth-limiting. ${ }^{12}$

The microbial biofilm may promote or deter successive stages of biofouling. Further colonisation is determined by the surface chemistry, micro-topography, and presence of microbial products. ${ }^{9,13}$ In the case of promotion, the biofilm provides food and may contain chemical attractants, encouraging the settlement of secondary colonisers: protozoans (i.e. unicellular eukaryotes) and microalgal spores. These microorganisms, characteristic of the third biofouling stage, attach with polysaccharide and protein glues and add to the biofilm within one week of immersion of the substrate in seawater. This leads to the increased capture of more organisms. The fourth and final stage of biofouling occurs within a few weeks of submersion and involves colonisation by the larvae of soft- and hard-fouling macroorganisms (tertiary colonisers). ${ }^{3,7,8}$ These larvae become fixed to the surface and grow, developing into a community of macroalgae and marine invertebrates. ${ }^{4}$ Previous reports suggest that the inhibition of biofilm development may result in a surface unsuitable for the attachment of macrofouler larvae, and, therefore, disruption of biofilm formation may reduce biofouling. ${ }^{14}$ However, the biofouling process is dynamic rather than successive, and a causal relationship between fouling stages should not necessarily be assumed. For instance, blocking biofilm formation will not prevent all 
macrofouling; experiments have shown positive, negative, and neutral effects of bacterial biofilms on algal spore and invertebrate larvae attachment. ${ }^{15}$ As over 4000 species of marine fouling organisms exist, the final biofouling community is highly complex and diverse. ${ }^{3}$

The composition of the biofouling community is dependent upon parameters related to the submerged object, as well as the seawater, geographical location, season, competition, and predation. ${ }^{3,15,16}$ For example, the biofouling of ship hulls is influenced by the hull geometry, depth of submersion, cruising speed at sea, length of time in port, and the intensity of sunlight at the hull's surface. It is well-known that the majority of organisms responsible for the fouling of ship hulls cannot adhere to ships traveling above 4-5 knots (7-9 kph). Therefore, slow, idle ships that spend a long time in port are more vulnerable to biofouling than high-speed ships that are rarely in port. The temperature, dissolved salts, density, $\mathrm{pH}$, oxygen concentration, and salinity of seawater also affect biofouling. ${ }^{3,16}$ In particular, the temperature, salinity, and density vary with latitude, providing an explanation for the diversity of biofouling in different geographical locations. For instance, the fouling species in tropical and equatorial regions differ from those in cold and temperate regions. Although, certain species have adapted to a wide range of environmental conditions and are ubiquitous. ${ }^{3}$ Biofouling communities are difficult to remove once established, and biofouling remains a worldwide problem for shipping industries. ${ }^{17}$

\subsubsection{Negative Consequences of Biofouling}

The fouling of ship hulls (Figure 1.2) results in a reduction of speed and manoeuvrability, as well as increased fuel consumption and accelerated corrosion. ${ }^{17}$ As a ship moves through the water, the drag resulting from water moving over the hull (i.e. skin friction) opposes the forward motion of the ship. To maximise performance, the drag must be minimised, meaning that biofouling on the wetted hull area must be limited. ${ }^{16} \mathrm{~A}$ study by Schultz ${ }^{18}$ calculated power penalties caused by different fouling types and coverage on the hull of a mid-sized naval surface combatant operating at cruising or near maximum speed, and it was determined that even a light slime layer on the ship's hull would require an approximately $11 \%$ increase in shaft power relative to a clean hull. Heavy calcareous fouling was predicted to necessitate an $86 \%$ increase in shaft power. A separate study by Schultz et $a l .{ }^{19}$ analysed the overall cost of hull fouling of a mid-sized naval surface ship (Arleigh Burke-class destroyer DDG-51). Expenditures included hull cleaning, fuel, hull coatings, and hull coating application and removal. These expenses totalled an estimated US\$56 million per year for the entire DDG-51 class (56 ships as of 2011), the primary cost being increased fuel consumption due to frictional drag. Fuel expenses for a fouled ship can be $30-40 \%$ greater than for an unfouled ship, and, in total, increased fuel 
consumption due to biofouling is estimated to cost the US Navy greater than US\$1 billion per year. ${ }^{20}$ A study for International Paint by Tobias Boren ${ }^{21}$ reported that an unprotected very large crude carrier with $40,000 \mathrm{~m}^{2}$ underwater area can, in less than six months, gather $150 \mathrm{~kg}$ of fouling per $\mathrm{m}^{2}$. By using an antifouling coating on the vessel, the study reported fuel savings of 39,429 tonnes over 15 years and, consequently, cost savings of US\$28.5 million. ${ }^{22}$ The performance and economic penalties from biofouling are staggering, but they are not the only negative consequences of fouling on ship hulls.

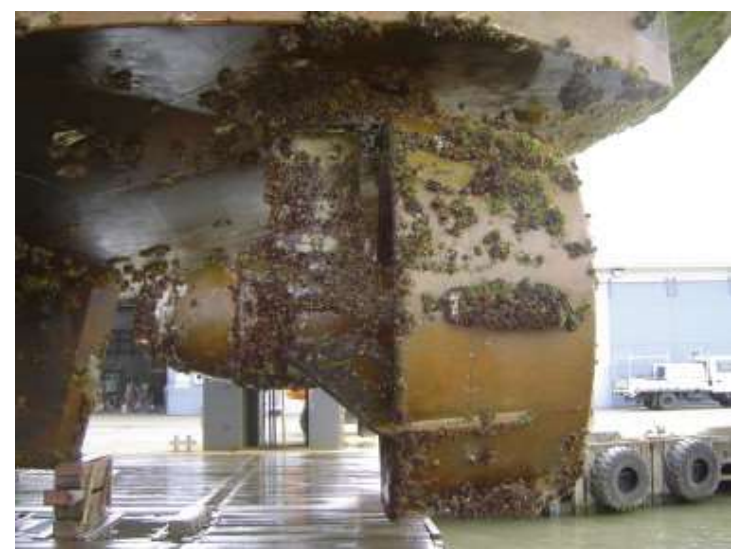

Figure 1.2. Biofouling on ship hulls. ${ }^{23}$

Reprinted from Marine Pollution Bulletin, 62(3), Dafforn, K. A., Lewis, J. A. \& Johnston E. L., “Antifouling strategies: History and regulation, ecological impacts and mitigation", 453-465, Copyright (C) 2011, with permission from Elsevier.

Another negative consequence of fouled ship hulls is that they have been directly responsible for the introduction of invasive species and, thus, are a threat to biosecurity. ${ }^{5}$ For example, ship traffic is the main route by which non-indigenous marine species are introduced to New Zealand, and hull fouling is a significant mechanism for these introductions. Merchant vessels constitute $75 \%$ of international ship traffic to New Zealand but typically have little biofouling. In contrast, slower-moving commercial vessels, such as tugs and barges, constitute less than $1 \%$ of New Zealand's international ship traffic but can be heavily fouled and, consequently, a biosecurity risk. ${ }^{24,25}$ For instance, an invasive colonial ascidian, Didemnum vexillum (i.e. carpet sea squirt), was carried on the hull of a heavily fouled barge and introduced to Shakespeare Bay, New Zealand, which is an international shipping port adjacent to an important mussel aquaculture region. ${ }^{24,26}$ In a study by Hopkins and Forrest ${ }^{24}$, the hull fouling on seven slow-moving commercial vessels (five barges, two tugs) in New Zealand ports was sampled over one year, and, out of the 29 identified taxa, $24 \%$ were indigenous to New Zealand, $17 \%$ were non-indigenous, and 59\% were classified as unknown. Such biosecurity threats, as well as losses of efficiency and exorbitant expenses, attributed to biofouling have prompted the continuous development of antifouling technology for ship hulls. 


\subsection{Antifouling Coatings}

\subsubsection{History of Antifouling Technology}

Written records of the methods employed to mitigate the adverse effects of hull fouling date back as early as the $7^{\text {th }}$ century B.C., and antifouling technology has been in development since that time (Table 1.2). ${ }^{3}$ The Greek philosopher Plutarch (45-125 A.D.) was the first to document the negative impact of biofouling on ship performance and that, by scraping away the slime, algae, and filth from a ship's sides, the ship could move more easily through the water. ${ }^{20,27}$ Ancient civilisations used wax, tar, and asphalt on wooden hulls to prevent biofouling. The Carthaginians and Phoenicians (700 B.C.) were said to have used pitch and, possibly, copper sheathing, and they are credited by some sources as being the first to use copper for antifouling purposes, although this is contentious. Similarly, the Greeks and Romans (200 B.C. -45 A.D.) used lead sheathing secured with copper nails. Metallic sheathing remained a popular antifouling technique, and a British patent granted to William Beale in 1625 is the first official acknowledgement of copper's efficacy as an antifoulant. The first authenticated use of copper sheathing is by the British 32-gun frigate HMS Alarm (Figure 1.3) in 1758, for which the sheathing provided protection against shipworms and fouling during its deployment to the West Indies. However, the corrosion of iron when in contact with copper was noted. Copper was widely used by the British Navy in the 1780s, but, following the introduction of iron ships in the late- $18^{\text {th }}$ century, copper sheathing was nearly abandoned due to its dangerously corrosive effects on iron. In the early- $19^{\text {th }}$ century while studying the corrosion process, Sir Humphrey Davy discovered that the mechanism of action by which copper prevents biofouling is dependent upon copper's dissolution in seawater. This further complicated the use of copper sheathing, since strategies proposed to prevent corrosion did not allow for the dissolution of copper. Thus, alternative antifouling methods were explored; specifically, antifouling paints were developed in the mid-19 ${ }^{\text {th }}$ century (Table 1.3). ${ }^{3,4,27,28}$

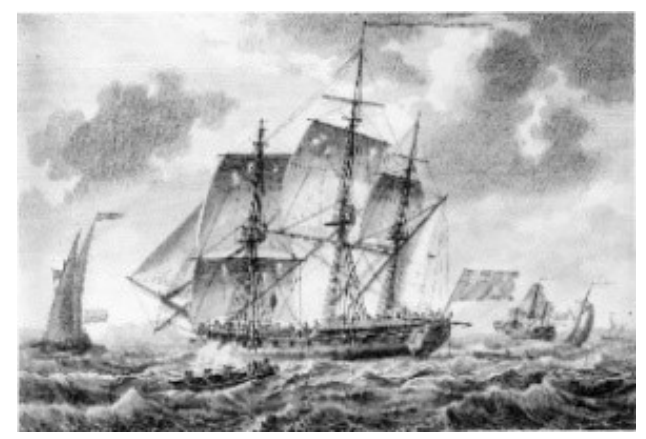

Figure 1.3. British 32-gun frigate HMS Alarm. Image: https://commons.wikimedia.org/wiki/File:HMS_Alarm_(1758).jpg. 
Table 1.2. Antifouling products used prior to the mid-19 $9^{\text {th }}$ century (adapted from Almeida et $a l .^{3}$ ).

\begin{tabular}{|c|c|c|}
\hline Approximate Time Period & Civilisation/Navigator & Antifouling Product \\
\hline Oldest & Oldest & Wax, tar, asphalt \\
\hline 700 B.C. & Phoenicians, Carthaginians & Pitch, possibly copper sheathing \\
\hline 700 B.C. & Phoenicians & Lead sheathing, tallow \\
\hline 500 B.C. & Phoenicians & Coatings of sulfur and arsenic mixed with oil \\
\hline 300 B.C. & Greeks & Wax, tar, lead sheathing \\
\hline 200 B.C. -45 A.D. & Romans, Greeks & Lead sheathing with copper nails \\
\hline 10 A.D. & Vikings & Seal tar \\
\hline 45-125 A.D. & Plutarch & Scraping slime, algae, and filth from the ship's sides \\
\hline \multirow{2}{*}{$13^{\text {th }}-15^{\text {th }}$ centuries } & Several & Pitch and mixtures with resin, oils, or tallow \\
\hline & Columbus & Pitch and tallow \\
\hline $1618-1625$ & Various & $\begin{array}{l}\text { Copper, possibly with a mixture of powdered iron, cement, and a copper compound (copper } \\
\text { sulfide or copper arsenic ore) }\end{array}$ \\
\hline \multirow[b]{2}{*}{$18^{\text {th }}$ century } & & Sacrificial wooden sheathing on a layer of animal hair and pitch \\
\hline & Various & $\begin{array}{l}\text { Wooden sheathing covered with mixtures of tar, fat, sulfur, and pitch, with numerous metallic } \\
\text { nails arranged with their heads forming a type of metallic sheathing }\end{array}$ \\
\hline 1758 & English (Frigate HMS Alarm) & Copper sheathing (abandoned for causing galvanic corrosion of iron nails) \\
\hline 1786 & English & Copper sheathing, using copper and zinc alloy nails \\
\hline Early-19 ${ }^{\text {th }}$ century & English & Sir Humphrey Davy demonstrated that copper dissolution in seawater prevented fouling \\
\hline \multirow[t]{2}{*}{ 1758-1816 } & \multirow[t]{2}{*}{ Various } & $\begin{array}{l}\text { Sheathing of lead, zinc, arsenic, nickel, and galvanised steel and alloys of tin, zinc, and } \\
\text { antimony followed by copper-plated wood sheathing }\end{array}$ \\
\hline & & Non-metallic sheathing (ebonite, rubber, enamel, cork, etc.) \\
\hline 1862 & Various & Wood sheathing covered with copper sheathing (abandoned due to cost) \\
\hline Mid-19 $9^{\text {th }}$ century & Various & $\begin{array}{l}\text { Paints containing a toxicant (copper, arsenic, or mercury oxides) dispersed in a polymeric } \\
\text { binder (shellac, linseed oil, colophony) }\end{array}$ \\
\hline
\end{tabular}


Table 1.3. Antifouling paints used on steel hulls after the mid-19 ${ }^{\text {th }}$ century (adapted from Almeida et al. ${ }^{3}$ ).

\begin{tabular}{|c|c|c|c|}
\hline \multirow[t]{2}{*}{ Product Type } & \multirow[t]{2}{*}{ First Used } & \multicolumn{2}{|c|}{ Main Components } \\
\hline & & Binder & Pigment/Biocide \\
\hline First paints & Mid-19 $19^{\text {th }}$ century & Linseed oil, shellac varnish, tar, resins & Copper, arsenic, or mercury oxides \\
\hline Application of insulating primer under antifouling paint & 1847 & $\begin{array}{l}\text { Linseed oil, shellac varnish, tar, and } \\
\text { resins; preliminary insulating varnish } \\
\text { coating }\end{array}$ & Copper, arsenic, or mercury oxides \\
\hline "Hot plastic paints" & 1860 & $\begin{array}{l}\text { Metallic soap composition, } \\
\text { colophony }\end{array}$ & $\begin{array}{l}\text { Copper sulfate, other copper } \\
\text { compounds }\end{array}$ \\
\hline Antifouling paint & 1863 & Tar & Copper oxide \\
\hline Rust preventer & Late- $19^{\text {th }}$ century & $\begin{array}{l}\text { Shellac primer and shellac antifouling } \\
\text { paint }\end{array}$ & Different toxicants \\
\hline Spirit varnish paints & $1908-1926$ & Grade A "Gum Shellac" & $\begin{array}{l}\text { Red mercury oxide or zinc oxide, } \\
\text { zinc dust and India red }\end{array}$ \\
\hline "Cold plastic paints" & 1926 & $\begin{array}{l}\text { Coal tar or coal tar + colophony, } \\
\text { shellac varnish, synthetic resins }\end{array}$ & Copper or mercury oxides \\
\hline First organo-metallic paints & $1950-1960$ & Acrylic esters or others & Copper compounds \pm co-biocides \\
\hline Soluble matrix & 1950 & Colophony and others & $\begin{array}{l}\text { Copper, arsenic, zinc, mercury, or } \\
\text { iron oxides }\end{array}$ \\
\hline Insoluble matrix or contact paint & 1955 & $\begin{array}{l}\text { Acrylic resins, vinyl resins, } \\
\text { chlorinated rubber polymers }\end{array}$ & $\begin{array}{l}\text { Copper and zinc oxides } \pm \text { organo- } \\
\text { metallic compounds }\end{array}$ \\
\hline Self-polishing paints containing tin & $1974-1985$ & $\begin{array}{l}\text { Acrylic polymer with TBT groups } \\
\text { bonded to main chain by ester } \\
\text { binders (copolymer) }\end{array}$ & $\begin{array}{l}\text { Zinc oxide and insoluble pigments } \\
\text { or copper oxide, tri-organo-tin, and } \\
\text { co-biocides }\end{array}$ \\
\hline
\end{tabular}


Antifouling paints on ship hulls minimise biofouling and, therefore, frictional drag leading to increased fuel costs. In general, a paint is composed of a varnish and pigment (Figure 1.4). The varnish includes additives, the film-former (i.e. binder), and the solvent/diluent. Additives are substances that are added to the paint to perform special jobs, such as additives functioning as antifoulants (i.e. toxicants, biocides). When the paint is in its liquid form, it may contain chemical ingredients of the binder that will polymerise during the process of curing. When the paint is dry and cured, the binder is the resultant polymer (e.g. acrylic, polyurethane, epoxy) or the resin (e.g. rosin or colophony, shellac) that binds together all of the other paint components. The mixture of the binder and liquid is referred to as the vehicle for the pigment. The primary, coloured pigment, which is made up of fine organic (e.g. phthalocyanin blue) or inorganic (e.g. titanium dioxide) particles, has protective and decorative functions, providing colour, opacity, and other visual effects. An inexpensive extender (i.e. filler) consisting of coarse inorganic particles is often used in conjunction with the primary pigment to achieve a desired effect at a lower cost (e.g. calcium carbonate as a matting agent with titanium dioxide). ${ }^{29,30}$ The first antifouling paints were based on the dispersal of a toxicant in a polymeric binder. $^{3}$

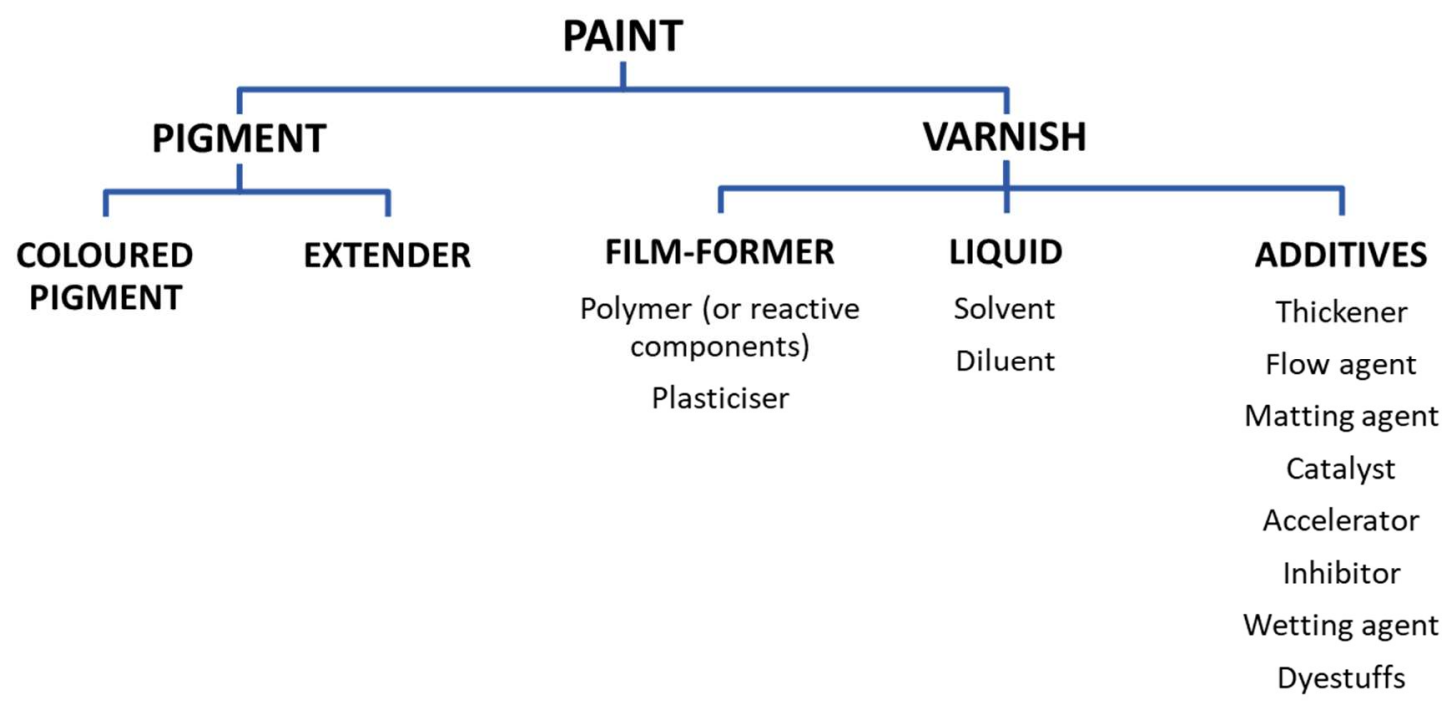

Figure 1.4. Paint components (adapted from Introduction to Paint Chemistry). ${ }^{29}$

Following renewed interest in antifouling coatings in the mid- $19^{\text {th }}$ century, the number of such products increased rapidly. Popular compositions utilised copper, arsenic, or mercury oxides as pigments or antifouling additives dispersed in the linseed oil, shellac varnish, tar, or other resins serving as coating binders (Table 1.3). However, since the toxicants were in direct contact with the 
ship hull, corrosion was still a problem for the first steel hulls, and, consequently, primers (i.e. undercoats) emerged to protect the hulls. The first organometallic paints containing tin, mercury, arsenic, and other metals appeared in the mid- $20^{\text {th }}$ century. $3,27,28$

\subsubsection{Biocide-Based Antifouling Coatings}

The new biocide-based antifouling coatings began to be classified according to their toxicant releasemechanism, and the three main classifications still in use are soluble matrix (i.e. ablative, erodible, controlled depletion), insoluble matrix (i.e. contact), and self-polishing paints (Table 1.3, Figure 1.5). Soluble matrix paints incorporate seawater-soluble binders based on rosins (i.e. colophony) and their derivatives and toxic pigments, such as copper, iron, or zinc oxides. Because of their solubility in seawater, they have poor mechanical strength and erode constantly, assuring protection against antifouling for only 12-15 months. Insoluble matrix paints contain binders that are insoluble in seawater (e.g. vinyl, acrylic, epoxy, or chlorinated rubber polymers) and contain high concentrations of antifoulants (e.g. copper and zinc oxides \pm organometallic compounds). As successive toxicant layers are dissolved or eroded by seawater penetrating the film, toxicants deeper in the coating have farther to diffuse, leading to a gradual decrease in the toxicant release rate and antifouling efficacy over time. Therefore, although these paints are mechanically strong, they have a short antifouling lifetime (12-24 months). ${ }^{3,28}$

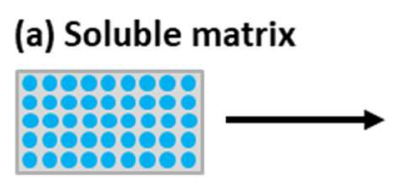

(b) Insoluble matrix
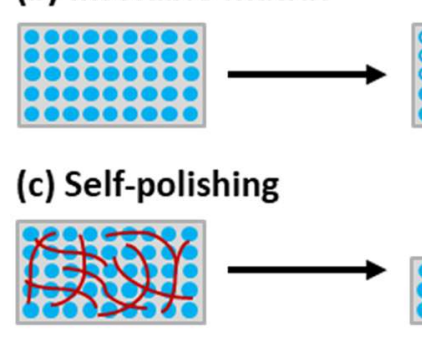

- Biocide oEmpty space 2Copolymer
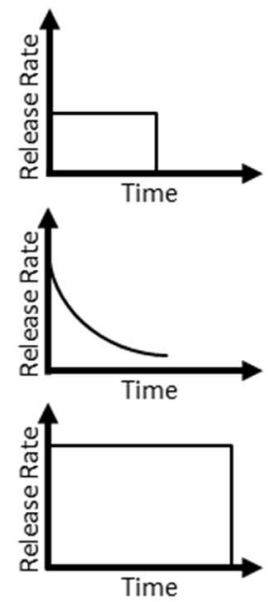

Figure 1.5. Classifications of biocide-based antifouling paints: (a) soluble matrix, (b) insoluble matrix, and (c) self-polishing paints (adapted from Yang et al. ${ }^{31}$ ). 
Self-polishing paints have a hydrophobic matrix composed of a biocide linked to a polymer, and hydrolysis in the coating results in release of the biocide. A widely used organometallic paint of this type, introduced in 1974, contained tributyltin (TBT) groups bonded to the acrylic polymer chain (derived from methyl methacrylate) by hydrolytically unstable ester bonds, resulting in the slow, controlled release of TBT in seawater over time (Scheme 1.1). With the dissolution of pigment particles and consequent release of TBT from increased exposure to seawater, the surface of the film would become brittle and erode to expose a fresh layer of the active coating (self-polishing effect). The TBT self-polishing paint had a much longer lifetime, being up to five years, and was hugely successful in preventing biofouling. ${ }^{2-4,23,28}$

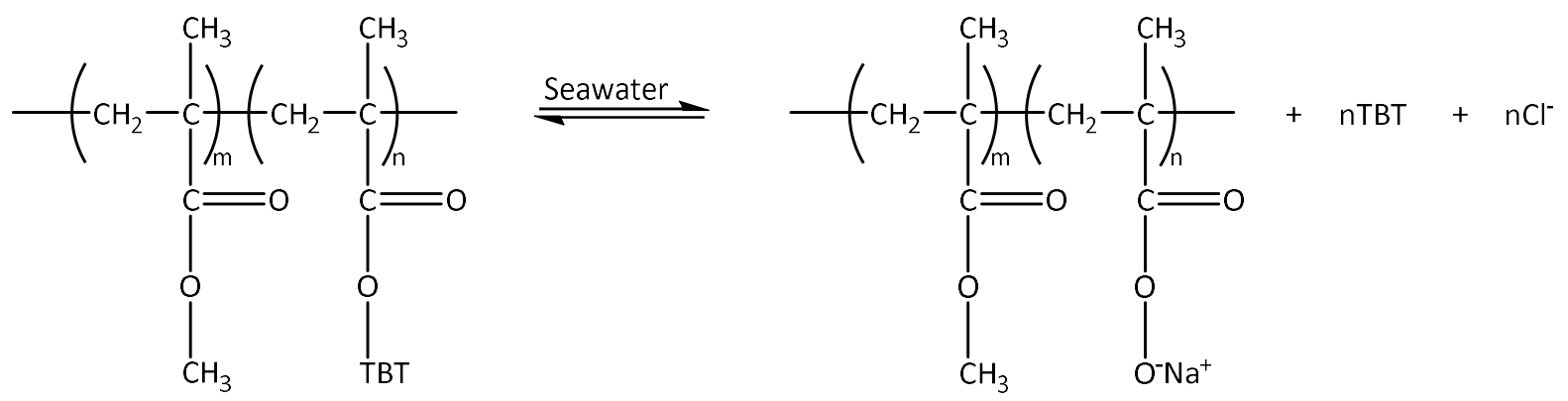

Scheme 1.1. Hydrolysis of TBT from the acrylic polymer (methyl methacrylate) in a TBT self-polishing paint. $^{3}$

Following their introduction in 1974, TBT self-polishing paints became enormously popular, and their use was widespread - so much so that, in 1999 , it was estimated that $70 \%$ of all commercial ships were coated with them, saving US\$2.4 billion per year in fuel and other costs. ${ }^{3}$ TBT is the most effective broad-spectrum antifoulant ever developed, having toxicity to molluscs, crustaceans, algae, and fish. ${ }^{2,23}$ For years, it seemed like hull fouling would be a problem of the past, but, as soon as the early 1980s, the profoundly negative effects of TBT on non-target organisms were noted. ${ }^{23}$ TBT leaching from coated ship hulls was environmentally damaging, for example, inducing sex changes in sea snails and dogwhelks. 2,22,32 Consequently, the use of TBT was restricted further and further until it was finally banned from use in coatings on all vessels after 1 January 2008 by the International Maritime Organization. Copper has replaced TBT as the biocide in many antifouling paints, and, with over $90 \%$ of US Navy ships coated with a copper ablative (i.e. soluble matrix) paint, copper-based antifouling coatings are the current standard in antifouling technology. ${ }^{2,23,33}$

Copper was viewed as a safe alternative to TBT because historical precedents exist for its use as an antifoulant, it is an essential trace element for all plants and animals, and it is naturally present in 
seawater. ${ }^{3}$ Antifouling coatings usually incorporate copper as metallic copper, cuprous oxide $\left(\mathrm{Cu}_{2} \mathrm{O}\right)$, or copper thiocyanate (CuSCN), and such copper-impregnated coatings designed to slowly release copper vary in their copper content from $20 \%$ to $76 \% .{ }^{8,34}$ Since seawater is oxygenated, the released copper is rapidly oxidised to $\mathrm{Cu}(\mathrm{II})$, the primary biocidal form. Yet, $\mathrm{Cu}(\mathrm{II})$ is not an efficacious antifoulant for all marine foulers, and, from most to least, the order of $\mathrm{Cu}(\mathrm{II})$-sensitivity among fouling organisms is as follows: microorganisms, invertebrates, algae, and macrophytes (i.e. aquatic plants). Additionally, the copper ions released from the paint often become bound to natural ligands in seawater, further reducing their toxicity. ${ }^{3}$

In copper-based antifouling coatings, formulations for the inert matrix and delivery are variable (e.g. copolymers, self-polishing, epoxy-based, ablative), differing in the passive flux of copper according to the matrix. However, all formulations require periodic (monthly) cleaning of the ship hull. ${ }^{2,8,34}$ Due to the copper tolerance of some algal species (e.g. Enteromorpha sp.), the majority of copper antifouling paints also contain a booster biocide, often an herbicide detrimental to the growth of photosynthetic organisms, to target these algae. The most common commercially available booster biocides include Irgarol 1051, Diuron, Zineb, Sea-Nine 211, Chlorothalonil, and metallic compounds zinc pyrithione and copper pyrithione, and these biocides are meant to be less harmful to the environment than copper. ${ }^{3,8,23,28} \mathrm{~A}$ toxicity study by Amara et $a l .^{8}$ reported that, out of the common booster biocides, Diuron and Irgarol 1051 seem to be the least environmentally harmful, while Chlorothalonil and Sea-Nine 211 seem to be the most. Conversely, a study by Voulvoulis et al. ${ }^{35}$ identified Zineb and zinc pyrithione as being less environmentally harmful than Diuron and Irgarol 1051, so the toxicity effects of the booster biocides have yet to be fully elucidated. ${ }^{3}$ Although copper coatings are an effective method to mitigate fouling, the leaching of copper from these antifouling paints has become an environmental concern because of the toxic effects of elevated copper concentrations on non-target organisms. ${ }^{36}$

Widespread prohibition of copper-based paints has not yet occurred, but they are increasingly being banned due to concerns about copper leaching, especially in sensitive ecosystems. ${ }^{22,37}$ For instance, antifouling paints leaching excessive amounts of copper have been restricted by the Swedish Chemicals Agency, which regards all copper-based paints as unacceptable for use on leisure boats $(<12 \mathrm{~m})$ in the vulnerable Baltic Sea. ${ }^{37}$ Despite copper being an essential trace element, copper concentrations higher than physiologically necessary are toxic to many marine organisms, including those not targeted by antifouling measures. ${ }^{2}$ For instance, Andersson and Kautsky ${ }^{38}$ found that even low concentrations of copper (e.g. $2.5 \mathrm{ppb}$ ) in brackish water could have detrimental effects on the reproduction of Fucus vesiculosus (i.e. bladderwrack), a key species in the Baltic Sea. This is particularly concerning because the release of $F$. vesiculosus gametes coincides with the launching of newly 
painted leisure boats, which are major contributors ( $30 \%)$ to the total copper burden in Swedish inland and coastal waters. ${ }^{38,39}$ Additionally, Ytreberg, Karlsson, and Eklund ${ }^{40}$ reported that, in semienclosed areas with high boat traffic, copper leached from antifouling paints may attain toxic concentrations for the non-target macroalga Ceramium tenuicorne. In places with little water exchange, such as marinas and harbours, therefore, elevated copper levels are a concern. ${ }^{23}$ The average concentration of copper in seawater is approximately $2 \mathrm{ppb}$, but concentrations up to $22 \mathrm{ppb}$ in a marina in San Diego Bay and 114 ppb in the coastal waters of India have been recorded, for instance. ${ }^{41,42}$ Furthermore, antifouling paint particles shed during periodic hull maintenance (e.g. scraping, sandpapering, water blasting) and winter storage pollute the soil with copper at concentrations often exceeding national guidelines, possibly leaching into ground water. ${ }^{1,36}$ Given the substantial diversity of fouling organisms ( $>4000$ species), a major challenge for biocide-based antifouling paints is finding an antifoulant that reduces target species but spares non-target species. Due to this challenge and the uncertain future of copper-impregnated coatings, the marine coatings industry desires alternatives to biocide-based antifouling paints. ${ }^{15}$

\subsubsection{Biocide-Free Coatings}

Biocide-free coatings utilise the surface physico-chemical and bulk materials properties (e.g. elastic modulus, frictional coefficient) of the film to either deter adherence of fouling organisms or reduce the strength with which they do attach, allowing for removal by shear forces as the ship moves through the water or is mechanically cleaned. This approach is dependent upon the molecular interactions between the surface of the film and the adhesive polymers (e.g. EPS) secreted by the fouling organisms. Thus, biocide-free coatings employ two different strategies, resulting in them being classified as antifouling or fouling-release coatings. Biocide-free antifouling coatings are unconducive to colonisation by fouling organisms and, therefore, prevent initial attachment, while fouling-release coatings weaken the interaction between the foulers' secreted adhesive polymers and the film surface after initial attachment. However, it should be noted that these two strategies are not mutually exclusive. ${ }^{15}$

\subsubsection{Hydrophilic Antifouling Coatings}

Hydrophilic coatings are a common type of biocide-free antifouling coating, containing hydrophilic polymer brushes that prevent or reduce the initial attachment of proteins, bacteria, and marine 
foulers. Their preparation usually involves the application of a monolayer of polyethylene glycol (PEG) brushes to a surface by grafting with a, typically, siloxane linker (Figure 1.6). ${ }^{22}$ PEG is the most commonly used polymer to stop protein adsorption to surfaces, and it is thought that the flexibility and large steric repulsion forces of the PEG chains discourage the approach of foulers. ${ }^{43,44} \mathrm{~A}$ study by Roosjen et al. ${ }^{45}$ reported a reduction in the attachment of bacteria (Staphylococcus epidermidis, Pseudomonas aeruginosa) and yeast (Candida albicans, Candida tropicalis) to a PEG brush-coated surface, with the initial attachment rate and number of adherent bacteria decreasing with increasing brush-length. Despite their success in reducing bacterial colonisation and preventing protein adsorption, these PEG coatings are of limited use, as they are quite fragile and even minor surface defects drastically reduce their efficacy. Additional drawbacks are that they are sensitive towards hydrolysis and oxidation. ${ }^{22}$

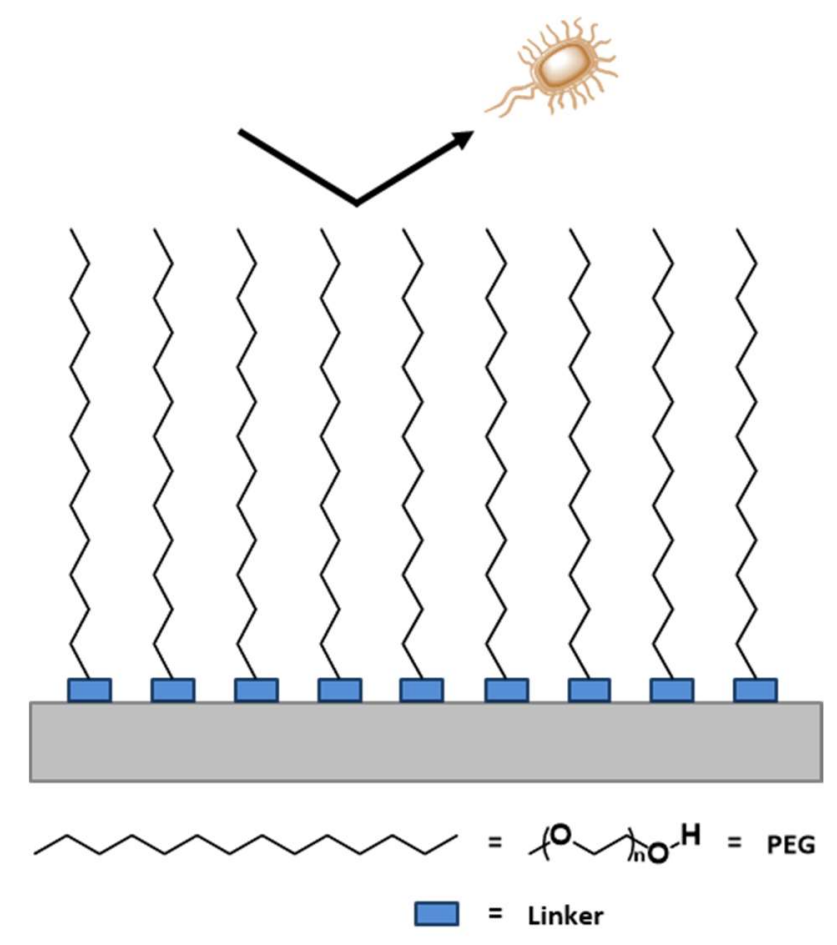

Figure 1.6. Hydrophilic antifouling coating with PEG brushes attached to the surface by a linker. ${ }^{22}$

\subsubsection{Fouling-Release Coatings}

Fouling-release coatings have an ultra-smooth, low-friction, hydrophobic surface that results in weak interactions between the surface and the foulers' adhesive polymers, enabling biofouling removal by shear forces (Figure 1.7). The smooth, glossy surface results in a decrease in frictional drag, allowing 
for ships to achieve maximal speeds with minimal fuel consumption, and the hydrophobicity of the surface discourages the adhesion of polar molecules (e.g. adhesive proteins). Fouling-release coatings also have a low surface energy and low elastic modulus, providing little resistance to the removal of fouling organisms. Surface energy, determined by contact angle measurements, is the excess energy of surface molecules compared to those in the coating interior, $3,15,22,28,46$ and the elastic modulus is the resistance of the film to elastic (i.e. "springy") deformation. ${ }^{47}$ Other coating requirements to prevent adhesion are as follows:

1) A flexible, linear backbone,

2) A surface that is smooth at the molecular level, which precludes infiltration by biological adhesives,

3) A large number of active groups capable of moving freely over the surface, imparting the desired surface energy,

4) A thickness capable of controlling fracture mechanics (e.g. crack propagation) at the surface/adhesive interface,

5) High molecular mobility in the surface-active sidechains and backbone, and,

6) In addition to the requirements above, molecules that are stable in seawater for long periods of time..$^{48}$

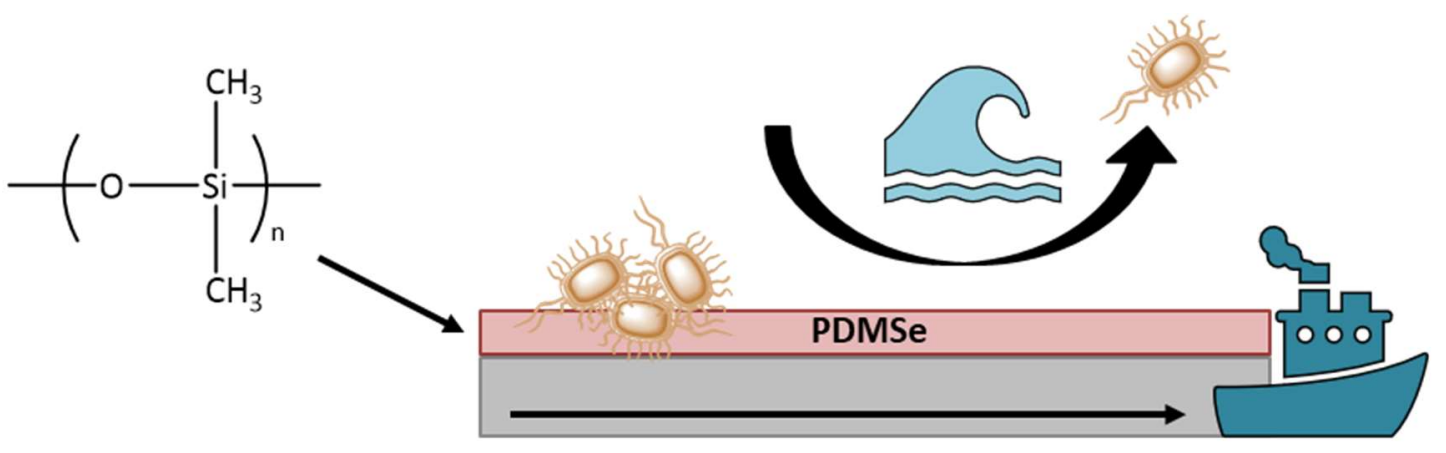

Figure 1.7. Detachment of marine foulers from a PDMSe-based fouling-release coating by shear forces as the vessel moves through the water. ${ }^{28}$

The only two types of materials that satisfy all of these requirements are fluoropolymers and silicones. Currently, the majority of commercial fouling-release coatings incorporate poly(dimethylsiloxane) elastomers (PDMSe; i.e. elastic polymers; Figure 1.7), which are hydrophobic and nonpolar and have a low surface energy and low microroughness. Due to the conformational mobility of their surfaces, 
they appear as "moving targets" to functional groups of the adhesive polymers, deterring adhesion, and their lifetime is approximately two years. Fouling-release coatings are regarded as the best of the biocide-free coatings, yet they have several disadvantages. They need the ship to be moving through the water at a minimum speed of 22 knots ( $41 \mathrm{kph}$ ) to detach fouling organisms, and, accordingly, they are most effective for high-speed vessels and may be inappropriate for slower vessels that spend a long time in port. They are also expensive, demonstrate poor substrate-adhesion, and are soft and easily damaged. ${ }^{3,15,22,28,46}$ As a result, the search continues for other environmentally friendly alternatives to biocide-based antifouling coatings.

\subsubsection{Recent Developments}

Numerous antifouling strategies have been inspired by natural phenomena, since many plants and animals are resistant to fouling. Some of the recent developments in environmentally friendly antifouling technology, many of them bioinspired, are discussed below.

\subsubsection{Biomimetic Topographies}

Multiple studies over the past 50 years have demonstrated that surface topography in the micrometre range influences cellular attachment. ${ }^{49-54}$ For example, in a study by Carman et al. ${ }^{55}$, the microscale topography (e.g. diamondlike riblets) of the naturally antifouling skin of fast-moving sharks was replicated on the surface of a PDMSe coating. This biomimetic coating, Sharklet $\mathrm{FF}^{\mathrm{TM}}$, was tested for the settlement of zoospores of the soft-fouling, green alga Ulva linza, which is a model system for biofouling experiments. It was found that zoospore settlement on Sharklet $\mathrm{AF}^{\mathrm{TM}}$ was reduced by approximately $85 \%$. Other biomimetic surfaces have duplicated the topographies of the skin of pilot whale Globicephala melas and surfaces of fouling-resistant plants. ${ }^{56}$ For instance, Fu et al. ${ }^{57}$ duplicated the ridge-like surface morphology of the leaves of mangrove tree Sonneratia apetala, as these leaves are practically immune to biofouling. Following settlement tests with tubeworm larvae, it was confirmed that the replica could prevent biofouling.

\subsubsection{Superhydrophobic Surfaces}

Bioinspired superhydrophobic surfaces have been developed for antifouling purposes. Superhydrophobic surfaces have high contact angles $\left(>150^{\circ}\right)$ and, therefore, are very difficult to wet. 
They rely on the trapping of air and resist fouling by dramatically reducing contact between the surface and the fouler. The most famous example of this surface type in nature is the lotus leaf, on which water moves freely to collect contaminants and then roll off the surface. This self-cleaning mechanism (i.e. lotus leaf effect) is a result of the superhydrophobicity of the surface. The leaf's surface is coated in low surface energy waxes and has two structural levels: microscale mounds decorated with nanoscale hair-like structures (hydrophobic hydrocarbon tubules). Due to the entrapment of a stable air cushion by the hierarchical micro/nanostructures, interactions between the surface and water are substantially reduced, precluding the attachment of fouling organisms. ${ }^{15,44,49,58}$ The multilevel structure of the lotus leaf has been replicated, for instance, by Zorba et al. ${ }^{59}$ through ultrafast (femtosecond) irradiation of a silicon surface, producing an artificial surface equal to the lotus leaf in its ability to repel water.

\subsubsection{Zwitterionic Polymers}

Zwitterionic polymers, containing both cationic and anionic groups, are well-known for deterring protein adsorption and have been used extensively in antifouling materials. The deterrence of protein adsorption is attributed to the electrostatically induced hydration layer serving as a barrier to proteins; the superhydrophilic surface prefers to bind water molecules, rather than biological material. Zwitterionic polymers are classified as polybetaines, which have positive and negative charges in the same monomer unit, or polyampholytes, which have positive and negative charges in a 1:1 ratio on separate monomer units. ${ }^{2,15,31,44,60,61}$ In a study by Zhang et al. ${ }^{62}$, poly(sulfobetaine methacrylate) brushes were grafted onto glass surfaces, and these grafted surfaces inhibited the attachment of zoospores of the green alga Ulva and cells of the diatom Navicula. It was also demonstrated that the attachment strength of Ulva sporelings was low.

\subsubsection{Amphiphilic Polymers}

In an effort to combine the antifouling properties of hydrophobic and hydrophilic coatings, amphiphilic polymer coatings possessing both hydrophobic and hydrophilic blocks have been prepared. Fouling organisms are known to have diverse preferences regarding the hydrophobicity of a surface, and such an ambiguous surface is expected to "confuse" foulers during attachment and settlement, leading to a reduction in biofouling. ${ }^{2,15,31,43,61}$ Gudipati et al. ${ }^{63}$ published the first example of an amphiphilic marine antifouling coating, which was prepared by crosslinking diamino-terminated PEG (hydrophilic) and hyperbranched fluoropolymers (hydrophobic). The same lab group later 
demonstrated reduced adsorption of proteins and lipopolysaccharides and reduced settlement of Ulva zoospores in compositions with high concentrations of PEG $(45-55 \% \mathrm{w} / \mathrm{w}) .{ }^{15,64}$

\subsubsection{Enzyme-Based Coatings}

In recent years, enzyme-based antifouling coatings have gained attention, as they are thought to be harmless to the environment. The enzymes interfere with biofilm formation by two mechanisms: 1) quenching bacterial quorum sensing signals and 2) degrading the EPS matrix of the biofilm..$^{44,65}$ Intercellular communication between bacterial cells in a biofilm occurs via quorum sensing, during which the concentration of signalling molecules (i.e. autoinducers) secreted by bacteria in the biofilm increases with increasing cell density. Once the autoinducer concentration reaches a certain threshold, the quorum sensing bacteria respond as a group by altering their gene expression. ${ }^{44,66}$ Most autoinducers (e.g. $\mathrm{N}$-acyl-homoserine lactones, AHLs) are involved in biofilm formation and maturation and the regulation of bacterial virulence factors. Therefore, enzymes capable of quenching these signals (e.g. AHL lactonases, $\mathrm{AHL}$ acylases) have been incorporated in coatings to prevent bacterial colonisation. ${ }^{44}$

Coatings have also been prepared by incorporating enzymes with the ability to degrade the biofilm matrix. The EPS matrix, composed of polysaccharides, proteins, nucleic acids, and lipids, is secreted by microorganisms in the biofilm and plays a vital role in adhesion to the substrate. Consequently, enzymes, such as proteases (to degrade the proteins) and glycosylases (to degrade the polysaccharides), have been included in coatings to hinder bacterial attachment. ${ }^{44,67}$ For instance, Tasso et $a l .{ }^{68}$ surface-functionalised maleic anhydride copolymer thin films with protease Subtilisin A and discovered that the coating reduced the adhesion strength of diatom Navicula perminuta cells and the settlement and adhesion strength of green alga Ulva linza zoospores. Unfortunately, enzymebased coatings have limitations to their use because they are less stable in warm marine environments (e.g. the tropics). ${ }^{67}$

\subsubsection{Quorum Sensing Inhibitors}

The use of quorum sensing inhibitors in antifouling materials has been studied as another method for interfering with bacterial quorum sensing and, subsequently, reducing microfouling. Numerous marine organisms have evolved mechanisms to block bacterial quorum sensing, and these mechanisms include the inhibition of autoinducer production, enzymatic degradation of autoinducers 
(as previously discussed), inhibition of DNA transcription, and competition with or suppression of autoinducer receptors. ${ }^{69,70}$ For example, red alga Delisea pulchra produces halogenated furanones with similar structures to $\mathrm{AHL}$ autoinducers produced by marine bacteria. Accordingly, these $\mathrm{AHL}$ mimics displace $A H L$ from its receptor, reducing bacterial adhesion and swarming. ${ }^{69-72}$ Such quorum sensing inhibitors could serve as additives in antifouling coatings. In fact, Ozcelik et al. ${ }^{73}$ incorporated a synthetic quorum sensing inhibitor (5-methylene-1-(prop-2-enoyl)-4-(2-fluorophenyl)-dihydropyrrol2-one) in a PEG-based coating, and biofilm formation by Staphylococcus aureus and Pseudomonas aeruginosa was reduced on the prepared coating. It should be emphasised that, even though such inhibitors reduce colonisation by microorganisms, they would not necessarily prevent all biofouling.

\subsubsection{Bacteria}

Marine bacteria colonising a surface secrete many different bioactive compounds to inhibit fouling by other marine organisms (micro- and macro-foulers), securing them a competitive advantage. For example, marine bacterium Pseudoalteromonas tunicata (strain D2) produces at least four bioactive, extracellular molecules with activity against a variety of marine foulers. ${ }^{2}$ Egan et al. $^{74}$ identified one of these molecules, which is heat-sensitive, polar, and 3-10 kDa in size, and this compound inhibited the germination of spores from the red alga Polysiphonia sp. and the common green alga Ulva lactuca. Therefore, research has been conducted regarding the incorporation of such bioactive natural products - or the bacteria that produce them - in antifouling coatings. Although, since these compounds often rapidly degrade in the marine environment, work needs to be done to extend their lifetimes when formulated in antifouling coatings. ${ }^{2,75}$ 


\subsection{Thesis ObJective: CoORdination of Cu(II) In SEAWATER to Create a Rechargeable Antifouling CoAting}

Despite the extensive research concerning "green" antifouling technology, copper-based antifouling coatings remain the current standard. Many of the mechanisms recently in consideration to combat biofouling have yet to overcome challenges associated with their commercialisation, such as high cost, instability, and a lack of broad-spectrum activity. Therefore, at this time, a comparable product has not achieved widespread commercial success, and the field is open to new possibilities. The project herein proposes a novel antifouling strategy that utilises copper as a biocide but circumvents the environmental concerns about copper leaching.

\subsubsection{Concept}

For this work, it has been proposed that antifouling technology can be developed to create marine paints that draw their biocidal ingredient, metals such as copper, zinc, cadmium, and chromium, from the sea. This is a revolutionary concept: no currently used antifouling paints draw their active component from seawater. Although the biocidal mode of action of these paints would be the same as existing paints pre-charged with metals, the environmental concerns and tightening regulations regarding metal-leaching would no longer be applicable; such paints would self-prime and selfregenerate from pre-existing metals in seawater. No new metals would be introduced to the marine environment. The technology would work by covalently binding ligands capable of selective metal ion complexation to components (e.g. binder, additives) in paint systems (Figure 1.8). High ligand concentrations in the paint would be required, and, at these high concentrations, the ligands would, in principle, capture enough of the metal to reach concentrations toxic to fouling organisms, thus preventing biofouling when applied to ship hulls. The project will initially focus on $\mathrm{Cu}(\mathrm{II})$ as the biocidal agent since it is currently the primary active form in copper-based antifouling paints and its efficacy has been well-documented. The specific aim of this project is to create a rechargeable, $\mathrm{Cu}$ (II)-based antifouling coating for use in the marine environment, but it has been acknowledged that such a coating would also have applications in the medical field, as nosocomial (i.e. hospital-acquired) infections and biofilm formation on implanted devices (e.g. artificial prostheses, catheters, dental implants, etc.) remain serious problems that could be mitigated by effective antimicrobial coatings. ${ }^{76}$ 


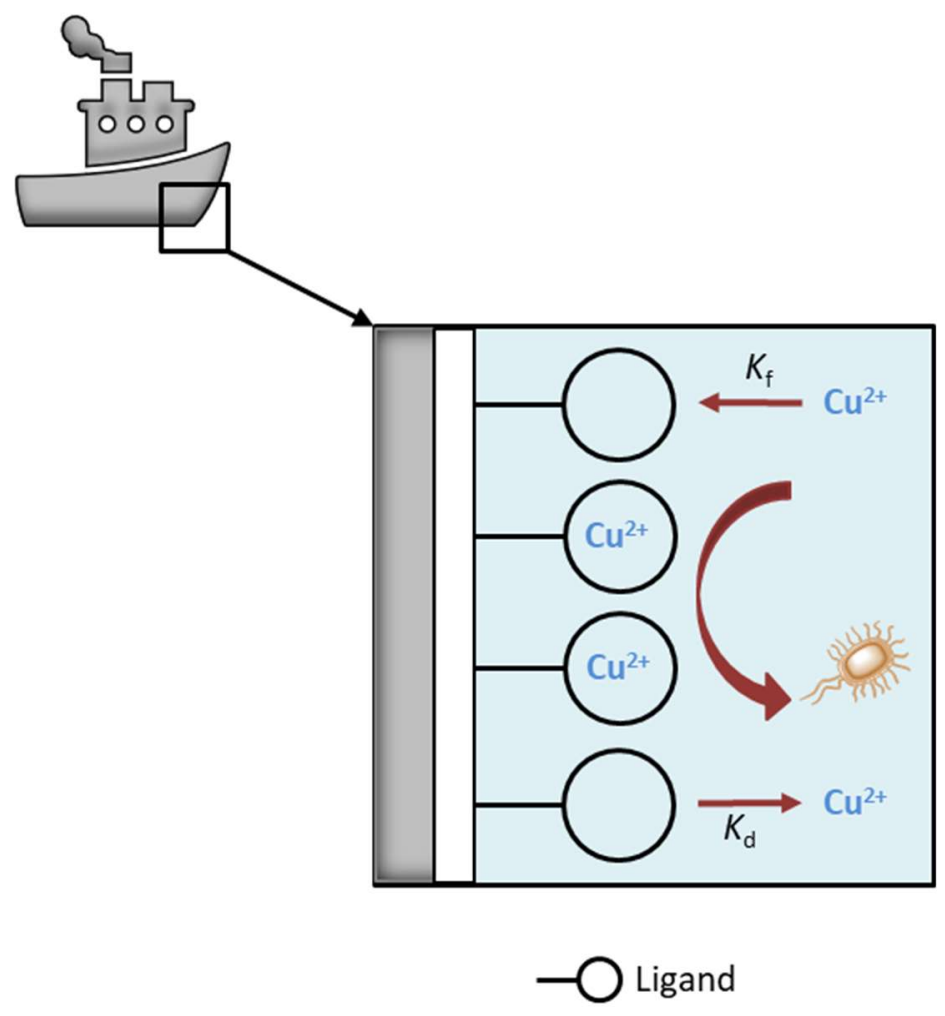

Figure 1.8. The goal of this project is to create a rechargeable $\mathrm{Cu}(\mathrm{II})$ coating by incorporating $\mathrm{Cu}(\mathrm{II})$ selective ligands to bind $\mathrm{Cu}(\mathrm{II})$, making the coating antimicrobial and antifouling. This depends on the $\mathrm{Cu}(\mathrm{II})$ flux at the surface, which is related to the complex's formation $\left(K_{\mathrm{f}}\right)$ and dissociation $\left(K_{\mathrm{d}}\right)$ constants.

Previous research supports the viability of this route of inquiry. For instance, Lindén et al. ${ }^{77}$ were the first to report on the use of polyethyleneimine (PEI)-based materials to scavenge $\mathrm{Cu}(\mathrm{II})$ from seawater for antifouling purposes. These glutaraldehyde (GA) crosslinked PEI, nano-thin coatings have demonstrated the efficient and preferential accumulation of $\mathrm{Cu}(\mathrm{II})$ from seawater, achieving a maximum $\mathrm{Cu}(\mathrm{II})$-loading of $2 \% \mathrm{w} / \mathrm{w} .{ }^{77}$ Further work by this group has concerned the effect of the conditioning layer between the GA-PEI coating and seawater and of competing ligands in seawater on $\mathrm{Cu}(\mathrm{II})$-binding. ${ }^{78}$ They recognised that the flux of $\mathrm{Cu}(\mathrm{II})$ at the seawater/coating interface is necessary for antimicrobial activity (Figure 1.8) and could be impeded by the presence of the conditioning layer. This flux is dependent on the strength of the $\mathrm{Cu}(\mathrm{II})$ complex, which is described by its formation $\left(K_{\mathrm{f}}\right)$ and dissociation $\left(K_{\mathrm{d}}\right)$ constants (Equation 1.1), but the group acknowledged that the release of $\mathrm{Cu}(\mathrm{II})$ may require an artificial intervention, such as an electrochemical stimulus. ${ }^{77,79}$ Furthermore, considering that $>99 \%$ of the $\mathrm{Cu}(\mathrm{II})$ dissolved in seawater is already strongly complexed by unidentified organic ligands with formation constants in the range of $10^{9}-10^{14}$, the ligand in the coating would have to be a stronger $\mathrm{Cu}(\mathrm{II})$-chelator than the natural ligands in seawater 
(i.e. $\left.K_{\mathrm{f}}>10^{14}\right) \cdot{ }^{80}$ To model the conditioning layer and competing ligands, the GA-PEI film was coated with polysaccharides alginate and/or carrageenan, and the uptake of $\mathrm{Cu}(\mathrm{II})$ at the concentrations of contaminated harbours in artificial seawater was tested in the presence of competing-ligand ethylene diamine tetraacetic acid (EDTA). It was discovered that, in seawater, the polysaccharide layer swelled, allowing $\mathrm{Cu}(\mathrm{II})$ to diffuse readily, and GA-PEI outcompeted strong $\mathrm{Cu}(\mathrm{II})$-chelator EDTA for $\mathrm{Cu}(\mathrm{II}){ }^{78}$ In regard to this work, the research group has applied for a patent. ${ }^{81}$ Building on these studies, Elmas et al. ${ }^{82}$ explored the controlled, electrochemical release of $\mathrm{Cu}(\mathrm{II})$ from crosslinked, porous PEI coated on conducting carbon cloth electrodes, and they reported that the coating could store and release $\mathrm{Cu}(\mathrm{II})$ in multiple cycles via the application of only $1 \mathrm{~mA} / \mathrm{cm}^{2}$ of current density through the electrode in artificial seawater. These results are encouraging and provide evidence for the feasibility of this project.

$$
M+L \rightleftharpoons M L \quad K_{f}=\frac{[M L]}{[M][L]} \quad K_{d}=\frac{[M][L]}{[M L]}
$$

Equation 1.1. The formation $\left(K_{\mathrm{f}}\right)$ and dissociation $\left(K_{\mathrm{d}}\right)$ constants of a metal-ligand complex $(\mathrm{M}=$ metal, $L=$ ligand).

\subsubsection{Practical Considerations}

In a preliminary approach to this project, there are questions that must be answered before proceeding:

1) How is a $\mathrm{Cu}(\mathrm{II})$ ligand designed?

2) What factors influence the stability of the $\mathrm{Cu}(\mathrm{II})$ complex, and how is the $\mathrm{Cu}(\mathrm{II})$ complex characterised?

3) What are the options for formulating the $\mathrm{CU}(\mathrm{II})$ ligand in a coating?

4) What are the surface toxicity mechanisms of $\mathrm{Cu}(I I)$ ?

\subsubsection{Design of Cu(II) Ligands}

Since many other transition metal ions exist in seawater, the ligand chosen or designed and synthesised for this project must be selective for $\mathrm{Cu}(\mathrm{II})$. Selectivity depends on matching the "hardness" or "softness" of the Lewis acids (i.e. metal ions, e.g. $\mathrm{Cu}(\mathrm{II})$ ) and Lewis bases (i.e. donor atoms of ligands), according to the Hard Soft Acid Base (HSAB) theory proposed by Ralph G. Pearson in 1963. In a coordination compound, the coordinate bond between the metal ion and the donor atom 
of the ligand displays a certain polarity, dictating the ionic character of the bond. Small, highly charged Lewis acids have a high surface charge density, which results in their electron clouds being drawn inward and, thus, less efficient at the orbital overlap necessary for covalent bonding. Such ions are classified as "hard acids" and have a preference for ionic bonding. "Hard bases" are weakly polarisable and highly electronegative. In contrast, large, low-charged Lewis acids with a low surface charge density are classified as "soft"; their more diffuse and expanded electron clouds are better-suited for orbital overlap, so they have a preference for covalent bonding. "Soft bases" are highly polarisable and weakly electronegative. According to the "like prefers like" principle of the HSAB theory, hard ligands prefer hard metals, and soft ligands prefer soft metals. $\mathrm{Cu}(\mathrm{II})$ is classified as "borderline" (Table 1.4), meaning that it is between hard and soft, and it usually forms $4-6$ coordinate bonds with hard bases (e.g. carbonyl and carboxylate oxygens, amines), borderline bases (e.g. azoles, pyridine), and/or sulfur. ${ }^{83-87}$

Table 1.4. Examples of hard, soft, and intermediate ligands and metal ions (adapted from Introduction to Coordination Chemistry ${ }^{84}$ and Hancock and Martell ${ }^{85}$ ).

\begin{tabular}{|c|c|c|}
\hline Hard & Borderline & Soft \\
\hline $\begin{array}{l}\text { Ligands } \\
\mathrm{F}^{-}, \mathrm{O}^{2-}, \mathrm{HO}^{-}, \mathrm{H}_{2} \mathrm{O}, \mathrm{HOR}, \mathrm{RO}^{-} \\
\mathrm{R}_{2} \mathrm{O}, \mathrm{CH}_{3} \mathrm{CO}_{2}^{-}, \mathrm{RCO}_{2}^{-}, \mathrm{NH}_{3}, \mathrm{NR}_{3}, \\
\mathrm{RNH}_{2}, \mathrm{RCN} \mathrm{Cl}^{-}, \mathrm{NO}_{3}^{-}, \mathrm{CO}_{3}{ }^{2-} \text {, } \\
\mathrm{SO}_{4}{ }^{2-}, \mathrm{PO}_{4}^{3-}, \mathrm{ClO}_{4}^{-}\end{array}$ & $\begin{array}{l}\mathrm{Br}^{-}, \mathrm{RS}^{-}, \mathrm{SO}_{3}{ }^{2-}, \mathrm{NO}_{2}^{-}, \mathrm{N}_{3}^{-}, \mathrm{N}_{2} \\
\mathrm{SCN}^{-}, \mathrm{C}_{6} \mathrm{H}_{5} \mathrm{NH}_{2}, \mathrm{C}_{5} \mathrm{H}_{5} \mathrm{~N}, \mathrm{C}_{3} \mathrm{~N}_{2} \mathrm{H}_{4}\end{array}$ & $\begin{array}{l}\mathrm{PR}_{3}, \mathrm{RSH}, \mathrm{R}_{2} \mathrm{~S}, \mathrm{RS}, \mathrm{Se} \mathrm{R}_{2}, \mathrm{AsR}_{3} \\
\mathrm{CNR}, \mathrm{CN}^{-}, \mathrm{SCN}^{-}, \mathrm{CO}, \mathrm{I}^{-}, \mathrm{H}^{-}, \mathrm{R}^{-} \\
\mathrm{C}_{2} \mathrm{H}_{4}, \mathrm{~S}_{2} \mathrm{O}_{3}^{2-}\end{array}$ \\
\hline $\begin{array}{l}\text { Metal lons } \\
\mathrm{Mo}^{5+}, \mathrm{Ti}^{4+}, \mathrm{V}^{4+}, \mathrm{Sc}^{3+}, \mathrm{Cr}^{3+}, \mathrm{Fe}^{3+} \\
\mathrm{Co}^{3+}, \mathrm{Al}^{3+}, \mathrm{Eu}^{3+}, \mathrm{Ga}^{3+}, \mathrm{Cr}^{2+} \\
\mathrm{Mn}^{2+}, \mathrm{Ca}^{2+}, \mathrm{Mg}^{2+}, \mathrm{Be}^{2+}, \mathrm{Sr}^{2+} \\
\mathrm{Ba}^{2+}, \mathrm{K}^{+}, \mathrm{Na}^{+}, \mathrm{Li}^{+}, \mathrm{H}^{+}\end{array}$ & $\begin{array}{l}\mathrm{Sb}^{3+}, \mathrm{Bi}^{3+}, \mathrm{Rh}^{3+}, \mathrm{Fe}^{2+}, \mathrm{Co}^{2+}, \mathrm{Ni}^{2+}, \\
\mathrm{Cu}^{2+}, \mathrm{Zn}^{2+}, \mathrm{Pb}^{2+}, \mathrm{Sn}^{2+}\end{array}$ & $\begin{array}{l}\mathrm{Pt}^{4+}, \mathrm{Te}^{4+}, \mathrm{Pd}^{2+}, \mathrm{Pt}^{2+}, \mathrm{Hg}^{2+}, \mathrm{Cd}^{2+}, \\
\mathrm{Cu}^{+}, \mathrm{Hg}^{+}, \mathrm{Rh}^{+}, \mathrm{Ag}^{+}, \mathrm{Au}^{+}, \mathrm{Br}^{+}, \mathrm{I}^{+}, \\
\mathrm{Tl}^{+}\end{array}$ \\
\hline
\end{tabular}

Well-documented $\mathrm{Cu}(\mathrm{II})$ ligands include polyamines, D-penicillamine, and EDTA. Macrocyclic (e.g. cyclen, cyclam) and linear (e.g. triethylenetetramine, TETA) polyamines (Figure 1.9) are known to be highly selective chelators of $\mathrm{Cu}(\mathrm{II})$ over other transition metals. ${ }^{88,89} \mathrm{In}$ fact, TETA has been used for more than two decades as an orphan drug in the treatment of Wilson's disease. ${ }^{88,90}$ Wilson's disease is caused by a genetic mutation that leads to impaired copper detoxification in the liver and an accumulation of copper, primarily $\mathrm{Cu}(\mathrm{II})$, to toxic levels in the body. ${ }^{86} \mathrm{D}$-Penicillamine is also used to treat Wilson's disease, but it is a less specific transition metal chelator. ${ }^{88}$ It was introduced as a treatment in 1956 before TETA, and it is used worldwide, whereas TETA is mainly used in the US. ${ }^{86}$ 
Both drugs bind excess copper and promote its urinary excretion. ${ }^{91}$ EDTA is relatively non-specific in its binding, yet $\mathrm{Fe}(\mathrm{III}), \mathrm{Al}(\mathrm{III})$, and $\mathrm{Cu}(\mathrm{II})$ form the most stable chelates. ${ }^{92}$

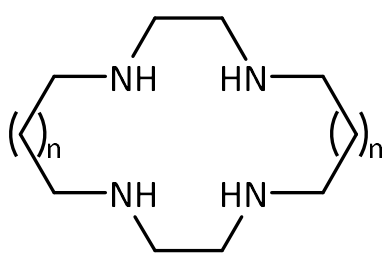

(a)<smiles>CC(C)(S)[C@H](N)C(=O)O</smiles>

(c)<smiles>NCCNCCNCCN</smiles>

(b)<smiles>O=C(O)CN(CCN(CC(=O)O)CC(=O)O)CC(=O)O</smiles>

(d)

Figure 1.9. Examples of well-documented $\mathrm{Cu}(\mathrm{II})$ ligands: $(\mathrm{a})$ cyclen $(n=0)$ or cyclam $(n=1),(b)$ TETA, (c) D-penicillamine, and (d) EDTA.

\subsubsection{Cu(II) Complexes: Stability and Characterisation}

In addition to the match in "hardness" between the metal ion and ligand's donor atoms, the stability of the metal complex is influenced by other factors dependent on the metal ion and the ligand. Regarding the metal ion, these factors include size, charge, and crystal field effects. A high formation (i.e. stability) constant is favoured by a high surface charge density, or, in other words, small, highly charged ions produce more stable metal complexes. For a series of transition metal ions (octahedral) with a $2+$ charge, a general trend in the value of $K_{\mathrm{f}}$ exists, regardless of the ligand:

$$
\mathrm{Mn}^{2+}<\mathrm{Fe}^{2+}<\mathrm{Co}^{2+}<\mathrm{Ni}^{2+}<\mathrm{Cu}^{2+}>\mathrm{Zn}^{2+} .
$$

According to this trend, called the natural order of stabilities (i.e. Irving-Williams series), $\mathrm{Cu}$ (II) forms the most stable complexes, irrespective of the ligand. The position of $\mathrm{Zn}(\mathrm{II})$ within the series is variable. The stability of $\mathrm{Cu}(\mathrm{II})$ complexes may be explained, in part, by the trend of ionic radii:

$$
\mathrm{Mn}^{2+}>\mathrm{Fe}^{2+}>\mathrm{Co}^{2+}>\mathrm{Ni}^{2+}<\mathrm{Cu}^{2+}<\mathrm{Zn}^{2+} .
$$

However, $\mathrm{Cu}(\mathrm{II})$ (electron configuration $[\mathrm{Ar}] 3 \mathrm{~d}^{9}$ ), like other metals with an incomplete set of $\mathrm{d}$ electrons, receives a contribution to its stability from the crystal field stabilisation energy (CFSE) ${ }^{84}$ 
Crystal field theory (CFT) is a model that describes, upon formation of the metal ion-ligand complex, the effect of the ligands' electronic charges on the energies of the electrons in the d-orbitals of the metal ion. The CFSE is the additional stability that arises from placement of the metal ion in the crystal field produced by a set of ligands, and it is due to splitting of the d-orbitals in the ligand field. ${ }^{93}$ For example, most d-block metals, including $\mathrm{Cu}(\mathrm{II})$, form six-coordinated complexes with an octahedral coordination geometry (Figure 1.10). Because of this geometry, the $d_{x^{2}-y^{2}}$ and $d_{z^{2}}$ orbitals of $\mathrm{Cu}(I I)$ experience repulsive forces due to their alignment with the ligands and, consequently, are higher in energy than the $d_{x y}, d_{x z}$, and $d_{y z}$ orbitals. The octahedral geometry of $\mathrm{Cu}(I I)$ experiences Jahn-Teller distortions in which either the equatorial bonds are longer than the axial (i.e. compression) or the axial bonds are longer than the equatorial (i.e. elongation; more common), and these deformations are more stable than the regular octahedral geometry. ${ }^{94,95}$ This enhancement of the CFSE explains the exceptional stability of $\mathrm{Cu}(\mathrm{II})$ complexes. $^{84}$

$\mathrm{Cu}(\mathrm{II}): \mathrm{d}^{9}$

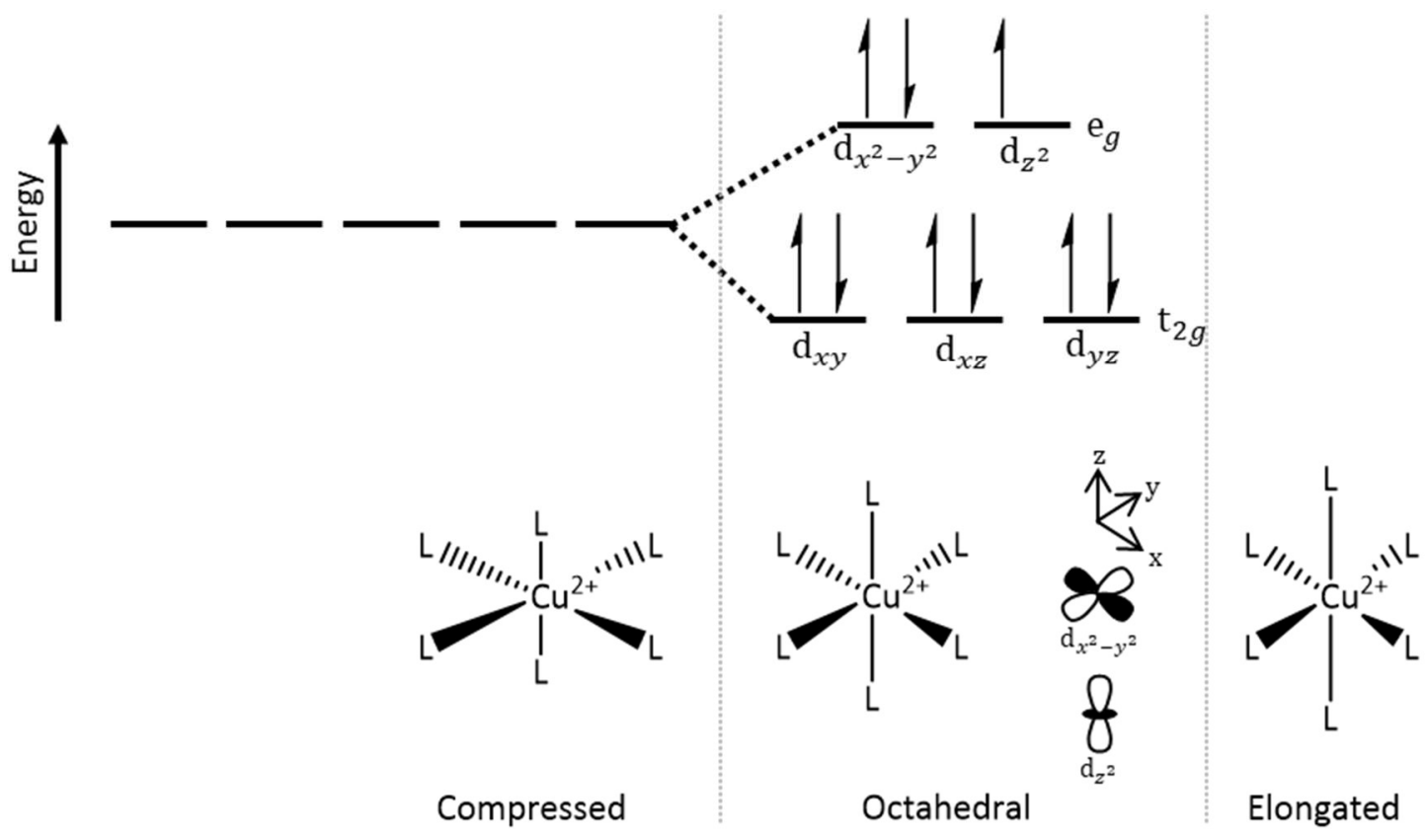

Figure 1.10. Crystal field splitting and structure of $\mathrm{Cu}(\mathrm{II})$ when complexed by ligands (L) in a sixcoordinate, octahedral geometry, which may be elongated or compressed due to Jahn-Teller distortions. ${ }^{96}$

The factors influencing metal complex stability that are related to the ligand include base strength, the chelate effect, chelate ring size, and steric strain. First, as the base strength (i.e. affinity for $\mathrm{H}^{+}$) of a ligand increases, its affinity for hard metal ions increases, and, therefore, the stability of the resultant 
metal complex increases. ${ }^{84}$ Second, the chelate effect is the observation that a metal complex formed with a bidentate or polydentate ligand has a higher $K_{\mathrm{f}}$ than a metal complex formed with an equal number of monodentate ligands containing the same donor atom (Figure 1.11). ${ }^{97,98}$ A monodentate ligand binds a metal ion through one donor atom. When one ligand contains two donor atoms that bind the metal ion, it is called bidentate, and, when three or more donor atoms bind, the ligand is called polydentate. Complexes formed from bidentate and polydentate ligands are referred to as chelate complexes. ${ }^{99}$ Third, the size of the chelate ring also influences stability, and saturated, fivemembered rings form the most stable complexes with first-row d-block metals (Figure 1.12). Finally, when coordinating a metal ion, ligands containing large, bulky groups that interact sterically yield less stable complexes. ${ }^{84}$<smiles>C1CN[C+]2(N1)NCCN2</smiles><smiles>CN[C+](NC)(NC)NC</smiles>

$$
K_{\mathrm{f}}\left[\mathrm{Cd}(\mathrm{en})_{2}\right]^{2+} \quad>\quad K_{\mathrm{f}}\left[\mathrm{Cd}\left(\mathrm{NH}_{2} \mathrm{Me}\right)_{4}\right]^{2+}
$$

Figure 1.11. Example of the chelate effect. Ethylenediamine (en) is bidentate, and methylamine $\left(\mathrm{NH}_{2} \mathrm{Me} ; \mathrm{Me}=\right.$ methyl) is monodentate. ${ }^{97}$
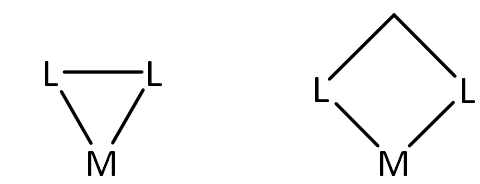

3-membered
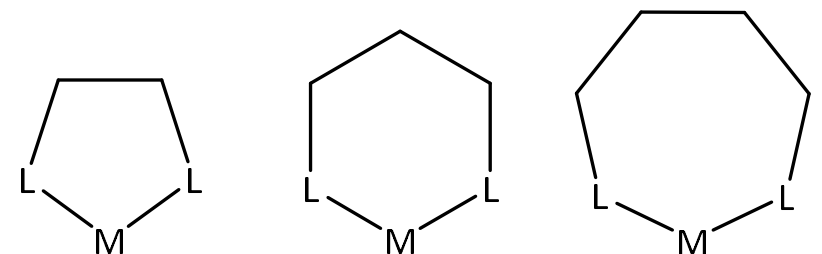

7-membered

Figure 1.12. Trend of stability depending on chelate ring size for first-row $d$-block metals $(M=$ metal, $\mathrm{L}=$ ligand $).{ }^{84}$

An understanding of the factors that influence the stability of a coordination compound is essential for designing and tuning the ligand in this project, as the ligand must be a stronger $\mathrm{Cu}(\mathrm{II})$-chelator than the natural ligands in seawater (i.e. $\left.K_{\mathrm{f}}>10^{14}\right){ }^{80}$ The formation constant of a metal complex can be 
measured via numerous methods, including spectrophotometry, potentiometry, pH metric equilibrium studies, competitive equilibria, cation exchange resins, liquid-liquid partitioning, and nuclear magnetic resonance (NMR) spectroscopy. To determine $K_{\mathrm{f}}$ by spectrophotometric methods, the ultraviolet-visible (UV-Vis) spectra of a series of solutions of the ligand (L) and metal (M), for which the mole ratio $\left(\frac{[L]}{[\mathrm{M}]+[L]}\right)$ varies from zero to one, are measured. ${ }^{100}$ Nowadays, the data are analysed by computer programs, such as the HYPERQUAD suite of programs by Gans et al. ${ }^{101}$, that calculate $K_{\mathrm{f}}$.

Spectrophotometric methods are also used to construct Job plots to determine the stoichiometry of metal complexes (i.e. 1:1 M:L, 1:2 M:L, etc.). This is done by mixing aliquots of two equimolar solutions in such a manner that the mole ratio $\left(\frac{[L]}{[\mathrm{M}]+[L]}\right)$ varies from zero to one but the total analytical concentration of metal plus ligand remains constant $([\mathrm{M}]+[\mathrm{L}]=k, k=$ constant). The absorbance of each solution is measured at a selected wavelength and plotted as a function of the mole ratio. The resultant curve is called a Job plot, and the peak absorbance of the curve reveals the metal:ligand ratio of the complex in solution. For example, as in Figure 1.13, a maximum at 0.5 for the mole ratio indicates that the stoichiometry of the complex is 1:1 metal:ligand. The requirements that must be met for a Job plot to be valid are as follows: conformance to Beer's law, one predominant complex, constant total analytical concentration of metal plus ligand, and constant $\mathrm{pH}$ and ionic strength. ${ }^{102,103}$ For the current project, knowledge of the stoichiometry of the complex in solution is important for planning the formulation of the ligand in a coating, recognising that this stoichiometry might change once the ligand is immobilised in a film.

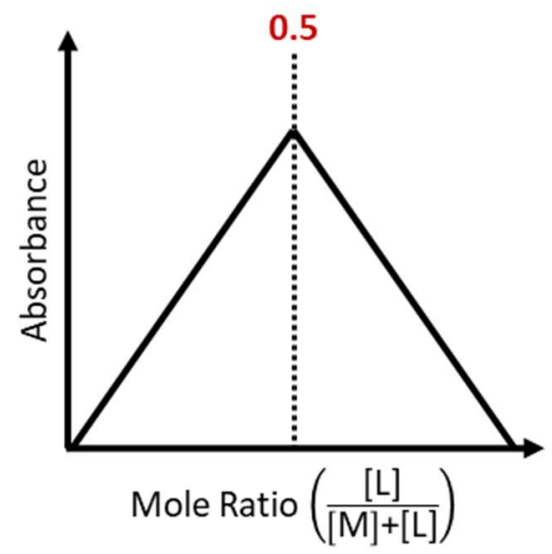

Figure 1.13. Example Job plot of a metal complex with 1:1 metal:ligand stoichiometry. 
Various analytical techniques are employed to further characterise $\mathrm{CU}(\mathrm{II})$ complexes. For instance, infrared (IR) spectroscopy is used to identify functional groups in a complex, and mass spectrometry (MS) measures a coordination compound's mass-to-charge ratio $(\mathrm{m} / \mathrm{z})$. Electronic transitions are observed using UV-Vis spectroscopy. CHNS elemental analysis gives the elemental concentrations of carbon, hydrogen, nitrogen and sulfur, while the $\mathrm{Cu}(\mathrm{II})$ concentration is determined via inductively coupled plasma mass spectrometry (ICP-MS), inductively coupled plasma optical emission spectroscopy (ICP-OES), or scanning electron microscopy/energy dispersive X-ray spectroscopy (SEMEDS). Other common techniques include molar conductivity, magnetic susceptibility, and thermal analyses (e.g. thermogravimetric analysis, differential thermal analysis). X-ray crystallography (XRC) is one of the most useful techniques, as it reveals the structure and geometry of the complex. ${ }^{104-108}$ Accordingly, these are all techniques that can be considered for the characterisation of $\mathrm{Cu}(\mathrm{II})$ complexes in this project. However, the use of NMR spectroscopy is limited due to the paramagnetic nature of $\mathrm{Cu}(\mathrm{II})$. Paramagnetic ions, atoms, or molecules have unpaired electrons (e.g. $\left.\mathrm{d}^{9} \mathrm{Cu}(\mathrm{II})\right)$ and, thus, permanent magnetic moments, causing the broadening of NMR signals. ${ }^{108,109}$

\subsubsection{Formulation of Cu(II) Ligands in Coatings}

Several routes exist for incorporating the $\mathrm{Cu}(\mathrm{II})$ ligand in a coating. As seen in Figure 1.4, a paint is composed of a binder, additives, and pigments carried in a solvent, ${ }^{29}$ and all of these components present an opportunity for functionalisation with the ligand. Although, in the literature, the more popular route seems to be the incorporation of the ligand in the polymeric binder. ${ }^{41,79,87,110,111}$ For example, Trojer et al. ${ }^{87}$ reported methods for affixing pendant azole ligands, which are strong $\mathrm{Cu}(\mathrm{II})$ chelators, to polymers for protection against fouling organisms. El-Wahab et al. ${ }^{112}$ simply add-mixed arylhydrazone ligand o-methoxybenzaldehyde benzoylhydrazone and its metal complexes in a polyurethane coating, and the film containing the $\mathrm{Cu}(\mathrm{II})$ complex had antimicrobial activity against Gram-positive and -negative bacteria and fungi. Evidence also exists that pigments can be functionalised with small molecules. For instance, González-Rodríguez et al. ${ }^{113}$ modified the surface of titanium dioxide with glutaric acid to improve the dispersion of these pigments in waterborne paints. Therefore, these are all routes that can be explored in this project for formulating the $\mathrm{Cu}(\mathrm{II})$ ligand in a coating, but the initial focus should be on the binder. 


\subsubsection{Mechanisms of Cu(II) Surface Toxicity}

In this work, an understanding of the mechanisms of $\mathrm{Cu}(\mathrm{II})$ surface toxicity to marine foulers is vital because it guides the coating design towards more effective antifouling formulations. After centuries of use as a disinfectant, copper and its alloys, being able to kill $99.9 \%$ of pathogenic bacteria in $2 \mathrm{~h}$, were officially recognised in 2008 by the Environmental Protection Agency (EPA) as the first effective metallic antimicrobial agents. ${ }^{114}$ Although copper is an essential trace element in the majority of living organisms, it is toxic at high concentrations. ${ }^{1,115} \mathrm{Cu}(\mathrm{II})$ is considerably less cytotoxic than $\mathrm{Cu}(\mathrm{I})$, but oxidising conditions (e.g. aerobic, aqueous) favour the formation of $\mathrm{Cu}(\mathrm{II}) \cdot{ }^{116,117}$ Copper compounds and elemental copper have a broad spectrum of antimicrobial activity against fungi, viruses, and bacteria by several mechanisms, such as enzyme inhibition (e.g. through iron displacement in ironsulfur clusters), disrupting plasma membrane integrity, degrading DNA, and causing oxidative damage to DNA and proteins by reactive oxygen species (ROS). ${ }^{117,118}$ Upon exposure to copper ions, the likely sequence of events is membrane damage followed by the rapid influx of copper ions and, subsequently, oxidative damage, cell death, and DNA degradation (Figure 1.14). Therefore, the antimicrobial effect of copper surfaces has been linked to their ability to release copper ions, ${ }^{114,115,117,119-121}$ and designing a system that allows the flux of $\mathrm{Cu}(\mathrm{II})$ at the surface is crucial for the success of this project.

A

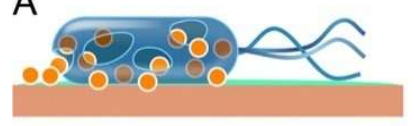

C

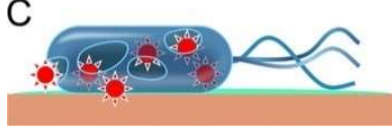

$\mathrm{B}$
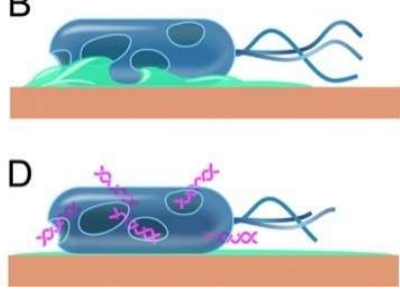

Figure 1.14. Sequence of events upon copper contact: (a) ionic copper causes cell damage, (b) the cell membrane ruptures, leading to the expulsion of cytoplasmic content, (c) ROS are generated and cause further cell damage, and (d) plasmid and genomic DNA are degraded (reproduced with permission: copyright (C 2011, American Society for Microbiology). ${ }^{115}$

The exposure of microfoulers to copper results in a reduction of biofilm biomass, but the effect on macrofoulers is indirect and less understood. In a biofilm, copper is sequestered by components of the EPS, and the copper concentration in the biofilm rises until reaching a plateau. This copper can be retained by the biofilm for up to four weeks and is referred to as the "copper legacy". Macrofoulers seem to be more affected by this copper legacy than by direct exposure to copper: the presence of 
copper changes the structure and composition of the microfouler biofilm and, consequently, macrofouler settlement, as it is mediated by chemical and biological characteristics of the biofilm. Due to differences in copper tolerance, the copper legacy selects for surface colonisation by more coppertolerant species, but this mechanism is not well-understood. ${ }^{122}$ For the current project, knowledge of these toxicity and antifouling mechanisms of $\mathrm{Cu}(\mathrm{II})$ directs the focus of attention on the microfouling stage.

\subsubsection{Strategy}

In light of all of these practical considerations, a multistage strategy emerges for meeting the goals of this project (Table 1.5). In the first stage, a ligand must be chosen based on selectivity and stability. Since $\mathrm{Cu}(\mathrm{II})$ is classified as borderline, the donor atoms should be hard bases, borderline bases, and/or sulfur to ensure the ligand is selective for $\mathrm{Cu}(\mathrm{II})$. The ligand must also be a stronger $\mathrm{Cu}(\mathrm{II})$-chelator than the natural ligands in seawater (i.e. $K_{\mathrm{f}}>10^{14}$ ), and stability may be tuned by altering the ligand's base strength, denticity, chelate ring size, or steric strain. The $\mathrm{Cu}(\mathrm{II})$ complex will be characterised via the discussed methods and analytical techniques (e.g. Job plot, IR, MS, etc.). The second stage of the project will involve formulation of the ligand into a coating by modifying the polymeric binder, additives, or pigments, focusing first on the binder. The third stage will require the development and implementation of methodology to test the ability of the coating to coordinate and retain $\mathrm{Cu}(\mathrm{II})$ and to test the coating's subsequent antimicrobial activity. The results of the antimicrobial assay are intended to be reflective of the $\mathrm{Cu}$ (II) surface toxicity in the microfouling phase of biofouling. These relatively quick tests are important because they serve as the first filters for identifying a lead product. In the fourth and final stage of the project, time-permitting, the antifouling ability of this lead product will be tested in the marine environment.

Table 1.5. Stages of this project.

\section{Stage Description}

$1 \quad$ Ligand chosen based on selectivity and stability

2 Incorporation of the ligand in a coating (binder, additives, pigments)

3

Development and implementation of the methodology to test the coating's $\mathrm{Cu}(\mathrm{II})$ loading and antimicrobial activity

Test the antifouling ability of the lead product in the marine environment 


\section{SElECTION AND SyNTheSiS OF Cu(II) LigANDS}

\subsection{BACKGROUND}

Chapter 1 (Section 1.3.2) discussed general considerations in the design of $\mathrm{Cu}(\mathrm{II})$ ligands (e.g. donor atom selection, chelate ring size, etc.), but, in the present work, the selection or design and synthesis of $\mathrm{Cu}(\mathrm{II})$ ligands must also be guided by the intended application. The application is to imbue a coating with antifouling properties through incorporation of the ligand and subsequent $\mathrm{Cu}(\mathrm{II})$-loading from seawater to the requisite concentration. Therefore, it is essential that the ligand maintain its selectivity, stability, and performance both when it is formulated in a coating and when it is exposed to the marine environment. Furthermore, selection of the ligand should also be considered from the perspective of financial viability. Large quantities of the ligand should be cheaply accessible, meaning that it is synthesised in only a few high-yield steps, ideally, from non-hazardous reagents and solvents (e.g. water). Once synthesised, the product should be able to be easily manipulated for incorporation in a paint. This requires the ligand to have a functionalisable handle, such as an amine or hydroxyl functional group, for possible covalent modification of paint components. For instance, formulation of the ligand in the paint may involve covalent alteration of the paint binder or additives with the ligand, which is a route that could be chosen for numerous reasons, including increased ligandretention in the paint. A ligand with a solubility profile compatible with such manipulations would also be beneficial, since highly insoluble compounds can be more difficult to characterise and modify. Following retention in a coating, the immobilised ligand should perform similarly to its unbound state, ${ }^{123}$ remaining in the proper orientation to chelate $\mathrm{Cu}(\mathrm{II})$, and interaction of the immobilised ligand with the metal ions in seawater is essential.

To be successful in the marine environment, an ideal $\mathrm{Cu}(I I)$-chelator must meet certain criteria. First, the ligand's $\mathrm{Cu}(\mathrm{II})$-selectivity should be retained in the presence of the significantly higher concentrations of alkali and alkaline earth metals in seawater. ${ }^{123}$ As seen in Figure 2.1, the composition of seawater is complex, consisting of solids, gases, colloids, and dissolved solutes. The dissolved solutes are organic and inorganic, and the inorganic solutes consist of major (>1 ppm) and minor $(<1 \mathrm{ppm})$ metals. The major metals in seawater, in order from most to least prevalent, are alkali and alkaline earth metals $\mathrm{Na}, \mathrm{Mg}, \mathrm{Ca}, \mathrm{K}$, and $\mathrm{Sr}$, ranging from $10.76 \mathrm{~g} / \mathrm{kg}$ (= parts per thousand) for $\mathrm{Na}$ to $0.0078 \mathrm{~g} / \mathrm{kg}$ (7.8 ppm) for Sr (Table 2.1). In contrast, $\mathrm{Cu}(2 \mu \mathrm{g} / \mathrm{L} \approx 2 \mathrm{ppb})$, present as $\mathrm{Cu}(\mathrm{II})$, and other transition metals are minor components in seawater (Table 2.2). ${ }^{42}$ So, for this project, the ligand is required to still be selective for $\mathrm{Cu}(\mathrm{II})$ in the presence of all the major and minor metal ions in seawater. As previously discussed (Chapter 1, Section 1.3.2.1), a factor influencing the selectivity and stability of 
metal complexes is the match between the metal ion and donor atom(s) of the ligand, according to the "like prefers like" principle of the HSAB theory. This theory classifies $\mathrm{Cu}(I I)$ as borderline, preferring nitrogen donors. Other transition metal ions in seawater classified as borderline include $\mathrm{Co}(\mathrm{II}), \mathrm{Ni}(\mathrm{II})$, $\mathrm{Zn}(\mathrm{II})$, and $\mathrm{Pb}(\mathrm{II})$. Conversely, alkali and alkaline earth metal ions, such as the major metal ions in seawater (i.e. $\mathrm{Na}(\mathrm{I}), \mathrm{Mg}(\mathrm{II}), \mathrm{Ca}(\mathrm{II}), \mathrm{K}(\mathrm{I})$, and $\mathrm{Sr}(\mathrm{II})$ ), are classified as hard and prefer oxygen donors. The disparity in donor preference between $\mathrm{Cu}(\mathrm{II})$ and the alkali and alkaline earth, as well as some of the transition, metal ions can, thus, be exploited in the design and selection of a $\mathrm{Cu}(\mathrm{II})$-chelator, while additional factors will need to be considered for the other borderline transition metal ions. ${ }^{42,83-85}$

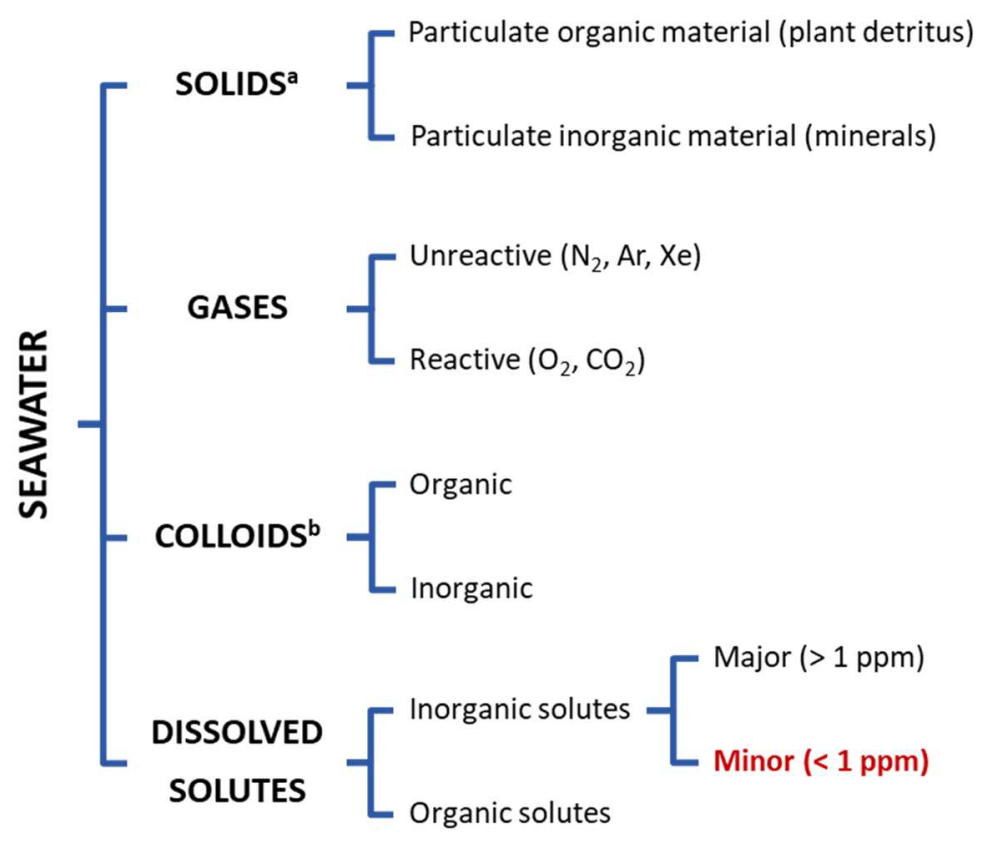

Figure 2.1. Major components of seawater (adapted from Practical Handbook of Marine Science). ${ }^{42}$ ${ }^{a}$ Material that does not pass through a $0.45 \mu \mathrm{m}$ filter. ${ }^{b}$ Passes through a $0.45 \mu \mathrm{m}$ filter but is not dissolved. 
Table 2.1. Major metals in seawater (alkali and alkaline earth metals). ${ }^{a}$

\begin{tabular}{lccc}
\hline \multirow{2}{*}{ Metal } & Probable Species & \multicolumn{2}{c}{${\text { Concentration }(\mathrm{g} / \mathrm{kg})^{\mathrm{b}}}^{\mathrm{b}}$} \\
\cline { 3 - 4 } & & Average & Range \\
\hline $\mathrm{Ca}$ & $\mathrm{Ca}^{2+}$ & 0.4119 & $0.4098-0.4134$ \\
$\mathrm{~K}$ & $\mathrm{~K}^{+}$ & 0.399 & $0.393-0.405$ \\
$\mathrm{Mg}$ & $\mathrm{Mg}^{2+}$ & 1.297 & $1.292-1.301$ \\
$\mathrm{Na}$ & $\mathrm{Na}^{+}$ & 10.76 & $10.72-10.80$ \\
$\mathrm{Sr}$ & $\mathrm{Sr}^{2+}$ & 0.0078 & $0.0074-0.0079$ \\
\hline
\end{tabular}

adapted from Practical Handbook of Marine Science. ${ }^{42}$

${ }^{b}$ Normalised to $3.5 \%$ salinity.

Table 2.2. Minor metals in seawater (>0.01 $\mu \mathrm{g} / \mathrm{L}$; transition metals). ${ }^{\mathrm{a}}$

\begin{tabular}{|c|c|c|c|}
\hline \multirow[t]{2}{*}{ Metal } & \multirow[t]{2}{*}{ Probable Species } & \multicolumn{2}{|c|}{ Concentration $(\mu \mathrm{g} / \mathrm{L}, \approx \mathrm{ppb})^{b}$} \\
\hline & & Average & Range \\
\hline $\mathrm{Ag}$ & $\mathrm{AgCl}_{2}^{-}$ & 0.29 & $0.055-1.5$ \\
\hline $\mathrm{Au}$ & $\mathrm{AuCl}_{2}^{-}$ & 0.068 & - \\
\hline $\mathrm{Cd}$ & $\mathrm{CdCl}_{2}^{-}$ & 0.113 & $0.02-0.25$ \\
\hline Co & $\mathrm{Co}^{2+}, \mathrm{CoCO}_{3}$ & 0.27 & $0.035-4.1$ \\
\hline $\mathrm{Cr}$ & $\mathrm{CrO}_{4}^{2-}$ & 0.3 & $0.23-0.43$ \\
\hline $\mathrm{Cu}$ & $\mathrm{CuCO}_{3}$ & 2 & $0.2-4.0$ \\
\hline $\mathrm{Fe}$ & $\mathrm{Fe}(\mathrm{OH})_{3}$ & 6.6 & $0.1-62$ \\
\hline $\mathrm{Hg}$ & $\mathrm{HgCl}_{4}^{2-}$ & 0.03 & - \\
\hline $\mathrm{Mn}$ & $\mathrm{Mn}^{2+}$ & 1.5 & $0.2-8.6$ \\
\hline Mo & $\mathrm{MoO}_{4}{ }^{2-}$ & 1 & $0.24-12.2$ \\
\hline $\mathrm{Nb}$ & $\mathrm{NbCO}_{3}{ }^{+}$ & 0.01 & $0.01-0.02$ \\
\hline $\mathrm{Ni}$ & $\mathrm{NiCO}_{3}$ & 5.4 & $0.43-43$ \\
\hline $\mathrm{Pb}$ & $\mathrm{PbCO}_{3}$ & 0.06 & $0.02-0.4$ \\
\hline Sc & $\mathrm{Sc}(\mathrm{OH})_{3}$ & 0.04 & - \\
\hline $\mathrm{Ti}$ & $\mathrm{Ti}(\mathrm{OH})_{4}$ & 1 & - \\
\hline V & $\mathrm{HVO}_{4}{ }^{2-}, \mathrm{H}_{2} \mathrm{VO}_{4}^{-}$ & 2.5 & $2.0-3.0$ \\
\hline W & $\mathrm{WO}_{4}^{2-}$ & 0.1 & - \\
\hline Y & $\mathrm{YCO}_{3}{ }^{+}$ & 0.0133 & $0.0112-0.0163$ \\
\hline $\mathrm{Zn}$ & $\mathrm{Zn}^{2+}, \mathrm{ZnOH}^{+}$ & 6.5 & $2-18$ \\
\hline $\mathrm{Zr}$ & $\mathrm{Zr}(\mathrm{OH})_{4}$ & 0.026 & - \\
\hline
\end{tabular}

adapted from Practical Handbook of Marine Science. ${ }^{42}$

${ }^{\mathrm{b}}$ The concentration in $\mu \mathrm{g} / \mathrm{L}$ is approximately equal to the concentration in $\mathrm{ppb}$, since the density of seawater is $1.025 \mathrm{~g} / \mathrm{mL}^{42}$ 
The second requirement to be a successful $\mathrm{Cu}(\mathrm{II})$-chelator in the marine environment is that the ligands and $\mathrm{Cu}(\mathrm{II})$ complexes must be stable in seawater's typical $\mathrm{pH}$ range. The $\mathrm{pH}$ of seawater is dependent on the cycle of dissolved carbon dioxide in seawater: 1) gaseous carbon dioxide in the atmosphere becomes dissolved in seawater, 2) the aqueous carbon dioxide is utilised for photosynthesis by marine plants but also equilibrates with water to produce carbonic acid, dissociating sequentially to form bicarbonate and carbonate ions, 3) solid calcium carbonate is generated and used by marine plants and animals to make shells, and 4) aqueous carbon dioxide is regenerated at greater depths from the oxidative destruction of organic matter. Carbonic acid, a weak acid, and its associated anions, bicarbonate and carbonate ions, act as a pH buffer system and stabilise the ocean pH at approximately 8.1. Although, the pH of deep waters can be as low as 7.5 due to the regeneration of carbon dioxide from the breakdown of organic matter. ${ }^{124,125}$ The state of protonation of donor atoms in the $\mathrm{Cu}(\mathrm{II})$ ligand should be considered, since, for instance, neutral amine groups coordinate $\mathrm{Cu}(\mathrm{II})$ more readily than ammonium salts. But, as seawater is slightly alkaline, this is not as much of a concern. For example, Chouyyok et al. ${ }^{126}$ prepared three nanoporous sorbents with diamine functionalities to chelate the $\mathrm{Cu}(\mathrm{II})$ in natural waters and found that, in seawater, $\mathrm{Cu}(\mathrm{II})$-binding increased with increasing $\mathrm{pH}$ until it was at peak absorption at the $\mathrm{pH}$ of seawater (i.e. 8.1). However, in the present work, the stability of the ligand alone, as well as the stability of its $\mathrm{Cu}$ (II) complex, should also be appraised; in particular, the ligand should be hydrolytically stable at the $\mathrm{pH}$ of seawater and non-toxic. After all, a hydrolytically unstable ligand introducing new, toxic components into seawater from an antifouling paint would unequivocally fail to meet the specifications of the project.

To summarise, in the context of the application for this work, the ideal ligand should:

1) Be cheaply prepared in large quantities,

2) Be easily manipulated for incorporation in a coating,

3) Perform similarly to its unbound state when immobilised in a coating,

4) Retain its $\mathrm{Cu}(\mathrm{II})$-selectivity in the presence of the major and minor metal ions in seawater, and

5) Be hydrolytically stable at $\mathrm{pH} 7.5-8.1$, the $\mathrm{pH}$ range of seawater, and form stable complexes. ${ }^{123}$ 


\subsection{Polyacylhydrazone: Dilevulinoyl Diethylene Glycol (DILEvDEG)/ADIPIC ACID DIHYDRAZIDE (ADH)}

The present study was inspired by previous, foundational work by our research group in which a water-soluble polyacylhydrazone synthesised from dilevulinoyl diethylene glycol (DiLevDEG) and adipic acid dihydrazide (ADH) was successfully incorporated as part of the resin in a commercial acrylic paint (Figure 2.2). This DiLevDEG/ADH polyacylhydrazone was of interest due to its suitability for formulation in waterborne coatings and its derivation from renewable components, making it a "greener" film-forming additive than its petrochemical-derived counterparts. Evaporation of water from a concentrated $(50 \% \mathrm{w} / \mathrm{v})$, aqueous (aq) solution of DiLevDEG/ADH yielded a film that was glassy and hard, yet flexible. Consequently, the aqueous DiLevDEG/ADH polymer solution was used to substitute $30-50 \% \mathrm{w} / \mathrm{w}$ of the crosslinking resin in an acrylic paint, and the resultant coating performed well in standard industry tests for gloss, adherence, and hardness and displayed moderate water resistance. ${ }^{127}$ The group noted that the acylhydrazone functionality $(-\mathrm{C}(=\mathrm{O})-\mathrm{NH}-\mathrm{N}=\mathrm{CR}-)$ of DiLevDEG/ADH had the potential to coordinate $\mathrm{Cu}$ (II) (Figure 2.3) in a five-membered chelate ring by bidentate coordination through the carbonyl oxygen and azomethinic nitrogen atom, inspiring the idea that ligands could be incorporated in paint systems to impart antimicrobial activity following $\mathrm{Cu}(\mathrm{II})$-chelation.

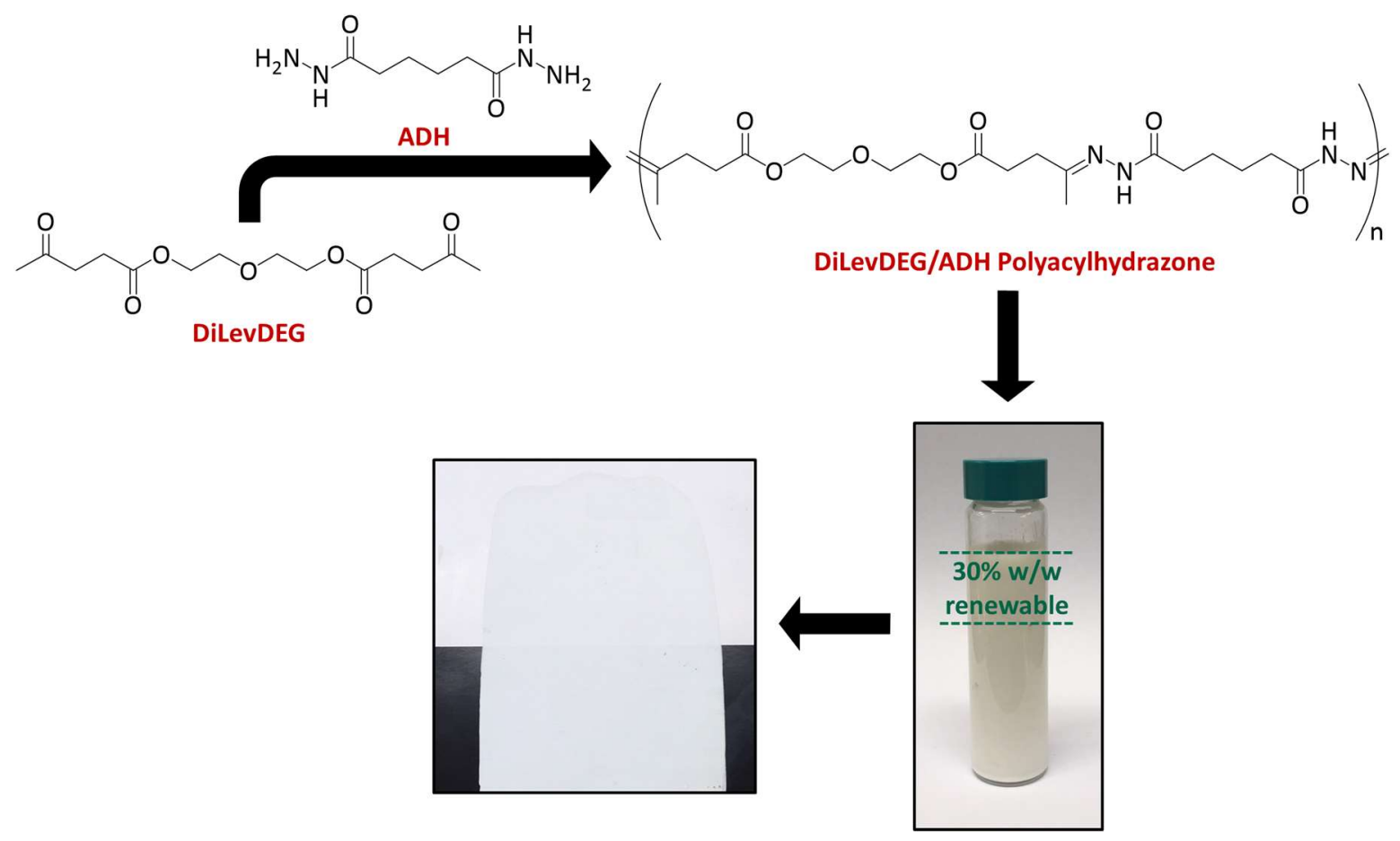

Figure 2.2. The DiLevDEG/ADH polyacylhydrazone, synthesised from DiLevDEG and ADH, substituted $30 \% \mathrm{w} / \mathrm{w}$ of the resin in a commercial acrylic paint with renewable components. ${ }^{127}$ 


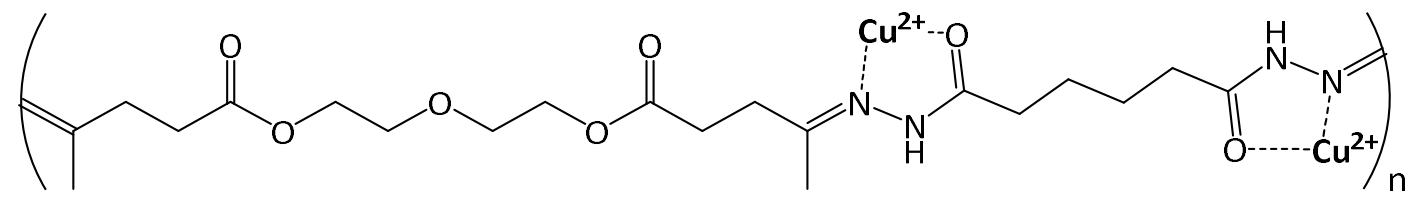

Figure 2.3. Proposed $\mathrm{Cu}(I I)$-chelation by the acylhydrazone functionality in DiLevDEG/ADH.

Since DiLevDEG/ADH had already been successfully formulated into a paint, it seemed reasonable to begin the present work by using this polyacylhydrazone as a proof-of-concept, even though, being a novel polymer, its affinity and selectivity for $\mathrm{Cu}(\mathrm{II})$ were unknown. The first stage of work required the synthesis of DiLevDEG/ADH and confirmation of its ability to chelate $\mathrm{Cu}(\mathrm{II})$. The second stage involved formulation of DiLevDEG/ADH into the commercial acrylic paint and evaluation of the coating's $\mathrm{Cu}(\mathrm{II})$ loading when exposed to a $\mathrm{Cu}(\mathrm{II})$ salt solution and its subsequent antimicrobial activity. The first stage will be discussed below, and the second stage will be discussed in Chapter 3 (Section 3.2).

The DiLevDEG/ADH polyacylhydrazone was synthesised in two steps, following the procedures of Daines et al. ${ }^{127}$ (Scheme 2.1). DiLevDEG was produced in the first step in a Fischer esterification reaction by heating diethylene glycol (DEG), $p$-toluenesulfonic acid (PTSA) monohydrate, and levulinic acid under reduced pressure and collecting the generated water by distillation. Cessation of water production signalled completion of the reaction, and DiLevDEG was isolated as an orange, viscous oil. In the second step, formation of the polyacylhydrazone proceeded by adding ADH to a solution of DiLevDEG in water, giving a clear, viscous solution of $\sim 50 \% \mathrm{w} / \mathrm{v}$ solids.<smiles>OCCOCCO</smiles>

DEG

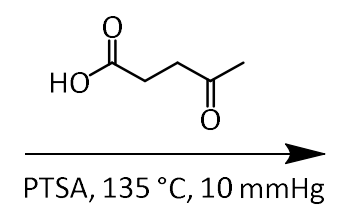<smiles>CCOCCOC(=O)CCC(C)=O</smiles><smiles>NNC(=O)CCCCC(=O)O</smiles><smiles>CCOC(=O)CCC(=O)OCCOCCOC(=O)CCC(=O)N/N=C(/C)CCC(=O)N/N=C(/C)CCC(=O)OCC</smiles>

Scheme 2.1. Synthesis of the DiLevDEG/ADH polyacylhydrazone. 
NMR spectral data provided evidence for the synthesis of DiLevDEG/ADH, being in good agreement with the reference spectra supplied by Daines. ${ }^{127}$ In particular, the carbonyl and methyl regions of the ${ }^{13} \mathrm{C}$ NMR spectrum contained useful signals to confirm generation of the product (Figure 2.4). Upon formation of the polyacylhydrazone, methyl $\mathbf{C 7}$ shifted upfield from $\delta_{c} 30.0 \mathrm{ppm}$ to $\delta_{c} 16.2 / 15.8 \mathrm{ppm}$ (C3). Doubled signals were thought to be due to rotamers caused by hindered rotation around the $\mathrm{C}-\mathrm{N}$ amide bond. The signal at $\delta_{\mathrm{C}} 163.1 \mathrm{ppm}$ was assigned as $\mathrm{C2}$, the carbon in the $\mathrm{C}=\mathrm{N}$ bond, and the signal for DiLevDEG ketone C6, either as the unreacted monomer or end-group, was observed at $\delta_{c} 213.5 \mathrm{ppm}$. MS studies previously conducted in our group revealed that the polymeric structure never terminated with the free hydrazide, and the signal for $\mathbf{C} \mathbf{1}$ was never observed in the ${ }^{13} \mathrm{C}$ NMR spectrum of DiLevDEG/ADH. ${ }^{128}$ Likewise, $\mathbf{C 1}$ was absent from the ${ }^{13} \mathrm{C}$ NMR spectrum of DiLevDEG/ADH prepared in the current study. The large signal for $\mathbf{C} 4$ at $\delta_{c} 175.3 \mathrm{ppm}$ was due to overlap with a signal attributed to a C5 rotamer. ${ }^{128}$ The ${ }^{13} \mathrm{C}$ NMR data supported the formation of the acylhydrazone functionality, which was then tested for its ability to chelate $\mathrm{Cu}(\mathrm{II})$.

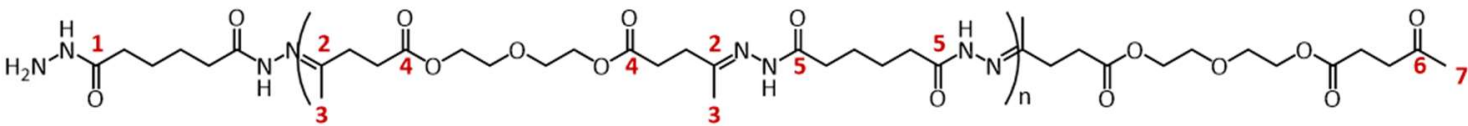

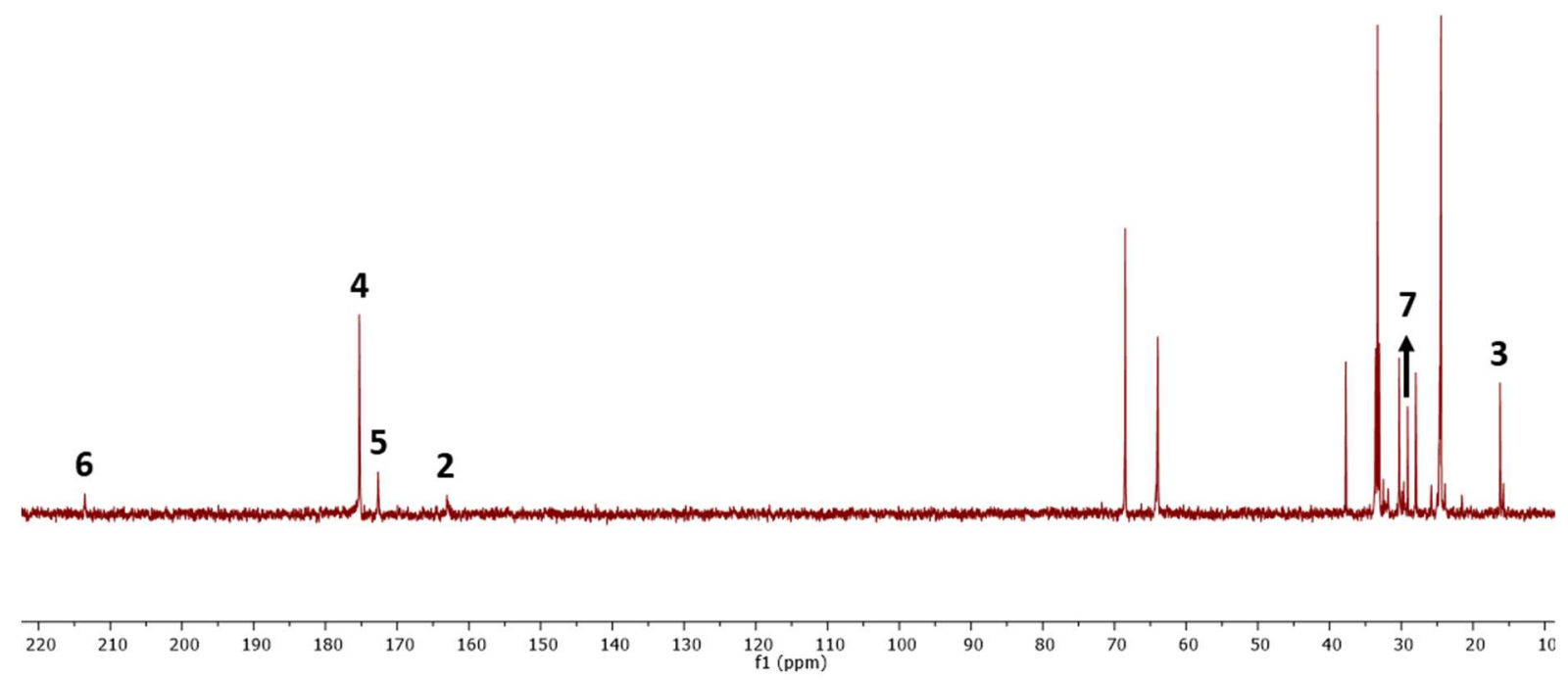

Figure 2.4. ${ }^{13} \mathrm{C}$ NMR spectrum (crude; $\mathrm{D}_{2} \mathrm{O}$ ) of the DiLevDEG/ADH polyacylhydrazone with select carbon assignments. 
Following the addition of the $\mathrm{Cu}(\mathrm{II})$ salt, the vibrant colour change of the DiLevDEG/ADH solution was indicative of $\mathrm{Cu}(\mathrm{II})$-coordination by the polyacylhydrazone. Once copper(II) nitrate was added, the pale-yellow, aqueous solution of DiLevDEG/ADH changed to a teal colour (Figure 2.5). The striking, characteristic colours of transition metal complexes are due to $d-d$ transitions, which involve the excitation of an electron from a lower-energy to a higher-energy d-orbital (i.e. $t_{2 g} \rightarrow e_{g}$ ) by a photon containing energy equal to the difference between the two d-orbitals $\left(\Delta_{0}\right)$. This difference often falls in the visible region of the electromagnetic spectrum. For example, $\mathrm{Cu}(\mathrm{II})$ has a total of nine electrons in its d-orbitals (Chapter 1, Figure 1.10), and, when one of the electrons in a lower-energy d-orbital absorbs energy in the visible region from a photon and is promoted, the observed colour of the $\mathrm{Cu}(\mathrm{II})$ complex is from the transmitted wavelengths. ${ }^{93,129}$ UV-Vis spectroscopy is used to record this absorption for $d-d$ transitions in transition metal complexes. Accordingly, the UV-Vis spectra of aqueous solutions of DiLevDEG/ADH, copper(II) nitrate, and DiLevDEG/ADH post-Cu(II)-addition (Cu(II)-DiLevDEG/ADH) were measured.

(a)

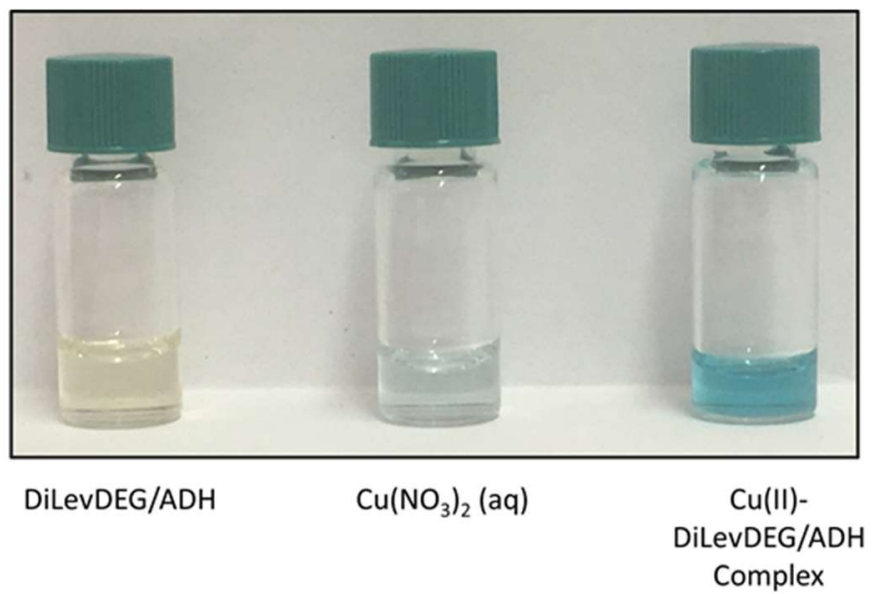

(b)

$\mathrm{Cu}(\mathrm{II}): \mathrm{d}^{9}$

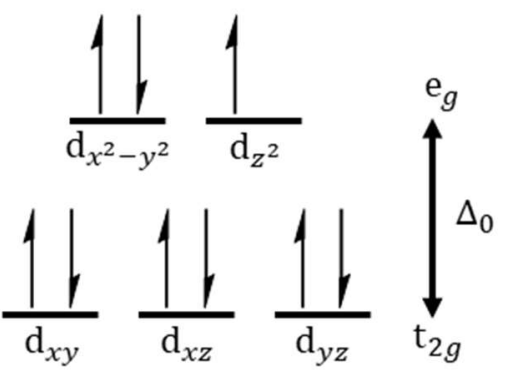

Figure 2.5. Relationship between the colour of $\mathrm{Cu}(\mathrm{II})$ complexes and the electron configuration of

$\mathrm{Cu}(\mathrm{II})$ : (a) colour change of the aq DiLevDEG/ADH solution upon the addition of $\mathrm{Cu}\left(\mathrm{NO}_{3}\right)_{2}$, which is caused by (b) $d-d$ transitions ( $t_{2 g} \rightarrow \mathrm{e}_{g} ; \Delta_{0}=$ crystal field splitting)..$^{93}$ 
The UV-Vis spectra provided evidence of $\mathrm{CU}(\mathrm{II})$-chelation by the DiLevDEG/ADH polyacylhydrazone (Figure 2.6). An aqueous solution of DiLevDEG/ADH absorbed at lower wavelengths, beginning around $420 \mathrm{~nm}$ (i.e. violet light), in its spectrum, which accounted for the pale-yellow colour of the solution. Copper(II) nitrate (aq) absorbed red light with $\lambda_{\max } \geq 800 \mathrm{~nm}$, appearing pale-blue. Upon the addition of copper(II) nitrate, the aqueous DiLevDEG/ADH solution absorbed at both higher $\left(\lambda_{\max }=782 \mathrm{~nm}\right)$ and lower (shoulder starting below $\sim 470 \mathrm{~nm}$ ) wavelengths; the absorption of red/orange light with $\lambda_{\max }=782 \mathrm{~nm}$ was due to $\mathrm{d}$-d transitions in the complex. ${ }^{130}$ Blue/green light $(\sim 500 \mathrm{~nm})$ was transmitted, and, thus, $\mathrm{Cu}(\mathrm{II})$-DiLevDEG/ADH appeared to be a teal colour. Therefore, the UV-Vis data and observations of a colour change after $\mathrm{Cu}(\mathrm{II})$-addition substantiated the hypothesised $\mathrm{Cu}(\mathrm{II})$ coordination by DiLevDEG/ADH and completed the first stage of work for evaluating this polyacylhydrazone as a proof-of-concept. The second stage, involving formulation into a paint and subsequent performance testing, is addressed in the next chapter (Chapter 3, Section 3.2).

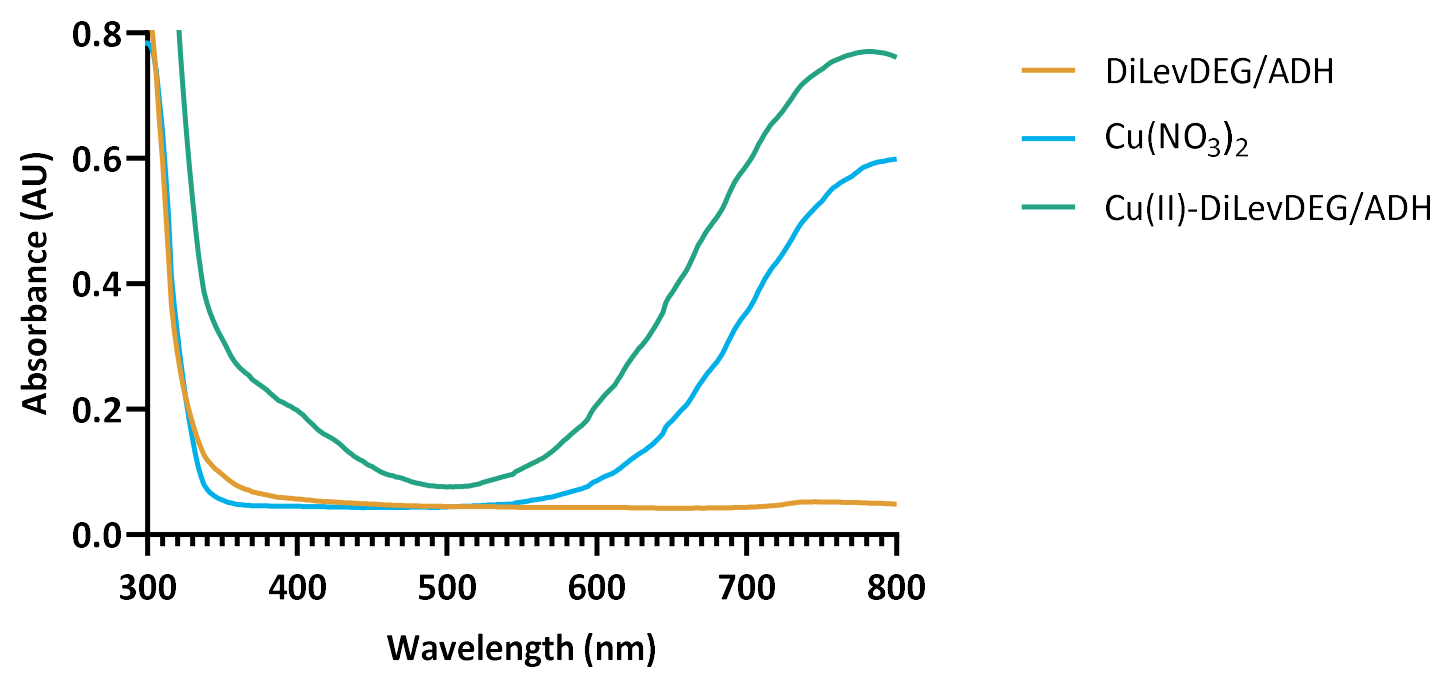

Figure 2.6. UV-Vis spectra of DiLevDEG/ADH, $\mathrm{Cu}\left(\mathrm{NO}_{3}\right)_{2}$, and $\mathrm{Cu}(\mathrm{II})$-DiLevDEG/ADH. 


\subsection{SIMPLE ACYLHYDRAZONES}

In light of the demonstrated ability of a polyacylhydrazone to coordinate $\mathrm{Cu}(\mathrm{II})$ and its successful formulation into a paint, acylhydrazones were proposed as a candidate ligand class to be evaluated for suitability in antifouling coatings. There is evidence in the literature of transition metalacylhydrazone complexes possessing antimicrobial activity, even when incorporated in a coating. For instance, in a study by El-Wahab ${ }^{131}$, acylhydrazone ligand $o$-hydroxyacetophenone benzoylhydrazone (HBH; Figure 2.7a) and its complexes with $\mathrm{Co}(\mathrm{II}), \mathrm{Cr}(\mathrm{III})$, and Fe(III) were added (1-2\% w/w) to an epoxy resin. These coatings were antifouling and displayed antimicrobial activity against Gram-positive and Gram-negative bacteria and fungi, with the metal complexes having higher activities than uncoordinated $\mathrm{HBH}$. The coating additives had no effect on the flexibility, hardness, or adhesion of the epoxy resin. Furthermore, there is also evidence in the literature of $\mathrm{Cu}(\mathrm{II})$-acylhydrazone complexes, specifically, having comparable antimicrobial activities to complexes with other transition metals. For example, Rodríguez-Argüelles et al. ${ }^{132}$ synthesised and characterised complexes of an acylhydrazone ligand, 2-thiophenecarbonyl hydrazone of 3-isatin $\left(\mathrm{H}_{2} \mathrm{~L}^{1}\right.$; Figure $\left.2.7 \mathrm{~b}\right)$, bound to $\mathrm{Zn}(\mathrm{II})$, $\mathrm{Cu}(\mathrm{II}), \mathrm{Ni}(\mathrm{II})$, and $\mathrm{Co}(\mathrm{II})$, and $\left[\mathrm{Cu}\left(\mathrm{HL}^{1}\right)_{2}\right]$ had antibacterial activity comparable to $\left[\mathrm{Zn}\left(\mathrm{HL}^{1}\right)_{2}\right],\left[\mathrm{Ni}\left(\mathrm{HL}^{1}\right)_{2}\right]$, and $\left[\mathrm{Co}\left(\mathrm{HL}^{1}\right)_{2}\right]$ against both Gram-positive and Gram-negative bacteria. Therefore, considering the work with DiLevDEG/ADH in the present study and the literature evidence of $\mathrm{Cu}(\mathrm{II})$-coordinated acylhydrazones having antimicrobial activity, a series of monoacylhydrazones and acyldihydrazones, as well as copper complexes of particular ligands of interest, were synthesised and characterised to better understand the chemical and physical properties of acylhydrazones.

(a)<smiles>C/C(=N\NC(=O)c1ccccc1)c1ccccc1O</smiles>

(b)

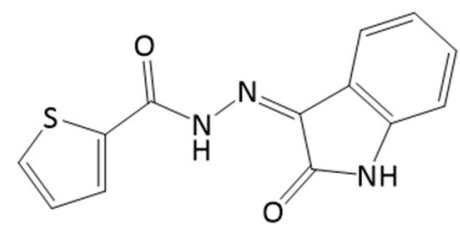

Figure 2.7. Structures of acylhydrazone ligands (a) $\mathrm{HBH}$ and (b) $\mathrm{H}_{2} \mathrm{~L}^{1}$.

\subsubsection{Synthesis}

The acylhydrazones in Scheme $\mathbf{2 . 2}$ were synthesised in carbonyl addition reactions, following the facile, general procedure by Zha and You ${ }^{133}$ in which the hydrazide was added to a methanol or ethanol 
solution containing the aldehyde or ketone. A catalytic amount of glacial acetic acid was then added dropwise to adjust to $\mathrm{pH} 4-5$, and the reaction mixture was heated to reflux temperature. Hydrazone formation progressed via protonation of the carbonyl oxygen of the aldehyde or ketone $(-R C=\underline{0})$ and subsequent nucleophilic attack at its electrophilic carbonyl carbon $(-R \underline{C}=0)$ by the primary amine of the hydrazide $\left(-\mathrm{C}(=\mathrm{O})-\mathrm{NH}-\mathrm{NH}_{2}\right) \cdot{ }^{134}$ Loss of water and formation of the $\mathrm{C}=\mathrm{N}$ double bond yielded the acylhydrazone product as a white or yellow precipitate, which was isolated by vacuum filtration and washed.

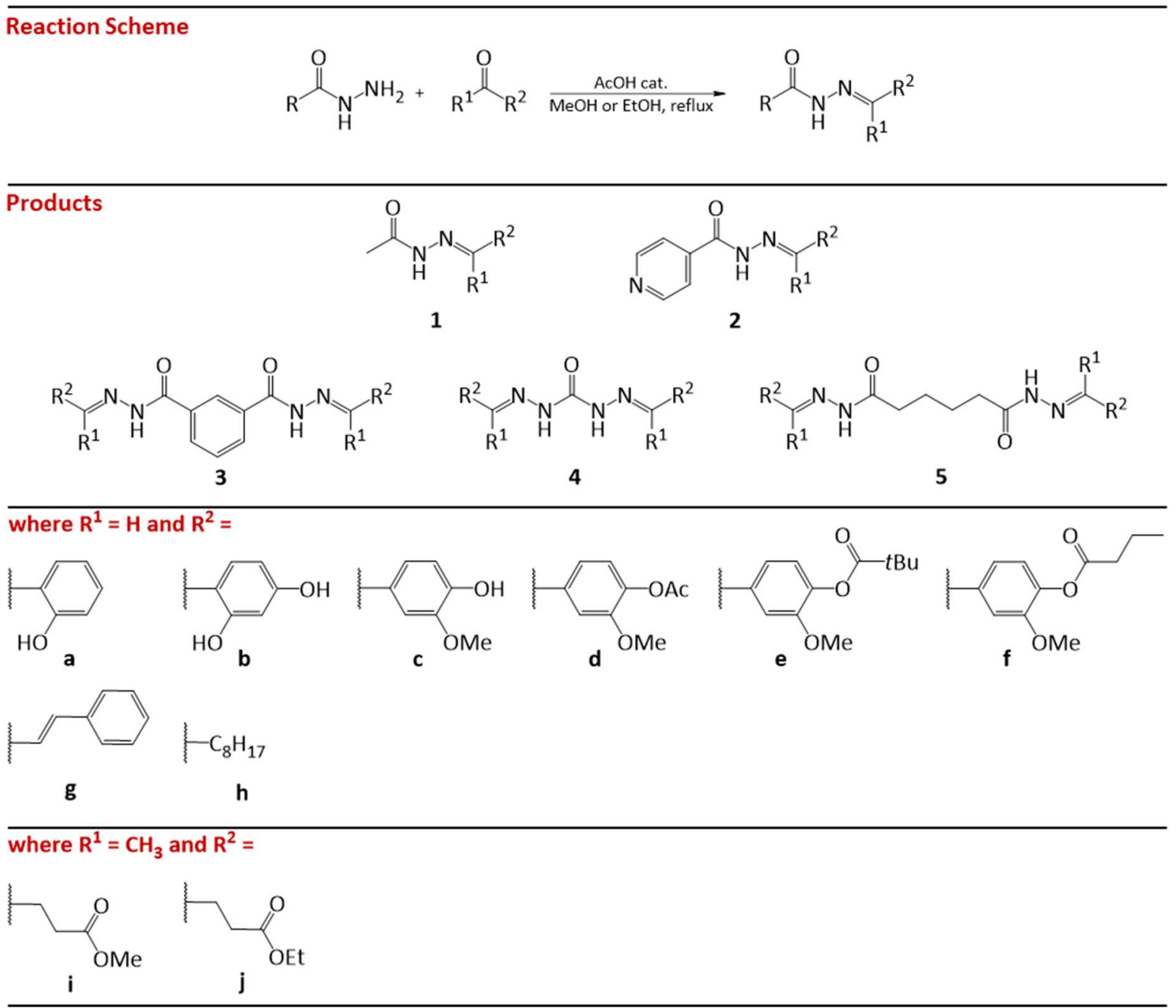

Scheme 2.2. General scheme for the synthesis of acylhydrazones (top; $R, R^{1}, R^{2}$ : same or different). The synthesised acylhydrazones are as follows: $\mathbf{1 a}-\mathbf{b}, \mathbf{1 i - j}, \mathbf{2 a - b}, \mathbf{3 c}-\mathbf{f}, \mathbf{3 h}, \mathbf{4 c}, \mathbf{4 g}, \mathbf{5 c}$, and $\mathbf{5 h}-\mathbf{j}$.

Successful preparation of acylhydrazones $\mathbf{1 a}-\mathbf{b}, \mathbf{1 i}-\mathbf{j}, \mathbf{2 a}-\mathbf{b}, \mathbf{3 c}-\mathbf{f}, \mathbf{3 h}, \mathbf{4 c}, \mathbf{4 g}, \mathbf{5 c}$, and $\mathbf{5 h}-\mathbf{j}$ was confirmed by high-resolution mass spectrometry (HRMS) and NMR spectroscopy, and these data, as well as 
reaction yields, are reported in the Experimental (Section 6.2.2.2). The NMR spectra are included in the Appendix (Section 8.1). In general, synthesis was evidenced by distinct acylhydrazone resonance $(-\mathrm{C}(=\mathrm{O})-\mathrm{NH}-\mathrm{N}=\underline{\mathrm{C}} \mathrm{R}-)$ in the ${ }^{13} \mathrm{C}$ NMR spectrum around $\delta_{\mathrm{C}} 140-155 \mathrm{ppm}$ and resonance in the ${ }^{1} \mathrm{H}$ NMR spectrum consistent with the amide proton $(-\mathrm{C}(=\mathrm{O})-\mathrm{N} \underline{\mathrm{H}}-\mathrm{N}=\mathrm{CR}-)$ around $\delta_{\mathrm{H}} 10-12 \mathrm{ppm}$ and $\delta_{H}$ 9-10 ppm for aldehyde- and ketone-derived acylhydrazones, respectively. Typically, exchange of the amide proton in the ${ }^{1} \mathrm{H}$ NMR spectra of the acylhydrazones was not observed, since the majority were measured in an aprotic solvent. Acylhydrazones $\mathbf{1 j}, \mathbf{3 d} \mathbf{d} \mathbf{f}, \mathbf{3} \mathbf{h}$, and $\mathbf{5 h}$ were novel compounds.

\subsubsection{Isomers}

It is known that acylhydrazones can exist in several geometric and conformational forms. The possible geometric $(E / Z)$-isomerism with respect to the $\mathrm{C}=\mathrm{N}$ double bond and conformational $\left(E^{\prime} / Z^{\prime}\right)$-isomerism from hindered rotation around the $\mathrm{C}-\mathrm{N}$ amide bond can result in four different configurations (EE', $E Z^{\prime}, Z E^{\prime}, Z Z^{\prime}$; Figure 2.8), which have been previously reported. ${ }^{135-139}$ Rotamers (i.e. ( $\left.{ }^{\prime} / Z^{\prime}\right)$-isomers) are observed as doubled ${ }^{1} \mathrm{H}$ and ${ }^{13} \mathrm{C}$ NMR signals at a probe temperature of $30{ }^{\circ} \mathrm{C}$ that, subsequently, coalesce at $90{ }^{\circ} \mathrm{C}$ (Figure 2.9). In the present study, peak assignment in the NMR spectra of both aldehyde- and ketone-derived acylhydrazones, specifically of $\mathbf{1 a - b}, \mathbf{2} \mathbf{a}-\mathbf{b}, \mathbf{5} \mathbf{c}$, and $\mathbf{5 h}-\mathbf{j}$ (Appendix, Tables 8.1-2, 8.5-6, 8.14-17), was often complicated by the presence of rotamers. Although, not every possible conformational or geometric isomer was seen in the NMR spectra of every acylhydrazone: isomers are favoured for multiple reasons, including intramolecular and intermolecular hydrogen bonds and experimental conditions (e.g.temperature, solvent). For example, the $(E)$-isomer of $\mathbf{1} \mathbf{a}-\mathbf{b}$ and $\mathbf{2} \mathbf{a}-\mathbf{b}$ could theoretically be stabilised by an intramolecular $\mathrm{N} \cdots \mathrm{H}-\mathrm{O}$ hydrogen bond, making it favoured over the (Z)-isomer (Figure 2.10). ${ }^{140-142}$<smiles>[R]C=NN([2H])C([R])=O</smiles><smiles>[R]C=NN([2H])C([R1])=O</smiles><smiles>[R]C(=O)N([2H])N=C([R])[R]</smiles><smiles>[R]C(=O)N([Z])N=C([R1])[Z20]</smiles>

Figure 2.8. Possible geometric (E/Z)-isomers and $\left(E^{\prime} / Z^{\prime}\right)$-rotamers of acylhydrazones (adapted from Daines et $\left.a l^{127}\right)$. 

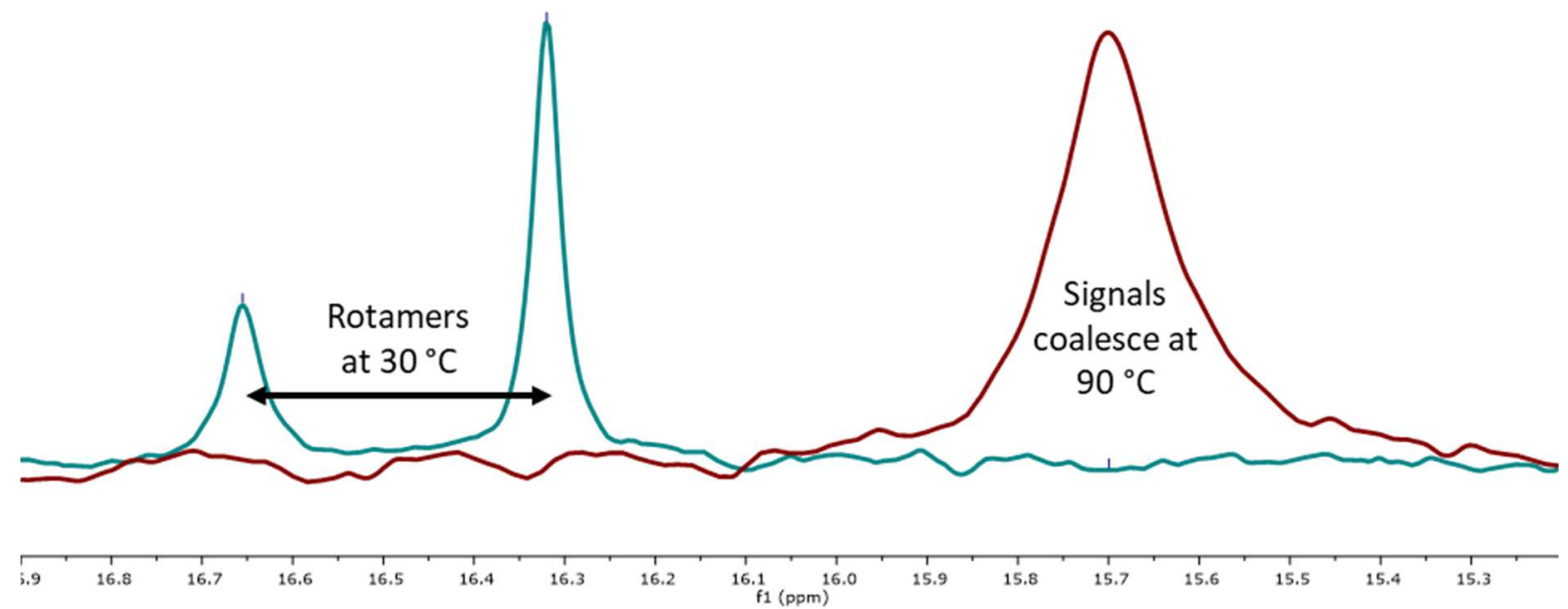

Figure 2.9. Signals attributed to rotamers in a ${ }^{13} \mathrm{C}$ NMR spectrum (5i: $\mathbf{C 1}$ ).

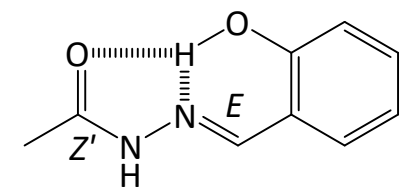<smiles></smiles>

Figure 2.10. Examples of intramolecular hydrogen bonding in monoacylhydrazone 1a (one geometric isomer $(E)$ and $\left(E^{\prime} / Z^{\prime}\right)$-rotamers). ${ }^{141,142}$

$\left(E^{\prime} / \mathrm{Z}^{\prime}\right)$-Rotamers, but only one geometric isomer, were observed in the ${ }^{1} \mathrm{H}$ and ${ }^{13} \mathrm{C} N \mathrm{NR}$ spectra of both aldehyde- and ketone-derived acylhydrazones. For instance, regarding ADH- and aromatic aldehydederived acyldihydrazone 5c (Figure 2.11), two signals in the ${ }^{13} \mathrm{C}$ NMR spectrum in a ratio of approximately 1:1 that were assigned as the hydrazone carbonyl resonances, $\mathbf{C} \mathbf{1}^{\prime}$, appeared at $\delta_{c} 173.9$ and $168.1 \mathrm{ppm}$ (probe temperature $30^{\circ} \mathrm{C}$; Appendix, Figure 8.16); these merged to a single resonance observed at $\delta_{\mathrm{c}} 173.4 \mathrm{ppm}$ when spectra were obtained at a probe temperature of $90{ }^{\circ} \mathrm{C}$. Likewise, the two ${ }^{1} \mathrm{H}$ NMR signals at $\delta_{\mathrm{H}} 11.14$ and $11.02 \mathrm{ppm}$ for the amide $\mathrm{N}-\mathrm{H}$ proton coalesced at $\delta_{\mathrm{H}} 10.69 \mathrm{ppm}$ at $90{ }^{\circ} \mathrm{C}$. These signals were, therefore, attributed to the $\left(E^{\prime} / Z^{\prime}\right)$-rotameric forms of $\mathbf{5 c}$. 
Only one geometric isomer was observed, and it was assigned as being in the $(E)$-configuration with respect to the $\mathrm{C}=\mathrm{N}$ moiety, since it is noted in the literature that, generally, acylhydrazones derived from aldehydes adopt the (E)-configuration. ${ }^{136,139,143}$ Furthermore, acylhydrazones, like $5 c$, synthesised from aromatic aldehydes are known to prevailingly exist as the $(E)$-isomer due to steric hindrance. ${ }^{136,137,139}$

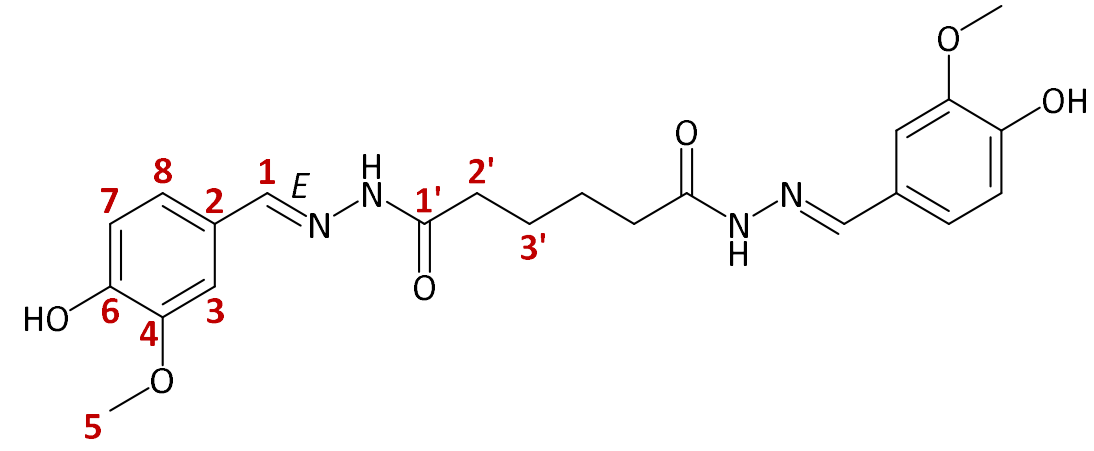

Figure 2.11. Aldehyde-derived acyldihydrazone 5c.

Similar results to those obtained for $\mathrm{ADH}$ - and aldehyde-derived acyldihydrazone $\mathbf{5 c}$ were observed for an $\mathrm{ADH}$ - and ketone-derived acyldihydrazone: $\mathbf{5 i}$ (Figure 2.12). Rotamers were observed as a doubling of ${ }^{13} \mathrm{C}$ NMR signals in a $2: 1$ ratio at $30^{\circ} \mathrm{C}$, and coalescence of the doubled signals was observed when spectra were recorded at $90{ }^{\circ} \mathrm{C}$. For example, the doubled ${ }^{13} \mathrm{C}$ NMR signals at $\delta_{\mathrm{c}} 16.7$ (minor) and 16.3 (major) ppm for the hydrazone methyl groups (C1; Figure 2.9; Appendix, Figure 8.18) and $\delta_{c} 51.2$ (minor) and 51.1 (major) ppm for the methoxy groups (C6) collapsed into single signals at $\delta_{\mathrm{c}} 15.7 \mathrm{ppm}$ and $\delta_{\mathrm{c}} 50.6 \mathrm{ppm}$, respectively. Evidence for the location of the bonds involved in generating rotamers was provided by the chemical shift difference $(\Delta \delta)$ between the major and minor resonances attributed to rotameric forms of each carbon in the ${ }^{13} \mathrm{C} N \mathrm{NR}$ spectrum, as shown in Figure 2.12 and Table 2.3. The difference in the chemical shift between rotamer signals was greater closer to the $\mathrm{C}-\mathrm{N}$ amide bond, which is the bond of hindered rotation generating rotamers (e.g. $\Delta \delta_{\mathrm{c}} 6.2 \mathrm{ppm}$ for $\mathbf{C 1}^{\prime}$ ). Again, only one predominant geometric isomer was observed, and the chemical shift data were consistent with the $(E)$-configuration. For instance, in a study by Palla et al. ${ }^{139}$, the chemical shift of a methyl carbon analogous to $\mathbf{C 1}$ was approximately $\delta_{c} \sim 17 \mathrm{ppm}$ in the $(E)$-configuration but $\delta_{c} \sim 25 \mathrm{ppm}$ in the (Z)-configuration. In the present study, the chemical shift of C1 was $\delta_{c} 16.3 \mathrm{ppm}$, suggesting the likely presence of the $(E)$-isomer. Additionally, it is reported that, for acylhydrazones, the (Z)-isomer is thermodynamically less-favoured without stabilisation, such as through intramolecular hydrogen bonding. ${ }^{144,145}$ 


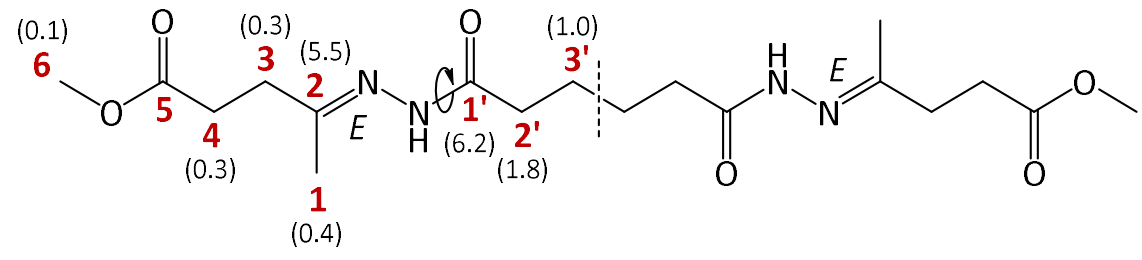

Figure 2.12. Ketone-derived acyldihydrazone $\mathbf{5 i}\left(\Delta \delta_{c}\right.$ between major and minor species at $30{ }^{\circ} \mathrm{C}$ in parentheses; adapted from Daines et al. ${ }^{127}$ ).

Table 2.3. ${ }^{13} \mathrm{C}$ NMR data for $\mathbf{5 i}$ (refer to Figure 2.12 above; adapted from Daines et al. ${ }^{127}$ ).

\begin{tabular}{lccc}
\hline Carbon & $\delta_{c}$ Major $(p p m)$ & $\delta_{c}$ Minor $(p p m)$ & $\Delta \delta_{c} \mid$ Major - Minor $\mid(p p m)$ \\
\hline $1^{\prime}$ & 174.5 & 168.3 & 6.2 \\
\hline $2^{\prime}$ & 32.0 & 33.8 & 1.8 \\
\hline $3^{\prime}$ & 24.1 & 25.1 & 1.0 \\
\hline 1 & 16.3 & 16.7 & 0.4 \\
\hline 2 & 149.9 & 155.4 & 5.5 \\
3 & 32.7 & 33.0 & 0.3 \\
4 & 29.4 & 29.7 & 0.3 \\
\hline 5 & 172.8 & - & - \\
\hline 6 & 51.1 & 51.2 & 0.1 \\
\hline
\end{tabular}

\subsubsection{Solubility}

In general, the acylhydrazone products were only soluble in dipolar aprotic solvents, such as dimethylformamide (DMF) and dimethylsulfoxide (DMSO). However, this solubility profile was undesirable due to the ability of these solvents to form complexes with $\mathrm{Cu}(\mathrm{II})$ and the complications to further analysis, manipulation, and development. As previously discussed, an ideal ligand should be easily manipulated for formulation in a coating, and highly insoluble compounds do not meet this requirement. The derivatisation of vanillin by acylation with acetic, trimethylacetic, and butyric anhydride (d, e, and $\mathbf{f}$, respectively) was explored as a method of altering the solubility profile of acylhydrazone 3c. Yet, the solubility profiles of products $\mathbf{3 d}, \mathbf{3 e}$, and $\mathbf{3 f}$ did not differ from underivatised 3c (Scheme 2.2): they were all only soluble in DMF and DMSO. The acylhydrazones with more desirable solubility profiles were monoacylhydrazone $\mathbf{1 a}$ and acyldihydrazone $\mathbf{3 h}$ (both soluble in chloroform containing $10 \% \mathrm{v} / \mathrm{v}$ methanol; Figure $\mathbf{2 . 1 3 a , b}$ ) and monoacylhydrazones $\mathbf{1} \mathbf{i}-\mathbf{j}$ (soluble in chloroform; Figure 2.13c,d). Efforts were focused on 1a, which is better-known in the literature than 
3h, $\mathbf{1 i}$, and $\mathbf{1 j}$. It was thought that, since $\mathbf{1 a}$ and its $\mathrm{Cu}(\mathrm{II})$ complex are already well-characterised, the methodology used in this study for characterisation of $\mathrm{Cu}(\mathrm{II})$ complexes could be validated by comparing results to those reported in the literature before progressing to lesser-known or novel ligands. Therefore, 1a served as a representative acylhydrazone ligand, and experiments were performed to characterise its $\mathrm{Cu}(\mathrm{II})$ complex.

(a)<smiles>CC(=O)N/N=C/c1ccccc1O</smiles>

(c)<smiles>COC(=O)CC/C(C)=N/NC(C)=O</smiles>

(b)

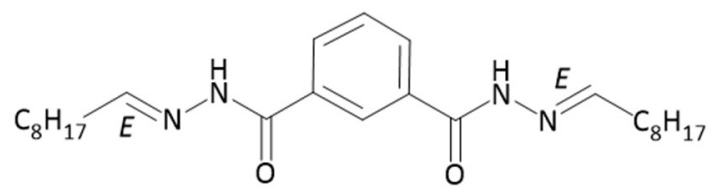

(d)

Figure 2.13. Ligands (a) $1 \mathrm{a}$, (b) $3 \mathrm{~h},(\mathrm{c}) \mathbf{1 i}$, and (d) $1 \mathbf{j}$.

\subsubsection{Cu(II)-Ligand Complexes}

Previous research has assessed the $\mathrm{Cu}(\mathrm{II})$-chelating ability of monoacylhydrazone $\mathbf{1 a}$ and the characteristics and antimicrobial activity of its $\mathrm{Cu}(\mathrm{II})$ complex. For instance, the geometry, stoichiometry, and formation constant $\left(K_{\mathrm{f}}\right.$; Equation 2.1) of the $\mathrm{Cu}(\mathrm{II})-\mathbf{1 a}$ complex have been reported. ${ }^{146,147}$ Furthermore, Yousef Ebrahimipour et al. ${ }^{148}$ demonstrated that $1 \mathrm{a}$ and the mixed-ligand $\mathrm{Cu}(\mathrm{II})$ complex with 1a and phenanthroline have antimicrobial activity against Escherichia coli, Staphylococcus aureus, Pseudomonas aeruginosa, and Candida albicans, with the $\mathrm{Cu}(\mathrm{II})$ complex being more active. In the present work, the $\mathrm{Cu}(\mathrm{II})-1 \mathrm{a}$ complex was characterised by employing the techniques described in Chapter 1 (Section 1.3.2.2; e.g. MS, UV-Vis, Job plot, $K_{\mathrm{f}}$ ). Unfortunately, the paramagnetic nature of $\mathrm{Cu}(\mathrm{II})$ precluded the use of NMR spectroscopy as a convenient analytical tool for characterisation. Method validation involved the generation of a Job plot to determine the metal:ligand stoichiometry and involved the attempted calculation of the $K_{f}$ of the $\mathrm{Cu}(\mathrm{II})$ complex. Since these are both known for $\mathbf{1 a}$, agreement between reported values in the literature and measured values in this study would allow for confidence when employing the same processes for novel ligands. 


$$
M+L \rightleftharpoons M L \quad K_{f}=\frac{[M L]}{[M][L]}
$$

Equation 2.1. The formation constant $\left(K_{\mathrm{f}}\right)$ of a metal-ligand complex $(\mathrm{M}=$ metal, $\mathrm{L}=$ ligand $)$.

For MS analysis, the $\mathrm{Cu}(\mathrm{II})-1 \mathrm{a}$ complex was prepared by adding one equivalent of copper(II) chloride to ligand 1a, a light yellow solid, in methanol. The green, precipitated complex was isolated and analysed by electrospray ionisation (ESI)-HRMS. ESI-HRMS showed the formation of a complex with a molecular formula consistent with a 1:1 $\mathrm{Cu}(\mathrm{II})$ : ligand complex minus a proton, giving the complex $\mathrm{a}+1$ charge (calcd for $\mathrm{C}_{9} \mathrm{H}_{9} \mathrm{CuN}_{2} \mathrm{O}_{2}[\mathrm{M}-\mathrm{H}]^{+}$239.9960; found 239.9963).

As shown in Figure 2.14, a Job plot was constructed for the $\mathrm{Cu}(\mathrm{II})-\mathbf{1 a}$ complex. This was done by combining aliquots of equimolar solutions of $1 \mathrm{a}$ and copper(II) nitrate in 9:1 v/v chloroform:methanol in a manner that kept the total number of moles constant (moles $1 \mathrm{a}+$ moles $\mathrm{Cu}\left(\mathrm{NO}_{3}\right)_{2}=k$ ) while varying the metal:ligand molar ratio. The addition of tetrabutylammonium nitrate $(0.1 \mathrm{M})$ ensured a constant ionic strength, and the absorbance of the complex was measured at $\lambda_{\max }=388 \mathrm{~nm}$.

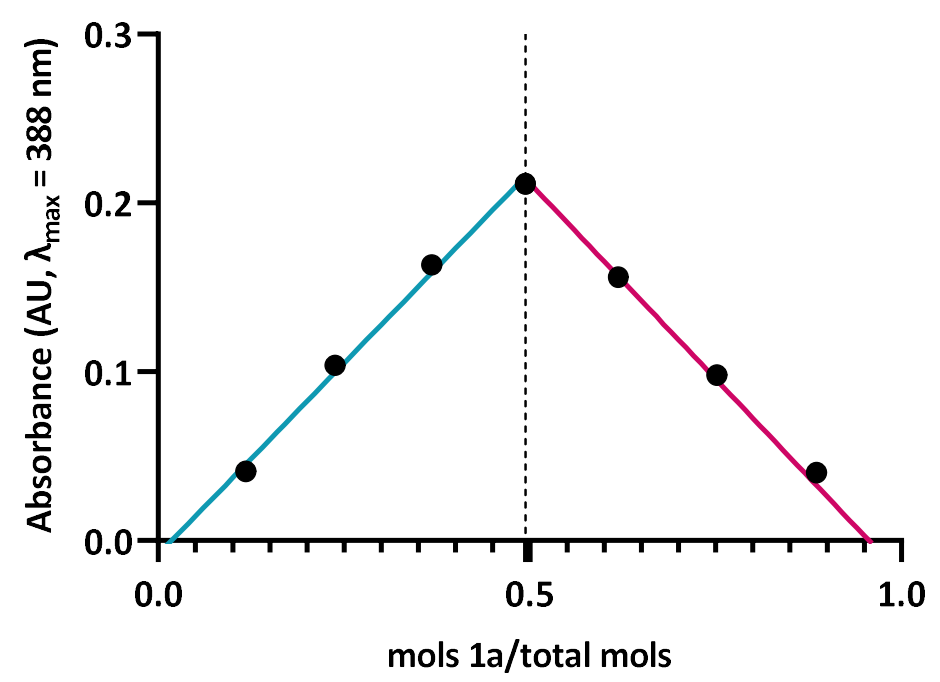

Figure 2.14. Job plot obtained by varying the molar ratio of ligand 1 a to copper(II) nitrate in 9:1 v/v chloroform:methanol ( $0.1 \mathrm{M}$ tetrabutylammonium nitrate). The absorbance of the complex was measured at $\lambda_{\max }=388 \mathrm{~nm}$.

The Job plot revealed that the molar ratio of 1 a to the total number of moles was 0.494 , suggesting that the metal:ligand ratio of $\mathrm{Cu}(\mathrm{II}): 1 \mathrm{a}$ was $1: 1$, which is consistent with reports in the literature. ${ }^{146,147}$ 
However, it has also been reported that $1 \mathrm{a}$ is capable of forming dimeric complexes with $\mathrm{Cu}$ (II) (i.e. 1:2 $\mathrm{Cu}(I): 1 a)$. The experiment should be repeated with a different value for $k$ and verified at a different wavelength: these are important crosschecks for evaluating the reliability of the data. ${ }^{102}$ Knowledge of the $\mathrm{Cu}(\mathrm{II})$ complex's stoichiometry is essential for understanding its performance (e.g. $\mathrm{Cu}(\mathrm{II})$ loading) when incorporated in a coating and for evaluating the strength of the interaction between the metal and the ligand, which is reported as the $K_{\mathrm{f}}$ of the complex and is another quantity of interest.

Since the formation constant of the $\mathrm{Cu}(\mathrm{II})-\mathbf{1 a}$ complex has been reported in the literature $\left(3.76 \times 10^{4}\right),{ }^{146,147,149} 1 \mathrm{a}$ was used in an attempt to develop and verify a method for measuring the $K_{\mathrm{f}}$ of the $\mathrm{Cu}(\mathrm{II})$-ligand complexes spectrophotometrically, which could then be applied to the synthesised ligands with unknown $K_{\mathrm{f}}$ values. It was thought that these values would provide a system for establishing lead compounds for further development: it was hypothesised that a relatively lower $K_{\mathrm{f}}$, within certain parameters (e.g. still higher than for the natural ligands in seawater), would be desirable, resulting in $\mathrm{Cu}(\mathrm{II})$ being more readily released and, therefore, able to exert its antimicrobial effect maximally. Comparison of relative $K_{\mathrm{f}}$ values of the same ligand complexed with different metal ions would reveal metal ion-ligand selectivity as well. To measure the $K_{\mathrm{f}}$ of $1 \mathrm{a}$, the absorption spectra of $0.100 \mathrm{mM}$ solutions of $1 \mathrm{a}$ in 9:1 v/v chloroform:methanol containing concentrations of copper(II) nitrate ranging from $0-0.500 \mathrm{mM}$ were measured .

The two absorbing chemical species in the UV-Vis spectra were ligand 1a at $\sim 323 \mathrm{~nm}$ and coordination complex Cu(II)-1a at $\sim 388 \mathrm{~nm}$, and, as the $\mathrm{Cu}(\mathrm{II})$ concentration increased, the absorbance at $\sim 388 \mathrm{~nm}$ increased and the absorbance at $\sim 323 \mathrm{~nm}$ decreased. Isosbestic points were observed at $\sim 308 \mathrm{~nm}$ and $\sim 346 \mathrm{~nm}$ (Figure 2.15). Isosbestic points are the points in an absorption spectrum at which the curves of absorbance vs wavelength for the two principle absorbing components, such as the ligand and transition metal complex, intersect, meaning that they have identical molar absorption coefficients. They are used in the determination of formation constants, and consistent isosbestic points indicate that the reaction proceeds without the formation of an intermediate or multiple products. ${ }^{150,151}$ In the present study, an expanded view of the regions in the absorption spectra containing the supposed isosbestic points revealed that the curves do not all intersect precisely at these points (Figure 2.16). Through correspondence with Dr Peter Gans, co-author of Hyperquad, it was surmised that multiple complexes, likely CuL and $\mathrm{CuL}_{2}$ and possibly a third species, were contributing to the spectra, resulting in the absence of consistent isosbestic points at $~ 308 \mathrm{~nm}$ and $\sim 346 \mathrm{~nm} .{ }^{152}$ Multiple species in solution complicate the calculation of $K_{\mathrm{f}}$, making the use of software, such as HypSpec, necessary to process the data efficiently. Due to the lack of access to this software, among other reasons, it was concluded that a different approach to ligand-selection was required. 


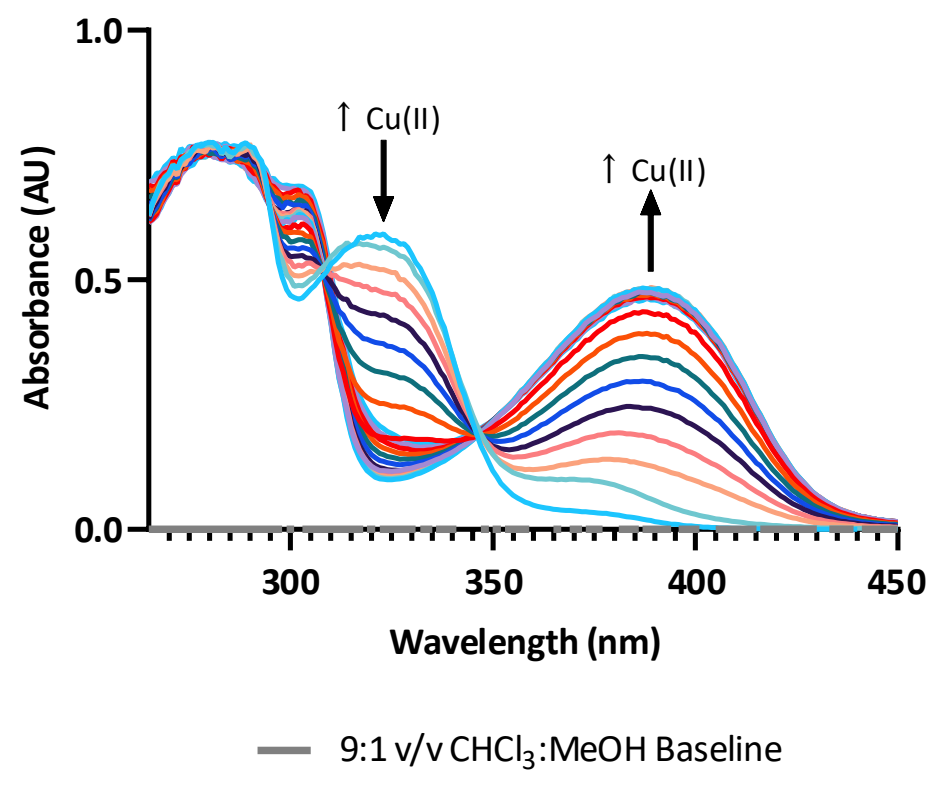

Figure 2.15. Absorption spectra of $0.100 \mathrm{mM}$ solutions of $1 \mathrm{a}$ and increasing concentrations of copper(II) nitrate in 9:1 v/v chloroform:methanol.

(a)

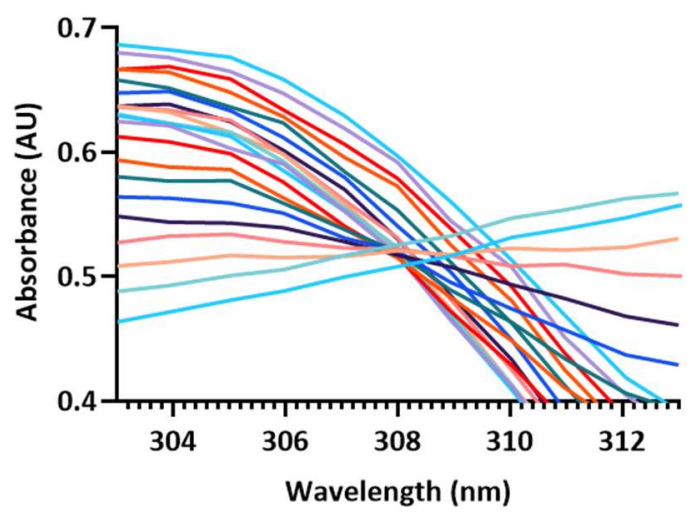

(b)

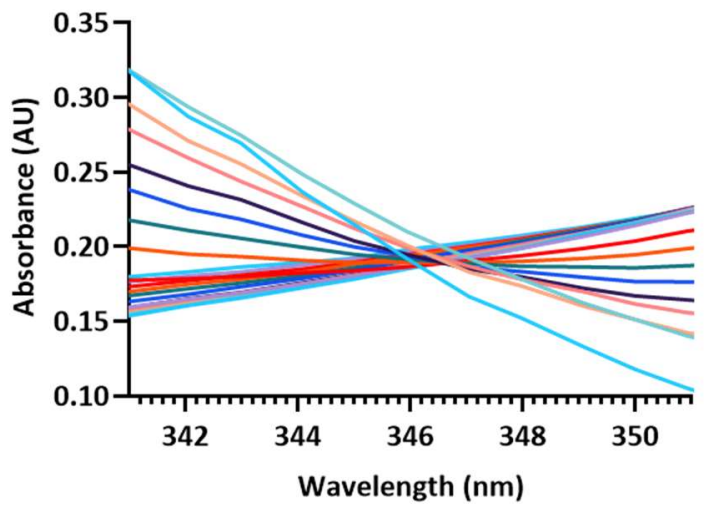

Figure 2.16. Expanded views of the regions in Figure 2.15 containing isosbestic points: (a) $\sim 308 \mathrm{~nm}$ and (b) $~ 346 \mathrm{~nm}$.

\subsubsection{Conclusions}

For multiple reasons, it was decided that a different ligand class should be pursued. First, the insolubility of the acylhydrazones was problematic. Most of them were only soluble in dipolar aprotic solvents, such as DMF and DMSO, which made characterisation of the ligand and its $\mathrm{Cu}(\mathrm{II})$ complex 
difficult. Many of the techniques and experiments (e.g. UV-Vis for Job plot, etc.) require dissolution of the $\mathrm{CU}(\mathrm{II})$ complex in a solvent for characterisation. Since both DMF and DMSO can coordinate $\mathrm{Cu}(\mathrm{II})$, experiments could be affected by ligand-competition for $\mathrm{Cu}(\mathrm{II})$. Also, when planning future stages of the project in which the acylhydrazone would be formulated into a coating, it was clear that the solubility limitations would restrict options, thus failing the requirement that the ligand be easily manipulated for incorporation in a coating. For instance, it would be difficult to incorporate an insoluble acylhydrazone into a paint binder. A few of the acylhydrazones were also soluble in chloroform or solutions of chloroform and methanol (e.g. 1a, $\mathbf{3 h}, \mathbf{1 i - j}$ ), but this was not ideal either, as these solvents would be impossible to incorporate in a modern coating due to solvent restrictions. ${ }^{153}$

Second, the acylhydrazones have complex solution chemistry with multiple possible forms and isomers - not all of which are properly configured for $\mathrm{Cu}(\mathrm{II})$-coordination (e.g. (E')-rotamer of 1a, Figure 2.10). Once affixed in a coating, there would be challenges in ensuring the correct ligandorientation, especially given the dynamicity of acylhydrazones. This dynamicity includes different constitutional states (Figure 2.17), which are a result of the reversibility of acylhydrazone formation under acid-catalysed, mild conditions. Hydrolysis of an acylhydrazone in the presence of other hydrazides or aldehydes/ketones can generate a new acylhydrazone via recombination, ${ }^{144,145,154-157}$ and this has been observed by our research group when working with aqueous solutions of the DiLevDEG/ADH polyacylhydrazone. ${ }^{128}$ Such dynamicity complicates the ligand design, formulation, and product characterisation, making the ligand class less attractive for a proof-of-concept. It is unknown if the acylhydrazones would be stable or properly orientated for $\mathrm{Cu}(\mathrm{II})$-chelation in the film, and this potentially violates the condition that the ligand perform similarly to its unbound state when immobilised in a coating.

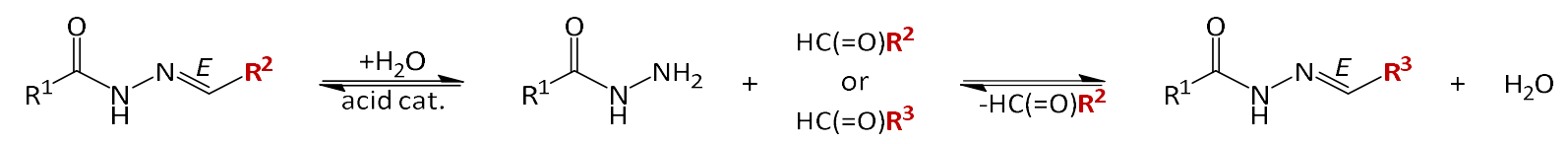

Figure 2.17. Example of different constitutional states in an acylhydrazone. ${ }^{145}$

Finally, it was acknowledged that simpler, better-characterised ligand systems were available and that, to meet the project goals, it was wise to begin with as few unknowns as possible, bearing in mind that the complexity increases upon formulation into a paint. Pursuing the acylhydrazones would necessitate complicated measurements already addressed in the literature for other ligand classes. 
For instance, most of the acylhydrazones would require calculation of the $K_{\mathrm{f}}$ of their $\mathrm{Cu}(\mathrm{II})$ complexes $\left(K_{\mathrm{Cu}(I I) L}\right)$ and experiments to determine if they are selective for $\mathrm{Cu}(I I)$. The $K_{\mathrm{Cu}(I I) L}$ is important for confirming that the selected ligand is a stronger $\mathrm{Cu}(\mathrm{II})$-chelator than the natural ligands in seawater, and $\mathrm{Cu}(\mathrm{II})$-selectivity is important because there are many transition metal ions in seawater at concentrations comparable to $\mathrm{Cu}(\mathrm{II})$ with which acylhydrazones are able to form stable complexes. ${ }^{132,158,159} \mathrm{An}$ ideal ligand should retain its $\mathrm{Cu}(\mathrm{II})$-selectivity and form stable $\mathrm{Cu}(\mathrm{II})$ complexes in seawater, so it seemed wiser to select a ligand class for which there was already evidence that it could fulfil such requirements. In light of all of these considerations, it was decided a new ligand class should be explored, and tetraaza/oxatriaza macrocycles were selected. Although, since it was already successfully formulated into a coating, it was still sensible to progress the DiLevDEG/ADH polyacylhydrazone to the second stage of testing in which the $\mathrm{Cu}(\mathrm{II})$-loading and antimicrobial activity of the paint would be evaluated. 


\subsection{TetraAza/OXatriaza MACROCyClES: CyClEN}

Tetraaza/oxatriaza macrocycles $\mathbf{6 - 1 5}$, as in Figure $\mathbf{2 . 1 8}$, were selected as a new ligand class due to their $\mathrm{Cu}(\mathrm{II})$-selectivity, antimicrobial activity, favourable $K_{\mathrm{Cu}(\mathrm{II}) \mathrm{L}}$ values, and more convenient solubility profiles. It has been demonstrated that 6-15 are selective for $\mathrm{Cu}(\mathrm{II})$ over multiple other transition metal ions, including $\mathrm{Mn}(\mathrm{II}), \mathrm{Co}(\mathrm{II}), \mathrm{Ni}(\mathrm{II}), \mathrm{Zn}(\mathrm{II}), \mathrm{Cd}(\mathrm{II})$, and $\mathrm{Pb}(\mathrm{II}) .{ }^{89}$ They form very stable $1: 1$ complexes and display inertness to demetallation due to the macrocyclic effect. ${ }^{76,89,160-164}$ The macrocyclic effect is the increased stability of complexes between metal ions and macrocyclic ligands, compared to their open-chain counterparts. For instance, the $\log \left(K_{\mathrm{Cu}(I) L)}\right)$ values for cyclen (11) and its open-chain counterpart, triethylenetetramine, are 23.3 and 20.0, respectively. ${ }^{165-168}$ There are also examples in the literature of tetraaza macrocyclic compounds displaying antimicrobial activity when complexed with $\mathrm{Cu}(\mathrm{II}) .{ }^{169-171}$ This antimicrobial activity has even been retained after fixation of the $\mathrm{Cu}(\mathrm{II})$ complex to a surface. For example, a study by Pallavicini et al. ${ }^{76}$ reported the grafting of the [13]ane $\mathrm{N}_{4}(13)$ $\mathrm{Cu}(\mathrm{II})$ complex to glass and quartz surfaces and the significant antimicrobial effect of these surfaces, despite their inertness to demetallation.

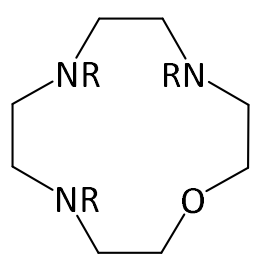

$\mathrm{R}=\mathrm{H},[12]$ ane $_{3} \mathrm{O}, 6$ $\mathrm{R}=\mathrm{CH}_{3}, \mathrm{Me}_{3}[12] \operatorname{aneN}_{3} \mathrm{O}, 7$

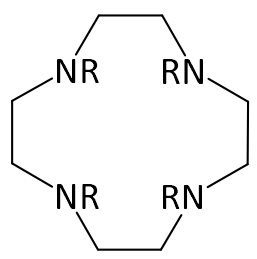

$\mathrm{R}=\mathrm{H}$, cyclen, [12] ane $\mathrm{N}_{4}, \mathbf{1 1}$

$\mathrm{R}=\mathrm{CH}_{3}, \mathrm{Me}_{4}[12] \mathrm{aneN}_{4}, 12$

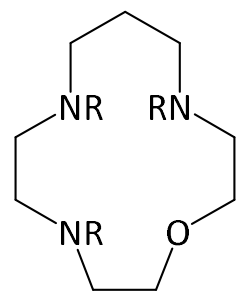

$\mathrm{R}=\mathrm{H},[13]$ ane $_{3} \mathrm{O}, 8$

$\mathrm{R}=\mathrm{H},[14]$ ane $\mathrm{N}_{3} \mathrm{O}, 9$

$\mathrm{R}=\mathrm{CH}_{3}, \mathrm{Me}_{3}[14]$ ane $\mathrm{N}_{3} \mathrm{O}, 10$

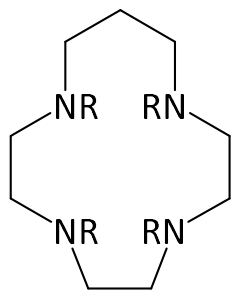

$\mathrm{R}=\mathrm{H},\left[13\right.$ aneN $_{4}, \mathbf{1 3}$

$\mathrm{R}=\mathrm{H}$, cyclam, [14]ane $\mathrm{N}_{4}, 14$

$\mathrm{R}=\mathrm{CH}_{3}, \mathrm{Me}_{4}[14]$ aneN $_{4}, 15$

Figure 2.18. Tetraaza/oxatriaza macrocycles (6-15)..$^{89}$ 
Ligands 6-15, as well as their $\mathrm{Cu}(\mathrm{II})$ complexes, are well-known in the literature: as seen in Table 2.4, all of the $\log \left(K_{\mathrm{f}}\right)$ values for the $\mathrm{Cu}(\mathrm{II})$-ligand complexes have been previously reported and range from $13.60(10)$ to $26.5(14) .{ }^{89}$ This range of $\log \left(K_{\mathrm{f}}\right)$ values is particularly desirable because it indicates that all but one of the macrocyclic ligands are stronger $\mathrm{Cu}(\mathrm{II})$-chelators than the organic $\mathrm{Cu}(\mathrm{II})$ complexes in seawater (i.e. $\log \left(K_{\mathrm{f}}\right)>13.8$ for diethylene triamine pentaacetic acid (DTPA)-type ligand, Table $\mathbf{2 . 4}$ "Seawater Complex"). ${ }^{80,172,173}$ Since the majority of dissolved copper in seawater is complexed with organic ligands (e.g. DTPA- or EDTA-type), macrocyclic ligands 6-15 may, therefore, be strong enough $\mathrm{Cu}(\mathrm{II})$-chelators to displace these organic ligands in seawater. ${ }^{80}$ Also, crystal structures of the $\mathrm{Cu}(\mathrm{II})$ macrocyclic ligand complexes have been published, providing further valuable information, and the ligands and complexes are known to have convenient solubility profiles..$^{89,174}$ For example, cyclen (11) is water-soluble. To begin, cyclen was chosen specifically out of the series to serve as a model compound for a proof-of-concept and development of methodology.

Table 2.4. Stability constants $\left(K_{\mathrm{ML}}=[\mathrm{ML}] /[\mathrm{M}][\mathrm{L}] ; \mathrm{M}=\right.$ metal, $\mathrm{L}=$ ligand) of metal complexes of the macrocyclic compounds in Figure 2.18 at $I=0.10 \mathrm{~mol} / \mathrm{dm}^{3}\left(\mathrm{KNO}_{3}\right)$ and $25.0 \pm 0.1^{\circ} \mathrm{C} .{ }^{89}$

\begin{tabular}{|c|c|c|c|c|c|c|c|}
\hline Metal ion & $\mathrm{Mn}^{2+}$ & $\mathrm{Co}^{2+}$ & $\mathrm{Ni}^{2+}$ & $\mathrm{Cu}^{2+}$ & $\mathrm{Zn}^{2+}$ & $\mathrm{Cd}^{2+}$ & $\mathrm{Pb}^{2+}$ \\
\hline Ligand & $\begin{array}{l}\log \\
\left(K_{\mathrm{Mn}(1) L}\right)\end{array}$ & $\begin{array}{l}\text { Log } \\
\left(K_{\text {co }(I I) L)}\right.\end{array}$ & $\begin{array}{l}\log \\
\left(K_{\mathrm{Ni}(I) L)}\right)\end{array}$ & $\begin{array}{l}\log \\
(\text { Kcu(II)L) }\end{array}$ & $\begin{array}{l}\log \\
(K \mathrm{zn}(1(1) L)\end{array}$ & $\begin{array}{l}\log \\
(K \operatorname{cod}((1) L)\end{array}$ & $\begin{array}{l}\log \\
\left(\mathrm{KPb}_{\mathrm{P}(\mathrm{I}) \mathrm{L}}\right)\end{array}$ \\
\hline 6 & $5.85(1)$ & $10.541(7)$ & $12.36(3)$ & $15.63(1)$ & $10.43(7)$ & $10.69(1)$ & 11.54(1) \\
\hline 7 & - & $10.30(3)$ & $11.42(4)$ & $14.98(4)$ & $10.006(8)$ & $10.23(1)$ & $10.53(1)$ \\
\hline 8 & $3.96(9)$ & $9.29(1)$ & $11.37(6)$ & $16.61(4)$ & $9.80(1)$ & $9.32(3)$ & $8.84(3)$ \\
\hline 9 & - & $8.87(4)$ & $9.7(1)$ & $15.4(2)$ & $8.9(2)$ & $7.13(1)$ & 7.30(1) \\
\hline 10 & - & $5.8(1)$ & $6.41(7)$ & $13.60(3)$ & $6.36(2)$ & $4.7(3)$ & $6.61(2)$ \\
\hline 11 & - & $13.8^{175}$ & $16.4^{176}$ & $23.3^{165}$ & $16.2^{177}$ & $14.3^{177}$ & $15.9^{177}$ \\
\hline 12 & - & - & - & $18.37^{178}$ & $14.04^{178}$ & $13.06^{178}$ & $13.91^{178}$ \\
\hline 13 & - & $14.28^{175}$ & $17.98^{176}$ & $24.36^{165}$ & $15.6^{177}$ & $12.71^{165}$ & $13.48^{165}$ \\
\hline 14 & - & $12.71^{175}$ & $22.2^{179}$ & $26.5^{165}$ & $15.5^{177}$ & $11.23^{165}$ & $10.83^{165}$ \\
\hline 15 & - & $7.58^{180}$ & $8.65^{180}$ & $18.3^{180}$ & $10.35^{180}$ & $9.0^{180}$ & - \\
\hline $\begin{array}{l}\text { Seawater } \\
\text { Complex }^{80,172,173}\end{array}$ & - & - & - & $\begin{array}{l}13.8^{\mathrm{a}} \\
11.7^{\mathrm{b}}\end{array}$ & $\begin{array}{l}10.7^{\mathrm{a}} \\
9.4^{\mathrm{b}}\end{array}$ & - & - \\
\hline
\end{tabular}


Cyclen (11) was chosen to be a model compound for several reasons. First, cyclen is commercially available and relatively inexpensive, saving time that would have been spent on synthesis to focus on its formulation and antifouling performance. One goal of the present work is to establish parameters for ligand-selection (e.g. $\log \left(K_{\mathrm{Cu}(\mathrm{IIIL})}\right)$ and the $\mathrm{Cu}(\mathrm{II})$-loading and presentation of the complex in a coating, helping to create a framework for future work. A well-known, readily available ligand increases the likelihood of meeting this goal but also of having a more commercially viable product down the track, since it is fully characterised and less expensive than a specialised ligand with a lengthy synthesis. This meets the requirement that the ligand be cheaply prepared in large quantities. Second, being water-soluble, cyclen is more easily manipulated for characterisation and incorporation in a coating. Third, cyclen is selective for $\mathrm{Cu}(\mathrm{II})$ over other transition metal ions (e.g. $\mathrm{Co}(\mathrm{II}), \mathrm{Ni}(\mathrm{II}), \mathrm{Zn}(\mathrm{II})$, $\mathrm{Cd}(\mathrm{II}), \mathrm{Pb}(\mathrm{II}))$ and has a high $\log \left(K_{\mathrm{Cu}(\mathrm{III})}\right)(23.3)$, meaning that the $\mathrm{Cu}(\mathrm{II})$-cyclen complex is stable and resistant to demetallation. ${ }^{89}$ This fulfils the selectivity and stability criteria for the project's ideal ligand. Because the flux of bioavailable metal ions from the surface is necessary for antimicrobial activity, ${ }^{115}$ it was hypothesised that a relationship may exist between the stability of the complex and surface toxicity. In the particular case of antifouling paints, the ligands would still need to outcompete the natural ligands in seawater, establishing the $\log \left(K_{\mathrm{Cu}(I I) L}\right)$ minimum value at $13.8 .80,172,173$ Within the series of tetraaza/oxatriaza macrocycles (Figure 2.18), cyclen has one of the highest $\log \left(K_{\mathrm{Cu}(I I) L}\right)$ values (Table 2.4). Consequently, determining if such a strong ligand could still exert an antimicrobial effect when affixed in a coating was of interest. Finally, the literature concerning cyclen and its derivatives, as well as their transition metal complexes, is vast, and precedents exist for numerous applications, including their use as radiopharmaceuticals (e.g. imaging probes for positron emission tomography $(P E T))$, antimicrobial agents, and generators of nitric oxide when affixed in polymeric coatings. ${ }^{110,164,170,181}$ This is evidence that cyclen immobilised in a coating could retain its ability to chelate $\mathrm{Cu}(\mathrm{II})$. Therefore, cyclen meets all of the requirements listed in Section $\mathbf{2 . 1}$ for an ideal ligand, and a solid base of work exists to support the current project. In the present study, initial experiments were completed to observe the coordination of $\mathrm{Cu}(\mathrm{II})$ by cyclen.

Formation of the $\mathrm{Cu}(\mathrm{II})$-cyclen complex was confirmed by both HRMS and UV-Vis spectroscopy. It is known that cyclen chelates $\mathrm{Cu}(\mathrm{II})$ in the trans-I conformation, with $\mathrm{Cu}(\mathrm{II})$ rising above the plane of the donor atoms (Figure 2.19). ${ }^{89,167,182}$ Cyclen (white solid) and copper(II) chloride (green solid, 2.2 eq) were added to methanol and stirred for $2 \mathrm{~h}$. Then, the dark blue, precipitated $\mathrm{Cu}(\mathrm{II})$-cyclen complex was isolated and analysed by ESI-HRMS. ESI-HRMS data were consistent with a 1:1 $\mathrm{Cu}(\mathrm{II})$ :cyclen complex minus a proton, imparting a +1 charge (calcd for $\mathrm{C}_{8} \mathrm{H}_{19} \mathrm{CuN}_{4}[\mathrm{M}-\mathrm{H}]^{+}$234.0906; found 234.0916). An aqueous, equimolar solution of cyclen and copper(II) nitrate was prepared, and the UV-Vis spectrum of the resultant, dark blue complex was measured (Figure 2.20). Due to 
d-d transitions, the complex absorbed from approximately $430 \mathrm{~nm}$ to $800 \mathrm{~nm}\left(\lambda_{\max }=590 \mathrm{~nm}\right)$, only transmitting blue light and, thus, accounting for the complex's dark blue colour. An aqueous solution of only copper(II) nitrate absorbed red light with $\lambda_{\max } \geq 800 \mathrm{~nm}$ and appeared pale-blue. Both of these experiments demonstrated $\mathrm{Cu}(\mathrm{II})$-chelation by the cyclen ligand.

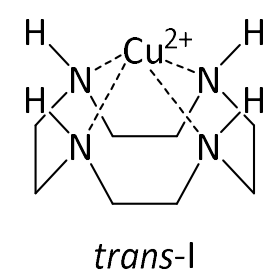

Figure 2.19. $\mathrm{Cu}(\mathrm{II})$-chelation by cyclen in the trans-I conformation. ${ }^{167}$

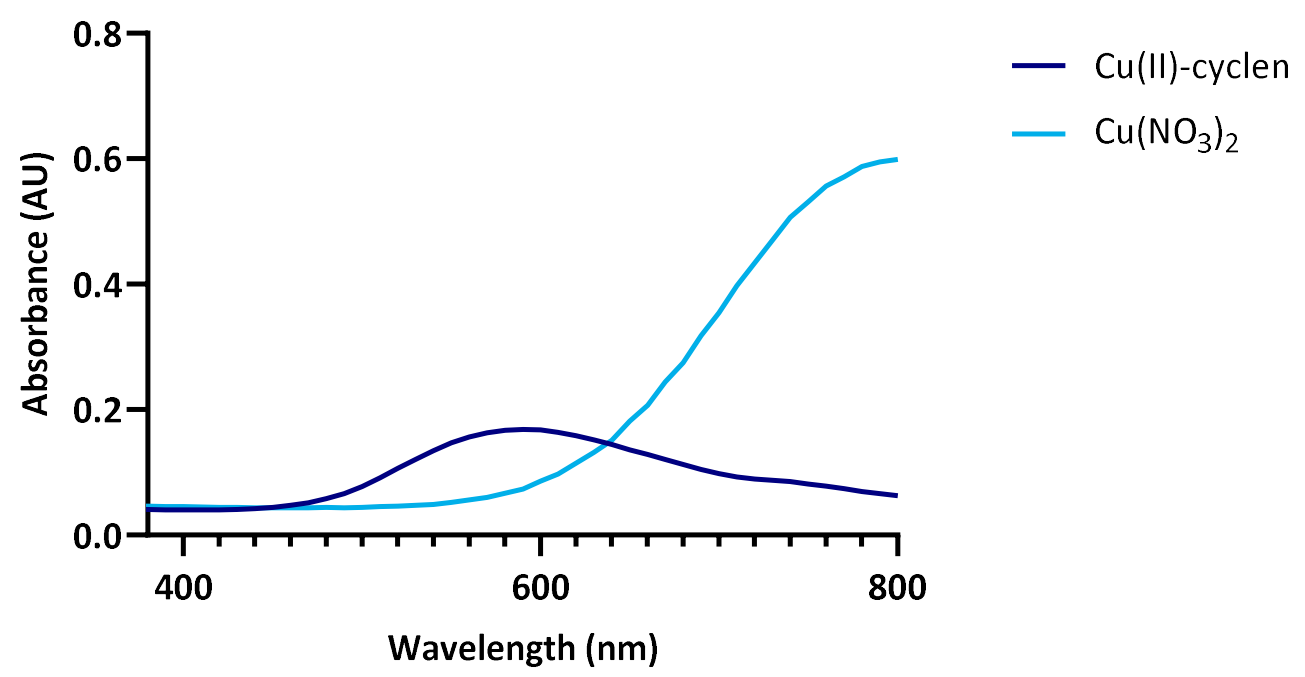

Figure 2.20. UV-Vis spectra of $\mathrm{Cu}(\mathrm{II})$-cyclen and $\mathrm{Cu}\left(\mathrm{NO}_{3}\right)_{2}$. 


\subsection{SUMMARY AND FUTURE WORK}

In the present study, the suitability of two ligand classes, acylhydrazones (e.g. DiLevDEG/ADH, 1a) and tetraaza macrocycles (e.g. cyclen), was considered for the proposed antifouling application. The project developed out of the work by our research group in which the DiLevDEG/ADH polyacylhydrazone replaced $30-50 \% \mathrm{w} / \mathrm{w}$ of the acrylic resin in a commercial coating, producing a more environmentally friendly paint with functionality capable of $\mathrm{Cu}(\mathrm{II})$-chelation (Figure 2.2). ${ }^{127}$ Continuing from this research, it was hypothesised that the DiLevDEG/ADH-substituted paint could charge itself with $\mathrm{Cu}$ (II) from an aqueous solution and, thus, become antifouling. In the current work, testing of this hypothesis was designed to be completed in two stages: 1) DiLevDEG/ADH would be synthesised and its $\mathrm{Cu}(\mathrm{II})$-coordination demonstrated, and 2) DiLevDEG/ADH would be formulated into the commercial acrylic paint and evaluated for $\mathrm{Cu}(\mathrm{II})$-loading and antimicrobial activity after exposure to an aqueous solution of $\mathrm{Cu}(\mathrm{II})$. The first stage was completed in this chapter; DiLevDEG/ADH was successfully synthesised (Figure 2.4), and the ability of this polyacylhydrazone to chelate $\mathrm{CU}(\mathrm{II})$ was confirmed via UV-Vis spectroscopy (Figure 2.6) and the observation of a colour change upon the addition of $\mathrm{Cu}(\mathrm{II})$ (Figure 2.5). The second stage of testing will be addressed in Chapter 3 (Section 3.2), and, based on the results, this coating may serve as the project's proof-ofconcept. To search for other ligand candidates, simple acylhydrazones were synthesised.

A series of monoacylhydrazones and acyldihydrazones was successfully synthesised (Scheme 2.2) and characterised, including select $\mathrm{Cu}(\mathrm{II})$ complexes. $\left(E^{\prime} / Z^{\prime}\right)$-Rotamers, but only one geometric isomer $(E)$, were observed in the NMR spectra of both aldehyde- and ketone-derived acylhydrazones. In general, the acylhydrazones were only soluble in DMF and DMSO, but one ligand with a more favourable solubility profile (1a) was progressed to complexation with $\mathrm{Cu}(\mathrm{II})$. A Job plot was constructed and an experiment was conducted to determine the $K_{\mathrm{Cu}(I I) L}$ of the $\mathrm{Cu}(\mathrm{II})-1 \mathrm{a}$ complex, which is known in the literature. This was done in an attempt to verify the methodology for later use on lesser-known acylhydrazones, and future experiments may pursue such $K_{\mathrm{Cu}(I I) L}$ measurements. However, due to the insolubility and complex solution chemistry of the acylhydrazones, as well as the acknowledgement of the existence of more suitable candidates, a different ligand class was pursued.

The new ligand class proposed for the project was tetraaza/oxatriaza macrocycles (Figure 2.18). Specifically, cyclen was selected out of the series to serve as a model ligand because it meets all of the project's requirements: it is commercially available, relatively inexpensive, selective for $\mathrm{Cu}(\mathrm{II})$ over other transition metals, and well-known in the literature. Formation of the $\mathrm{Cu}(\mathrm{II})$-cyclen complex was confirmed by HRMS and UV-Vis spectroscopy. The Cu(II)-cyclen complex, with a high $\log \left(K_{\mathrm{Cu}(I I) L}\right)(23.3)$, is stable, resistant to demetallation, and able to outcompete the natural ligands in seawater. Since it 
has been reported that the flux of copper ions is necessary for surface toxicity, ${ }^{114,115}$ it seemed prudent to determine if such stable complexes could still exert an antimicrobial effect when bound to a surface. In future work, a ligand with a lower $\log \left(K_{\mathrm{Cu}(I I) L}\right)$ from the series of tetraaza/oxatriaza macrocycles (e.g. 6) could be synthesised, incorporated into a coating, and tested for antimicrobial activity, comparing these results to cyclen to determine if activity increases with decreasing complex-stability. Alternatively, other ligand classes (e.g. polyamines, EDTA-type, etc.) could be explored. 


\section{INCORPORATION OF CU(II)-LIGAND COMPLEXES IN COATING Binders}

\subsection{BACKGROUND}

Chapter 2 discussed the selection and synthesis of $\mathrm{Cu}(\mathrm{II})$ ligands (e.g. DiLevDEG/ADH, cyclen) to function as antifoulants in a coating, and the first explored route for formulation in a paint was incorporating these ligands in coating binders. As shown in Figure 3.1, the paint binder is the filmforming resin or polymer that binds together all of the other components, such as the coloured pigments, extenders, and additives. It is either dissolved in the paint or suspended as a colloid, and this binder/liquid mixture is referred to as the vehicle for the pigment. Binders are classified according to their molecular weight: low molecular weight binders require further chemical reactions to solidify in a film, but high molecular weight binders will form solid films without further reactions. Examples of low and high molecular weight film-formers are listed in Table 3.1. ${ }^{29,30}$

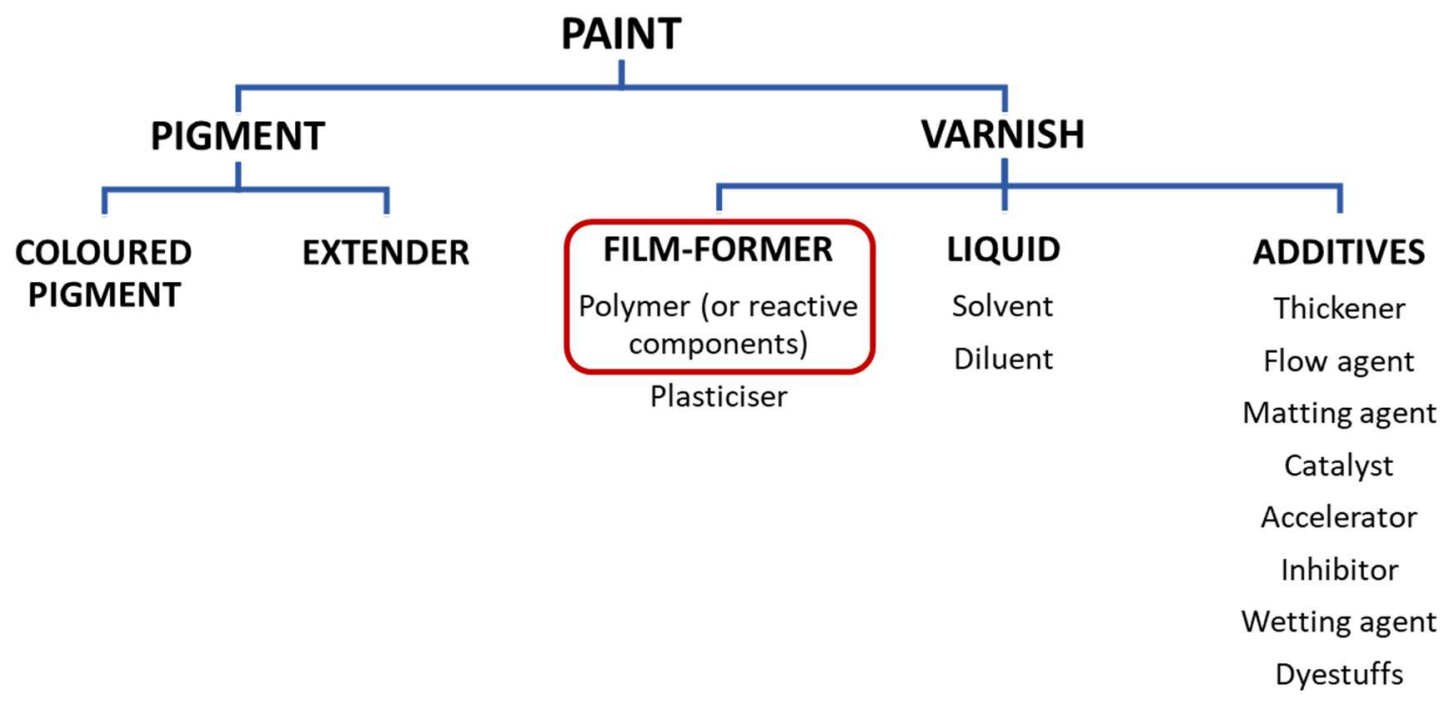

Figure 3.1. Paint components (adapted from Introduction to Paint Chemistry). ${ }^{29}$ 
Table 3.1. Low and high molecular weight binders (adapted from Paint and Surface Coatings). ${ }^{30}$

\begin{tabular}{ll}
\hline Low Molecular Weight & High Molecular Weight \\
\hline Oleoresinous binders & Nitrocellulose \\
Alkyds & Solution vinyls \\
Polyurethanes & Solution acrylics \\
Urethane oils & Non-aqueous dispersion polymers \\
Amino resins & Polyvinyl acetate \\
Phenolic resins & Acrylic \\
Epoxide resins & Styrene/butadiene \\
Unsaturated polyesters & \\
Chlorinated rubber &
\end{tabular}

In the work by Daines et al. ${ }^{127}$ in our research group, the DiLevDEG/ADH polyacylhydrazone was used to substitute a proportion of the crosslinking resin, classified as high molecular weight, in a commercial acrylic paint. Acrylic polymers display exceptional strength, flexibility, gloss, clarity, and weather and chemical resistance, leading to their widespread use..$^{30,183}$ In general, the polymers and copolymers (i.e. polymer made from more than one type of monomer) ${ }^{29}$ of methacrylic and acrylic acid are referred to as acrylic resins (Figure 3.2). Copolymers of the corresponding esters with styrene, butadiene, or vinyl acetate are also sometimes called acrylic resins, but the term "acrylic" is usually reserved for resins dominated by the methacrylic or acrylic structures. ${ }^{183}$ Acrylic polymers form via chain addition polymerisation in which the high molecular weight polymer is generated through initiation by an active species (e.g. radical) breaking bonds in the monomer, propagation of the reactive centre at the end of the chain, and, finally, termination of the reactive centre (Figure 3.3). This differs from stepwise polymerisation in which monomers, each containing two reactive functional groups, first form dimers, and the molecules continue to link together, resulting in an increase in average molecular weight over time. Stepwise polymerisation is the mechanism by which, for example, polyurethane and epoxy resins are produced. ${ }^{29,30,184}$<smiles>C=CC(=O)O</smiles>

acrylic acid<smiles>CCCC(=O)O</smiles>

polyacrylate<smiles>C=C(C)C(=O)O</smiles>

methacrylic acid<smiles>CC(C)(C)C(=O)O</smiles>

polymethacrylate

Figure 3.2. Structures of acrylic acid, the monomer of polyacrylate, and methacrylic acid, the monomer of polymethacrylate. 

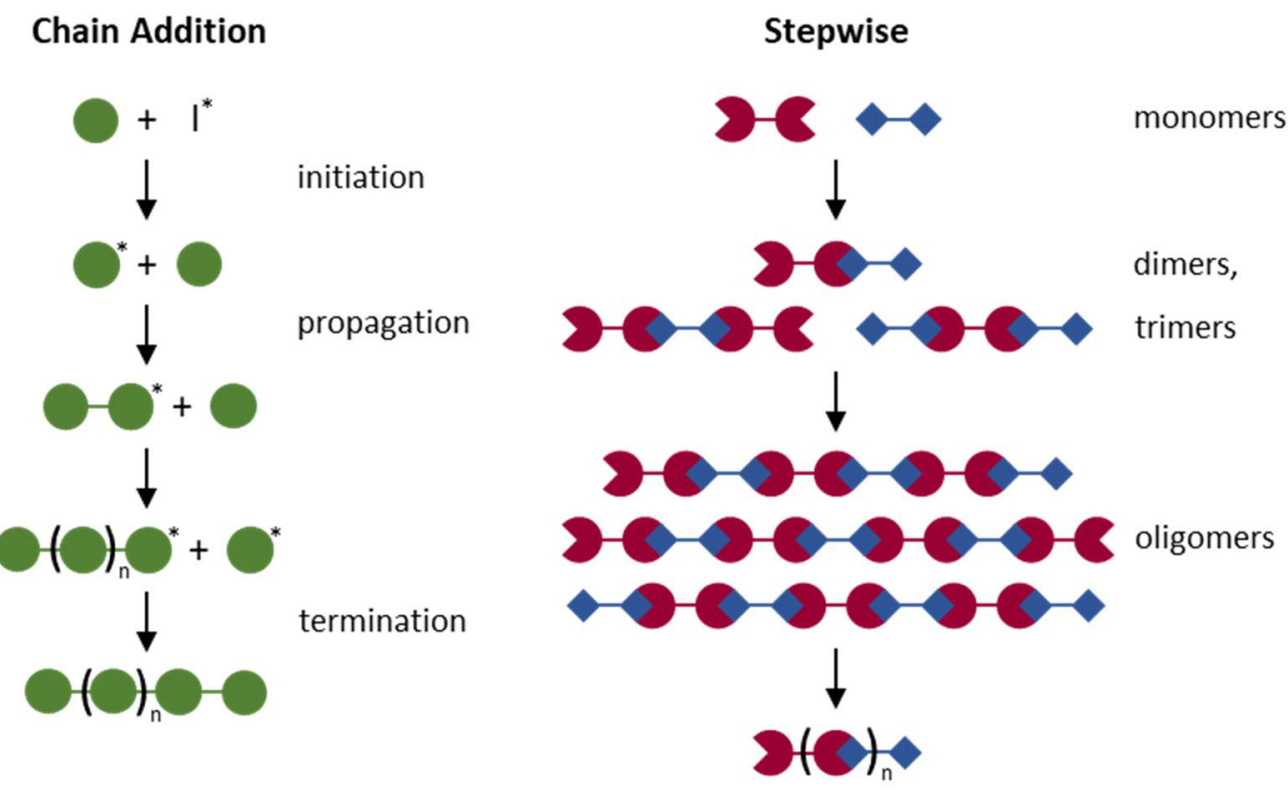

Figure 3.3. Chain addition vs stepwise polymerisation $\left(I^{*}=\right.$ initiator, radical; adapted from Bossion et al. $\left.{ }^{184}\right)$.

Polyurethane and epoxy resins, both low molecular weight, are common binders in marine paints. ${ }^{30,185}$ Polyurethanes are used extensively in the coatings industry, as they are tough, flexible, and exhibit excellent substrate adhesion and resistance to chemicals, abrasion, and corrosion. ${ }^{29,183}$ They are characterised by carbamate, also referred to as urethane, linkages $(-\mathrm{HN}-(\mathrm{C}=\mathrm{O})-\mathrm{O}-)$ but may contain other functional groups (e.g. esters, ethers, ureas, amides). ${ }^{183}$ The urethane linkage is typically formed by the reaction between an isocyanate group $(\mathrm{R}-\mathrm{N}=\mathrm{C}=\mathrm{O})$ and a hydroxyl group. Thus, polyurethanes are produced by the reaction of a polymeric isocyanate or diisocyanate with a polyol (Scheme 3.1). ${ }^{186}$ Epoxy resins, on the other hand, are popular due to their toughness, corrosion and chemical resistance, thermal stability, and superior substrate adhesion and electrical properties. ${ }^{30,183}$ They contain one or more epoxy groups, which react in their preparation or crosslinking; preparation of an epoxy resin usually involves the reaction of bisphenol A with epichlorohydrin (Scheme 3.2). The resultant polymer is a reactive intermediate, and, to function as a film-former, this resin requires curing (i.e. crosslinking) via the reaction of curing agents (i.e. hardeners, e.g. polyamines) with pendant hydroxyl groups and/or terminal epoxy groups. The result of the curing process is a film with a crosslinked, three-dimensional network. ${ }^{29,30,183}$ 


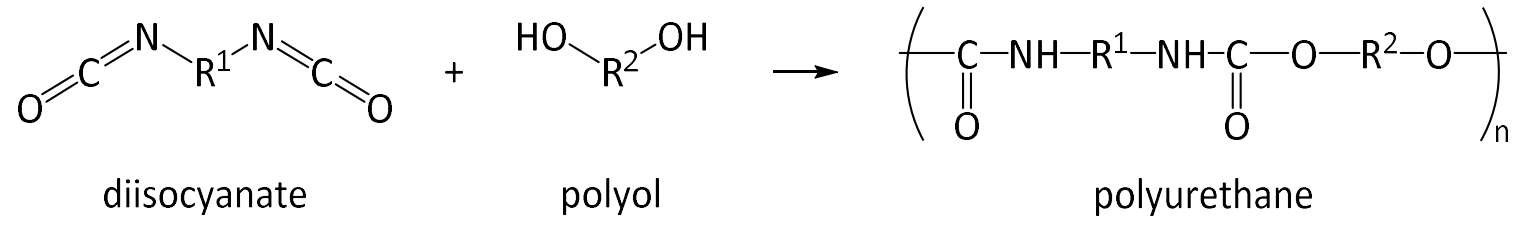

Scheme 3.1. Reaction of a diisocyanate with a polyol to produce a polyurethane.
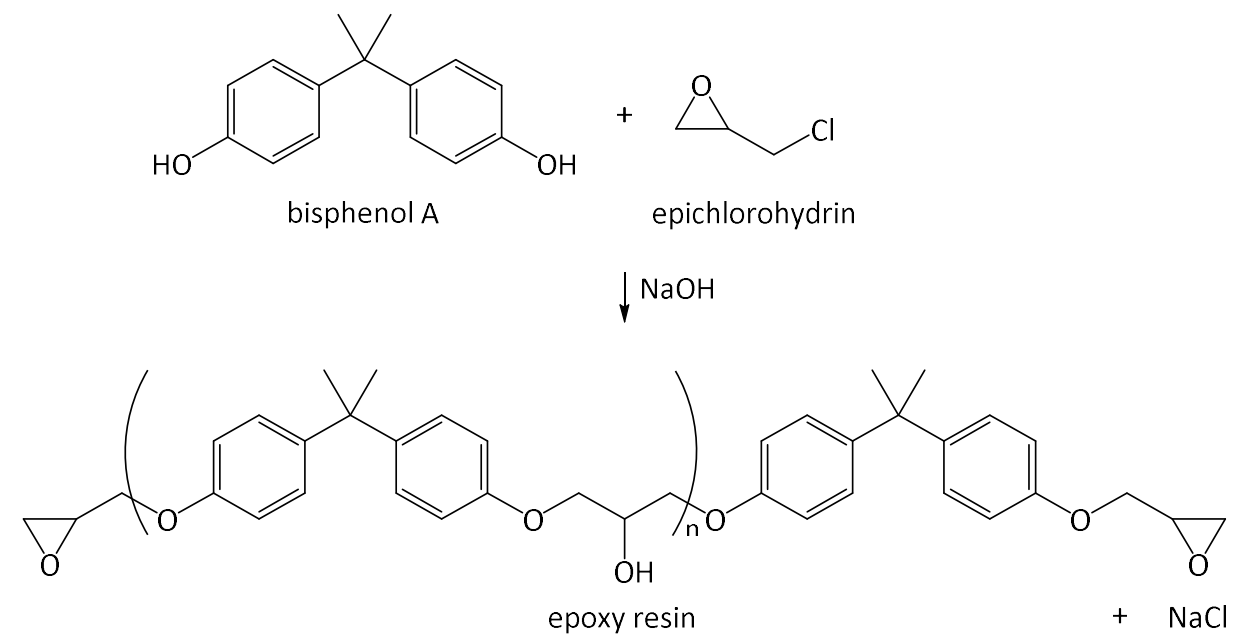

$\downarrow \mathrm{H}_{2} \mathrm{~N}^{-\mathrm{R}} \backslash \mathrm{NH}_{2}$<smiles>CCC(COc1ccc(C(C)(C)c2ccc(OC(CC)(CC)C(CNPNC)OC[C@H](C)O)cc2)cc1)OCC(C)O</smiles>

Scheme 3.2. Reaction of bisphenol A with epichlorohydrin to produce an epoxy resin, which then reacts with an amine curing agent and other epoxy groups to form the crosslinked coating (adapted from Introduction to Paint Chemistry). ${ }^{29}$

There are multiple examples in the literature of the incorporation of $\mathrm{Cu}(\mathrm{II})$ ligands in both polyurethane and epoxy resins. ${ }^{110,111,187-194}$ For instance, Hwang and Meyerhoff ${ }^{110}$ tethered the $\mathrm{Cu}(\mathrm{II})$ cyclen complex, serving as a nitric oxide (NO)-generating catalyst, to a medical grade polyurethane (Tecophilic, SP-93A-100). Biomedical device coatings capable of releasing NO are desirable, as they improve the biocompatibility of the device, and NO generation was observed when the $\mathrm{Cu}(\mathrm{II})$-cyclen complex-linked polyurethane was in contact with fresh animal whole blood. In a study by Xie et al. ${ }^{188}$, 
marine antifoulant $\mathrm{N}$-(2,4,6-trichlorophenyl) maleimide was linked as a pendant group to a polyurethane with poly(dimethylsiloxane) main chains, a polymer used in antifouling, and this surface successfully prevented biofouling by marine organisms (e.g. bacterium Micrococcus luteus, diatom Navicula, barnacle cyprids).

There has been much interest in the incorporation of transition metal complexes in epoxy systems due to the improved physical properties of the coatings, and such complexes have been used as curing agents, catalysts, and cure accelerators. ${ }^{189}$ For example, Kurnoskin ${ }^{111,190-192}$ synthesised metalliferous epoxy chelate polymers from bisphenol A diglycidyl ether (DGEBA), a constituent of epoxy resins, and transition metals (e.g. $\mathrm{Cu}(\mathrm{II}), \mathrm{Co}(\mathrm{II}), \mathrm{Ni}(\mathrm{II}), \mathrm{Zn}(\mathrm{II}), \mathrm{Cd}(\mathrm{II}), \mathrm{Fe}(\mathrm{III}), \mathrm{Mn}(\mathrm{IV}))$ coordinated to aliphatic amines (e.g. ethylenediamine, diethylenetriamine, triethylenetetramine), which functioned as curing agents. Inclusion of the transition metal complexes resulted in increased strength and, for the $\mathrm{Cu}(\mathrm{II})$ containing polymers in particular, increased water and heat resistance. Given this evidence of the successful formulation of metal-ligand complexes into coating binders, the inclusion of $\mathrm{Cu}$ (II) ligands in polyurethanes and epoxy systems, as well DiLevDEG/ADH in the acrylic polymer, was explored. 


\subsection{POLYACYLHYDRAZONE DiLEvDEG/ADH-SUBSTITUTED Resin in a COMmercial ACrylic PAint}

In the aforementioned, foundational work by Daines et al. ${ }^{127}$ in our research group (Chapter 2, Section 2.2), 30-50\% w/w of the crosslinking resin in a commercial acrylic paint was substituted with polyacylhydrazone DiLevDEG/ADH, creating from renewable sources a paint that performed well in standard industry tests. Since DiLevDEG/ADH contains functionality capable of $\mathrm{Cu}(\mathrm{II})$-chelation (Chapter 2, Figure 2.3), it was speculated that the paint might serve as the current project's proof-ofconcept, being a candidate antifouling coating, and two stages of evaluation were proposed. The first stage involved the synthesis of DiLevDEG/ADH and confirmation of its ability to bind $\mathrm{Cu}(\mathrm{II})$, and the successful completion of this stage was discussed in Chapter 2. The second stage involved the formulation of DiLevDEG/ADH into the acrylic paint, the quantification of $\mathrm{Cu}(\mathrm{II})$-loading on the coating surface, and an assessment of the subsequent antimicrobial activity (Figure 3.4). The results from the second stage are discussed in the present chapter.

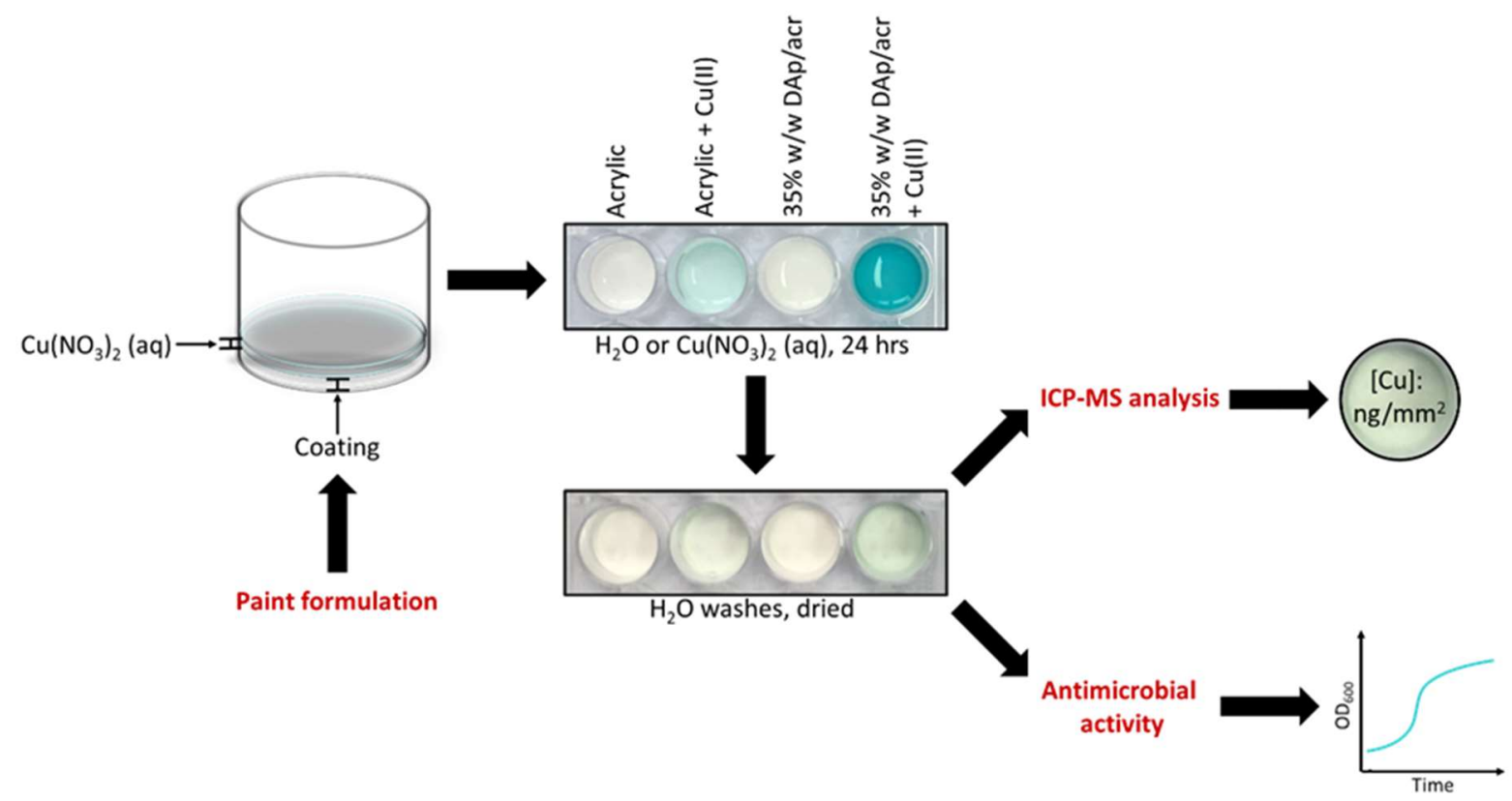

Figure 3.4. Methodology for assessing the $\mathrm{Cu}(\mathrm{II})$-chelating ability of the acrylic and DAp/acr paints and their impact on bacterial growth ( $\pm \mathrm{Cu}(I I)$; second stage of testing). 
The workflow for the second stage of assessments is outlined in Figure 3.4. Initially, the commercial acrylic and DiLevDEG/ADH polyacylhydrazone/acrylic (DAp/acr) paints were formulated, and the bottoms of wells or vials were coated with the paints. Once the paints cured, they were exposed to a $\mathrm{Cu}$ (II) salt solution for $24 \mathrm{~h}$. After $24 \mathrm{~h}$, the coatings were washed, dried, and assessed for either their ability to retain $\mathrm{Cu}(\mathrm{II})$ or their impact on bacterial growth, with and without $\mathrm{Cu}(\mathrm{II})$. The amount of $\mathrm{Cu}(\mathrm{II})$ sequestered by each coating, as well as the amount of $\mathrm{Cu}$ (II) leaching from it into the bacterial growth medium, was quantified via inductively coupled plasma mass spectrometry (ICP-MS), a trace metal technique capable of rapid, multi-element determinations. ICP-MS has detection limits in the sub parts per trillion (ppt) but can also measure in the high parts per million (ppm). For ICP-MS analysis, a sample (diluted as needed) is pumped into a nebulizer and converted into a fine aerosol with argon gas, which is introduced to an argon plasma for ionisation. The positively charged ions then pass into the mass spectrometer to be separated and counted. ${ }^{195}$ For the growth assays, the growth of a bacterial culture in contact with the painted surfaces was monitored, and, by pairing the ICP-MS and growth assay results, the effects of the paint surfaces' levels of $\mathrm{Cu}(\mathrm{II})$-loading and $\mathrm{Cu}(\mathrm{II})$-leaching on bacterial growth could be evaluated.

\subsubsection{Formulation}

The DAp/acr paint was formulated as in Daines et al. ${ }^{127}: 35 \% \mathrm{w} / \mathrm{w}$ of the ADH-crosslinked dispersion polymer in a commercial acrylic paint (LR200) supplied by Resene Paints Ltd. (Table 3.2) was substituted with the aqueous solution of $\sim 50 \% \mathrm{w} / \mathrm{v}$ DiLevDEG/ADH prepared in Chapter 2 (Section 2.2). For comparison, the acrylic paint was also prepared according to the conventional recipe. The percent substitution of the resin was chosen to be $35 \% \mathrm{w} / \mathrm{w}$ because, although the work by Daines et al. ${ }^{127}$ showed that substitution up to $50 \% \mathrm{w} / \mathrm{w}$ was possible, a lower level of substitution (e.g. $30 \% \mathrm{w} / \mathrm{w}$ ) improved properties in the final paint. These properties included gloss, hardness, water resistance, and adherence to standard paint test-cards. Due to the inclusion of titanium dioxide as a pigment, both the acrylic and DAp/acr paints were white and opaque, but the DAp/acr paint was notably less viscous than the acrylic paint. Following formulation, the paints were exposed to $\mathrm{Cu}(\mathrm{II})$ and tested for their $\mathrm{Cu}(\mathrm{II})$-retention and impact on bacterial growth. 
Table 3.2. Composition of the commercial acrylic paint used in this study. ${ }^{a}$

Millbase

Titanium dioxide

Water, rheology modifier, dispersant, surfactant, MPG ${ }^{b}$, defoamer, biocide, alkali

\section{Dispersion mixture}

Dispersion polymer ${ }^{c}$

Coalescent, surface wetter, MPG ${ }^{\text {, }}$, defoamer, biocide, alkali

\footnotetext{
a Proprietary composition and specific components confidential to Resene Paints Ltd.

${ }^{b}$ Monopropylene glycol.

${ }^{\mathrm{c}}$ Resin $=\mathrm{ADH}$-crosslinked dispersion polymer (45\% w/w).
}

\subsubsection{Growth Assay: E. coli NZRM 3647}

The effects of the acrylic and DAp/acr paints $( \pm \mathrm{Cu}(I I))$ on the growth of $E$. coli NZRM 3647 were determined in an assay developed in our research group. This assay was developed in response to concerns about the industry-accepted protocol used to test surfaces for antimicrobial activity: International Organisation for Standardisation (ISO) 22196 (Japanese Industry Standard (JIS) Z 2801). ${ }^{196-198}$ In ISO 22196, standard inocula of E. coli or S. aureus are delivered in a suspension to test and control surfaces, which are then covered with a plastic film and incubated for $24 \pm 1 \mathrm{~h}$ at $35 \pm 1{ }^{\circ} \mathrm{C}$ and $>90 \%$ relative humidity. Viable bacteria are recovered and enumerated at 0 and $24 \mathrm{~h}$ to calculate antimicrobial activity. ${ }^{199}$ This protocol is favoured by manufacturers because it allows ample time for the biocide to diffuse from the coating and exert an effect, confirming antimicrobial activity prior to commercialisation of the product. ${ }^{196,198}$ Yet, there are concerns about the reproducibility of the method. For instance, Wiegand et al. ${ }^{197}$ completed a round robin test that employed the ISO 22196 procedure to elucidate the antimicrobial activity of standard materials. Eight different laboratories participated and reported different results in the first round of testing. Furthermore, in unpublished work in our research team, the reproducibility of results derived from utilisation of the ISO 22196 protocol to determine the antimicrobial activities of different paints was evaluated and found to be poor. ${ }^{200}$ Consequently, a more robust method for measuring the antimicrobial activity of a coating was developed.

The new growth assay developed in our research group generated reproducible results and was more high-throughput, requiring less time per sample. For consistency with previous work on ISO 22196, the bacterium used for testing was E. coli NZRM 3647 (serotype 0157:H7, verocytotoxin negative). In 
the assay, the bottoms of wells in a 24-well plate were coated evenly with the paint, which was allowed to cure and then was washed with water and dried. A standard inoculum of E. coli NZRM 3647 was delivered to each well, and the plate was incubated at $37^{\circ} \mathrm{C}$. The optical density (OD, $\left.600 \mathrm{~nm}\right)$ of the culture in each well was measured at different time points up until $24 \mathrm{~h}$, generating growth curves of the bacterium. Such growth curves are typically sigmoidal, as bacterial growth proceeds through successive phases (Figure 3.5). Immediately following inoculation of a medium, bacteria do not divide, and growth is nil. But, this lag phase is followed by a phase of exponential growth, which eventually plateaus at a maximum population density. In the stationary phase, either growth halts or the growth and death rates of bacterial cells become equal, resulting in no overall growth. As nutrients are depleted, however, bacteria enter a phase of death, and the cells lyse. Although growth curves plot the logarithm ( $\log _{10}$ ) of bacterial density vs time, $\mathrm{OD}_{600}$, a measurement of light scattering, is generally proportional to bacterial density and serves as its proxy in growth experiments, as in the present study. ${ }^{201,202}$

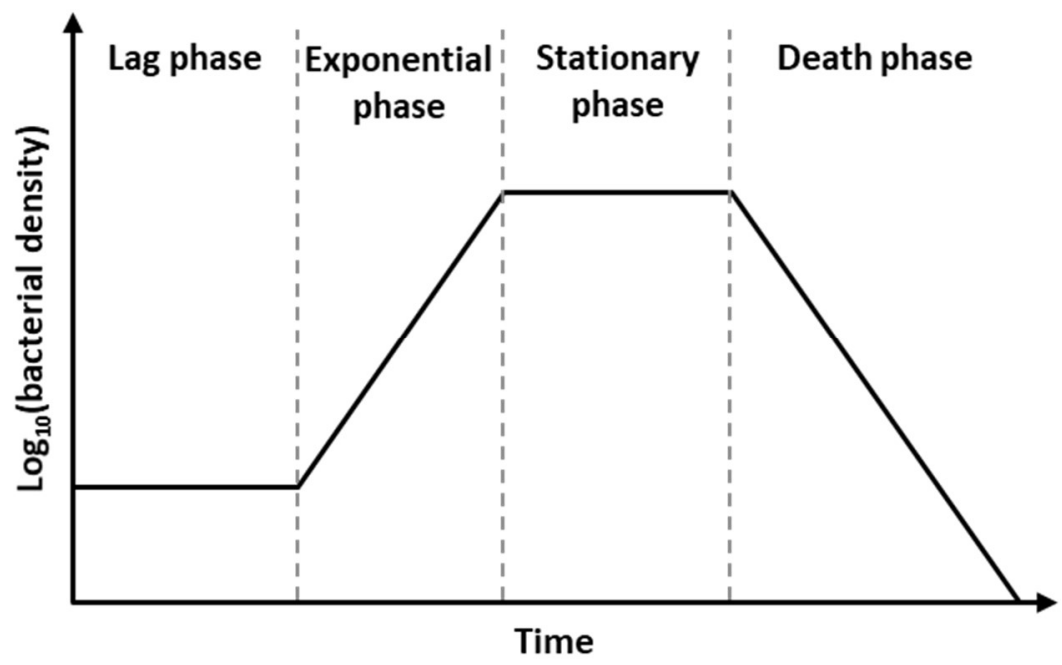

Figure 3.5. Example of a bacterial growth curve. ${ }^{202}$

The developed assay was employed to assess the growth of $E$. coli NZRM 3647 when in contact with the acrylic $( \pm \mathrm{Cu}(\mathrm{II}))$ paint, $35 \% \mathrm{w} / \mathrm{w}$ DAp/acr $( \pm \mathrm{Cu}(I I))$ paint, and a commercial marine paint. The marine paint (Altex Yacht and Boat Paint, Ablative Antifouling No. 5; 40-50\% w/w $\mathrm{Cu}_{2} \mathrm{O}$ ) was included for comparison to a commercial antifouling standard from Resene Paints Ltd., the collaborating paint manufacturer who supplied the acrylic paint. Uncoated wells served as growth controls, and the assay was repeated in triplicate for each coating. In order to determine the effect of $\mathrm{Cu}(\mathrm{II})$-loading on 
bacterial growth, the acrylic and DAp/acr paints were prepared for testing with and without exposure to $\mathrm{CU}(\mathrm{II})$ (Appendix, Figure 8.20). $\mathrm{Cu}(\mathrm{II})$-loading was achieved via exposure to a dilute, aqueous $\mathrm{Cu}(\mathrm{II})$ salt solution for $24 \mathrm{~h}$ (Figure 3.6). These conditions were selected as a starting point because the ultimate application of the coating will require it to bind dilute $\mathrm{Cu}(\mathrm{II})$ in seawater. It was observed that, in the DAp/acr-coated wells, the $\mathrm{Cu}(\mathrm{II})$ salt solution changed from a light blue to teal colour (Figure 3.6, left " $35 \% \mathrm{w} / \mathrm{w} \mathrm{DAp} / \mathrm{acr}+\mathrm{Cu}(\mathrm{II})$ "), suggesting that ligands may have leached from the paint into the supernatant to form $\mathrm{Cu}(\mathrm{II})$ complexes. ${ }^{129}$

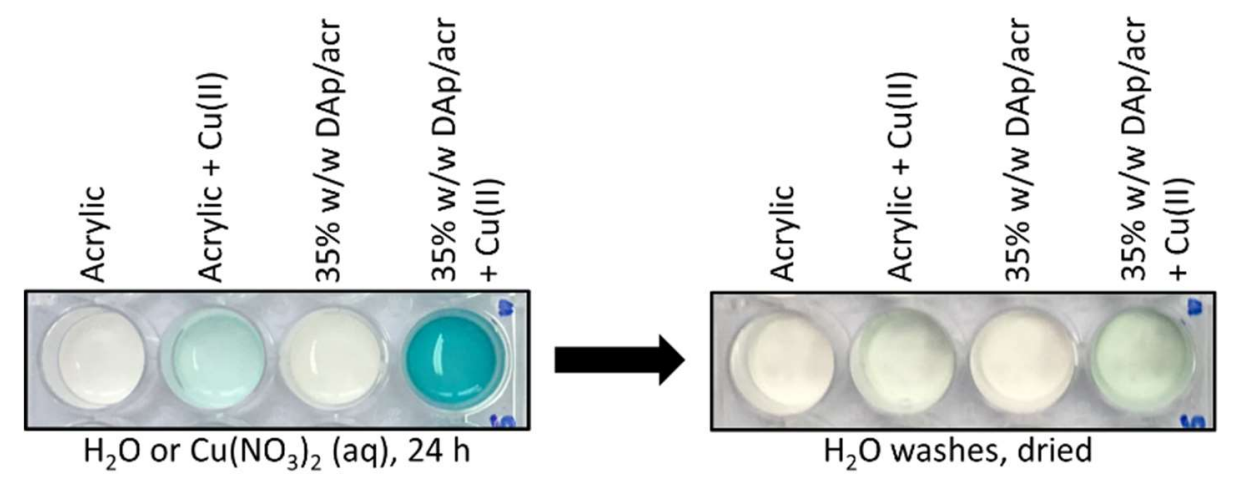

Figure 3.6. $\mathrm{Cu}(\mathrm{II})$-loading process for acrylic and DAp/acr paints.

In the assay, growth inhibition was observed in the wells coated with, in order from least- to mostinhibiting, the acrylic, marine, Cu(II)-loaded 35\% w/w DAp/acr (“DAp/acr + Cu(II)"), 35\% w/w DAp/acr, and $\mathrm{Cu}(\mathrm{II})$-loaded acrylic ("Acrylic + $\mathrm{Cu}(\mathrm{II})$ ") paints (Figure 3.7). Bacterial growth in the uncoated wells adhered to the classic sigmoidal curve, plateauing at a higher $\mathrm{OD}_{600}$ than the other test samples. Growth in the wells coated with the acrylic paint was also sigmoidal but plateaued at a lower $\mathrm{OD}_{600}$ than in the uncoated wells, indicating some inhibitory effect by leached components. Yet, growth in these wells was the least-impacted out of all the coated wells. In contrast, the "Acrylic + $\mathrm{Cu}(\mathrm{II})$ " paint had the greatest inhibitory effect on bacterial growth; the overall growth was net negative, with alternating stages of death and growth. The DAp/acr paint had the second-greatest inhibitory effect. For the other copper-containing paints (e.g. "Marine Paint", "DAp/acr + $\mathrm{Cu}(I I)$ )), the inhibitory effects were approximately equivalent, being only marginally greater for the $\mathrm{Cu}(\mathrm{II})$-loaded $\mathrm{DAp} / \mathrm{acr}$ paint, and alternating phases of slowed/negative and positive growth were observed. Such alternating phases were unsurprising, given that the copper coatings were expected to release $\mathrm{Cu}(\mathrm{II})$ into the growth medium and, subsequently, cause cell lysis. ${ }^{115}$ 


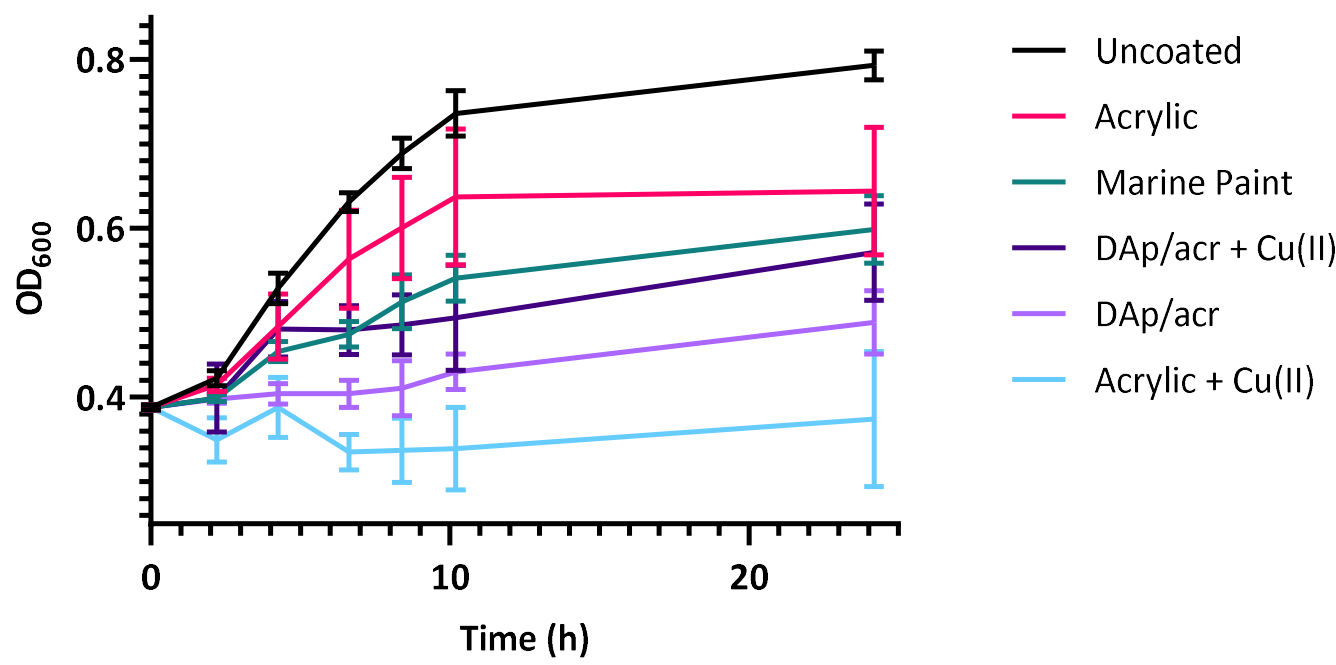

Figure 3.7. Growth curves $\left(\mathrm{OD}_{600}\right.$ vs time) of $E$. coli NZRM 3647 in uncoated wells and wells coated with the acrylic $( \pm \mathrm{Cu}(I I)), 35 \% \mathrm{w} / \mathrm{w}$ DAp/acr $( \pm \mathrm{Cu}(I I))$, and marine paints. The error bars show the standard deviation (SD) from three biological replicates (br).

Regarding the order of inhibition, the assay results were not as predicted. It had been anticipated that both the marine and $\mathrm{Cu}(I I)$-loaded DAp/acr paints would have higher surface concentrations of copper than the $\mathrm{Cu}(\mathrm{II})$-loaded acrylic paint, which is not known to have functionality for stable $\mathrm{Cu}(\mathrm{II})$ complexation, and that they would, therefore, have a more detrimental effect on the growth of $E$. coli NZRM 3647. So, it was unexpected that the "Acrylic + $\mathrm{Cu}(I I)$ " coating had a greater inhibitory impact than both the marine paint, which is $40-50 \% \mathrm{w} / \mathrm{w}$ cuprous oxide, and the "DAp/acr + $\mathrm{Cu}(\mathrm{II})$ " paint, which has $35 \% \mathrm{w} / \mathrm{w}$ of its binder substituted with a polymer capable of binding $\mathrm{Cu}(\mathrm{II})$. Due to the presence of $\mathrm{Cu}(\mathrm{II})$ ligands in the DAp/acr paint, it was hypothesised that the "DAp/acr + $\mathrm{Cu}(\mathrm{II})$ " paint bound significantly more but released less $\mathrm{Cu}(\mathrm{II})$ than the "Acrylic + $\mathrm{Cu}(\mathrm{II})$ " paint, on which the $\mathrm{Cu}(\mathrm{II})$ would be only weakly associated. Since the release of copper ions from a surface is necessary for antimicrobial activity, this could explain the greatest growth inhibition being in the "Acrylic + $\mathrm{Cu}(\mathrm{II})$ " wells. Regarding the marine paint, it was hypothesised that, like "DAp/acr + Cu(II)", the surface released less copper than the "Acrylic + $\mathrm{Cu}(I I)$ " paint. It was also unexpected that E. coli NZRM 3647 would exhibit the second-greatest growth defect in the presence of the DAp/acr paint, a paint without copper. But, there was evidence of ligands, perhaps the DiLevDEG/ADH polyacylhydrazone, leaching from the coating, and such leached components may have their own antimicrobial activity. To test these hypotheses, the amounts of copper bound to and released from the surfaces of the 
"Acrylic $+\mathrm{Cu}(I I)$ " and "DAp/acr + $\mathrm{Cu}(I I)$ " paints, as well as released from the surface of the marine paint, were quantified. Additionally, the DAp/acr paint was tested for the leaching of DiLevDEG/ADH.

\subsubsection{Quantification of Cu(II)-Leaching}

To quantify the copper leaching from the coatings during the growth assay, the amounts of copper leached from the $\mathrm{Cu}(\mathrm{II})$-loaded commercial acrylic and 35\% w/w DAp/acr paints, as well as the marine paint, into the nutrient broth after $24 \mathrm{~h}$ were measured. As previously described for the growth assay, this was done by coating the well-bottoms of a 24-well plate with the acrylic, DAp/acr, and marine paints and loading some of the cured acrylic and DAp/acr paints with $\mathrm{Cu}(I I)$ (Appendix, Figure 8.20). Then, nutrient broth was added to uncoated wells and the wells coated with the acrylic $( \pm \mathrm{Cu}(I I))$, DAp/acr $( \pm \mathrm{Cu}(I I))$, and marine paints. The uncoated wells and wells coated with the acrylic and DAp/acr paints but unexposed to $\mathrm{Cu}(\mathrm{II})$ served as blanks. After incubating the plate for $24 \mathrm{~h}$ at $37^{\circ} \mathrm{C}$, the nutrient broth from each well was removed and diluted for ICP-MS analysis to quantify the amount of copper, likely in the oxidised $\mathrm{Cu}(\mathrm{II})$ form, released from the coated surfaces into the growth medium.

The copper results (Table 3.3) supported the hypothesis that the extent of growth inhibition of E. coli NZRM 3647 in the assay was related to the amount of $\mathrm{Cu}(\mathrm{II})$ released by the copper-containing paints. The marine paint leached the least amount of copper $(25.2 \pm 0.6 \mu \mathrm{g})$. This provided an explanation for it having a smaller impact on bacterial growth than the other copper paints but was surprising, given its high copper content. Although, cuprous oxide $\left(\mathrm{Cu}_{2} \mathrm{O}\right)$, the biocidal agent in the marine paint, is poorly water-soluble, and its dissolution rate depends on the particle size, $\mathrm{pH}$, temperature, and presence of other ligands or ions in solution. When exposed to oxygen in solution, $\mathrm{Cu}(\mathrm{I})$ is rapidly oxidised to $\mathrm{Cu}(\mathrm{II}) .{ }^{203-205}$ The $\mathrm{Cu}(\mathrm{II})$-loaded acrylic paint had the greatest inhibitory effect on bacterial growth and released the most copper ( $91 \pm 5 \mu \mathrm{g}$ ) out of all of the tested coatings. The $\mathrm{Cu}(\mathrm{II})$-loaded DAp/acr paint, which had a slightly greater inhibitory growth effect than the marine paint, released more copper than the marine paint but less than the "Acrylic + $\mathrm{Cu}(\mathrm{II})$ " paint (39 $\pm 5 \mu \mathrm{g})$. Thus, for the copper coatings, increased growth inhibition corresponded to increased $\mathrm{Cu}(I I)$-leaching, as hypothesised. This is in agreement with the vital role of surface-released copper ions in the proposed mechanism of contact killing for copper surfaces: in the literature, it is proposed that copper ions released from a surface cause damage to the bacterial membrane and then enter the cell, resulting in oxidative damage, cell death, and DNA degradation. ${ }^{115}$ Additionally, Macomber and Imlay ${ }^{206}$ found that $E$. coli W3110 exhibited a growth defect at $\mathrm{Cu}(\mathrm{II})$ concentrations greater than $8 \mu \mathrm{M}$ in a minimal 
salts medium containing glucose after $10 \mathrm{~h}$ at $37^{\circ} \mathrm{C}$. As the $\mathrm{Cu}(\mathrm{II})$-leaching results from the coatings in the present study are an order of magnitude higher (i.e. $\mathrm{mM} v s \mu \mathrm{M}$ ), it is reasonable that the "Acrylic + $\mathrm{Cu}(\mathrm{II})$ ", "DAp/acr + $\mathrm{Cu}(\mathrm{II})$ ", and marine paints negatively affected the growth of E. coli NZRM 3647 in the assay. In light of these $\mathrm{Cu}(\mathrm{II})$-leaching results, measuring the $\mathrm{Cu}(\mathrm{II})$-loading on the surface of the acrylic and DAp/acr paints was of interest, since it was hypothesised that the DAp/acr paint would bind more but release less $\mathrm{Cu}(\mathrm{II})$.

Table 3.3. Amounts of copper leached from the $\mathrm{Cu}(\mathrm{II})$-loaded commercial acrylic and $35 \% \mathrm{w} / \mathrm{w}$ DAp/acr paints, as well as the marine paint, into the nutrient broth after $24 \mathrm{~h}$.

\begin{tabular}{lccc}
\hline Coating & ppm Cu & $\mu^{a}$ & $\mathrm{mM} \mathrm{Cu}^{\mathrm{a}}$ \\
\hline Acrylic $+\mathrm{Cu}(\mathrm{II})$ & $180 \pm 10$ & $91 \pm 5$ & $2.9 \pm 0.2$ \\
DAp/acr + Cu(II) & $80 \pm 10$ & $39 \pm 5$ & $1.2 \pm 0.2$ \\
Marine Paint & $50 \pm 1$ & $25.2 \pm 0.6$ & $0.79 \pm 0.02$ \\
\hline
\end{tabular}

${ }^{\mathrm{a}}$ Mean $\pm \mathrm{SD}, \mathrm{n}=3 . \mathrm{ppm}=\mu \mathrm{g} / \mathrm{mL}, \mu \mathrm{g} / \mathrm{g}$.

\subsubsection{Quantification of Cu(II)-Loading}

The amounts of $\mathrm{Cu}(\mathrm{II})$ retained by the commercial acrylic and 35\% w/w DAp/acr paints were quantified (Figure 3.4). These paints were used to coat the bottoms of custom-made glass vials that had dimensions matching the wells from the 24-well plate in the growth assay so that the copper results could be directly related to the assay; it was acknowledged that the depth and exposed surface area of the coating would vary in differently sized vials and, thus, affect $\mathrm{Cu}(\mathrm{II})$-loading. The paints were left to cure until they were no longer tacky, and then an aliquot of a dilute, aqueous $\mathrm{Cu}(\mathrm{II})$ salt solution was added to each vial. In the DAp/acr-coated vials, again, the supernatant changed from a light blue to teal colour - presumably, from $\mathrm{Cu}(\mathrm{II})$ ligands leached from the paint. After $24 \mathrm{~h}$, the vials were washed with water and dried. Following exposure to $\mathrm{Cu}(\mathrm{II})$, the surface of the acrylic paint was light green, while the $35 \%$ w/w DAp/acr paint surface was a slightly darker shade of green (as in Figure 3.6, right). The $\mathrm{Cu}(\mathrm{II})$-loaded coatings were digested in concentrated nitric acid and diluted for copper analysis via ICP-MS.

To verify that the digestion procedure was sufficient to release all of the copper into solution, two acrylic paint samples were spiked with a known amount of copper. For the first sample, the copper was homogeneously mixed in the paint before using it to coat the bottom of a vial. For the second sample, the copper was added to the surface of the already-cured paint. The acrylic paint without 
added copper served as a blank, and the reported results are blank-subtracted. Post-digestion of these coatings, ICP-MS $(\mathrm{Cu})$ analysis revealed that the percent error between the theoretical and actual amounts of copper were $7 \%$ and $4 \%$ for the mixed and surface-spiked samples, respectively (Table 3.4). The copper-spiked surface was more representative of the $\mathrm{Cu}(I I)$-loaded paints, and the percent error calculated from its measured copper content was deemed acceptable. Therefore, the copper results validated the overall efficacy of the digestion procedure.

Table 3.4. ICP-MS (Cu) results of acrylic paint spike study.

\begin{tabular}{lccc} 
Sample & Actual $\mu \mathrm{g} \mathrm{Cu}$ in Coating & Theoretical $\mu \mathrm{g} \mathrm{Cu}$ in Coating & \% Error \\
\hline Mixed & 11.8 & 11.1 & 7 \\
Surface & 10.8 & 10.4 & 4 \\
\hline
\end{tabular}

The $\mathrm{Cu}(\mathrm{II})$-loading results for the acrylic and $35 \% \mathrm{w} / \mathrm{w}$ DAp/acr paints were reported as the amount of copper normalised by the mass of the cured paint in the vial and by the surface area of the paint exposed to the $\mathrm{Cu}(\mathrm{II})$ salt solution (Table 3.5). This was done to account for variations in the sample preparation (e.g. mass, surface area), allowing for comparisons between coatings. Although, such normalisations could be misleading, as the depth of $\mathrm{Cu}(\mathrm{II})$-penetration in the coating is unknown (e.g. normalisation by surface area when not all of the copper is necessarily at the surface). Four batches of each paint were prepared and analysed in triplicate on different days to assess the reproducibility of the method for samples both within a batch and between independent batches. Within a batch, the copper results were relatively reproducible, with the percent relative standard deviation (RSD) between the triplicate measurements being, at most, 15\%. However, between batches, the results were not consistently reproducible. A one-way ANOVA with Tukey's post hoc test revealed no statistically significant differences $(p>0.05)$ between the results for Batch 1 vs 2 and Batch 3 vs 4 , but the results for Batches $1 / 2$ vs Batches 3/4 were different. In fact, the percent difference between the mean results for Batches $1 / 2$ vs Batches $3 / 4$ was, in some cases, greater than $60 \%$. Furthermore, it was uncertain which paint could retain the most copper, since the mean batch results ranged from 1450-2700 $\mu \mathrm{g} \mathrm{Cu} / \mathrm{g}\left(730-1400 \mathrm{ng} \mathrm{Cu} / \mathrm{mm}^{2}\right)$ and 1300-2020 $\mu \mathrm{g} \mathrm{Cu} / \mathrm{g}$ (600-920 ng Cu/mm²) for the acrylic and DAp/acr paints, respectively. 
Table 3.5. Amounts of copper retained by different batches of the commercial acrylic and $35 \% \mathrm{w} / \mathrm{w}$ DAp/acr paints.

\begin{tabular}{|c|c|c|c|}
\hline Coating & Batch \# & $\mu g \mathrm{Cu} / \mathrm{g}$ Coating ${ }^{\mathrm{a}}$ & $\mathrm{ng} \mathrm{Cu} / \mathrm{mm}^{2}$ Coating ${ }^{a, b}$ \\
\hline Acrylic + Cu(II) & 1 & $2500 \pm 200$ & $1200 \pm 100$ \\
\hline Acrylic + Cu(II) & 2 & $2700 \pm 300$ & $1400 \pm 200$ \\
\hline Acrylic + Cu(II) & 3 & $1500 \pm 100$ & $740 \pm 90$ \\
\hline Acrylic + Cu(II) & 4 & $1450 \pm 80$ & $730 \pm 40$ \\
\hline $35 \% \mathrm{w} / \mathrm{w}$ DAp/acr $+\mathrm{Cu}(\mathrm{II})$ & 1 & $1300 \pm 200$ & $620 \pm 90$ \\
\hline $35 \% \mathrm{w} / \mathrm{w} \mathrm{DAp} / \mathrm{acr}+\mathrm{Cu}(\mathrm{II})$ & 2 & $1300 \pm 100$ & $600 \pm 80$ \\
\hline $35 \% \mathrm{w} / \mathrm{w}$ DAp/acr $+\mathrm{Cu}(\mathrm{II})$ & 3 & $1820 \pm 10$ & $880 \pm 40$ \\
\hline $35 \% \mathrm{w} / \mathrm{w}$ DAp/acr $+\mathrm{Cu}(\mathrm{II})$ & 4 & $2020 \pm 40$ & $920 \pm 20$ \\
\hline
\end{tabular}

${ }^{a}$ Mean $\pm S D, n=3$.

${ }^{\mathrm{b}}$ Surface area $\left(\mathrm{mm}^{2}\right)$ exposed to $\mathrm{Cu}(\mathrm{II})$ salt solution. Calculated as in the Appendix (Figure 8.22).

Because the same procedure was followed to prepare and test the coatings, the source of variability in the copper results between paint batches was unclear. The only difference between batches was that the paint cure-times were inconstant, ranging from 17-66 h. Rather than standardising the length of time the paints were allowed to cure, each batch was left at $50{ }^{\circ} \mathrm{C}$ until, at least, all of the samples in the batch were no longer tacky, but sometimes they were left longer. Since triplicate samples within batches were cured for the same time-length, this could explain the absence of such variability between these replicates. Also, $\mathrm{Cu}(\mathrm{II})$-loading experiments for the batches were conducted on different days over the course of two months. Thus, the samples could have been exposed to different atmospheric conditions (e.g. humidity) as well, further impacting the curing process. It is probable that the parameters and extent of curing notably affect $\mathrm{Cu}(\mathrm{II})$-loading in a paint. For example, a softer, not yet fully cured paint may allow deeper penetration of the $\mathrm{Cu}(\mathrm{II})$ salt solution and, consequently, bind more $\mathrm{Cu}(\mathrm{II})$.

Regarding the growth assay, the curing conditions (e.g. time, temperature) of the paints in the wells of the 24-well plate were different from the conditions in the $\mathrm{Cu}(\mathrm{II})$-loading experiments. Although, similarly, the paints were allowed to cure until they were no longer tacky. The variability of the copper results between paint batches, possibly due to differences in curing, suggested that the data should only be related semi-quantitatively to the growth assay, meaning that the copper concentrations in the coated vials were not necessarily equivalent to what those had been in the coated wells. Accordingly, it may be concluded that, in the growth assay, similar amounts of $\mathrm{Cu}(\mathrm{II})$ were retained by the acrylic and $35 \% \mathrm{w} / \mathrm{w}$ DAp/acr paints post-exposure to the $\mathrm{Cu}(\mathrm{II})$ salt solution and that the $\mathrm{Cu}(\mathrm{II})$ 
concentrations were in the $\mathrm{mg} / \mathrm{g}\left(\mu \mathrm{g} / \mathrm{mm}^{2}\right)$ range. This evidence contradicted the hypothesis that the DAp/acr paint, with the $\mathrm{Cu}(\mathrm{II})$ ligand incorporated at $35 \% \mathrm{w} / \mathrm{w}$, would retain significantly more $\mathrm{Cu}(\mathrm{II})$ than the acrylic paint. Since $\mathrm{Cu}(\mathrm{II})$-leaching was measured for samples in triplicate within a single batch, batch variability was also expected to affect the accuracy of these results, but the relative order was not expected to change. Both the leaching and loading of $\mathrm{Cu}(\mathrm{II})$ in the DAp/acr paint were likely affected by the additional leaching of a $\mathrm{Cu}(\mathrm{II})$ ligand, the identity of which was determined in a separate experiment.

\subsubsection{DiLevDEG/ADH Polyacylhydrazone-Leaching}

An experiment was conducted to confirm that the $\mathrm{Cu}(\mathrm{II})$ ligand leaching from the $35 \% \mathrm{w} / \mathrm{w}$ DAp/acr paint was the DiLevDEG/ADH polyacylhydrazone. This leaching was suspected when, upon the addition of the aqueous $\mathrm{Cu}$ (II) salt solution to the DAp/acr-coated well or vial, the supernatant changed colour from light blue to teal, which is consistent with the $d-d$ transitions in $\mathrm{Cu}(I I)$ complexes. ${ }^{129}$ DiLevDEG/ADH is water-soluble and present at a high concentration in the paint, making it a probable candidate for the leachate. The identity of the leachate was confirmed by applying the DAp/acr paint to the bottom of a well in a 24-well plate, allowing it to cure, adding deuterium oxide $\left(D_{2} O\right)$ to the well for $24 \mathrm{~h}$, and then measuring the ${ }^{13} \mathrm{C}$ NMR spectrum of the $D_{2} \mathrm{O}$ supernatant and comparing it to that of DiLevDEG/ADH.

The ${ }^{13} \mathrm{C}$ NMR spectrum of the leachate revealed that the DiLevDEG/ADH polyacylhydrazone binder was indeed leaching out of the DAp/acr paint. As seen in Figure 3.8, the signals in the reference spectrum of DiLevDEG/ADH were present in the spectrum of the leachate. For instance, the signals for C2 $(\underline{\mathrm{C}}=\mathrm{N})$, doubled due to rotamers, were observed at $\delta_{\mathrm{c}} 163.0$ and $159.1 \mathrm{ppm}$, and the signal for $\mathbf{C 5}$,

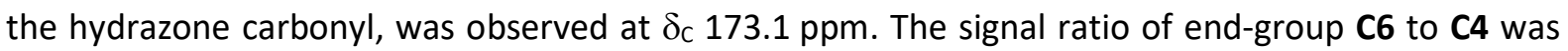
approximately 1:11 in the reference spectrum but 1:4 in the leachate spectrum, indicating that the polyacylhydrazone was releasing small molecules and/or monomers into solution. This was unsurprising, as our research group has observed that the acylhydrazone bond is dynamic, breaking and reforming in aqueous solutions. ${ }^{128}$ Because signals unattributed to DiLevDEG/ADH were also present in the leachate's spectrum, it was apparent that other compounds leached from the DAp/acr paint as well, but these compounds were not identified, as the remainder of the paint composition is confidential to Resene Paints Ltd. Undoubtedly, the leaching of this paint's binder impacted the binding and release of $\mathrm{Cu}(\mathrm{II})$ and, subsequently, bacterial growth. For example, in the assay with $E$. coli NZRM 3647, the growth defect was greater in the DAp/acr wells without $\mathrm{Cu}(I I)$ than with. It is possible that the leached polyacylhydrazone had an antimicrobial effect that was mitigated by binding $\mathrm{Cu}(\mathrm{II})$, 
since the formation of such complexes could even promote DiLevDEG/ADH's retention in the paint. In addition to these effects, the leaching of DiLevDEG/ADH had serious implications regarding the suitability of the DAp/acr paint as the project's proof-of-concept.
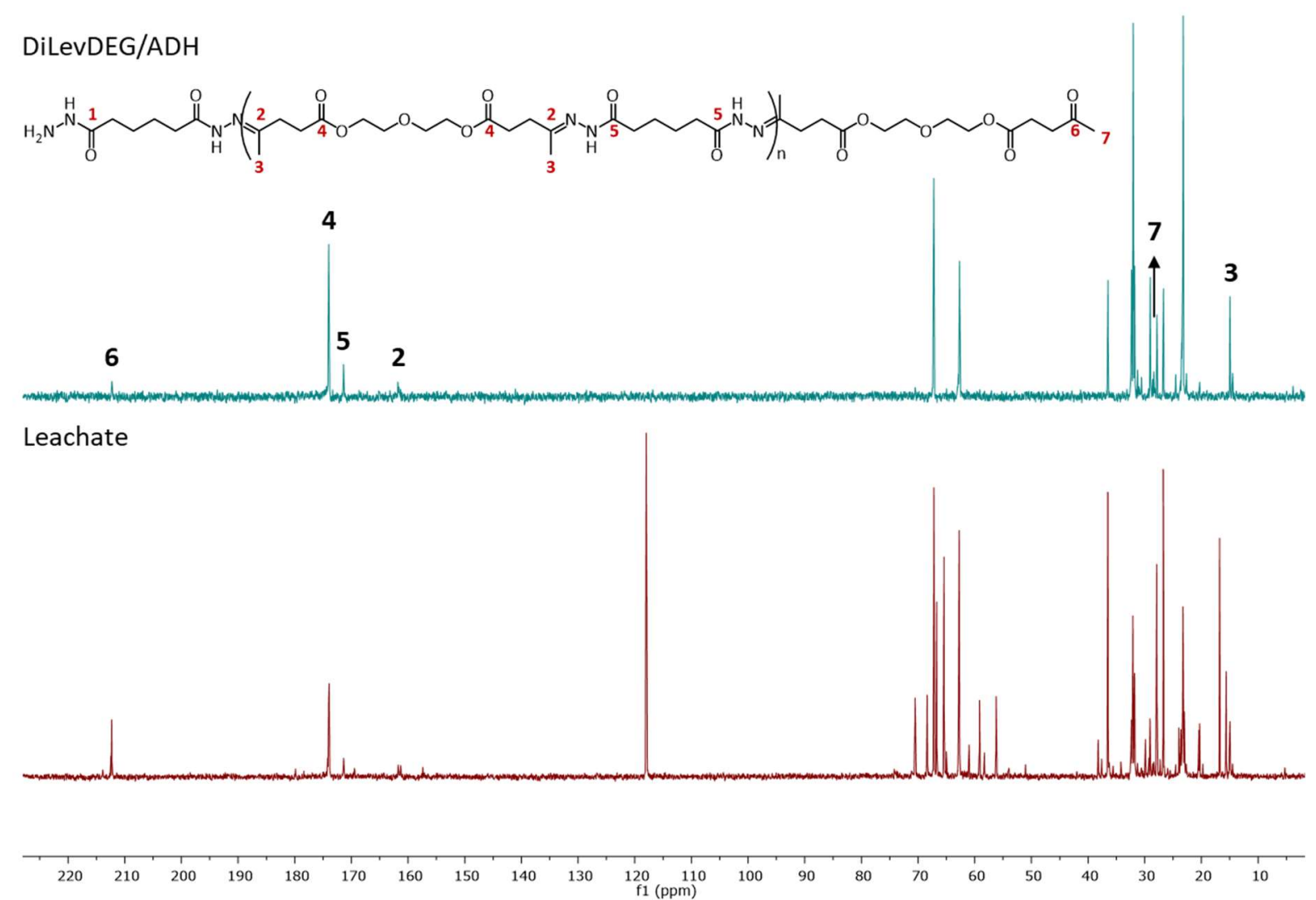

Figure 3.8. ${ }^{13} \mathrm{C}$ NMR spectra (crude; $\mathrm{D}_{2} \mathrm{O}$ ) of the leachate and DiLevDEG/ADH polyacylhydrazone with select carbon assignments $\left(\delta_{c} 118.0 \mathrm{ppm}=\right.$ acetonitrile $)$.

\subsubsection{Conclusions}

Cumulatively, the $\mathrm{Cu}(\mathrm{II})$-leaching, $\mathrm{Cu}(\mathrm{II})$-loading, polyacylhydrazone-leaching, and growth assay results revealed that the DAp/acr paint was unsuitable as an antifouling coating. As previously stated in Chapter 2 (Section 2.1), an ideal ligand to serve as the project's proof-of-concept must be stable and not introduce any new, possibly toxic, components into seawater from the antifouling paint. The DAp/acr paint failed to meet this criterion: it leached small molecules and/or monomers from the DiLevDEG/ADH polyacylhydrazone binder into the aqueous solution (Figure 3.8), inhibiting bacterial growth (Figure 3.7). This suggested that DiLevDEG/ADH may have some associated toxicity. In fact, the DAp/acr paint had an even more negative impact on the growth of $E$. coli NZRM 3647 than the 
$\mathrm{CU}$ (II)-loaded DAp/acr paint, which could be explained by enhanced retention of DiLevDEG/ADH in the paint through $\mathrm{CU}(\mathrm{II})$-coordination. Furthermore, the $\mathrm{Cu}(\mathrm{II})$-loaded commercial acrylic paint inhibited growth to a greater extent than any of the other tested coatings, including the DAp/acr ( $\pm \mathrm{Cu}(\mathrm{II}))$ paints and the industry-standard marine paint.

It was discovered that the order of growth inhibition for the copper-containing paints (i.e. from most to least: "Acrylic + $\mathrm{Cu}(I I)$ ", "DAp/acr + CU(II)", "Marine Paint") was due to the relative amounts of copper released by the coatings, rather than the surface concentrations of copper. Both the acrylic and DAp/acr paints bound similar amounts of $\mathrm{Cu}(\mathrm{II})$ in the range of $0.1-0.3 \% \mathrm{w} / \mathrm{w}$ (Table 3.5), while the marine paint was $40-50 \% \mathrm{w} / \mathrm{w}$ cuprous oxide. Yet, the marine paint had less of an inhibitory effect than either coating. Upon completion of the $\mathrm{Cu}(\mathrm{II})$-leaching experiment, however, it was shown the there was a direct relationship between the degree of growth inhibition and the amount of copper released from the coating into the bacterial culture. For example, the "Acrylic + $\mathrm{Cu}(I I)$ " paint released the most copper and had the largest inhibitory effect (Table 3.3), and the marine paint released the least copper and had the smallest inhibitory effect. When considering these results, it became clear that the results of the growth assay would not be translatable to antifouling in the marine environment.

The growth assay was deemed an inappropriate proxy for testing the antifouling performance of coatings because it determined the antimicrobial activity of leached components, rather than surface activity. The copper concentrations measured in the bacterial cultures would not be achieved in the marine environment; the leached copper would be greatly diluted by seawater, with the concentration of copper ions being highest at the surface and decreasing in a gradient. The flux of copper ions immediately at the surface has a role in antifouling, but this is not what is reflected in the assay. Since the copper ions are not continuously removed from the culture, the copper concentration is allowed to increase until reaching an equilibrium as copper-leaching progresses, exerting a greater inhibitory effect on bacterial growth. This does not provide any understanding of the surface dynamics or proclivity of the paint to be colonised by bacteria. Therefore, it was decided that the growth assay should no longer be used to assess and rank potential antifouling coatings and that an assay testing bacterial adherence to the coatings would be more suitable. 


\subsection{CU(II) LIGANDS IN AN EPOXY COATING}

With the abandonment of the DAp/acr paint and acylhydrazone ligands, efforts were focused on the incorporation of other $\mathrm{Cu}(\mathrm{II})$ ligands, particularly tetraaza macrocycle cyclen (Table 3.6), into a coating binder. Marine paints commonly use polyurethanes or epoxies as polymeric resins. ${ }^{185}$ Therefore, several combinations of amine and/or alcohol components with Desmodur ${ }^{\circledR} \mathrm{W}$ (Covestro), a cycloaliphatic diisocyanate, were trialled to produce polyurea or polyurethane coatings, respectively (Experimental, Scheme 6.6, Table 6.4). However, these combinations resulted in polymers that were unamenable to further manipulation or in no observable polymerisation at all (Appendix, Table 8.18), meaning that the reactions were either too fast or too slow/did not occur. This was undesirable, as it precluded spreading of the polymer into a film. Therefore, in consultation with Resene Paints Ltd., an epoxy system was selected to meet our requirements. ${ }^{207}$

The selected, two-pack epoxy system is composed of commercial epoxy resin Epikote ${ }^{\mathrm{TM}} 235$ (Resolution Performance Products) and commercial hardener Ancamine ${ }^{\circledR} 2459$ (Air Products and Chemicals, Inc.). Epikote ${ }^{\mathrm{TM}}$ Resin 235 contains no diluent and is a blend of bisphenol A (BPA) and F (BPF) resins derived from bisphenol $A$ or $F$ and epichlorohydrin (Table 3.6). It is generally used in solvent-free, high-solids coatings in the building and civil engineering industries, and its weight per epoxide (WPE), which is the weight of resin per one gram-equivalent of epoxy groups, is $177-182 \mathrm{~g} /$ eq. $^{208-210}$

$$
W P E=\frac{\text { molecular weight of epoxy resin }}{\text { number of epoxy groups }}
$$

Ancamine ${ }^{\circledR} 2459$ Curing Agent consists of a ketimine-protected polyamine (Table 3.6), and moisture is necessary for its deprotection, freeing each amine to react with an epoxy group (Scheme 3.3). Consequently, its cure-time is relatively slow. This is an attractive feature for the purposes of the current project, as it allows time for manipulation of the epoxy system (e.g. able to coat a surface with a film). Other attractive features of Ancamine ${ }^{\circledR} 2459$ include its usage in marine coatings and its high polyamine content $(60 \% \mathrm{w} / \mathrm{w})^{211}$; since polyamines can function as $\mathrm{Cu}(\mathrm{II})$ ligands, ${ }^{130}$ coatings derived from Ancamine ${ }^{\circledR} 2459$ may already be capable of $\mathrm{Cu}(\mathrm{II})$-sequestration. Despite its high amine content, Ancamine ${ }^{\circledR} 2459$ yields low-blush films. ${ }^{211}$ Blushing is caused by the sorption of carbon dioxide and moisture by the hygroscopic amine scavengers under conditions of high humidity or cool ambient temperatures and appears as white patches, making a clear coating look hazy or milky. ${ }^{212}$ The amine hydrogen equivalent weight (AHEW) of Ancamine ${ }^{\circledR} 2459$ is $101 \mathrm{~g} / \mathrm{eq}$, representing the weight of hardener per one gram-equivalent of active hydrogens in the polyamine. The number of active hydrogens is the number of amine hydrogens. ${ }^{209,211}$ 


$$
\text { AHEW }=\frac{\text { molecular weight of amine }}{\text { number of active hydrogen atoms of amine }}
$$

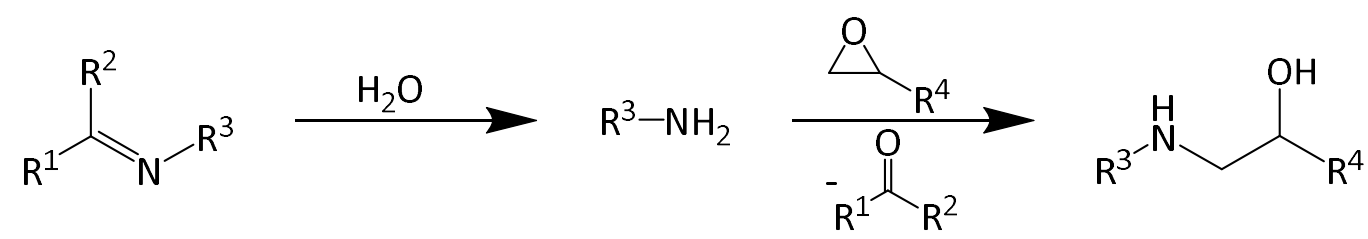

Scheme 3.3. Curing process for Ancamine ${ }^{\circledR}$ 2459: ketimine deprotection of an amine, which then reacts with an epoxide. A side product of the deprotection is a volatile ketone.

In order to obtain the best performance from the two-pack epoxy system, Epikote ${ }^{\mathrm{TM}} 235$ and Ancamine ${ }^{\circledR} 2459$ should be mixed in stoichiometric proportions so that there is one active amine hydrogen per epoxy group. ${ }^{209}$ Both the WPE and AHEW are crucial in this calculation. For example, the WPE of Epikote ${ }^{\mathrm{TM}} 235$ is $177-182 \mathrm{~g} /$ eq epoxy group, and the AHEW of Ancamine ${ }^{\circledR} 2459$ is $101 \mathrm{~g} / \mathrm{eq}$ amine hydrogen. This means that 177-182 g of Epikote ${ }^{\mathrm{TM}} 235$ are required to react completely with $101 \mathrm{~g}$ of Ancamine ${ }^{\circledR}$ 2459. Typically, these stoichiometric proportions are represented by the per hundred resin (phr), which is the weight of hardener required to mix stoichiometrically with $100 \mathrm{~g}$ of epoxy resin (example calculation in the Appendix, Scheme 8.1).

$$
p h r=\frac{A H E W}{W P E} \times 100
$$

Once the phr was known, two types of alterations to the epoxy system were explored: 1) modification of the composition, identity, and/or proportion of hardener, and 2) surface-functionalisation of undercured epoxy coatings. The epoxy resin, hardeners, and surface ligands are described in Table 3.6, and all of the tested combinations are displayed in Table 3.7. In addition to Ancamine ${ }^{\circledR} 2459$, the other polyamine hardeners used to prepare coatings with Epikote ${ }^{\mathrm{TM}} 235$ were $\mathrm{Cu}(\mathrm{II})$ ligands cyclen, cyclam, and diethylenetriamine (DETA). As previously discussed in Chapter 2 (Section 2.4), tetraaza macrocycles, including cyclen and cyclam, selectively bind $\mathrm{Cu}(\mathrm{II})$ in highly stable 1:1 complexes. ${ }^{89,167}$ DETA is also popular in the literature as a tridentate, nitrogen-donor ligand, forming two fivemembered chelate rings with $\mathrm{Cu}(\mathrm{II}) .{ }^{213-215} \mathrm{Cyclen}$, cyclam, and DETA, as well as branched PEI, were also used to functionalise the surfaces of undercured Ancamine ${ }^{\circledR}$ 2459/Epikote ${ }^{\mathrm{TM}} 235$ coatings. Branched PEI is a polymer consisting of repeating $-\left(\mathrm{C}_{2} \mathrm{H}_{5} \mathrm{~N}\right)-$ units. Its primary, secondary, and tertiary amines are present in an approximately 1:1:1 ratio, and it has demonstrated a strong preference for $\mathrm{Cu}(\mathrm{II}) \cdot{ }^{76,77,216}$ 
Table 3.6. Descriptions of epoxy resin, hardeners, and surface ligands used in this study.

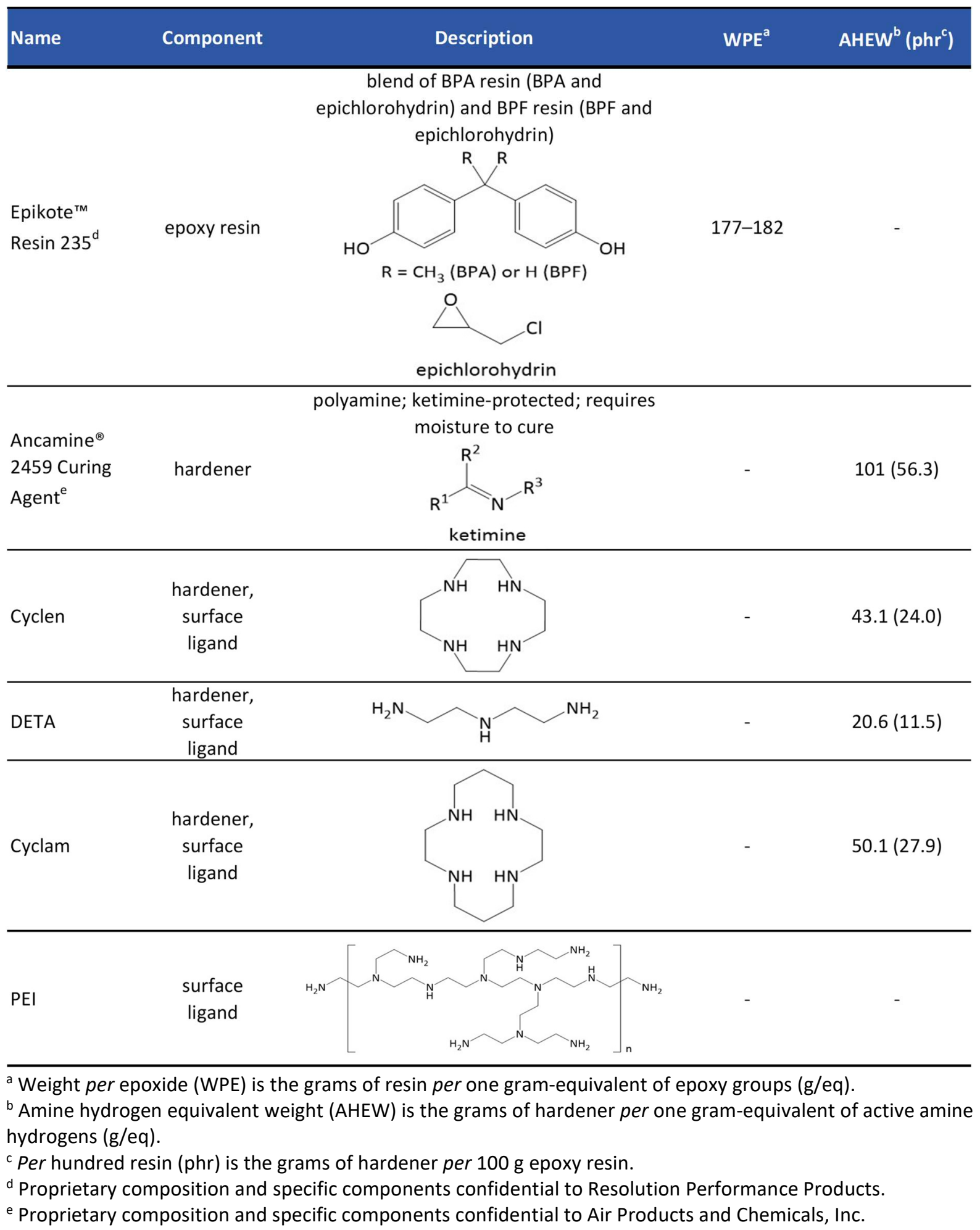


Table 3.7. Epoxy coatings prepared with epoxy resin Epikote ${ }^{\mathrm{TM}} 235$ and the following hardeners: Ancamine ${ }^{\circledR} 2459$, cyclen, DETA, and cyclam.

\begin{tabular}{|c|c|c|}
\hline Hardener & $\begin{array}{c}\text { Surface } \\
\text { Functionalisation }\end{array}$ & $\%$ w/w Overcure (+)/Undercure $(-)^{a}$ \\
\hline Ancamine ${ }^{\circledR} 2459$ & - & $\begin{array}{c}-50 \%,-40 \%,-30 \%,-20 \%,-10 \% \\
\text { stoichiometric, }+10 \%,+20 \%,+50 \%\end{array}$ \\
\hline Ancamine ${ }^{\circledR} 2459$ & cyclam & $-50 \%,-40 \%,-30 \%,-20 \%,-10 \%$ \\
\hline Ancamine ${ }^{\circledR} 2459$ & cyclen & $-40 \%$ \\
\hline Ancamine $^{\circledR} 2459$ & DETA & $-40 \%,-30 \%$ \\
\hline Ancamine ${ }^{\circledR} 2459$ & PEI & $-40 \%$ \\
\hline Ancamine ${ }^{\circledR} 2459$, cyclen & - & $+10 \%,+20 \%\left(\right.$ cyclen $\left.^{b}\right)$ \\
\hline Ancamine $^{\circledR} 2459$, DETA & - & $+10 \%,+20 \%\left(\mathrm{DETA}^{\mathrm{c}}\right)$ \\
\hline Cyclen & - & $-20 \%,-10 \%$, stoichiometric, $+10 \%,+20 \%$ \\
\hline DETA & - & $-20 \%,-10 \%$, stoichiometric, $+10 \%,+20 \%$ \\
\hline DETA $(75 \%)$, cyclen $(25 \%)^{d}$ & - & stoichiometric \\
\hline DETA $(50 \%)$, cyclen $(50 \%)^{\mathrm{e}}$ & - & stoichiometric \\
\hline DETA $(25 \%)$, cyclen $(75 \%)^{f}$ & - & stoichiometric \\
\hline DETA $(25 \%)$, cyclen $(100 \%)^{g}$ & - & $+25 \%$ \\
\hline DETA $(100 \%)$, cyclen $(25 \%)^{\mathrm{h}}$ & - & $+25 \%$ \\
\hline Cyclam & - & $-20 \%$, stoichiometric, $+20 \%$ \\
\hline \multicolumn{3}{|c|}{$\begin{array}{l}\text { a The coatings in bold were surface-functionalised, and the coatings in red were determined to be high Cu( } \\
\text { loading films based on colour intensity. } \\
\text { b Stoichiometric amount of Ancamine }{ }^{\circledR} 2459 \text { but overcured with cyclen. } \\
\text { c Stoichiometric amount of Ancamine }{ }^{\circledR} 2459 \text { but overcured with DETA. } \\
{ }^{d} \text { Enough DETA and cyclen added as hardeners to react with } 75 \% \text { and } 25 \% \text { of the epoxy groups, respectively. } \\
\text { e Enough DETA and cyclen added as hardeners to each react with half of the epoxy groups. } \\
\text { f Enough DETA and cyclen added as hardeners to react with } 25 \% \text { and } 75 \% \text { of the epoxy groups, respectively. } \\
\text { g Stoichiometric amount of cyclen but overcured with DETA. } \\
\text { h Stoichiometric amount of DETA but overcured with cyclen. }\end{array}$} \\
\hline
\end{tabular}


The epoxy coatings prepared from these hardeners and surface-functionalising ligands were evaluated qualitatively and, in some cases, quantitatively for their appearance, mechanical properties, and ability to retain $\mathrm{Cu}(\mathrm{II})$ (Appendix, Table 8.19). Each coating was applied to a black and white brushout card using the standard draw-down method with a bar film applicator (Experimental, Figure 6.2). Then, the coatings were analysed by Fourier-transform mid infrared (FT-MIR) spectroscopy and, in some cases, near infrared (NIR) spectroscopy. Both techniques utilise the interaction of IR radiation with the sample to identify key bonds. However, MIR spectroscopy uses the region from 400$4000 \mathrm{~cm}^{-1}$ containing the fundamental molecular vibrations, while NIR absorptions $\left(4000-12500 \mathrm{~cm}^{-1}\right)$ reflect the overtones and combinations of the fundamental vibrations. ${ }^{217-219}$ Following analysis by IR spectroscopy, an aliquot of a dilute $\mathrm{Cu}(\mathrm{II})$ salt solution was added to each coating surface for $2-6.5 \mathrm{~h}$. The coatings were washed with water, and any colour changes or retention were recorded. An example of this process is seen in Figure 3.9 in which $\mathrm{CU}(\mathrm{II})$ was added to the surface of a coating of Epikote $^{\mathrm{TM}} 235$ and Ancamine ${ }^{\circledR} 2459$ prepared in stoichiometric proportions and applied to a brushout card via the draw-down method. Select coatings were tested for their $\mathrm{Cu}(\mathrm{II})$-loading via ICP-MS.

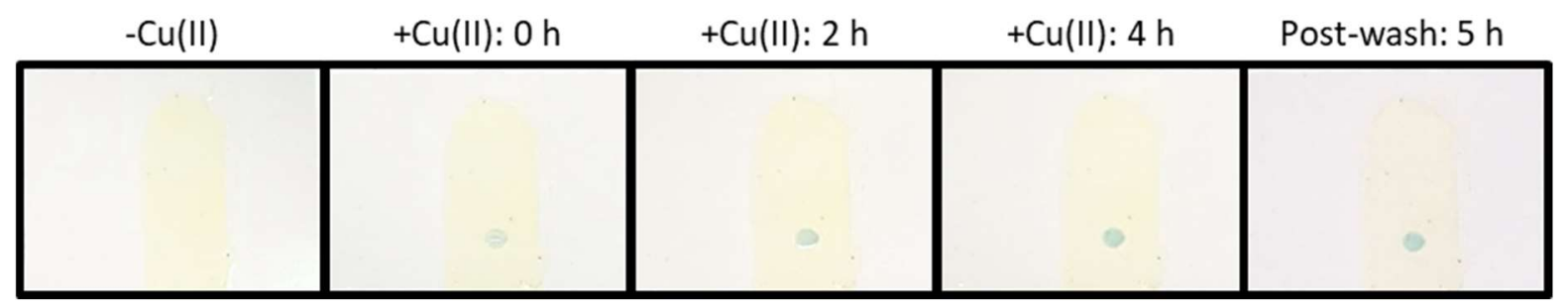

Figure 3.9. Coating composed of Epikote ${ }^{\mathrm{TM}} 235$ and Ancamine ${ }^{\circledR} 2459$ in stoichiometric proportions applied to a brushout card via the draw-down method. $\mathrm{Cu}(I I)$ was added to the surface ("+Cu(II)", blue spot) and washed off after $5 \mathrm{~h}$.

\subsubsection{Modifications of the Hardener Component}

\subsubsection{Epikote ${ }^{T M} 235$ and Ancamine ${ }^{\circledR} 2459$}

The hardener component of the two-pack epoxy system was modified either by changing the identity/composition of the hardener or altering the proportion of hardener used to react with Epikote $^{\mathrm{TM}}$ 235. Initially, a range of super- or sub-stoichiometric amounts of Ancamine ${ }^{\circledR} 2459$ $( \pm 50 \% \mathrm{w} / \mathrm{w})$ were added to Epikote ${ }^{\mathrm{TM}} 235$ to generate overcured $(+)$ or undercured $(-)$ coatings, respectively. It was thought that overcured coatings would have more free amine groups, a fraction 
of which might be presented at the surface, capable of binding $\mathrm{Cu}(\mathrm{II})$. Conversely, undercured coatings would have more free epoxy groups at the surface capable of reacting with a prospective $\mathrm{Cu}(I I)$ ligand, thus functionalising the surface. Curing is commonly monitored by MIR spectroscopy using the band at $\sim 915 \mathrm{~cm}^{-1}$ attributed to the $\mathrm{C}-\mathrm{O}$ stretching vibrations of the epoxy groups in the resin (Table 3.8). ${ }^{218,220}$ The bands corresponding to the amine groups in the hardener are of little use, as they overlap with other bands: the $\mathrm{N}-\mathrm{H}$ stretching band $\left(3300-3500 \mathrm{~cm}^{-1}\right)$ overlaps with the $\mathrm{O}-\mathrm{H}$ stretching band $\left(\sim 3500 \mathrm{~cm}^{-1}\right)$ from the opened epoxy rings, and the $\mathrm{N}-\mathrm{H}$ deformation band (1490-1650 cm $\mathrm{cm}^{-1}$ ) overlaps with the $\mathrm{C}-\mathrm{C}$ and $\mathrm{C}=\mathrm{C}$ stretching bands $\left(1509 \mathrm{~cm}^{-1}\right.$ and $1609 \mathrm{~cm}^{-1}$, respectively) of the aromatic rings of BPA and BPF. ${ }^{218,220-222}$ However, both the primary amine group bands and epoxy group bands are distinct in an NIR spectrum. For the epoxy groups of the resin, the combination band of the second overtone of epoxy ring stretching with the fundamental $\mathrm{C}-\mathrm{H}$ stretching is observed at $4530 \mathrm{~cm}^{-1}$, while, for the primary amine groups of the hardener, the combination band of $\mathrm{N}-\mathrm{H}$ bending and stretching is observed at $4900-5000 \mathrm{~cm}^{-1}$. The overtones of the primary and secondary amine groups overlap at $6500 \mathrm{~cm}^{-1}$. 221 Thus, the degree of curing in an epoxy coating can be determined by assessing the relative sizes of the epoxy group band by MIR and NIR spectroscopy and the amine group band by NIR spectroscopy.

In the present work, the undercuring and overcuring $( \pm 50 \% \mathrm{w} / \mathrm{w})$ of the Ancamine ${ }^{\circledR} 2459$ / Epikote $^{\mathrm{TM}} 235$ coatings were confirmed by MIR and NIR spectroscopy. The epoxy peak area at $914 \mathrm{~cm}^{-1}$ in the MIR spectra (Figure 3.10) increased with increasingly undercured coatings (i.e. $+50 \%<$ stoichiometric $\approx-10 \%<-20 \%<-30 \%<-40 \%<-50 \%$ ). Likewise, this trend was observed in the NIR spectra of the coatings, using the epoxy peak at $4533 \mathrm{~cm}^{-1}$. Yet, the amine group bands at 4900-5000 and $6500 \mathrm{~cm}^{-1}$, which are commonly used to monitor the curing of epoxy resins, ${ }^{218,221,222}$ were not present in the NIR spectra. Since both the MIR and NIR spectra contained well-defined, discrete epoxy group peaks and neither contained such peaks for the amine groups in the hardener, there was no clear advantage in analysing the coatings via NIR spectroscopy. Furthermore, FT-MIR spectroscopy with attenuated total reflectance (ATR) sampling is more of a surface technique than NIR spectroscopy, which can be considered a bulk measurement, and, in this study, changes to the surface were of interest. Accordingly, it was decided that future analyses of epoxy group content should be conducted by FT-MIR (i.e. FT-IR) spectroscopy alone. 
Table 3.8. Expected bands in the MIR and NIR spectra of an epoxy coating. ${ }^{218,220-222}$

\begin{tabular}{|c|c|c|c|}
\hline Epoxy Component & Region & Frequency $\left(\mathrm{cm}^{-1}\right)$ & Assignment \\
\hline Resin & MIR & 915 & C-O stretching of epoxy groups \\
\hline Hardener & MIR & $1490-1650 ; 3300-3500$ & $\mathrm{~N}-\mathrm{H}$ deformation; $\mathrm{N}-\mathrm{H}$ stretching \\
\hline Resin & NIR & 4530 & $\begin{array}{l}\text { combination band of second overtone of epoxy ring stretching } \\
\text { with fundamental } \mathrm{C}-\mathrm{H} \text { stretching }\end{array}$ \\
\hline Hardener & NIR & $4900-5000 ; 6500$ & $\begin{array}{c}\text { combination band of } \mathrm{N}-\mathrm{H} \text { bending and stretching }\left(1^{\circ} \text { amine); }\right. \\
\text { overlapping overtones of } 1^{\circ} \text { and } 2^{\circ} \text { amines }\end{array}$ \\
\hline
\end{tabular}


(a)

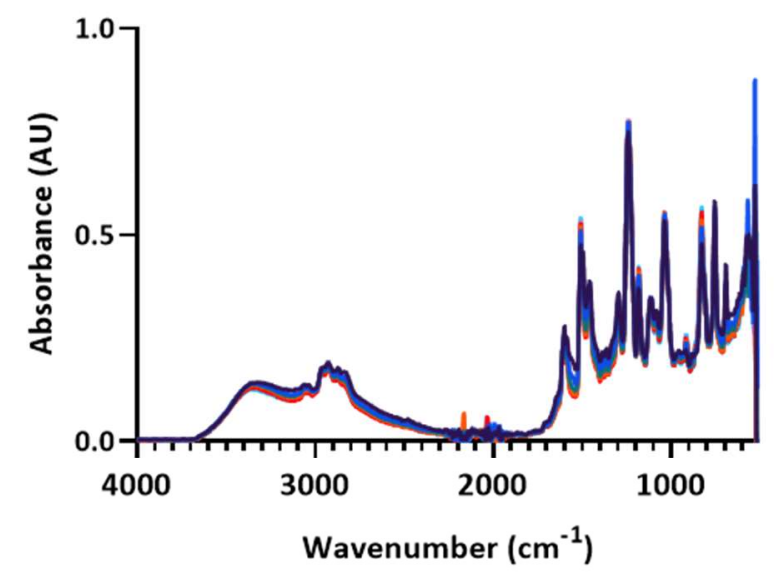

(b)

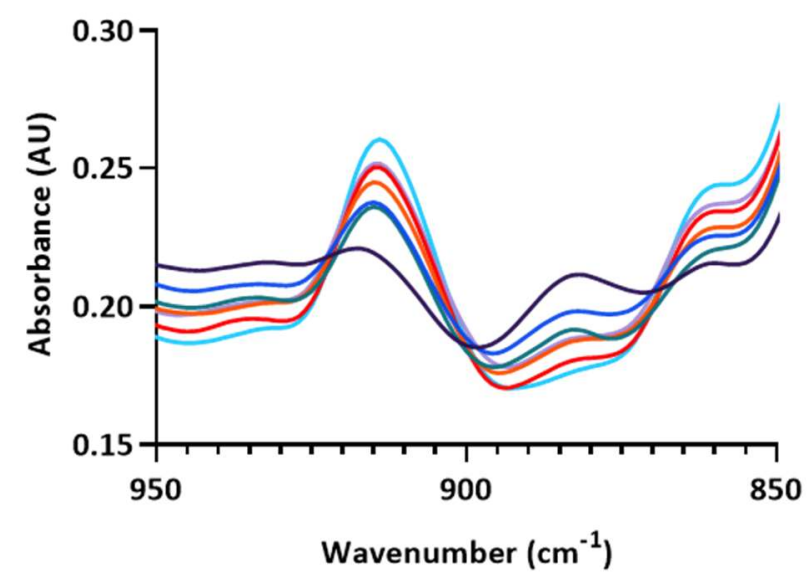

(c)

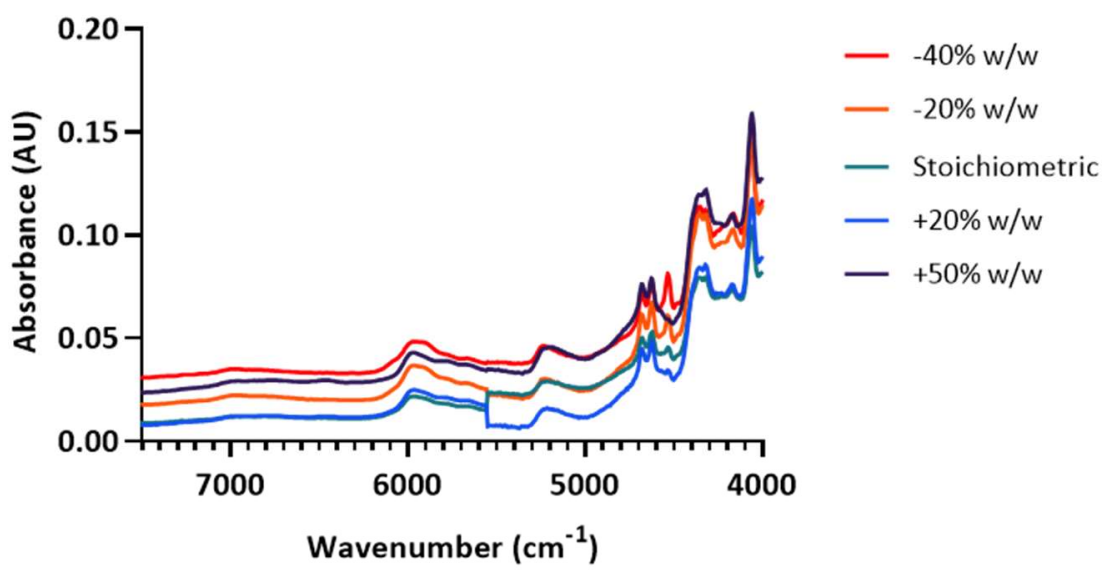

(d)

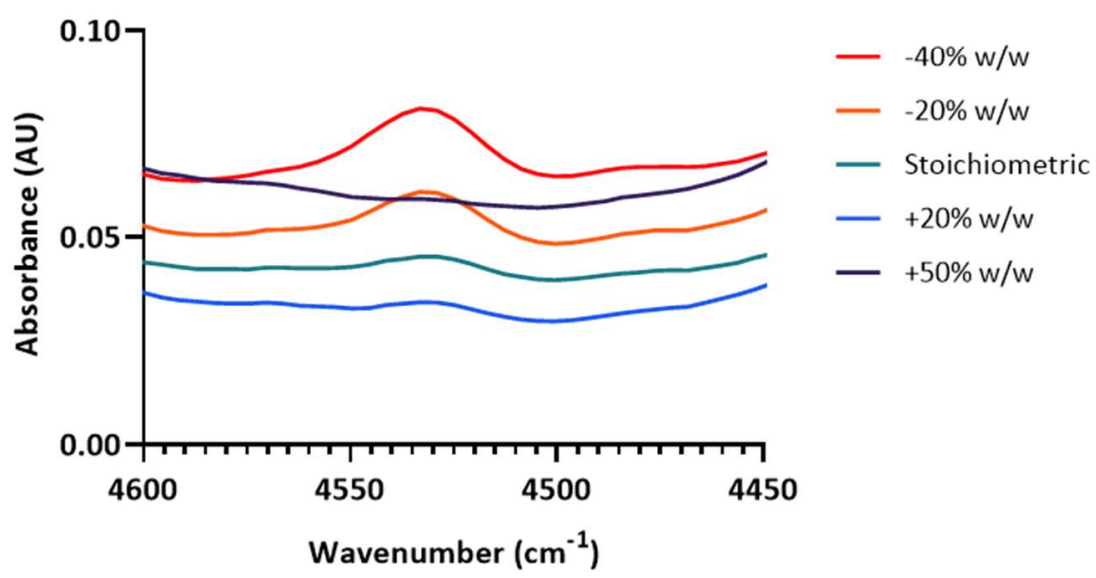

Figure 3.10. FT-MIR (a, b) and NIR (c, d) spectra of overcured (+) and undercured (-) Epikote ${ }^{\mathrm{TM}}$ 235/Ancamine ${ }^{\circledR} 2459$ coatings: (a) and (c) are the full spectra, and (b) and (d) show an expanded view of the bands due to the epoxy group ( $915 \mathrm{~cm}^{-1}$ for (b), $\sim 4530 \mathrm{~cm}^{-1}$ for (d)). 
In addition to epoxy group content, the Ancamine ${ }^{\circledR} 2459 /$ Epikote $^{\text {TM }} 235$ films were evaluated qualitatively for their appearance, hardness, ease of draw-down, and colour following the addition of $\mathrm{Cu}$ (II) (Table 3.9). Undercuring of the coating by $50 \% \mathrm{w} / \mathrm{w}$ yielded unfavourable mechanical properties: the coating was soft and patchy. The coatings undercured by $30-40 \% \mathrm{w} / \mathrm{w}$ were also patchy but were hard and flexible, and the remaining coatings had no obvious differences compared to the stoichiometrically composed film. An aliquot from a dilute, aqueous $\mathrm{CU}(\mathrm{II})$ salt solution was added to each coating and washed off after $5 \mathrm{~h}$ (Figure 3.11). The post-wash retention of a blue colour at the site of the $\mathrm{Cu}(\mathrm{II})$-addition was noted for all films, but the blue colour did not appear to change between the initial addition of the aliquot and $5 \mathrm{~h}$ later, except, perhaps, increasing in intensity over time. As colour change is often indicative of metal-coordination, the lack thereof was suggestive that the colour retention may be due to adsorption rather than chelation. After these preliminary experiments, the same process was repeated for other hardener compositions with Epikote ${ }^{\mathrm{TM}} 235$.

Table 3.9. Properties of epoxy coatings prepared with epoxy resin Epikote ${ }^{\mathrm{TM}} 235$ and hardener Ancamine ${ }^{\circledR} 2459$.

\begin{tabular}{|c|c|c|c|c|c|c|}
\hline Hardener & $\begin{array}{c}\% \text { Over (+)/ } \\
\text { Undercure (-) }\end{array}$ & Appearance & $\begin{array}{l}\text { Softness/ } \\
\text { Hardness }\end{array}$ & $\begin{array}{c}\text { Ease of } \\
\text { Draw-Down }\end{array}$ & $\begin{array}{c}\text { Colour Change } \\
\text { Upon Cu(II)- } \\
\text { Addition (Y/N) }\end{array}$ & $\begin{array}{c}\text { Colour } \\
\text { Intensity }\end{array}$ \\
\hline $\begin{array}{l}\text { Ancamine }^{\circledR} \\
2459\end{array}$ & $-50 \%$ & $\begin{array}{l}\text { yellow, clear, } \\
\text { splotchy }\end{array}$ & soft & splotchy & - & - \\
\hline $\begin{array}{l}\text { Ancamine }^{\circledR} \\
2459\end{array}$ & $-40 \%$ & yellow, clear & $\begin{array}{c}\text { hard, } \\
\text { flexible }\end{array}$ & $\begin{array}{l}\text { slightly } \\
\text { splotchy }\end{array}$ & $\mathrm{N}$ - retention & weak \\
\hline $\begin{array}{l}\text { Ancamine }^{\circledR} \\
2459\end{array}$ & $-30 \%$ & yellow, clear & $\begin{array}{c}\text { hard, } \\
\text { flexible }\end{array}$ & $\begin{array}{l}\text { slightly } \\
\text { splotchy }\end{array}$ & $\mathrm{N}$ - retention & medium \\
\hline $\begin{array}{l}\text { Ancamine }^{\circledR} \\
2459\end{array}$ & $-20 \%$ & yellow, clear & $\begin{array}{c}\text { hard, } \\
\text { flexible }\end{array}$ & $\begin{array}{c}\text { easy, } \\
\text { uniform }\end{array}$ & $\mathrm{N}$ - retention & medium \\
\hline $\begin{array}{l}\text { Ancamine }^{\circledR} \\
2459\end{array}$ & $-10 \%$ & yellow, clear & $\begin{array}{c}\text { hard, } \\
\text { flexible }\end{array}$ & $\begin{array}{c}\text { easy, } \\
\text { uniform }\end{array}$ & $\mathrm{N}$ - retention & medium \\
\hline $\begin{array}{l}\text { Ancamine }^{\circledR} \\
2459\end{array}$ & stoichiometric & yellow, clear & $\begin{array}{c}\text { hard, } \\
\text { flexible }\end{array}$ & $\begin{array}{c}\text { easy, } \\
\text { uniform }\end{array}$ & $\mathrm{N}$ - retention & medium \\
\hline $\begin{array}{l}\text { Ancamine }^{\circledR} \\
2459\end{array}$ & $+10 \%$ & yellow, clear & $\begin{array}{c}\text { hard, } \\
\text { flexible }\end{array}$ & $\begin{array}{c}\text { easy, } \\
\text { uniform }\end{array}$ & $\mathrm{N}$ - retention & medium \\
\hline $\begin{array}{l}\text { Ancamine }^{\circledR} \\
2459\end{array}$ & $+20 \%$ & yellow, clear & $\begin{array}{c}\text { hard, } \\
\text { flexible }\end{array}$ & $\begin{array}{c}\text { easy, } \\
\text { uniform }\end{array}$ & $\mathrm{N}$ - retention & medium \\
\hline $\begin{array}{l}\text { Ancamine }^{\circledR} \\
2459\end{array}$ & $+50 \%$ & yellow, clear & $\begin{array}{l}\text { hard, } \\
\text { brittle }\end{array}$ & $\begin{array}{c}\text { easy, } \\
\text { uniform }\end{array}$ & $\mathrm{N}$ - retention & medium \\
\hline
\end{tabular}




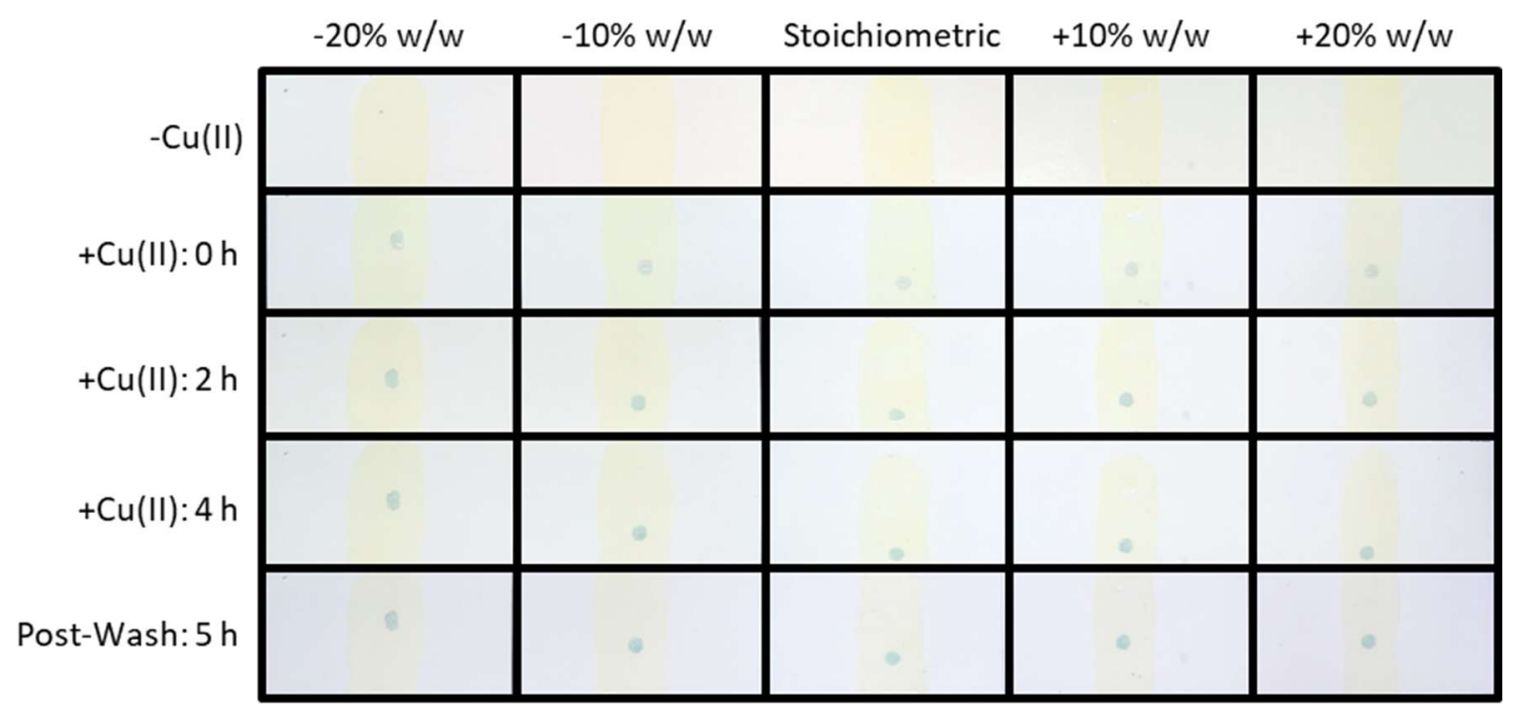

Figure 3.11. Example of monitoring the $\mathrm{Cu}(\mathrm{II})$-addition to under/overcured $( \pm 20 \% \mathrm{w} / \mathrm{w})$ Ancamine $^{\circledR} 2459 /$ Epikote $^{\text {TM }} 235$ coatings.

\subsubsection{Epikote $^{T M} 235$ and Cu(II) Ligands}

As shown in Table 3.10, the other polyamine hardeners or hardener combinations trialled with Epikote $^{\text {TM }} 235$ were Ancamine ${ }^{\circledR}$ 2459/cyclen, Ancamine ${ }^{\circledR}$ 2459/DETA, DETA/cyclen, cyclen, DETA, and cyclam. Regarding the FT-IR spectra of the Ancamine ${ }^{\circledR}$ 2459/Epikote ${ }^{\mathrm{TM}} 235$ coatings overcured with cyclen and DETA (Appendix, Figures 8.23-24), no epoxy group peak was observed. However, this peak was present at $\sim 915 \mathrm{~cm}^{-1}$ in the spectra of all of the other coatings (Appendix, Figures 8.25-28), regardless of the level of overcuring with hardener. Although the hardener is, in theory, supposed to react completely with the resin in the stoichiometric compositions, the freedom of movement and reaction rate decrease as the polymer crosslinks and builds up molecular weight, which is due to unreacted components becoming separated and unable to react. ${ }^{223}$ Therefore, in practice, the reaction does not progress to completion. This explains the presence of the epoxy group band in the stoichiometric and overcured, as well as the undercured, coatings.

The FT-IR spectra of the films prepared from Epikote ${ }^{\mathrm{TM}}$ 235/cyclen and Epikote ${ }^{\mathrm{TM}}$ 235/cyclam showed a trend of increasing epoxy group peak area with increased undercuring (Appendix, Figures 8.26, 28), but no clear trend was observable in the remaining spectra, which were of coatings using DETA as a hardener. It was unknown whether this was due to the coating preparation (e.g. inhomogeneous mixing) or structure of DETA. DETA has two primary amines and one secondary, while cyclen and cyclam both contain only secondary amines. The less sterically hindered primary amines react faster than secondary amines, ${ }^{224}$ so the reaction with DETA might be expected to first produce a linear 
polymer. Thus, crosslinking via the secondary amines could be limited due to steric and diffusional restrictions, leaving unreacted epoxy and amine groups. Indeed, a study by Morgan ${ }^{223}$ reported that epoxy coatings prepared from DGEBA (Figure 3.12) cured with DETA were not highly crosslinked, and it was suggested that either few epoxy group/secondary amine reactions occurred and/or DGEBA underwent homopolymerisation. In the present work, the epoxy groups in the resin have the same reactivities, and, unlike DETA, the (secondary) amines in cyclen and cyclam have the same reactivities. ${ }^{224}$ Therefore, the reactive functional groups in the resin and cyclen/cyclam will react at the same rate.

Table 3.10. Epoxy coatings prepared with Epikote ${ }^{\mathrm{TM}} 235$ and $\mathrm{Cu}(\mathrm{II})$ ligands as hardeners.

\begin{tabular}{|c|c|}
\hline Hardener & $\%$ w/w Overcure $(+) /$ Undercure $(-)^{a}$ \\
\hline Ancamine ${ }^{\circledR} 2459$, cyclen & $+10 \%,+20 \%\left(\right.$ cyclen $\left.^{b}\right)$ \\
\hline Ancamine ${ }^{\circledR} 2459$, DETA & $+10 \%,+20 \%\left(D^{2} A^{c}\right)$ \\
\hline Cyclen & $-20 \%,-10 \%$, stoichiometric, $+10 \%,+20 \%$ \\
\hline DETA & $-20 \%,-10 \%$, stoichiometric, $+10 \%,+20 \%$ \\
\hline $\operatorname{DETA}(75 \%)$, cyclen $(25 \%)^{d}$ & stoichiometric \\
\hline DETA $(50 \%)$, cyclen $(50 \%)^{\mathrm{e}}$ & stoichiometric \\
\hline $\operatorname{DETA}(25 \%)$, cyclen $(75 \%)^{f}$ & stoichiometric \\
\hline DETA (25\%), cyclen (100\%)g & $+25 \%$ \\
\hline DETA $(100 \%)$, cyclen $(25 \%)^{h}$ & $+25 \%$ \\
\hline Cyclam & $-20 \%$, stoichiometric, $+20 \%$ \\
\hline
\end{tabular}

a The coatings in red were determined to be high $\mathrm{Cu}(\mathrm{II})$-loading films based on colour intensity.

b Stoichiometric amount of Ancamine ${ }^{\circledR} 2459$ but overcured with cyclen.

c Stoichiometric amount of Ancamine ${ }^{\circledR} 2459$ but overcured with DETA.

${ }^{d}$ Enough DETA and cyclen added as hardeners to react with $75 \%$ and $25 \%$ of the epoxy groups, respectively.

e Enough DETA and cyclen added as hardeners to each react with half of the epoxy groups.

${ }^{f}$ Enough DETA and cyclen added as hardeners to react with $25 \%$ and $75 \%$ of the epoxy groups, respectively.

${ }^{g}$ Stoichiometric amount of cyclen but overcured with DETA.

${ }^{\mathrm{h}}$ Stoichiometric amount of DETA but overcured with cyclen.<smiles>CC(C)(C)OC(C)(C)C</smiles>

Figure 3.12. Structure of DGEBA. 
The films using DETA and DETA/cyclen as curing agents had undesirable physical and mechanical properties but displayed $\mathrm{Cu}(\mathrm{II})$-binding. The films were milky white, indicative of blushing, and patchy and sticky when DETA made up a greater portion of the hardener (Appendix, Table 8.19). At the site of $\mathrm{Cu}(\mathrm{II})$-addition to these coatings, however, a striking surface colour change to cobalt blue occurred, signifying metal-coordination, and the cobalt blue colour was retained after washing the surface with water (Figure 3.13; Appendix, Figure 8.31). The Ancamine ${ }^{\circledR} 2459 /$ Epikote $^{\mathrm{TM}} 235$ films overcured with DETA were hard, brittle, and uniform. Following the addition of $\mathrm{Cu}(\mathrm{II})$ though, they also retained their colour change post-wash (Appendix, Figure 8.30). This was observed for the cyclen and cyclam coatings as well: the films containing cyclen retained the dark blue colour (Appendix, Figures 8.29, 31-32), and the films containing cyclam retained the light purple colour (Appendix, Figure 8.33). The colour changes of these surfaces with ligands incorporated all matched the colours of the corresponding $\mathrm{Cu}(\mathrm{II})$-ligand complexes in solution, providing further qualitative evidence that the surface colour changes may be attributed to metal chelation. Inclusion of tetraaza macrocycles cyclen and cyclam in the films made them hard and brittle, and their degree of uniformity was variable. The brittleness could be explained by the relative inflexibility of the macrocyclic structures, compared to linear, alkyl chains, or, more likely, by a greater degree of crosslinking in the coating, which occurs at multiple, crosslinkable points with limited flexibility (e.g. secondary amines in cyclen and cyclam). All of these properties of the Epikote ${ }^{\text {TM }} 235$ films prepared with different hardeners were considered, and the coatings with the most intense colour changes upon $\mathrm{Cu}(\mathrm{II})$-addition and -retention post-wash were noted. Then, functionalisation of the surfaces of undercured films was explored.

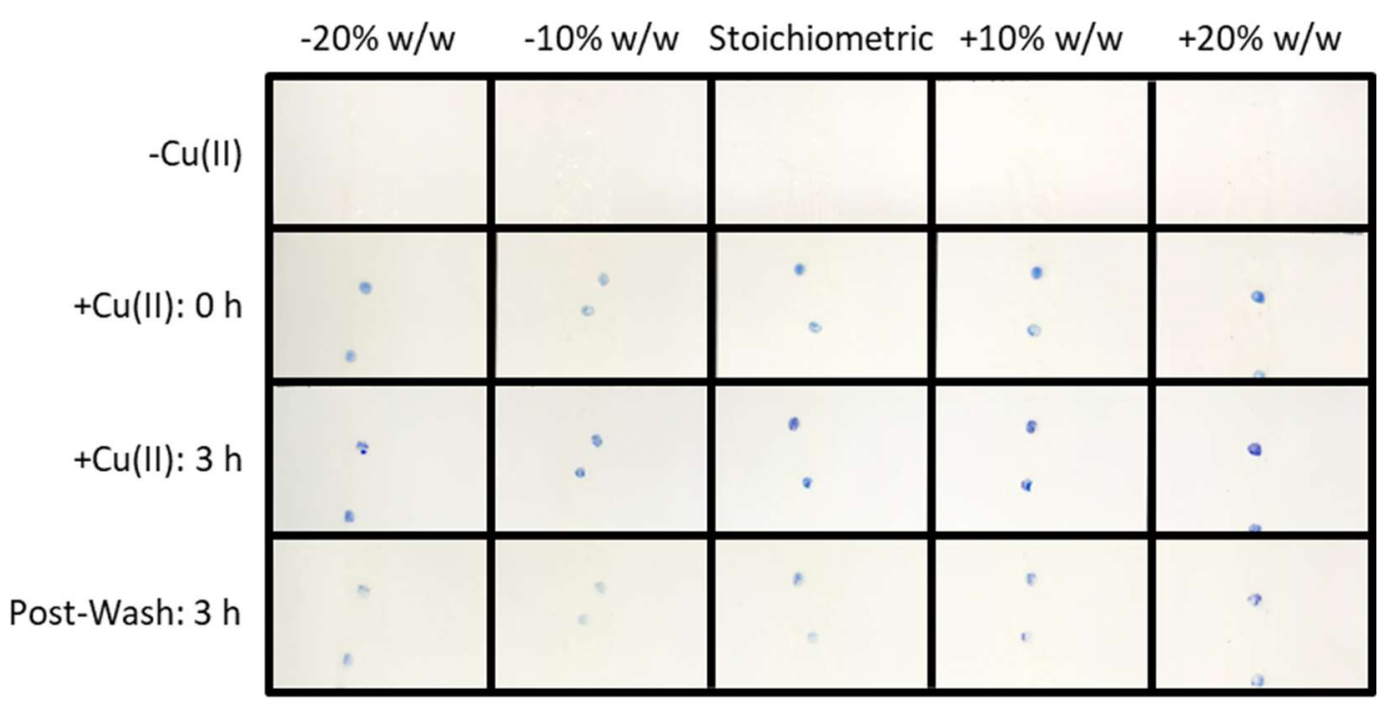

Figure 3.13. Monitoring the $\mathrm{Cu}(\mathrm{II})$-addition to coatings prepared from Epikote ${ }^{\mathrm{TM}} 235$ and DETA in stoichiometric proportions or under/overcured $( \pm 20 \% \mathrm{w} / \mathrm{w})$ with DETA. 


\subsubsection{Surface-Functionalisation}

As in Table 3.11, the surfaces of Epikote ${ }^{\mathrm{TM}} 235$ films undercured by $10-50 \% \mathrm{w} / \mathrm{w}$ with Ancamine ${ }^{\circledR} 2459$ were functionalised with cyclam, cyclen, DETA, or PEI. It was proposed that the undercured coatings would have epoxy groups at the surface available for reaction with the amine groups of the $\mathrm{Cu}(\mathrm{II})$ ligands. Cyclam and cyclen were dissolved in water/methanol and water, respectively, and the solutions were used to cover defined surface areas of the films. DETA and PEI were added neat. After 16-24 h, the coatings were rinsed with water, analysed by FT-IR spectroscopy, and evaluated for their $\mathrm{Cu}(\mathrm{II})$-coordinating ability. The epoxy group band was still present at $\sim 915 \mathrm{~cm}^{-1}$ in the FT-IR spectra of the cyclam- and cyclen-modified surfaces (Appendix, Figures 8.34-35), which could be indicative of incomplete surface-functionalisation. This was unsurprising, as secondary amines are less reactive and both cyclen and cyclam were diluted in solution. However, although FT-IR ATR spectroscopy is considered to be a surface-sensitive technique, the ATR samples down to a depth of $<2 \mu \mathrm{m},{ }^{225}$ so it was difficult to determine if the epoxy peak was the result of sampling below the modified surface layer and/or the result of incomplete reaction at the surface. But, the addition of $\mathrm{Cu}(\mathrm{II})$ to the functionalised areas and the subsequent colour changes (e.g. light purple for cyclam, dark blue for cyclen; Appendix, Figures 8.36-37) demonstrated that some functionalisation had taken place. Generally, the cyclam- and cyclen-modified films were yellow, clear, hard, and flexible, but a few of them were brittle (Appendix, Table 8.19). It is possible that the brittleness was due to crosslinking with cyclen/cyclam at the surface.

Table 3.11. Epoxy coatings prepared with Epikote $^{\mathrm{TM}} 235$ and Ancamine ${ }^{\circledR} 2459$ and surfacefunctionalised with $\mathrm{Cu}(\mathrm{II})$ ligands.

\begin{tabular}{lcc}
\hline Hardener & Surface Functionalisation & $\%$ w/w Overcure $(+) /$ Undercure $(-)^{a}$ \\
\hline Ancamine $^{\circledR} 2459$ & cyclam & $-50 \%,-40 \%,-30 \%,-20 \%,-10 \%$ \\
Ancamine $^{\circledR} 2459$ & cyclen & $-40 \%$ \\
Ancamine $^{\circledR} 2459$ & DETA & $-40 \%,-30 \%$ \\
Ancamine $^{\circledR} 2459$ & PEI & $-40 \%$ \\
\hline
\end{tabular}

a The coatings in red were determined to be high $\mathrm{Cu}(I I)$-loading films based on colour intensity.

In contrast, surface-modification of the undercured Ancamine ${ }^{\circledR} 2459 /$ Epikote $^{\text {TM }} 235$ coating with DETA and PEI demonstrated $\mathrm{Cu}(\mathrm{II})$-chelation and complete functionalisation by FT-IR spectroscopy, yet the physical properties of the film were undesirable. No epoxy group peak was observed at $\sim 915 \mathrm{~cm}^{-1}$ in the FT-IR spectra, meaning that the surface and ATR-sampled underlying layers had no unreacted 
epoxy groups (Appendix, Figure 8.35). Since DETA and PEI were applied to the surface neat and contain the more reactive primary amines, it seemed reasonable that they would react more extensively with the surface than cyclam and cyclen. Also, it is likely that DETA and PEI penetrated the film more deeply, as it was noted that their application softened the films. Aliquots of the dilute, aqueous $\mathrm{Cu}$ (II) salt solution delivered to the modified areas of the film yielded striking colour changes at the surface from light yellow to dark blue (Appendix, Figure 8.37). The PEl-functionalised surface retained the colour change particularly well post-wash, but both coating preparations were noted for the intensity of their colour changes. These experiments would have been encouraging, if not for the negative effects of functionalisation on the coating's physical properties: modification with DETA and PEI softened the coating and made it appear matte in the areas of application. Modification with PEI also resulted in significant blushing. Regardless, the DETA- and PEl-functionalised surfaces, as well as the other coatings notable for their post-wash colour retention, were progressed to quantification of the amount of copper bound to the surface.

\subsubsection{Quantification of Cu(II)-Loading}

Due to the intensity of the colour retained after the addition of $\mathrm{Cu}(\mathrm{II})$ and subsequent washes, the coatings in Table $\mathbf{3 . 1 2}$ were selected for quantification of their $\mathrm{Cu}(\mathrm{II})$-loading abilities. It was thought that colour intensity would increase with increased $\mathrm{Cu}(\mathrm{II})$-binding and, therefore, would be valuable as a preliminary, qualitative assessment of the capacity of each film to chelate $\mathrm{Cu}(\mathrm{II})$. The procedure for measuring the surface $\mathrm{Cu}(\mathrm{II})$ was similar to the procedure in Section 3.2.4 used to quantify the $\mathrm{Cu}(\mathrm{II})$-loading for the commercial acrylic and 35\% w/w DAp/acr paints. A standard amount of each prepared epoxy coating was delivered to evenly coat the bottom of a glass vial; glass vials of the same size were used in every experiment to keep the depth and exposed surface area of each coating consistent. Then, the coating was allowed to cure, the mass and exposed surface area of the coating were recorded, and an aliquot of a dilute, aqueous $\mathrm{Cu}$ (II) salt solution was added. Water was added to the vials serving as blanks. After $24 \mathrm{~h}$, the coatings were washed with water, dried, digested in concentrated nitric acid, and diluted for copper analysis via ICP-MS. The reported copper results are blank-subtracted.

Given the poor reproducibility of the $\mathrm{Cu}(\mathrm{II})$-binding results for the acrylic and DAp/acr paints (Table 3.5), the first $\mathrm{Cu}(\mathrm{II})$-loading experiment for the epoxies utilised the stoichiometric Ancamine ${ }^{\circledR}$ 2459/Epikote ${ }^{\mathrm{TM}} 235$ coating to validate the method, making the alterations suggested in Section 3.2.4. To explain the previous irreproducibility of the method, it was hypothesised that the variability was 
due to shifting cure conditions (i.e. time, temperature, humidity), and it was suggested that these parameters be kept constant in future experiments. Accordingly, in this experiment, all coatings were cured for $24 \mathrm{~h}$ at $50{ }^{\circ} \mathrm{C}$ and $50 \%$ humidity. The samples were prepared in triplicate, and the experiment was repeated on three different days. Thus, the variability within a set of measurements and between sets could be determined (Table 3.13).

Table 3.12. Epoxy coatings prepared with Epikote ${ }^{\mathrm{TM}} 235$ and to be measured for $\mathrm{Cu}(\mathrm{II})$-loading.

\begin{tabular}{lcc}
\hline Hardener & Surface Functionalisation & $\%$ w/w Overcure (+)/Undercure (-) \\
\hline Ancamine $^{\circledR} 2459$ & DETA & $-40 \%,-30 \%$ \\
Ancamine $^{\circledR} 2459$ & PEI & $-40 \%$ \\
Ancamine $^{\circledR} 2459$, cyclen & - & $+10 \%,+20 \%\left(\right.$ cyclen $\left.^{\mathrm{a}}\right)$ \\
Cyclen & - & $+20 \%$ \\
DETA & - & $+20 \%$ \\
DETA $(25 \%)$, cyclen $(100 \%)^{b}$ & - & $+25 \%$ \\
DETA $(100 \%)$, cyclen $(25 \%)^{c}$ & - & $+25 \%$ \\
\hline
\end{tabular}

a Stoichiometric amount of Ancamine ${ }^{\circledR} 2459$ but overcured with cyclen.

${ }^{\mathrm{b}}$ Stoichiometric amount of cyclen but overcured with DETA.

'Stoichiometric amount of DETA but overcured with cyclen.

Table 3.13. Amount of copper retained by the Ancamine ${ }^{\circledR} 2459 /$ Epikote $^{\mathrm{TM}} 235$ coating prepared in stoichiometric proportions.

\begin{tabular}{lcccc}
\hline Replicate $^{\mathrm{a}}$ & Hardener & $\begin{array}{c}\% \text { w/w Overcure (+)/ } \\
\text { Undercure(-) }\end{array}$ & $\begin{array}{c}\mu \mathrm{\mu g} \mathrm{Cu} / \mathrm{g} \\
\text { Coating }^{\mathrm{b}}\end{array}$ & $\begin{array}{c}\mathrm{ng} \mathrm{Cu} / \mathrm{mm}^{2} \\
\text { Coating }^{\mathrm{b}, \mathrm{c}}\end{array}$ \\
\hline 1 & Ancamine $^{\circledR} 2459$ & stoichiometric & $5 \pm 1$ & $4 \pm 1$ \\
2 & Ancamine $^{\circledR} 2459$ & stoichiometric & $3 \pm 1$ & $3 \pm 1$ \\
3 & Ancamine $^{\circledR} 2459$ & stoichiometric & $7 \pm 1$ & $6 \pm 1$ \\
\hline
\end{tabular}

a The experiment was repeated three times (three different days).

${ }^{\mathrm{b}}$ Mean $\pm \mathrm{SD}, \mathrm{n}=3$.

c Surface area $\left(\mathrm{mm}^{2}\right)$ exposed to $\mathrm{Cu}(\mathrm{II})$ salt solution. Calculated as in the Appendix (Figure 8.38).

As seen in Table 3.13, the Ancamine ${ }^{\circledR}$ 2459/Epikote ${ }^{\mathrm{TM}} 235$ coatings (stoichiometric) retained very little copper $\left(<10 \mu \mathrm{g} / \mathrm{g}\right.$ and $\left.\mathrm{ng} / \mathrm{mm}^{2}\right)$. A one-way ANOVA with Tukey's post hoc test revealed no statistically significant differences $(p>0.05)$ between the results for replicates 1 and 2 and replicates 1 and 3, but the results for replicates 2 and 3 were different. Due to the extremely low $\mathrm{Cu}(\mathrm{II})$-loading in these coatings, variability in the results was unsurprising, as slight differences in coating preparation would have a larger relative impact on the results. This made it difficult to determine if standardising cure 
conditions improved the precision of the results. However, a few tentative conclusions were drawn from the $\mathrm{Cu}(\mathrm{II})$-loading experiments in this chapter (Table 3.5, Table 3.13):

1) The order of magnitude of each copper result is likely accurate, which, for the most part, is an acceptable degree of accuracy for the purposes of the project,

2) It is probable that differences in curing can significantly impact $\mathrm{Cu}(\mathrm{II})$-loading and -retention, so tight control of the curing conditions in such experiments is advisable, and

3) The percent relative standard deviation within a set of measurements is generally $\leq 20 \%$ (with the exception of replicate 2, Table 3.13).

Therefore, this method and the results obtained following its implementation are still useful for relative comparisons of $\mathrm{Cu}(\mathrm{II})$-loading in coatings, especially when the coatings are prepared on the same day, but it is important to be aware of the limitations.

The next experiment was to confirm a direct relationship between surface colour intensity and $\mathrm{Cu}(\mathrm{II})$ binding. The epoxy coatings selected for this experiment were Epikote ${ }^{\mathrm{TM}} 235$ cured with cyclen superstoichiometrically $(+20 \% \mathrm{w} / \mathrm{w})$, sub-stoichiometrically $(-20 \% \mathrm{w} / \mathrm{w})$, and stoichiometrically. As the amount of cyclen in the coatings increased, the intensity of the colour change post $\mathrm{Cu}(\mathrm{II})$-addition increased (Figure 3.14).

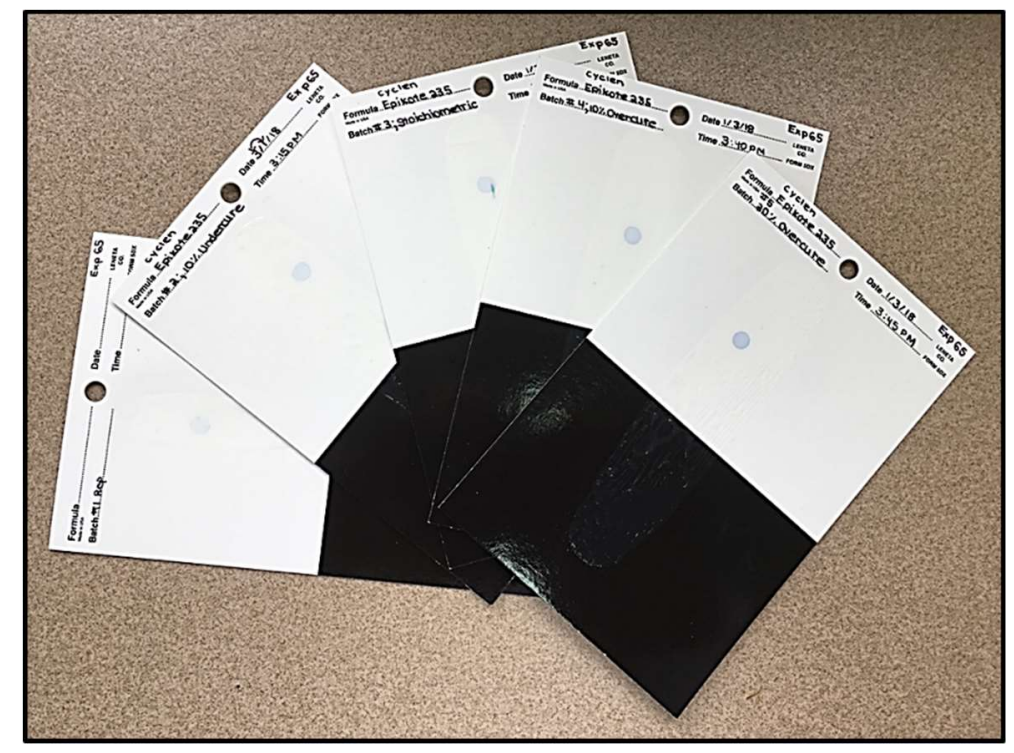

Figure 3.14. $\mathrm{CU}(\mathrm{II})$-addition to coatings prepared from Epikote ${ }^{\mathrm{TM}} 235$ and cyclen in stoichiometric proportions or under/overcured $( \pm 20 \% \mathrm{w} / \mathrm{w}$ ) with cyclen (increasing amount of cyclen from left to right). 
Measurement of the $\mathrm{Cu}(\mathrm{II})$-loading for these epoxies revealed that the amount of copper retained by the surface increased with increasing amounts of cyclen (Table 3.14), confirming the direct relationship between colour intensity and $\mathrm{Cu}(\mathrm{II})$-loading. Furthermore, the increment by which the amount of bound copper increased from the $-20 \% \mathrm{w} / \mathrm{w}$ to the stoichiometric coating was nearly equal to the increment by which the amount increased from the stoichiometric to the $+20 \% \mathrm{w} / \mathrm{w}$ coating. This was encouraging, as the increment by which the amount of cyclen between these coatings increased was equivalent. Upon substantiation of this relationship, the $\mathrm{Cu}(\mathrm{II})$-binding by the coatings selected based on colour intensity was evaluated.

Table 3.14. Amounts of copper retained by coatings prepared from Epikote ${ }^{\mathrm{TM}} 235$ and cyclen in stoichiometric proportions or under/overcured $( \pm 20 \% \mathrm{w} / \mathrm{w})$ with cyclen.

\begin{tabular}{llcccc}
\hline Number & Hardener & $\begin{array}{c}\text { \% w/w Overcure (+)/ } \\
\text { Undercure (-) }\end{array}$ & $\begin{array}{c}\mu \mathrm{g} \mathrm{Cu} / \mathrm{g} \\
\text { Coating }^{\mathrm{a}}\end{array}$ & $\begin{array}{c}\mathrm{ng} \mathrm{Cu} / \mathrm{mm}^{2} \\
\text { Coating }^{\mathrm{a}, \mathrm{b}}\end{array}$ \\
\hline 1 & cyclen & $-20 \%$ & 19 & 20 \\
2 & & cyclen & stoichiometric & 66 & 75 \\
& $\Delta_{2-1}$ & & & 47 & 55 \\
\hline 3 & & cyclen & $+20 \%$ & 111 & 127 \\
& $\Delta_{3-2}$ & & & 45 & 52 \\
\hline
\end{tabular}

a Estimated variability $\pm 20 \%$, but 2-3 significant figures reported for $\Delta$ calculations.

${ }^{\text {b }}$ Surface area $\left(\mathrm{mm}^{2}\right)$ exposed to $\mathrm{Cu}(\mathrm{II})$ salt solution. Calculated as in the Appendix (Figure 8.38).

In addition to Epikote ${ }^{\mathrm{TM}} 235$ overcured $(+20 \% \mathrm{w} / \mathrm{w})$ with cyclen (Table 3.14), the other coatings determined to be high $\mathrm{Cu}(\mathrm{II})$-loading based on colour intensity and selected for quantification of bound copper were as follows:

1) Epikote ${ }^{\mathrm{TM}} 235$ undercured with Ancamine ${ }^{\circledR} 2459(-30,-40 \% \mathrm{w} / \mathrm{w})$ and surface-functionalised with PEI and DETA,

2) Epikote ${ }^{\mathrm{TM}} 235$ cured stoichiometrically with Ancamine ${ }^{\circledR} 2459$ then overcured with cyclen $(+10-20 \% \mathrm{w} / \mathrm{w})$,

3) Epikote ${ }^{\mathrm{TM}} 235$ overcured with DETA (+20\% w/w),

4) Epikote ${ }^{\mathrm{TM}} 235$ cured stoichiometrically with cyclen then overcured with DETA ( $\left.+25 \% \mathrm{w} / \mathrm{w}\right)$, and

5) Epikote $^{\mathrm{TM}} 235$ cured stoichiometrically with DETA then overcured with cyclen $(+25 \% \mathrm{w} / \mathrm{w})$.

However, attempts to employ the method to measure the copper retained by the PEI- and DETAfunctionalised epoxies (1) failed. The addition of PEI and DETA neat to the undercured Ancamine ${ }^{\circledR} 2459$ /Epikote ${ }^{\mathrm{TM}} 235$ surfaces resulted in gel-like coatings that would not harden. This 
softening of the coatings had also been observed for the PEI- and DETA-modified surfaces prepared via the draw-down method. Consequently, these coatings were not pursued, but the procedure was successfully employed to measure the $\mathrm{Cu}(I I)$-loading for the other epoxies (2-5), the results of which

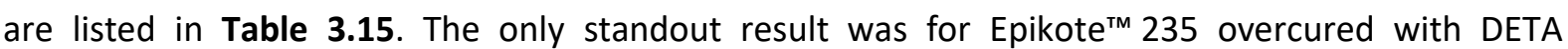
$(+20 \% \mathrm{w} / \mathrm{w})$, which bound approximately an order of magnitude more copper $(3300 \mu \mathrm{g} / \mathrm{g} \approx 0.3 \% \mathrm{w} / \mathrm{w}$, $3000 \mathrm{ng} / \mathrm{mm}^{2}$ ) than the other coatings. This was about one-third of the available copper. The amount of copper retained by the other epoxies was $<0.05 \% \mathrm{w} / \mathrm{w}$ (i.e. $<500 \mu \mathrm{g} / \mathrm{g}$ ).

Table 3.15. Amounts of copper retained by coatings determined to be high $\mathrm{Cu}(\mathrm{II})$-loading based on colour intensity. Prepared with epoxy resin Epikote ${ }^{\mathrm{TM}} 235$.

\begin{tabular}{|c|c|c|c|}
\hline Hardener & $\begin{array}{c}\% \text { w/w Overcure (+)/ } \\
\text { Undercure (-) }\end{array}$ & $\begin{array}{l}\mu \mathrm{g} \mathrm{Cu} / \mathrm{g} \\
\text { Coating }^{\mathrm{a}}\end{array}$ & $\begin{array}{l}\text { ng Cu/ } \mathrm{mm}^{2} \\
\text { Coating }\end{array}$ \\
\hline Ancamine ${ }^{\circledR} 2459$, cyclen & $+10 \%$ (cyclen) & 480 & 420 \\
\hline Ancamine ${ }^{\circledR} 2459$, cyclen & $+20 \%$ (cyclen) & 450 & 410 \\
\hline DETA & $+20 \%$ & 3300 & 3000 \\
\hline DETA (25\%), cyclen (100\%) & $+25 \%$ & 240 & 210 \\
\hline DETA (100\%), cyclen (25\%) & $+25 \%$ & 330 & 300 \\
\hline
\end{tabular}

${ }^{a}$ Estimated variability $\pm 20 \%$.

${ }^{\text {b }}$ Surface area $\left(\mathrm{mm}^{2}\right)$ exposed to $\mathrm{Cu}(\mathrm{II})$ salt solution. Calculated as in the Appendix (Figure 8.38).

\subsubsection{Conclusions}

Known $\mathrm{Cu}(\mathrm{II})$ ligands cyclen, cyclam, DETA, and PEI were used as curing agents or surface-modifiers of epoxy coatings prepared with resin Epikote ${ }^{\mathrm{TM}} 235$ and, in select cases, hardener Ancamine ${ }^{\circledR} 2459$ (Table 3.7). When serving as hardeners, they were used in super-stoichiometric, sub-stoichiometric, and stoichiometric proportions to over/undercure the films or adhere to the manufacturers' specifications. FT-IR spectroscopy was determined to be useful for monitoring the curing process, and the spectra revealed that the epoxy group band at $\sim 915 \mathrm{~cm}^{-1}$ was present for all of the films except those surface-functionalised with DETA or PEI, overcured by $50 \% \mathrm{w} / \mathrm{w}$ with Ancamine ${ }^{\circledR} 2459$, and cured stoichiometrically with Ancamine ${ }^{\circledR} 2459$ but overcured with cyclen or DETA by $10-20 \% \mathrm{w} / \mathrm{w}$. This suggested that, since polymerisation separates reactive components, reaction with every epoxy group is atypical, requiring an excess of hardener. The films using tetraaza macrocycles cyclen and cyclam as curing agents were hard and brittle, which was unsurprising given the relative rigidity of the molecular structures and the expectation of high crosslinking in the film. In contrast, the films using the more flexible, linear polyamine DETA as a hardener were often soft and sticky. To a certain extent, 
these trends were also identified in the surface-modifications; functionalisation with the tetraaza macrocycles (i.e.cyclen and cyclam) resulted in hard and, occasionally, brittle films, while functionalisation with the linear polyamines (i.e. DETA, PEI) often softened the coatings.

Upon delivering an aliquot of a $\mathrm{Cu}(\mathrm{II})$ salt solution to the surfaces of the films prepared or surfacefunctionalised with cyclen, cyclam, DETA, and PEI, a colour change was observed, signalling Cu(II)coordination. Those with the most intense colour retention were noted (Table 3.7), and the amounts of copper retained by these coatings were quantified. The method for quantification of the $\mathrm{Cu}(\mathrm{II})$ loading was evaluated, and the direct relationship between colour intensity and copper content was affirmed. As before, the amount of copper was normalised by the mass of the cured epoxy and the surface area exposed to the $\mathrm{Cu}(\mathrm{II})$ salt solution. Although, it was acknowledged that the depth of the $\mathrm{Cu}(\mathrm{II})$-penetration was still unknown. The coatings that were surface-modified with DETA and PEI were not amenable to the method established for determining $\mathrm{Cu}(\mathrm{II})$-loading, as DETA and PEI softened the coatings until they became gel-like. Therefore, these coatings were not pursued. The Epikote $^{\mathrm{TM}} 235 /$ DETA ( $+20 \% \mathrm{w} / \mathrm{w}$ ) epoxy bound an order of magnitude more copper ( $\sim 0.3 \% \mathrm{w} / \mathrm{w}$ ) than the remaining coatings, which bound $<0.05 \% \mathrm{w} / \mathrm{w}$ copper (Table 3.15). Assuming the copper was bound at the surface, the Epikote ${ }^{\text {TM }} 235 /$ DETA coating bound $4.7 \times 10^{-6} \mathrm{~mol} \mathrm{Cu} / \mathrm{cm}^{2}$, while the other coatings bound $<6.5 \times 10^{-7} \mathrm{~mol} \mathrm{Cu} / \mathrm{cm}^{2}$. These amounts are very low but substantially higher than the $3.8 \times 10^{-10} \mathrm{~mol} \mathrm{Cu} / \mathrm{cm}^{2}$ bound by PEI-functionalised glass with low microbicidal activity for $E$. coli ATCC 10356 in a study by Dacarro et al. ${ }^{216}$, suggesting that the level of $\mathrm{Cu}(\mathrm{II})$ retained by these epoxies might be high enough to exert an antimicrobial effect.

Despite the ability of the highlighted coatings to sequester low levels of copper, the physical properties of these coatings were unfavourable, eliminating them as suitable candidates for this project. The Epikote $^{\text {TM }}$ 235/DETA coating, which retained the most $\mathrm{Cu}(\mathrm{II})$, was soft and sticky, and, when attempting to spread the epoxy in a uniform film by the draw-down method, the resultant film was patchy and uneven. Thus, the coating would be unsuitable for antifouling applications: covering a surface evenly with such a coating would be infeasible, and a sticky coating would likely result in increased adherence by biofouling organisms. The coatings prepared with combinations of Ancamine ${ }^{\circledR} 2459$ and cyclen as hardeners were brittle, and those prepared with combinations of DETA and cyclen were brittle, patchy, and sometimes sticky. The DETA/cyclen coatings also exhibited blushing. It became clear that alterations to the paint binders would significantly impact the physical and mechanical properties of the coatings and, therefore, limit the type and amount of hardener that could be added to increase $\mathrm{Cu}(\mathrm{II})$-loading. As a high capacity to bind $\mathrm{Cu}(\mathrm{II})$ is essential for this work, alternative routes to include $\mathrm{Cu}(\mathrm{II})$ ligands, specifically cyclen, in a paint were pursued. 


\subsection{SUMMARY AND FUTURE WORK}

In continuation of the work in the previous chapter (Chapter 2, Section 2.2), the DiLevDEG/ADH polyacylhydrazone was formulated into a commercial acrylic paint, substituting $35 \% \mathrm{w} / \mathrm{w}$ of the binder, and this paint was assessed for its ability to bind $\mathrm{Cu}(\mathrm{II})$ and its subsequent effect on bacterial growth. The $35 \% \mathrm{w} / \mathrm{w}$ DAp/acr and commercial acrylic paints sequestered comparable amounts of $\mathrm{Cu}$ (II) (Table 3.5), but the $\mathrm{Cu}(\mathrm{II})$-loaded acrylic paint had a much more negative impact on the growth of E. coli NZRM 3647 (Figure 3.7), as it leached over twice the amount of copper into the bacterial culture (Table 3.3). A direct relationship between growth inhibition and the amount of copper leached into solution was substantiated, meaning that, instead of surface activity, the growth assay was measuring the antimicrobial activity of leached components. As a result, it was decided that a bacterial adherence assay, rather than the growth assay, would be more useful for initial evaluation of the antifouling performance of coatings, and this is explored in Chapter 5. When exposing the DAp/acr paint to a $\mathrm{Cu}(\mathrm{II})$ salt solution, the colour change of the solution indicated that a component had leached from the paint to form a $\mathrm{Cu}$ (II) complex. It was assumed that this component was DiLevDEG/ADH, since it is water-soluble and composes a significant percentage of the paint, and the release of small molecules and/or monomers from the polyacylhydrazone was confirmed by ${ }^{13} \mathrm{C} N M R$ spectroscopy (Figure 3.8). Consequently, it was decided that a paint which leaches its binder when exposed to water was not useful for the purposes of the project, and further work with the DAp/acr paint was discontinued. Alternative ligands and methods for their incorporation into a paint binder were pursued.

Along with commercial hardener Ancamine ${ }^{\circledR} 2459$, cyclen, cyclam, DETA, and PEI were used as curing agents and/or surface-modifiers in a two-pack epoxy system with resin Epikote ${ }^{\text {TM }} 235$. Multiple combinations were trialled (Table 3.7) by preparing films via the draw-down method, recording their physical and mechanical properties, measuring their FT-IR spectra to assess unreacted epoxy group content, and adding $\mathrm{Cu}(\mathrm{II})$ to evaluate binding to the incorporated ligands (Appendix, Table 8.19). $\mathrm{Cu}(I I)$-loading was quantified in the films recorded as having an intense colour change post-addition of $\mathrm{Cu}(\mathrm{II})$. The copper data was reported by normalising the amount of copper by the mass of the cured coating or the surface area, but it was recognised that this was misleading, given that the depth of copper penetration in each coating was unknown. In future experiments, depth-profiling via laser ablation (LA) ICP-MS could be employed to analyse such surfaces for trace metals, providing a better picture of the copper distribution in the film. ${ }^{226}$ Contact angle measurements to determine the wettability of the coatings would also be a useful characterisation technique. ${ }^{227}$ Out of the coatings noted for their colour retention, Epikote ${ }^{\mathrm{TM}} 235$ overcured with DETA $(+20 \% \mathrm{w} / \mathrm{w})$ retained the most 
copper (Table 3.15). Yet, this epoxy, as well as the others, was unsuitable as an antifouling coating because of its undesirable physico-mechanical properties. The realisation that changes to the binder would be restricted due to the negative impact on coating properties led to the consideration of different methods for incorporating $\mathrm{Cu}(\mathrm{II})$ ligands in a paint. 


\section{INCORPORATION OF CU(II)-LIGAND COMPLEXES IN CoATING AdDiTives}

\subsection{BACKGROUND}

Given the limitations of incorporating the $\mathrm{Cu}(\mathrm{II})$-ligand complex into the paint binder, the other option explored was incorporating it as an additive. In general, the simplest paint formulations are usually composed of pigments dispersed in a binder and carried by a solvent. Such simple systems are usually unsatisfactory products, often characterised by defects in the liquid paint and dry film. Some of these defects are as follows: pigment settlement, the formation of a paint skin in the can, bubble retention on application, the appearance of depressions in the film (i.e. cissing), the development of a wrinkled surface (i.e. shrivelling), excessive, vertical flow of a paint causing an uneven coating (i.e. sagging), colour differences in the film from pigment separation post-application (i.e. floating), and permanent colour change once an applied paint is subject to shear (i.e. flooding). While a number of the defects may be overcome by reformulation of the paint, other defects require the incorporation of small quantities (0.001-5\%) of additives as a remedy (Figure 4.1)..$^{29,30}$

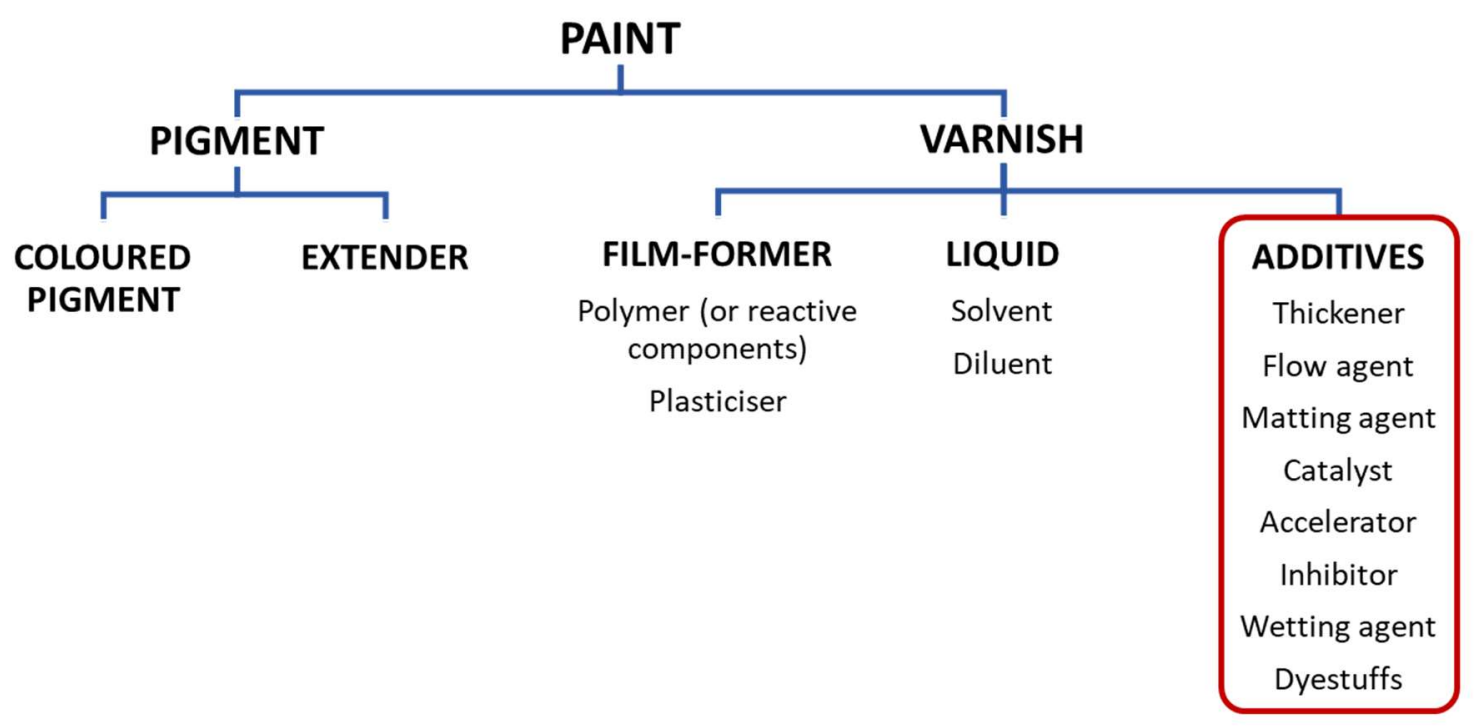

Figure 4.1. Paint components (adapted from Introduction to Paint Chemistry). ${ }^{29}$ 
Silica is a low-cost, common additive and extender that can enhance multiple coating properties. For instance, silica can act as an anti-settling agent. When fine silica particles ( $15 \mathrm{~nm}$ diameter, a few percent loading) are dispersed in a paint, they produce a pseudo-plastic effect due to the surface forces and their relatively large surface area (e.g. 190-460 m²/g). A pseudo-plastic paint is shearthinning, meaning that the viscosity decreases as the shear-rate increases. This is beneficial because, while the paint is at rest (e.g. sitting in a can), its higher viscosity prevents pigment settlement, but, when the paint is applied, the shearing action of, for example, the brush results in a decrease in viscosity for easy application. The viscosity increases again once the shearing action ceases, thus preventing sagging of the coating on the substrate. ${ }^{29,30}$ The addition of silica (nano, micro, fumed) to a coating has also been shown to improve its hardness, abrasion and scratch resistance, tensile strength, and weatherability, as well as imparting superhydrophobic properties and acting as a matting agent and rheology modifier. ${ }^{228-231}$ Accordingly, silica additives are found in multiple coating types, including antifouling coatings. ${ }^{232,233}$

The properties of silica are tuneable via functionalisation, and there is evidence that modified silica particles enhance the antifouling performance of marine paints. For example, in a study by Michailidis et al. ${ }^{234}$, mesoporous silica nanoparticles (MSNs) were functionalised with quaternary ammonium salts (QASs), which have antimicrobial activity due to the interaction of their positive charge with negatively charged bacterial cell membranes. The QAS-modified MSNs were formulated into a coating, and the coating was tested for its antifouling performance against a control in a field test in the northern Red Sea. Incorporation of the QAS-modified MSNs in the coating reduced the biofouling coverage from $39 \%$ to below $10 \%$, suggesting that modified silica particles could serve as functional fillers in antifouling coatings. Therefore, it was decided that the functionalisation of silica with $\mathrm{Cu}(\mathrm{II})$-ligand cyclen (Chapter 2, Section 2.4) should be explored for formulation as an additive into an antifouling paint.

The incorporation of cyclen in the coating through the modification of silica, rather than as the free ligand, was preferable for a few reasons. First, given the water-solubility of cyclen, leaching into seawater was probable, but its attachment to a coating filler was considered to be a strategy for more permanent retention in the paint. ${ }^{234}$ Second, it was unknown how free cyclen would be presented at the coating surface and if it would be accessible to copper ions for complexation. In contrast, it is known that silica tends to float to the surface of a coating in large aggregates composed of numerous particles, which would result in the exposure of silica-attached cyclen to copper ions in seawater at the coating-liquid interface. ${ }^{29}$ Lastly, previous experiments demonstrated the reactivity of cyclen with 
other paint components, such as the epoxy resin. Such undesirable interactions are more likely to occur with free cyclen.

The majority of studies involving silica functionalised with macrocycles, such as cyclen and cyclam (Figure 4.2), were conducted by Guilard ${ }^{123,235-241}$ and Corriu $235,237,239,240,242-245$, but a number of others $^{246-251}$ have contributed to the literature as well. These materials are mainly used for gas adsorption, catalysis, or chromatography. ${ }^{252}$ However, their suitability as adsorbents for the removal of metal ions from wastewater, industrial effluents, and tap water is also recognised; silica is an excellent supporting material for this purpose due to its high surface area, mechanical and chemical stability, low cost, amenability to modification, and commercial availability. ${ }^{123,236,253}$ These examples provide further support for the idea that cyclen-functionalised silica can be utilised for the sequestration of copper ions from seawater. Furthermore, work by Díaz-García et al. ${ }^{254}$ demonstrated that $\mathrm{CU}(\mathrm{II})$-complexed, ligand-modified silica materials similar to those proposed have moderate antimicrobial activity against E. coli, indicating that such materials are capable of both chelating copper ions and, subsequently, exerting an antimicrobial effect.

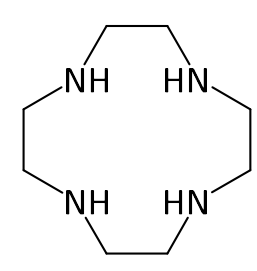

Cyclen

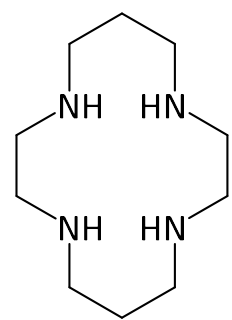

Cyclam

Figure 4.2. Structures of macrocycles cyclen and cyclam.

Although the literature addressing the functionalisation of silica with macrocycles is substantial, the preparation methods and product characterisation techniques are variable. At times, assumptions are made about the product without satisfactory final characterisation, and, in multiple cases, characterisation data of the modified silica are incomplete or absent. For instance, Puranik et al..$^{248}$ and Bagnoud et al. ${ }^{255}$ infer the generation of the desired product from its ability to retain $\mathrm{Cu}(\mathrm{II})$, rather than from direct measurements and characterisation of the modified silica. For those studies in which data are reported, common characterisation techniques of the silica include FT-IR spectroscopy, CHN elemental analysis, microscopy, solid-state cross-polarisation magic angle spinning nuclear magnetic 
resonance spectroscopy (CP-MAS NMR), and Brunauer, Emmett, and Teller (BET) surface area measurements. Frequently used techniques for copper quantification include X-ray fluorescence (XRF) and inductively coupled plasma optical emission spectroscopy (ICP-OES). The literature shows that silica-bound cyclam has typically been prepared either by grafting or sol-gel synthesis. No standard synthetic procedure or characterisation strategy is followed, which makes direct comparisons difficult and reliable data sparse. Consequently, in the present work, methods were investigated, evaluated, and developed for silica modification with cyclen and subsequent characterisation of the products. 


\subsection{Silica FUNCTIONALISATION}

As in Scheme 4.1, two methods were considered for the functionalisation of silica with the cyclen ligand. In Method 1, trialkoxysilane $\left(\mathrm{RSi}\left(\mathrm{OR}^{1}\right)_{3}\right.$ ) (3-glycidyloxypropyl)trimethoxysilane (GLYMO) hydrolyses to yield the corresponding silanols. Siloxane linkages form in a condensation reaction between the silanol groups of GLYMO and silica $\left(\mathrm{SiO}_{2}\right)$, producing GLYMO-functionalised silica $\left(\mathrm{GmSiO}_{2}\right) .{ }^{256} \mathrm{GLYMO}$ is a commercial silane coupling reagent capable of bonding with both organic and inorganic materials and, therefore, acting as an intermediary linker to modify the inorganic matrix of silica with a terminal epoxide functional group (Figure 4.3). In turn, the terminal epoxide can react with organic compounds, ${ }^{257}$ as in the next step of Method 1 in which the epoxide of $\mathrm{GmSiO}_{2}$ reacts with a secondary amine of cyclen by the $\mathrm{S}_{\mathrm{N}} 2$ mechanism to produce cyclen-GLYMO-silica $\left(\mathrm{CnGmSiO}_{2}\right)$. This approach was used by Bagnoud et $a .^{255}$ and Gros et al. ${ }^{241}$ to bond tetraazacycloalkanes cyclam and cyclen (Figure 4.2), respectively, to silica for $\mathrm{Cu}(\mathrm{II})$-retention as the stationary phase in chromatography.

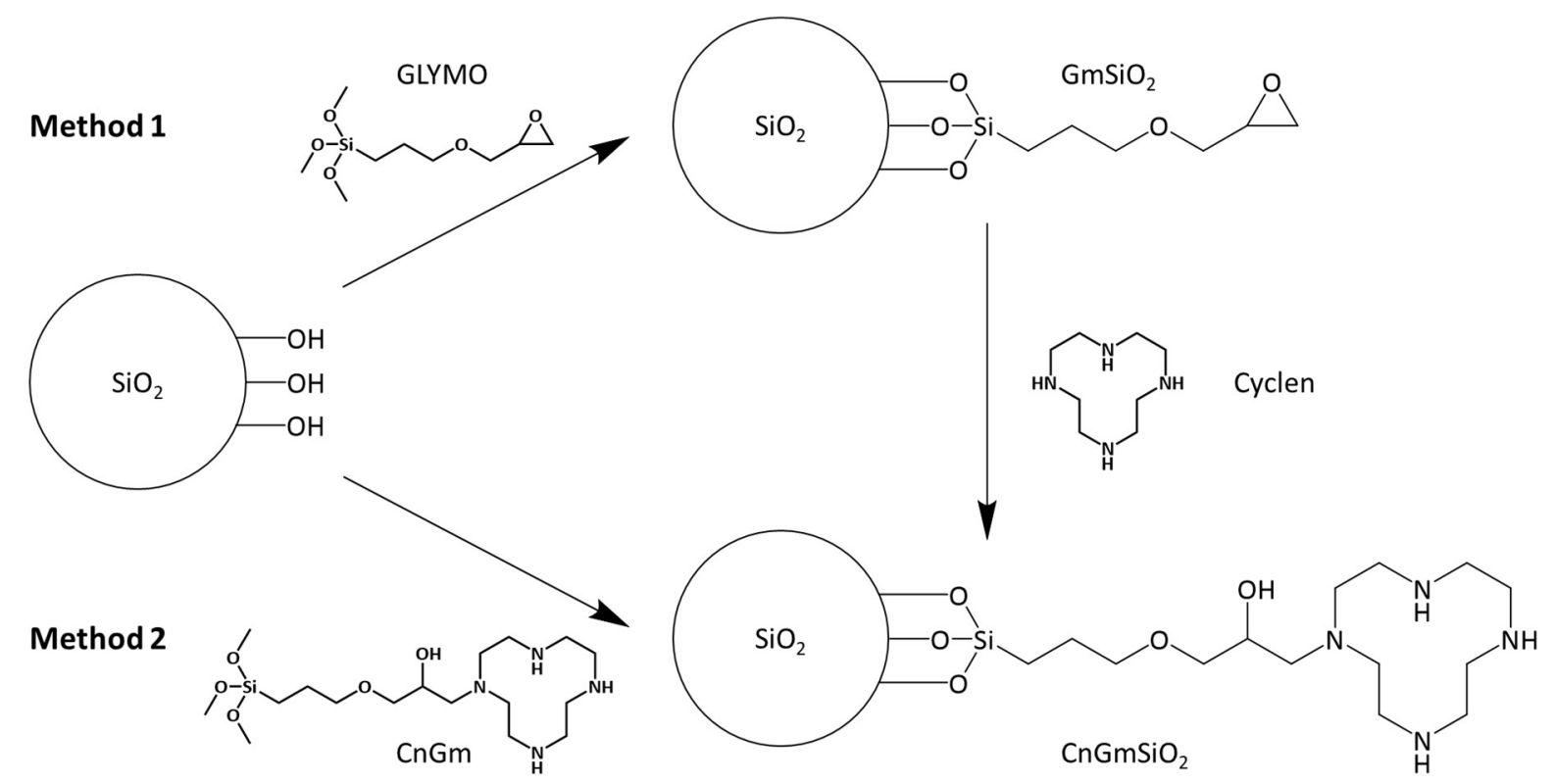

Scheme 4.1. Two silica functionalisation methods. Method 1: Reaction between GLYMO and the silanol groups of silica $\left(\mathrm{SiO}_{2}\right)$ produces GLYMO-functionalised silica $\left(\mathrm{GmSiO}_{2}\right)$, and then the reaction between $\mathrm{GmSiO}_{2}$ and cyclen produces cyclen-GLYMO-silica $\left(\mathrm{CnGmSiO}_{2}\right)$. Method 2: GLYMO and cyclen react first to produce cyclen-GLYMO $(\mathrm{CnGm})$, and then $\mathrm{CnGm}$ reacts with silica to produce $\mathrm{CnGmSiO}_{2}$. 


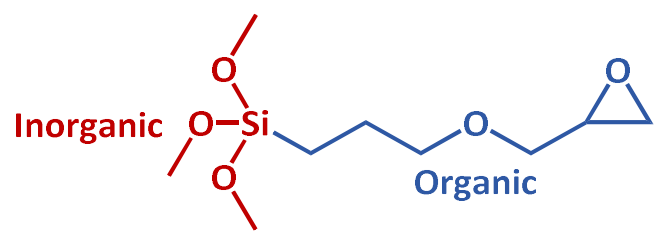

Figure 4.3. The organic and inorganic components of silane coupling agent GLYMO.

In Method 2, the order of reactions differs from Method 1. The first reaction is between cyclen and GLYMO to produce cyclen-GLYMO ( $\mathrm{CnGm})$, and then reaction of $\mathrm{CnGm}$ with silica produces $\mathrm{CnGmSiO}_{2}$. An advantage of performing the synthesis in this order is that the product of the first reaction, $\mathrm{CnGm}$, may be characterised by NMR spectroscopy. When "pre-loading" $\mathrm{CnGm}$ with $\mathrm{Cu}(\mathrm{II}), \mathrm{Cu}(\mathrm{II})$-chelation by $\mathrm{CnGm}$ results in the formation of a dark blue complex $(\mathrm{Cu}(\mathrm{II})-\mathrm{CnGm})$, which serves as a visual confirmation of the silica modification with $\mathrm{Cu}(\mathrm{II})-\mathrm{CnGm}$ (i.e. the functionalised silica is blue). Characterisation becomes more difficult once the compounds are bonded to silica, and techniques are limited to, for example, IR spectroscopy, CHN elemental analysis, and gravimetric analyses. Furthermore, since cyclen may become adsorbed to silica, this inverted synthetic approach allows for confidence that the reaction between cyclen and GLYMO has occurred because of the ability to more fully characterise the product. The second step of Method 1 brings a measure of uncertainty as to whether or not changes in mass or the IR spectrum are simply due to cyclen adsorption onto $\mathrm{GmSiO}_{2}$ - i.e. non-covalent attachment. Method 2 was employed by Bereczki et al. ${ }^{258}$ to create reusable catalysts by functionalising mesoporous silica aerogels with $\mathrm{Cu}(\mathrm{II})$-cyclen complexes and by Barreto et al. ${ }^{259}$ to modify magnetite nanoparticles with cyclen for ${ }^{64} \mathrm{Cu}(\mathrm{II})$-labelling, generating superparamagnetic nanoparticles for tumour detection by positron emission tomography (PET) or magnetic resonance imaging (MRI).

The silica used in the experiments in the present study was high-purity grade silica gel with a $60 \AA$ pore size, 220-440 mesh particle size, and 35-75 $\mu \mathrm{m}$ particle size. It was selected to serve as a model compound since the material is less dusty than finer silica, mitigating the safety hazard via inhalation. In this work, the silica gel will be referred to simply as silica. The ratio of GLYMO to silica (mmol GLYMO/g silica) for functionalisation was based on the ratio of $n$-octadecyldimethylchlorosilane to silica (0.991 mmol modifier/g silica) used by Ortega et al. ${ }^{260}$ to synthesise reversed-phase silica gel. Gros et al. ${ }^{241}$ advised against pre-treatment of the silica with strong acids due to concerns about the introduction of structural changes, and, therefore, such pre-treatment was not conducted in the present study. However, the silica was dried before use, except when stated otherwise. 


\subsubsection{Method 1}

\subsubsection{Synthesis of $\mathrm{GmSiO}_{2}$}

Following the first step of Method 1, four procedures for the synthesis of $\mathrm{GmSiO}_{2}$ were evaluated (Scheme 4.2), and the reaction conditions and outcomes are summarised in Table 4.1. Recovery of unreacted GLYMO was used to determine the theoretical amount of GLYMO attached to the silica, which was compared to the corresponding mass increase of the silica post-reaction. The identity of the recovered material was confirmed by ${ }^{1} \mathrm{H}$ NMR spectroscopy. The maximum loading of GLYMO onto the silica (i.e. mmol GLYMO functionalising $1 \mathrm{~g} \mathrm{SiO}_{2}$ ) was calculated from these data, and the percent functionalisation was calculated by comparing against the supposed maximum level of $0.991 \mathrm{mmol}$ modifier/g $\mathrm{SiO}_{2}$ from the literature. ${ }^{260}$ This maximum level is, presumably, not a robust measure because it would depend on the size of the silica, its porosity, surface area, etc., and, although the specifications of the silica gel used by Ortega et $a l^{260}$ are similar to those for the silica gel used in the present work, they are not identical. Nevertheless, the maximum level was still used as a crude measure of success. The product from each procedure was characterised by measuring the IR spectrum, and these data, in concert with the gravimetric data, were used to assess the extent of reaction.
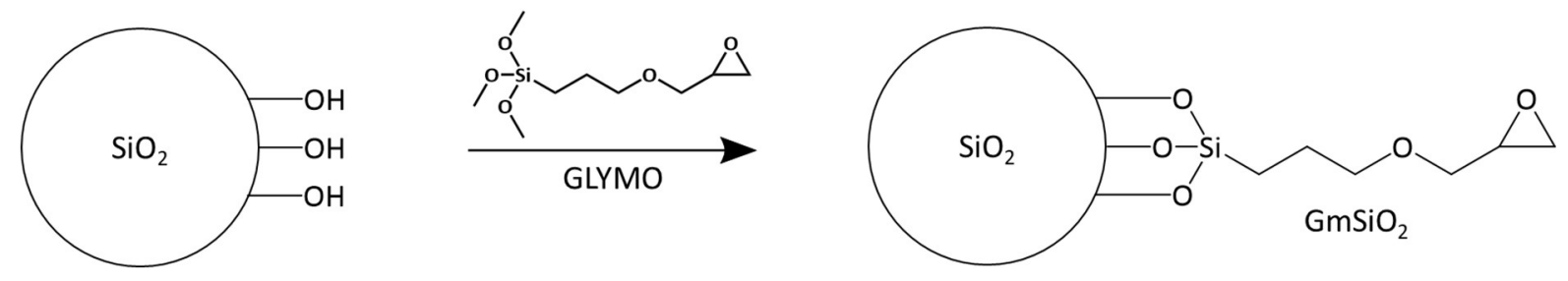

Scheme 4.2. Synthesis of $\mathrm{GmSiO}_{2}$.

Different temperatures, solvent amounts, and reactant stoichiometries were trialled in Procedures

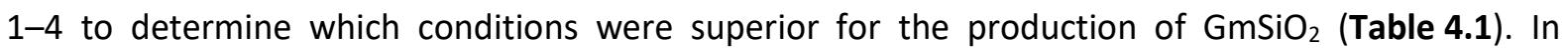
Procedure 1, $1.2 \mathrm{mmol} \mathrm{GLYMO}_{\mathrm{g} \mathrm{SiO}}$ in anhydrous toluene under argon were reacted for $24 \mathrm{~h}$ at room temperature, and the white silica product was filtered, washed, and dried. The clear, colourless filtrate was concentrated via rotary evaporation to obtain the mass of unreacted GLYMO, which was 99\% of the starting amount. From this amount, the loading was calculated to be $0.01 \mathrm{mmol}$ $\mathrm{GLYMO} / \mathrm{g} \mathrm{SiO}_{2}$. This was well below the literature amount $(0.991 \mathrm{mmol} / \mathrm{g}),{ }^{260}$ suggesting a percent functionalisation of, at most, $1 \%$. 
Table 4.1. Reaction conditions and outcomes in Procedures 1-4 for $\mathrm{GmSiO}_{2}$ synthesis.

\begin{tabular}{|c|c|c|c|c|}
\hline Conditions & Procedure 1 & Procedure 2 & Procedure 3 & Procedure 4 \\
\hline $\mathrm{SiO}_{2}(\mathrm{~g})$ & 0.91 (dry) & 1.02 (dry) & 1.00 (wet) & 2.50 (dry) \\
\hline GLYMO (g, mmol) & $0.27,1.1$ & $2.1,9.1$ & $4.3,18$ & $3.7,16$ \\
\hline Anhydrous toluene ( $\mathrm{mL}$ ) & 5 & 4 & - & 35 \\
\hline Triethylamine $(\mu \mathrm{L})$ & - & - & - & 75 \\
\hline Temperature $\left({ }^{\circ} \mathrm{C}\right)$ & $20(\mathrm{RT})$ & 80 & 100 & 110.6 (reflux) \\
\hline Time (h) & 24 & 72 & 69 & 42 \\
\hline Outcomes & Procedure 1 & Procedure 2 & Procedure 3 & Procedure 4 \\
\hline GLYMO filtrate (g) & 0.27 & 2.0 & 3.8 & 2.7 \\
\hline GLYMO - GLYMO filtrate (g) & 0.003 & 0.2 & 0.5 & 1.0 \\
\hline \% GLYMO recovered & 99 & 92 & 89 & 73 \\
\hline $\mathrm{GmSiO}_{2}(\mathrm{~g})$ & 0.97 & 1.18 & 1.40 & 3.17 \\
\hline $\mathrm{GmSiO}_{2}-\mathrm{SiO}_{2}(\mathrm{~g})$ & 0.06 & 0.16 & 0.40 & 0.67 \\
\hline $\mathrm{GmSiO}_{2}: \mathrm{mmol}^{\mathrm{GLYMO} / \mathrm{g} \mathrm{SiO}}{ }_{2}$ & 0.01 & 0.65 & 1.7 & 1.1 \\
\hline$\%$ Functionalisation $^{a}$ & 1 & 65 & 170 & 110 \\
\hline
\end{tabular}

The reaction in Procedure 2 was conducted with $8.9 \mathrm{mmol} \mathrm{GLYMO} / \mathrm{g} \mathrm{SiO}_{2}$ in anhydrous toluene under argon for 3 days at $80^{\circ} \mathrm{C}$, and recovery of $92 \%$ of the GLYMO in the filtrate, along with the corresponding mass increase of the silica, revealed a maximum loading of $0.65 \mathrm{mmol} \mathrm{GLYMO} / \mathrm{g} \mathrm{SiO}_{2}$ or $65 \%$ of the literature value. GLYMO and silica ( $18 \mathrm{mmol} \mathrm{GLYMO} / \mathrm{g} \mathrm{SiO}_{2}$, silica not dried) were reacted neat for 3 days at $100{ }^{\circ} \mathrm{C}$ in Procedure 3. GLYMO recovery was $89 \%$, and the loading was calculated to

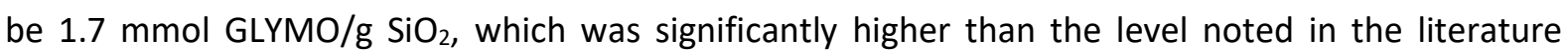
(170\%). The final procedure, Procedure 4, followed protocols by Bagnoud et al. ${ }^{255}$ and Gros et al. ${ }^{241}$, and $6.4 \mathrm{mmol} \mathrm{GLYMO} / \mathrm{g} \mathrm{SiO}_{2}$ were reacted in anhydrous toluene and triethylamine $(0.2 \% \mathrm{v} / \mathrm{v}$, base catalyst) under argon for 2 days at reflux temperature. The percent recovery of the GLYMO starting material was 73\%, and the loading was $1.1 \mathrm{mmol} \mathrm{GLYMO} / \mathrm{g} \mathrm{SiO}_{2}(110 \%)$.

To ensure that undesirable starting material transformations did not occur under the reaction conditions, the identity of the material recovered from the filtrate in each procedure was confirmed by comparing its ${ }^{1} \mathrm{H}$ NMR spectrum to the ${ }^{1} \mathrm{H}$ NMR spectrum of GLYMO (Figure 4.4). GLYMO may undergo, at least, two major transformations observable by ${ }^{1} \mathrm{H}$ NMR spectroscopy: 1) the epoxide can open, and 2) the methoxy groups can hydrolyse. For the opening of the epoxide, the disappearance of the peak at $\delta_{H} 3.14 \mathrm{ppm}$, attributed to the proton attached to the secondary carbon in the ring $(\mathbf{H} \mathbf{2}$, Figure 4.4), was a simple way to monitor the ring-opening. Hydrolysis of the methoxy groups was 
monitored by observing the disappearance of the peak at $\delta_{H} 3.57 \mathrm{ppm}$ (methyl protons, $\mathbf{H 7}$ ) and the appearance of methanol in the spectrum $\left(\delta_{\mathrm{H}} 3.49 \mathrm{ppm}\right.$ for $\mathrm{C}_{3} \mathrm{OH}$ in chloroform- $\left.d, \mathrm{CDCl}_{3}\right) .{ }^{261}$

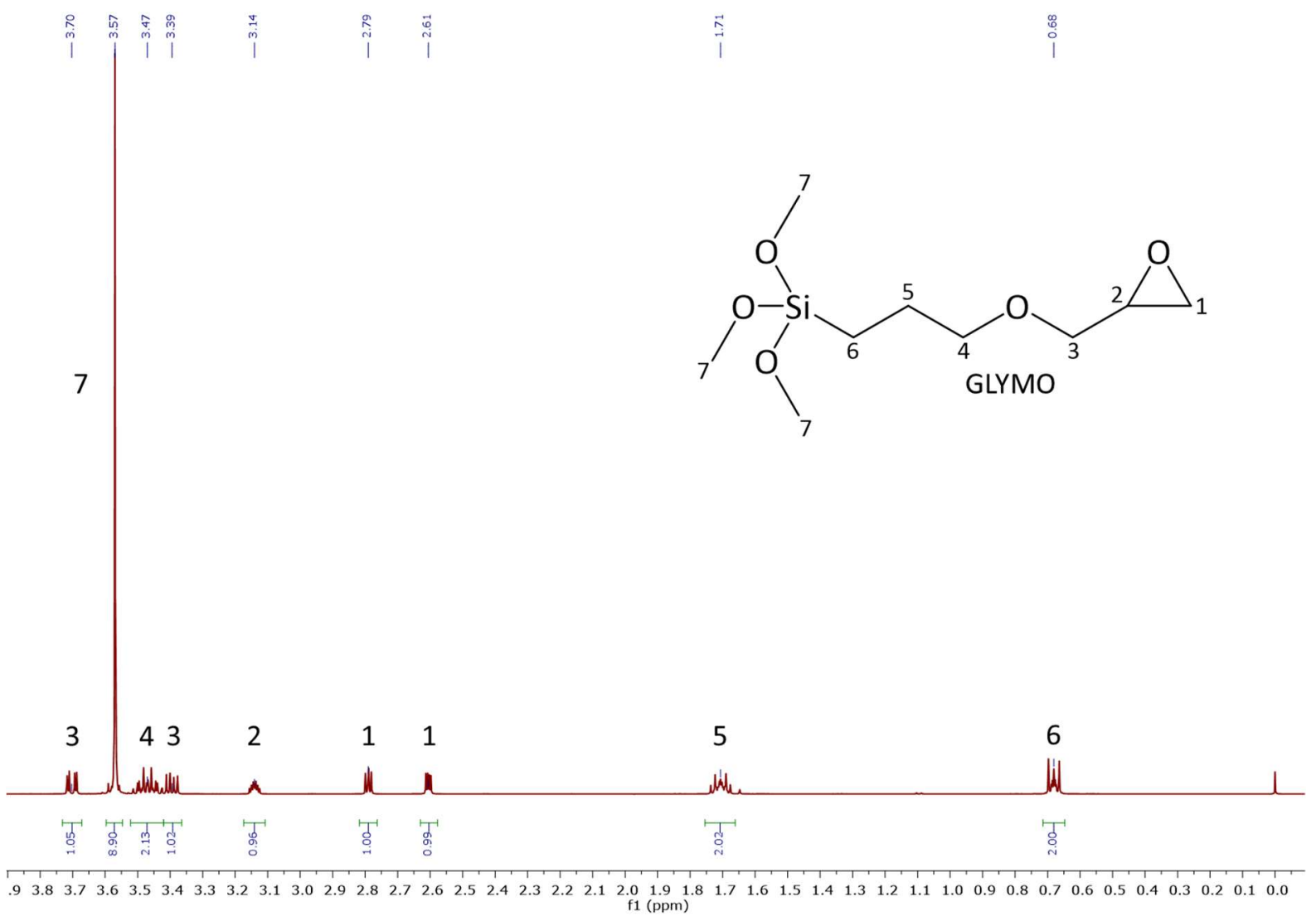

Figure 4.4. ${ }^{1} \mathrm{H}$ NMR spectrum $\left(\mathrm{CDCl}_{3}\right)$ of $\mathrm{GLYMO}$ and peak assignments.

As shown in Figure 4.5, the ${ }^{1} \mathrm{H}$ NMR spectra confirmed that the isolated filtrate material in Procedures 1-4 was indeed unreacted GLYMO. In all cases, the peak at $\delta_{H} 3.14 \mathrm{ppm}$ was observed, indicating that the epoxide was still present. The only spectrum with differences from the GLYMO starting material was that of the Procedure 3 filtrate, in which it appeared that some of the methoxy groups had hydrolysed: the peak at $\delta_{H} 3.57 \mathrm{ppm}$ decreased. Also, rather than being a clear, colourless, viscous liquid, as in the other procedures, the Procedure 3 filtrate material was a yellow colour. Presumably, this relates to the hydrolysis of the Si-OMe groups, which would be consistent with the use of the "wet" silica. 


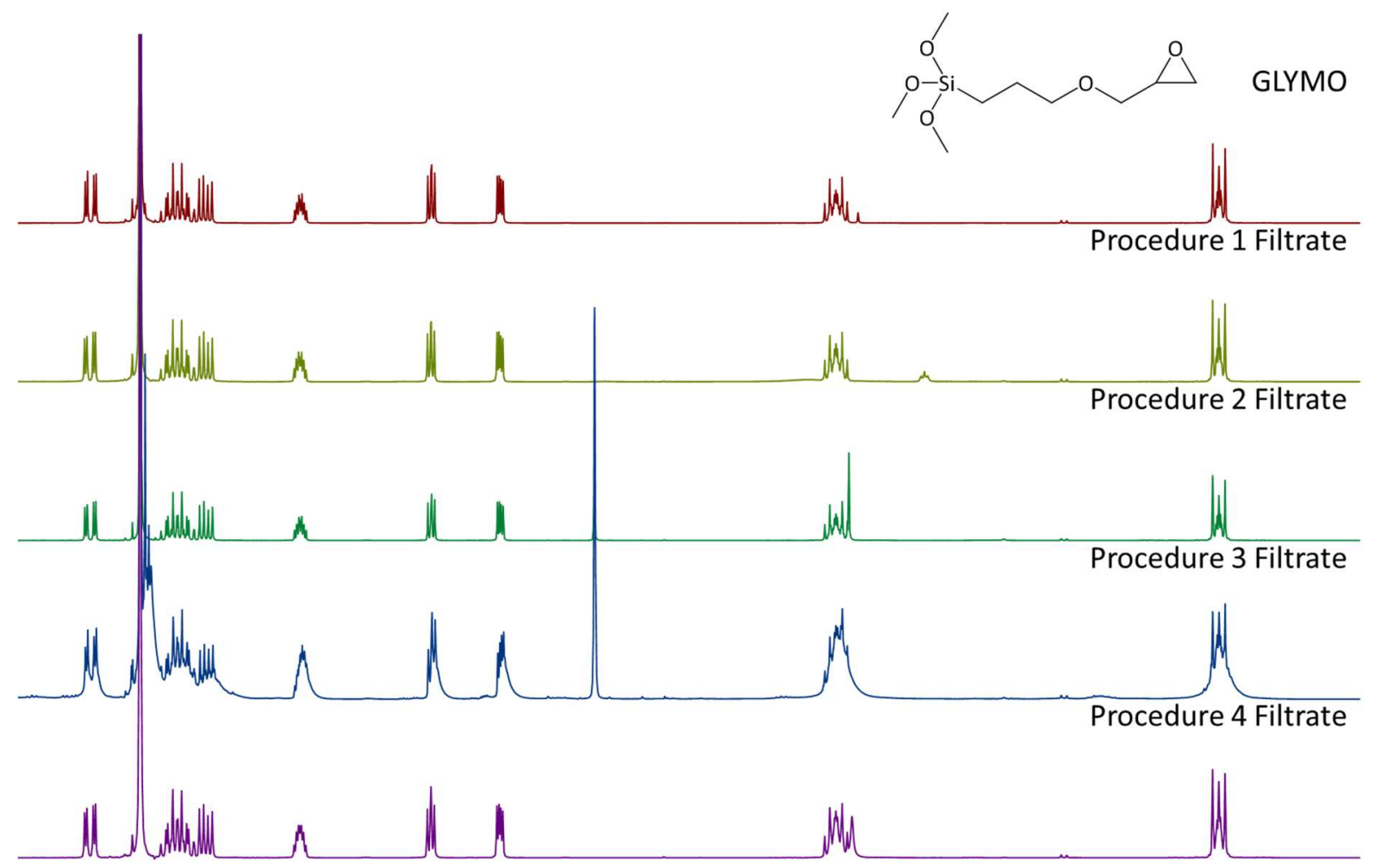

$\begin{array}{llllllllllllllllllllllllllllllllllllllllllllllll}3.8 & 3.7 & 3.6 & 3.5 & 3.4 & 3.3 & 3.2 & 3.1 & 3.0 & 2.9 & 2.8 & 2.7 & 2.6 & 2.5 & 2.4 & 2.3 & 2.2 & 2.1 & 2.0 & 1.9 & 1.8 & 1.7 & 1.6 & 1.5 & 1.4 & 1.3 & 1.2 & 1.1 & 1.0 & 0.9 & 0.8 & 0.7 & 0.6 & 0.5 & 0.4\end{array}$

Figure 4.5. ${ }^{1} \mathrm{H}$ NMR spectra $\left(\mathrm{CDCl}_{3}\right)$ of the filtrates from Procedures $1-4$ compared to GLYMO (toluene $\mathrm{PhC}_{3}$ at $\left.\delta_{H} 2.35 \mathrm{ppm}\right)$.

The IR spectra of starting materials GLYMO and silica and the purported $\mathrm{GmSiO}_{2}$ products from Procedures 1-4 were recorded to evaluate the extent of reaction (Figure 4.6). The corresponding peak data and assignments are shown in Table 4.2. If the GLYMO-functionalisation of silica occurred, the following changes in the IR spectrum would, theoretically, be expected to confirm the production of $\mathrm{GmSiO}_{2}: 1$ ) the $\mathrm{Si}-\mathrm{OH}$ stretching peak around $950 \mathrm{~cm}^{-1}$ and $\mathrm{O}-\mathrm{H}$ stretching peak at $3351 \mathrm{~cm}^{-1}$ due to the silanol groups of silica would decrease in intensity or disappear, 2) the $\mathrm{C}-\mathrm{O}$ stretching peak attributed to the GLYMO epoxy ring at $908-920 \mathrm{~cm}^{-1}$ would appear, and 3) additional peaks due to GLYMO $\left(2943,2841,1467,1254,1191\right.$, and $\left.1076 \mathrm{~cm}^{-1}\right)$ would appear. ${ }^{218,221,222,262}$ In actuality, the O-H stretching peak $\left(3351 \mathrm{~cm}^{-1}\right)$ was much too broad for use as a reaction marker, and the only perceptible evidence of modified silica was the appearance of the epoxy ring C-O peak $\left(908-920 \mathrm{~cm}^{-1}\right)$. For example, the spectra of the $\mathrm{GmSiO}_{2}$ from Procedures 2-4 displayed very weak epoxy ring peaks, and the $\mathrm{Si}-\mathrm{OH}$ stretching peak was absent in the Procedures 3 and 4 spectra. Otherwise, the spectra of the products from Procedures 2-4 matched the IR spectrum of the silica starting material. The spectrum 
of the Procedure 1 product showed no evidence of silica functionalisation, being nearly identical to that of the silica starting material.

(a)

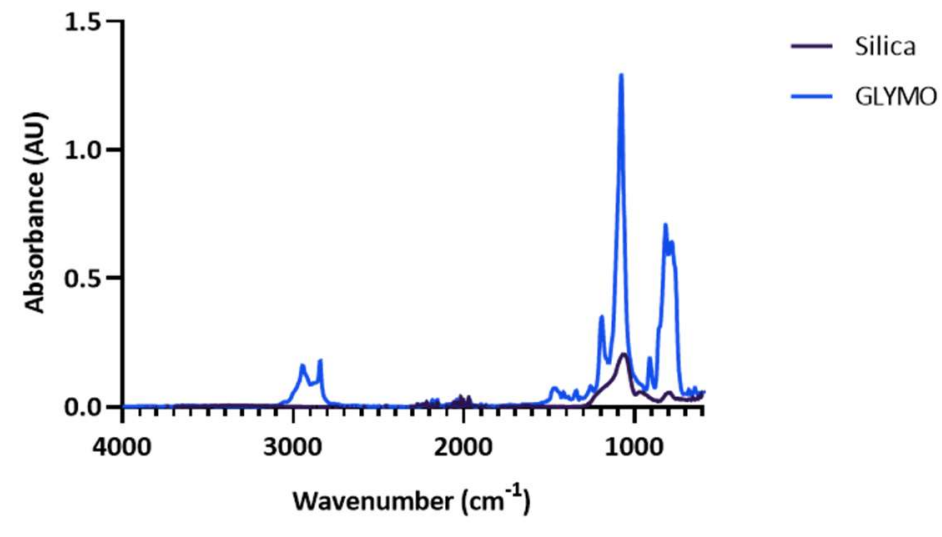

(b)

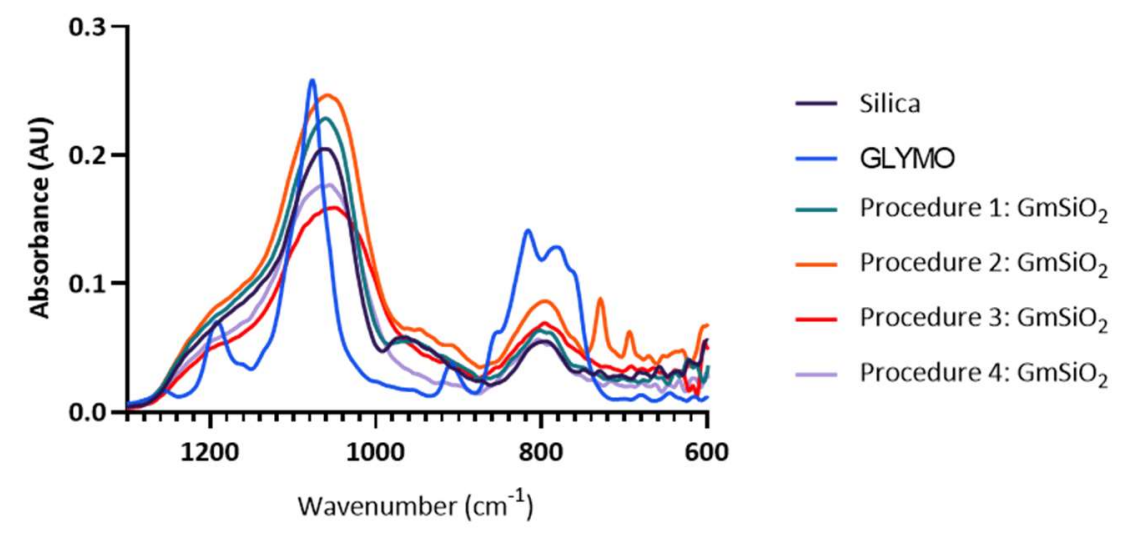

(c)

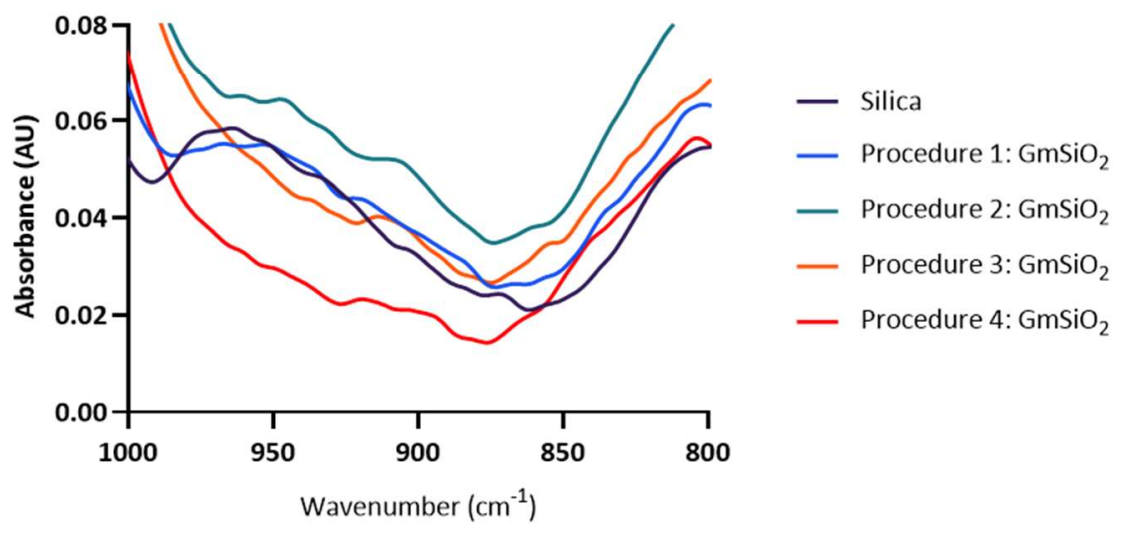

Figure 4.6. IR spectra of (a) silica and GLYMO starting materials, (b) silica, GLYMO, and purported $\mathrm{GmSiO}_{2}$ products from Procedures 1-4, and (c) silica and purported $\mathrm{GmSiO}_{2}$ products from Procedures 1-4, expanded view of the epoxide $\mathrm{C}-\mathrm{O}$ region $\left(908-920 \mathrm{~cm}^{-1}\right)$. 


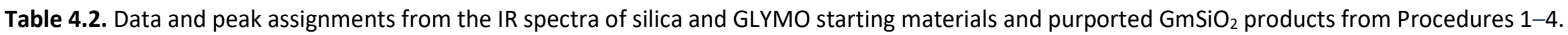

\begin{tabular}{|c|c|c|c|c|c|c|}
\hline $\begin{array}{c}\text { GLYMO } \\
\text { Frequency }\left(\mathrm{cm}^{-1}\right) \\
\end{array}$ & $\begin{array}{c}\text { Silica } \\
\text { Frequency }\left(\mathrm{cm}^{-1}\right) \\
\end{array}$ & $\begin{array}{c}\text { Procedure } 1 \\
\text { GmSiO }_{2} \\
\text { Frequency }\left(\mathrm{cm}^{-1}\right)\end{array}$ & $\begin{array}{c}\text { Procedure 2 } \\
\text { GmSiO }_{2} \\
\text { Frequency }\left(\mathrm{cm}^{-1}\right)\end{array}$ & $\begin{array}{c}\text { Procedure } 3 \\
\mathrm{GmSiO}_{2} \\
\text { Frequency }\left(\mathrm{cm}^{-1}\right)\end{array}$ & $\begin{array}{c}\text { Procedure } 4 \\
\mathrm{GmSiO}_{2} \\
\text { Frequency }\left(\mathrm{cm}^{-1}\right) \\
\end{array}$ & Assignment $^{218,221,222,262}$ \\
\hline & 3351 (w, vbr) & & & & & $\mathrm{O}-\mathrm{H}$ stretching \\
\hline $2943(\mathrm{~m})$ & & & & & & $\mathrm{C}-\mathrm{H}$ stretching in $\mathrm{Si}-\mathrm{O}-\mathrm{CH}_{3}$ and $\mathrm{CH}_{2}$ \\
\hline $2841(m)$ & & & & & & $\mathrm{C}-\mathrm{H}$ stretching in $\mathrm{Si}-\mathrm{O}-\mathrm{CH}_{3}$ and $\mathrm{CH}_{2}$ \\
\hline $1467(\mathrm{vw})$ & & & & & & $\mathrm{C}-\mathrm{H}$ in-plane rocking in $\mathrm{Si}-\mathrm{O}-\mathrm{CH}_{3}$ and $\mathrm{CH}_{2}$ \\
\hline $1254(v w)$ & & & & & & Si-C bending \\
\hline $1191(\mathrm{~m})$ & & & & & & $\mathrm{C}-\mathrm{O}$ stretching of aliphatic ether \\
\hline \multirow[t]{3}{*}{1076 (vs) } & & & & & & Si-O-C stretching \\
\hline & $1063(s, b r)$ & $1060(s, b r)$ & $1058(\mathrm{~s}, \mathrm{br})$ & 1049 (s, br) & 1066 (s, br) & Si-O-Si asymmetric stretching \\
\hline & $964(m, b r)$ & $953(\mathrm{~m}, \mathrm{br})$ & $948(v w, b r)$ & & & $\mathrm{Si}-\mathrm{OH}$ stretching \\
\hline $909(w)$ & & & $908(v w, b r)$ & $914(w, b r)$ & $920(v w, b r)$ & C-O stretching in epoxy ring \\
\hline \multirow[t]{4}{*}{$816(s)$} & & & & & & Si-C stretching \\
\hline & 794 (m, br) & $801(\mathrm{~m}, \mathrm{br})$ & 797 (m, br) & 796 (m, br) & $801(\mathrm{~m}, \mathrm{br})$ & Si-O-Si symmetric stretching \\
\hline & & & $729(\mathrm{~m})$ & & & \\
\hline & & & $694(w)$ & & & \\
\hline
\end{tabular}


Together, the GLYMO-loading data and the IR spectra of the products from Procedures 1-4 were reviewed to decide which synthetic route and $\mathrm{GmSiO}_{2}$ product should be carried forward for the second step of Method 1, which was the synthesis of $\mathrm{CnGmSiO}_{2}$. Procedure 1 was eliminated from consideration because the IR data yielded no evidence of functionalisation, and the loading was, at most, $0.01 \mathrm{mmol}$ GLYMO/g SiO 2 (1\%), as $99 \%$ of the GLYMO was unreacted. The loading data further narrowed the choices to between Procedures 3 and 4 , since both were well above the literature value of $0.991 \mathrm{mmol}$ GLYMO/g SiO 2 (1.7 and $1.1 \mathrm{mmol} \mathrm{GLYMO} / \mathrm{g} \mathrm{SiO}$, respectively). ${ }^{260}$ Procedure 2 had a

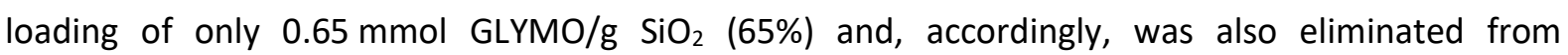
consideration. In Procedure 3, the ${ }^{1} \mathrm{H}$ NMR spectrum and colour change (i.e. yellow instead of colourless) of the GLYMO filtrate indicated that some of the methoxy groups had hydrolysed, and this would be expected to result in self-condensation (Scheme 4.3), as well as the desired condensation reaction with the silica silanol groups. For that reason, a large, concentrated excess of GLYMO, as in Procedure 3 (18 $\mathrm{mmol} \mathrm{GLYMO}_{\mathrm{g} \mathrm{SiO}}$ ), is likely problematic because it could lead to polymerisation. Therefore, Procedure 4 was selected, and the protocols reported by Bagnoud et al. ${ }^{255}$ and Gros et al..$^{241}$ continued to be followed for the synthesis of $\mathrm{CnGmSiO}_{2}$.

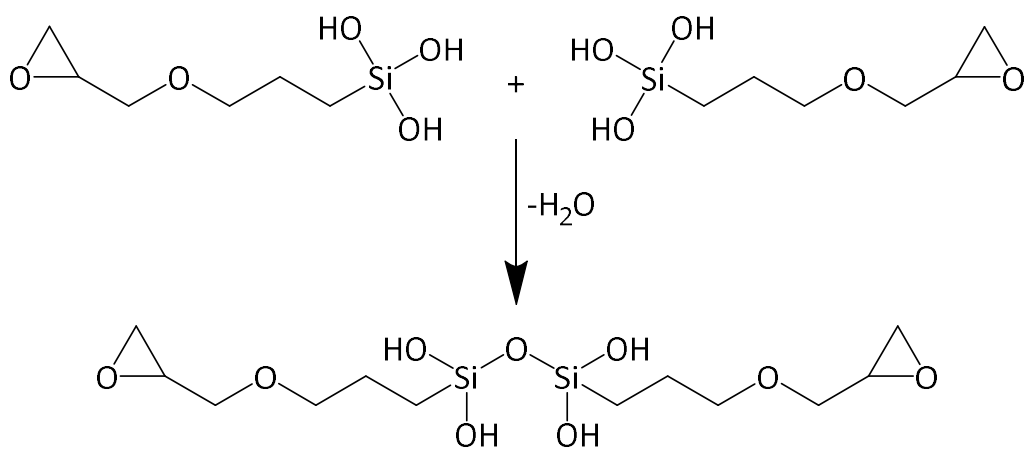

Scheme 4.3. Self-condensation of GLYMO.

\subsubsection{Synthesis of $\mathrm{CnGmSiO}_{2}$}

Continuing with the Procedure 4 product, end-capping of $\mathrm{GmSiO}_{2}$ (Scheme 4.4) was undertaken by heating in trimethylchlorosilane (TMCS). This step was recommended by Gros et al. ${ }^{241}$ because free silanol groups are able to complex metal ions, complicating future measurements of the efficiency of the system to bind $\mathrm{Cu}(\mathrm{II})$. However, there was no increase in product mass, suggesting that the endcapping reaction did not occur. In fact, the product mass decreased by $0.6 \%$. The IR spectrum of the putative end-capped $\mathrm{GmSiO}_{2}$ (ec- $\mathrm{GmSiO}_{2}$ ) was virtually identical to the $\mathrm{GmSiO}_{2}$ starting material, apart from the absence of the epoxide $\mathrm{C}-\mathrm{O}$ peak at $920 \mathrm{~cm}^{-1}$ (Figure 4.7, Table 4.3). This was contrary to 
expectations, since modification with TMCS should yield bands in the IR spectrum at 2977 and 2912 ( $\mathrm{CH}_{3}$ stretching), 1415 and 1260 ( $\mathrm{CH}_{3}$ deformation), 857 and 767 ( $\mathrm{CH}_{3}$ rocking), and 700 and 640 ( $\mathrm{Si}-\mathrm{C}$ stretching) $\mathrm{cm}^{-1}{ }^{263}$ None of these bands were observed in the IR spectrum of ec- $\mathrm{GmSiO}_{2}$, and, thus, it was concluded that the reaction did not take place. In fact, the decrease in mass and disappearance of the epoxy ring peak in the IR spectrum were indicative of GLYMO hydrolysis.

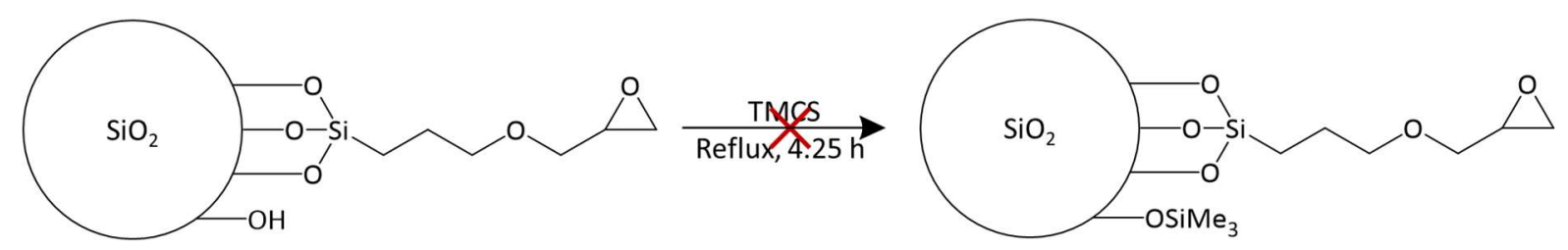

Scheme 4.4. Attempted end-capping of $\mathrm{GmSiO}_{2}$.

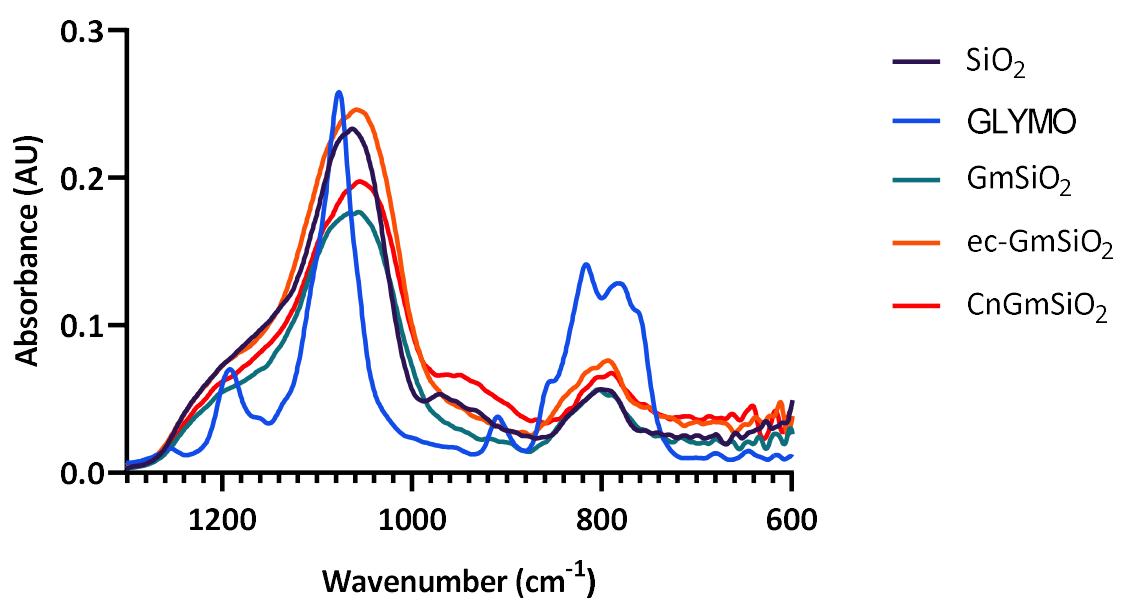

Figure 4.7. IR spectra of $\mathrm{GmSiO}_{2}$ (Procedure 4), ec- $\mathrm{GmSiO}_{2}$, and $\mathrm{CnGmSiO}_{2}$.

Table 4.3. Data and peak assignments from the IR spectra of $\mathrm{GmSiO}_{2}$ (Procedure 4), ec- $-\mathrm{GSiO}_{2}$, and $\mathrm{CnGmSiO}_{2}$.

\begin{tabular}{|c|c|c|c|c|}
\hline $\begin{array}{c}\text { Silica } \\
\text { Frequency }\left(\mathrm{cm}^{-1}\right)\end{array}$ & $\begin{array}{c}\text { Procedure } 4 \\
\mathrm{GmSSiO}_{2} \\
\text { Frequency }\left(\mathrm{cm}^{-1}\right)\end{array}$ & $\begin{array}{c}\text { Procedure } 4 \\
\text { ec-GmSiO } 2 \\
\text { Frequency }\left(\mathrm{cm}^{-1}\right)\end{array}$ & $\begin{array}{c}\text { Procedure } 4 \\
\mathrm{CnGmSiO}_{2} \\
\text { Frequency }\left(\mathrm{cm}^{-1}\right)\end{array}$ & Assignment \\
\hline $3351(w, b r)$ & & & & $\mathrm{O}-\mathrm{H}$ stretching \\
\hline $1063(\mathrm{~s}, \mathrm{br})$ & $1066(s, b r)$ & $1059(\mathrm{~s}, \mathrm{br})$ & $1055(\mathrm{~s}, \mathrm{br})$ & $\mathrm{Si}-\mathrm{O}-\mathrm{Si}$ asymmetric stretching \\
\hline \multirow[t]{2}{*}{$964(m, b r)$} & & & $950(m, b r)$ & $\mathrm{Si}-\mathrm{OH}$ stretching \\
\hline & $920(\mathrm{vw}, \mathrm{br})$ & & & $\mathrm{C}-\mathrm{O}$ stretching in epoxy ring \\
\hline $794(m, b r)$ & $801(m, b r)$ & 793 (m, br) & 789 (m, br) & Si-O-Si symmetric stretching \\
\hline
\end{tabular}


The synthesis of $\mathrm{CnGmSiO}_{2}$ (Scheme 4.5) proceeded by heating ec- $\mathrm{GmSiO}_{2}$ in an aqueous cyclen solution and then washing the product with dilute hydrochloric acid. Post-reaction, the product mass increased only $0.17 \%$, and, if this mass increase was due to the addition of cyclen to $\mathrm{GmSiO}_{2}$, then the reaction efficiency was only $1 \%$. Like ec- $\mathrm{GmSiO}_{2}$, the epoxy ring peak was absent in the IR spectrum of purported $\mathrm{CnGmSiO}_{2}$ (Figure 4.7, Table 4.3). Also, a peak at $950 \mathrm{~cm}^{-1}$, attributed to $\mathrm{Si}-\mathrm{OH}$ stretching, appeared. The $\mathrm{N}-\mathrm{H}$ stretching band at $\sim 3500 \mathrm{~cm}^{-1}$ from the addition of cyclen was absent. ${ }^{264}$ So, there was no definitive evidence of the formation of $\mathrm{CnGmSiO}_{2}$, and the practically negligible mass increase of the product suggested that the reaction occurred to a very small extent, if at all.

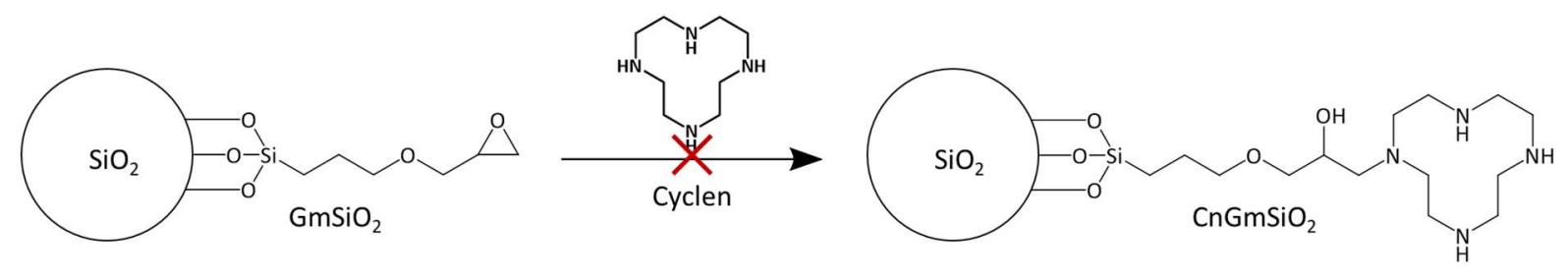

Scheme 4.5. Attempted synthesis of $\mathrm{CnGmSiO}_{2}$.

\subsubsection{Conclusions}

The functionalisation of silica with $\mathrm{CnGm}$ via Method 1 proceeded, first, with the synthesis of $\mathrm{GmSiO}_{2}$ by Procedure 4, which was selected as the best protocol out of Procedures 1-4. The end-capping of Procedure $4 \mathrm{GmSiO}_{2}$ and subsequent synthesis of $\mathrm{CnGmSiO}_{2}$ were both unsuccessful, as determined by IR spectroscopy. There are several possible explanations for the differences between the IR spectra of the supposed ec- $\mathrm{GmSiO}_{2}$ and $\mathrm{CnGmSiO}_{2}$ products and the $\mathrm{GmSiO}_{2}$ starting material. For instance, the disappearance of the epoxy ring peak in the IR spectra of ec- $\mathrm{GmSiO}_{2}$ and $\mathrm{CnGmSiO}_{2}$ could be due to hydrolysis of some of the GLYMO from the silica. After all, a decrease in product mass was observed in the end-capping reaction, and the $\mathrm{Si}-\mathrm{OH}$ stretching peak reappeared for $\mathrm{CnGmSiO}_{2}$. Yet, it is likely that the epoxy ring and $\mathrm{Si}-\mathrm{OH}$ stretching peaks are both too weak and too broad to be diagnostic, and additional characterisation techniques, such as CHN elemental analysis, are necessary. The characterisation challenges in Method 1 led to its abandonment, in favour of the synthetic route in Method 2. 


\subsubsection{Method 2}

\subsubsection{Synthesis of $\mathrm{Cn}(\mathrm{Gm})_{n}$}

Following the first step of Method 2, six protocols were trialled to synthesise $\mathrm{Cn}(\mathrm{Gm})_{\mathrm{n}}$ (Scheme 4.6). It was acknowledged that $\mathrm{Cn}(\mathrm{Gm})_{n}$ could be mono- $(\mathrm{CnGm})$, di- $\left(\mathrm{Cn}(\mathrm{Gm})_{2}\right)$, tri- $\left(\mathrm{Cn}(\mathrm{Gm})_{3}\right)$, or tetrasubstituted $\left(\mathrm{Cn}(\mathrm{Gm})_{4}\right)$. Under basic conditions, the ring-opening reaction of an epoxide proceeds by the $\mathrm{S}_{\mathrm{N}} 2$ mechanism, and, in the case of an asymmetric epoxide, the favoured site of nucleophilic attack is the less substituted carbon, since such reactions are governed by steric considerations. ${ }^{265}$ Regarding the synthesis of $\mathrm{Cn}(\mathrm{Gm})_{n}$, cyclen has basic nitrogen atoms that serve as nucleophiles to attack GLYMO at $\mathbf{C 1}$, the less substituted carbon. Purification of the product was expected to be complicated by the tendency of such compounds to self-polymerise, which contraindicates heating and concentration under vacuum, and to functionalise silica, which contraindicates chromatographic purification with silica gel. It was preferable, therefore, to find a procedure in which the reaction went to completion, as evidenced by ${ }^{1} \mathrm{H}$ NMR spectroscopy, and in which any excess starting reactant could be removed by trituration or future washes. The reaction conditions, outcomes, and procedure references are summarised in Table 4.4.

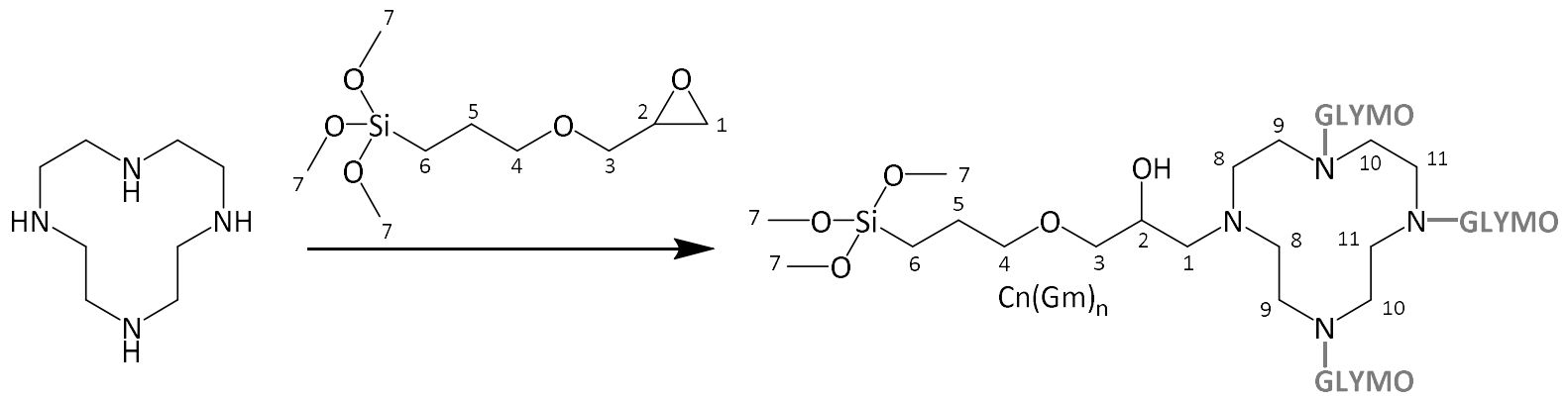

Scheme 4.6. Synthesis of $\mathrm{Cn}(\mathrm{Gm})_{n}$.

The protocol by Bereczki et al. ${ }^{258}$ was followed in Procedure 1 for $\mathrm{Cn}(\mathrm{Gm})_{4}$ synthesis. GLYMO (3.9 eq) was added to cyclen dissolved in 2:1 methanol:water, and the reaction was stirred for $5 \mathrm{~h}$ at room temperature. Concentration of the crude reaction mixture for NMR analysis yielded an intractable polymer. So, test reactions were set up in NMR tubes using deuterated solvents (2:1 methanol- $d_{4}$, $\mathrm{CD}_{3} \mathrm{OD}: \mathrm{D}_{2} \mathrm{O}$ ) to monitor the reaction. ${ }^{1} \mathrm{H}$ NMR spectra of the test reactions revealed that, after $24 \mathrm{~h}$, the majority of GLYMO (62\%) remained unreacted. Thus, an alternative method was sought. 
Table 4.4. Reaction conditions and outcomes in Procedures 1-6 for $\mathrm{Cn}(\mathrm{Gm})_{n}$ synthesis.

\begin{tabular}{|c|c|c|c|c|c|c|}
\hline Conditions & $\begin{array}{c}\text { Procedure } \\
1 \\
\end{array}$ & $\begin{array}{c}\text { Procedure } \\
2 \\
\end{array}$ & $\begin{array}{c}\text { Procedure } \\
3 \\
\end{array}$ & $\begin{array}{c}\text { Procedure } \\
4\end{array}$ & $\begin{array}{c}\text { Procedure } \\
5\end{array}$ & $\begin{array}{c}\text { Procedure } \\
6 \\
\end{array}$ \\
\hline GLYMO (mmol) & 4.4 & 1.1 & 2.3 & 1.1 & 1.1 & 8.9 \\
\hline Cyclen (mmol, eq) & $\begin{array}{l}1.1 \\
0.26\end{array}$ & $\begin{array}{l}4.8, \\
4.3\end{array}$ & $\begin{array}{l}0.56 \\
0.25\end{array}$ & $\begin{array}{l}1.2, \\
1.1\end{array}$ & $\begin{array}{l}1.2 \\
1.1\end{array}$ & $\begin{array}{l}9.7 \\
1.1\end{array}$ \\
\hline $\mathrm{Al}(\mathrm{OTf})_{3}(\mathrm{mmol})$ & - & - & - & 0.1 & 0.1 & - \\
\hline Solvent & $\begin{array}{c}2: 1 \\
\mathrm{MeOH}: \mathrm{H}_{2} \mathrm{O}\end{array}$ & toluene & toluene & $\mathrm{CHCl}_{3}$ & $\mathrm{CDCl}_{3}$ & $\mathrm{CHCl}_{3}$ \\
\hline Volume solvent (mL) & 23.8 & 7.5 & 10 & 2 & 2 & 16 \\
\hline Temperature $\left({ }^{\circ} \mathrm{C}\right)$ & 20 (RT) & $\begin{array}{c}110.6 \\
\text { (reflux) }\end{array}$ & $\begin{array}{c}110.6 \\
\text { (reflux) }\end{array}$ & $20(\mathrm{RT})$ & 20 (RT) & 20 (RT) \\
\hline Time & $5 \mathrm{~h}$ & $24 \mathrm{~h}$ & $97.5 \mathrm{~h}$ & $90 \mathrm{~h}$ & $69 \mathrm{~h}$ & $8-10$ days \\
\hline Outcomes & $\begin{array}{c}62 \% \\
\text { unreacted } \\
\text { GLYMO }\end{array}$ & inconclusive & $\begin{array}{c}\text { intractable } \\
\text { solid }\end{array}$ & $\begin{array}{c}\text { intractable } \\
\text { solid }\end{array}$ & $\begin{array}{c}6 \% \\
\text { unreacted } \\
\text { GLYMO }\end{array}$ & $\begin{array}{c}\text { no } \\
\text { unreacted } \\
\text { GLYMO }\end{array}$ \\
\hline References & $\begin{array}{c}\text { Bereczki et } \\
\text { al. }^{258}\end{array}$ & $\begin{array}{c}\text { Barreto et } \\
\text { al. } .^{259}\end{array}$ & $\begin{array}{l}\text { Barreto et } \\
\text { al. }^{259}\end{array}$ & $\begin{array}{c}\text { Williams } \\
\text { and } \\
\text { Cullen }^{266}\end{array}$ & $\begin{array}{c}\text { Williams } \\
\text { and } \\
\text { Cullen }^{266}\end{array}$ & - \\
\hline
\end{tabular}

Procedures 2 and 3 followed the protocol by Barreto et al. ${ }^{259}$ In Procedure 2, GLYMO was added to an excess of cyclen ( $4.3 \mathrm{eq}$ ) in toluene, and the reaction was heated to reflux temperature for $24 \mathrm{~h}$. Cooling of the reaction mixture resulted in the precipitation of much of the excess cyclen, which was removed by filtration. To ensure the removal of any remaining, unreacted cyclen, the filtrate was evaporated to dryness, dissolved in chloroform, and washed with aqueous sodium hydroxide (0.1 M). Drying over magnesium sulfate, filtration, and concentration yielded the presumed $\mathrm{CnGm}$ product as a colourless oil. The ${ }^{1} \mathrm{H}$ NMR spectrum of cyclen in chloroform- $d$ showed a singlet at $\delta_{H} 2.67 \mathrm{ppm}$ $\left(-\mathrm{CH}_{2}-\right)$, since all of the methylene protons in the molecule are chemically equivalent. Consequently, the appearance of multiple signals from $\delta_{H} 2.90-2.40 \mathrm{ppm}$ in the ${ }^{1} \mathrm{H}$ NMR spectrum $\left(\mathrm{CDCl}_{3}\right)$ of the product suggested coupling of the macrocycle to GLYMO (Experimental, Section 6.4.1.2.1). However, neither $\mathrm{Cn}(\mathrm{Gm})_{\mathrm{n}}$ nor its possible derivations from the loss of methyl groups were observed in the product's mass spectrum. Rather, there was evidence of GLYMO self-condensation. For example, the peak at $m / z 603.3$ could be due to the condensation of three GLYMO molecules, protonation, and the loss of a methyl group, as shown in Figure 4.8. The conflicting ${ }^{1} \mathrm{H}$ NMR and MS data regarding the formation of $\mathrm{Cn}(\mathrm{Gm})_{\mathrm{n}}$ led to another attempt based on the same procedure but, this time, with a small excess of GLYMO.

In Procedure 3, GLYMO (4.0 eq) was added to cyclen in toluene, and the reaction was heated to reflux temperature for 4 days. Concentration of the product under vacuum yielded an intractable polymer, attributed to GLYMO self-polymerisation. Therefore, it was decided that the procedure should always 
use an excess of cyclen, instead of GLYMO, and that the reaction mixture should not be heated, since heating drives the condensation reaction. An excess of cyclen was also preferable because it is more easily removed than GLYMO by trituration or washes of the final $\mathrm{CnGmSiO}_{2}$ material. Furthermore, it was better for avoiding the synthesis of $\mathrm{GmSiO}_{2}$, rather than desired product $\mathrm{CnGmSiO}_{2}$, in the following step.

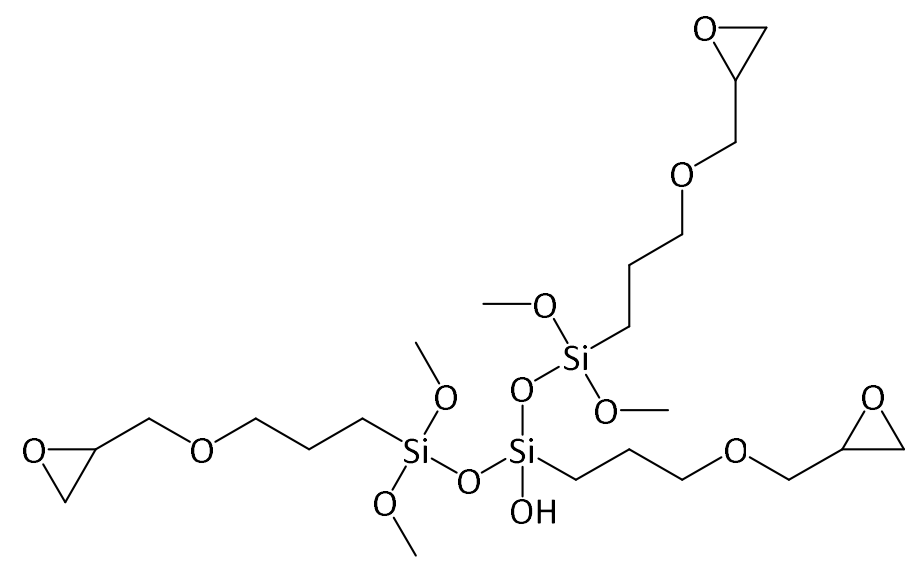

Figure 4.8. Potential structure of self-polymerised GLYMO $\left(\mathrm{m} / \mathrm{z} 603.2[\mathrm{M}+\mathrm{H}]^{+}\right)$.

In the study by Williams and Cullen ${ }^{266}$ that was referred to for Procedures 4 and 5 , aluminium triflate $\left(\mathrm{Al}(\mathrm{OTf})_{3}\right)$ served as an effective Lewis acid catalyst for the ring-opening reaction of an epoxide with an aliphatic amine, and the reaction was regioselective for nucleophilic attack by the amine at the less hindered carbon of the epoxide, in accordance with the $\mathrm{S}_{\mathrm{N}} 2$ reaction mechanism. This mechanism was thought to be favoured due to chelation of the metal by the two oxygen atoms of the glycidyl ether (Figure 4.9); chelation diminishes the partial positive charge at the epoxide's more highly substituted carbon and, consequently, decreases reactivity, allowing steric hindrance to play a greater, discriminant role. ${ }^{266,267}$<smiles></smiles>

Figure 4.9. Proposed mechanism of nucleophilic attack on the less hindered carbon of the metalchelating (Al(OTf) $)_{3}$ glycidyl ether. ${ }^{267}$ 
In Procedure 4, GLYMO was added to cyclen (1.1 eq) and AI(OTf) 3 (10 mol\%) in anhydrous chloroform under dry conditions and an argon atmosphere. The reaction mixture was stirred at room temperature for 4 days and then was quenched by the addition of aqueous sodium bicarbonate. Extraction with dichloromethane, washing with water, and concentration of the organic layers produced an intractable polymer. As a result, it was decided that concentration of the reaction mixture under vacuum while heating should be avoided, and the protocol was repeated with this modification in Procedure 5.

The reaction in Procedure 5 was monitored via ${ }^{1} \mathrm{H}$ NMR spectroscopy by diluting an aliquot of the crude reaction mixture in chloroform- $d$ (Figure 4.10). As shown in Figure 4.11, progress of the ringopening reaction was determined by observing the disappearance of the peak at $\delta_{H} 3.14 \mathrm{ppm}$, attributed to the proton attached to $\mathbf{C 2}$ in the ring ( $\mathrm{H}$ in the figure; see Scheme $\mathbf{4 . 6}$ for numbering), and the appearance of the peak at $\delta_{H} 3.89 \mathrm{ppm}$, attributed to the downfield shift of that same proton once the epoxide opened. After approximately 3 days, integration of these two peaks revealed that only $6 \%$ of GLYMO remained unreacted, and the mass spectrum contained peaks for the tri$\left.\left(\mathrm{m} / \mathrm{z} 441.4\left[\mathrm{Cn}(\mathrm{Gm})_{3}+2 \mathrm{H}\right]^{+2}\right]\right)$, di- $\left(\mathrm{m} / \mathrm{z} 645.4\left[\mathrm{Cn}(\mathrm{Gm})_{2}+\mathrm{H}\right]^{+}\right)$, and mono-functionalised $(\mathrm{m} / z 409.3$ $\left[\mathrm{CnGm}+\mathrm{H}^{+}\right)$product, as well as for excess cyclen $\left(\mathrm{m} / \mathrm{z} 173.2[\text { cyclen }+\mathrm{H}]^{+}\right)$. This evidence, therefore, was initially indicative of the formation of $\mathrm{Cn}(\mathrm{Gm})_{n}$. Yet, given that cyclen is capable of metal-chelation, there were concerns about using a Lewis acid catalyst, since the free ligand was desirable. So, the same reaction was performed without $\mathrm{Al}(\mathrm{OTf})_{3}$ in Procedure 6.

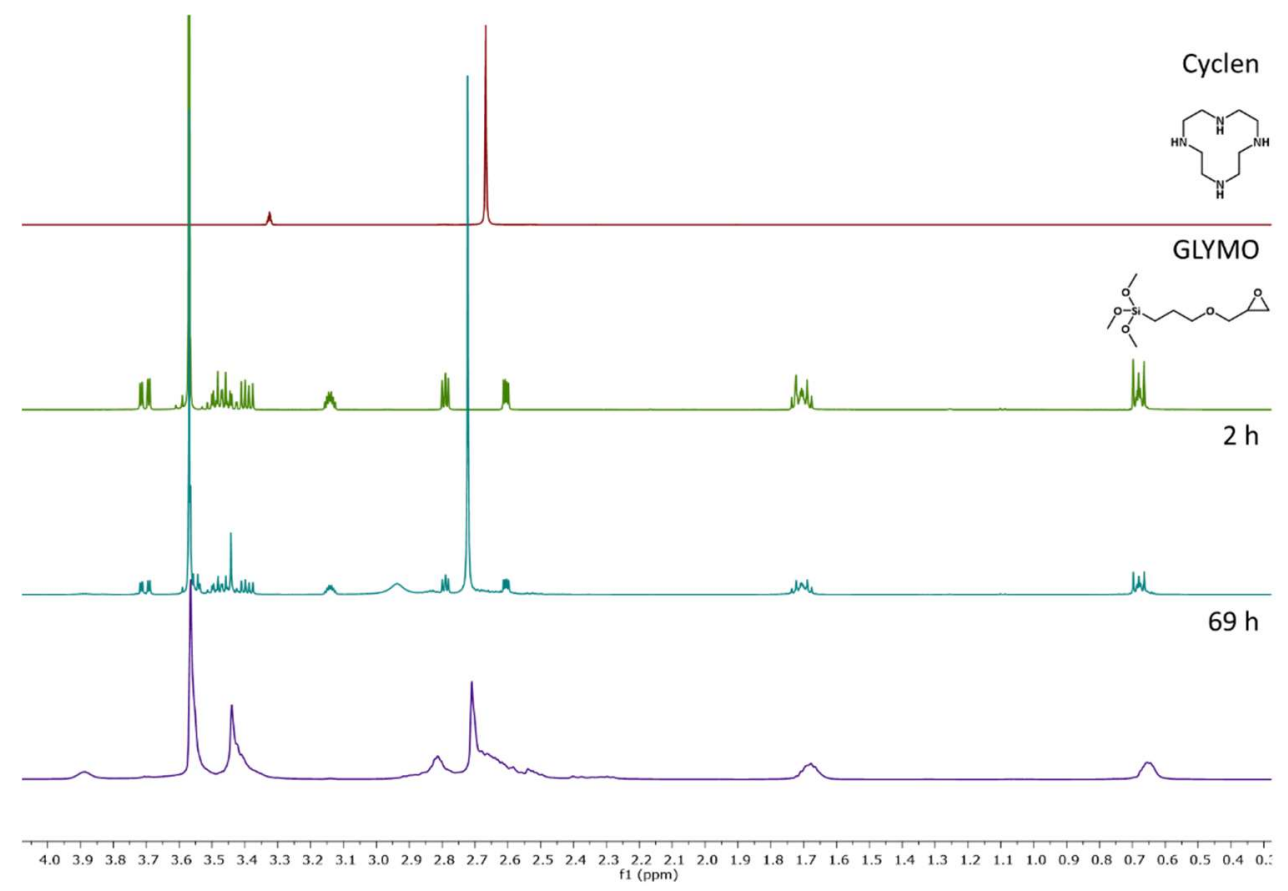

Figure 4.10. ${ }^{1} \mathrm{H}$ NMR spectra $\left(\mathrm{CDCl}_{3}\right)$ of cyclen, GLYMO, and samples at 2 and $69 \mathrm{~h}$ from the $\mathrm{Cn}(\mathrm{Gm})_{\mathrm{n}}$ Procedure 5 reaction mixture. 


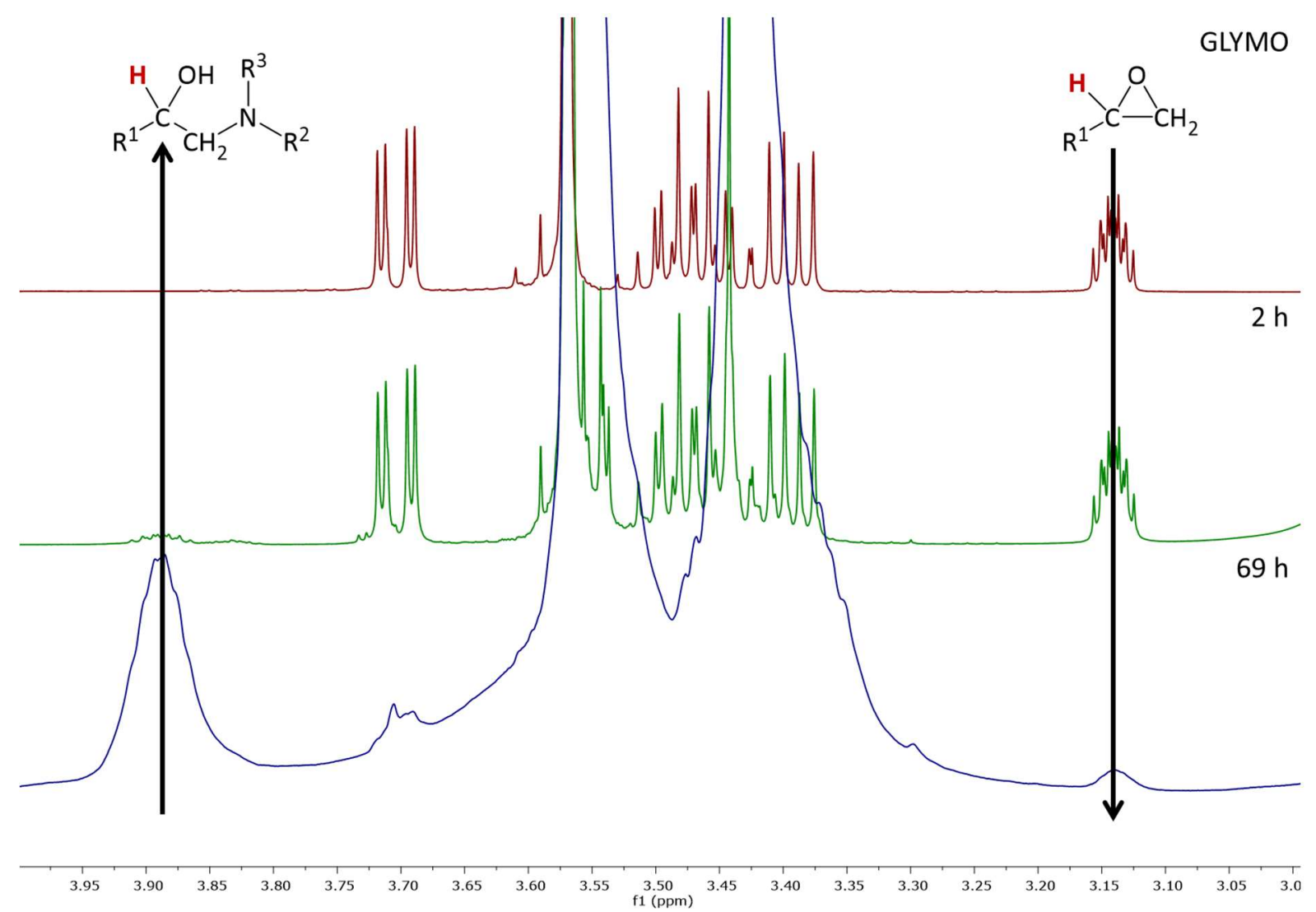

Figure 4.11. ${ }^{1} \mathrm{H}$ NMR spectra $\left(\mathrm{CDCl}_{3}, \delta_{\mathrm{H}} 4.00-3.00 \mathrm{ppm}\right)$ of $\mathrm{GLYMO}$ and the samples at 2 and $69 \mathrm{~h}$ from the $\mathrm{Cn}(\mathrm{Gm})_{n}$ Procedure 5 reaction mixture, showing the change in the spectrum with the progression of the ring-opening reaction. $\mathrm{H}$ is the proton responsible for the indicated peak. 
The reaction in Procedure 6 commenced with the addition of GLYMO to cyclen (1.1 eq) in anhydrous chloroform at room temperature under dry conditions and an argon atmosphere and was monitored by ${ }^{1} \mathrm{H}$ NMR spectroscopy, as in Procedure 5, for 10 days. As shown in Figure 4.12, the peak at $\delta_{\mathrm{H}} 3.14 \mathrm{ppm}$ disappeared in 5-6 days, indicating that the ring-opening reaction of GLYMO was complete. The high-resolution mass spectrometry (HRMS) data confirmed the presence of the $\mathrm{CnGm}$ product (calcd for $\mathrm{C}_{17} \mathrm{H}_{41} \mathrm{~N}_{4} \mathrm{O}_{5} \mathrm{Si}[\mathrm{CnGm}+\mathrm{H}]^{+}$409.2846; found 409.2843). Although the ring-opening reaction was monitored by observing the downfield shift of the $\mathbf{H} \mathbf{2}$ peak from $\delta_{\mathrm{H}} 3.14 \mathrm{ppm}$ to $\delta_{H} 3.89 \mathrm{ppm}$, this did not prove that a bond had formed between a secondary amine of cyclen and the less hindered carbon (C1) of the GLYMO epoxy group. Therefore, in order to confirm the identity of the product with complete certainty, full characterisation by NMR spectroscopy was conducted.

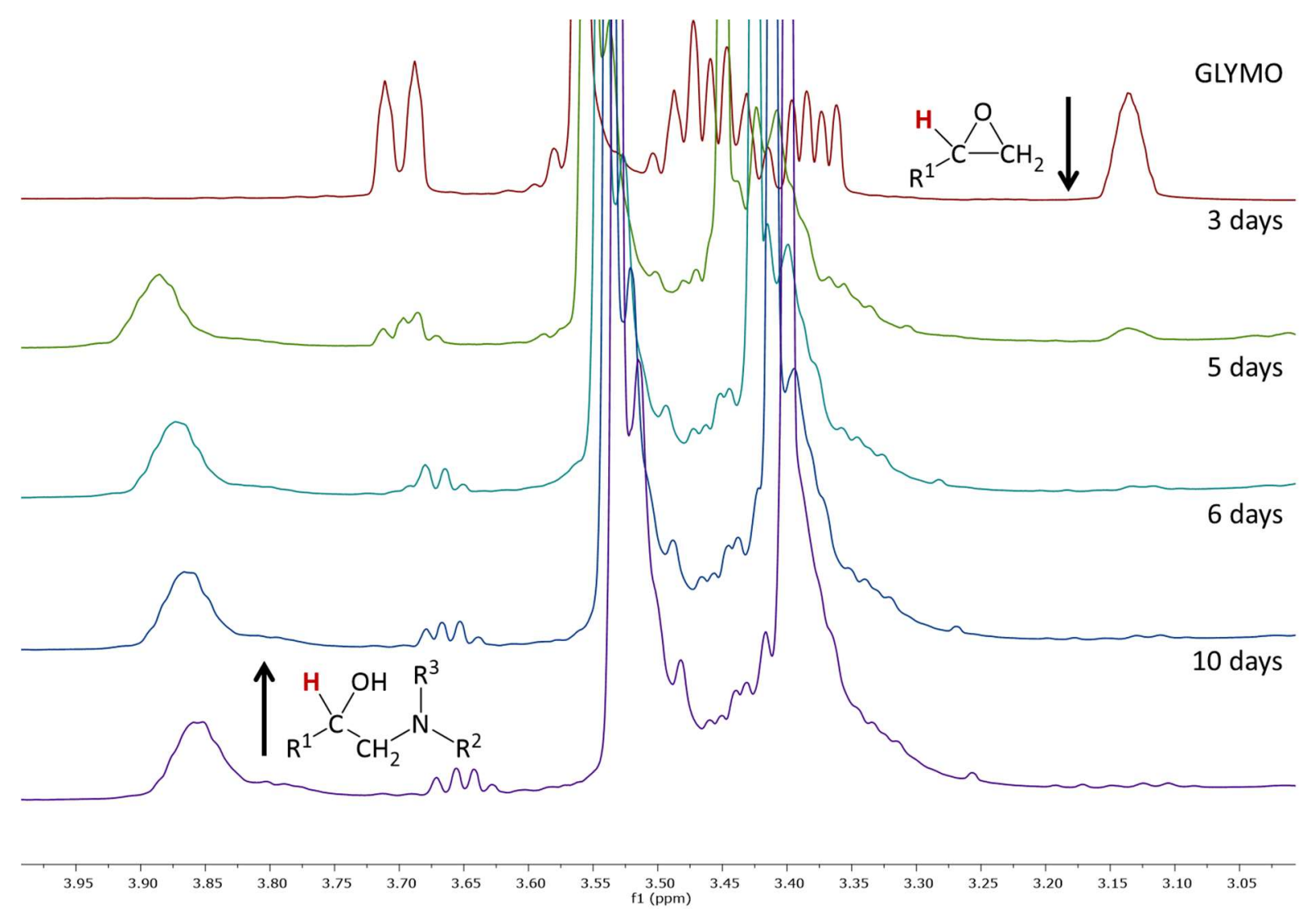

Figure 4.12. ${ }^{1} \mathrm{H}$ NMR spectra $\left(\mathrm{CDCl}_{3}, \delta_{\mathrm{H}} 4.00-3.00 \mathrm{ppm}\right)$ of GLYMO and the samples at 3, 5, 6, and 10 days from the $\mathrm{Cn}(\mathrm{Gm})_{n}$ Procedure 6 reaction mixture, showing the change in the spectrum with the progression of the ring-opening reaction. $\mathbf{H}$ is the proton responsible for the indicated peak. 
NMR analyses (HSQC; $\left({ }^{1} \mathrm{H},{ }^{13} \mathrm{C}\right)$ - and $\left.\left({ }^{1} \mathrm{H},{ }^{15} \mathrm{~N}\right)-\mathrm{HMBC} ; \mathrm{COSY}\right)$ of the product from Procedure 6 were completed to confirm the linkage of cyclen and GLYMO. An aliquot from the crude reaction mixture was used for the characterisation, since it had been decided that concentration and purification of $\mathrm{Cn}(\mathrm{Gm})_{\mathrm{n}}$ were contraindicated and that the reaction mixture, as is, should be used for subsequent steps to avoid self-polymerisation. The peaks in the ${ }^{1} \mathrm{H}$ and ${ }^{13} \mathrm{C}$ NMR spectra (Appendix, Figures 8.39-41, Table 8.20) were assigned by approximations from the HSQC correlations in Figure 4.13. The $\left({ }^{1} \mathrm{H},{ }^{13} \mathrm{C}\right)-\mathrm{HMBC}$ spectrum (Figure 4.14) revealed that $\mathbf{H} \mathbf{1}$ of $\mathrm{Cn}(\mathrm{Gm})_{n}$ correlated to $\mathbf{C 2}$ and C3 of the GLYMO component of the molecule, as well as to the methylene carbons of the macrocycle ( $\mathbf{C 8}$ and $\mathbf{C 9}, \mathbf{C 1 0}$, or $\mathbf{C 1 1}$ ). This was conclusive evidence for the formation of desired product $\mathrm{Cn}(\mathrm{Gm})_{\mathrm{n}}$. Additionally, the $\left({ }^{1} \mathrm{H},{ }^{15} \mathrm{~N}\right)-\mathrm{HMBC}$ spectrum (Appendix, Figure 8.42) contained correlations between the amines and $\mathbf{H 1}$ and $\mathbf{H 8 - 1 1}$, which was further evidence of the coupling of cyclen to GLYMO. The spin-coupling in the $\left({ }^{1} \mathrm{H},{ }^{1} \mathrm{H}\right)$-COSY spectrum (Appendix, Figure 8.43) was consistent with the assignment.

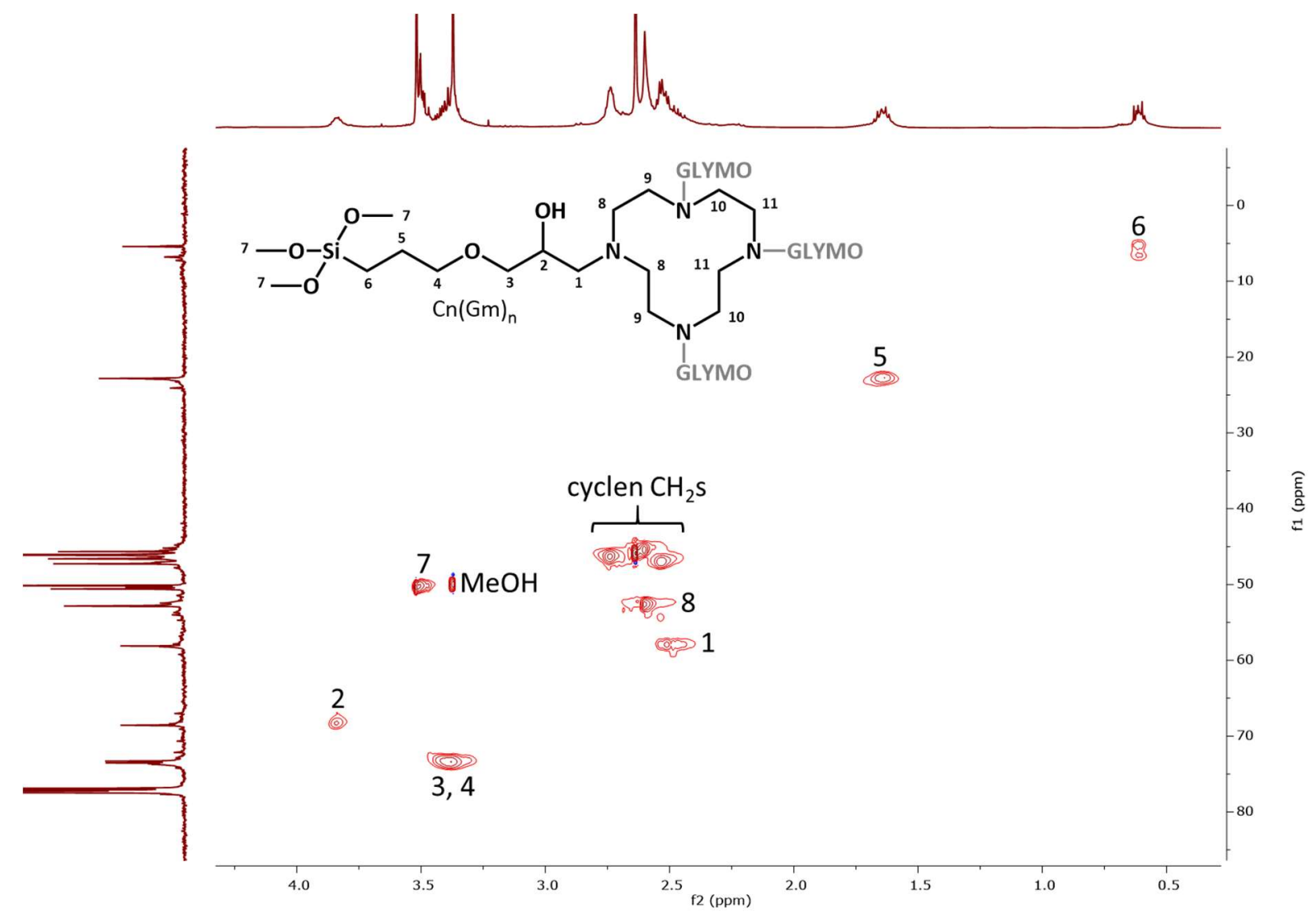

Figure 4.13. 2D HSQC NMR spectrum $\left(\mathrm{CDCl}_{3}\right)$ of $\mathrm{Cn}(\mathrm{Gm})_{n}$ from Procedure 6 with peak assignments. 


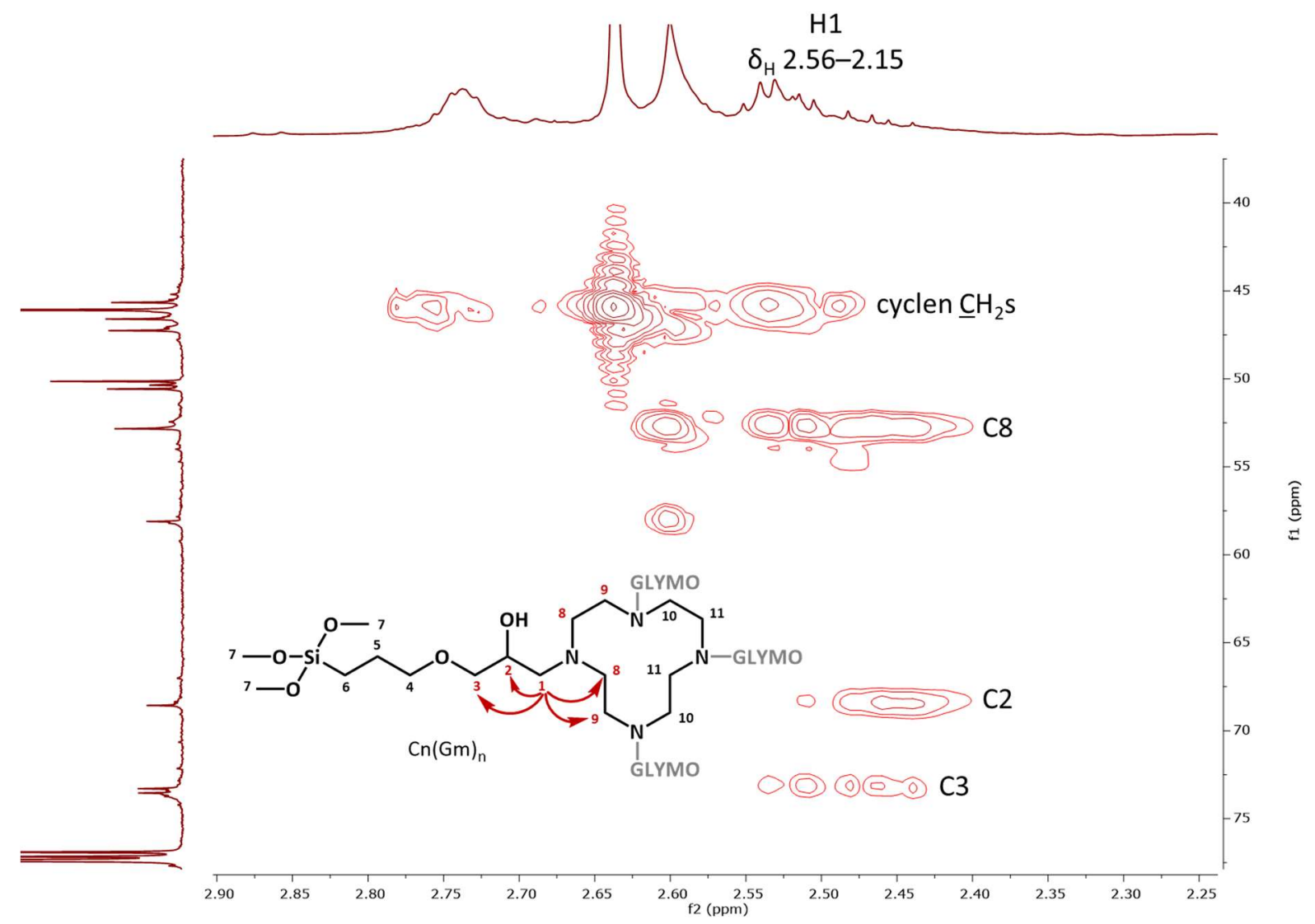

Figure 4.14. $2 \mathrm{D}\left({ }^{1} \mathrm{H},{ }^{13} \mathrm{C}\right)-\mathrm{HMBC} N M R$ spectrum $\left(\mathrm{CDCl}_{3}\right)$ of $\mathrm{Cn}(\mathrm{Gm})_{\mathrm{n}}$ from Procedure 6 with peak assignments.

The ${ }^{29} \mathrm{Si}$ NMR spectrum (Appendix, Figure 8.44) of the product showed two peaks $\left(\delta_{\mathrm{si}}-41.8 \mathrm{ppm},-50.2 \mathrm{ppm}\right)$. This indicated that two products had formed: $\mathrm{CnGm}$ and either $\mathrm{CnGm}$ with hydrolysed methoxy groups or $\mathrm{Cn}(\mathrm{Gm})_{2}$. The signal at $\delta_{\mathrm{si}}-41.8 \mathrm{ppm}$ was probably caused by $\mathrm{CnGm}$, since the chemical shift was consistent with previously reported data for GLYMO $\left(\delta_{\mathrm{Si}}-42.1 \mathrm{ppm}\right) .{ }^{268}$ The observation of methanol (Figure 4.13) and the reduction in size of the peak at $\delta_{H} 3.52 \mathrm{ppm}$ in the ${ }^{1} \mathrm{H}$ NMR spectrum revealed that methoxy group hydrolysis had occurred, to an extent, suggesting that water was still present under the "dry" conditions of the experiment. However, the mass spectrum also revealed the presence of $\mathrm{Cn}(\mathrm{Gm})_{2}\left(\mathrm{~m} / \mathrm{z} 645.4\left[\mathrm{Cn}(\mathrm{Gm})_{2}+\mathrm{H}\right]^{+}\right)$. The presence of these multiple species (i.e. $\mathrm{CnGm}, \mathrm{CnGm}$ with hydrolysed methoxy groups, $\left.\mathrm{Cn}(\mathrm{Gm})_{2}\right)$ in the ${ }^{1} \mathrm{H}$ NMR spectrum was the likely cause of the broad signals observed (Appendix, Figure 8.39).

Based on the refinements made to the six procedures for $\mathrm{Cn}(\mathrm{Gm})_{n}$ synthesis, multiple guidelines were established for an optimised protocol. First, to avoid the self-condensation reaction of GLYMO, heating and concentration under vacuum of the reaction mixture should be avoided. Second, an excess of 
cyclen, rather than GLYMO, is preferable due to easier removal by trituration or washing, in addition to self-polymerisation concerns. In Procedure 6, $\mathrm{CnGm}$ is the dominant product, in opposition to the multi-functionalised cyclen products $\left(\mathrm{Cn}(\mathrm{Gm})_{2}, \mathrm{Cn}(\mathrm{Gm})_{3}, \mathrm{Cn}(\mathrm{Gm})_{4}\right)$. This is desirable because monofunctionalised product maximises the amount of ligand attached to the silica; multi-functionalised cyclen would react with more silica silanol groups per ligand. It has been reported that increasing the number of arms attached to the macrocycle increases its chemical resistance to degradation, but this also has a dramatic, negative effect on its $\mathrm{Cu}(\mathrm{II})$-binding properties due to rigidity constraints. ${ }^{252}$ Third, the ring-opening reaction using a Lewis acid catalyst goes to completion faster than the same reaction without the catalyst: $\sim 3$ days vs 5-6 days. However, utilisation of a metal catalyst may be inadvisable because of the metal-chelating ability of cyclen. Fourth, the epoxide opening may be monitored by ${ }^{1} \mathrm{H}$ NMR spectroscopy by observing the downfield shift from $\delta_{H} 3.14 \mathrm{ppm}$ to $\delta_{H} 3.89 \mathrm{ppm}$ of the peak attributed to H2 (Scheme 4.6). Lastly, Procedure 6 should be followed to synthesise $\mathrm{CnGm}$, and the crude reaction mixture should be progressed directly to the next step.

\subsubsection{Synthesis of $\pm \mathrm{Cu}(\mathrm{II})-\mathrm{CnGmSiO}$}

As shown in Scheme 4.7, silica modification with Procedure $6 \mathrm{CnGm}$ is a multi-step process. First, $\mathrm{CnGm}$ may be chelated to $\mathrm{Cu}(\mathrm{II})$ to produce, after hydrolysis of the methoxy groups and silica functionalisation, "pre-loaded" $\mathrm{Cu}(\mathrm{II})-\mathrm{CnGmSiO}_{2}$. This is useful for comparing the copper content of pre-loaded $\mathrm{Cu}(\mathrm{II})-\mathrm{CnGmSiO}{ }_{2}$ to $\mathrm{CnGmSiO}_{2}$ exposed to a $\mathrm{Cu}$ (II) solution (i.e. "post-loaded", "CnGmSiO $2+\mathrm{Cu}(\mathrm{II})$ "). The methoxy groups of $\pm \mathrm{Cu}(\mathrm{II})-\mathrm{CnGm}$ can be hydrolysed under acidic or basic conditions. However, acidic conditions are less desirable due to concerns about the decomplexation of $\mathrm{Cu}(\mathrm{II})$ from the cyclen ligand upon protonation of the amines. ${ }^{241}$ The addition of silica to $\pm \mathrm{Cu}$ (II)-CnGm and heating drives the hydrolytic reaction to completion, and heating and concentration under vacuum promotes the dehydration/condensation reaction between $\pm \mathrm{Cu}(\mathrm{II})-\mathrm{CnGm}$ and the silica silanol groups by removing water. Finally, the $\pm \mathrm{Cu}(\mathrm{II})-\mathrm{CnGmSiO}$, product is washed by Soxhlet extraction with water for $24 \mathrm{~h}$ to remove unbound impurities, such as excess cyclen. It is generally assumed that anything remaining after this extraction is covalently bound, and hydrolysis of the siloxane linkages is said to be unlikely. ${ }^{207}$ 


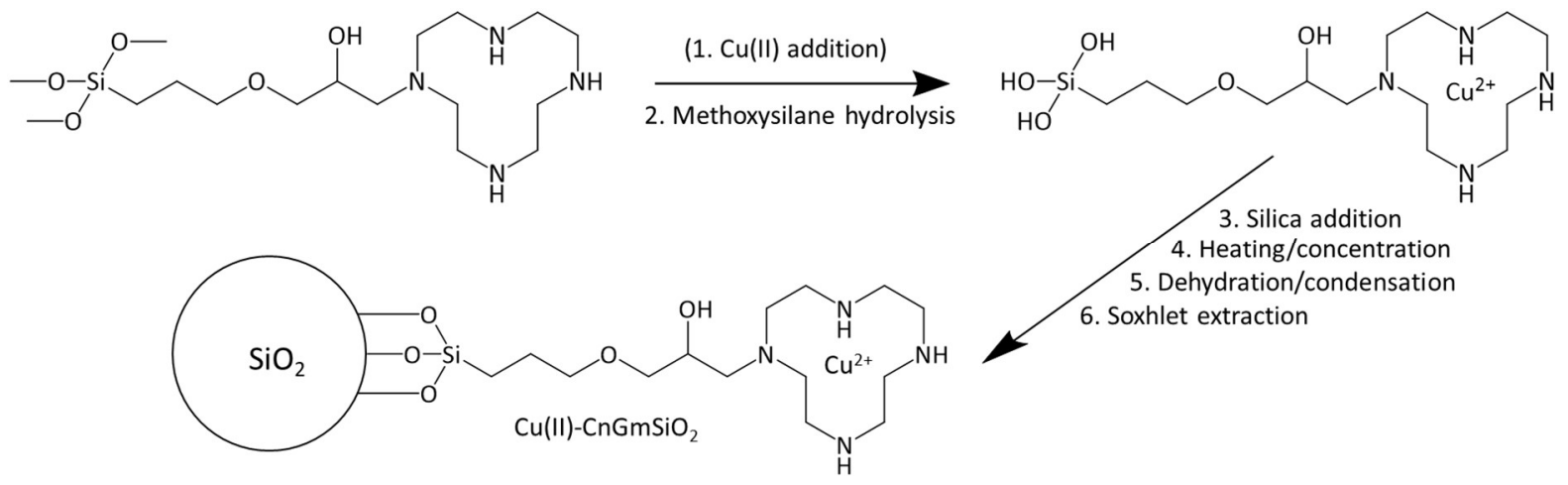

Scheme 4.7. Synthesis of $\pm \mathrm{Cu}(\mathrm{II})-\mathrm{CnGmSiO}_{2}$. In order to synthesise $\mathrm{Cu}(\mathrm{II})-\mathrm{CnGmSiO}{ }_{2}, \mathrm{Cu}(\mathrm{II})$ may be added first to produce the $\mathrm{Cu}(\mathrm{II})-\mathrm{CnGm}$ complex (1). Then, hydrolysis of the methoxysilane groups (2) is followed by the addition of silica (3) and heating and concentration (4) to drive the dehydration/condensation reaction (5). Finally, the $\pm \mathrm{Cu}(\mathrm{II})-\mathrm{CnGmSiO}{ }_{2}$ product is washed via Soxhlet extraction (6).

As listed in Table 4.5, four batches of $\pm \mathrm{Cu}(\mathrm{II})-\mathrm{CnGmSiO}_{2}$ were prepared: Batches 1 and 2 were preloaded with $\mathrm{Cu}(\mathrm{II})\left(\mathrm{Cu}(\mathrm{II})-\mathrm{CnGmSiO}_{2}\right)$, and Batches 3 and 4 were the free, uncoordinated ligand $\left(\mathrm{CnGmSiO}_{2}\right)$. Also, Batches 2-4 were synthesised following the same procedure. Batch 1 was synthesised following a different procedure (A vs B).

Table 4.5. Batches of $\pm \mathrm{Cu}(\mathrm{II})-\mathrm{CnGmSiO} 2$ prepared.

\begin{tabular}{lccc}
\hline Batch \# & $\pm \mathrm{Cu}(\mathrm{II})$ & Product & Procedure \\
\hline 1 & + & $\mathrm{Cu}(\mathrm{II})-\mathrm{CnGmSiO}_{2}$ & A \\
2 & + & $\mathrm{Cu}(\mathrm{II})-\mathrm{CnGmSiO}_{2}$ & B \\
3 & - & $\mathrm{CnGmSiO}$ & $\mathrm{B}$ \\
4 & - & $\mathrm{CnGmSiO}_{2}$ & B \\
\hline
\end{tabular}

For Batch 1, an excess of copper(II) nitrate (aq, light blue solution) was added to the $\mathrm{CnGm}$ reaction mixture in chloroform from Procedure 6, and complexation was evidenced by the colour change of the reaction mixture from colourless to dark blue. The $\mathrm{Cu}(\mathrm{II})-\mathrm{CnGm}$ complex was extracted into the aqueous layer by washing the organic layer with water until the chloroform was no longer blue, and analysis of the organic layer by ${ }^{1} \mathrm{H}$ NMR spectroscopy $\left(1: 1 \mathrm{v} / \mathrm{v} \mathrm{CDCl} 3: \mathrm{CD}_{3} \mathrm{OD}\right)$ confirmed that very little $\mathrm{CnGm}$ remained in the organic phase. To hydrolyse the $\mathrm{CnGm}$ methoxy groups under basic conditions, ammonium hydroxide was added to the isolated, dark blue aqueous layer to give a final concentration 
of $0.05 \% \mathrm{v} / \mathrm{v}$ ammonium hydroxide in water, and the mass spectrum of a sample of this solution revealed the presence of $\mathrm{Cu}(\mathrm{II})-\mathrm{CnGm}$, less the three methoxy groups $\left(\mathrm{m} / \mathrm{z} 428.1\left[\mathrm{Cu}(\mathrm{II})-\mathrm{CnGm}-3 \mathrm{CH}_{3}\right.\right.$ $\left.+2 \mathrm{H}]^{+}, 214.6\left[\mathrm{Cu}(\mathrm{II})-\mathrm{CnGm}-3 \mathrm{CH}_{3}+3 \mathrm{H}\right]^{2+}\right)$. The addition of silica, concentration of the reaction mixture under vacuum while heating, and Soxhlet extraction yielded the royal blue $\mathrm{Cu}(\mathrm{II})-\mathrm{CnGmSiO}{ }_{2}$ product (Figure 4.15). In contrast, the exposure of unmodified silica to an aqueous $\mathrm{Cu}(\mathrm{II})$ solution did not result in any colour change: the silica remained white.

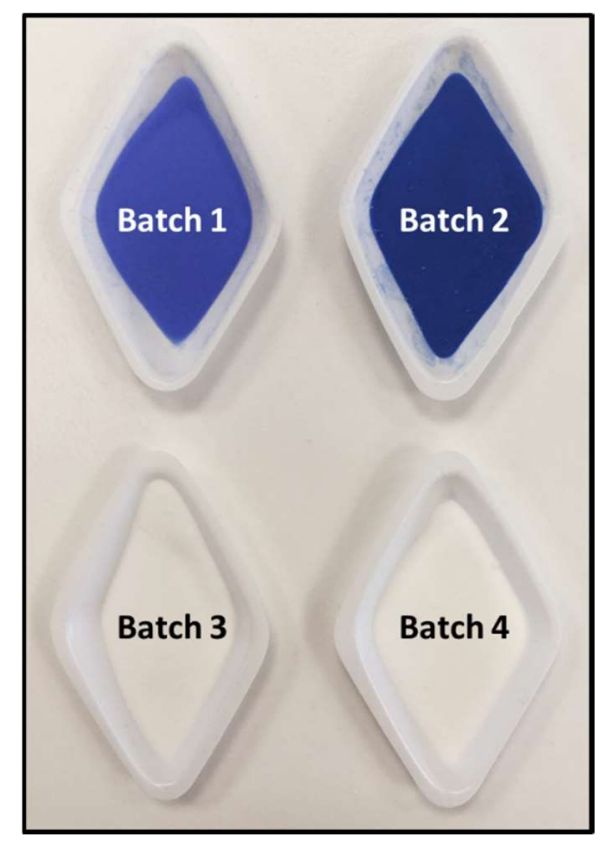

Figure 4.15. $\pm \mathrm{Cu}(\mathrm{II})-\mathrm{CnGmSiO} 2$ products from Batches $1-4$.

Batch $1 \mathrm{Cu}(\mathrm{II})-\mathrm{CnGmSiO}_{2}$ was submitted for $\mathrm{CHN}$ elemental analysis and copper quantification by ICP-MS. CHN analysis of the product revealed a percent functionalisation of 32\% (Table 4.6), which was calculated based on the measured $\% \mathrm{~N}$ value compared to the theoretical maximum (i.e. if all of the Procedure $6 \mathrm{CnGm}$ functionalised the silica). To determine if the ratio of $\mathrm{C}$ to $\mathrm{N}$ was as expected for $\mathrm{Cu}(\mathrm{II})-\mathrm{CnGmSiO}$, the expected $\% \mathrm{~N}(0.60 \%)$ was calculated from the measured $\% \mathrm{C}$ and compared to the measured $\% \mathrm{~N}(0.47 \%)$, yielding a $-22 \%$ error. The difference between the measured and theoretical \%N was unsurprising, given that some $\mathrm{Cn}(\mathrm{Gm})_{2}$ was also produced in the Procedure 6 reaction, as evidenced by MS data, and that calculations were based on the mono-functionalised product $(\mathrm{CnGm})$. The ICP-MS results (Table 4.7) revealed that the sample was $0.48 \% \mathrm{w} / \mathrm{w} \mathrm{Cu}$, which was $91 \%$ of the theoretical value $(0.53 \% \mathrm{w} / \mathrm{w} \mathrm{Cu})$. This theoretical value was calculated from the theoretical maximum adjusted for the percent functionalisation. Again, the theoretical maximum was based on the modification of silica with all of the $\mathrm{Cu}(\mathrm{II})-\mathrm{CnGm}$. 
Table 4.6. $\mathrm{CHN}$ elemental analysis and percent functionalisation results for $\mathrm{Batch} 1 \mathrm{Cu}(\mathrm{II})-\mathrm{CnGmSiO}$, as well as the percent error between the expected and measured \%N.

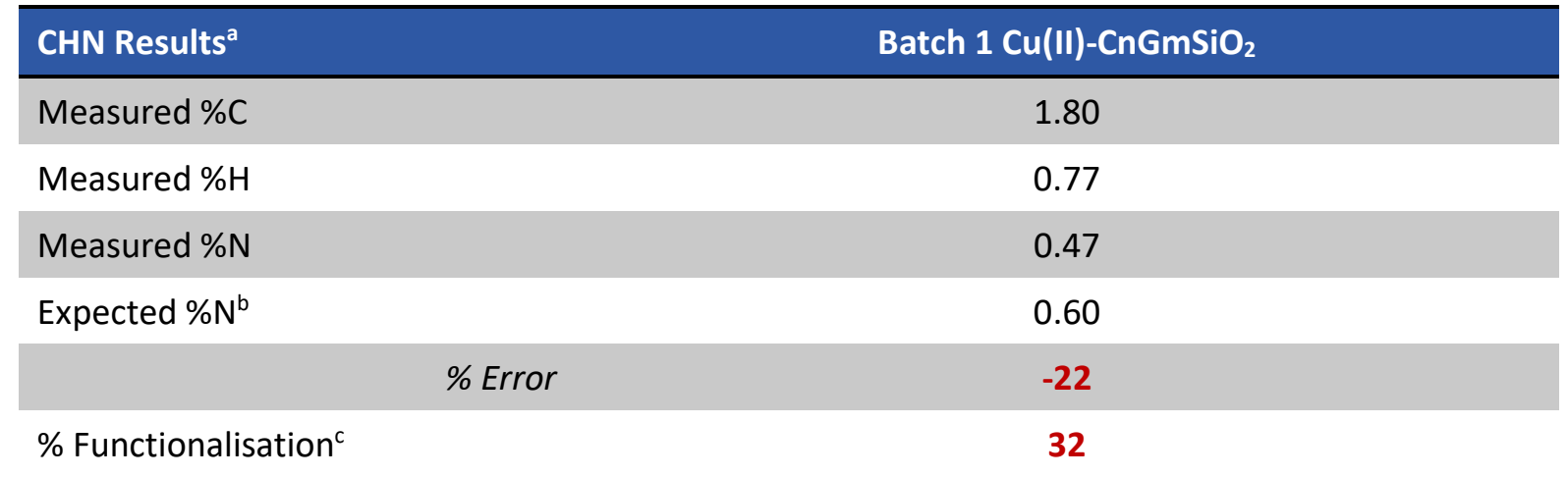

\footnotetext{
a Reported as the mean of duplicate measurements within $\leq 0.30 \%$ of each other.

${ }^{b}$ Calculated from the measured \%C data.

${ }^{c}$ Calculated based on the measured $\% \mathrm{~N}$ value compared to the theoretical maximum.
}

Table 4.7. ICP-MS (Cu) results for Batch $1 \mathrm{Cu}(\mathrm{II})-\mathrm{CnGmSiO}_{2}$ compared to the calculated theoretical $\% \mathrm{w} / \mathrm{wCu}$.

\section{ICP-MS Results}

Measured \% w/w $\mathrm{Cu}^{\mathrm{a}}$

Theoretical \% w/w $\mathrm{Cu}^{\mathrm{b}}$

\section{Batch $1 \mathrm{Cu}(\mathrm{II})-\mathrm{CnGmSiO}{ }_{2}$}

\subsection{8}

\subsection{3}

\footnotetext{
${ }^{a}$ Measured in triplicate. The percent error of the measurement is $\pm 5 \%$.

${ }^{\mathrm{b}}$ Calculated based on the percent functionalisation data (Table 4.6) and theoretical maximum.
}

Given the low percent functionalisation for Batch $1(32 \%)$, experiments were conducted to gauge the extent of hydrolysis of the GLYMO methoxy groups (Scheme 4.8) under the conditions in the procedure and to explore alternative conditions. Failure of the methoxy groups to hydrolyse would preclude subsequent silica modification. Thus, incomplete hydrolysis would result in a lower percent functionalisation, and it was hypothesised that the conditions employed might be insufficient for complete hydrolysis. As shown in Figure 4.16, hydrolysis was tracked via ${ }^{1} \mathrm{H}$ NMR spectroscopy by observing the decrease over time in the intensity of the peak attributed to the methoxy protons $\left(\delta_{\mathrm{H}} 3.57 \mathrm{ppm},-\mathrm{Si}\left(\mathrm{OC}_{3}\right)_{3}\right)$ and, inversely, by observing an increase in the methanol peak $\left(\delta_{\mathrm{H}} 3.47 \mathrm{ppm}\right.$ in $\left.\mathrm{CDCl}_{3}, \mathrm{CH}_{3} \mathrm{OH}\right) .{ }^{261}$ The hydrolytic stability of GLYMO was initially tested in chloroform at room temperature and in methanol at $60{ }^{\circ} \mathrm{C}$. GLYMO was stable in chloroform for the 6 days during which it was monitored, but the methoxy groups hydrolysed completely after heating in methanol for $40 \mathrm{~h}$. It was useful to know that the CnGm product would be stable in chloroform for, at least, 6 days. Then, the hydrolysis conditions (Table 4.8) used for Batch 1 (0.05\% v/v ammonium hydroxide, water, RT) 
were tested, and it was found that, under these conditions, $78 \%$ of the methoxy groups were hydrolysed within $0.2 \mathrm{~h}$ (Figure 4.17). However, hydrolysis was still incomplete after $4.7 \mathrm{~h}$, which may account, in part, for the low percent functionalisation. Therefore, it was decided that other hydrolysis conditions should be tested to optimise the method.

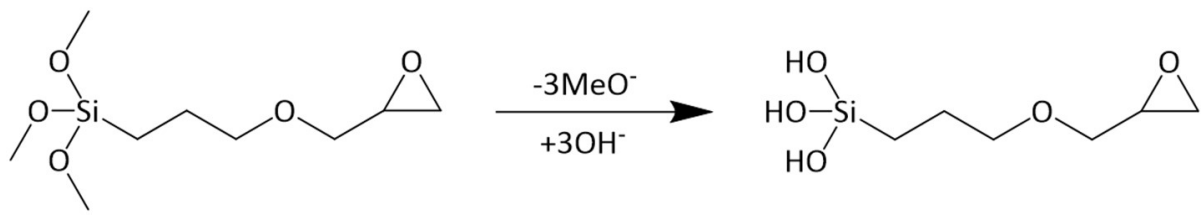

Scheme 4.8. Hydrolysis of GLYMO methoxysilane groups.

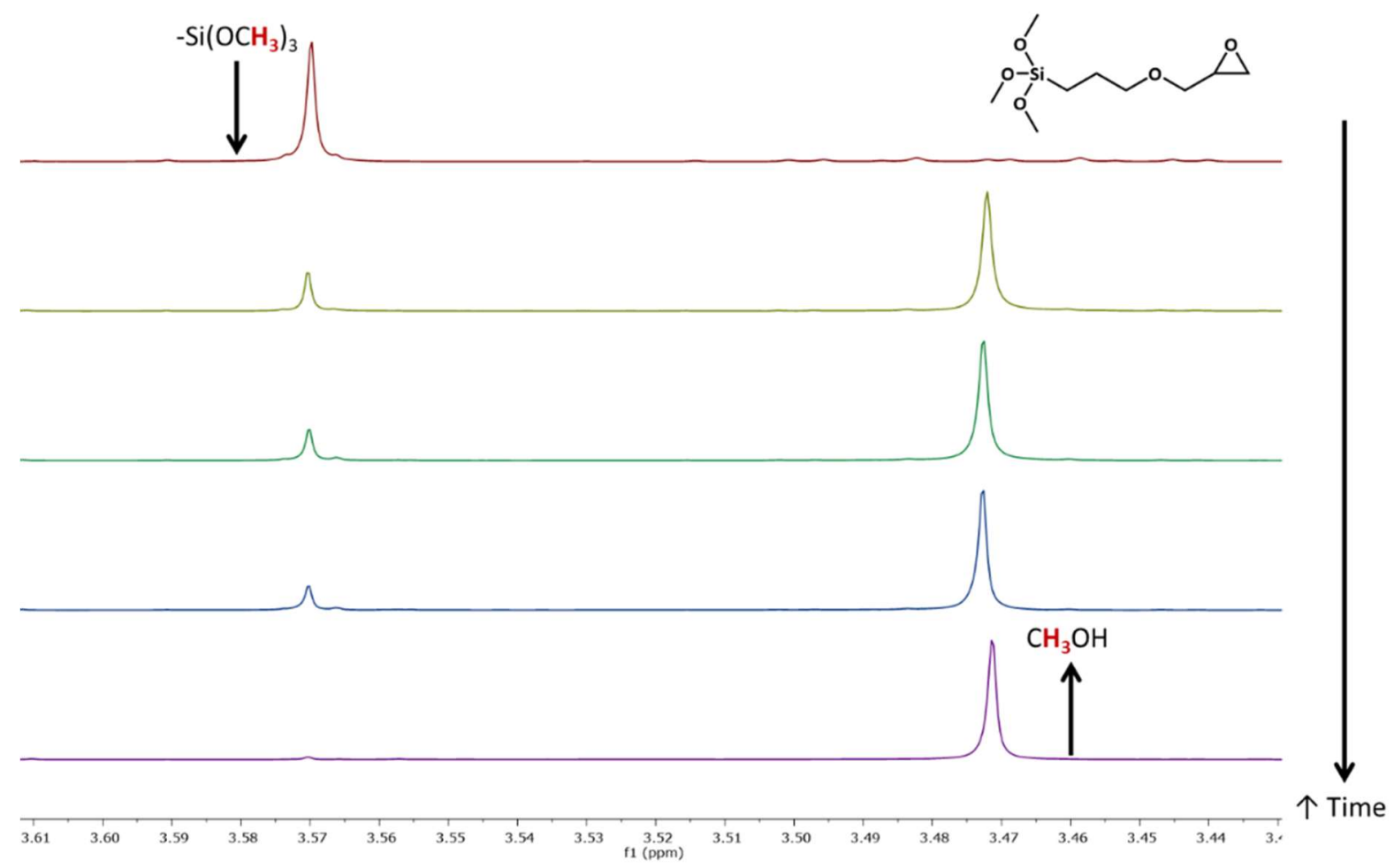

Figure 4.16. ${ }^{1} \mathrm{H}$ NMR spectra $\left(\mathrm{CDCl}_{3}\right)$ of $\operatorname{GLYMO}\left(\delta_{\mathrm{H}} 3.61-3.43 \mathrm{ppm}\right)$, following methoxy group hydrolysis over time. 
Table 4.8. Conditions for GLYMO hydrolysis experiments. The concentration of GLYMO was approximately $63 \mathrm{mM}$.

\begin{tabular}{|c|c|c|c|}
\hline Conditions & $\%$ v/v $\mathrm{NH}_{4} \mathrm{OH}$ & Solvent & Temperature $\left({ }^{\circ} \mathrm{C}\right)$ \\
\hline Batch 1 & 0.05 & $\mathrm{D}_{2} \mathrm{O}$ & 20 (RT) \\
\hline Lu, $0.4 \%$ & 0.04 & $\mathrm{CD}_{3} \mathrm{OD}, 1.6 \% \mathrm{v} / \mathrm{v} \mathrm{H} \mathrm{H}_{2} \mathrm{O}$ & 60 \\
\hline Lu, $1 \%$ & 1 & $\mathrm{CD}_{3} \mathrm{OD}, 3.6 \% \mathrm{v} / \mathrm{v} \mathrm{H} \mathrm{H}_{2} \mathrm{O}$ & 60 \\
\hline Batches 2-4 & 1 & $\mathrm{CD}_{3} \mathrm{OD}, 3.7 \% \mathrm{v} / \mathrm{v} \mathrm{H}_{2} \mathrm{O}, 11 \% \mathrm{v} / \mathrm{v} \mathrm{CHCl}_{3}$ & 60 \\
\hline
\end{tabular}

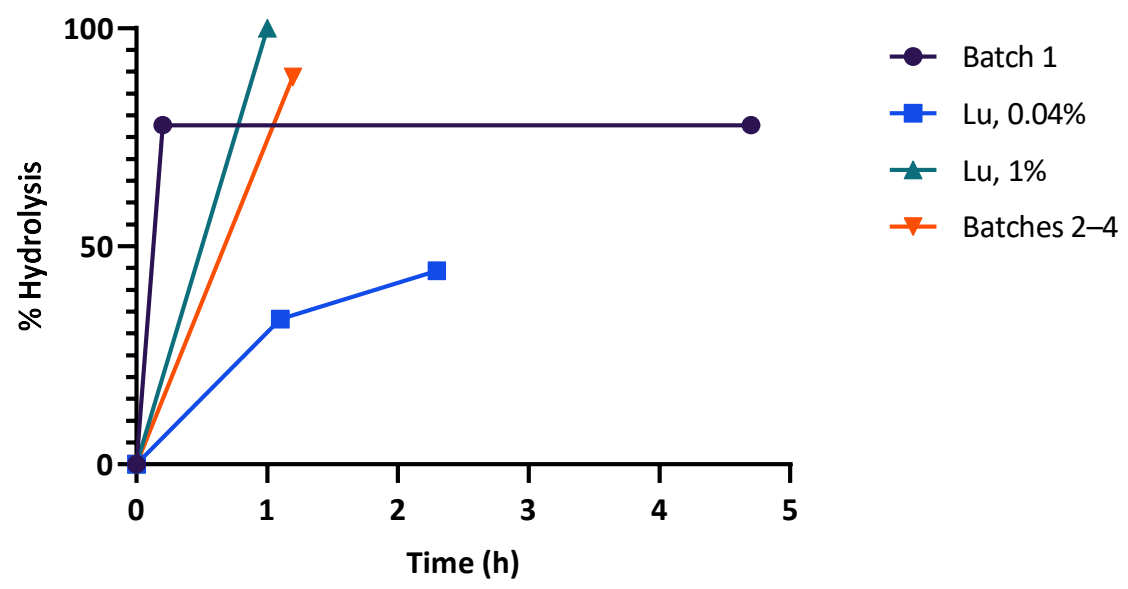

Figure 4.17. GLYMO hydrolysis over time under the sets of conditions reported in Table 4.8.

Aspects of the procedure by $\mathrm{Lu}^{269}$ were utilised for the next three sets of hydrolysis conditions tested, as shown in Table 4.8. First, GLYMO ( $63 \mathrm{mM})$ in 0.04\% v/v ammonium hydroxide in methanol and water $(1.6 \% \mathrm{v} / \mathrm{v})$ only partly hydrolysed $(44 \%$, Figure 4.17$)$ after $2.3 \mathrm{~h}$ heating at $60^{\circ} \mathrm{C}$. Consequently, the percentage $(\mathrm{v} / \mathrm{v})$ of ammonium hydroxide was increased from $0.04 \%$ to $1 \%$. This resulted in complete hydrolysis after $1 \mathrm{~h}$, and, therefore, this set of conditions was chosen for subsequent silica modification experiments. Since the $\mathrm{CnGm}$ reaction mixture would be introduced as a solution in chloroform to the basic mixture containing silica, the same set of conditions was tested again with the appropriate amount of chloroform $(11 \% \mathrm{v} / \mathrm{v})$, and almost complete hydrolysis of the methoxy groups was observed after $1.2 \mathrm{~h}$. Self-polymerisation of GLYMO was not observed. It was decided that this set of hydrolysis conditions was acceptable for the synthesis of $\pm \mathrm{Cu}(\mathrm{II})-\mathrm{CnGmSiO}_{2}$, in the hope of improving the percent functionalisation relative to Batch 1. 
Following the outcomes from the GLYMO hydrolysis experiments, Batch 2 of $\mathrm{Cu}(\mathrm{II})-\mathrm{CnGmSiO}{ }_{2}$ was prepared. An excess of copper(II) nitrate in methanol was added to the Procedure $6 \mathrm{CnGm}$ reaction mixture in chloroform. The colour change of the reaction mixture from colourless to dark blue indicated the formation of the $\mathrm{Cu}(\mathrm{II})-\mathrm{CnGm}$ complex, which was confirmed by HRMS (calcd for $\mathrm{C}_{17} \mathrm{H}_{39} \mathrm{CuN}_{4} \mathrm{O}_{5} \mathrm{Si}[\mathrm{Cu}(\mathrm{II})-\mathrm{CnGm}-\mathrm{H}]^{+} 470.1986$; found 470.1978). Then, the $\mathrm{Cu}(\mathrm{II})-\mathrm{CnGm}$ reaction mixture was added to silica in a basic water/methanol solution, resulting in a final concentration of $1 \% \mathrm{v} / \mathrm{v}$ ammonium hydroxide in methanol and water $(3.6 \% \mathrm{v} / \mathrm{v})$. The mixture was heated at $60^{\circ} \mathrm{C}$ for $1 \mathrm{~h}$ while stirring to hydrolyse the methoxysilane groups. Subsequent heating and concentration under vacuum of the mixture promoted the dehydration/condensation reactions, and Soxhlet extraction of the product yielded dark blue $\mathrm{Cu}(\mathrm{II})-\mathrm{CnGmSiO}$. Batches 3 and 4 were prepared in the same manner, but without the addition of $\mathrm{Cu}(\mathrm{II})$, to produce $\mathrm{CnGmSiO}_{2}$ (Figure 4.15) as a white solid. The $\mathrm{Cu}(\mathrm{II})$-chelating ability of the $\mathrm{CnGmSiO}_{2}$ from Batches 3 and 4 was tested by immersing the ligand-functionalised silica in an aqueous copper(II) nitrate solution for $24 \mathrm{~h}$ and then washing the now-blue product. This "postloaded" Batches 3 and $4 \mathrm{CnGmSiO}_{2}$ with $\mathrm{Cu}(\mathrm{II})$, rather than pre-loading the modified silica, as for Batches 1 and 2.

In order to determine if a comparable amount of cyclen ligand could be retained by the silica without the GLYMO linker, the adsorption of $\mathrm{Cu}(\mathrm{II})$-cyclen to the silica was tested. An excess of copper(II) nitrate was added to a basic, aqueous solution of cyclen. Silica was added to the dark blue $\mathrm{Cu}$ (II)-cyclen complex, and the mixture was concentrated under vacuum while heating and washed by Soxhlet extraction with water for 1 day. The light blue product was compared to Batches 1-4 to determine if covalent attachment of the cyclen ligand to silica via the GLYMO linker was necessary, since polyamine compounds are already well-retained by silica through acid/base interactions.

Batches 1-4 and the silica with adsorbed $\mathrm{Cu}(\mathrm{II})$-cyclen were submitted for $\mathrm{CHN}$ elemental analysis, and the results are tabulated (Table 4.9). Unmodified silica was submitted as a blank, and its \% $\mathrm{C}$ and $\% \mathrm{~N}$ results were below the detection limit. Batch 1 had a much lower percent functionalisation than Batches $2-4: 32 \%$ vs $73 \%, 75 \%$, and $88 \%$, respectively. Therefore, it was concluded that the procedure employed for Batch 1 was significantly less effective than the new procedure followed for Batches 2-4. Furthermore, these results suggested that the new procedure was fairly reproducible: the percent relative standard deviation (RSD) between the $\% \mathrm{~N}$ results for Batches 2-4 was approximately $11 \%$. The $\mathrm{CHN}$ results for the $\mathrm{Cu}(\mathrm{II})$-cyclen-adsorbed silica indicated that $<17 \%$ of the $\mathrm{Cu}(\mathrm{II})$-cyclen was retained by the silica, suggesting that covalent attachment of the ligand through the GLYMO linker was necessary for retention. 
Table 4.9. $\mathrm{CHN}$ elemental analysis and percent functionalisation/adsorption results for silica, Batches 1-4 $\pm \mathrm{Cu}(\mathrm{II})-\mathrm{CnGmSiO}$, and adsorbed $\mathrm{Cu}(\mathrm{II})$-cyclen.

\begin{tabular}{|c|c|c|c|c|}
\hline Product & $\% \mathrm{C}^{\mathrm{a}}$ & $\% \mathrm{H}^{\mathrm{a}}$ & $\% \mathrm{~N}^{\mathrm{a}}$ & $\begin{array}{c}\text { \% Functionalisation/ } \\
\text { Adsorption }^{b}\end{array}$ \\
\hline Silica & $<0.30$ & 0.96 & $<0.30$ & - \\
\hline Batch $1 \mathrm{Cu}(\mathrm{II})-\mathrm{CnGmSiO}{ }_{2}$ & 1.80 & 0.77 & 0.47 & 32 \\
\hline Batch $2 \mathrm{Cu}(\mathrm{II})-\mathrm{CnGmSiO}{ }_{2}$ & 4.17 & 0.92 & 1.18 & 73 \\
\hline Batch $3 \mathrm{CnGmSiO}_{2}$ & 4.52 & 1.22 & 1.24 & 75 \\
\hline Batch $4 \mathrm{CnGmSiO}_{2}$ & 4.81 & 0.94 & 1.46 & 88 \\
\hline Adsorbed Cu(II)-cyclen & 0.53 & 0.87 & $<0.30$ & $<17$ \\
\hline
\end{tabular}

${ }^{a}$ Reported as the mean of duplicate measurements within $\leq 0.30 \%$ of each other.

${ }^{b}$ Calculated based on the measured $\% \mathrm{~N}$ value compared to the theoretical maximum.

The percent error between the expected and measured $\% \mathrm{~N}$ from the $\mathrm{CHN}$ results was also calculated (Table 4.10). As before, the expected \% $\mathrm{N}$ was calculated from the measured \%C. Out of Batches 1-4, at $-22 \%$, Batch 1 had the largest error, and, at $-9 \%$, Batch 4 had the smallest. All of the $\pm \mathrm{Cu}(\mathrm{II})-\mathrm{CnGmSiO} 2$ samples had a lower measured $\% \mathrm{~N}$ than expected, and, as before, this was unsurprising due to the presence of multi-functionalised, as well as mono-functionalised, $\mathrm{Cn}(\mathrm{Gm})_{\mathrm{n}}$ in the reaction mixture. The percent error could not be calculated for the $\mathrm{Cu}(\mathrm{II})$-cyclen-adsorbed silica, as the measured $\% \mathrm{~N}$ was below the detection limit.

Table 4.10. Percent error between the expected and measured $\% \mathrm{~N}$ from $\mathrm{CHN}$ results.

\begin{tabular}{|c|c|c|c|}
\hline Product & Measured \%N & Expected \% $\mathrm{N}^{\mathrm{a}}$ & \% Error \\
\hline Batch $1 \mathrm{Cu}(\mathrm{II})-\mathrm{CnGmSiO}{ }_{2}$ & 0.47 & 0.60 & -22 \\
\hline Batch $2 \mathrm{Cu}(\mathrm{II})-\mathrm{CnGmSiO} 2$ & 1.18 & 1.39 & -15 \\
\hline Batch $3 \mathrm{CnGmSiO}_{2}$ & 1.24 & 1.51 & -18 \\
\hline Batch $4 \mathrm{CnGmSiO}_{2}$ & 1.46 & 1.60 & -9 \\
\hline Adsorbed Cu(II)-cyclen & $<0.3$ & 0.17 & - \\
\hline
\end{tabular}

${ }^{a}$ Calculated from the measured \%C data (Table 4.9).

To characterise the materials further, the copper content of pre-loaded and post-loaded $\mathrm{CnGm}$ modified silica from Batches 1-4, as well as the $\mathrm{Cu}(\mathrm{II})$-cyclen-adsorbed silica, was measured by ICP-MS and compared to the calculated theoretical \% w/w Cu (Table 4.11). As it has been reported that free silanol groups are capable of metal ion-complexation, ${ }^{241}$ the ability of unfunctionalised silica to retain 
$\mathrm{Cu}$ (II) was also determined. The samples were prepared for ICP-MS analysis by digestion in concentrated hydrofluoric acid and then nitric acid $(9 \mathrm{M})$ and dilution in nitric acid $(3 \% \mathrm{v} / \mathrm{v})$ to bring the samples into the range of the standards. Batches 1 and $2 \mathrm{Cu}(\mathrm{II})-\mathrm{CnGmSiO}{ }_{2}$ were both pre-loaded with $\mathrm{Cu}(\mathrm{II})$ in the first step of the functionalisation procedures. However, the measured copper content of Batch $1(0.48 \% \mathrm{w} / \mathrm{w})$ was much lower than that of Batch $2(2.60 \% \mathrm{w} / \mathrm{w})$. This served as further evidence that the new procedure for Batches 2-4 was superior to the Batch 1 procedure. The measured amount of copper in Batch 2 was almost twice the calculated theoretical amount $(1.33 \% \mathrm{w} / \mathrm{w})$, but the cause for this disparity remains unclear. The copper content in Batches 3 and 4 $\mathrm{CnGmSiO}_{2}$ was measured before post-loading with $\mathrm{Cu}(\mathrm{II})$. Thus, they served as blanks, and copper was only detected at the level of parts per million. After exposure to an aqueous copper(II) nitrate solution for $24 \mathrm{~h}$ (i.e. post-loading with $\mathrm{Cu}(\mathrm{II}):$ " $\mathrm{CnGmSiO} 2+\mathrm{Cu}(\mathrm{II})$ ”), the measured copper contents of Batches 3 and 4 were $0.96 \% \mathrm{w} / \mathrm{w}$ and $1.02 \% \mathrm{w} / \mathrm{w}$, respectively. These values agreed well, but both were lower than the calculated theoretical \% w/w Cu and lower than pre-loaded Batch 2. It is possible that the $\mathrm{Cu}(\mathrm{II})$ complexes formed more easily in solution, as in for the pre-loading, than in the aqueous silica mixture, as in for the post-loading. In fact, previous studies in the literature found that harsh conditions (2-3 $\mathrm{h}$ refluxing in ethanol with an excess of $\mathrm{Cu}(\mathrm{II}))$ were necessary for complete metalation of cyclam-modified silica. ${ }^{240,243,252}$

Table 4.11. ICP-MS (Cu) results for silica $\pm \mathrm{Cu}(\mathrm{II})$, Batches $1-4 \pm \mathrm{Cu}(\mathrm{II})-\mathrm{CnGmSiO}_{2}$, and adsorbed $\mathrm{Cu}(\mathrm{II})$-cyclen compared to the calculated theoretical \% w/w Cu.

\begin{tabular}{|c|c|c|c|}
\hline Product & Measured \% w/w Cu & $\%$ RSD & Theoretical \% w/w Cu ${ }^{\circ}$ \\
\hline Silica (unexposed to $\mathrm{Cu}(I I))^{b}$ & $<0.0001$ & - & 0 \\
\hline Silica (exposed to $\mathrm{Cu}(\mathrm{II})$ ) & 0.06 & 1.0 & - \\
\hline Batch $1 \mathrm{Cu}(\mathrm{II})-\mathrm{CnGmSiO}{ }_{2}^{\mathrm{c}}$ & 0.48 & $d$ & 0.53 \\
\hline Batch $2 \mathrm{Cu}(\mathrm{II})-\mathrm{CnGmSiO}_{2}$ & 2.60 & 0.3 & 1.33 \\
\hline Batch $3 \mathrm{CnGmSiO}_{2}$ & 0.00 & 0.4 & 0 \\
\hline Batch $3 \mathrm{CnGmSiO}_{2}+\mathrm{Cu}(\mathrm{II})$ & 0.96 & 0.3 & 1.41 \\
\hline Batch $4 \mathrm{CnGmSiO}_{2}$ & 0.00 & 1.6 & 0 \\
\hline Batch $4 \mathrm{CnGmSiO}_{2}+\mathrm{Cu}(\mathrm{II})$ & 1.02 & 0.3 & 1.65 \\
\hline Adsorbed Cu(II)-cyclen ${ }^{b}$ & 0.28 & $d$ & 0.35 \\
\hline
\end{tabular}

\footnotetext{
${ }^{a}$ Calculated based on the percent functionalisation/adsorption data (Table 4.9) and theoretical maximum.

${ }^{b}$ Measured in duplicate.

c Measured in triplicate.

${ }^{d}$ The percent error of the measurement is $\pm 5 \%$.
} 
In the present work, the copper content for the $\mathrm{Cu}(\mathrm{II})$-cyclen-adsorbed silica was $0.28 \% \mathrm{w} / \mathrm{w}$, which was $80 \%$ of the theoretical amount and even lower than Batch 1 . This further reinforced the necessity of covalent attachment. The copper content of unfunctionalised silica before and after exposure to an aqueous copper(II) nitrate solution was $<0.0001 \% \mathrm{w} / \mathrm{w}$ and $0.06 \% \mathrm{w} / \mathrm{w}$, respectively. Thus, it was demonstrated that, even without modification, silica is able to retain a relatively significant amount of $\mathrm{Cu}(\mathrm{II})$.

\subsubsection{Conclusions}

A successful method was developed for the functionalisation of silica with $\pm \mathrm{Cu}(\mathrm{II})-\mathrm{CnGm}$, and a simple process was established for characterisation of the product. Two methods with differing approaches were trialled (Scheme 4.1): in Method 1, silica was modified with GLYMO and then reacted with cyclen, but, in Method 2, cyclen and GLYMO were reacted first, followed by silica modification with CnGm. Method 2 was found to be superior to Method 1 for the synthesis of $\pm \mathrm{Cu}(\mathrm{II})-\mathrm{CnGmSiO}$. The main advantage of Method 2 was that the product of the first reaction, $\mathrm{CnGm}$, could be characterised by NMR spectroscopy. In Method 1, characterisation of the products was complicated by their attachment to the inorganic silica matrix, and it was difficult to prove that, first, the GLYMO linker was covalently bound to the silica and, second, that the cyclen ligand was covalently bound to GLYMO. Following Method 2, the six procedures for $\mathrm{Cn}(\mathrm{Gm})_{n}$ synthesis (Table 4.4) established guidelines in order to avoid GLYMO self-condensation and to generate free-ligand $\mathrm{CnGm}$ as the major product. It was decided that the crude $\mathrm{CnGm}$ (Procedure 6) reaction mixture in chloroform should be used for subsequent silica functionalisation. Multiple sets of conditions for hydrolysis of the methoxysilane groups were tested (Table 4.8), and the results led to the establishment of a fairly reproducible silica modification procedure with a percent functionalisation consistently greater than 70\% (Batches 2-4). Pre-loading the $\mathrm{CnGmSiO}_{2}$ with $\mathrm{Cu}(\mathrm{II})$ yielded a \% w/w $\mathrm{Cu}$ more than twice that achieved by postloading (Batch 2: $2.60 \%$ vs Batch 3: $0.96 \%$ and Batch 4: 1.02\%). Finally, the poor retention of $\mathrm{Cu}(\mathrm{II})$-cyclen adsorbed onto silica demonstrated the necessity of covalent attachment of the ligand to silica through a linker.

The loading of the macrocycle and copper on the silica from Batches 2-4 was comparable to previous studies (Table 4.12). Prior works achieved macrocycle concentrations ranging from $0.20-0.93 \mathrm{mmol} / \mathrm{g}$ silica. In comparison, the macrocycle-loading for Batches $2-4$ was $0.21-0.26 \mathrm{mmol} / \mathrm{g}$ silica, which, albeit at the lower end, was within the range reported in the literature. Similarly, the copper content $(0.15-0.41 \mathrm{mmol} / \mathrm{g}$ silica) was within the reported range $(0.03-0.78 \mathrm{mmol} / \mathrm{g}$ silica). This indicated that 
the new, optimised method for $\pm \mathrm{Cu}(\mathrm{II})-\mathrm{CnGmSiO}_{2}$ synthesis could generate products with similar ligand- and copper-loading to those in the literature.

Table 4.12. Loading of the macrocycle and copper on silica.

\begin{tabular}{|c|c|c|}
\hline Samples in This Work & Loading (mmol macrocycle/g silica) ${ }^{\mathrm{a}}$ & Loading (mmol Cu/g silica) ${ }^{b}$ \\
\hline Batch $1 \mathrm{Cu}(\mathrm{II})-\mathrm{CnGmSiO}{ }_{2}$ & 0.08 & 0.08 \\
\hline Batch $2 \mathrm{Cu}(\mathrm{II})-\mathrm{CnGmSiO}{ }_{2}$ & 0.21 & 0.41 \\
\hline Batch $3 \mathrm{CnGmSiO}_{2}+\mathrm{Cu}(\mathrm{II})$ & 0.22 & 0.15 \\
\hline Batch $4 \mathrm{CnGmSiO}_{2}+\mathrm{Cu}(\mathrm{II})$ & 0.26 & 0.16 \\
\hline Adsorbed Cu(II)-cyclen & 0.05 & 0.04 \\
\hline References & Loading (mmol macrocycle/g silica) & Loading (mmol Cu/g silica) \\
\hline Bagnoud et al..$^{255}$ & - & 0.20 \\
\hline Gros et al..$^{241}$ & $0.33-0.44$ & $0.054-0.31$ \\
\hline Bereczki et al. ${ }^{258}$ & - & $0.21-0.33$ \\
\hline Barreto et al. ${ }^{259}$ & $0.20-0.24$ & - \\
\hline Goubert-Renaudin et al. ${ }^{252}$ & $0.25-0.63$ & $0.03-0.35$ \\
\hline Corriu et al. ${ }^{243}$ & $0.48-0.93$ & $0.45-0.78$ \\
\hline Dubois et al. ${ }^{238}$ & $0.38-0.81$ & $0.35-0.75$ \\
\hline Cuenot et al. ${ }^{123}$ & $0.30-0.61$ & - \\
\hline
\end{tabular}

a Calculated from the measured $\% \mathrm{~N}$ values in Table 4.9.

${ }^{\mathrm{b}}$ Calculated from the measured \% w/w Cu values in Table 4.11. 


\subsection{InCORPORATION OF SILICA IN A COATING}

To incorporate the functionalised silica products into a coating, it was suggested that the silica be applied to an uncured resin, which would then be allowed to cure with silica adhered to the surface. In other words, the resin would glue the silica to the substratum. A similar procedure is implemented in industry for textured paints. ${ }^{207}$ The intention was to evaluate bacterial adherence to the coatings with the biocidal ingredient at the surface, which was desirable for a couple of reasons. First, part of the motivation for modifying silica with the ligand was because it is known that silica tends to float to the surface of a coating, ${ }^{29}$ meaning that the bacteria would, ideally, be exposed to the biocide at the coating/water interface anyway. Second, it was assumed that the maximum effect of the biocide on bacterial adherence would be achieved when the surface is purely the biocide, rather than diluted throughout a coating. Therefore, if bacterial adherence to a surface composed of the putative biocide was uninhibited, then it could be concluded that the modified silica would likely be unsuccessful as an antifouling agent. This method, however, is complicated by the propensity of bacteria to colonise rough surfaces, such as the proposed silica surfaces.

The positive relationship between surface roughness and bacterial colonisation has a few explanations. The initial adhesion of bacteria is facilitated on rough surfaces because they provide shelter from shear forces that would, otherwise, remove the bacteria, thus allowing time for the attachment to become irreversible. Rough surfaces also have a greater surface area for bacterial adhesion and are difficult to clean. ${ }^{270}$ Consequently, increased bacterial adherence to the rough silica coatings might be hypothesised. But, the increase in colonisation by bacteria with the roughening of a surface does not always hold true; if the biocide at the surface is at the appropriate concentration, even if the surface is rough, bacterial adhesion will be deterred. For example, in a study by Kozlovsky et al. ${ }^{271}$, chlorhexidine (CHX), a chemical antiplaque agent, was adsorbed to both sandblasted, acid-etched rough and machined-smooth titanium disks, and the antibacterial activity of the disks was assessed against Streptococcus mutans, a human odontopathogen. ${ }^{272}$ It was found that the greater surface area of the rough disk provided more adsorption sites for $\mathrm{CHX}$, and, as a result, the rough titanium surface demonstrated improved antimicrobial performance compared to the smooth surface. Therefore, in this work, the aim of the functionalised silica coatings was to achieve a high enough copper concentration at the silica surface via the $\mathrm{Cu}(\mathrm{II})$-ligand to be antimicrobial, despite the roughness of the surface.

A two-pack epoxy, composed of a commercial hardener and epoxy resin, was chosen as the resin on which to apply the $\pm \mathrm{Cu}(\mathrm{II})-\mathrm{CnGmSiO}$ 2 products. It was chosen due to familiarity with its use, curing process, and properties (Chapter 3, Section 3.3). The hardener (Ancamine ${ }^{\circledR} 2459$ Curing Agent) and 
epoxy resin (Epikote ${ }^{\mathrm{TM}}$ 235) were mixed manually, according to the manufacturers' specifications, and the viscous, dark yellow mixture was applied by the standard draw-down technique with a bar film applicator (Experimental, Figure 6.2) to one side of a black, plastic sheet (vinyl chloride/acetate copolymer, Leneta). The silica was applied to a defined surface area of the still-uncured epoxy resin with an inverted funnel so that, at least, one layer of silica covered the resin, and the coating was left to cure at room temperature overnight. The following day, the same procedure was repeated for the second side of the substratum. Once the second side had cured, the silica/epoxy $\left(\mathrm{SiO}_{2} / \mathrm{epx}\right)$-type coatings were brushed, washed with water, and dried to remove non-adhered particles, and squares $(23 \mathrm{~mm} \times 23 \mathrm{~mm}$ ) coated on both sides were cut from the material for immediate bacterial adherence testing. The four silica samples incorporated into coatings for testing were as follows: 1) Batch 4 $\mathrm{CnGmSiO}_{2}$ (free ligand), 2) Batch $4 \mathrm{CnGmSiO}_{2}+\mathrm{Cu}(\mathrm{II})$ (post-loaded), 3) Batch $2 \mathrm{Cu}(\mathrm{II})-\mathrm{CnGmSiO}$ (preloaded), and 4) unmodified, commercial silica $\left(\mathrm{SiO}_{2}\right)$. Batch $4 \mathrm{CnGmSiO} 2$ was arbitrarily chosen over Batch $3 \mathrm{CnGmSiO}_{2}$, but, in retrospect, Batch 3 would have been more appropriate due to its elemental composition being closer to Batch $2 \mathrm{Cu}(\mathrm{II})-\mathrm{CnGmSiO}_{2}$ (Table 4.9). To post-load $\mathrm{CnGmSiO}_{2}$ with $\mathrm{Cu}(\mathrm{II})$, the cut-out squares coated on both sides with $\mathrm{CnGmSiO}_{2}$ were submerged for $24 \mathrm{~h}$ in an aqueous solution containing an excess of copper(II) nitrate, washed with water, dried, and immediately tested for bacterial adherence. The prepared squares are shown in Figure $\mathbf{4 . 1 8}$ (top left corner), and the bacterial adherence results are discussed in Chapter 5 (Sections 5.3-4).

Although the silica application on each square sample was, unsurprisingly, inhomogeneous, the goal of the application was to simply cover the underlying epoxy resin with silica so that the bacteria were only exposed to the silica. Magnified images of the $\mathrm{SiO}_{2} /$ epx-type coatings were obtained by scanning electron microscopy (SEM; Appendix, Figures 8.45-47), and SEM images of the squares at 200x magnification (Figure 4.18) revealed the desired surface coverage. Homogeneous application of the silica would require more specialised equipment. Differences in the numbers of layers of silica adhered to the coatings would likely affect bacterial adhesion results if the total surface area of the square was variable and the biocide (i.e. $\mathrm{Cu}(\mathrm{II}))$ was ineffective. But, it was thought that, if the biocide was effective, relative differences between the coatings with and without $\mathrm{Cu}(\mathrm{II})$ would still be observable, since the differences would need to be orders of magnitude to be meaningful.

In tandem with imaging the squares, the elemental compositions of the coatings were obtained via scanning electron microscopy/energy dispersive X-ray spectroscopy (SEM-EDS; Table 4.13). The EDS spectra are in the Appendix (Figures 8.48-51). EDS is an elemental analysis technique based on the ionisation of atoms in a sample by an incident electron beam, resulting in the emission of characteristic $\mathrm{X}$-rays as the atoms return to their ground state. The X-ray photons are detected, and a proportional output signal is generated to create an X-ray spectrum of counts or intensity vs X-ray energy 
(Figure 4.19). The information contained in the spectrum is both qualitative and semi-quantitative: the peak position in the spectrum (i.e. the X-ray energy) identifies the element, while the peak area is proportional to the atomic percent of the particular element in the irradiated area. ${ }^{273,274}$ The data are considered semi-quantitative because physical standards are not measured. Rather, the standardless analysis refers to libraries of standard spectra for the calculations. ${ }^{275}$

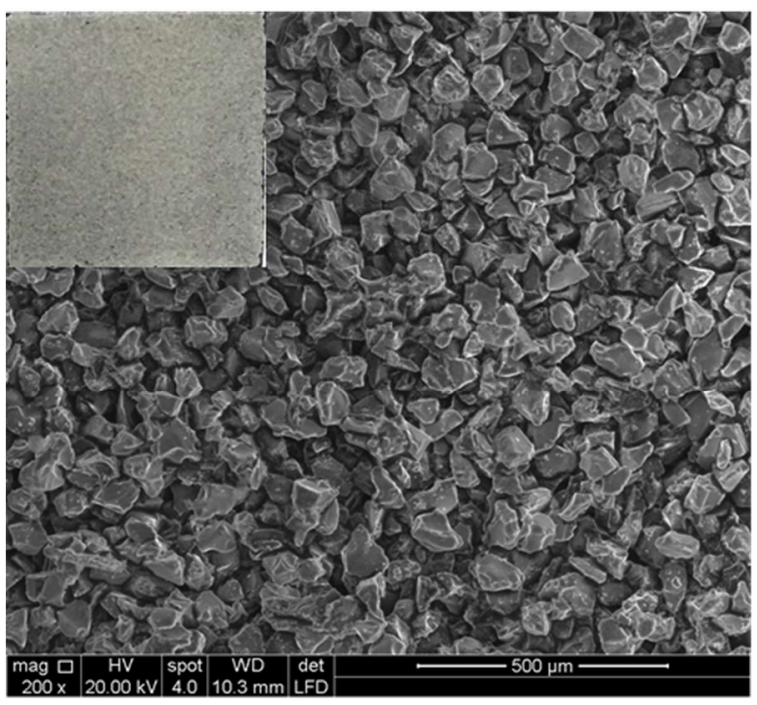

$\mathrm{SiO}_{2}$

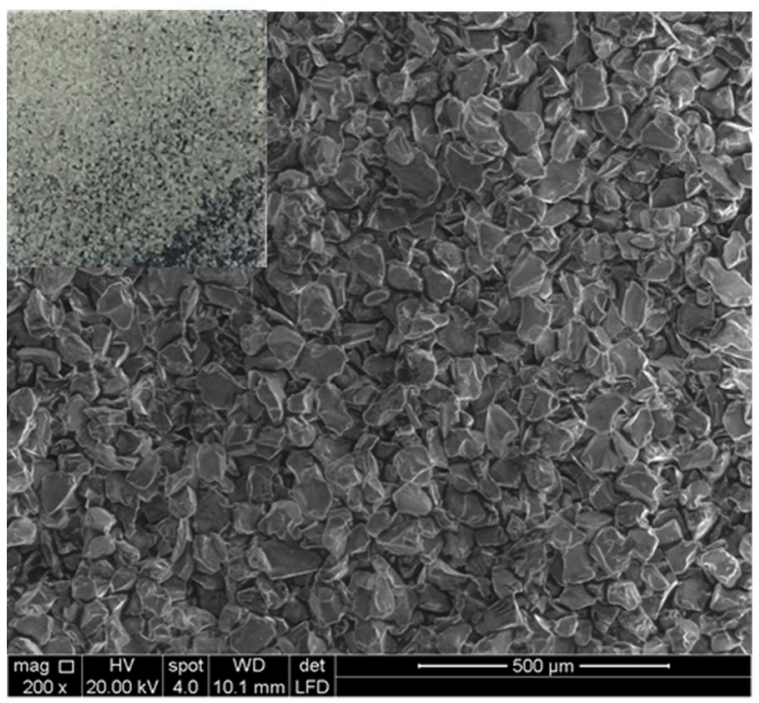

$\mathrm{CnGmSiO}_{2}$

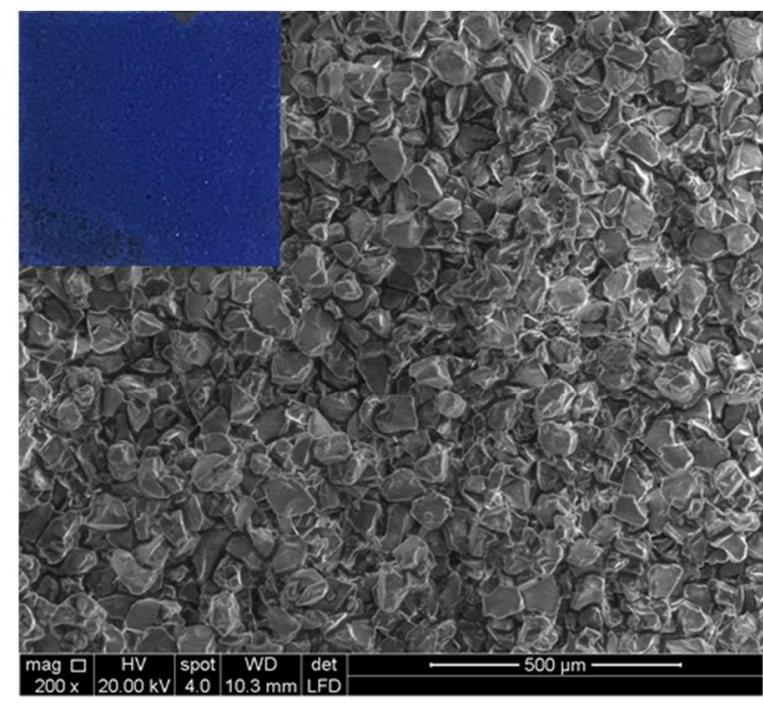

$\mathrm{Cu}(\mathrm{II})-\mathrm{CnGmSiO}{ }_{2}$

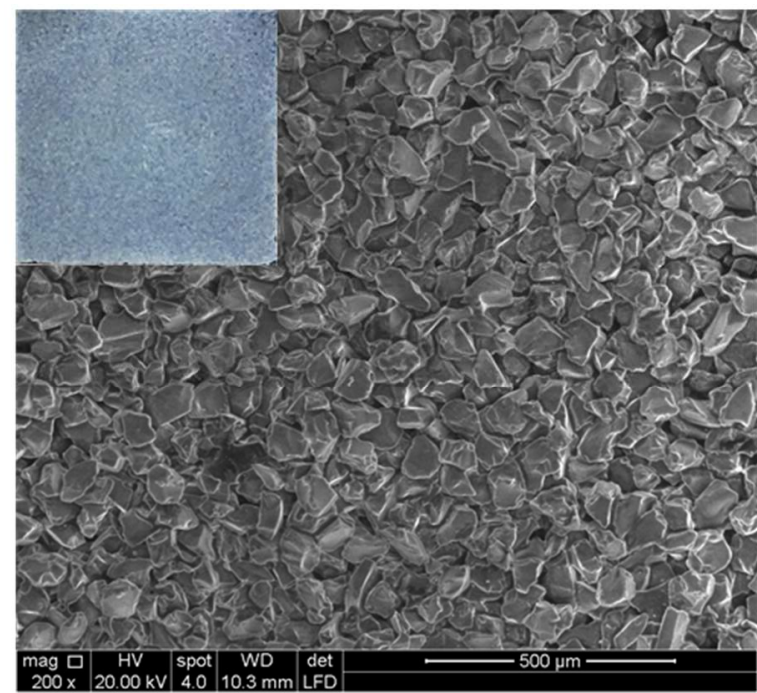

$\mathrm{CnGmSiO}_{2}+\mathrm{Cu}(\mathrm{II})$

Figure 4.18. SEM secondary electron images at $200 \times$ magnification of the $\mathrm{SiO}_{2} / \mathrm{epx}$-type squares to be tested for bacterial adherence (HV $20 \mathrm{kV}$, spot size 4, WD $10 \mathrm{~mm}$, LFD). The coatings include $\mathrm{SiO}_{2} /$ epx, Batch $2 \mathrm{Cu}(\mathrm{II})-\mathrm{CnGmSiO}_{2} / \mathrm{epx}$, Batch $4 \mathrm{CnGmSiO}_{2} / \mathrm{epx}$, and Batch $4 \mathrm{CnGmSiO}+\mathrm{Cu}(\mathrm{II}) / \mathrm{epx}$, and pictures of these coatings are in the top left corner of each SEM image. 
Table 4.13. EDS results for Batch $2 \mathrm{Cu}(\mathrm{II})-\mathrm{CnGmSiO}_{2} / \mathrm{epx}$, Batch $4 \mathrm{CnGmSiO} / \mathrm{epx}$, Batch $4 \mathrm{CnGmSiO}_{2}+$ $\mathrm{Cu}(\mathrm{II}) / \mathrm{epx}$, and $\mathrm{SiO}_{2} / \mathrm{epx}$.

\begin{tabular}{|c|c|c|}
\hline \multicolumn{3}{|c|}{ Batch $2 \mathrm{Cu}(\mathrm{II})-\mathrm{CnGmSiO} / \mathrm{epx}$} \\
\hline Element & Weight \% & Atomic \% \\
\hline $\mathrm{C} \mathrm{K}$ & $45 \pm 4$ & $55 \pm 5$ \\
\hline N K & $4.0 \pm 0.7$ & $4.2 \pm 0.8$ \\
\hline $\mathrm{OK}$ & $36 \pm 4$ & $33 \pm 3$ \\
\hline Si K & $13.9 \pm 0.6$ & $7.3 \pm 0.4$ \\
\hline Cu K & $1.4 \pm 0.2$ & $0.32 \pm 0.06$ \\
\hline \multicolumn{3}{|c|}{ Batch $4 \mathrm{CnGmSiO}_{2} / \mathrm{epx}$} \\
\hline Element & Weight \% & Atomic \% \\
\hline $\mathrm{CK}$ & $43 \pm 4$ & $53 \pm 5$ \\
\hline N K & $3.9 \pm 0.7$ & $4.2 \pm 0.8$ \\
\hline $\mathrm{OK}$ & $38 \pm 4$ & $35 \pm 4$ \\
\hline Si K & $15.7 \pm 0.6$ & $8.3 \pm 0.4$ \\
\hline \multicolumn{3}{|c|}{ Batch $4 \mathrm{CnGmSiO}_{2}+\mathrm{Cu}(\mathrm{II}) / \mathrm{epx}$} \\
\hline Element & Weight \% & Atomic \% \\
\hline $\mathrm{C} \mathrm{K}$ & $40 \pm 4$ & $50 \pm 5$ \\
\hline N K & $4.7 \pm 0.8$ & $5.0 \pm 0.9$ \\
\hline $\mathrm{OK}$ & $39 \pm 4$ & $37 \pm 4$ \\
\hline Si K & $16.1 \pm 0.8$ & $8.6 \pm 0.5$ \\
\hline Cu K & $1.0 \pm 0.2$ & $0.23 \pm 0.06$ \\
\hline \multicolumn{3}{|c|}{$\mathrm{SiO}_{2} / \mathrm{epx}$} \\
\hline Element & Weight \% & Atomic \% \\
\hline C K & $42 \pm 4$ & $53 \pm 5$ \\
\hline $\mathrm{OK}$ & $40 \pm 4$ & $38 \pm 4$ \\
\hline Si K & $17.3 \pm 0.5$ & $9.3 \pm 0.3$ \\
\hline
\end{tabular}




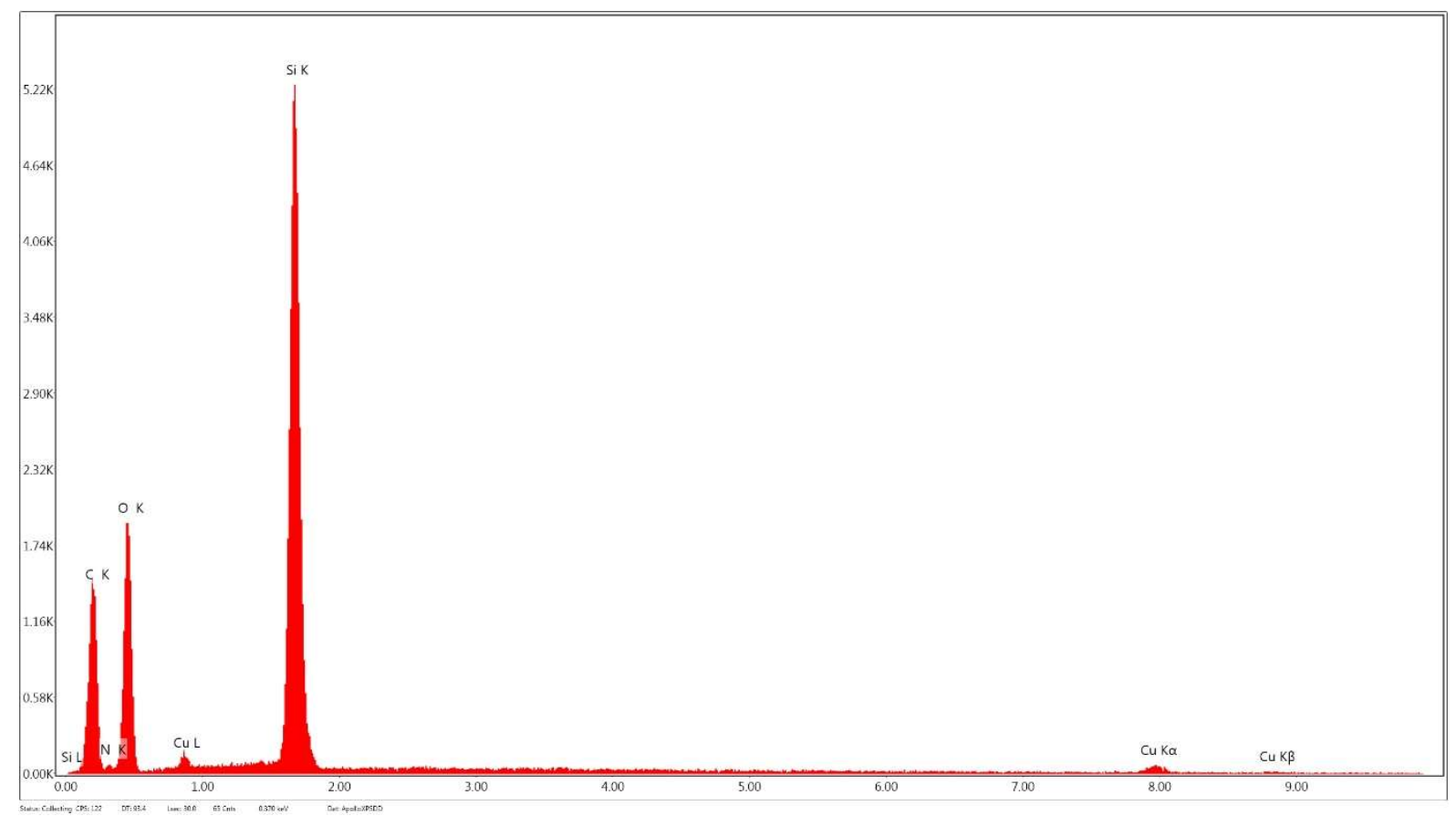

Figure 4.19. Example EDS spectrum (counts vs keV).

As predicted, all of the modified-silica coatings were found to be composed of $\mathrm{C}, \mathrm{O}, \mathrm{N}$, and $\mathrm{Si}$, with the $\mathrm{Cu}(\mathrm{II})-\mathrm{CnGmSiO} / \mathrm{epx}$ and $\mathrm{CnGmSiO}_{2}+\mathrm{Cu}(\mathrm{II}) /$ epx coatings also containing $\mathrm{Cu}$ (Table 4.13). Hydrogen could not be detected by this technique because it has no core electrons - only a valence electron that participates in chemical bonding, meaning that it will not produce X-rays. ${ }^{275,276}$ The major constituents were $\mathrm{C}, \mathrm{O}$, and $\mathrm{Si}$, and the minor constituents were $\mathrm{N}$ and $\mathrm{Cu}$. $\mathrm{A}$ larger percentage of the elemental composition than expected was carbon, with a weight percent between $40 \%$ and $50 \%$. However, the accumulation of carbon as a contaminant on the sample surface in an electron microscope is common, making carbon measurements unreliable. ${ }^{277}$ For Batch $4 \mathrm{CnGmSiO}_{2}+$ $\mathrm{Cu}(\mathrm{II}) / \mathrm{epx}$, the copper results were comparable to those obtained by ICP-MS (Table 4.11), but, in the case of Batch $2 \mathrm{Cu}(\mathrm{II})-\mathrm{CnGmSiO}_{2} / \mathrm{epx}$, the results were not as comparable: 1.02\% w/w (ICP-MS) vs 1.0\% (SEM-EDS) for $\mathrm{CnGmSiO}_{2}+\mathrm{Cu}(\mathrm{II}) / \mathrm{epx}$ and $2.60 \% \mathrm{w} / \mathrm{w}$ (ICP-MS) vs $1.4 \% \mathrm{w} / \mathrm{w}$ (SEM-EDS) for $\mathrm{Cu}(\mathrm{II})-\mathrm{CnGmSiO} / \mathrm{epx}$. Any consistency was actually somewhat surprising, given the inhomogeneity of the samples and the different sampling methods of the two techniques. The samples were prepared for ICP-MS by total digestion of the material, and, consequently, the results are presented as $\% \mathrm{w} / \mathrm{w}$ $\mathrm{Cu}$ in the bulk material. In contrast, the onion-shaped interaction volume sampled by SEM-EDS (Figure 4.20) had a width and depth of up to a few micrometres. Since the functionalised silica was heterogeneous, with the ligand and copper on the surface and an interior of silicon dioxide, it was thought that the elemental composition results by EDS would be skewed by the sampling method. 


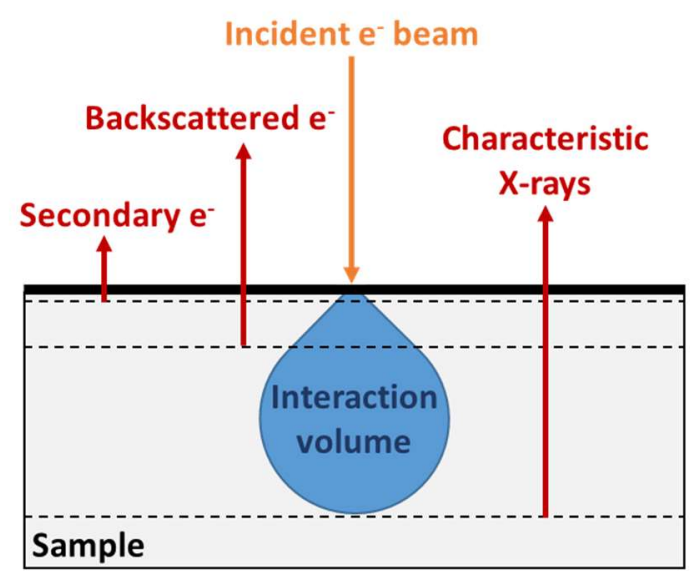

Figure 4.20. Interactions between the sample and the incident electron ( $\left.\mathrm{e}^{-}\right)$beam..$^{278,279}$

The quantitative results were even more questionable due to specimen geometry effects, which are variations in the topography of the sample. These variations in shape and inclination of the surface relative to the incident electron beam modify the measured $\mathrm{X}$-ray intensities. For accurate quantitative analyses by EDS, it is assumed that the only factor affecting X-ray intensity is the composition, and sample surfaces are polished to a surface roughness below $50 \mathrm{~nm}$. If this condition is violated, the error associated with each measurement can be so large that it renders the compositional results unusable. ${ }^{280,281}$ The topography of the $\mathrm{SiO}_{2} /$ epx-type coatings was rough and likely impacted the quantitative results. Considering all of the complications, it was decided that SEM-EDS was not an appropriate technique for determining the elemental compositions of the silica coatings with accuracy, but the qualitative results still had value. It was not visibly apparent that these complications in the analyses of the silica coatings were applicable to the commercial marine paint (Altex Yacht and Boat Paint, Aurora Red, Ablative Antifouling No. 5; 40-50\% $\mathrm{Cu}_{2} \mathrm{O}$ ) used as a benchmark comparison throughout this study, and, so, the marine paint was analysed by SEM-EDS.

SEM-EDS analysis was completed to better-understand the topography and copper distribution at the surface of the marine paint. The marine paint served as the commercial standard against which the silica coatings were compared - particularly in the microbiological assays. Therefore, as with the silica coatings, it was reasonable to examine the physical and chemical features of the coating's surface, since these properties are responsible for the outcome of the bacteria-surface interaction. A sample was prepared for analysis by applying one coat of the marine paint via the standard draw-down technique with a bar film applicator (Experimental, Figure 6.2) to the substratum. SEM images of the cured paint (Figure 4.21) revealed a heterogeneous surface containing areas of high and low copper content. Different areas of the surface were sampled for EDS measurements, and it was found that a 
couple of these areas had a \% w/w Cu (1.3-1.5\%, Areas 1 and 2, Table 4.14) comparable to the $\mathrm{Cu}(\mathrm{II})$ containing silica coatings. However, there was also an area that was $37.4 \% \mathrm{w} / \mathrm{w} \mathrm{Cu}$ (Area 3), and this high-copper area, as well as others, appeared as a white spot in the SEM image. These results were unsurprising, as the marine paint is a dispersion of cuprous oxide in solvent, zinc oxide, rosincolophony, and thiram. Given the commercial success of the paint, the inhomogeneity of the copper distribution does not appear to significantly impact its efficacy, and fouling organisms do not colonise the low-copper areas. The marine paint was tested with the silica coatings in a bacterial adherence assay, and the results are reported in Chapter 5 (Sections 5.3-4).

(a)

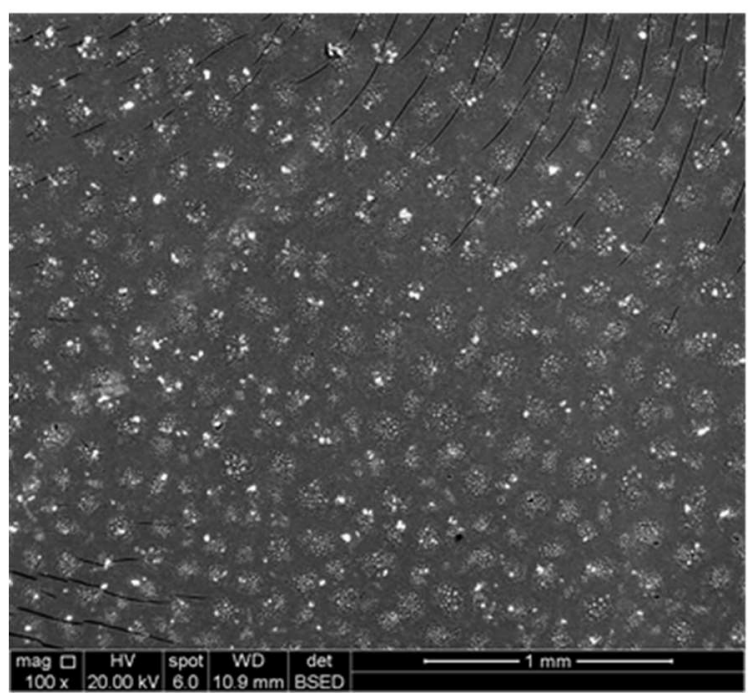

(b)

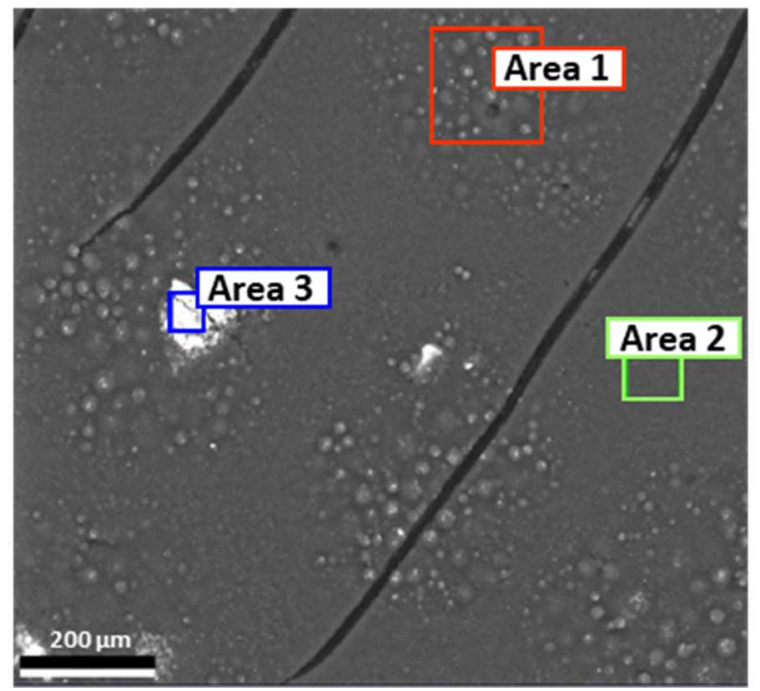

Figure 4.21. SEM backscatter electron images of the marine paint at (a) 100x magnification (HV $20 \mathrm{kV}$, spot size 6, WD $11 \mathrm{~mm}, \mathrm{BSED}$ ) and (b) sampling three areas for elemental analysis by EDS (white in the images $=$ areas of high copper content).

Table 4.14. EDS results for the marine paint at the sampled areas in Figure 4.21.

\begin{tabular}{lccc}
\hline Area & Element & Weight \% & Atomic \% \\
\hline 1 & Cu K & $1.5 \pm 0.2$ & $0.31 \pm 0.04$ \\
2 & Cu K & $1.3 \pm 0.2$ & $0.27 \pm 0.04$ \\
3 & Cu K & $37.4 \pm 0.9$ & $11.2 \pm 0.3$ \\
\hline
\end{tabular}




\subsection{SUMMARY AND FUTURE WORK}

In this work, a simple, optimised, reproducible method, with a similar macrocycle-loading and coppercapacity to macrocycle-functionalised silica from other studies, was developed for the synthesis of $\pm \mathrm{Cu}(\mathrm{II})-\mathrm{CnGmSiO}_{2}$. Despite the numerous examples in the literature, this chemistry was non-trivial: the challenges included characterisation and avoiding GLYMO self-condensation. In the literature, characterisation of the modified silica was, at times, incomplete or based solely on product performance. The characterisation process developed in this work (e.g. NMR spectroscopy for $\mathrm{CnGm}$, $\mathrm{CHN}$ elemental analysis and ICP-MS for $\pm \mathrm{Cu}(\mathrm{II})-\mathrm{CnGmSiO}_{2}$ ) was straightforward and allowed for confidence in the identities of the products. The literature procedures referenced also failed to mention the difficulties of working with silane coupling agents like GLYMO. For example, Barreto et al. ${ }^{259}$ recommended heating cyclen and GLYMO in toluene at reflux temperature to synthesise $\mathrm{CnGm}$. However, implementation of this protocol resulted in an intractable solid from the polymerisation of GLYMO. The developed procedure circumvented or overcame these challenges, but there are still areas for improvement.

Although the macrocycle- and copper-loadings of the $\pm \mathrm{Cu}(\mathrm{II})-\mathrm{CnGmSiO}_{2}$ synthesised via this method were within reported ranges, both were at the lower end (Table 4.12). So, future work could explore a few alterations to improve the method. First, the amount of modifier per gram silica could be increased. Second, Soxhlet extraction could be conducted in a non-aqueous solvent. Despite reassurances that hydrolysis of the modifier from the silica was unlikely during water extraction, ${ }^{207}$ it would be worthwhile to verify the claim by, instead, using a solvent like acetonitrile for the extraction. Finally, different types of silica (e.g. MSNs) could be modified with cyclen for formulation into a paint. All of these alterations may increase the macrocycle-loading and, subsequently, the copper-loading of the material, which might be essential for preventing bacterial adherence.

To test its effect on bacterial adherence, the modified silica was incorporated in a coating by application to the surface of an uncured epoxy resin on a plastic substrate. Squares coated on both sides in this manner were appraised in a bacterial adherence assay described in the following chapter. The goal was to achieve a high enough copper concentration at the surface to discourage colonisation by bacteria, which is one of the first steps of biofouling. Squares coated with the following silica materials were prepared for evaluation: Batch $4 \mathrm{CnGmSiO}_{2}$, Batch $4 \mathrm{CnGmSiO}_{2}+\mathrm{Cu}(\mathrm{II})$, Batch 2 $\mathrm{Cu}(\mathrm{II})-\mathrm{CnGmSiO}$, and unmodified, commercial silica. Bacterial adherence to these coatings was tested, and the results are reported in the following chapter (Chapter 5, Sections 5.3-4). 


\section{BACTERial AdHerence to CoAtings}

\subsection{BACKGROUND}

In Chapter 3 (Section 3.2.6), it was concluded that the method employed for determining the antimicrobial activity of coatings was inappropriate for the purposes of this project; the assay measured the effect of leachates on bacterial growth, which, although of interest, is not a measurement of surface activity. Rather, the propensity of bacteria to adhere to a coating is more directly relevant to antifouling technology. ${ }^{282}$ In the microfouling stage of biofouling, unicellular microorganisms, including bacteria, adhere to surfaces immersed in seawater and form complex biofilms. ${ }^{15}$ The control of biofilm formation is a main goal for biofouling mitigation because these biofilms produce vital cues for the settlement of larvae of sessile marine organisms. ${ }^{9}$ For an antifouling coating to be effective, colonisation of the surface by fouling organisms must be deterred. Therefore, measurements of this deterrence at the microfouling stage better-model the desired effect of antifouling coatings, and it was decided that an assay to test bacterial adherence to coatings should be developed and used for the $\mathrm{SiO}_{2} /$ epx-type coatings. However, assay development first necessitated a review of the attachment process and factors influencing adherence.

Despite extensive study, the process and mechanisms of bacterial attachment are not yet wellunderstood, but mechanistic models based on physico-chemical interactions between the bacterium and the substrate have been developed. The Derjaguin-Landau-Verwey-Overbeek (DLVO) model is a commonly used mechanistic model that describes adhesion as a two-step process: a reversible physical step followed by an irreversible molecular/cellular step. When planktonic (i.e. free-living) bacteria are transported to a conditioned surface, the DLVO model describes the reversible adsorption of cells as a balance between attractive Van der Waals forces and repulsive electrostatic interactions of the negatively charged cells and substrate. For the bacteria to remain attached, the attractive forces must be greater than the repulsive. Once the cells overcome the electrostatic repulsion and are in close contact $(<1-2 \mathrm{~nm})$ with the substrate, hydrogen bonding and dipole, ionic, and hydrophobic interactions further promote adhesion. Molecular reactions between the substrate and bacterial surface structures (e.g. flagella, fimbriae, pili) occur, making the attachment irreversible. ${ }^{283-286}$

Factors influencing attachment are the substrate, conditioning film, hydrodynamics, the medium, and the cell surface (Figure 5.1). Regarding the substrate, microbial colonisation usually increases with increasing surface roughness due to a larger surface area and protection from shear forces. Studies provide conflicting reports about the effect of surface hydrophobicity, but many conclude that the 
attachment of bacteria to hydrophobic materials proceeds more rapidly than to hydrophilic, since hydrophobic interactions between the cell surface and substrate promote adherence. ${ }^{284,287}$ The electrostatic charge, microtopography, surface free energy, vulnerability to wear, and wettability are additional properties of the substrate that impact colonisation..$^{12}$ Once the substrate is submerged in an aqueous medium, it becomes coated in polymers, and the rate and extent of bacterial attachment, as well as the species of bacteria present at the surface, depend on the chemical nature of this conditioning film. ${ }^{12,287}$

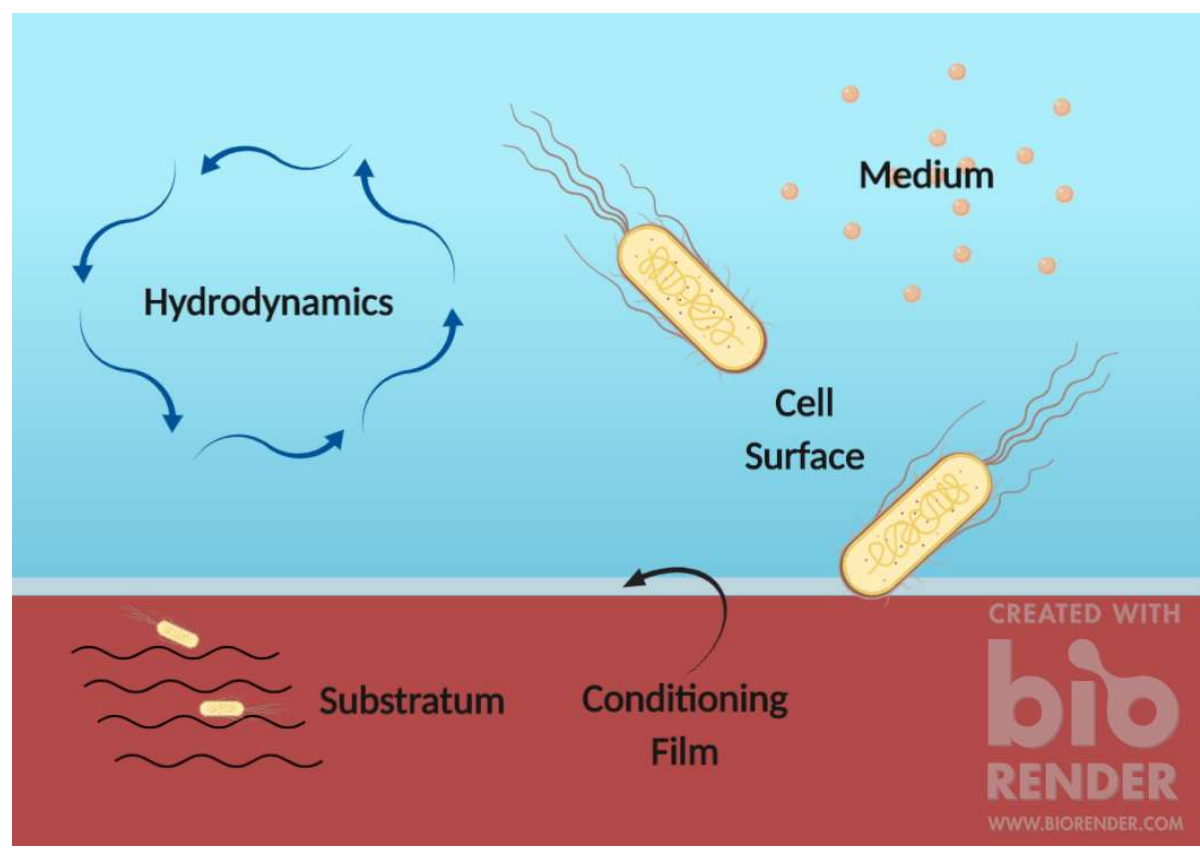

Figure 5.1. Factors that affect bacterial surface attachment (created with Biorender.com).

Transport of bacteria from the medium to the conditioned surface depends on hydrodynamics, including the velocity characteristics of the medium. An increase in velocity results in greater turbulence and mixing, and, consequently, bacteria associate with the surface rapidly until shear forces are sufficient for removal. ${ }^{287,288}$ When the velocity of the medium is low, the surface-association of bacteria is largely dependent on the size of the microorganism and its motility (i.e. presence of fimbriae and flagella). Additional characteristics of the medium that affect adherence are nutrient concentration, temperature, ionic strength, and $\mathrm{pH}$, and other important properties of the bacteria are cell surface hydrophobicity and the production of adhesins, including EPS. Although most bacteria are negatively charged, they still contain hydrophobic components on their surface (e.g. fimbriae) that promote adhesion. ${ }^{287}$ 
All of these attachment conditions may be summarised as follows: 1) surfaces are more prone to colonisation if they are rough, hydrophobic, and coated by a conditioning film; 2) the medium promotes surface adhesion with increasing velocity, temperature, and nutrient concentration, provided they do not exceed a certain threshold; and 3) fimbriae, flagella, EPS, and other adhesins are surface components of bacteria that play a significant role in attachment. ${ }^{287}$ Since the adhesion mechanisms are poorly understood and testing methodologies suffer from a lack of standardisation, it should be noted that conflicting reports do exist about the effects of many of these factors on attachment. ${ }^{284}$ For example, a study by Dewanti and Wong ${ }^{289}$ reported the promotion of biofilm formation by E. coli (serotype 0157:H7) under nutrient-limiting conditions. However, it is generally acknowledged that all of the factors addressed do play a role, so they were considered during assay development and implementation.

In order to quantify bacterial attachment to the coatings in this work, establishment of the testing framework was necessary. It was decided that E. coli NZRM 3647 (O157:H7, verocytotoxin negative) would be used in the method development of this assay due to the ease with which it can be cultured and for the sake of continuity with previous experiments (Chapter 3, Section 3.2.2). E. coli often serves as a representative Gram-negative bacterium for testing antimicrobial activity because it is easy to culture; it has a fast growth rate and simple nutritional requirements. Marine bacteria typically grow more slowly than $E$. coli, ${ }^{290}$ and, thus, the use of $E$. coli for the timely completion of method development seemed logical. However, this was done with the intent of also testing the coatings with marine bacteria once the protocol was established and validated, and marine bacterium Vibrio harveyi NZRM 2698 (ATCC 14126, NCIMB 1280, type strain) was selected. V. harveyi is a Gram-negative, bioluminescent, coastal marine bacterium that has been isolated from fouled surfaces in the marine environment and used in antifouling assays. ${ }^{290-294}$

Many antifouling assays incubate the coated substrate in a culture of bacteria under static conditions and then examine the samples for bacterial colonisation, often by enumeration of the adhered microbes. ${ }^{14,282,295-298}$ Although, under a threshold, agitation can promote adhesion, the shear forces present in a dynamic marine environment are sufficient to remove biofouling from marine objects (e.g. boats), and, to a greater extent, biofouling occurs when these objects are stationary. ${ }^{299}$ Therefore, the assay in the present work was designed so that plastic squares coated on both sides with the potential antifouling coating would be submerged in the liquid bacterial culture without agitation, allowing cells time to adhere before enumeration. This was modelled after the work by Mendonça et al. ${ }^{300}$ in which the adhesion of E. coli O157:H7 to stainless steel and poly(vinyl chloride) covered by thick or thin cloths was tested by their incubation in diluted cultures of E. coli, removal, 
washing, transfer to a fresh solution, vortexing to detach cells, and enumeration of viable, adherent cells via the drop plate method.

Once the framework for the assay was established, procedural details, such as the medium, length of incubation, and processes of detachment and enumeration, were chosen based on bacterial adherence protocols in the literature. These preliminary conditions and processes were assessed during method development of the assay with E. coli NZRM 3647, but it was recognised that some of the conditions (e.g. medium) would need to be altered for testing the adherence of $V$. harveyi NZRM 2698 to coated samples. Similar E. coli attachment assays by Danese et al. ${ }^{301}$ and O'Toole et al. ${ }^{302}$ utilised M63 minimal medium as the diluent for an overnight culture of $E$. coli because adherence was promoted under the nutrient-poor conditions. ${ }^{303}$ Then, the test substrate was submerged in the diluted culture. In contrast, assays with marine bacterium $V$. harveyi required a highsalt (1-2\% w/w) medium, such as Luria marine broth (LMB) or artificial seawater. ${ }^{291,293-295,304}$ Regarding the length of incubation in the diluted culture of $E$. coli, multiple studies required incubation of the substrate for $24 \mathrm{~h}$ before rinsing it and detaching the bacteria. ${ }^{301,302,305}$ Detachment was achieved by vortexing 1-2 min and/or sonication, ${ }^{300,305-307}$ and agar plating, specifically by the drop plate method, was performed to determine the colony forming units (CFU) per $\mathrm{cm}^{2}$ of the substrate. ${ }^{300,305}$ For the drop plate method (Figure 5.2), aliquots $(10 \mu \mathrm{L}$ ) of each dilution were dispensed in small, separate drops on agar plates and then were allowed to absorb into the agar, followed by incubation of the plate and colony-counting within the drops. The countable dilution yielded 3-30 colonies per $10 \mu \mathrm{L}$ drop. ${ }^{308}$ All of these details were incorporated for establishment of the initial testing protocol, which then required experimental development and validation.

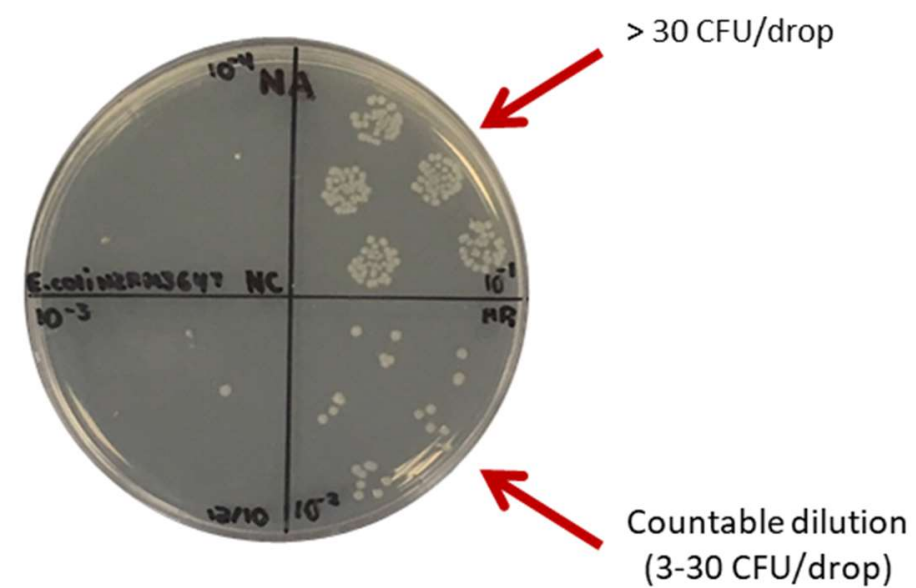

Figure 5.2. Enumeration of $E$. coli NZRM 3647 cells via the drop plate method. 


\subsection{METHOD DEVELOPMENT WITH E. COLI NZRM 3647}

In summary, the proposed bacterial adherence assay (Figure 5.3), which is a composite of the processes and conditions in multiple literature procedures, ${ }^{300-302,305-308}$ would begin with dilution to $\mathrm{OD}_{600} 0.05$ of an overnight (16 h) culture of E. coli NZRM 3647 in M63 minimal medium. As in Chapter 4 (Section 4.3), the coated, plastic test square $(23 \mathrm{~mm} \times 23 \mathrm{~mm}$ or $25 \mathrm{~mm} \times 25 \mathrm{~mm}$ ) would be prepared by applying a single layer of the test coating via the draw-down technique to both sides of a vinyl chloride/acetate copolymer, allowing each side to cure overnight, and cutting out a square to the desired dimensions. This would be followed by submersion of the prepared square in the diluted culture and static incubation for $24 \mathrm{~h}$ at $37^{\circ} \mathrm{C}$ to allow the bacteria time to adhere. Then, the square would be taken from the culture, washed with saline (i.e. $0.85 \% \mathrm{w} / \mathrm{w}$ aq $\mathrm{NaCl}$ ) to remove the planktonic cells, and transferred to a saline solution containing $0.05 \% \mathrm{v} / \mathrm{v}$ Tween 20 . Tween 20 is a nonionic surfactant that is included to prevent bacterial agglomeration. ${ }^{309}$ The solution would be vortexed and sonicated to detach the bacteria adhered to the square, and serial dilutions of the detachment solution, followed by the drop plate method, would be completed to determine the $\mathrm{CFU} / \mathrm{cm}^{2}$ on the test square. For a coating to be deemed bactericidal (i.e. kills bacteria), $\mathrm{a} \geq 3 \log _{10}$ reduction in the $\mathrm{CFU} / \mathrm{cm}^{2}$ is required. ${ }^{310}$

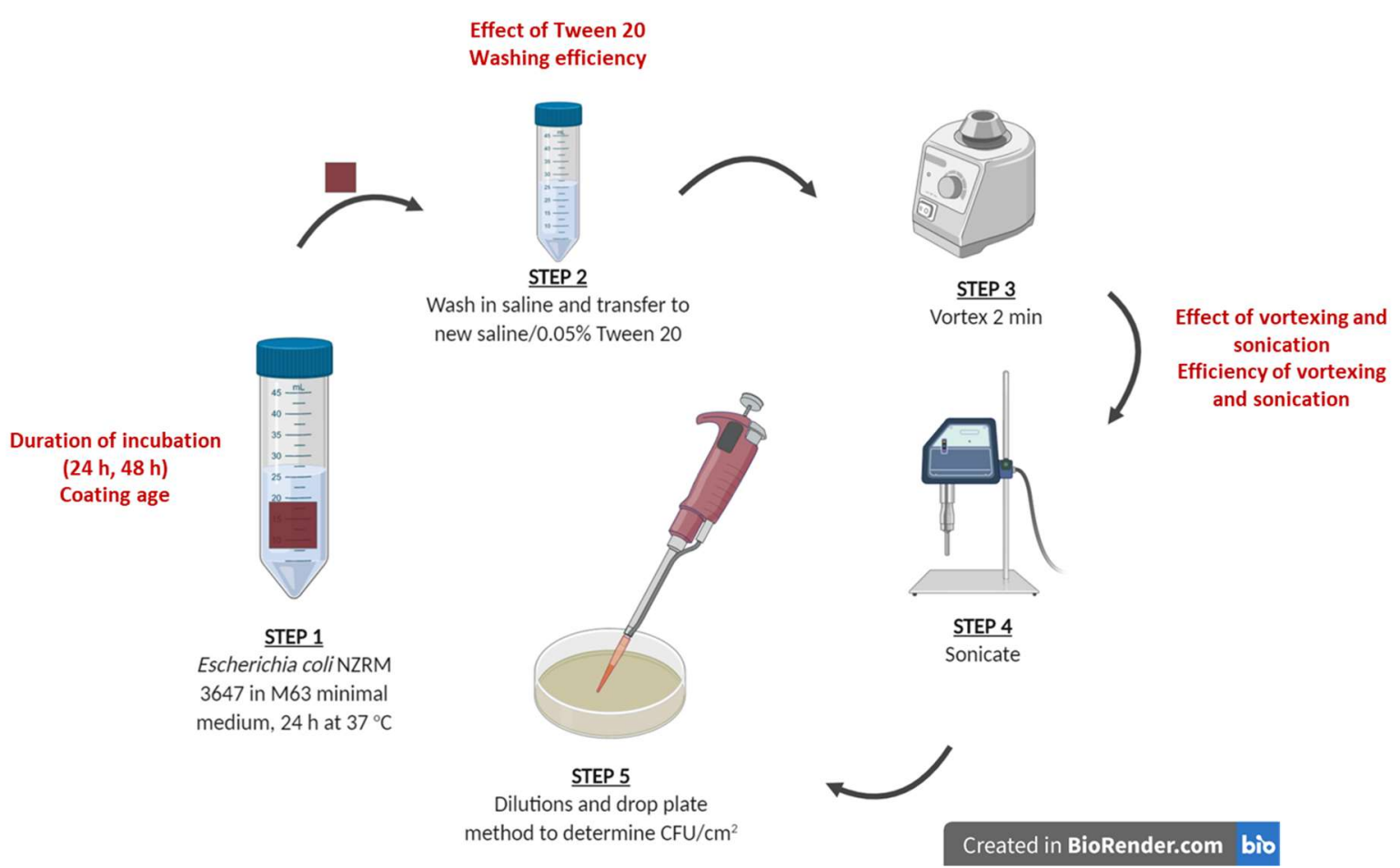

Figure 5.3. Steps of the bacterial adherence assay and, in red, areas to be addressed for method development (created with Biorender.com). 
The goal of method development was to optimise conditions for the attachment and recovery of viable, adherent cells so that bactericidal effects would be detectable. In order to develop and validate this method, the following aspects of the protocol, shown in red in Figure 5.3, were evaluated:

1) The duration of incubation of the test square in the bacterial culture ( $24 \mathrm{~h}$ vs $48 \mathrm{~h}$ ),

2) The effect of coating age on bacterial adherence,

3) The effect of Tween 20 on bacteria,

4) The efficiency of the saline wash in removing planktonic bacteria,

5) The combined effect of vortexing and sonication on bacteria, and

6) The combined efficiency of vortexing and sonication ( \pm Tween 20 ) for the detachment of bacteria.

\subsubsection{Duration of Incubation}

Although multiple studies set the incubation period of the test sample in the diluted $E$. coli culture at $24 \mathrm{~h}$, most of those studies also allowed for the extension of this time period to $\geq 48 \mathrm{~h}$. ${ }^{301,302,305}$ Therefore, in order to assess the difference in the numbers of viable, adherent cells after $24 \mathrm{~h}$ vs $48 \mathrm{~h}$, an experiment was conducted in which one uncoated square was incubated for $24 \mathrm{~h}$ and the other for $48 \mathrm{~h}$ (Figure 5.4a). Uncoated squares were used because they had the least variability between prepared samples. Following incubation, both squares were rinsed, and the adherent cells were enumerated.

(a)

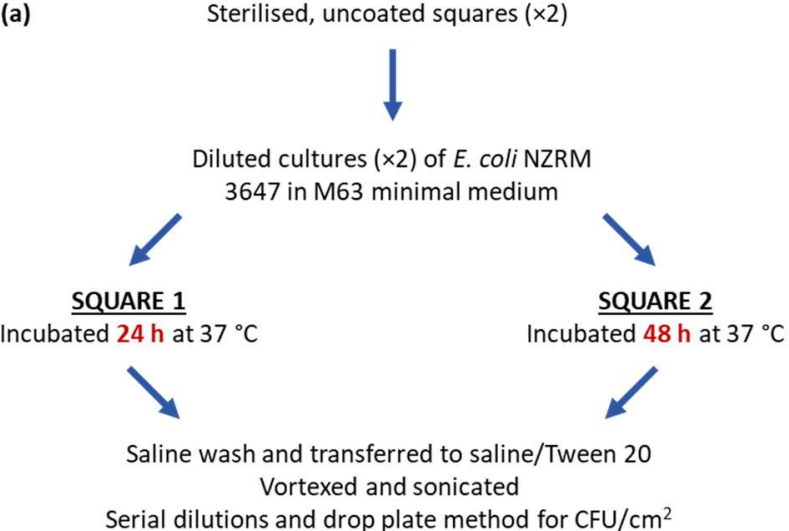

(b)

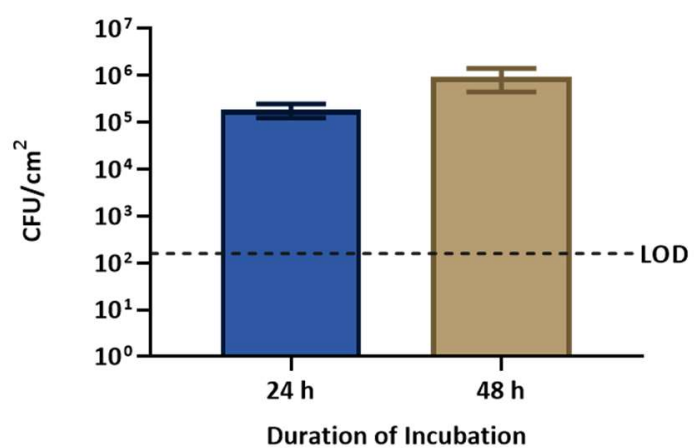

Figure 5.4. Testing the effect of the duration of incubation on bacterial adherence ( $24 h$ vs $48 h$ ): (a) procedure and (b) results of CFU/ $\mathrm{cm}^{2}$ on uncoated squares obtained after incubation for $24 \mathrm{~h}$ and $48 \mathrm{~h}$. The bars represent the mean of five technical replicates (tr), and the error bars show the SD. The limit of detection (LOD) is $160 \mathrm{CFU} / \mathrm{cm}^{2}$. 
As shown in Figure 5.4b, incubation of the uncoated square for $48 \mathrm{~h}$ yielded an approximately fivefold increase in the number of adherent cells, compared to incubation for $24 \mathrm{~h}$. Because the increase was $<1 \log _{10}$ over an additional $24 \mathrm{~h}$, it was decided that the total incubation time should remain at $24 \mathrm{~h}$. The shorter assay was more practical, since the turnaround time was quicker, and lengthening the incubation time did not seem to drastically increase the number of attached cells. Growth curves of E. coli NZRM 3647 in nutrient broth and M63 minimal medium revealed that the bacteria were in the stationary phase after $14 \mathrm{~h}$ and $20 \mathrm{~h}$, respectively (Appendix, Figure 8.52).

\subsubsection{Coating Age}

It was acknowledged that variability in the coating age could confound the assay results, given that fresh coatings are likely to be more antimicrobial than older coatings. Fresh coatings contain potential antimicrobial ingredients that wash out, evaporate, or wear away over time. ${ }^{207}$ To determine if the coating age was a parameter in the adherence assay that should be kept constant, fresh and twoweek-old coatings were tested and the results compared. The coatings assessed were the same ones from Chapter 3: the marine paint (Section 3.2.2; Altex Yacht and Boat Paint, Aurora Red, Ablative Antifouling No. 5; 40-50\% $\mathrm{Cu}_{2} \mathrm{O}$ ), the commercial, two-pot epoxy resin (Section 3.3; Epikote ${ }^{\mathrm{TM}} 235$ and Ancamine $^{\circledR}$ 2459), and the commercial acrylic paint (Section 3.2.1; LR200, Resene Paints Ltd.). Uncoated squares served as controls. The epoxy resin was included because it was used to prepare the $\mathrm{SiO}_{2}$ /epx-type coatings in Chapter 4 (Section 4.3), and the marine paint was included because it was the commercial antifouling standard to which the coatings produced in this work were compared. Following the usual procedure (Figure 5.5a), the experiment was repeated three times, and the viable, adherent cells on the fresh and two-week-old test squares were enumerated.

The results revealed no statistically significant differences between the average numbers of viable, adherent bacteria per $\mathrm{cm}^{2}$ on the fresh versus the two-week-old coatings (Figure 5.5b), even when the data were normalised by the results for the uncoated squares (Figure 5.5c). However, for all samples, a trend was observable in which the numbers of CFUs on the two-week-old coatings were $1 \log _{10}$ higher than on the fresh coatings. This was consistent with the expectation that the fresh coatings would be more antimicrobial and affirmed that the coating age could be a confounding factor if not controlled. Therefore, it was decided that bacterial adherence to the coatings should be measured immediately after overnight curing, since the shorter coating preparation time was preferable. However, it should be kept in mind that the antifouling activity of such coatings may decrease over time. 
(a)

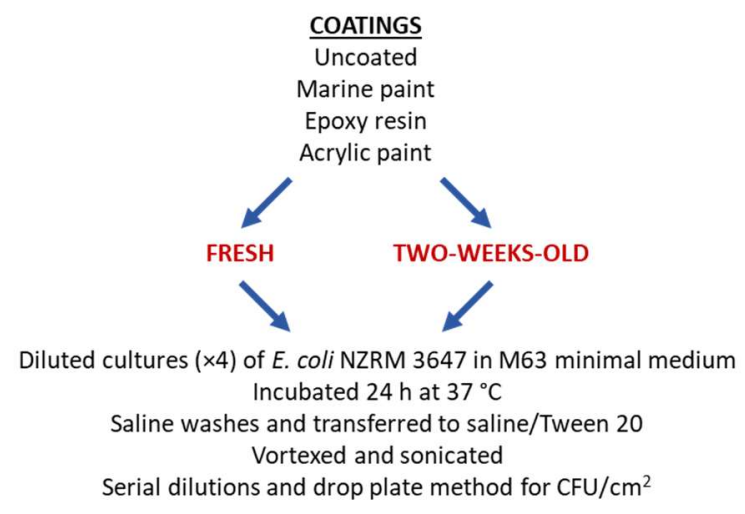

(b)

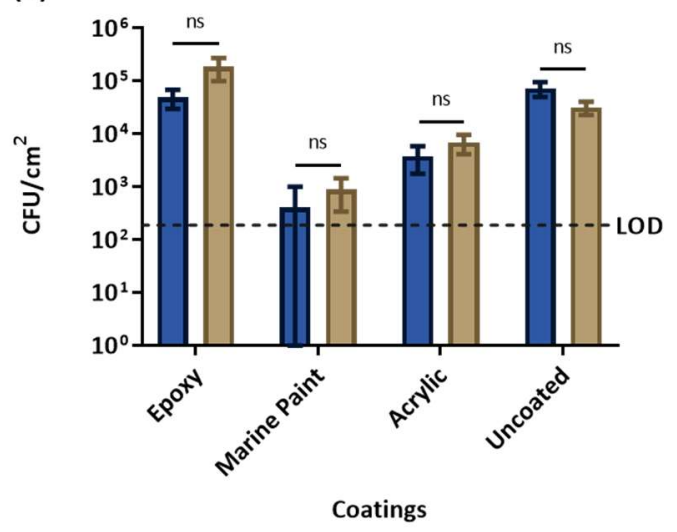

(c)

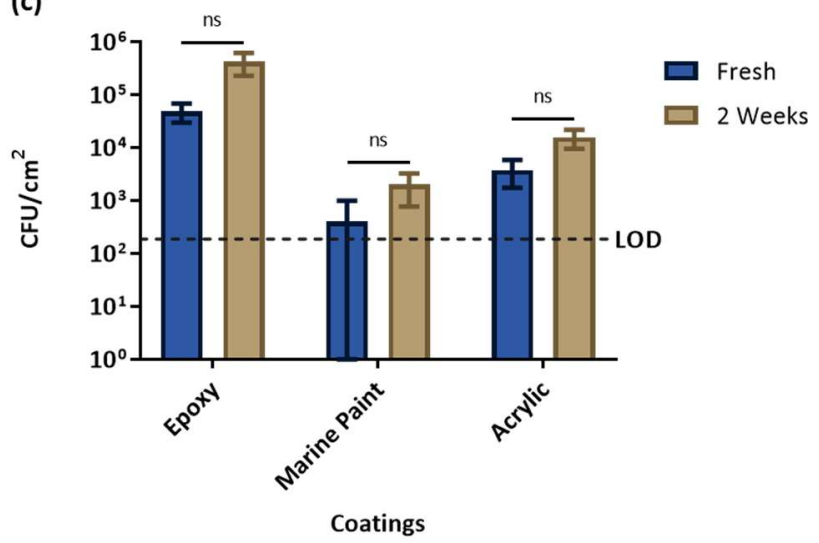

Figure 5.5. Testing the effect of coating age on bacterial adherence: (a) procedure and (b)/(c) results of $\mathrm{CFU} / \mathrm{cm}^{2}$ on fresh and two-week-old test squares coated with the epoxy resin, marine paint, and acrylic paint. Uncoated squares served as controls. (b) Un-normalised data and (c) data normalised by the "Uncoated" results. The bars represent the mean of three biological replicates (br), and the error bars show the SD. To determine if the means from the "Fresh" and "2 Weeks" data sets were significantly different from each other, a two-sample, two-tail Student's $t$-test was conducted, and the difference was considered to be significant when the $p$-value was $<0.05$ (ns = not significant; LOD = $\left.189 \mathrm{CFU} / \mathrm{cm}^{2}\right)$.

\subsubsection{Effect of Tween 20}

The addition of nonionic detergent Tween 20 (T20) to the detachment solution was recommended as standard practice to disaggregate the bacteria prior to agar plating, ${ }^{311}$ thus ensuring that each bacterial colony represented one CFU. So, the inclusion of Tween 20 was assessed to confirm that it would not negatively affect the assay results. The concern was that bacteria already stressed by Tween 20 would be killed by subsequent detachment methods, since the use of Tween 20 at or above a concentration of $0.1 \% \mathrm{v} / \mathrm{v}$ has been shown to impair bacterial growth. ${ }^{309}$ Consequently, it was 
recommended that Tween 20 be included at $0.05 \% \mathrm{v} / \mathrm{v},{ }^{311}$ and an experiment was performed to determine the effect of Tween 20 in the detachment solution on the assay results. This was done by incubating two uncoated squares in diluted cultures of E. coli, rinsing, and transferring them to detachment solutions with or without $0.05 \% \mathrm{v} / \mathrm{v}$ Tween 20 , followed by vortexing and agar plating (Figure 5.6a). The results showed no difference in the number of viable bacteria attached to the squares, whether or not Tween 20 was used in the detachment solution (Figure 5.6b). Therefore, the inclusion of $0.05 \% \mathrm{v} / \mathrm{v}$ Tween 20 did not negatively impact the assay results, and its addition to the detachment solutions continued. Although, it acknowledged that, when testing antimicrobial surfaces, it would be important to confirm that Tween 20 did not amplify the antibacterial effect, as the cells exposed to the coating would likely be more fragile and vulnerable to lysis.

(a)

Sterilised, uncoated squares $(\times 2)$

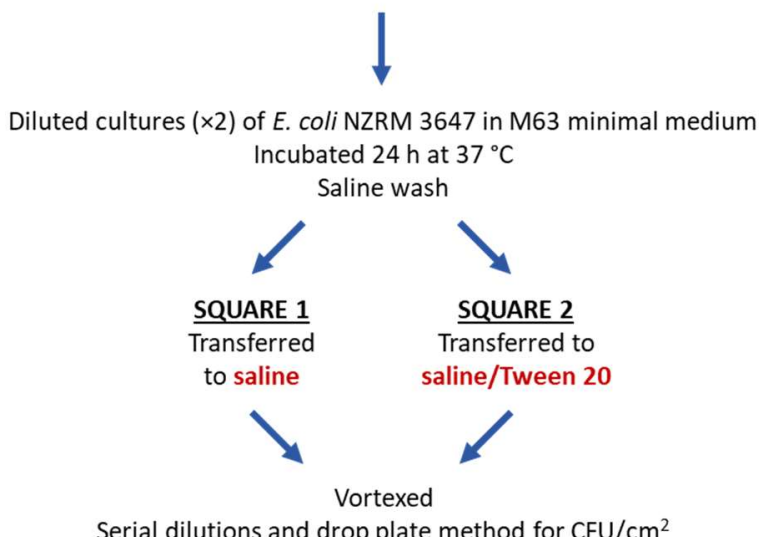

(b)

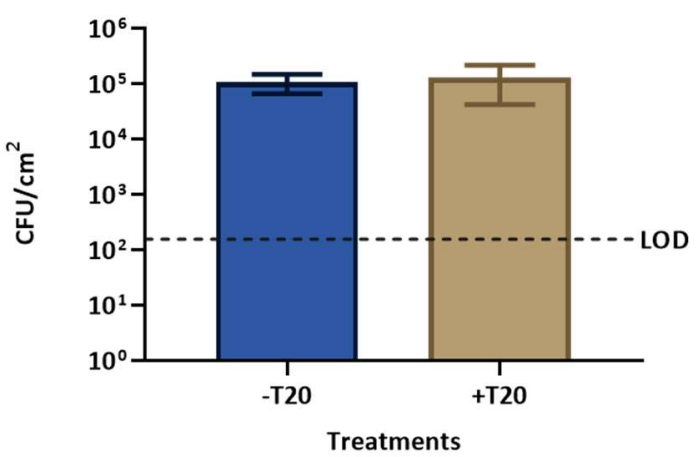

Figure 5.6. Testing the effect of Tween 20 in the bacterial adherence assay: (a) procedure and (b) results of $\mathrm{CFU} / \mathrm{cm}^{2}$ on uncoated squares obtained after detachment in solutions with and without $0.05 \% \mathrm{v} / \mathrm{v}$ Tween 20 . The bars represent the mean $(\operatorname{tr}=5) \pm S D\left(L O D=160 \mathrm{CFU} / \mathrm{cm}^{2}\right)$.

\subsubsection{Washing Efficiency}

In the assay's proposed protocol, the test squares are washed with saline to remove planktonic $E$. coli, leaving only the adherent cells. Thus, an experiment was performed to assess the washing efficiency via the enumeration of cells in successive saline washes of an uncoated sample, comparing the counts to those obtained after vortexing and sonicating the sample for the detachment of adherent cells. It was thought that the counts from the washes would be from planktonic cells carried over from the $24 \mathrm{~h}$ culture in M63 minimal medium and from some cells detaching from the surface due to the shear forces experienced by the sample upon delivery and removal from each saline solution. It was also thought that, if the samples were subjected to multiple rinses before bacterial detachment, higher 
CFUs in the detachment solution than in the previous wash would confirm that bacteria were remaining attached to the substrate throughout the short washes. The uncoated sample was incubated in a diluted culture of $E$. coli in M63 minimal medium and, after $24 \mathrm{~h}$, transferred to the first saline wash (Figure 5.7a). It was transferred to a second saline wash and then to the saline/Tween 20 solution for the detachment of cells via vortexing and sonication.

(a)

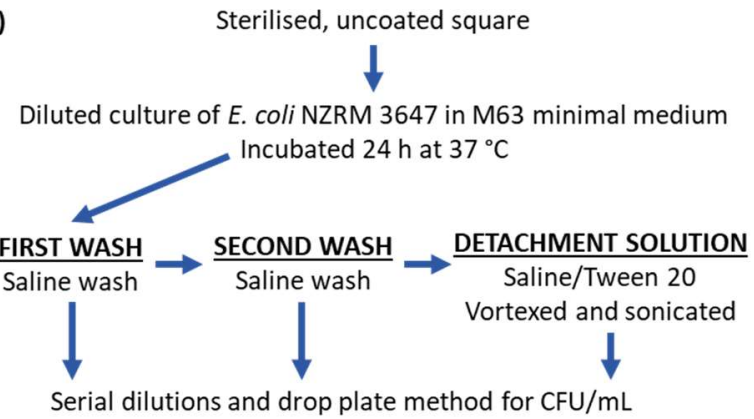

(b)

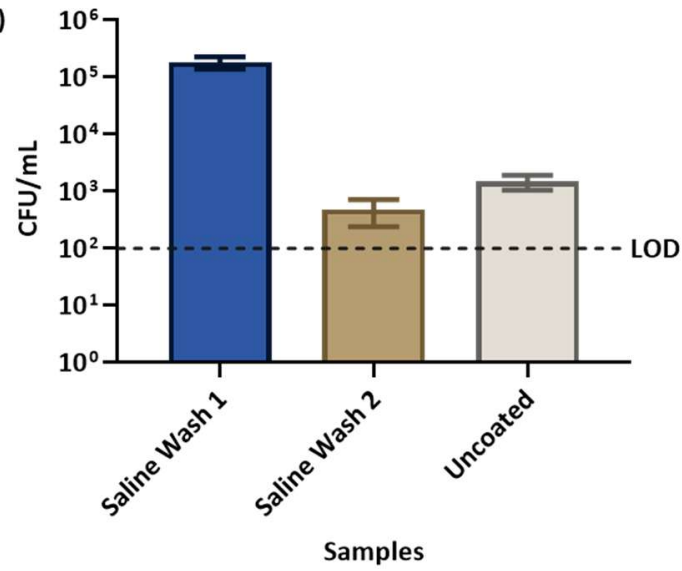

Figure 5.7. Enumeration of bacterial counts in the saline washes: (a) procedure and (b) results of $\mathrm{CFU} / \mathrm{mL}$ in saline washes and the detachment solution containing an uncoated square. The bars represent the mean $(\operatorname{tr}=5) \pm S D(L O D=100 \mathrm{CFU} / \mathrm{mL})$.

The cell counts in the detachment solution containing the uncoated sample were an order of magnitude higher than the counts in the second wash (Figure 5.7b). "Saline Wash 2" contained only $\sim 500 \mathrm{CFU} / \mathrm{mL}$, which was $2-3 \log _{10}$ fewer cells than "Saline Wash 1". This meant that few cells were carried over from the first wash or detached from the sample in the second wash, and it was decided that one wash in the assay was sufficient. Cells remained adhered to the substrate through multiple, short saline rinses until detachment by vortexing and sonication, which confirmed that the assay was working as intended. Repetition of the experiment with an $\mathrm{SiO}_{2} / \mathrm{epx}$-type coating, which has very different physico-chemical properties, yielded similar results (Appendix, Figure 8.53). A certain background count of CFUs from the carryover of planktonic cells was expected, leading to some variability in the results. However, to look for a bactericidal effect, the coating containing the biocide (i.e. $\mathrm{Cu}(\mathrm{II}))$ was to be compared to the control (i.e. coating without $\mathrm{Cu}(\mathrm{II})$ ) and the industry-standard marine paint. A successful coating would need a $\geq 3 \log _{10}$ reduction in the $\mathrm{CFU} / \mathrm{cm}^{2}$, compared to the control, or perform similarly to the marine paint. Therefore, it was reasoned that such large differences would still be apparent, despite the variability of the assay. 


\subsubsection{Effect of Vortexing and Sonication}

Since vortexing (V) plus sonication (S) was proposed as a detachment method in the adherence assay, an experiment was completed to test its effect on planktonic $E$. coli. This was done to confirm that the combined technique of vortexing for $2 \mathrm{~min}$ and sonicating for $8 \mathrm{~s}$ did not negatively affect cell viability and, thus, skew bacterial counts. The sonication conditions were derived from a study by Merritt et al. ${ }^{306}$ ( $8 \mathrm{~s}, 40 \%$ amplitude). In the present experiment, a diluted culture of $E$. coli was incubated $24 \mathrm{~h}$, an aliquot was removed, and then the culture was vortexed and sonicated (Figure 5.8a). The culture and the aliquot taken before vortexing and sonication were serially diluted and plated. The means (CFU/mL) from the two data sets were within one standard deviation of each other (Figure 5.8b), and it was concluded that the combined effect of vortexing and sonication on the viability of planktonic cells in the $24 \mathrm{~h}$ culture was likely neutral. Like Tween 20, however, it was recognised that exposure to an antimicrobial surface could predispose the bacterial cells to injury from vortexing and sonication.

(a)

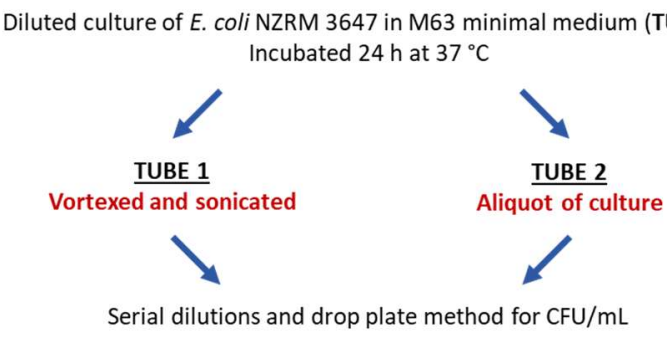

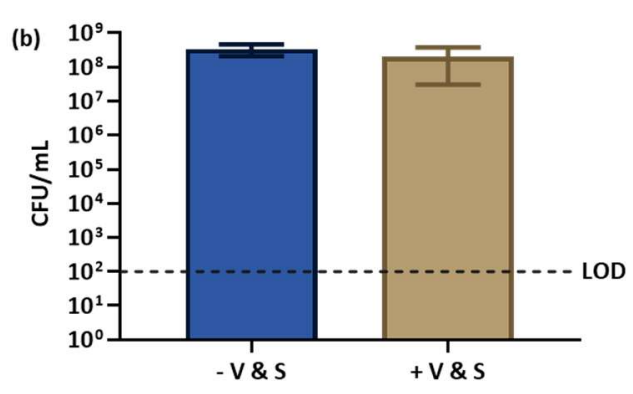

Treatments

Figure 5.8. Testing the combined effect of vortexing and sonication on planktonic bacteria: (a) procedure and (b) results of CFU/mL in a vortexed and sonicated culture (" $+\mathrm{V} \& \mathrm{~S}$ ") and a culture that was neither vortexed nor sonicated (“-V \& S"). The bars represent the mean $(\operatorname{tr}=10) \pm S D(L O D=$ $100 \mathrm{CFU} / \mathrm{mL})$.

\subsubsection{Efficiency of Vortexing and Sonication ( \pm Tween 20)}

In addition to the effect of vortexing plus sonication on planktonic bacteria, the efficiency of the technique as a detachment method was considered. The detachment methods were evaluated by comparing the numbers of viable, adherent bacteria recovered after vortexing and vortexing plus sonication to the control, which was neither vortexed nor sonicated. Three uncoated squares were incubated in dilute cultures of E. coli, washed, and transferred to solutions of saline/Tween 20 
(Figure 5.9a). The first square received no further treatment ("Control": "-V \& S"), but the second square was vortexed ("Vortex $1 x^{\prime \prime}:$ " $V 1 \times$ "), removed, transferred to a fresh saline/Tween 20 solution, and vortexed again ("Vortex $2 x^{\prime \prime}:$ " $+\vee 2 x$ "). The second round of vortexing was done to ascertain if vortexing once was sufficient to remove the vast majority of adherent cells. The third square was both vortexed and sonicated ("Vortex \& Sonicate": "+V \& S"). The "Control", "Vortex 1x", "Vortex 2x", and "Vortex \& Sonicate" solutions were serially diluted and plated to count colonies.

(a)

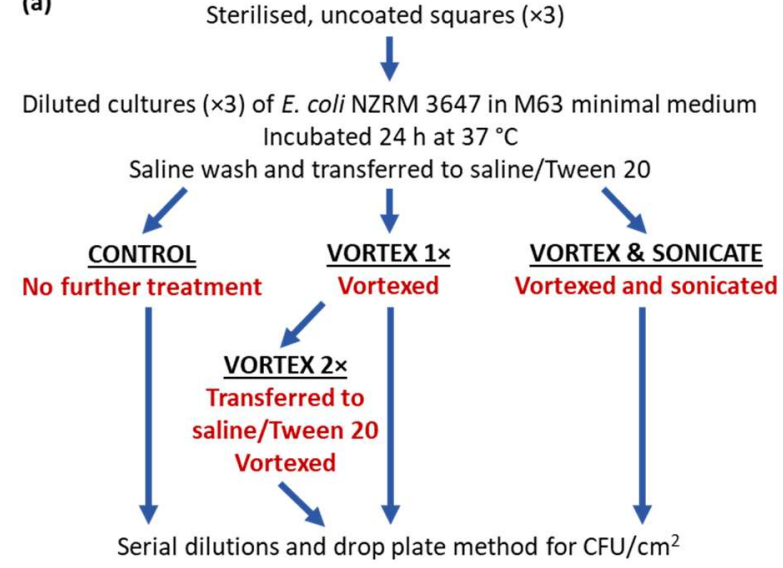

(b)

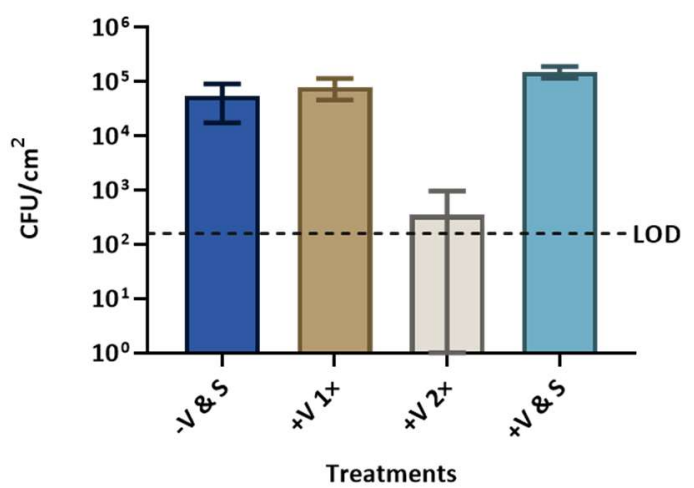

Figure 5.9. Testing the efficiency of vortexing and sonication with Tween 20 in the removal of adherent bacteria from uncoated squares: (a) procedure and (b) results of $\mathrm{CFU} / \mathrm{cm}^{2}$ on uncoated squares obtained after vortexing (once, " $+V 1 x^{\prime \prime} ;$ twice, " $+V 2 x$ "), vortexing and sonicating ("+V \& $S$ "), and neither vortexing nor sonicating ("-V \& S"). The bars represent the mean $(\operatorname{tr}=5) \pm S D(L O D=$ $\left.160 \mathrm{CFU} / \mathrm{cm}^{2}\right)$.

The number of CFUs in the "Vortex $2 x$ " solution was at the assay's limit of detection, meaning that the second round of vortexing yielded relatively few additional counts. Vortexing once plus sonication yielded higher average counts than vortexing alone, which yielded higher average counts than the control (Figure 5.9b). However, the experiment needed to be repeated with biological replicates to identify any truly significant differences. Regarding the control, a considerable number of bacteria were detached just upon transference of the square to saline/Tween 20. Consequently, a role for Tween 20 in detachment was postulated, and an experiment was conducted to elucidate the true effects of vortexing plus sonication and Tween 20.

An experiment was completed to determine if differences in bacterial counts after vortexing plus sonication with or without Tween 20 were statistically significant. This was done by incubating three uncoated squares in diluted cultures of $E$. coli in M63 minimal medium, and, after incubation, washing 
them with saline (Figure 5.10a). Then, the first and second squares were transferred to saline/Tween 20 solutions, and the first square was vortexed and sonicated. The third square was delivered to a fresh saline solution. All square-containing solutions were serially diluted, and the dilutions were plated.

(a)

Sterilised, uncoated squares $(\times 3)$

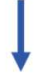

Diluted cultures $(\times 3)$ of $E$. coli NZRM 3647 in M63 minimal medium Incubated $24 \mathrm{~h}$ at $37^{\circ} \mathrm{C}$

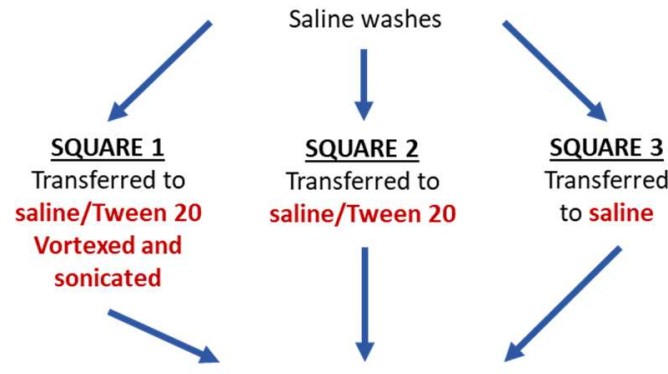

Serial dilutions and drop plate method for $\mathrm{CFU} / \mathrm{cm}^{2}$ (b)

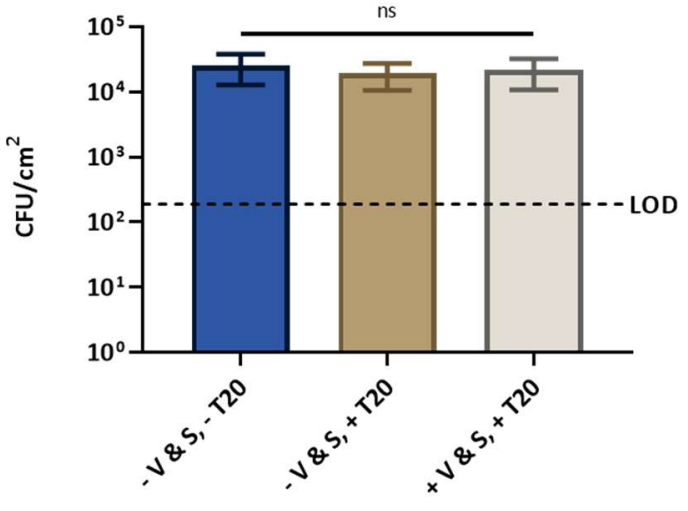

Treatments

Figure 5.10. Testing the efficiency of vortexing and sonication with or without Tween $\mathbf{2 0}$ in the removal of adherent bacteria from uncoated squares: (a) procedure and (b) results of $\mathrm{CFU} / \mathrm{cm}^{2}$ on uncoated squares obtained under the conditions of \pm vortexing and sonication ("V \& S") and \pm Tween 20 ("T20"). The bars represent the mean ( $b r=6$ for " $-V \& S,+T 20$ ", br $=3$ for all others) \pm SD. To determine if the means from the data sets were significantly different from each other, a one-way analysis of variance (ANOVA) with Tukey's post hoc test was conducted, and the difference was considered to be significant when the $p$-value was $<0.05$ ( $\mathrm{ns}=$ not significant; LOD $=189 \mathrm{CFU} / \mathrm{cm}^{2}$ ).

The differences in the viable counts between the three solutions were not statistically significant (Figure 5.10b). This meant that vortexing and sonicating an uncoated square in saline/Tween 20 yielded the same counts as from simply transferring the square to saline or saline/Tween 20, which was surprising. The experimental design was based on the premise that $E$. coli would attach to the squares within $24 \mathrm{~h}$ and require removal via vortexing and, possibly, sonication, and, as seen in a previous experiment (Figure 5.7b), cells remained attached to an uncoated sample through multiple washes until their removal by vortexing and sonication. It was hypothesised that $E$. coli does not strongly adhere to the vinyl chloride/acetate copolymer and that the cells easily detach from the surface. The cells could have detached upon delivery of the uncoated sample to the detachment solution, during the prolonged time period between delivery and dilutions $(0.5-1 \mathrm{~h})$, or from gentle inversion of the tube for the homogeneous distribution of cells before serial dilutions. This experiment 
was repeated, in part, with a coated sample to determine if a substrate with different physicochemical properties would require vortexing and sonication for cellular detachment.

To test if vortexing plus sonication and Tween 20 aid the detachment of cells from a coated substrate, the same experiment (Figure 5.10) was repeated for samples coated with $\mathrm{SiO}_{2} / \mathrm{epx}$ (Experimental, Section 6.4.2). The rough $\mathrm{SiO}_{2} / \mathrm{epx}$ coating was chosen because of its vastly different physico-chemical properties, compared to the smooth, uncoated vinyl chloride/acetate copolymer. Two $\mathrm{SiO}_{2} / \mathrm{epx}-$ coated squares were incubated in diluted $E$. coli cultures in M63 minimal medium, and, after $24 \mathrm{~h}$, the squares were rinsed with saline (Figure 5.11a). The first square was transferred to a saline/Tween 20 solution, vortexed, and sonicated, and the second square was delivered to fresh saline and gently inverted for the homogeneous distribution of cells. The cell counts in both solutions were enumerated.

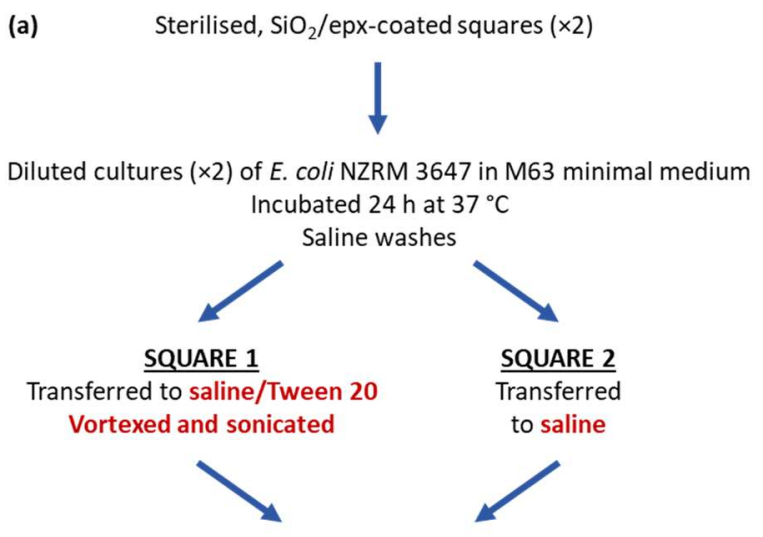

Serial dilutions and drop plate method for $\mathrm{CFU} / \mathrm{cm}^{2}$ (b)

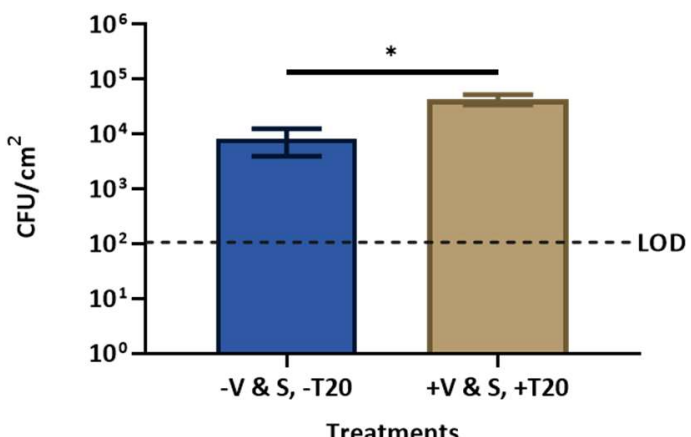

Treatments

Figure 5.11. Testing the efficiency of vortexing and sonication with or without Tween 20 in the removal of adherent bacteria from $\mathrm{SiO}_{2} /$ epx-coated squares: (a) procedure and (b) results of $\mathrm{CFU} / \mathrm{cm}^{2}$ on $\mathrm{SiO}_{2} /$ epx-coated squares obtained under the conditions of \pm vortexing and sonication ("V \& S") and \pm Tween 20 ("T20"). The bars represent the mean $(b r=3) \pm S D$. To determine if the means from the data sets were significantly different from each other, a two-sample, two-tail Student's $t$-test was conducted, and the difference was considered to be significant when the $p$-value was $<0.05\left(* p<0.05 ; \mathrm{LOD}=106 \mathrm{CFU} / \mathrm{cm}^{2}\right)$.

As shown in Figure $\mathbf{5 . 1 1 b}$, the average CFUs in the solution containing the first square (" $\mathrm{V} \& \mathrm{~S},+\mathrm{T} 2 \mathrm{C}^{\prime \prime}$ ) were an order of magnitude higher than the average CFUs in the solution containing the second square ("-V \& S, -T20"), and the difference in counts between the two was statistically significant $(p<0.05)$. This indicated that, unlike the uncoated sample, vortexing and sonicating the saline/Tween 20 solution 
aided the detachment of cells from the $\mathrm{SiO}_{2} / \mathrm{epx}$-coated samples. It is unknown if this was due to more and/or stronger interactions at the molecular level between the surface and bacteria or if the rough surface topography simply protected cells from removal by shear forces. In either case, it was decided that the use of vortexing plus sonication as a detachment method and Tween 20 in the detachment solution should be continued.

\subsubsection{Conclusions}

Multiple aspects of the designed procedure were probed for development and validation (Figure 5.3). It was decided that $24 \mathrm{~h}$ was an adequate incubation period and that the coatings should be tested directly after application to the substrate. Yet, further testing would be necessary to determine the antifouling efficacy of each coating over time, as it is likely to decrease. Assessment of washing efficiency revealed that one sample wash was adequate but that a certain background count from planktonic cells was expected. However, it was thought that they would make a relatively insignificant contribution to the total, depending on the order of magnitude of the result. In this experiment, it was also demonstrated that cells adhered to the substrate and remained attached during multiple rinses, verifying that, to a certain extent, the assay was working as anticipated. When used in the assay, the effect of Tween 20 was neutral, and the combination of vortexing and sonication did not seem to affect the viability of planktonic cells in the $24 \mathrm{~h}$ culture.

It appeared that vortexing and sonication were unnecessary for bacterial detachment from uncoated samples: there was not a statistically significant difference in counts between the uncoated sample that was vortexed and sonicated in saline/Tween 20 and the uncoated sample that was simply transferred to the detachment solution, with or without Tween 20. In order to evaluate adherence to a substrate with different physico-chemical properties, the experiment was repeated with an $\mathrm{SiO}_{2}$ /epx-coated sample, comparing samples with and without vortexing plus sonication and Tween 20. The significantly higher counts recovered from the vortexed and sonicated sample in Tween 20 suggested that, unlike for the uncoated substrate, the detachment methods were necessary for the removal of adherent cells from the rough $\mathrm{SiO}_{2} / \mathrm{epx}$-coated substrate. With the completion of the method development, the refined assay protocol was used to test bacterial attachment to the $\mathrm{SiO}_{2}$ /epx-type coatings prepared in Chapter 4 (Section 4.3). 


\subsection{ADHERENCE OF E. COLI NZRM 3647 TO SIO $/$ /EPX-TYPE COATINGS}

\subsubsection{Adherence Assay}

The $\mathrm{SiO}_{2} /$ epx-coated squares (Figure 5.12, pictured right) were prepared for the bacterial adherence assay as described in Chapter 4 (Section 4.3) and tested immediately after preparation. These coatings included Batch $4 \mathrm{CnGmSiO}_{2} /$ epx (free ligand), Batch $4 \mathrm{CnGmSiO}_{2}+\mathrm{Cu}($ II)/epx (post-loaded), Batch 2 $\mathrm{Cu}(\mathrm{II})-\mathrm{CnGmSiO} / \mathrm{epx}$ (pre-loaded), and unfunctionalised $\mathrm{SiO}_{2} / \mathrm{epx}$. The adherence results were compared to the industry-standard marine paint (Altex Yacht and Boat Paint, Aurora Red, Ablative Antifouling No. $5 ; 40-50 \% \mathrm{Cu}_{2} \mathrm{O}$ ) because such paints are the current standard in antifouling technology. For the functionalised silica coatings to be viable alternatives and worthy of pursuit, they would need to have similar activity to or better activity than the marine paint, or they would need a $\geq 3 \log _{10}$ reduction in the $\mathrm{CFU} / \mathrm{cm}^{2}$ on the biocide-containing coatings (i.e. $\mathrm{CnGmSiO}_{2}+\mathrm{Cu}(\mathrm{II}) / \mathrm{epx}$, $\mathrm{Cu}(I I)-\mathrm{CnGmSiO}_{2} / \mathrm{epx}$ ), compared to the controls (i.e. epoxy, $\mathrm{SiO}_{2} / \mathrm{epx}, \mathrm{CnGmSiO}_{2} / \mathrm{epx}$ ). The attachment of bacteria to the uncoated substrate (vinyl chloride/acetate copolymer) and epoxy resin used to glue the silica to the plastic (Epikote ${ }^{\mathrm{TM}} 235$ and Ancamine ${ }^{\circledR}$ 2459; Chapter 3, Section 3.3) was also tested. Each test square was incubated for $24 \mathrm{~h}$ at $37^{\circ} \mathrm{C}$ in an overnight culture (16 h) of E. coli diluted with M63 minimal medium to $\mathrm{OD}_{600} 0.05$. After $24 \mathrm{~h}$, the squares were rinsed with saline and then transferred to saline/Tween 20 solutions in which they were vortexed and sonicated. The detachment solutions were serially diluted, and the dilutions were plated to count colonies. The experiment was independently repeated three times for each coating.

Interestingly, $\mathrm{Cu}(\mathrm{II})-\mathrm{CnGmSiO}_{2} / \mathrm{epx}$ had the greatest number of viable, adherent bacteria, and this result was statistically different from the adherence results for all of the other coatings but $\mathrm{SiO}_{2} / \mathrm{epx}$ (Figure 5.12). The number of bacteria attached to $\mathrm{Cu}(\mathrm{II})-\mathrm{CnGmSiO}_{2} / \mathrm{epx}$ was approximately six-fold higher than the number for $\mathrm{CnGmSiO}_{2} / \mathrm{epx}$ and $\mathrm{CnGmSiO}_{2}+\mathrm{Cu}(\mathrm{II}) / \mathrm{epx}$ and approximately twice the number for $\mathrm{SiO}_{2} / \mathrm{epx}$. This was unexpected, given that $\mathrm{Cu}(\mathrm{II})-\mathrm{CnGmSiO}$ had the highest $\% \mathrm{w} / \mathrm{w}$ copper (2.6 \pm 0.3; Table 5.1) out of all of the $\mathrm{SiO}_{2}$ /epx-type coatings. The counts were not significantly different between the following samples: $\mathrm{CnGmSiO}_{2} / \mathrm{epx}, \mathrm{CnGmSiO}_{2}+\mathrm{Cu}(\mathrm{II}) / \mathrm{epx}, \mathrm{SiO}_{2} / \mathrm{epx}$, epoxy resin, and uncoated. These results showed that there was no deterrence of bacterial adhesion on any of the functionalised silica coatings, including the $\mathrm{Cu}(\mathrm{II})$ biocide-containing $\mathrm{CnGmSiO}_{2}+\mathrm{Cu}(\mathrm{II}) / \mathrm{epx}$ and $\mathrm{Cu}(\mathrm{II})-\mathrm{CnGmSiO} / \mathrm{epx}$ coatings. For the marine paint, the $\mathrm{CFU} / \mathrm{cm}^{2}( \pm \mathrm{SD})$ was at the LOD of the assay, and none of the other coatings had comparable activity. 


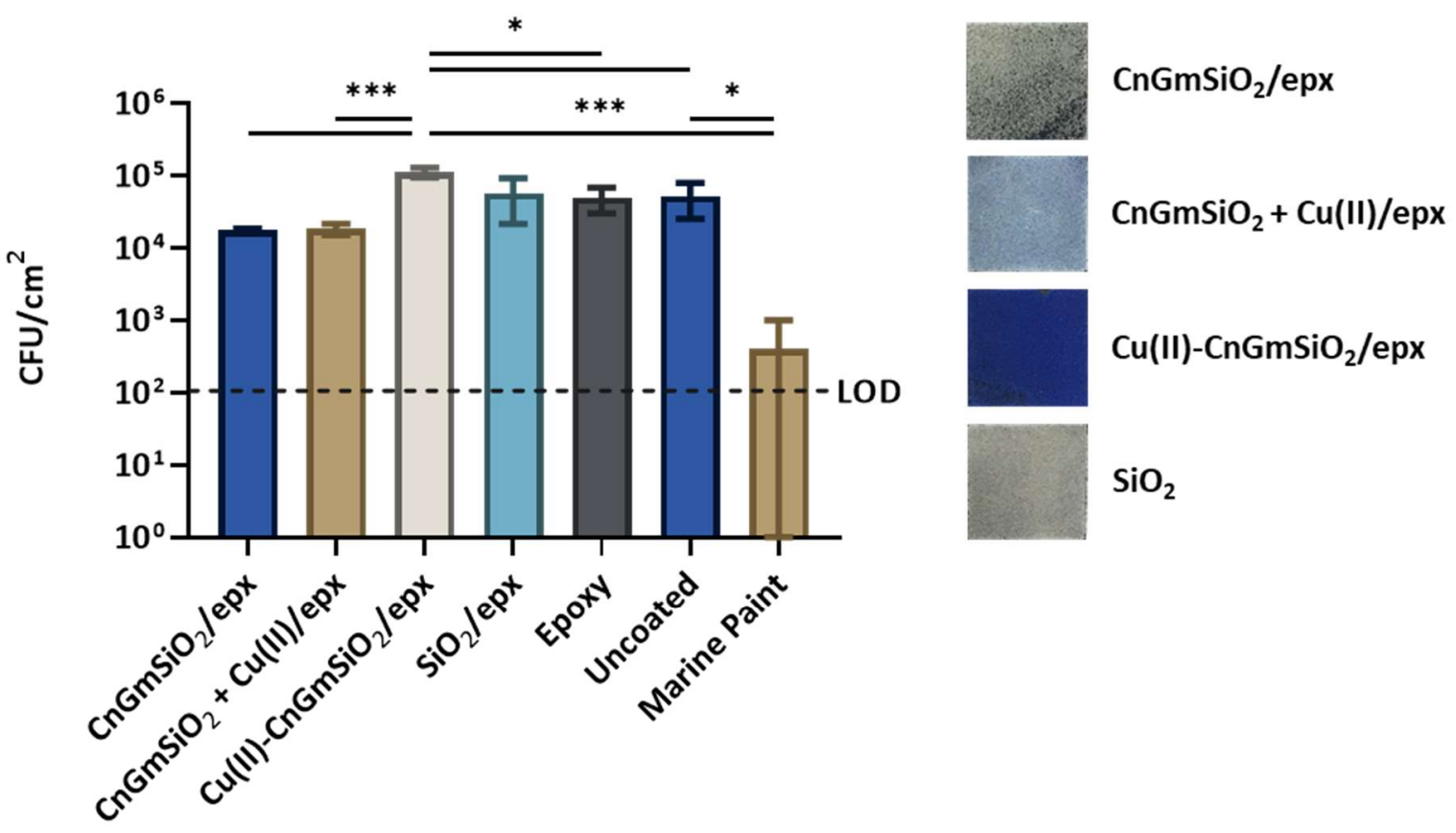

Coatings

Figure 5.12. Results of $E$. coli NZRM $3647 \mathrm{CFU} / \mathrm{cm}^{2}$ on the $\mathrm{SiO}_{2} /$ epx-type coatings (pictured right), as well as on uncoated squares, the epoxy resin, and the marine paint. The bars represent the mean ( $b r=6$ for "Uncoated", $b r=3$ for all others) \pm SD. To determine if the means from the data sets were significantly different from each other, a one-way ANOVA with Tukey's post hoc test was conducted, and the difference was considered to be significant when the $p$-value was $<0.05\left({ }^{*} p<0.05\right.$, ${ }^{* *} p<0.01,{ }^{* * *} p<0.001 ; \mathrm{LOD}=106 \mathrm{CFU} / \mathrm{cm}^{2}$ ). 
Table 5.1. The $\mathrm{OD}_{600}$ of the $E$. coli cultures after $24 \mathrm{~h}$ incubation and the copper content of the test squares, as well as the copper-leachate concentrations after $24 \mathrm{~h}$.

\begin{tabular}{lccc}
\hline Coating & $\mathrm{OD}_{600}(24 \mathrm{~h}, \mathrm{M63})^{\mathrm{a}}$ & $\% \mathrm{w} / \mathrm{w} \mathrm{Cu}^{\mathrm{b}}$ & $\mathrm{ppm}(\boldsymbol{\mu M}){\text { Leached } \mathrm{Cu}^{\mathrm{a}}}^{\mathrm{a}}$ \\
\hline $\mathrm{CnGmSiO}_{2} / \mathrm{epx}$ & $0.119 \pm 0.007$ & 0 & - \\
$\mathrm{CnGmSiO}_{2}+\mathrm{Cu}(\mathrm{II}) / \mathrm{epx}$ & $0.057 \pm 0.003$ & $1.0 \pm 0.3^{\mathrm{a}}$ & $2.3 \pm 0.1(36 \pm 2)^{\mathrm{c}}$, \\
& & & $12 \pm 2(190 \pm 30)^{\mathrm{d}}$ \\
$\mathrm{Cu}(\mathrm{II})-\mathrm{CnGmSiO}_{2} / \mathrm{epx}$ & $0.072 \pm 0.003$ & $2.6 \pm 0.3^{\mathrm{a}}$ & $3.6 \pm 0.3(57 \pm 5)^{\mathrm{c}}$, \\
$\mathrm{SiO}_{2} / \mathrm{epx}$ & $0.116 \pm 0.004$ & 0 & $6.0 \pm 0.4(94 \pm 6)^{\mathrm{d}}$ \\
Epoxy & $0.151 \pm 0.008$ & - & - \\
Uncoated & $0.148 \pm 0.009$ & - & - \\
Marine Paint & $0.101 \pm 0.006$ & $40-50$ & $3.3 \pm 0.5(52 \pm 8)^{\mathrm{c}}$, \\
\hline
\end{tabular}

${ }^{a}$ Mean \pm SD. Experiment repeated $3 \times$.

${ }^{\mathrm{b}} \% \mathrm{w} / \mathrm{w} \mathrm{Cu}$ in the (un)functionalised silica component or in the marine paint.

${ }^{c} \mathrm{M} 63$ minimal medium without $E$. coli NZRM 3647.

${ }^{\mathrm{d}}$ M63 minimal medium with E. coli NZRM 3647.

In order to investigate possible factors impacting the adherence results, the amount of copper leaching from each copper-containing coating into the medium during the $24 \mathrm{~h}$ incubation was measured - both in the presence and absence of $E$. coli (Table 5.1). The mechanisms of the contact killing of bacteria on a copper surface are not fully understood, but there is a consensus in the literature that copper ions released from the surface play a role. ${ }^{115,117,121,312}$ It is proposed that, following damage to the bacterial cell membrane, the influx of copper ions into the cell causes oxidative damage by ROS and enzyme inhibition, leading to cell death and DNA degradation. ${ }^{115,117}$ In culture, copper toxicity is due to the displacement of iron by copper in iron-sulfur cluster dehydratases, which are essential for the branched-chain biosynthesis of amino acids. ${ }^{206}$

Given the direct relationship between bactericidal effects and copper ion concentration, the concentrations of copper leachate from $\mathrm{CnGmSiO}_{2}+\mathrm{Cu}(\mathrm{II}) / \mathrm{epx}, \mathrm{Cu}(\mathrm{II})-\mathrm{CnGmSiO}_{2} / \mathrm{epx}$, and the marine paint were determined by incubating the test squares in M63 minimal medium statically for $24 \mathrm{~h}$ at $37^{\circ} \mathrm{C}$. This experiment was conducted with and without $E$. coli in order to determine if the presence of bacteria affected copper-leaching. After $24 \mathrm{~h}$ incubation of the samples with E. coli, each culture was filter-sterilised and centrifuged, and the supernatant was isolated for analysis. All solutions were diluted, as needed, with dilute nitric acid for copper quantification by ICP-MS. Although the copper concentrations in the marine paint $(40-50 \% \mathrm{w} / \mathrm{w})$ and $\mathrm{Cu}(\mathrm{II})-\mathrm{CnGmSiO}_{2}(2.6 \pm 0.3 \% \mathrm{w} / \mathrm{w})$ were vastly different, the concentrations of copper leachate in the absence of $E$. coli were statistically equivalent: $3.3 \pm 0.5 \mathrm{ppm}(52 \pm 8 \mu \mathrm{M})$ and $3.6 \pm 0.3 \mathrm{ppm}(57 \pm 5 \mu \mathrm{M})$, respectively (Table 5.1). The concentration of copper leached from $\mathrm{CnGmSiO}_{2}+\mathrm{Cu}(\mathrm{II}) / \mathrm{epx}$ was slightly lower $(2.3 \pm 0.1 \mathrm{ppm}, 36 \pm 2 \mu \mathrm{M})$ than the other coatings when bacteria were absent. However, the presence of bacteria resulted in increased 
copper-leaching for all samples, and $\mathrm{CnGmSiO}_{2}+\mathrm{Cu}(\mathrm{II}) /$ epx leached the most copper (12 $\pm 2 \mathrm{ppm}$, $190 \pm 30 \mu \mathrm{M})$, while the marine paint $(7.3 \pm 0.4 \mathrm{ppm}, 115 \pm 6 \mu \mathrm{M})$ and $\mathrm{Cu}(\mathrm{II})-\mathrm{CnGmSiO}_{2} / \mathrm{epx}$ $(6.0 \pm 0.4 \mathrm{ppm}, 94 \pm 6 \mu \mathrm{M})$ leached similar, lower amounts.

This increase in copper-leaching in the presence of $E$. coli could be due to the uptake of $\mathrm{Cu}(I I)$ from the surface by bacteria, since cations are attracted to the negatively charged lipopolysaccharide (LPS) in E. coli's outer membrane. ${ }^{313,314}$ Some of the $\mathrm{Cu}(\mathrm{II})$ at the surface of $\mathrm{CnGmSiO}_{2}+\mathrm{Cu}(\mathrm{II}) / \mathrm{epx}$ is likely adsorbed, rather than chelated, and, thus, is more easily sequestered from the surface, leading to a higher copper-leachate concentration. Tetraaza macrocycles (e.g. cyclen) form incredibly stable $\mathrm{Cu}(\mathrm{II})$ complexes with enhanced inertness to demetallation, ${ }^{76}$ but it is possible that bacteria have a role in competition with the cyclen-functionalised surfaces (e.g. $\mathrm{Cu}(\mathrm{II})-\mathrm{CnGmSiO}_{2} / \mathrm{epx}$ ) and the subsequent release of $\mathrm{Cu}(\mathrm{II})$. Although, studies have reported the protective effects of copper-chelators, such as EDTA, in the context of the contact killing of bacteria. ${ }^{115,314}$ Furthermore, even if the bacteria sequestered $\mathrm{Cu}(\mathrm{II})$, those bacteria were removed from solution prior to copper analysis by ICP-MS, and it is unknown if the copper would have been released back into solution before the removal of bacteria. Therefore, the cause of the increased leaching was not easily determined.

Comparison of the copper leachate data to copper toxicity data in the literature revealed that the copper concentrations in this work were high enough to cause injury to $E$. coli cells in culture (Table 5.2). For instance, Macomber and Imlay ${ }^{206}$ found that E. coli W3110 exhibited a growth defect when incubated for $10 \mathrm{~h}$ at $37^{\circ} \mathrm{C}$ in M9 minimal medium containing glucose as the sole carbon source and $\mathrm{Cu}(\mathrm{II})$ concentrations greater than $8 \mu \mathrm{M}$ (e.g. $16 \mu \mathrm{M}, 32 \mu \mathrm{M})$. After three days in inorganic carbon buffer $(\mathrm{pH} 7)$ at $4{ }^{\circ} \mathrm{C}$, Singh et al. ${ }^{315}$ observed that $\mathrm{Cu}(\mathrm{II})$ concentrations ranging from 9 to $16 \mu \mathrm{M}$ in culture were sufficient to cause $>84 \%$ injury and $63-83 \%$ lethality for all E. coli strains tested (TX432, E7, H10407, E6). All of the copper leachate concentrations in this study exceeded these thresholds (36-190 $\mu \mathrm{M}$, Table 5.1), and the growth of E. coli was inhibited in the presence of copper coatings, as evidenced by the optical densities of the sample-containing cultures after incubation for $24 \mathrm{~h}$ at $37^{\circ} \mathrm{C}$ in M63 minimal medium. Compared to the $\mathrm{CnGmSiO}_{2} / \mathrm{epx}$ control, the $\mathrm{OD}_{600}$ of the $24 \mathrm{~h}$ cultures decreased $52 \%$ and $39 \%$ when $\mathrm{CnGmSiO}_{2}+\mathrm{Cu}(\mathrm{II}) / \mathrm{epx}$ and $\mathrm{Cu}(\mathrm{II})-\mathrm{CnGmSiO}_{2} / \mathrm{epx}$, respectively, were present during incubation. And, compared to the uncoated control, the $\mathrm{OD}_{600}$ of the $24 \mathrm{~h}$ culture decreased $32 \%$ when the marine paint was present. The largest decrease in $\mathrm{OD}_{600}(52 \%$ for $\mathrm{CnGmSiO}_{2}+\mathrm{Cu}(\mathrm{II}) / \mathrm{epx}$ ) did not correspond to the coating that contained (40-50\% w/w for the marine paint) the most copper, but it did correspond to the coating that leached (190 $\pm 30 \mu \mathrm{M}$ for $\mathrm{CnGmSiO}_{2}$ $+\mathrm{Cu}(I I) / \mathrm{epx})$ the most copper in the presence of E. coli. This result is in agreement with previous results (Chapter 3, Section 3.2.3) in which there was a direct relationship between the degree of growth inhibition and the amount of copper leached from the coated surface. 
Table 5.2. Literature data of $\mathrm{Cu}(\mathrm{II})$ toxicity in cultures of $E$. coli.

\begin{tabular}{|c|c|c|c|c|}
\hline Reference & $\begin{array}{l}\text { [Cu(II)] Necessary for } \\
\text { Toxicity in Culture }\end{array}$ & Effect & E. coli Strain & Conditions \\
\hline Macomber and Imlay ${ }^{206}$ & $>8 \mu \mathrm{M}$ & growth defect & W3110 & $\begin{array}{l}\text { M9 minimal medium (glucose); } \\
\qquad 37^{\circ} \mathrm{C} ; 10 \mathrm{~h}\end{array}$ \\
\hline Grey and Steck ${ }^{316}$ & $100-500 \mu \mathrm{M}$ & $\begin{array}{c}\text { removal of all culturable cells } \\
(90 \% \text { lethality at } 500 \mu \mathrm{M})\end{array}$ & ES80 & saline; $25^{\circ} \mathrm{C} ; 10$ days \\
\hline Singh et al. ${ }^{315}$ & 9-16 $\mu \mathrm{M}$ & $\begin{array}{c}>84 \% \text { injury } \\
(63-83 \% \text { lethality })\end{array}$ & TX432, E7, H10407, E6 & $\begin{array}{l}\text { inorganic carbon buffer }(\mathrm{pH} 7) \\
\qquad 4^{\circ} \mathrm{C} ; 3 \text { days }\end{array}$ \\
\hline Santo et al. ${ }^{312}$ & $600 \mathrm{mM}$ & complete killing & W3110 & $\mathrm{CuCl}_{2}$ solutions $(\mathrm{aq}) ; 23^{\circ} \mathrm{C} ; 1 \mathrm{~min}$ \\
\hline
\end{tabular}


Regardless of the sample, the optical densities of all the cultures measured after $24 \mathrm{~h}$ incubation were unusually low, being at most 0.151 for the culture containing the epoxy-coated substrate (Table 5.1). The growth curve of $E$. coli NZRM 3647 in M63 minimal medium plateaued in the stationary phase by $20 \mathrm{~h}$ at $\mathrm{OD}_{600} \sim 1.5$ (Appendix, Figure 8.52) - nearly ten-times the highest $\mathrm{OD}_{600}$ in the assay. The two differences between the incubation conditions for the assay and growth curve were that, for the assay, the cultures were incubated under static conditions with test squares, while, for the growth curve, the cultures were incubated with periodic shaking but without test squares. Therefore, an experiment was completed in which cultures of E. coli NZRM 3647 in M63 minimal medium were incubated for $24 \mathrm{~h}$ at $37^{\circ} \mathrm{C}$, with and without shaking and with and without an uncoated test square (Experimental, Figure 6.13). The results clearly showed that the low optical densities in the assay were the consequence of incubation under static conditions (Table 5.3). This was likely due to poor aeration and/or the inhomogeneous distribution of nutrients in the culture, and it was also likely that the cells were not remaining suspended in culture, impacting the adherence results. Therefore, it was decided that, in future experiments, the cultures should be incubated with shaking, despite the multiple examples in the literature of antifouling assays performed under static conditions. ${ }^{14,282,295-298}$

Table 5.3. $\mathrm{OD}_{600}$ of cultures of $E$. coli NZRM 3647 in M63 minimal medium after incubation for $24 \mathrm{~h}$ at $37^{\circ} \mathrm{C}, \pm$ uncoated test squares and \pm shaking.

\begin{tabular}{ccc} 
Square $( \pm)$ & Shaking $( \pm)$ & OD $_{600}$ \\
\hline- & + & 0.958 \\
+ & + & 0.962 \\
- & - & 0.131 \\
+ & - & 0.139 \\
\hline
\end{tabular}

The copper leachate data for the marine paint were compared to a similar paint with a lower copper content in a study by Lindgren et al. $^{317}$ The reference paint used in the present study is a commercial marine antifouling paint composed of $40-50 \% \mathrm{w} / \mathrm{w}$ cuprous oxide in a rosin-based coating. After $24 \mathrm{~h}$ in M63 minimal medium, the amount of copper that leached from the marine paint in the presence of E. coli was $11 \mu \mathrm{g} / \mathrm{cm}^{2}$ (Table 5.4). Lindgren et al. ${ }^{317}$ prepared and tested the antifouling ability of a similar rosin-based paint, which was $8.5 \% \mathrm{w} / \mathrm{w}$ cuprous oxide and leached $3.04 \mu \mathrm{g} / \mathrm{cm}^{2}$ copper into the sea after $24 \mathrm{~h}$. Although the copper content in this paint was much lower than the commercial marine paint, they found that biofouling was still deterred, suggesting that antifouling performance could still be maintained with less copper and lower copper leachate concentrations. In the present work, $\mathrm{Cu}(\mathrm{II})-\mathrm{CnGmSiO}{ }_{2}$ and $\mathrm{CnGmSiO}_{2}+\mathrm{Cu}(\mathrm{II})$ contained $<3 \% \mathrm{w} / \mathrm{w}$ copper and leached 
2-10 $\mu \mathrm{g} \mathrm{Cu}(I I) / \mathrm{cm}^{2}$ when incorporated in a coating, but the adherence of $E$. coli was not deterred on these substrates as it was on the marine paint.

In the literature, surfaces functionalised with $\mathrm{Cu}(\mathrm{II})$-ligand complexes still had antimicrobial activity with surface and leached copper concentrations even lower than or equal to $\mathrm{Cu}(\mathrm{II})-\mathrm{CnGmSiO} 2 / \mathrm{epx}$ and $\mathrm{CnGmSiO}_{2}+\mathrm{Cu}(\mathrm{II}) / \mathrm{epx}$ (Table 5.4). For example, He et al. ${ }^{318}$ prepared a surface containing $~ 2.75 \% \mathrm{w} / \mathrm{w}$ copper by grafting a $\mathrm{Cu}(\mathrm{II})$-polydopamine (PDA) complex onto titanium, and, after $24 \mathrm{~h}$ immersed in phosphate-buffered saline, the concentration of $\mathrm{Cu}(\mathrm{II})$ released from the coating was $0.15-0.2 \mathrm{ppm}$. Antimicrobial activity was assessed by the enumeration of CFUs after incubation of the sample for $24 \mathrm{~h}$ in a culture of E. coli, and incubation with the modified titanium surface was lethal for $\sim 82.5 \%$ of the bacteria in the culture. In the present work, the \% w/w copper in $\mathrm{Cu}(\mathrm{II})-\mathrm{CnGmSiO}_{2}(2.6 \%)$ was almost equal to the PDA-modified titanium surface, which leached less $\mathrm{Cu}$ (II) $(0.15-0.2$ vs 3.6-6.0 ppm for $\left.\mathrm{Cu}(\mathrm{II})-\mathrm{CnGmSiO}_{2} / \mathrm{epx}\right)$. The \% w/w copper in $\mathrm{CnGmSiO}_{2}+\mathrm{Cu}$ (II) (1.0\%) was relatively lower, but $\mathrm{CnGmSiO}_{2}+\mathrm{Cu}(\mathrm{II}) / \mathrm{epx}$ also leached more $\mathrm{Cu}(\mathrm{II})(2.3-12 \mathrm{ppm})$ than the PDA-modified surface. Although the $\mathrm{Cu}(\mathrm{II})$ released by the silica coatings negatively affected bacterial growth in liquid culture, E. coli surface adherence was enhanced on $\mathrm{Cu}(\mathrm{II})-\mathrm{CnGmSiO}_{2} / \mathrm{epx}$ and unaffected on $\mathrm{CnGmSiO}_{2}+\mathrm{Cu}(\mathrm{II}) / \mathrm{epx}$. Therefore, bactericidal activity against planktonic bacteria in liquid culture due to the leaching of copper ions from a surface does not necessarily correspond to the deterrence of cellular attachment to that surface.

Copper leachate concentrations were measured because they were thought to be relevant to the bacterial adherence results, but there was not a straightforward relationship between the two. The marine paint and $\mathrm{Cu}(\mathrm{II})-\mathrm{CnGmSiO}_{2}$ /epx leached the same amount of copper (94-115 $\mu \mathrm{M}$, Table 5.1), but there was an almost $3 \log _{10}$ reduction in the number of viable, adherent bacteria when the test square was coated with the marine paint instead of $\mathrm{Cu}(\mathrm{II})-\mathrm{CnGmSiO}_{2} / \mathrm{epx}$ (Figure 5.12). The copper leachate concentration was high enough to cause injury to $E$. coli cells in culture but too low for complete killing (> $500 \mu \mathrm{M}$, Table 5.2). There is evidence in the literature of metal ions in solution effecting change in planktonic bacteria: Perrin et al. ${ }^{319}$ reported that a sub-inhibitory concentration of $\mathrm{Ni}(I I)(100 \mu \mathrm{M})$ encouraged biofilm formation in E. coli K-12. Therefore, it was hypothesised that, in the present work, the sub-lethal copper concentrations in liquid culture encouraged stressed bacteria to form biofilms on the coatings, but, in the case of the marine paint, the copper content at the surface $(40-50 \% \mathrm{w} / \mathrm{w})$ was sufficiently high to trigger the viable but nonculturable condition (i.e. viable bacteria that do not grow or divide) or to be bactericidal. In contrast, increased adherence to $\mathrm{Cu}(\mathrm{II})-\mathrm{CnGmSiO} / \mathrm{epx}$ was observed because either the surface copper content $(2.6 \% \mathrm{w} / \mathrm{w})$ was not above the threshold for lethality or the $\mathrm{Cu}(\mathrm{II})$ was not bioavailable, since it was complexed by a strong ligand. Therefore, this is a possible explanation for the enhanced adherence to $\mathrm{Cu}(\mathrm{II})-\mathrm{CnGmSiO} / \mathrm{epx}$. 
Table 5.4. Literature data from copper ion-containing coatings compared to data from this study.

\begin{tabular}{|c|c|c|c|c|c|}
\hline Reference & Surface & $\mathrm{Cu}$ in Coating & Cu Leachate & Leaching Conditions & Outcome \\
\hline $\begin{array}{l}\text { Lindgren } \\
\text { et al. }\end{array}$ & $\begin{array}{l}\mathrm{Cu}_{2} \mathrm{O} \text { in generic paint } \\
\text { (hard, rosin-based) }\end{array}$ & $8.5 \% \mathrm{w} / \mathrm{w}$ & $3.04 \mu \mathrm{g} / \mathrm{cm}^{2}$ & sea, $24 \mathrm{~h}$ & fouling deterred \\
\hline $\begin{array}{l}\text { Pallavicini } \\
\text { et al. }{ }^{313}\end{array}$ & $\begin{array}{l}\text { glass }\left(11.5 \mathrm{~cm}^{2}\right) \text { functionalised } \\
\text { with } \mathrm{Cu}(\mathrm{II}) \text {-cyclam complex }\end{array}$ & $\begin{array}{l}\sim 1.6 \times 10^{-9} \mathrm{~mol} / \mathrm{cm}^{2} \\
\quad(1.2 \mu \mathrm{g} \text { total })\end{array}$ & $<8 \% \mathrm{Cu}\left(8.1 \mathrm{ng} / \mathrm{cm}^{2}\right)$ & $\mathrm{H}_{2} \mathrm{O}, 48 \mathrm{~h}$ & $\begin{array}{l}\text { low microbicidal activity } \\
\text { for E. coli ATCC } 10356\end{array}$ \\
\hline $\begin{array}{l}\text { Dacarro } \\
\text { et al. }{ }^{216}\end{array}$ & $\begin{array}{c}\text { glass }\left(11.5 \mathrm{~cm}^{2}\right) \text { functionalised } \\
\text { with polyethyleneimine }\end{array}$ & $\begin{array}{c}3.8 \times 10^{-10} \mathrm{~mol} / \mathrm{cm}^{2} \\
(0.28 \mu \mathrm{g} \text { total })\end{array}$ & $<5 \% \mathrm{Cu}\left(1.2 \mathrm{ng} / \mathrm{cm}^{2}\right)$ & $\mathrm{H}_{2} \mathrm{O}, 24 \mathrm{~h}$ & $\begin{array}{l}\text { low microbicidal activity } \\
\text { for E. coli ATCC } 10356\end{array}$ \\
\hline $\begin{array}{l}\text { He et } \\
\text { al. }\end{array}$ & $\begin{array}{l}\mathrm{Cu}(\mathrm{II}) \text {-polydopamine grafted } \\
\text { onto titanium }\end{array}$ & $\sim 2.75 \% \mathrm{w} / \mathrm{w}$ & $0.15-0.2 \mathrm{ppm}$ & $\begin{array}{l}\text { phosphate-buffered } \\
\text { saline, } 24 \mathrm{~h}\end{array}$ & $\begin{array}{c}\sim 17.5 \% \text { survival rate for } \\
\text { E. coli }\end{array}$ \\
\hline This study & $\begin{array}{c}\mathrm{CnGmSiO}{ }_{2}+\mathrm{Cu}(\mathrm{II}) / \mathrm{epx} \\
\left(18.9 \mathrm{~cm}^{2}\right)\end{array}$ & $\begin{array}{c}1.0 \% \mathrm{w} / \mathrm{w} \\
(1.1 \mathrm{mg} \text { total })\end{array}$ & $\begin{array}{l}2.3 \mathrm{ppm}\left(3 \% \mathrm{Cu}, 2.0 \mu \mathrm{gg} / \mathrm{cm}^{2}\right)^{\mathrm{a}} \text {, } \\
12 \mathrm{ppm}\left(17 \% \mathrm{Cu}, 10 \mu \mathrm{g} / \mathrm{cm}^{2}\right)^{\mathrm{b}}\end{array}$ & $\begin{array}{l}\text { M63 minimal } \\
\text { medium, } 24 \mathrm{~h}\end{array}$ & $\begin{array}{l}\text { adherence of } E \text {. coli } \\
\text { NZRM } 3647 \text { unchanged }\end{array}$ \\
\hline This study & $\begin{array}{c}\mathrm{Cu}(\mathrm{II})-\mathrm{CnGmSiO}_{2} / \mathrm{epx} \\
\left(18.9 \mathrm{~cm}^{2}\right)\end{array}$ & $\begin{array}{c}2.6 \% \mathrm{w} / \mathrm{w} \\
(2.0 \mathrm{mg} \text { total })\end{array}$ & $\begin{array}{c}3.6 \mathrm{ppm}\left(3 \% \mathrm{Cu}, 3.1 \mu \mathrm{gg} / \mathrm{cm}^{2}\right)^{\mathrm{a}} \\
6.0 \mathrm{ppm}\left(7 \%, 5.2 \mu \mathrm{g} / \mathrm{cm}^{2}\right)^{\mathrm{b}}\end{array}$ & $\begin{array}{l}\text { M63 minimal } \\
\text { medium, } 24 \mathrm{~h}\end{array}$ & $\begin{array}{l}\text { increased adherence of } \\
\text { E. coli NZRM } 3647\end{array}$ \\
\hline This study & $\begin{array}{l}\text { Marine Paint } \\
\left(10.6 \mathrm{~cm}^{2}\right)\end{array}$ & $\begin{array}{c}40-50 \% \mathrm{w} / \mathrm{w} \\
(25-31 \mathrm{mg} \text { total) }\end{array}$ & $\begin{array}{l}3.3 \mathrm{ppm}\left(0.2 \% \mathrm{Cu}, 5.1 \mu \mathrm{gg} / \mathrm{cm}^{2}\right)^{\mathrm{a}} \text {, } \\
7.3 \mathrm{ppm}\left(0.6 \% \mathrm{Cu}, 11 \mu \mathrm{g} / \mathrm{cm}^{2}\right)^{\mathrm{b}}\end{array}$ & $\begin{array}{l}\text { M63 minimal } \\
\text { medium, } 24 \mathrm{~h}\end{array}$ & $\begin{array}{c}\text { decreased adherence of } \\
\text { E. coli NZRM } 3647\end{array}$ \\
\hline
\end{tabular}

${ }^{a}$ M63 minimal medium without E. coli NZRM 3647.

${ }^{b}$ M63 minimal medium with E. coli NZRM 3647. 
The aforementioned enhanced attachment was not observed for $\mathrm{CnGmSiO}_{2}+\mathrm{Cu}(\mathrm{II}) / \mathrm{epx}$, which had a lower copper content but higher copper leachate concentration in the presence of $E$. coli than $\mathrm{Cu}(\mathrm{II})-\mathrm{CnGmSiO} / \mathrm{epx}$. Instead of enhanced bacterial adherence, the viable counts from $\mathrm{CnGmSiO}_{2}+\mathrm{Cu}(\mathrm{II}) / \mathrm{epx}$ were equivalent to the control, $\mathrm{CnGmSiO}_{2} / \mathrm{epx}$. Two possible explanations were considered: first, the higher copper leachate concentration in the culture $(190 \mu \mathrm{M})$ likely resulted in increased killing or growth inhibition, as evidenced by the $24 \mathrm{~h}$ culture having the lowest $\mathrm{OD}_{600}$ (Table 5.1), and, second, it is possible that some of the $\mathrm{Cu}(\mathrm{II})$ in $\mathrm{CnGmSiO}_{2}+\mathrm{Cu}(\mathrm{II}) / \mathrm{epx}$ was adsorbed onto the silica surface during post-loading, rather than chelated, meaning that it was more weakly associated with the surface and, consequently, more bioavailable. The increased bioavailability of $\mathrm{Cu}(\mathrm{II})$ at the surface and in solution could have had a marginal antimicrobial effect, counteracting any inducement of biofilm formation.

The surface topography was also considered in regard to the results of the assay, but bacterial adherence appeared to be unaffected by different topographies. The surfaces of the $\mathrm{SiO}_{2} /$ epx-type coatings were rough, and, as previously discussed, increased surface roughness generally results in increased colonisation by bacteria. ${ }^{287}$ Nevertheless, the only coating of this type with increased bacterial attachment was $\mathrm{Cu}(\mathrm{II})-\mathrm{CnGmSiO}_{2} / \mathrm{epx}$. There was no observable difference in surface topography between the silica coatings, as evidenced by SEM (Chapter 4, Figure 4.18). So, increased adherence to $\mathrm{Cu}(\mathrm{II})-\mathrm{CnGmSiO}_{2} / \mathrm{epx}$ was not because it was the roughest out of all the tested coatings. The counts from $\mathrm{SiO}_{2} / \mathrm{epx}, \mathrm{CnGmSiO}_{2} / \mathrm{epx}$, and $\mathrm{CnGmSiO}_{2}+\mathrm{Cu}(\mathrm{II}) / \mathrm{epx}$ were statistically equivalent to each other and to the epoxy resin and uncoated sample (Figure 5.12), both of which were much smoother than the silica coatings.

\subsubsection{Conclusions}

Bacterial adherence to the $\mathrm{SiO}_{2}$ /epx-type coatings, epoxy coating, marine paint, and uncoated substrate was tested (Figure 5.12). All of the results were statistically equivalent, except for the marine paint and $\mathrm{Cu}(\mathrm{II})-\mathrm{CnGmSiO}_{2} / \mathrm{epx}$. There were approximately $2 \log _{10}$ fewer cells recovered from the marine paint, compared to the other coatings, and this result was at the LOD of the assay. In contrast, the number of adherent cells per $\mathrm{cm}^{2}$ was highest on $\mathrm{Cu}(\mathrm{II})-\mathrm{CnGmSiO}_{2} / \mathrm{epx}$, which could be due to low levels of copper in the culture precipitating biofilm formation by stressed cells. It has been reported that $10^{5}-10^{7}$ adherent cells per $\mathrm{cm}^{2}$ are required for biofilm formation to occur, ${ }^{300}$ and $\mathrm{Cu}(\mathrm{II})-\mathrm{CnGmSiO} / \mathrm{epx}$ is the only coating approaching this value. It is possible that E. coli NZRM 3647 does not readily form biofilms or requires more than $48 \mathrm{~h}$ for biofilm formation, and future adherence 
assays should be carried out with a different bacterial species (e.g. marine). Measurement of the amounts of copper leached from $\mathrm{CnGmSiO}_{2}+\mathrm{Cu}(\mathrm{II}) / \mathrm{epx}, \mathrm{Cu}(\mathrm{II})-\mathrm{CnGmSiO}_{2} / \mathrm{epx}$, and the marine paint in the presence and absence of $E$. coli revealed enhanced leaching in the presence of bacteria, and comparisons to copper toxicity data in the literature showed that the copper concentrations were high enough to cause injury to E. coli cells in culture (Table 5.2). The highest concentration of leached copper corresponded to the culture with the lowest $\mathrm{OD}_{600}\left(\mathrm{CnGmSiO}_{2}+\mathrm{Cu}(\mathrm{II}) / \mathrm{epx}: 12 \mathrm{ppm} \mathrm{Cu}\right.$, $\mathrm{OD}_{600}$ 0.057). However, all of the optical densities of the $E$. coli cultures in M63 minimal medium measured after $24 \mathrm{~h}$ incubation were unusually low, and it was discovered that this was due to incubating the cultures under static conditions. Therefore, for future testing, it was decided that the cultures should be shaken during incubation. Further investigations of the literature yielded examples of $\mathrm{Cu}$ (II) ligand-functionalised surfaces with antimicrobial activity - even at equivalent or lower surface and leached copper concentrations than $\mathrm{Cu}(\mathrm{II})-\mathrm{CnGmSiO}_{2} / \mathrm{epx}$ and $\mathrm{CnGmSiO}_{2}+\mathrm{Cu}(\mathrm{II}) / \mathrm{epx}$ (Table 5.4). Unfortunately, none of the $\mathrm{SiO}_{2}$ /epx-type coatings had comparable activity to the marine paint, but it should be noted that the marine paint also contains other antimicrobial ingredients (e.g. thiram) capable of leaching into the culture or exerting a bactericidal surface effect. ${ }^{320}$

There are several limitations and aspects of the assay that could seriously impact or skew the results. First, the assay is not high-throughput, being that it is quite lengthy and labour-intensive, and its precision is too low to identify anything less than $\log _{10}$-differences between coatings as statistically significant. Second, because the counts are reported as CFUs per unit surface area of the coatings $\left(e . g . \mathrm{cm}^{2}\right)$, the estimated surface area notably influences the results. For the relatively flat and smooth samples, such as the uncoated substrate, epoxy coating, and marine paint, the surface area was calculated as a square $\left(10.6 \mathrm{~cm}^{2}\right.$ for $23 \mathrm{~mm} \times 23 \mathrm{~mm}$, two-sided), but this was not valid for the rougher silica coatings. The surface area of the silica coatings $\left(18.9 \mathrm{~cm}^{2}\right.$ for $23 \mathrm{~mm} \times 23 \mathrm{~mm}$, two-sided) was estimated by treating the silica particles like spheres confined in a square (Appendix, Scheme 8.2), since the particle diameter is known to be 35-75 $\mu \mathrm{m}$. However, as seen by SEM (Chapter 4, Figure 4.18), the particles are not spherical, and the estimated surface area may be quite different from the true value, meaning that the $\mathrm{CFU} / \mathrm{cm}^{2}$ may be quite different as well. BET surface area analysis of Batch $4 \mathrm{CnGmSiO}_{2}$, Batch $2 \mathrm{Cu}(\mathrm{II})-\mathrm{CnGmSiO}_{2}$, and the unfunctionalised silica was conducted (Appendix, Table 8.21), but the results were not particularly useful, as the technique includes the surface area of the pores. E. coli (1-3 $\mu \mathrm{m}$ length, $0.4-0.7 \mu \mathrm{m}$ diameter) is too big to fit in the pores (60 Å diameter), ${ }^{321}$ so the surface area measured by BET analysis is not reflective of the surface area available for bacterial colonisation.

A third critique of the assay's design is that it only measures the number of viable bacteria that are attached to the coating; it does not reveal if bacteria have adhered and then died, but, in the context 
of biofouling, the remaining biomass can still be problematic. Unfortunately, staining is contraindicated because most stains would also be retained by the coating or substrate, which is supported by the result of staining adherent biomass with crystal violet (Appendix, Figure 8.54). Fourth, there were concerns that Tween 20 could have a synergistic effect with the antimicrobial coatings by making cells more vulnerable to lysis. To determine if a detergent is even necessary, Gramstaining should be performed to check for cellular aggregation. Also, the experiment testing the effect of Tween 20 (Section 5.2.3, Figure 5.6) could be repeated with a standard amount of $\mathrm{Cu}(\mathrm{II})$ (comparable to the leached $\mathrm{Cu}(\mathrm{II})$ concentrations) added to the $24 \mathrm{~h}$ cultures. Finally, there are concerns that the assay will not accurately predict coating performance in field experiments. For example, the copper leachate concentration may impact the assay's results, but it is unlikely to have an extensive effect in the ocean in which the copper will be greatly diluted. Despite these concerns, establishment of such an assay is vital for the project.

Even though it is acknowledged that the data from laboratory-based antifouling bioassays do not always correlate with the data from field experiments, these bioassays are still essential for the selection of a lead product. Field experiments in which the coatings are immersed in the ocean are expensive because they require large quantities of the test products and long immersion times. Consequently, proceeding directly from coating formulation to field experiments for each antifouling candidate is unfeasible, and numerous laboratory antifouling assays have been developed to serve as the first gate for narrowing down the candidates to a manageable number for field experiments. ${ }^{282}$ The adherence assay in this work is practical and relevant, and the assay, with the recommended adjustments, was progressed to test the adherence of marine bacterium V. harveyi NZRM 2698 to the $\mathrm{SiO}_{2} /$ epx-type coatings. 


\subsection{ADHERENCE ASSAY: V. HARVEYI NZRM 2698}

\subsubsection{Optimisation}

Prior to testing bacterial adherence to the $\mathrm{SiO}_{2} /$ epx-type coatings, the assay was optimised for marine bacterium $V$. harveyi NZRM 2698. Optimisation involved adjusting the growth conditions, such as the temperature to $30{ }^{\circ} \mathrm{C}$ and medium to Luria marine broth, and performing many of the same experiments conducted during the method development with E. coli NZRM 3647 (Figure 5.3). First, the effects of vortexing, sonication, and Tween $20(0.05 \% \mathrm{v} / \mathrm{v})$ on planktonic $V$. harveyi were assessed (Experimental, Figure 6.14), and it was found that the addition of Tween 20 to the culture before diluting for enumeration via the drop plate method resulted in a 3.5-4.5 $\log _{10}$ reduction in the $\mathrm{CFU} / \mathrm{mL}$, compared to the control (Figure 5.13a). Therefore, Tween 20 had a bactericidal effect on V. harveyi. Vortexing and/or sonication yielded similar counts to the control, meaning that the cells were unaffected by the vortexing and sonication conditions in the assay. For the samples without Tween 20, however, similar counts were recorded in multiple dilutions (e.g. $10^{-10}$ and $10^{-11}$ ), which is indicative of the transfer of agglomerated cells.
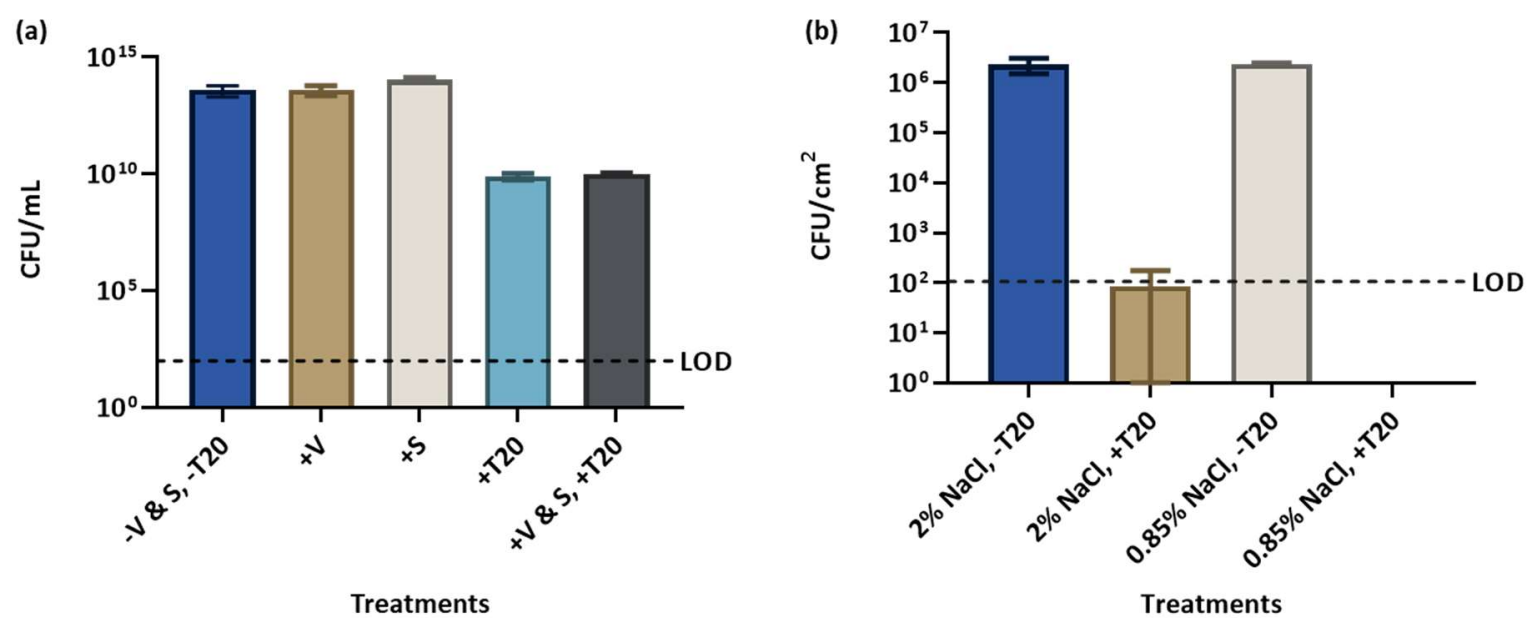

Figure 5.13. Optimisation of the bacterial adherence assay for $V$. harveyi NZRM 2698: (a) results of $\mathrm{CFU} / \mathrm{mL}$ in an untreated planktonic culture ("-V \& S, -T20") and planktonic cultures treated with vortexing $("+V ")$, sonication $\left("+S^{\prime \prime}\right)$, Tween $20("+T 20$ "), and vortexing plus sonication and Tween 20 ("+V \& S, $+\mathrm{T} 20$ "; $\mathrm{LOD}=100 \mathrm{CFU} / \mathrm{mL}$ ) and (b) results of $\mathrm{CFU} / \mathrm{cm}^{2}$ on $\mathrm{CnGmSiO}_{2} /$ epx-coated samples obtained using $\pm 0.05 \% \mathrm{v} / \mathrm{v}$ Tween 20 for deagglomeration and $0.85 \% \mathrm{vs} 2 \% \mathrm{w} / \mathrm{w} \mathrm{NaCl}$ for washes and dilutions $\left(\mathrm{LOD}=106 \mathrm{CFU} / \mathrm{cm}^{2}\right)$. The bars represent the mean $(\mathrm{tr}=5) \pm \mathrm{SD}$. 
Gram-staining revealed that cells of $V$. harveyi were indeed clumping. The aggregation of cells was observed after staining an overnight culture of $V$. harveyi (Figure 5.14a). Nonionic detergent Tween 20 $(0.05 \% \mathrm{v} / \mathrm{v})$ was added, and the culture was checked once more for clumping cells via Gram-staining. The clumps dispersed in the presence of Tween 20 (Figure 5.14b). Agglomerated cells could affect the results of the assay by making it difficult to deliver a standard inoculum for the $24 \mathrm{~h}$ culture and by propagation of the clumps through serial dilutions and plating, resulting in multiple cells being counted as a single colony. However, the use of Tween $20(0.05 \% \mathrm{v} / \mathrm{v})$ to mitigate clumping was contraindicated by its bactericidal effect on planktonic $V$. harveyi.

(a)

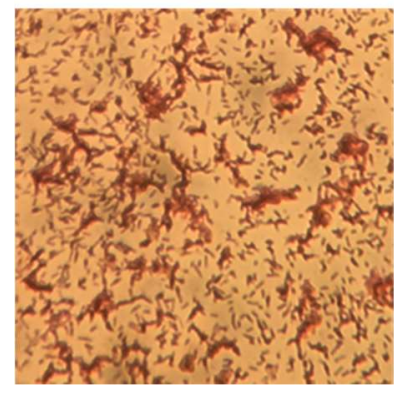

(b)

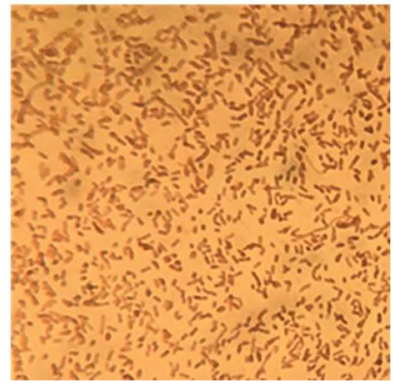

Figure 5.14. Gram-staining of a culture of $V$. harveyi NZRM 2698 in Luria marine broth: (a) before and (b) after the addition of Tween 20.

An experiment was conducted to determine the impact of Tween $20(0.05 \% \mathrm{v} / \mathrm{v})$ in the adherence assay when used to break up clumps 1) prior to inoculation for $24 \mathrm{~h}$ incubation of the $\mathrm{CnGmSiO}_{2} / \mathrm{epx}$ coated sample, 2) in the vortexed detachment solution, and 3) in the serial dilutions to be plated for counting (Experimental, Figure 6.15). $\mathrm{CnGmSiO}_{2} / \mathrm{epx}$, rather than the uncoated substrate, was used because testing the attachment of $V$. harveyi to the $\mathrm{SiO}_{2} /$ epx-type coatings was the ultimate goal, so optimising conditions with coatings of this type was reasonable. Also, in previous experiments (Figure 5.10), strong interactions did not seem to be promoted between E. coli and the uncoated substrate, and it was unknown if $V$. harveyi would behave similarly. Since both $\mathrm{CnGmSiO}_{2} / \mathrm{epx}$ and $\mathrm{SiO}_{2} /$ epx were negative controls for the $\mathrm{Cu}(\mathrm{II})$-containing $\mathrm{SiO}_{2} /$ epx-type coatings, they were used in the optimisation experiments. Treatment of the samples with Tween 20 resulted in a $4-5 \log _{10}$ reduction in the number of adherent cells (Figure 5.13b), and, for this reason, Tween 20 was not used in the adherence assay, despite concerns about cells aggregating. Time constraints did not allow for further optimisations regarding the use of alternative detergents or lower concentrations of Tween 20. In the same experiment, different concentrations of salt $(0.85 \%$ vs $2 \% \mathrm{w} / \mathrm{w} \mathrm{NaCl})$ in the 
aqueous rinses and diluent yielded equivalent bacterial counts (Figure 5.13b); this was checked because saline (i.e. $0.85 \% \mathrm{w} / \mathrm{w} \mathrm{NaCl}$ ) was normally used in the assay, but marine bacterium $V$. harveyi is cultured in high-salt media (e.g. $2 \% \mathrm{w} / \mathrm{w}$ in Luria marine broth).

The duration of incubation of a sample with $V$. harveyi and the efficiency of vortexing and sonication in the subsequent detachment of the cells were also evaluated (Experimental, Figure 6.16). $\mathrm{SiO}_{2} / \mathrm{epx}-$ coated samples were incubated $24 \mathrm{~h}$ or $48 \mathrm{~h}$ in diluted cultures of $V$. harveyi with shaking (Appendix,

Figure 8.55, stationary phase by $16 \mathrm{~h}$ ). To detach the adherent cells, the samples were treated with and without vortexing and/or sonication, and the efficacy of one round of vortexing vs two was assessed. Incubation for $48 \mathrm{~h}$ yielded 1-2 $\log _{10}$ fewer adherent cells than incubation for $24 \mathrm{~h}$ (Figure 5.15a), so $24 \mathrm{~h}$ remained the incubation time-length. The combination of vortexing and sonication as a detachment method resulted in approximately the same number of CFUs recovered from the substrate as vortexing alone, and one round of vortexing appeared to be sufficient for the removal of the majority of adherent cells. Therefore, it was decided that one round of vortexing alone was an adequate detachment method. The substrate not subjected to any detachment methods revealed that a percentage of the CFUs were attributable to the carryover of planktonic cells from washes or the detachment of weakly adhered cells.
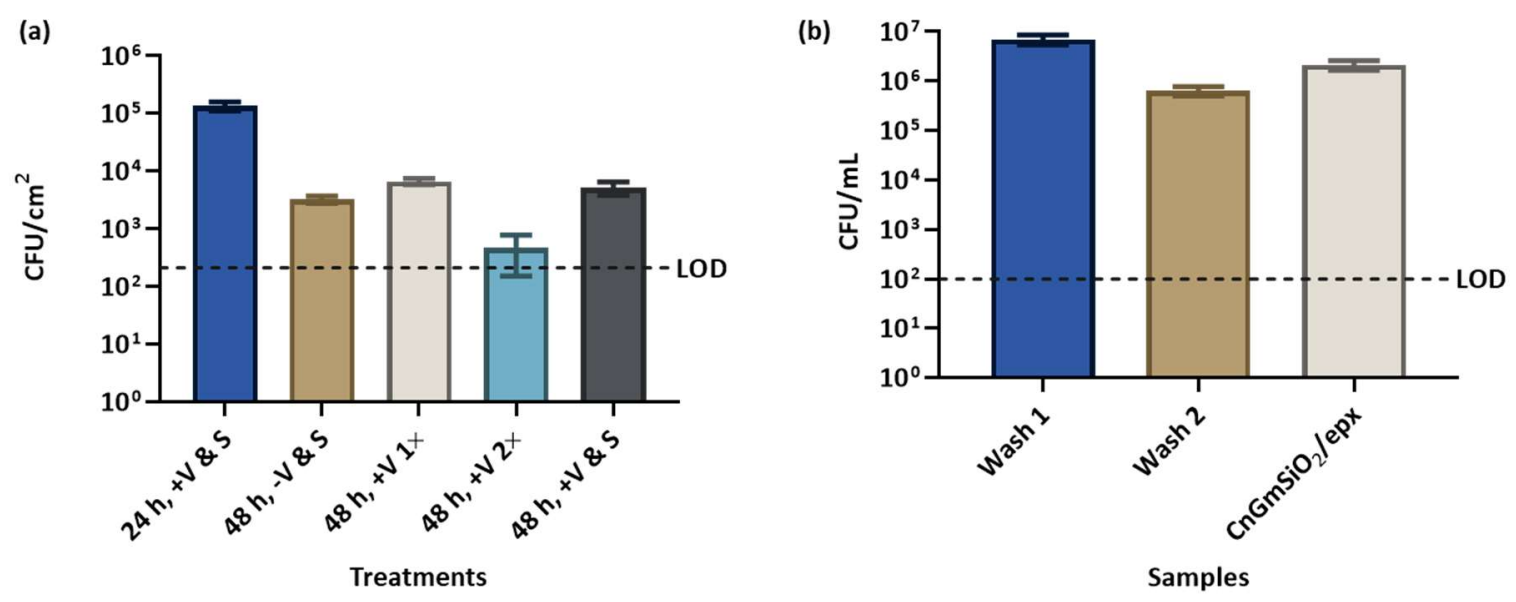

Figure 5.15. Optimisation of the bacterial adherence assay for V. harveyi NZRM 2698 (continued): (a) results of $\mathrm{CFU} / \mathrm{cm}^{2}$ on the $\mathrm{SiO}_{2} /$ epx-coated substrate after incubating $24 \mathrm{~h}$ vs $48 \mathrm{~h}$ and obtained under the conditions of \pm vortexing and sonication, vortexing once (" $1 \times$ "), and vortexing the same sample a second time (" $2 x^{\prime} ;$ LOD $=212 \mathrm{CFU} / \mathrm{cm}^{2}$ ) and (b) results of CFU $/ \mathrm{mL}$ in $2 \% \mathrm{w} / \mathrm{w} \mathrm{NaCl}$ washes and in the detachment solution containing a $\mathrm{CnGmSiO}_{2} / \mathrm{epx}$-coated square (LOD $=100 \mathrm{CFU} / \mathrm{mL}$ ). The bars represent the mean $(\operatorname{tr}=5) \pm S D$. 
An experiment was conducted, as in Figure 5.7, to enumerate the cells in successive washes of a $\mathrm{CnGmSiO}_{2} /$ epx-coated sample (Experimental, Figure 6.17). The cells were then detached from the sample via vortexing and enumerated. More bacteria were recovered from the detachment solution containing the sample than from the second wash, demonstrating that cells remained attached through multiple washes (Figure 5.15b). The number of cells in the second wash was $\sim 4 \log _{10}$ higher than the LOD of the assay. This would be problematic if the counts were indeed from the carryover of planktonic cells from the $24 \mathrm{~h}$ culture, rather than cells detaching from the surface, since the large number of carryover cells could mask effects of deterred adherence to the test samples. Thus, it would be possible to determine if the coatings were not bactericidal, but the reverse would be difficult, if not impossible. Unfortunately, this line of inquiry could not be pursued further due to time constraints. With the aforementioned limitations in mind, as well as the optimisations to the protocol, the adherence of $V$. harveyi to the $\mathrm{SiO}_{2} /$ epx-type coatings was tested.

\subsubsection{Adherence to $\mathrm{SiO}_{2} / \mathrm{epx}$-Type Coatings}

The attachment of marine bacterium $V$. harveyi NZRM 2698 to the $\mathrm{SiO}_{2} /$ epx-type coatings, epoxy coating, commercial marine paint, and uncoated substrate was assessed, following the optimised protocol. The samples were incubated with $V$. harveyi in a liquid culture while shaking for $24 \mathrm{~h}$ at $30^{\circ} \mathrm{C}$. After incubation, the samples were rinsed with an aqueous salt solution ( $2 \% \mathrm{w} / \mathrm{w} \mathrm{NaCl})$ and transferred to a fresh solution for detachment via vortexing. The detached cells were enumerated, yielding the number of adherent cells per $\mathrm{cm}^{2}$ for each sample. As shown in Figure 5.16, all of the differences in the $\mathrm{CFU} / \mathrm{cm}^{2}$ between samples were statistically significant $(p<0.01)$, with the following exceptions: the differences between the marine paint and the epoxy and $\mathrm{SiO}_{2} / \mathrm{epx}$ were not significant, and the difference between the uncoated substrate and $\mathrm{Cu}(\mathrm{II})-\mathrm{CnGmSiO} / \mathrm{epx}$ was not significant.

Unexpectedly, the fewest cells adhered to $\mathrm{SiO}_{2} / \mathrm{epx}$. The addition of unfunctionalised silica to the epoxy surface resulted in a $1 \log _{10}$ reduction in the number of adherent cells, compared to the epoxy. Functionalisation of the silica with $\mathrm{CnGm}$ yielded a $2 \log _{10}$ increase in the number of adherent cells, which was the largest number out of the tested coatings. Measurement of the optical densities in each $24 \mathrm{~h}$ culture revealed that the lowest $\mathrm{OD}_{600}$ was measured in the culture containing $\mathrm{SiO}_{2} / \mathrm{epx}$ $\left(\mathrm{OD}_{600} 0.71\right.$, Table 5.5), demonstrating that the presence of silica had a growth-inhibitory or killing effect. It is possible that cation sequestration by the weakly acidic $\left(p K_{\mathrm{a}} 4\right)$ silanol groups at the silica gel surface could have negatively impacted planktonic $V$. harveyi and cellular attachment. ${ }^{322,323}$ 
However, this antimicrobial effect was not observed by Sarkheil et al. ${ }^{324}$ in a study in which silver nanoparticles were immobilised on silica sand beads to create an antibacterial material against Vibrio sp. Persian1. Although the modified silica sand beads killed $100 \%$ of bacteria after $2 \mathrm{~h}$ contact, the unmodified silica sand beads had no such bactericidal activity.

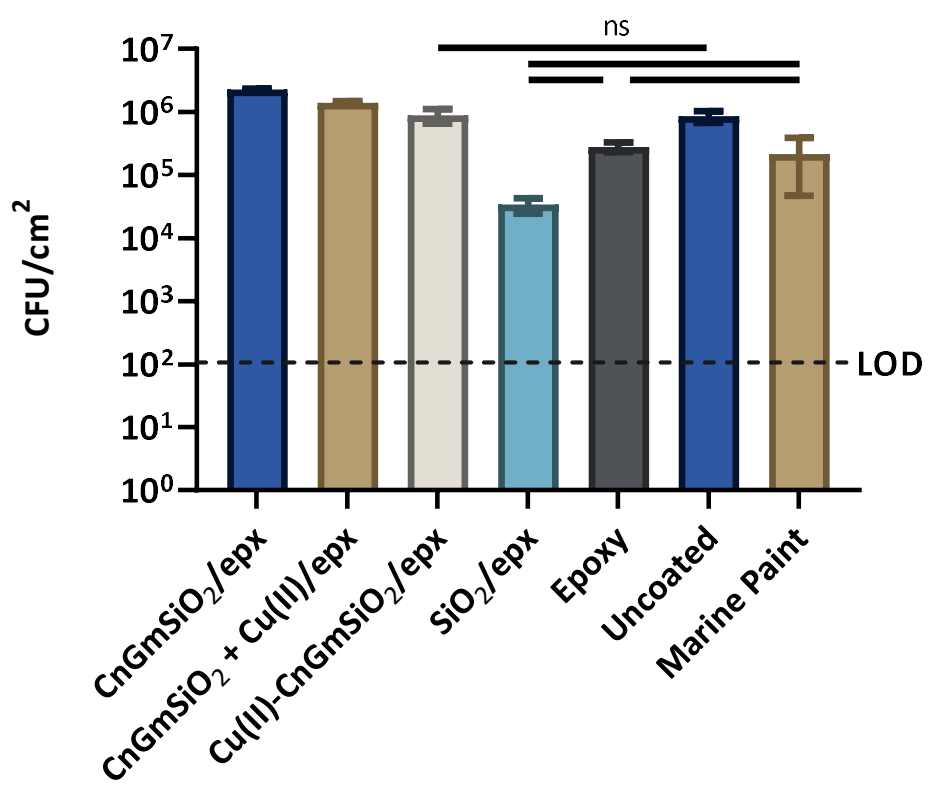

Coatings

Figure 5.16. Results of $V$. harveyi NZRM $2698 \mathrm{CFU} / \mathrm{cm}^{2}$ on the $\mathrm{SiO}_{2} /$ epx-type coatings, as well as on uncoated squares, the epoxy resin, and the marine paint. The bars represent the mean (br $=6$ for "SiO $2 /$ epx" and "Marine Paint", br = 3 for all others) \pm SD (LOD = $\left.106 \mathrm{CFU} / \mathrm{cm}^{2}\right)$. To determine if the means from the data sets were significantly different from each other, a one-way ANOVA with Tukey's post hoc test was conducted, and the difference was considered to be significant when the $p$-value was $<0.05$. Unless annotated as not significant $(\mathrm{ns})$, the results were significantly different $(p<0.01)$. 
Table 5.5. The $\mathrm{OD}_{600}$ of the $V$. harveyi cultures after $24 \mathrm{~h}$ incubation and the copper content of the test squares.

\begin{tabular}{lcc}
\hline Coating & OD $_{600}(24 \mathrm{~h}, \mathrm{LMB})^{\mathrm{a}}$ & $\% \mathrm{w} / \mathrm{w} \mathrm{Cu}^{\mathrm{b}}$ \\
\hline $\mathrm{CnGmSiO}_{2} / \mathrm{epx}$ & $1.22 \pm 0.05$ & 0 \\
$\mathrm{CnGmSiO}_{2}+\mathrm{Cu}(\mathrm{II}) / \mathrm{epx}$ & $0.95 \pm 0.03$ & $1.0 \pm 0.3^{\mathrm{a}}$ \\
$\mathrm{Cu}(\mathrm{II})-\mathrm{CnGmSiO}_{2} / \mathrm{epx}$ & $1.04 \pm 0.01$ & $2.6 \pm 0.3^{\mathrm{a}}$ \\
$\mathrm{SiO}_{2} / \mathrm{epx}$ & $0.71 \pm 0.04$ & 0 \\
Epoxy & $1.34 \pm 0.02$ & - \\
Uncoated & $1.46 \pm 0.05$ & - \\
Marine Paint & $1.23 \pm 0.12$ & $40-50$ \\
\hline $\begin{array}{l}\text { a Mean } \pm \text { SD. Experiment repeated 3x. } \\
\text { b } \% \text { w/w Cu in the (un)functionalised silica component or in the marine paint. }\end{array}$
\end{tabular}

In the present experiment, another unexpected result was for the marine paint, on which $>10^{5} \mathrm{CFU} / \mathrm{cm}^{2}$ were recovered. The result is somewhat suspicious, as the marine paint is known to be antifouling. It is uncertain if this result is truly reflective of the propensity of bacteria to colonise the surface or, rather, if the coating's activity is being masked by counts from planktonic cells carried over from washes; the antifouling ability of the marine paint or $\mathrm{SiO}_{2} / \mathrm{epx}$ might actually be greater than what is reflected in the assay. Yet, the results for coatings with significantly higher counts than the marine paint (e.g. uncoated, $\mathrm{CnGmSiO}_{2} / \mathrm{epx}, \mathrm{CnGmSiO}_{2}+\mathrm{Cu}(\mathrm{II}) / \mathrm{epx}, \mathrm{Cu}(\mathrm{II})-\mathrm{CnGmSiO}_{2} / \mathrm{epx}$ ) are likely accurate, in as much as they show that bacterial adherence is undeterred on these coatings. Further method development is required to ensure that the washes in future testing are efficient in removing planktonic cells.

\subsubsection{Conclusions}

The adherence assay was optimised for marine bacterium $V$. harveyi NZRM 2698. First, the effects of vortexing, sonication, and Tween $20(0.05 \% \mathrm{v} / \mathrm{v})$ on planktonic $V$. harveyi were evaluated, and it was found that cells were unaffected by vortexing and sonication. Gram-staining of a culture of $V$. harveyi revealed that the cells were aggregating, and, although Tween 20 dispersed the clumps, it was found to be bactericidal and was not used. In another experiment, more cells were recovered after an incubation time of $24 \mathrm{~h}$, compared to $48 \mathrm{~h}$, and one round of vortexing alone was found to be sufficient for the removal of surface-adherent cells. Enumeration of the cells in successive washes of $\mathrm{CnGmSiO}_{2} /$ epx and its detachment solution demonstrated that cells remained adhered through 
multiple rinses, but the large numbers of bacteria in the sample washes called into the question the washing efficiency. Had time permitted, further experimentation would have examined increasing the number of washes.

The optimised protocol was followed to test the adherence of $\mathrm{V}$. harveyi to the $\mathrm{SiO}_{2} / \mathrm{epx}$-type coatings, epoxy coating, marine paint, and uncoated substrate. Attachment was undeterred on the functionalised silica coatings, both with and without copper, and the highest average counts out of all the tested coatings were recovered from $\mathrm{CnGmSiO}_{2} /$ epx. Interestingly, the $\mathrm{CFU} / \mathrm{cm}^{2}$ were lowest on $\mathrm{SiO}_{2} / \mathrm{epx}$, rather than the marine paint. It was unknown if these results were a true reflection of the number of adherent cells or if bactericidal effects were being masked by planktonic cells carried over from the wash step. It is probable that carryover is more of a problem with $V$. harveyi than with E. coli due to cellular agglomeration. Therefore, future work should explore the use of a different detergent or a lower concentration of Tween 20 to disperse clumps, as well as exploring the benefit of additional wash steps. The $\mathrm{Cu}(\mathrm{II})$-leaching from the coatings into the shaken $24 \mathrm{~h}$ cultures of $\mathrm{V}$. harveyi in Luria marine broth should also be measured. 


\subsection{SUMMARY AND FUTURE WORK}

To rank antifouling products and select a lead coating, a bacterial adherence assay was developed using representative, Gram-negative bacterium E. coli NZRM 3647. In this assay, freshly coated squares were incubated with the bacteria in M63 minimal medium, and, after $24 \mathrm{~h}$, they were removed, rinsed, delivered to a saline/Tween 20 solution, vortexed, and sonicated. The CFUs in the detachment solutions were enumerated via the drop plate method to reveal the number of viable cells attached per $\mathrm{cm}^{2}$ of each coating. This procedure was employed to test the adherence of $E$. coli to the $\mathrm{SiO}_{2}$ /epx-type coatings prepared in Chapter 4 (Section 4.3). Surprisingly, $\mathrm{Cu}$ (II)-CnGmSiO $2 / e p x$ had the greatest number of viable, adherent bacteria, despite it having the highest $\% \mathrm{w} / \mathrm{w}$ copper out of the prepared coatings. The counts from $\mathrm{CnGmSiO}_{2} / \mathrm{epx}, \mathrm{CnGmSiO}_{2}+\mathrm{Cu}(\mathrm{II}) / \mathrm{epx}, \mathrm{SiO}_{2} / \mathrm{epx}$, the epoxy resin, and the uncoated sample were statistically equivalent, and the counts for the marine paint were at the LOD of the assay. The amount of copper released from each copper-containing coating (i.e. $\mathrm{Cu}(\mathrm{II})-\mathrm{CnGmSiO}_{2} / \mathrm{epx}, \mathrm{CnGmSiO}_{2}+\mathrm{Cu}(\mathrm{II}) / \mathrm{epx}$, and the marine paint) over the $24 \mathrm{~h}$ incubation period was measured. Interestingly, the amount of leached copper increased when bacteria were present in the culture, and the highest amount of leached copper corresponded to the $24 \mathrm{~h}$ culture with the lowest $\mathrm{OD}_{600}$ (i.e. $\mathrm{CnGmSiO}_{2}+\mathrm{Cu}(\mathrm{II}) / \mathrm{epx}$ ). With the validation of the assay, the samples were progressed to testing the propensity of a marine bacterium to colonise their surfaces.

The assay protocol was optimised to examine the attachment of marine bacterium $V$. harveyi NZRM 2698 to these coatings. The procedure for $V$. harveyi differed from the one for $E$. coli in the following ways: the bacteria were cultured in Luria marine broth at $30^{\circ} \mathrm{C}$, the samples were rinsed and detached in an aqueous salt solution ( $2 \% \mathrm{w} / \mathrm{w} \mathrm{NaCl}$ ) without Tween 20 , and vortexing alone was used as a detachment method. For the assay with $V$. harveyi, however, planktonic cells carried over from the rinses may have contributed more significantly to the total CFUs than in the assay with E. coli, possibly due to the tendency of $V$. harveyi cells to aggregate. Tween 20 dispersed clumps of cells but had a bactericidal effect and, therefore, was not used. Testing the adherence of $V$. harveyi to the $\mathrm{SiO}_{2}$ /epx-type coatings revealed that cellular attachment was deterred on $\mathrm{SiO}_{2} /$ epx but undeterred on $\mathrm{CnGmSiO}_{2} / \mathrm{epx}, \mathrm{CnGmSiO}_{2}+\mathrm{Cu}(\mathrm{II}) / \mathrm{epx}$, and $\mathrm{Cu}(\mathrm{II})-\mathrm{CnGmSiO}_{2} / \mathrm{epx}$. The possible contribution of agglomerated planktonic cells to the total cell count made it difficult to determine the actual magnitude of any attachment-deterrent effects.

The goals of future work should be to improve and expand the bacterial adherence assay and answer questions raised by this study. Since both E. coli and V. harveyi are Gram-negative bacteria, the colonisation of the surfaces by other, especially Gram-positive (e.g. Micrococcus luteus), marine bacteria should also be assessed, preferably using seawater as the growth medium. The enumeration 
of the viable, adherent cells is an indirect, laborious method for evaluating bacterial colonisation by a surface, and, without visual confirmation, it is difficult to have confidence that the assay is working as designed. The assay would greatly benefit from a different detection method, such as staining (e.g. live/dead) and microscopy (e.g. fluorescence, SEM), with which the surface colonisation could be observed directly. Although, the opacity and dye-retention of the samples complicates the implementation of these techniques. Instead of using dyes, the bioluminescence of $V$. harveyi could be exploited to determine the percent coverage of a surface, or fluorescent bacteria could be used. These adjustments would shorten the assay, making it more high-throughput. Another improvement to the assay would be to find an antifouling coating with a high copper content that does not contain other antimicrobial components, and this coating would be tested alongside the marine paint. Furthermore, it would be beneficial to determine the wettability of each surface via contact angle measurements.

Testing the adherence of both $\mathrm{E}$. coli and $\mathrm{V}$. harveyi to the $\mathrm{SiO}_{2} /$ epx-type coatings revealed that the functionalised silica coatings, with and without $\mathrm{Cu}(\mathrm{II})$, did not deter cellular attachment. Thus, stage four of the current project in which the antifouling ability of the lead product would be tested in the marine environment (Chapter 1, Table 1.5) is not achievable, and other antifouling candidates should be pursued in the future. Following the present line of inquiry, the functionalisation of different kinds of silica with different ligands (e.g. other ligands in the tetraaza/oxatriaza series; Chapter 2, Figure 2.18) could be pursued to create products with a range of $\mathrm{Cu}(\mathrm{II})$-loading and -leaching abilities, establishing the parameters of the $\mathrm{Cu}(\mathrm{II})$ flux required to make a surface antifouling. 


\section{EXPERIMENTAL}

\subsection{GENERAL}

\subsubsection{Materials and Procedures}

Anhydrous solvents were obtained commercially, and Milli-Q water was used throughout. The model ligand in this work, cyclen, was purchased from Chem-Impex International, Inc., and key precursor molecule ADH was supplied by Nuplex Industries. For silica functionalisation reactions, silica gel (highpurity grade, pore size $60 \AA$, 220-440 mesh particle size, 35-75 $\mu \mathrm{m}$ particle size; Sigma-Aldrich), unless otherwise stated, was dried for $24 \mathrm{~h}$ at $180^{\circ} \mathrm{C}$ under vacuum before use. The ratio of GLYMO to silica (mmol GLYMO/g silica) used in functionalisation reactions was based on the ratio of $n$-octadecyldimethylchlorosilane to silica ( $0.991 \mathrm{mmol}$ modifier/g silica) used by Ortega et al. ${ }^{260}$ to synthesise reversed-phase silica gel. All acids used for ICP-MS analysis were double-distilled at subboiling temperatures in a Teflon system in-house (School of Geography, Environment and Earth Sciences), starting from reagent grade acids to generate quality-controlled products equal to or better than Optima (Thermo Fisher) grade acids. The acids were distilled at the azeotropic concentration (i.e. concentrated for $\mathrm{HNO}_{3}$ and $6.02 \mathrm{M}$ for $\mathrm{HCl}$ ) and then diluted, as needed, using Milli-Q water (resistivity $>18.6 \mathrm{M} \Omega$ ), which was also regularly checked as a blank. Air-sensitive reactions were performed under an atmosphere of argon, and rotary evaporation was completed at $10 \mathrm{mmHg}$ and a suitable temperature. Reaction progress was monitored by NMR spectroscopy or normal-phase thinlayer chromatography (TLC) on aluminium sheets coated with silica gel $60 \mathrm{~F}_{254}$ (Merck) and visualised by staining or UV light. Flash chromatography was performed on a Reveleris ${ }^{\circledR}$ X2 flash chromatography system (BUCHI) using FlashPure Cartridges (BUCHI), silica $(50 \mu \mathrm{m})$, and a gradient of petroleum ether and ethyl acetate.

\subsubsection{Instrumentation, Equipment, and Analysis}

NMR spectra were recorded on a $500 \mathrm{MHz}$ Bruker NMR Spectrometer or $500 \mathrm{MHz}$ Jeol Resonance NMR Spectrometer in the indicated deuterated solvents. The ${ }^{1} \mathrm{H}$ NMR spectra were referenced to tetramethylsilane (TMS) or the solvent peak, and the ${ }^{13} \mathrm{C}$ NMR spectra were referenced to the solvent peak. The major solvent was referenced in mixed systems. NMR spectral data will be reported for key and novel compounds. Mass spectrometry was conducted on a Waters Q-TOF Premier ${ }^{\mathrm{TM}}$ Electrospray 
Mass Spectrometer with a MassLynx 4.1 operating system in positive mode. Samples were prepared for analysis by mixing in aqueous methanol containing trace formic acid. IR spectra were measured on a PerkinElmer Spectrum One FT-IR Spectrometer with a Universal ATR sampling accessory or on a Bruker Tensor II FT-IR Spectrometer with a Platinum ATR sampling accessory, and NIR spectra were measured on a PerkinElmer LAMBDA 1050 UV/Vis/NIR Spectrophotometer with a 3D WB Det. Module. A SpectraMax M4 Multi-Mode Microplate Reader (Molecular Devices) and Cary 50 Bio UV-Visible Spectrophotomer (Agilent) were used to obtain UV-Vis spectra, measure absorbance, or measure $\mathrm{OD}_{600}$. The functionalised silica products were submitted to The Campbell Microanalytical Laboratory (University of Otago; Dunedin, NZ) for $\mathrm{CHN}$ elemental and, in the cases of Batch $1 \mathrm{Cu}(\mathrm{II})-\mathrm{CnGmSiO} 2$ and the $\mathrm{Cu}(\mathrm{II})$-cyclen adsorbed onto silica, for ICP-MS analyses. CHN results were reported as duplicates within $0.30 \%$ of one another. All other metal analyses via ICP-MS were conducted in-house (School of Geography, Environment and Earth Sciences) on a Thermo Scientific Element 2 sector field ICP-MS. Images and the atomic compositions of coatings were obtained with an FEI Quanta 450 scanning electron microscope (tungsten filament) positioned at a $10 \mathrm{~mm}$ working distance and an Apollo X Silicon Drift Detector (EDAX) for SEM-EDS. Images were taken at 200× and 800× magnification with an electron beam spot size set at 4 and an accelerating voltage of $20 \mathrm{kV}$. EDS measurements were conducted at 200x magnification with the following parameters: spot size 6 (> $1000 \mathrm{cps}$ ), $20 \mathrm{kV}$ accelerating voltage, $30 \mathrm{~s}$ live time, $1.6 \mu \mathrm{s}$ amplitude time, $35-36^{\circ}$ take-off angle, and $129 \mathrm{eV}$ resolution. BET surface area analyses were conducted on a Flowsorb II 2300 Surface Area Analyser (Micromeritics) at $21.6^{\circ} \mathrm{C}$ and $1027 \mathrm{hPa}$. In bacterial adherence testing, sonication was carried out with a Bandelin Sonopuls Ultrasonic Homogeniser mini20 (2.0 sonotrode, 2.5 tip). Samples were centrifuged at 3000 relative centrifugal force (RCF) in a Rotina 380 centrifuge (Hettich).

\subsubsection{Bacterial Strains and Culture Conditions}

The strains of bacteria used in this work were Escherichia coli NZRM 3647 and Vibrio harveyi NZRM 2698 (Table 6.1), which were handled using aseptic technique and maintained by re-streaking from the seed stock every month for single colonies on agar plates. The plates were stored at $4{ }^{\circ} \mathrm{C}$, and the strains were stored at $-80^{\circ} \mathrm{C}$ in $25 \% \mathrm{v} / \mathrm{v}$ glycerol in liquid medium. E. coli was grown at $37^{\circ} \mathrm{C}$ in nutrient broth, on nutrient agar plates, or in M63 minimal medium. V. harveyi was grown at $30^{\circ} \mathrm{C}$ in Luria marine broth or on Luria marine agar plates. 
Table 6.1. Bacterial strains used in this study. ${ }^{325,326}$

\begin{tabular}{cccc}
\hline NZRM Strain \# & Name & Description & Source \\
\hline 3647 & Escherichia coli & $\begin{array}{c}\text { Biotech Australia - BTA 2850. O157 } \\
\text { verocytotoxin negative. Originally from The } \\
\text { Royal Children's Hospital, Melbourne }\end{array}$ & $\begin{array}{c}\text { Callaghan } \\
\text { Innovation, } \\
\text { Wellington, NZ }\end{array}$ \\
& Vibrio harveyi & 01/12/1995. & NZCC $^{\text {a }}$ \\
& & ATCC 14126 (NCIMB 1280). & Type strain. \\
\hline
\end{tabular}

a The New Zealand Reference Culture Collection: Medical Section, Environmental Science and Research.

\subsubsection{Bacterial Media Preparation}

All media were stored at $2-8{ }^{\circ} \mathrm{C}$ and handled using aseptic technique, which was implemented whenever a sterile environment was required. Sterilisation of media via autoclaving was conducted at $121^{\circ} \mathrm{C}$ for $20 \mathrm{~min}$.

\subsubsection{Nutrient Broth}

Nutrient broth was prepared as in ISO $22196 .{ }^{199}$ Beef Extract (3 g; Lab M), BBL ${ }^{\mathrm{TM}}$ Trypticase ${ }^{\mathrm{TM}}$ Peptone (10 g; BD Biosciences), and sodium chloride (5 g) were dissolved in double-distilled water (dd $\left.\mathrm{H}_{2} \mathrm{O} ; 1 \mathrm{~L}\right)$. The broth was adjusted to $\mathrm{pH} 7.0 \pm 0.2$ and autoclaved. Alternatively, dehydrated nutrient broth (13 g; Oxoid) was dissolved in $\mathrm{ddH}_{2} \mathrm{O}(1 \mathrm{~L})$, and the broth was adjusted to $\mathrm{pH} 7.4 \pm 0.2$ and autoclaved.

\subsubsection{Nutrient Agar}

Nutrient agar was prepared as in ISO 22196. ${ }^{199}$ Beef Extract (5 g; Lab M), BBL ${ }^{\mathrm{TM}}$ Trypticase ${ }^{\mathrm{TM}}$ Peptone (10 g; BD Biosciences), sodium chloride (5 g), and Bacto ${ }^{\mathrm{TM}}$ Agar (15 g; BD Biosciences) were dissolved in $\mathrm{ddH}_{2} \mathrm{O}(1 \mathrm{~L})$. The solution was adjusted to $\mathrm{pH} 7.1 \pm 0.1$ and autoclaved. Alternatively, dehydrated nutrient agar (28 g; Oxoid) was dissolved in $\mathrm{ddH}_{2} \mathrm{O}(1 \mathrm{~L})$, and the solution was adjusted to $\mathrm{pH} 7.4 \pm 0.2$ and autoclaved. Molten agar was delivered aseptically to $90 \mathrm{~mm}$ Petri dishes (25 $\mathrm{mL}$ per plate), and the agar plates were allowed to solidify at room temperature ( $15 \mathrm{~min})$. Before use, the plates were dried at $37^{\circ} \mathrm{C}$ or at room temperature in a laminar flow hood. 


\subsubsection{M63 Minimal Medium}

To prepare M63 minimal medium, ammonium sulfate $(2 \mathrm{~g})$, monobasic potassium phosphate (13.6 g), and ferrous sulfate heptahydrate $(0.5 \mathrm{mg})$ were dissolved in $\mathrm{ddH}_{2} \mathrm{O}(1 \mathrm{~L})$. The solution was adjusted to $\mathrm{pH} 7.0$ and autoclaved. After the solution cooled to below $50{ }^{\circ} \mathrm{C}$, filter-sterilised $20 \% \mathrm{v} / \mathrm{v}$ glycerol (aq; $10 \mathrm{~mL}$ ) and filter-sterilised $1 \mathrm{M}$ magnesium sulfate (aq; $1 \mathrm{~mL}$ ) were added. ${ }^{327}$

\subsubsection{Luria Marine Broth}

Luria marine broth was prepared as in Mire et al. ${ }^{304}$ : tryptone (10 g; Oxoid), yeast extract ( $5 \mathrm{~g}$; Oxoid), and sodium chloride $(20 \mathrm{~g})$ were dissolved in $\mathrm{ddH}_{2} \mathrm{O}(1 \mathrm{~L})$, and the solution was autoclaved.

\subsubsection{Luria Marine Agar}

Luria marine agar was prepared as in Mire et al. ${ }^{304}$ : tryptone (10 g; Oxoid), yeast extract ( $5 \mathrm{~g}$; Oxoid), sodium chloride $(20 \mathrm{~g})$, and bacteriological agar ( $15 \mathrm{~g}$; Oxoid) were dissolved in $\mathrm{ddH}_{2} \mathrm{O}(1 \mathrm{~L})$, and the solution was autoclaved. Molten agar was delivered aseptically to $90 \mathrm{~mm}$ Petri dishes $(25 \mathrm{~mL}$ per plate), and the agar plates were allowed to solidify at room temperature ( $15 \mathrm{~min})$. Before use, the plates were dried at $37^{\circ} \mathrm{C}$ or at room temperature in a laminar flow hood.

\subsubsection{Optical Density Measurements}

The optical densities of bacterial cultures were measured at $600 \mathrm{~nm}$ with a Unicam Helios UV-Vis spectrophotometer (Biolab Scientific Ltd.) in $1 \mathrm{~mL}$ cuvettes (1 cm path length). The appropriate growth medium was used as a blank and to dilute the sample, as needed.

\subsubsection{Growth Curves}

An overnight culture was prepared by inoculating the appropriate liquid medium ( $3 \mathrm{~mL}$ ) with a colony of the bacterium from an agar plate and incubating the culture for $16 \mathrm{~h}$ at the appropriate temperature with shaking. In a well of a 96-well plate (Thermo Scientific), fresh liquid medium was inoculated with overnight culture to $\mathrm{OD}_{600} 0.05$. Uninoculated medium served as a blank. The plate was incubated for $20.8 \mathrm{~h}$ at the appropriate temperature, and, every $5 \mathrm{~min}$, the $\mathrm{OD}_{600}$ of each well was recorded by a 
SPECTROstar ${ }^{\text {Nano }}$ plate reader (BMG LABTECH) after the plate was shaken $10 \mathrm{~s}$ (orbital) to generate a growth curve for the bacterium.

\subsubsection{Enumeration of Cells by the Drop Plate Method}

Ten-fold serial dilutions of the samples in sterile $0.85 \% \mathrm{w} / \mathrm{w}$ aq sodium chloride (i.e. saline; E. coli) or $2 \% \mathrm{w} / \mathrm{w}$ aq sodium chloride ( $\mathrm{V}$. harveyi) were performed to quantify the number of CFUs in a given volume or area by the drop plate method in which five aliquots ( $10 \mu \mathrm{L}$; technical replicates) of each dilution were delivered to and allowed to absorb into an agar plate. The plates were incubated overnight at the appropriate temperature until the colonies were countable, and the countable dilution contained 3-30 colonies per $10 \mu \mathrm{L}$ drop. The results from the technical replicates were averaged.

\subsubsection{Gram Staining}

Following the protocol published by the American Society for Microbiology, ${ }^{328}$ a loopful of fresh liquid culture was smeared on a microscope slide and allowed to air dry. Once the inoculum was dry, the cells were heat-fixed to the slide by passing it, cell side up, $3 \times$ through the top of a flame, warming the glass. The heat-fixed cells were dyed with crystal violet staining reagent for $1 \mathrm{~min}$ and then briefly $(<5 s)$ washed with $d_{d H_{2}} \mathrm{O}$. They were covered with Gram's iodine solution, a mordant to fix the dye, for $1 \mathrm{~min}$ and then briefly $\left(<5 \mathrm{~s}\right.$ ) washed with $\mathrm{dd}_{2} \mathrm{O}$. The slide was rinsed with $95 \% \mathrm{v} / \mathrm{v}$ ethanol (aq) for $<10 \mathrm{~s}$ to decolourise the cells and then rinsed with $\mathrm{ddH}_{2} \mathrm{O}(<5 \mathrm{~s})$ to stop the decolourisation. The cells were counterstained with safranin for $<30 \mathrm{~s}$ and then briefly $(<5 \mathrm{~s})$ rinsed with $\mathrm{dd}_{2} \mathrm{O}$. The slide was gently blotted dry, and the cells were examined under a light microscope.

\subsubsection{Graphing and Statistical Analyses}

Graphs were created and statistical analyses were performed using GraphPad Prism 8 (GraphPad Software, Inc.) or Microsoft ${ }^{\circledR}$ Excel $^{\circledR}$ for Office 365. The bacterial enumeration data were presented as the mean value \pm the standard deviation (SD), and statistical analyses were conducted when there were three or more biological replicates for each sample in the experiment. To determine if the means from two data sets were significantly different from each other, a two-sample, two-tail Student's $t$-test (unequal variance) was conducted, and the difference was considered to be significant when the 
$p$-value was $<0.05$. For the graphs, statistical significance was denoted as follows: ${ }^{*} p<0.05$, ** $p<0.01$, and $* * * p<0.001$. One-way analyses of variance (ANOVAs) were completed to determine if the differences between the means of two or more independent data sets were statistically significant, and Tukey's post hoc test was used to identify which groups were statistically different. 


\subsection{CHAPTER 2}

\subsubsection{Polyacylhydrazone: DiLevDEG/ADH}

Procedures by Daines et al. ${ }^{127}$ (Scheme 6.1) were followed for the synthesis of the DiLevDEG/ADH polyacylhydrazone.

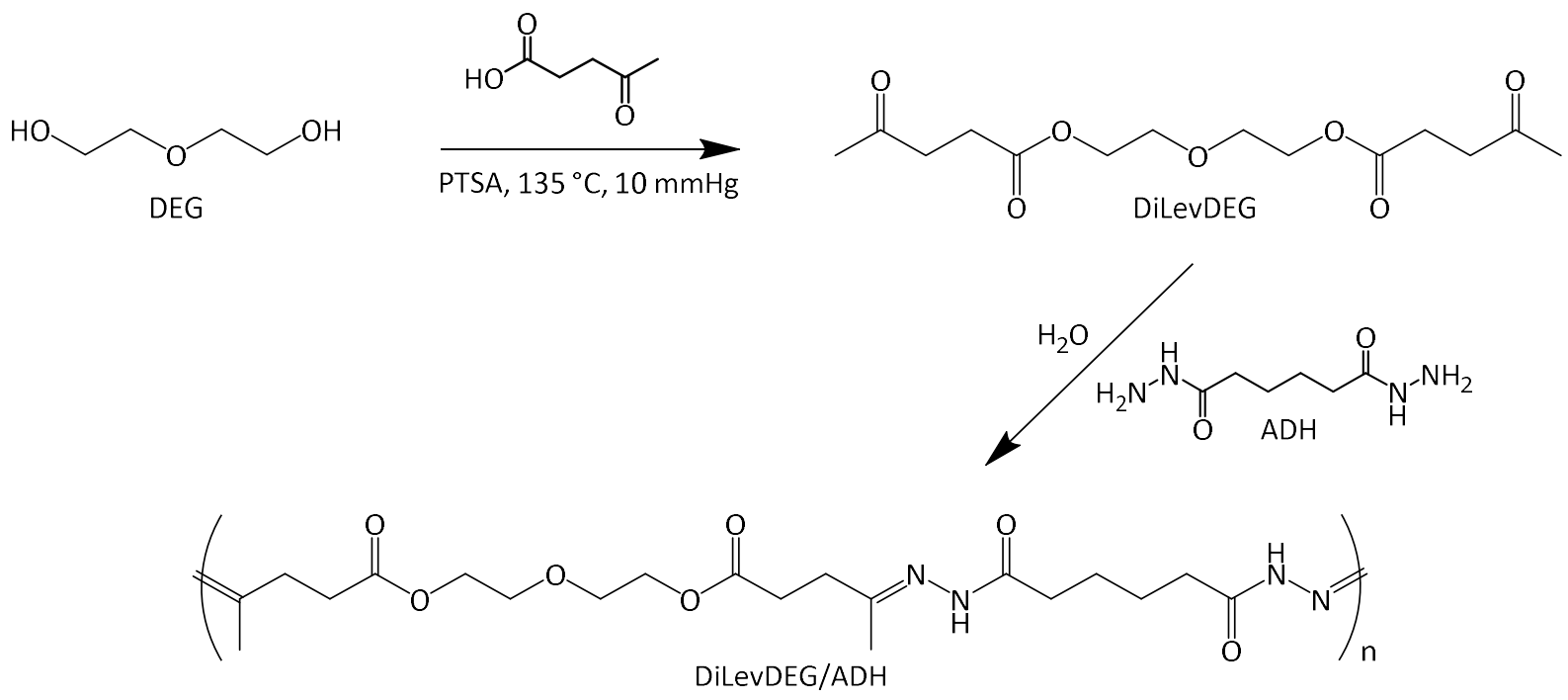

Scheme 6.1. Synthesis of DiLevDEG/ADH polyacylhydrazone.

\subsubsection{Synthesis of DiLevDEG}

Levulinic acid (61.91 g, $0.5225 \mathrm{~mol}, 1.4 \mathrm{eq})$, DEG ( $40 \mathrm{~g}, 0.37 \mathrm{~mol})$, and PTSA monohydrate $(0.208 \mathrm{~g}$, $1.18 \mathrm{mmol}, 0.003 \mathrm{eq})$ were heated at $135^{\circ} \mathrm{C}$ under reduced pressure $(10 \mathrm{mmHg})$. The water produced by the reaction was collected by distillation, and the reaction was determined to be complete when water production ceased (approximately $8 \mathrm{~h}$ ). The reaction mixture was cooled to room temperature, washed with water $(3 \times 100 \mathrm{~mL})$ and sodium chloride (saturated, aq; $100 \mathrm{~mL})$, dried over magnesium sulfate, and concentrated to give the product as an orange, viscous oil (56.81 g, 50\% yield). NMR spectral data were consistent with those in the literature. ${ }^{127}$

${ }^{1} \mathrm{H}$ NMR $\left(500 \mathrm{MHz}, \mathrm{CDCl}_{3}\right): \delta 4.23(\mathrm{t}, J=4.7 \mathrm{~Hz}, 4 \mathrm{H}), 3.69(\mathrm{t}, J=4.7 \mathrm{~Hz}, 4 \mathrm{H}), 2.76(\mathrm{t}, J=6.5 \mathrm{~Hz}, 4 \mathrm{H}), 2.61$ $(\mathrm{t}, J=6.5 \mathrm{~Hz}, 4 \mathrm{H}), 2.19(\mathrm{~s}, 6 \mathrm{H}) ;{ }^{13} \mathrm{C}$ NMR $\left(126 \mathrm{MHz}, \mathrm{CDCl}_{3}\right): \delta$ 206.6, 172.8, 69.2, 63.8, 38.0, 30.0, 28.1. 


\subsubsection{Synthesis of DiLevDEG/ADH}

$\operatorname{ADH}(1.298 \mathrm{~g}, 7.302 \mathrm{mmol}, 0.9 \mathrm{eq})$ was added to a solution of DiLevDEG $(2.453 \mathrm{~g}, 8.114 \mathrm{mmol})$ in water $(5 \mathrm{~mL})$ to give a clear, viscous solution of $\sim 50 \% \mathrm{w} / \mathrm{v}$ solids. To obtain NMR spectra, a test reaction was set up in an NMR tube by adding ADH (13 mg, $73 \mu \mathrm{mol}, 0.9$ eq) to a solution of DiLevDEG ( $25 \mathrm{mg}$, $83 \mu \mathrm{mol})$ in deuterium oxide $(1 \mathrm{~mL})$. NMR spectral data were consistent with previously recorded laboratory data.

${ }^{1} \mathrm{H}$ NMR $\left(500 \mathrm{MHz}, \mathrm{D}_{2} \mathrm{O}\right): \delta 4.58-4.18(\mathrm{~m}, 2 \mathrm{H}), 3.93-3.66(\mathrm{~m}, 2 \mathrm{H}), 3.02-2.92(\mathrm{~m}, 1 \mathrm{H}), 2.84-2.52(\mathrm{~m}$, $4 \mathrm{H}), 2.49-2.36(\mathrm{~m}, 1 \mathrm{H}), 2.35-2.21(\mathrm{~m}, 3 \mathrm{H}), 2.12-1.92(\mathrm{~m}, 2 \mathrm{H}), 1.80-1.55(\mathrm{~m}, 4 \mathrm{H}) ;{ }^{13} \mathrm{C} \mathrm{NMR}(126 \mathrm{MHz}$, $\left.D_{2} \mathrm{O}\right): \delta 213.5,175.3,172.7,163.1,68.5,64.0,37.8,33.7,33.6,33.4,33.3,33.1,32.5,31.8,30.3,29.9$, $29.7,29.1,28.0,25.8,24.8,24.7,24.6,24.5,23.9,21.6,16.2,15.8$.

\subsubsection{Simple Acylhydrazones}

\subsubsection{Synthesis of Precursor Compounds}

\subsection{Synthesis of Acethydrazide}

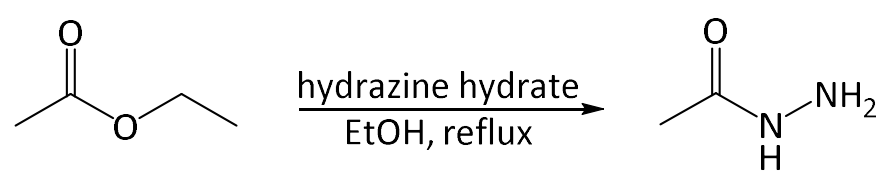

Scheme 6.2. Synthesis of acethydrazide.

Acethydrazide was synthesised following the procedure by Sadek et al. ${ }^{329}$ (Scheme 6.2). Hydrazine hydrate $(80 \% \mathrm{w} / \mathrm{w} ; 8.0 \mathrm{~mL}, 0.13 \mathrm{~mol})$ was added dropwise to ethanol $(5 \mathrm{~mL})$, and the solution was heated $\left(<50^{\circ} \mathrm{C}\right)$. Then, ethyl acetate $(20 \mathrm{~mL}, 0.20 \mathrm{~mol})$ was added dropwise to the solution, which was heated under reflux for $96 \mathrm{~h}$. The white, crystalline product was isolated via rotary evaporation and cooling to $0{ }^{\circ} \mathrm{C}(8.131 \mathrm{~g}, 108 \%$ yield, presence of contaminants in NMR spectra). Aside from the contaminants, NMR spectral data were consistent with those in the literature. ${ }^{329}$ 


\subsection{Synthesis of Methyl and Ethyl Levulinate}<smiles>[R]OC(=O)CCC(C)=O</smiles>

Scheme 6.3. Synthesis of methyl/ethyl levulinate $(R=-M e,-E t)$.

Methyl and ethyl levulinate were synthesised by following established procedures in the laboratory (Scheme 6.3), and the characterisation data were compared to previous records. Methyl levulinate was produced by adding methanol ( $31 \mathrm{~mL})$ to levulinic acid $(3.034 \mathrm{~g}, 25.35 \mathrm{mmol})$, PTSA monohydrate $(0.317 \mathrm{~g}, 1.79 \mathrm{mmol})$, and $3 \AA$ molecular sieves. The reaction mixture was heated under reflux for $44.5 \mathrm{~h}$. Upon reaction completion, the mixture was filtered and concentrated by rotary evaporation. Ethyl acetate $(10 \mathrm{~mL})$ was added to the concentrated reaction mixture, and the organic layer was washed with sodium chloride (saturated, aq; $1 \times 10 \mathrm{~mL}$ ) and dried over magnesium sulfate. The solvent was removed by rotary evaporation to yield the product as a clear, colourless liquid $(1.230 \mathrm{~g}, 37 \%$ yield). Following the same procedure, ethyl levulinate was generated by adding ethanol $(31 \mathrm{~mL})$ to levulinic acid (3.069 g, $25.64 \mathrm{mmol})$, PTSA monohydrate $(0.322 \mathrm{~g}, 1.82 \mathrm{mmol})$, and 3 Å molecular sieves (2.745 g, 74\% yield; clear, colourless liquid). NMR spectral data were consistent with previously recorded laboratory data.

\subsection{Derivatisation of Vanillin: Acylation with Acetic, Trimethylacetic, and Butyric Anhydride}<smiles>COc1cc(C=O)ccc1O</smiles>

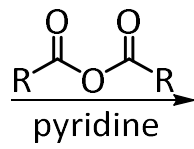<smiles>[R]C(=O)Oc1ccc(C=O)cc1OC</smiles>

Scheme 6.4. Synthesis of acylated vanillin $(R=-M e,-t B u,-P r)$. 
Acylated vanillin was synthesised following the procedure by Yang et al. ${ }^{330}$ (Scheme 6.4). Acetic, trimethylacetic, and butyric anhydride $(3 \mathrm{eq})$ were each added to separate solutions of vanillin $(1.0 \mathrm{~g}$, $6.4 \mathrm{mmol}$ ) in pyridine $(5 \mathrm{~mL})$. The reactions with acetic and butyric anhydride were stirred for $3 \mathrm{~h}$ at room temperature until they were determined to be complete via TLC. After $30 \mathrm{~h}$ stirring at room temperature, the reaction with trimethylacetic anhydride was still incomplete, as determined by TLC, so 4-dimethylaminopyridine $(10 \mathrm{mg}, 81 \mu \mathrm{mol})$ was added. The reaction was stirred at room temperature for an additional $23 \mathrm{~h}$ until completion. All reaction mixtures were diluted with diethyl ether $(50 \mathrm{~mL})$ and washed with hydrochloric acid $(10 \% \mathrm{v} / \mathrm{v}$, aq; $5 \times 10 \mathrm{~mL})$ and sodium chloride (saturated, aq; $1 \times 10 \mathrm{~mL}$ ), and the organic layers were dried over magnesium sulfate for $30 \mathrm{~min}$. The organic layers were then filtered and concentrated under vacuum to yield the crude products, which were purified by flash chromatography. Vanillin acetate was isolated as a cream-coloured solid $(0.935 \mathrm{~g}, 74 \%$ yield), vanillin pivalate was isolated as a white solid $(0.769 \mathrm{~g}, 51 \%$ yield), and vanillin butyrate was isolated as a brown liquid ( $>0.350 \mathrm{~g},>23 \%$ yield). NMR spectral data were consistent with those in the literature. ${ }^{330-332}$

\subsubsection{General Procedure for Synthesis of Acylhydrazones}

The acylhydrazones in Scheme 6.5 were synthesised following the general procedure by Zha and You. ${ }^{133}$ The hydrazide (1-5) was added to a solution of the aldehyde or ketone ( 1 eq for monoacylhydrazones, $\sim 2$ eq for acyldihydrazones) in methanol or ethanol. Glacial acetic acid (AcOH) was then added dropwise to adjust to $\mathrm{pH} 4-5$, and the reaction mixture was heated under reflux to yield a white or yellow precipitate, which was isolated by vacuum filtration and washed with methanol or ethanol $(2 \times 5 \mathrm{~mL})$. The acylhydrazones were generally soluble in dimethylformamide and dimethylsulfoxide. However, $1 \mathrm{a}$ and $\mathbf{3 h}$ were also soluble in chloroform containing $10 \% \mathrm{v} / \mathrm{v}$ methanol, and ligands $\mathbf{1 i}$ and $\mathbf{1 j}$ were soluble in chloroform. Ligand $\mathbf{5 h}$ was only poorly soluble in chloroform/methanol (10\% v/v). Refer to the Appendix (Figures 8.3-19, Table 8.1-17) for the NMR spectra and peak assignments. In describing the ${ }^{1} \mathrm{H}$ NMR data for molecules that display rotamers in solution, the convention followed is to list the two signals demonstrating the rotameric forms, then their multiplicity and the total value for which they integrate (e.g. $\delta 11.60 \& 11.21(\mathrm{~s}, 1 \mathrm{H}))$. 
Reaction Scheme

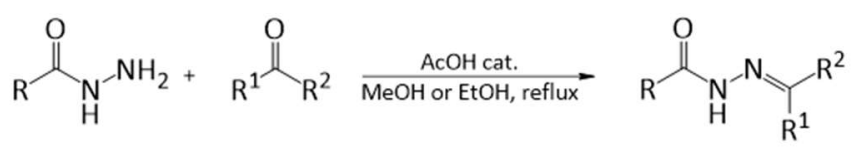

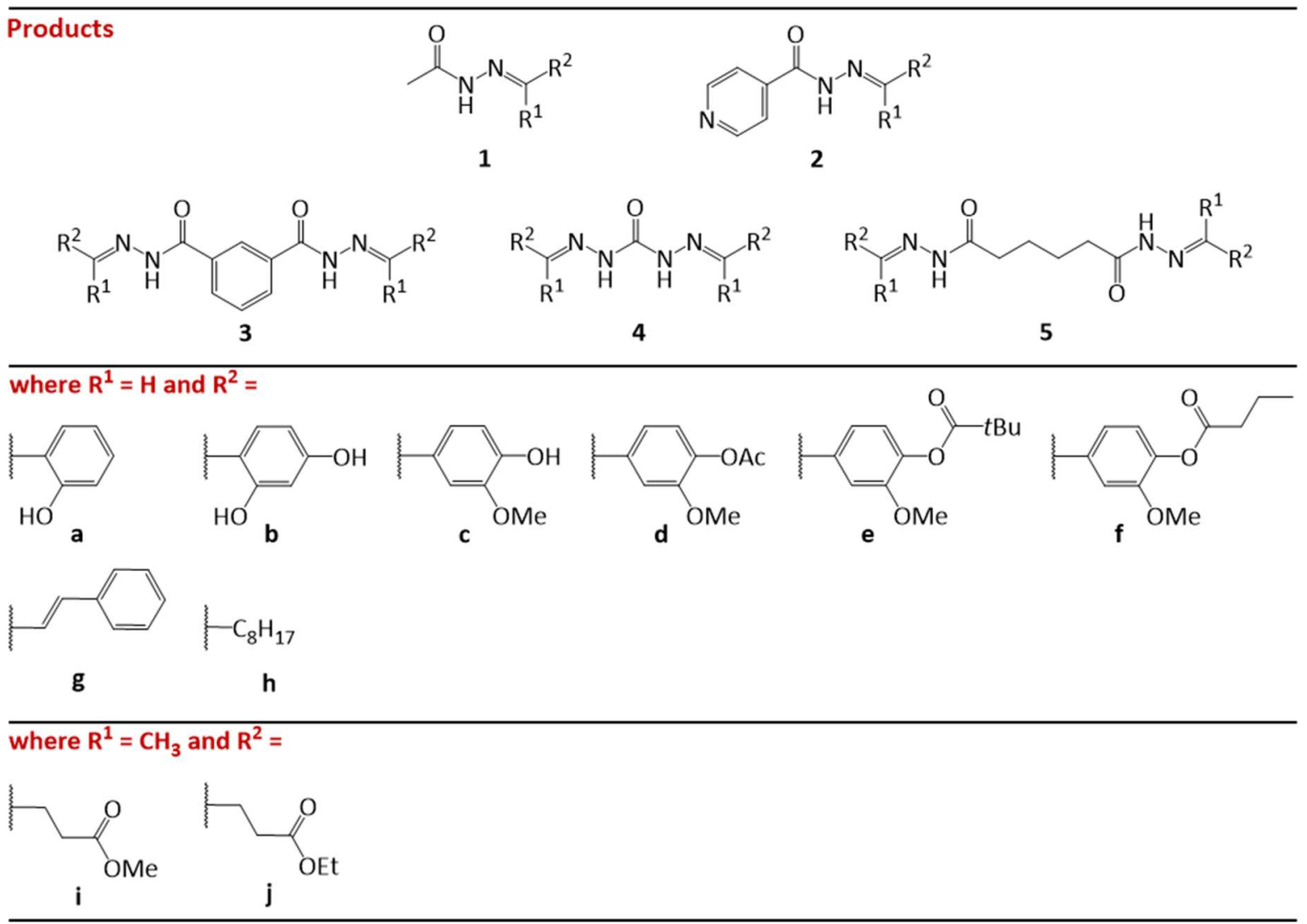

Scheme 6.5. General scheme for the synthesis of acylhydrazones (top; $R, R^{1}, R^{2}$ : same or different). The synthesised acylhydrazones are as follows: $\mathbf{1 a}-\mathbf{b}, \mathbf{1} \mathbf{i}-\mathbf{j}, \mathbf{2 a - b}, \mathbf{3 c - f}, \mathbf{3 h}, \mathbf{4 c}, \mathbf{4 g}, \mathbf{5 c}$, and $\mathbf{5 h}-\mathbf{j}$.

\subsection{Monoacylhydrazones}

Compound 1a was prepared by reacting acethydrazide $(0.490 \mathrm{~g}, 5.95 \mathrm{mmol})$ and salicylaldehyde $(0.753 \mathrm{~g}, 6.10 \mathrm{mmol})$ for $18 \mathrm{~h}$ in $5 \mathrm{~mL}$ methanol $\left(0.717 \mathrm{~g}, 64 \%\right.$ yield). ${ }^{1} \mathrm{H}$ NMR (500 MHz, DMSO- $\left.d_{6}\right)$ : $\delta 11.60 \& 11.21(\mathrm{~s}, 1 \mathrm{H}), 10.13(\mathrm{~s}, 1 \mathrm{H}), 8.34 \& 8.27(\mathrm{~s}, 1 \mathrm{H}), 7.62 \& 7.49(\mathrm{dd}, J=7.8,1.7 \mathrm{~Hz}, J=7.5,1.7 \mathrm{~Hz}$, $1 \mathrm{H}), 7.28 \& 7.24(\mathrm{td}, J=7.6,1.6 \mathrm{~Hz}, J=7.5,1.6 \mathrm{~Hz}, 1 \mathrm{H}), 6.94-6.83(\mathrm{~m}, 2 \mathrm{H}), 2.18 \& 1.98(\mathrm{~s}, 3 \mathrm{H}) ;{ }^{13} \mathrm{C} \mathrm{NMR}$ (126 MHz, DMSO-d $)$ : $\delta$ 171.4, 165.4, 157.3, 156.3, 146.3, 140.9, 131.1, 130.8, 129.4, 126.8, 119.9, 119.4, 119.2, 118.5, 116.3, 116.1, 21.3, 20.3. ESI-HRMS: calcd for $\mathrm{C}_{9} \mathrm{H}_{10} \mathrm{~N}_{2} \mathrm{O}_{2} \mathrm{Na}[\mathrm{M}+\mathrm{Na}]^{+}$201.0640; found 201.0641 . 
Compound 1b was prepared by reacting acethydrazide $(0.481 \mathrm{~g}, 5.84 \mathrm{mmol})$ and 2,4-dihydroxybenzaldehye $(0.861 \mathrm{~g}, 6.11 \mathrm{mmol})$ for $16 \mathrm{~h}$ in $10 \mathrm{~mL}$ ethanol $(0.812 \mathrm{~g}, 68 \%$ yield). ${ }^{1} \mathrm{H}$ NMR $\left(500 \mathrm{MHz}\right.$, DMSO-d $\left.d_{6}\right): \delta 11.39 \& 11.02(\mathrm{~s}, 1 \mathrm{H}), 9.89(\mathrm{~s}, 1 \mathrm{H}), 8.20 \& 8.13(\mathrm{~s}, 1 \mathrm{H}), 7.38 \& 7.25$ (d, $J=9.1 \mathrm{~Hz}, J=8.4 \mathrm{~Hz}, 1 \mathrm{H}), 6.37-6.27(\mathrm{~m}, 2 \mathrm{H}), 2.13 \& 1.94(\mathrm{~s}, 3 \mathrm{H}) ;{ }^{13} \mathrm{C} \mathrm{NMR}\left(126 \mathrm{MHz}, \mathrm{DMSO}-d_{6}\right): \delta 171.0$, $165.0,160.5,160.2$, 159.3, 158.1, 147.3, 142.3, 131.2, 128.8, 111.4, 110.4, 107.8, 107.6, 102.6, 102.4, 21.3, 20.3. ESI-HRMS: calcd for $\mathrm{C}_{13} \mathrm{H}_{11} \mathrm{~N}_{3} \mathrm{O}_{3}[\mathrm{M}+\mathrm{H}]^{+}$258.0879; found 258.0878 .

Compound 1i was prepared by reacting acethydrazide $(0.152 \mathrm{~g}, 1.85 \mathrm{mmol})$ and methyl levulinate $(0.274 \mathrm{~g}, 2.08 \mathrm{mmol})$ for $46 \mathrm{~h}$ in $6 \mathrm{~mL}$ methanol ( $0.159 \mathrm{~g}, 46 \%$ yield). ${ }^{1} \mathrm{H}$ NMR (500 MHz, $\left.\mathrm{CDCl}_{3}\right): \delta 8.84$ $(\mathrm{s}, 1 \mathrm{H}), 3.68(\mathrm{~s}, 3 \mathrm{H}), 2.63-2.57(\mathrm{~m}, 4 \mathrm{H}), 2.20(\mathrm{~s}, 3 \mathrm{H}), 1.87(\mathrm{~s}, 3 \mathrm{H}) ;{ }^{13} \mathrm{C} \mathrm{NMR}\left(126 \mathrm{MHz}, \mathrm{CDCl}_{3}\right): \delta 173.8$, 173.5, 149.6, 51.7, 33.3, 29.9, 20.4, 15.9. ESI-HRMS: calcd for $\mathrm{C}_{8} \mathrm{H}_{14} \mathrm{~N}_{2} \mathrm{O}_{3}[\mathrm{M}+\mathrm{H}]^{+}$187.1083; found 187.1087.

Compound $1 \mathbf{j}$ was prepared by reacting acethydrazide $(0.149 \mathrm{~g}, 1.81 \mathrm{mmol})$ and ethyl levulinate (0.305 g, $2.09 \mathrm{mmol}$ ) for $46 \mathrm{~h}$ in $6 \mathrm{~mL}$ ethanol (0.412 g, 39\% yield). ${ }^{1} \mathrm{H}$ NMR (500 MHz, $\mathrm{CDCl}_{3}$ ): $\delta 8.79$ (s, $1 \mathrm{H}), 4.13(\mathrm{q}, J=7.1 \mathrm{~Hz}, 2 \mathrm{H}), 2.62-2.58(\mathrm{~m}, 4 \mathrm{H}), 2.21(\mathrm{~s}, 3 \mathrm{H}), 1.86(\mathrm{~s}, 3 \mathrm{H}), 1.25(\mathrm{t}, J=7.1 \mathrm{~Hz}, 3 \mathrm{H})$; ${ }^{13} \mathrm{C} \mathrm{NMR}\left(126 \mathrm{MHz}, \mathrm{CDCl}_{3}\right): \delta 173.7,173.0,149.7,60.5,33.3,30.2,20.5,15.9,14.4$. ESI-HRMS: calcd for $\mathrm{C}_{9} \mathrm{H}_{16} \mathrm{~N}_{2} \mathrm{O}_{3}[\mathrm{M}+\mathrm{H}]^{+}$201.1239; found 201.1245.

Compound 2a was prepared by reacting isonicotinic hydrazide $(0.397 \mathrm{~g}, 2.87 \mathrm{mmol})$ and salicylaldehyde $(0.310 \mathrm{~mL}, 2.85 \mathrm{mmol})$ for $16 \mathrm{~h}$ in $10 \mathrm{~mL}$ ethanol $\left(0.600 \mathrm{~g}, 85 \%\right.$ yield). ${ }^{1} \mathbf{H}$ NMR (500 MHz, DMSO- $\left.d_{6}\right): \delta 12.28 \& 12.01(\mathrm{~s}, 1 \mathrm{H}), 11.09 \& 9.97(\mathrm{~s}, 1 \mathrm{H}), 8.81 \& 8.74(\mathrm{dd}, J=4.5,1.6 \mathrm{~Hz}, \mathrm{~d}$, $J=4.3 \mathrm{~Hz}, 2 \mathrm{H}), 8.69 \& 8.38(\mathrm{~s}, 1 \mathrm{H}), 7.85 \& 7.64(\mathrm{dd}, J=4.4,1.7 \mathrm{~Hz}, \mathrm{~d}, J=4.6 \mathrm{~Hz}, 2 \mathrm{H}), 7.61 \& 7.41$ (dd, $J=7.7,1.8 \mathrm{~Hz}, \mathrm{~d}, J=7.5 \mathrm{~Hz}, 1 \mathrm{H}), 7.33 \& 7.22(\mathrm{td}, J=7.4,1.6 \mathrm{~Hz}, \mathrm{t}, J=7.3,1 \mathrm{H}), 6.98-6.90$ \& 6.88-6.79 $(\mathrm{m}, 2 \mathrm{H}) ;{ }^{13} \mathrm{C}$ NMR $\left(126 \mathrm{MHz}\right.$, DMSO- $\left.d_{6}\right): \delta 161.3,157.5,156.5,150.4,149.6,149.0,143.2,140.0,131.7$, 131.1, 129.2, 127.1, 122.6, 121.5, 119.4, 118.7, 116.4, 116.1. ESI-HRMS: calcd for $\mathrm{C}_{13} \mathrm{H}_{11} \mathrm{~N}_{3} \mathrm{O}_{2}[\mathrm{M}+\mathrm{H}]^{+}$ 242.0930; found 242.0932.

Compound $\mathbf{2 b}$ was prepared by reacting isonicotinic hydrazide $(0.398 \mathrm{~g}, 2.87 \mathrm{mmol}$ ) and 2,4-dihydroxybenzaldehyde $(0.404 \mathrm{~g}, 2.87 \mathrm{mmol})$ for $16 \mathrm{~h}$ in $10 \mathrm{~mL}$ ethanol $(0.691 \mathrm{~g}, 89 \%$ yield). ${ }^{1}$ H NMR $\left(500 \mathrm{MHz}\right.$, DMSO- $\left.d_{6}\right): \delta 12.11 \& 11.82(\mathrm{~s}, 1 \mathrm{H}), 11.28(\mathrm{~s}, 1 \mathrm{H}), 10.00(\mathrm{~s}, 1 \mathrm{H}), 8.79 \& 8.72$ (dd, $J=4.4,1.6 \mathrm{~Hz}, \mathrm{~d}, J=5.3 \mathrm{~Hz}, 2 \mathrm{H}), 8.55 \& 8.24(\mathrm{~s}, 1 \mathrm{H}), 7.84 \& 7.61(\mathrm{dd}, J=4.5,1.5 \mathrm{~Hz}, \mathrm{~d}, J=5.1 \mathrm{~Hz}, 2 \mathrm{H})$, $7.37 \& 7.22(\mathrm{~d}, J=8.4 \mathrm{~Hz}, J=8.3 \mathrm{~Hz}, 1 \mathrm{H}), 6.39 \& 6.30-6.25(\mathrm{dd}, J=8.4,2.3 \mathrm{~Hz}, \mathrm{~m}, 1 \mathrm{H}), 6.35$ \& 6.30-6.25 $(\mathrm{d}, J=2.4 \mathrm{~Hz}, \mathrm{~m}, 1 \mathrm{H}) ;{ }^{13} \mathrm{C}$ NMR $\left(126 \mathrm{MHz}, \mathrm{DMSO}-d_{6}\right): \delta 161.0,160.9,159.5,158.2,150.3,150.0,149.7$, 144.4, 140.1, 131.2, 129.2, 122.5, 121.4, 110.4, 107.9, 102.7, 102.4. ESI-HRMS: calcd for $\mathrm{C}_{13} \mathrm{H}_{11} \mathrm{~N}_{3} \mathrm{O}_{3}$ $[\mathrm{M}+\mathrm{H}]^{+}$258.0879; found 258.0878. 


\subsection{Acyldihydrazones}

Compound 3c was prepared by reacting isophthalic dihydrazide $(0.396 \mathrm{~g}, 1.98 \mathrm{mmol})$ and vanillin (0.622 g, $3.97 \mathrm{mmol}$ ) for $18 \mathrm{~h}$ in $140 \mathrm{~mL}$ methanol (0.591 g, 64\% yield). ${ }^{1} \mathrm{H}$ NMR (500 MHz, DMSO- $d_{6}$ ): $\delta 11.82(\mathrm{~s}, 2 \mathrm{H}), 9.54(\mathrm{~s}, 2 \mathrm{H}), 8.42(\mathrm{~s}, 1 \mathrm{H}), 8.37(\mathrm{~s}, 2 \mathrm{H}), 8.09(\mathrm{~d}, J=7.7 \mathrm{~Hz}, 2 \mathrm{H}), 7.67(\mathrm{t}, J=7.7 \mathrm{~Hz}, 1 \mathrm{H})$, $7.34(\mathrm{~s}, 2 \mathrm{H}), 7.11(\mathrm{~d}, J=8.0 \mathrm{~Hz}, 2 \mathrm{H}), 6.86(\mathrm{~d}, J=8.1 \mathrm{~Hz}, 2 \mathrm{H}), 3.85(\mathrm{~s}, 6 \mathrm{H}) ;{ }^{13} \mathrm{C} \mathrm{NMR}\left(126 \mathrm{MHz}, \mathrm{DMSO}-d_{6}\right)$ : $\delta 162.4,149.1,148.8,148.1,133.9,130.5,128.7,126.8,125.6,122.2$, 115.5, 109.1, 55.6. ESI-HRMS: calcd for $\mathrm{C}_{24} \mathrm{H}_{22} \mathrm{~N}_{4} \mathrm{O}_{6}[\mathrm{M}+\mathrm{Na}]^{+} 485.1437$; found 485.1430 .

Compound 3d was prepared by reacting isophthalic dihydrazide $(0.138 \mathrm{~g}, 0.689 \mathrm{mmol})$ and 4-formyl2-methoxyphenyl acetate ( $0.270 \mathrm{~g}, 1.36 \mathrm{mmol})$ for $3 \mathrm{~h}$ in $50 \mathrm{~mL}$ methanol ( $0.312 \mathrm{~g}, 42 \%$ yield). ${ }^{1} \mathrm{H}$ NMR (500 MHz, DMSO- $\left.d_{6}\right): \delta 12.05(\mathrm{~s}, 2 \mathrm{H}), 8.49(\mathrm{~s}, 2 \mathrm{H}), 8.47(\mathrm{~s}, 1 \mathrm{H}), 8.13(\mathrm{~d}, J=7.5 \mathrm{~Hz}, 2 \mathrm{H}), 7.71(\mathrm{t}, J=7.6 \mathrm{~Hz}$, 1H), $7.49(\mathrm{~s}, 2 \mathrm{H}), 7.32(\mathrm{~d}, J=7.9 \mathrm{~Hz}, 2 \mathrm{H}), 7.20(\mathrm{~d}, J=8.1 \mathrm{~Hz}, 2 \mathrm{H}), 3.86(\mathrm{~s}, 6 \mathrm{H}), 2.28(\mathrm{~s}, 6 \mathrm{H}) ;{ }^{13} \mathrm{C}$ NMR $\left(126 \mathrm{MHz}, \mathrm{DMSO}-d_{6}\right): \delta 168.4,162.6,151.2,147.6,140.9,133.7,133.1,130.8,128.8,126.9,123.3$, 120.6, 109.9, 55.9, 20.4. ESI-HRMS: calcd for $\mathrm{C}_{28} \mathrm{H}_{26} \mathrm{~N}_{4} \mathrm{O}_{8}[\mathrm{M}+\mathrm{Na}]^{+} 569.1648$; found 569.1655.

Compound 3e was prepared by reacting isophthalic dihydrazide $(0.096 \mathrm{~g}, 0.48 \mathrm{mmol})$ and (4-formyl2-methoxy-phenyl) 2,2-dimethylpropanoate $(0.221 \mathrm{~g}, 0.935 \mathrm{mmol}$ ) for $5.5 \mathrm{~h}$ in $35 \mathrm{~mL}$ methanol $\left(0.231 \mathrm{~g}, 39 \%\right.$ yield). ${ }^{1} \mathrm{H}$ NMR (500 MHz, DMSO- $\left.d_{6}\right): \delta 12.05(\mathrm{~s}, 2 \mathrm{H}), 8.48(\mathrm{~s}, 2 \mathrm{H}), 8.46(\mathrm{~s}, 1 \mathrm{H}), 8.13$ (d, $J=7.5 \mathrm{~Hz}, 2 \mathrm{H}), 7.71(\mathrm{t}, J=7.6 \mathrm{~Hz}, 1 \mathrm{H}), 7.48(\mathrm{~s}, 2 \mathrm{H}), 7.32(\mathrm{~d}, J=7.9 \mathrm{~Hz}, 2 \mathrm{H}), 7.17(\mathrm{~d}, J=8.1 \mathrm{~Hz}, 2 \mathrm{H}), 3.85$ $(\mathrm{s}, 6 \mathrm{H}), 1.32$ (s, 18H); ${ }^{13} \mathrm{C}$ NMR (126 MHz, DMSO- $\left.d_{6}\right): \delta 175.6,162.6,151.3,147.6,141.3,133.7,133.0$, $130.8,128.8,126.9,123.2,120.6,110.0,56.0,38.6,26.8$. ESI-HRMS: calcd for $\mathrm{C}_{34} \mathrm{H}_{38} \mathrm{~N}_{4} \mathrm{O}_{8}[\mathrm{M}+\mathrm{Na}]^{+}$ 653.2587; found 653.2593 .

Compound $3 \mathbf{f}$ was prepared by reacting isophthalic dihydrazide $(0.053 \mathrm{~g}, 0.26 \mathrm{mmol})$ and 4-formyl-2methoxyphenyl butanoate $(0.131 \mathrm{~g}, 0.560 \mathrm{mmol})$ for $16 \mathrm{~h}$ in $20 \mathrm{~mL}$ methanol $(0.089 \mathrm{~g}, 56 \%$ yield). ${ }^{1} \mathrm{H}$ NMR (500 MHz, DMSO-d $\left.d_{6}\right): \delta 12.04(\mathrm{~s}, 2 \mathrm{H}), 8.49(\mathrm{~s}, 2 \mathrm{H}), 8.46(\mathrm{~s}, 1 \mathrm{H}), 8.13(\mathrm{~d}, J=7.3 \mathrm{~Hz}, 2 \mathrm{H}), 7.71(\mathrm{t}$, $J=7.4 \mathrm{~Hz}, 1 \mathrm{H}), 7.48(\mathrm{~s}, 2 \mathrm{H}), 7.32(\mathrm{~d}, J=7.7 \mathrm{~Hz}, 2 \mathrm{H}), 7.19(\mathrm{~d}, J=8.0 \mathrm{~Hz}, 2 \mathrm{H}), 3.85(\mathrm{~s}, 6 \mathrm{H}), 2.56(\mathrm{t}, J=$ $7.1 \mathrm{~Hz}, 4 \mathrm{H}), 1.68$ (sxt, $J=7.1 \mathrm{~Hz}, 4 \mathrm{H}$ ), 1.00 (t, $J=7.3 \mathrm{~Hz}, 6 \mathrm{H}$ ); ${ }^{13} \mathrm{C}$ NMR (126 MHz, DMSO- $d_{6}$ ): $\delta$ 170.9, $162.6,151.2,147.6,141.0,133.7,133.1,130.8,128.8,126.9,123.3,120.6,110.0,55.9,35.0,18.0$, 13.3. ESI-HRMS: calcd for $\mathrm{C}_{32} \mathrm{H}_{34} \mathrm{~N}_{4} \mathrm{O}_{8}[\mathrm{M}+\mathrm{H}]^{+} 603.2455$; found 603.2464 .

Compound $3 \mathrm{~h}$ was prepared by reacting isophthalic dihydrazide $(0.395 \mathrm{~g}, 1.97 \mathrm{mmol})$ and nonanal ( $0.750 \mathrm{~mL}, 4.14 \mathrm{mmol}$ ) for $17 \mathrm{~h}$ in $12 \mathrm{~mL}$ ethanol ( $0.577 \mathrm{~g}, 66 \%$ yield). ${ }^{1} \mathrm{H} \mathrm{NMR} \mathrm{(500} \mathrm{MHz,} \mathrm{CDCl}_{3} / 10 \% \mathrm{v} / \mathrm{v}$ $\mathrm{CD}_{3} \mathrm{OD}$ ): $\delta 11.10(\mathrm{~s}, 2 \mathrm{H}), 8.03(\mathrm{~s}, 1 \mathrm{H}), 7.91(\mathrm{~d}, J=7.7 \mathrm{~Hz}, 2 \mathrm{H}), 7.66(\mathrm{t}, J=5.7 \mathrm{~Hz}, 2 \mathrm{H}), 7.44(\mathrm{t}, J=7.7 \mathrm{~Hz}$, $1 \mathrm{H}), 2.37-2.24(\mathrm{~m}, 4 \mathrm{H}), 1.51(\mathrm{p}, J=7.6 \mathrm{~Hz}, 4 \mathrm{H}), 1.38-1.21(\mathrm{~m}, 2 \mathrm{H}), 0.88(\mathrm{t}, J=6.9 \mathrm{~Hz}, 6 \mathrm{H}) ;{ }^{13} \mathrm{C} \mathrm{NMR}$ 
(126 MHz, $\mathrm{CDCl}_{3} / 10 \%$ v/v CD $\mathrm{OD}$ ): $\delta$ 164.5, 154.5, 133.4, 131.0, 128.9, 125.8, 32.6, 31.8, 29.3, 29.3, 29.2, 26.6, 22.6, 14.0. ESI-HRMS: calcd for $\mathrm{C}_{26} \mathrm{H}_{42} \mathrm{~N}_{4} \mathrm{O}_{2}[\mathrm{M}+\mathrm{H}]^{+} 443.3386$; found 443.3390 .

Compound $4 \mathrm{c}$ was prepared by reacting carbohydrazide $(0.395 \mathrm{~g}, 4.30 \mathrm{mmol})$ and vanillin $(2.050 \mathrm{~g}$, $13.07 \mathrm{mmol}$ ) for $23 \mathrm{~h}$ in $140 \mathrm{~mL}$ methanol ( $1.101 \mathrm{~g}, 72 \%$ yield). ${ }^{1} \mathrm{H}$ NMR (500 MHz, DMSO- $d_{6}$ ): $\delta 10.43$ $(\mathrm{s}, 2 \mathrm{H}), 9.38(\mathrm{~s}, 2 \mathrm{H}), 8.06(\mathrm{~s}, 2 \mathrm{H}), 7.36(\mathrm{~s}, 2 \mathrm{H}), 7.08(\mathrm{~d}, \mathrm{~J}=8.0 \mathrm{~Hz}, 2 \mathrm{H}), 6.82(\mathrm{~d}, J=8.1 \mathrm{~Hz}, 2 \mathrm{H}), 3.84(\mathrm{~s}$, 6H); ${ }^{13}$ C NMR (126 MHz, DMSO- $\left.d_{6}\right): \delta 152.2,148.3,148.0,143.4,126.1,121.3,115.4,109.4,55.7$. ESI-HRMS: calcd for $\mathrm{C}_{17} \mathrm{H}_{18} \mathrm{~N}_{4} \mathrm{O}_{5}[\mathrm{M}+\mathrm{Na}]^{+}$381.1175; found 381.1170.

Compound $4 \mathrm{~g}$ was prepared by reacting carbohydrazide $(0.397 \mathrm{~g}, 4.32 \mathrm{mmol})$ and trans-cinnamaldehyde ( $1.170 \mathrm{~g}, 8.764 \mathrm{mmol})$ for $18 \mathrm{~h}$ in $140 \mathrm{~mL}$ methanol $\left(0.999 \mathrm{~g}\right.$, $73 \%$ yield). ${ }^{1} \mathrm{H}$ NMR (500 MHz, DMSO-d $d_{6}$ : $\delta 10.57$ (s, 2H), 7.97 (d, $\left.J=6.4 \mathrm{~Hz}, 2 \mathrm{H}\right), 7.57$ (d, $J=7.3 \mathrm{~Hz}, 4 \mathrm{H}$ ), 7.39 (t, $J=7.5 \mathrm{~Hz}$, $4 \mathrm{H}), 7.31(\mathrm{t}, J=7.3 \mathrm{~Hz}, 2 \mathrm{H}), 7.02-6.89(\mathrm{~m}, 4 \mathrm{H}) ;{ }^{13} \mathrm{C}$ NMR (126 MHz, DMSO- $\left.d_{6}\right): \delta 151.7,145.0,137.0$, 136.1, 128.8, 128.5, 126.7, 125.7. ESI-HRMS: calcd for $\mathrm{C}_{19} \mathrm{H}_{18} \mathrm{~N}_{4} \mathrm{O}[\mathrm{M}+\mathrm{Na}]^{+} 341.1378$; found 341.1376.

Compound 5c was prepared by reacting ADH (0.406 g, $2.26 \mathrm{mmol})$ and vanillin $(0.714 \mathrm{~g}, 4.55 \mathrm{mmol})$ for $40 \mathrm{~h}$ in $140 \mathrm{~mL}$ methanol (0.809 g, 82\% yield). ${ }^{1} \mathrm{H}$ NMR (500 MHz, DMSO- $\left.d_{6}\right): \delta 11.14$ \& 11.02 (d, $J=5.3 \mathrm{~Hz}, J=5.0 \mathrm{~Hz}, 2 \mathrm{H}), 9.45(\mathrm{~s}, 2 \mathrm{H}), 8.05 \& 7.86(\mathrm{~s}, 2 \mathrm{H}), 7.25 \& 7.22(\mathrm{~s}, 2 \mathrm{H}), 7.08-7.00(\mathrm{~m}, 2 \mathrm{H})$, 6.84-6.76 (m, 2H), $3.81 \& 3.77(\mathrm{~s}, 6 \mathrm{H}), 2.69-2.60 \& 2.26-2.17(\mathrm{~m}, 4 \mathrm{H}), 1.70-1.57(\mathrm{~m}, 4 \mathrm{H}) ;{ }^{13} \mathrm{C}$ NMR (126 MHz, DMSO- $\left.d_{6}\right): \delta 173.9,173.9,168.2,168.1,148.8,148.5,148.5,148.0,147.9,146.4,146.3$, $142.9,142.8,125.8,125.8,121.8,120.8,115.5,115.4,109.5,109.3,109.0,55.5,55.5,34.1$, 34.1, 31.8, 31.7, 25.0, 24.9, 24.2, 24.0. ESI-HRMS: calcd for $\mathrm{C}_{22} \mathrm{H}_{26} \mathrm{~N}_{4} \mathrm{O}_{6}[\mathrm{M}+\mathrm{Na}]^{+} 465.1750$; found 465.1752 .

Compound $5 \mathrm{~h}$ was prepared by reacting $\mathrm{ADH}(0.395 \mathrm{mmol}, 2.20 \mathrm{mmol})$ and nonanal $(0.810 \mathrm{~mL}$, $4.47 \mathrm{mmol}$ ) for $18 \mathrm{~h}$ in $12 \mathrm{~mL}$ ethanol (0.894 g, 95\% yield). ${ }^{1} \mathrm{H}$ NMR (500 MHz, CDCl $/ 5 \% \mathrm{v} / \mathrm{v} \mathrm{CD}{ }_{3} \mathrm{OD}$ ): $\delta 10.16,9.84,8.97, \& 8.94(\mathrm{~s}, 2 \mathrm{H}$, exchangeable), 7.46-7.39 \& 7.16-7.07 (m, 2H), 2.70-2.60 \& 2.28-2.20 ( $\mathrm{m}$, expected $4 \mathrm{H}$, overlapping HDO peak), 2.36-2.28 \& 2.28-2.20 (m, expected $4 \mathrm{H}$, overlapping HDO peak), 1.79-1.64 (m, 4H), 1.57-1.43 (m, 4H), 1.38-1.19 (m, 20H), 0.93-0.81 (m, 6H); ${ }^{13} \mathrm{C} \mathrm{NMR}\left(126 \mathrm{MHz}, \mathrm{CDCl}_{3} / 5 \%\right.$ v/v CD $\left.3 \mathrm{OD}\right): \delta 175.5,170.1,169.6,152.6,152.1,148.5,147.7,34.8,34.3$, 32.5, 32.4, 32.2, 32.0, 31.9, 29.7, 29.4, 29.4, 29.3, 29.2, 26.7, 26.4, 26.1, 25.2, 24.9, 24.4, 23.8, 22.7, 14.1. ESI-HRMS: calcd for $\mathrm{C}_{24} \mathrm{H}_{46} \mathrm{~N}_{4} \mathrm{O}_{2}[\mathrm{M}+\mathrm{Na}]^{+} 445.3518$; found 445.3519 .

Compound $5 \mathrm{i}$ was prepared by reacting $\mathrm{ADH}(0.201 \mathrm{~g}, 1.10 \mathrm{mmol})$ and methyl levulinate $(0.328 \mathrm{~g}$, $2.50 \mathrm{mmol}$ ) for $46 \mathrm{~h}$ in $6 \mathrm{~mL}$ methanol $\left(0.378 \mathrm{~g}, 87 \%\right.$ yield). ${ }^{1} \mathrm{H}$ NMR (500 MHz, DMSO- $\left.d_{6}\right): \delta 9.96$ \& $9.90(\mathrm{~s}, 2 \mathrm{H}), 3.58(\mathrm{~s}, 6 \mathrm{H}), 2.57-2.46\left(\mathrm{~m}\right.$, expected $8 \mathrm{H}$, overlapping DMSO- $d_{6}$ peak), 2.46-2.37 \& 2.24-2.17 (m, 4H), 1.84 \& $1.82(\mathrm{~s}, 6 \mathrm{H}), 1.60-1.45(\mathrm{~m}, 4 \mathrm{H}) ;{ }^{13} \mathrm{C}$ NMR $\left(126 \mathrm{MHz}, \mathrm{DMSO}-d_{6}\right): \delta 174.5$, 
$172.8,168.3,155.4,149.9,51.2,51.1,33.8,33.0,32.7,32.0,29.7,29.4,25.1,24.1,16.7,16.3$. ESI-HRMS: calcd for $\mathrm{C}_{18} \mathrm{H}_{30} \mathrm{~N}_{4} \mathrm{O}_{6}[\mathrm{M}+\mathrm{H}]^{+} 399.2238$; found 399.2230 .

Compound $5 \mathbf{j}$ was prepared by reacting $\operatorname{ADH}(0.201 \mathrm{~g}, 1.10 \mathrm{mmol})$ and ethyl levulinate $(0.372 \mathrm{~g}$, $2.55 \mathrm{mmol}$ ) for $46 \mathrm{~h}$ in $6 \mathrm{~mL}$ methanol (0.242 g, 52\% yield). ${ }^{1} \mathrm{H}$ NMR (500 MHz, DMSO- $\left.d_{6}\right): \delta 9.96$ \& $9.90(\mathrm{~s}, 2 \mathrm{H}), 4.09-3.99(\mathrm{~m}, 4 \mathrm{H}), 2.55-2.47$ (m, expected 8H, overlapping DMSO- $d_{6}$ peak), $2.47-2.40$ \& 2.22-2.18 (m, 4H), 1.84 \& $1.82(\mathrm{~s}, 6 \mathrm{H}), 1.59-1.46(\mathrm{~m}, 4 \mathrm{H}), 1.22-1.13(\mathrm{~m}, 6 \mathrm{H}) ;{ }^{13} \mathrm{C} \mathrm{NMR}(126 \mathrm{MHz}$, DMSO- $\left.d_{6}\right): \delta 174.4,172.3,168.2,155.3,149.9,59.7,59.6,33.8,33.1,32.7,32.0,30.0,29.6,25.1,24.1$, 16.6, 16.3, 14.0. ESI-HRMS: calcd for $\mathrm{C}_{20} \mathrm{H}_{34} \mathrm{~N}_{4} \mathrm{O}_{6}[\mathrm{M}+\mathrm{H}]^{+}$427.2557; found 427.2547.

\subsubsection{Preparation of $\mathrm{Cu}(\mathrm{II})$-Ligand Complexes}

\subsubsection{DiLevDEG/ADH}

The teal-coloured $\mathrm{Cu}(\mathrm{II})$-DiLevDEG/ADH complex was prepared by adding an aliquot of the DiLevDEG/ADH solution ( $50 \% \mathrm{w} / \mathrm{v} ; 56 \mu \mathrm{L}$ ) to copper(II) nitrate trihydrate (32 mg, $0.13 \mathrm{mmol}$ ) dissolved in water $(1 \mathrm{~mL})$.

\subsubsection{1a}

The $\mathrm{Cu}(\mathrm{II})-1 \mathrm{a}$ complex was prepared by stirring copper(II) chloride dihydrate $(0.043 \mathrm{~g}, 0.25 \mathrm{mmol})$ and ligand 1a $(0.045 \mathrm{~g}, 0.25 \mathrm{mmol})$ in methanol $(10 \mathrm{~mL})$. The green, precipitated $\mathrm{Cu}(\mathrm{II})$ complex was isolated and analysed by ESI-HRMS.

ESI-HRMS: calcd for $\mathrm{C}_{9} \mathrm{H}_{9} \mathrm{CuN}_{2} \mathrm{O}_{2}[\mathrm{M}-\mathrm{H}]^{+}$239.9960; found 239.9963.

\subsubsection{Cyclen}

The Cu(II)-cyclen complex was prepared by adding cyclen $(0.044 \mathrm{~g}, 0.25 \mathrm{mmol})$ and copper(II) chloride dihydrate $(0.091 \mathrm{~g}, 0.53 \mathrm{mmol}, 2.2 \mathrm{eq})$ to methanol $(10 \mathrm{~mL})$ and stirring for $2 \mathrm{~h}$. The dark blue, precipitated $\mathrm{Cu}(\mathrm{II})$-cyclen complex was isolated and analysed by ESI-HRMS.

ESI-HRMS: calcd for $\mathrm{C}_{8} \mathrm{H}_{19} \mathrm{CuN}_{4}[\mathrm{M}-\mathrm{H}]^{+}$234.0906; found 234.0916. 


\subsubsection{UV-Vis Spectrophotometry}

\subsubsection{DiLevDEG/ADH}

The UV-Vis spectra of DiLevDEG/ADH, the Cu(II)-DiLevDEG/ADH complex, and copper(II) nitrate (50 mM, aq) were recorded. The samples were diluted, as needed, with water for measurement.

\subsubsection{1a}

\subsection{Job Plot}

Aliquots of $100 \mu \mathrm{M}$ solutions of copper(II) nitrate and ligand 1a in 9:1 chloroform:methanol containing $0.1 \mathrm{M}$ tetrabutylammonium nitrate were added as shown in Table 6.2, and the absorption spectra from 240-1000 nm were measured. The absorbance at $388 \mathrm{~nm}$, corresponding to the $\mathrm{Cu}(\mathrm{II})-1 \mathrm{a}$ complex, was used in subsequent calculations to determine the metal:ligand stoichiometry.

Table 6.2. Aliquots $(\mathrm{mL})$ of $100 \mu \mathrm{M}$ solutions of $\mathrm{Cu}\left(\mathrm{NO}_{3}\right)_{2}$ and ligand $1 \mathrm{a}$.

\begin{tabular}{ccc}
\hline Solution & $\mathrm{mL} 100 \mu \mathrm{M} \mathrm{Cu}\left(\mathrm{NO}_{3}\right)_{2}$ & $\mathrm{~mL} 100 \mu \mathrm{M} \mathrm{1a}$ \\
\hline 1 & 0.00 & 2.00 \\
2 & 0.25 & 1.75 \\
3 & 0.50 & 1.50 \\
4 & 0.75 & 1.25 \\
5 & 1.00 & 1.00 \\
6 & 1.25 & 0.75 \\
7 & 1.50 & 0.50 \\
8 & 1.75 & 0.25 \\
9 & 2.00 & 0.00 \\
\hline
\end{tabular}

\subsection{Formation Constant $\left(K_{f}\right)$}

The UV-Vis spectra from $265-450 \mathrm{~nm}$ of solutions containing $0.100 \mathrm{mM}$ ligand $1 \mathrm{a}$ and $0,0.013,0.025$, $0.038,0.050,0.063,0.075,0.088,0.100,0.113,0.125,0.138,0.150,0.175,0.200,0.250,0.300,0.350$, $0.400,0.450$, and $0.500 \mathrm{mM}$ copper(II) nitrate in 9:1 v/v chloroform:methanol (100 mL total) were measured, as well as a baseline spectrum of 9:1 v/v chloroform:methanol. The $\lambda_{\max }$ of the $\mathrm{Cu}(\mathrm{II})-1 \mathrm{a}$ complex to be used for subsequent calculations of the $K_{\mathrm{f}}$ was determined to be $388 \mathrm{~nm}$. 


\subsubsection{Cyclen}

An aqueous solution of the dark blue $\mathrm{Cu}$ (II)-cyclen complex was prepared by adding copper(II) nitrate and cyclen to give final concentrations of $50.5 \mathrm{mM}$ each. The solution was diluted $100 x$, and its UV-Vis spectrum was recorded. The UV-Vis spectrum of copper(II) nitrate (50 mM, aq) was also measured. 


\subsection{CHAPTER 3}

\subsubsection{DiLevDEG/ADH-Substituted Resin in a Commercial Acrylic Paint}

As shown in Figure 6.1, a commercial acrylic paint with $35 \% \mathrm{w} / \mathrm{w}$ of its resin substituted with the DiLevDEG/ADH polyacylhydrazone (DAp/acr) was prepared and evaluated, along with the acrylic paint, for its ability to retain $\mathrm{Cu}(\mathrm{II})$ and the subsequent impact on bacterial growth.

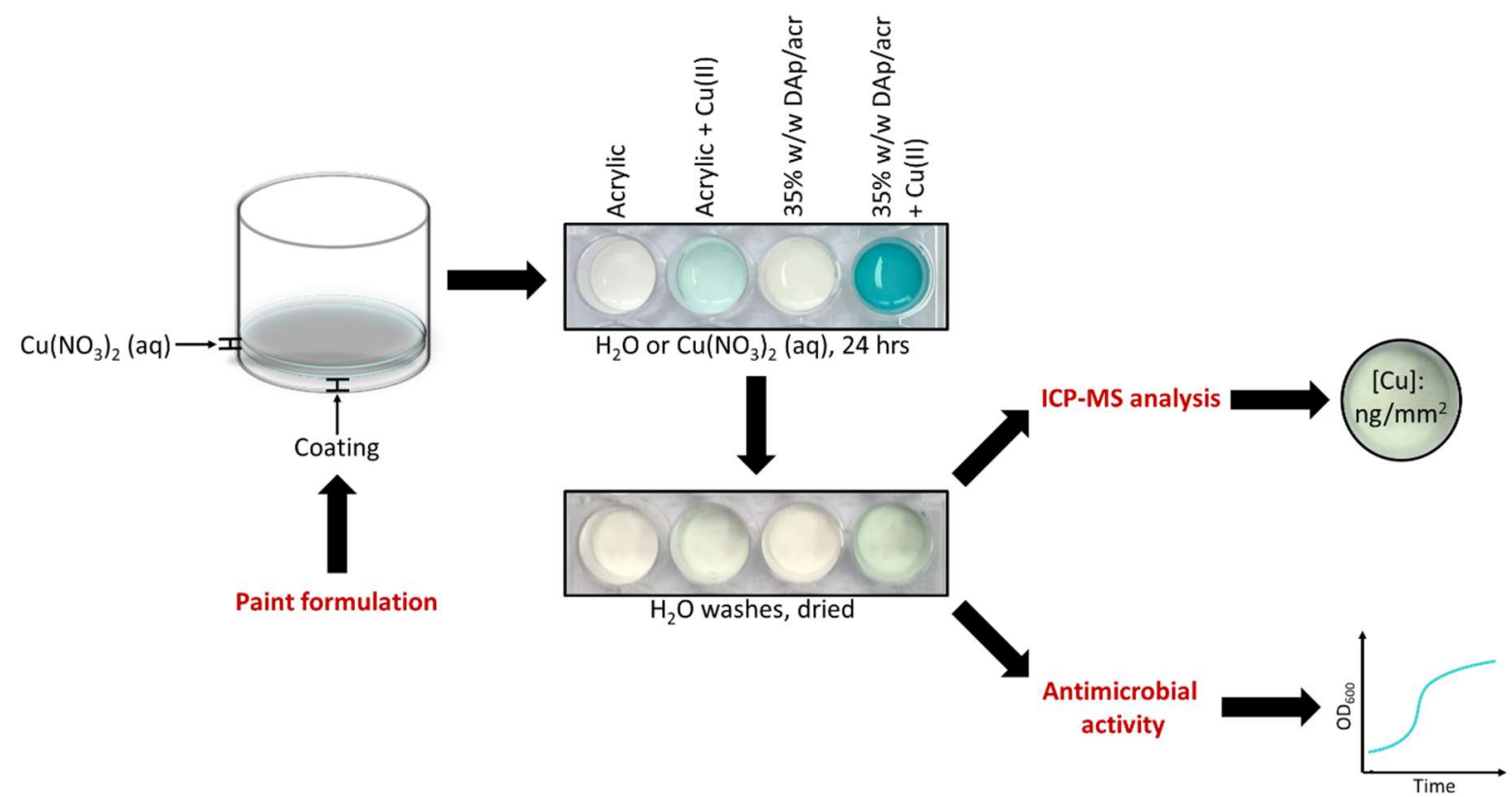

Figure 6.1. Methodology for assessing the $\mathrm{Cu}(\mathrm{II})$-chelating ability of the acrylic and DAp/acr paints and their impact on bacterial growth $( \pm \mathrm{Cu}(I I))$.

\subsubsection{Formulation}

The aqueous solution of $\sim 50 \% \mathrm{w} / \mathrm{v}$ DiLevDEG/ADH from Section 6.2.1.2 was used to substitute $35 \% \mathrm{w} / \mathrm{w}$ of the resin component in a commercial acrylic paint, the composition of which is tabulated (Table 6.3). The resin was an ADH crosslinked dispersion polymer. 
Table 6.3. Composition of the commercial acrylic paint used in this study. ${ }^{\text {a }}$

Millbase

Titanium dioxide

Water, rheology modifier, dispersant, surfactant, MPG ${ }^{b}$, defoamer, biocide, alkali

\section{Dispersion mixture}

Dispersion polymer ${ }^{c}$

Coalescent, surface wetter, MPG ${ }^{\text {, }}$, defoamer, biocide, alkali

\footnotetext{
a Proprietary composition and specific components confidential to Resene Paints Ltd.

${ }^{b}$ Monopropylene glycol.

${ }^{\mathrm{c}}$ Resin $=\mathrm{ADH}$ crosslinked dispersion polymer $(45 \% \mathrm{w} / \mathrm{w})$.
}

\subsubsection{Growth Assay: E. coli NZRM 3647}

Refer to Figure 6.1 and the Appendix (Figures 8.20 \& 8.21) for the plate setup. To wells in a 24-well plate (JET BIOFIL ${ }^{\circledR}$ ), $250 \mu \mathrm{L}$ of the commercial acrylic paint, 35\% w/w DAp/acr paint, or the commercial marine paint (Altex Yacht and Boat Paint, Ablative Antifouling No. 5; 40-50\% w/w $\mathrm{Cu}_{2} \mathrm{O}$ ) were delivered, and the plate was agitated to coat the well bottoms evenly. The marine paint was included to compare to an industry standard, and uncoated wells served as growth controls. The paints were cured at $25^{\circ} \mathrm{C}$ while shaking gently for $72 \mathrm{~h}$, at which point they were no longer tacky. Then, they were washed with water $(3 \times 500 \mu \mathrm{L})$ and air-dried. An aqueous solution of $\operatorname{copper}(I I)$ nitrate $(50 \mathrm{mM}$; $500 \mu \mathrm{L} /$ well) was added to a row of wells coated with the acrylic paint ("Acrylic $+\mathrm{Cu}(I I)$ ") and a row of wells coated with the DAp/acr paint ("DAp/acr + Cu(II)"; Figure 8.20). Water (500 $\mu \mathrm{L} /$ well) was added to a row of uncoated wells and to rows of wells coated with the acrylic, DAp/acr, and marine paints. After $24 \mathrm{~h}$, all wells were washed with water $(3 \times 500 \mu \mathrm{L})$, air-dried, and sterilised with UV light for $20 \mathrm{~min}$. The approximate surface area of the paint in each well was recorded. The prepared plates were used to determine the effects of the coatings on the growth of E. coli NZRM 3647, following the previously established laboratory procedure (unpublished data) ${ }^{200}$ : an overnight culture was prepared by inoculating nutrient broth $(3 \mathrm{~mL})$ with a colony of $E$. coli NZRM 3647 from a nutrient agar plate and incubating for $16 \mathrm{~h}$ at $37^{\circ} \mathrm{C}$ with shaking. This overnight culture was diluted $1: 1 \mathrm{v} / \mathrm{v}$ with nutrient broth, and an aliquot of the diluted culture ( $\left.500 \mu \mathrm{L} ; 1.3 \times 10^{8} \mathrm{CFU} / \mathrm{mL}\right)$ was added to each well. The plate was incubated at $37^{\circ} \mathrm{C}$, and the $\mathrm{OD}_{600}$ of the culture in each well was measure at $\mathrm{t}=0,2,4,6,8$, 10 , and $24 \mathrm{~h}$ to generate growth curves (Figure 8.21). The experiment was independently repeated three times. 


\subsubsection{Quantification of Cu(II)-Leaching}

As in Section 6.3.1.2, a coated, 24-well plate (JET BIOFIL ${ }^{\circledR}$ ) was prepared (Appendix, Figure 8.20): aliquots (250 $\pm 10 \mathrm{mg}$ ) of the commercial acrylic paint, 35\% w/w DAp/acr paint, and the commercial marine paint were delivered to wells in the 24-well plate, which was agitated to coat the well bottoms evenly. The paints were cured at $50^{\circ} \mathrm{C}$ for $20 \mathrm{~h}$, at which point they were no longer tacky. Then, they were washed with water $(4 \times)$ and air-dried. An aqueous solution of copper(II) nitrate (50 mM; $500 \mu \mathrm{L} /$ well) was added to three of the wells coated with the acrylic paint ("Acrylic + $\mathrm{Cu}(I I)$ ") and three of the wells coated with the DAp/acr paint ("DAp/acr + Cu(II)"). Water $(500 \mu \mathrm{L})$ was added to three uncoated wells and wells coated with the acrylic, DAp/acr, and marine paints (three wells per coating). The uncoated wells and water-exposed acrylic- and DAp/acr-coated wells served as blanks. After $24 \mathrm{~h}$, all wells were washed with water $(4 \times)$ and air-dried, and nutrient broth $(500 \mu \mathrm{L})$ was added to each well. The plate was incubated at $37^{\circ} \mathrm{C}$ for $24 \mathrm{~h}$. Then, the supernatant was removed from each well, diluted $10,000 \times$ with $3 \% \mathrm{v} / \mathrm{v}$ aq nitric acid, and analysed by ICP-MS to quantify the copper that had leached into the nutrient broth after $24 \mathrm{~h}$.

\subsubsection{Acrylic Paint Spike Study}

In order to validate the $\mathrm{Cu}(\mathrm{II})$-loading and quantification method, two samples were prepared with the commercial acrylic paint and a known amount of copper from an ICP-MS stock copper standard (Inorganic Ventures, $1001 \mu \mathrm{g} / \mathrm{mL} \mathrm{Cu}$ ). For the first sample, the copper was homogeneously mixed in a paint: the acrylic paint (200 $\pm 10 \mathrm{mg}$ ) was spiked with the copper standard $(10 \mu \mathrm{L}, 10.01 \mu \mathrm{g})$, mixed, and delivered to uniformly cover the bottom of a $7 \mathrm{~mL}$ glass vial that had been washed with water $(3 x)$ and air-dried. Then, the paint was cured at $50{ }^{\circ} \mathrm{C}$ overnight $(19 \mathrm{~h})$, at which point it was no longer tacky. For the second sample, the copper was added to the top of an already-cured paint: the acrylic paint ( $200 \pm 10 \mathrm{mg}$ ) was delivered to uniformly cover the bottom of a $7 \mathrm{~mL}$ glass vial that had been washed with water $(3 x)$ and air-dried. The paint was cured at $50{ }^{\circ} \mathrm{C}$ overnight $(19 \mathrm{~h})$, at which point it was no longer tacky. Then, an aliquot of the copper standard $(10 \mu \mathrm{L}, 10.01 \mu \mathrm{g})$ was added to the surface of the cured paint and left for $24 \mathrm{~h}$ at $50^{\circ} \mathrm{C}$. For both samples, the acrylic paint without copper served as a blank. To prepare all samples for ICP-MS $(\mathrm{Cu})$ analysis, the samples were digested in concentrated nitric acid $(70 \% \mathrm{v} / \mathrm{v}$, aq; $500 \mu \mathrm{L})$ for $3 \mathrm{~h}$ at $80^{\circ} \mathrm{C}$, and the digested solutions were diluted $2,500 \times$ with $1 \% \mathrm{v} / \mathrm{v}$ aq nitric acid to bring them into the range of the ICP-MS copper standards. 


\subsubsection{Quantification of Cu(II)-Loading}

Special-made glass vials with dimensions matching the wells of the 24-well plates in Section 6.3.1.2 (7.3 $\pm 0.1 \mathrm{~mm}$ radius) were washed with water $(3 x)$ and air-dried. An aliquot $(250 \pm 10 \mathrm{mg}$ ) of the acrylic paint or $35 \% \mathrm{w} / \mathrm{w}$ DAp/acr paint was delivered to the bottom of each vial, which was agitated for an even coating. Six vials coated with the acrylic paint and six vials coated with the DAp/acr paint were prepared. The paints were cured at $50^{\circ} \mathrm{C}$ until, at least, they were no longer tacky, and then they were washed with water ( $3 x)$ and air-dried. Aqueous copper(II) nitrate ( $50 \mathrm{mM} ; 500 \mu \mathrm{L} /$ vial) was added to three of the vials coated with acrylic paint and three of the vials coated with the DAp/acr paint. Water $(500 \mu \mathrm{L} /$ vial) was added to the remaining vials, which served as blanks. The vials sat at room temperature for $24 \mathrm{~h}$ and then were washed with water $(4 x)$ and air-dried. The surface area exposed to liquid (Appendix, Figure 8.22) and the mass of each coating were recorded. To prepare the samples for ICP-MS (Cu) analysis, concentrated nitric acid ( $70 \% \mathrm{v} / \mathrm{v}$, aq; $1 \mathrm{~mL})$ was added to each vial, and the vials were heated at $80^{\circ} \mathrm{C}$ for $3 \mathrm{~h}$ to digest the paint and release the copper into solution. The digested solutions were diluted $10,000 \times$ with $1 \% \mathrm{v} / \mathrm{v}$ aq nitric acid, bringing them into the range of the ICP-MS standards. If any particles were observed in a dilution, the sample was centrifuged for $10 \mathrm{~min}$ at 1788 RCF. The copper adsorbed onto or coordinated by each coating was quantified via ICP-MS analysis. The experiment was independently repeated four times. The cure-time was consistent within a batch of three replicates per coating but inconsistent between batches, varying from 16-67 h.

\subsubsection{DiLevDEG/ADH Polyacylhydrazone-Leaching}

The bottom of a well in a 24 -well plate (JET BIOFIL ${ }^{\circledR}$ ) was coated evenly via agitation with $260 \mathrm{mg}$ of the $35 \% \mathrm{w} / \mathrm{w}$ DAp/acr paint, which was cured at $50^{\circ} \mathrm{C}$ for $24 \mathrm{~h}$, at which point it was no longer tacky. Then, the cured paint was washed with water $(3 x)$ and air-dried, and deuterium oxide $(500 \mu \mathrm{L})$ was added to the well and left for $24 \mathrm{~h}$. The ${ }^{13} \mathrm{C}$ NMR spectrum of the deuterium oxide supernatant was measured to assess whether or not the DiLevDEG/ADH polyacylhydrazone was leaching out of the coating. The key signals below are in bold.

${ }^{13} \mathrm{C}$ NMR $\left(126 \mathrm{MHz}, \mathrm{D}_{2} \mathrm{O}\right): \delta$ 214.2, 214.0, 175.9, 175.7, 175.6, 173.1, 163.4, 163.0, 159.1, 119.7, 72.2, $72.1,70.1,68.9,68.9,68.4,67.1,67.0,66.7,64.6,64.5,62.7,60.9,60.0,57.9,55.7,52.8,39.9,39.3$, $38.2,37.3,35.9,34.1,33.8,33.8,33.5,33.0,31.6,31.0,30.8$, 30.3, 30.1, 29.7, 29.6, 29.0, 28.4, 26.2, $25.7,25.3,25.1,25.0,25.0,24.7,24.4,22.2,22.0,21.5,18.5,17.3,16.8,16.7,16.2,7.0$. 


\subsubsection{Polyurethane and Polyurea Coatings}

Diisocyanate Desmodur ${ }^{\circledast}$ W (Covestro) was added to the following amine or alcohol components to form polyurea or polyurethane coatings, respectively: DETA, triethylene glycol (TEG), and 1,2-dianilinoethane (Scheme 6.6). Reactant amounts, reaction conditions, and treatments are summarised in Table 6.4. Refer to the Appendix (Table 8.18) for the result of each combination.
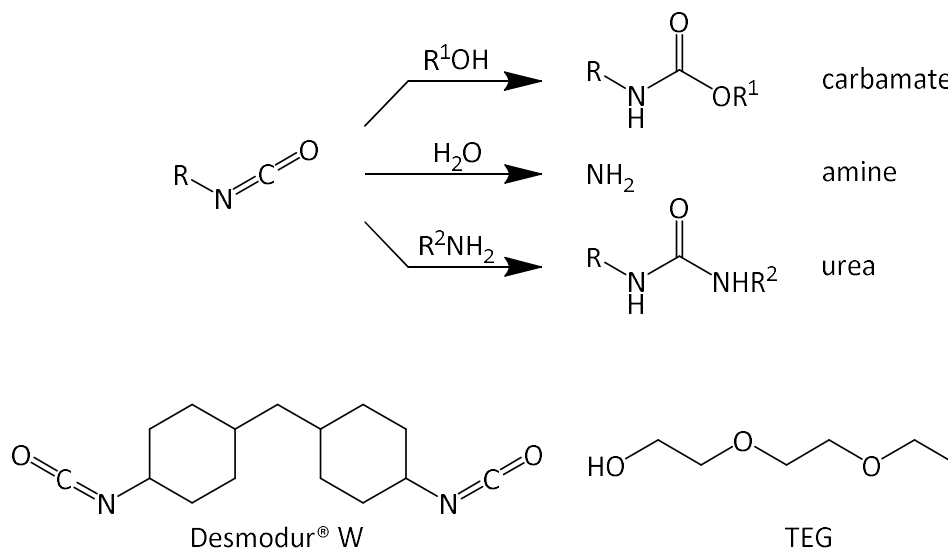<smiles>OCCOCCOCCO</smiles><smiles>NCCNCCN</smiles>

DETA<smiles>c1ccc(NCCNc2ccccc2)cc1</smiles>

Scheme 6.6. Isocyanate reactions and structures of Desmodur ${ }^{\circledast}$ W, TEG, DETA, and 1,2-dianilinoethane.

Table 6.4. Amounts of amine and/or alcohol component reacted with Desmodur ${ }^{\circledR} \mathrm{W}$ under the tabulated conditions.

\begin{tabular}{|c|c|c|c|}
\hline $\begin{array}{l}\text { Amine/Alcohol } \\
\text { Component }\end{array}$ & $\begin{array}{c}\text { mol Amine and/or } \\
\text { Alcohol Component per } \\
1 \text { mol Desmodur }{ }^{\oplus} \mathrm{W}\end{array}$ & $\begin{array}{l}\text { Amounts of Amine } \\
\text { and/or Alcohol } \\
\text { Component (mmol) }\end{array}$ & Conditions/Treatment \\
\hline DETA & 0.67 & 4.58 & $20^{\circ} \mathrm{C}(\mathrm{RT})$ \\
\hline DETA & 0.67 & 4.58 & $0{ }^{\circ} \mathrm{C}$ \\
\hline TEG & 0.99 & 3.8 & $\begin{array}{l}\text { draw-down; cured at } \\
70^{\circ} \mathrm{C} \text { for } 19 \mathrm{~h}\end{array}$ \\
\hline TEG, DETA & 0.90 (TEG); 0.071 (DTA) & 3.5 (TEG); 0.3 (DTA) & $20^{\circ} \mathrm{C}(\mathrm{RT})$ \\
\hline 1,2-Dianilinoethane & 0.99 & 3.85 & $\begin{array}{c}20^{\circ} \mathrm{C}(\mathrm{RT}), 1 \mathrm{~h} ; 1.5 \mathrm{~mL} \\
\mathrm{CHCl}_{3}\end{array}$ \\
\hline
\end{tabular}




\subsubsection{DETA}

Desmodur $^{\circledast} \mathrm{W}(1.77 \mathrm{~mL}, 6.86 \mathrm{mmol})$ was added to DETA $(0.500 \mathrm{~mL}, 4.58 \mathrm{mmol})$ and mixed manually at room temperature or $0{ }^{\circ} \mathrm{C}$. The reaction mixture solidified immediately.

\subsubsection{TEG}

Desmodur ${ }^{\oplus} \mathrm{W}(1.00 \mathrm{~mL}, 3.87 \mathrm{mmol})$ was added to TEG $(0.52 \mathrm{~mL}, 3.8 \mathrm{mmol})$ and mixed manually at room temperature. The reaction mixture was applied to a Leneta Form 5DX Brushout card using the standard draw-down method with a bar film applicator (1120/25/100, S232228, Sheen; Figure 6.2), and the film was cured at $70{ }^{\circ} \mathrm{C}$ for $19 \mathrm{~h}$.

\subsubsection{TEG/DETA}

Desmodur ${ }^{\circledast} \mathrm{W}(1.00 \mathrm{~mL}, 3.87 \mathrm{mmol})$ was added to TEG $(0.47 \mathrm{~mL}, 3.5 \mathrm{mmol})$ and DETA $(0.03 \mathrm{~mL}$, $0.3 \mathrm{mmol}$ ) and mixed manually at room temperature. The application of the reaction mixture to a Leneta Form 5DX Brushout card using the standard draw-down method with a bar film applicator (1120/25/100, S232228, Sheen; Figure 6.2) was attempted.

\subsubsection{1,2-Dianilinoethane}

Desmodur $^{\circledast} \mathrm{W}(1.00 \mathrm{~mL}, 3.87 \mathrm{mmol})$ was added to 1,2-dianilinoethane $(0.834 \mathrm{~g}, 3.85 \mathrm{mmol})$ in chloroform $(1.5 \mathrm{~mL})$ and stirred for $1 \mathrm{~h}$ at room temperature.

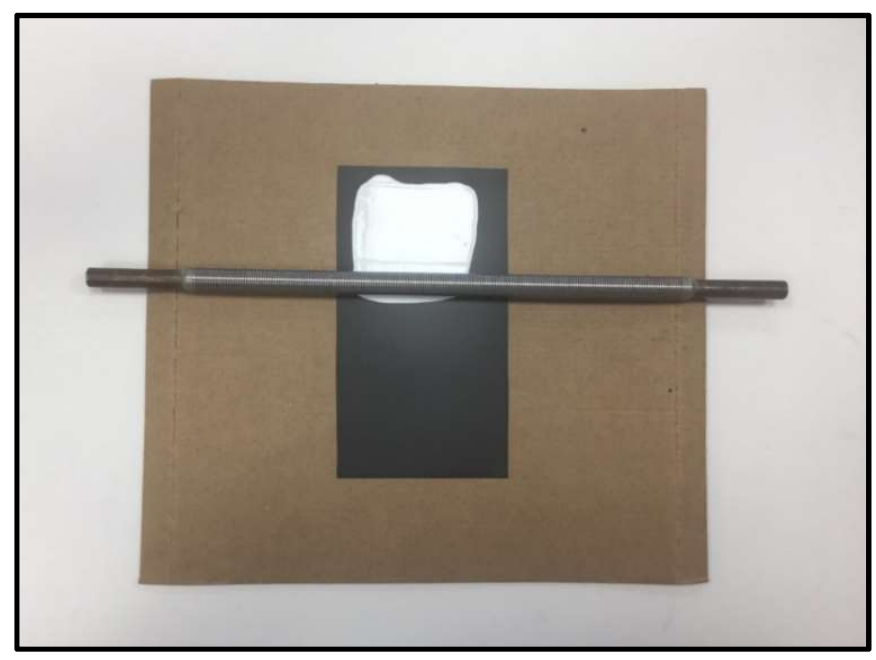

Figure 6.2. Demonstration of the standard draw-down technique with a bar film applicator. 


\subsubsection{Cu(II) Ligands in an Epoxy Coating}

The epoxy resin, hardeners, and surface ligands used in this study are described in Table 6.5, and the prepared epoxy coatings are listed in Table 6.6.

Table 6.5. Descriptions of epoxy resin, hardeners, and surface ligands used in this study.

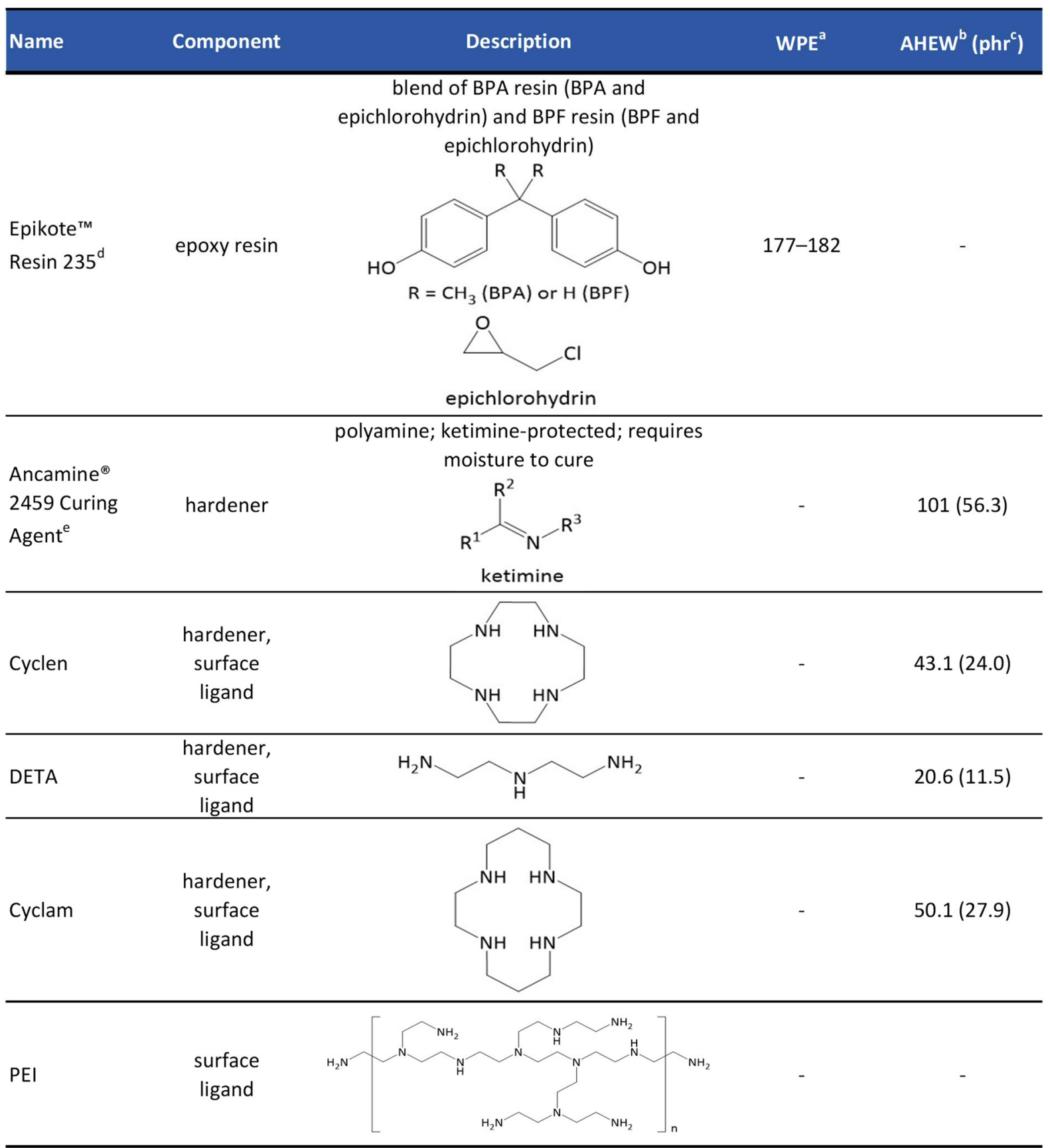

Weight per epoxide (WPE) is the grams of resin per one gram-equivalent of epoxy groups (g/eq).

${ }^{b}$ Amine hydrogen equivalent weight (AHEW) is the grams of hardener per one gram-equivalent of active amine hydrogens $(\mathrm{g} / \mathrm{eq})$.

c Per hundred resin (phr) is the grams of hardener per $100 \mathrm{~g}$ epoxy resin.

d Proprietary composition and specific components confidential to Resolution Performance Products.

e Proprietary composition and specific components confidential to Air Products and Chemicals, Inc. 
Table 6.6. Epoxy coatings prepared with epoxy resin Epikote ${ }^{\mathrm{TM}} 235$ and the following hardeners: Ancamine ${ }^{\circledR} 2459$, cyclen, DETA, and cyclam.

\begin{tabular}{|c|c|c|}
\hline Hardener & $\begin{array}{c}\text { Surface } \\
\text { Functionalisation }\end{array}$ & $\%$ w/w Overcure $(+) /$ Undercure $(-)^{a}$ \\
\hline Ancamine ${ }^{\circledR} 2459$ & - & $\begin{array}{c}-50 \%,-40 \%,-30 \%,-20 \%,-10 \% \\
\text { stoichiometric, }+10 \%,+20 \%,+50 \%\end{array}$ \\
\hline Ancamine ${ }^{\circledR} 2459$ & cyclam & $-50 \%,-40 \%,-30 \%,-20 \%,-10 \%$ \\
\hline Ancamine ${ }^{\circledR} 2459$ & cyclen & $-40 \%$ \\
\hline Ancamine ${ }^{\circledR} 2459$ & DETA & $-40 \%,-30 \%$ \\
\hline Ancamine $^{\circledR} 2459$ & PEI & $-40 \%$ \\
\hline Ancamine ${ }^{\circledR} 2459$, cyclen & - & $+10 \%,+20 \%\left(\right.$ cyclen $\left.^{b}\right)$ \\
\hline Ancamine $^{\circledR} 2459$, DETA & - & $+10 \%,+20 \%\left(\mathrm{DETA}^{\mathrm{c}}\right)$ \\
\hline Cyclen & - & $-20 \%,-10 \%$, stoichiometric, $+10 \%,+20 \%$ \\
\hline DETA & - & $-20 \%,-10 \%$, stoichiometric, $+10 \%,+20 \%$ \\
\hline DETA $(75 \%)$, cyclen $(25 \%)^{d}$ & - & stoichiometric \\
\hline DETA $(50 \%)$, cyclen $(50 \%)^{e}$ & - & stoichiometric \\
\hline DETA $(25 \%)$, cyclen $(75 \%)^{f}$ & - & stoichiometric \\
\hline DETA $(25 \%)$, cyclen $(100 \%)^{g}$ & - & $+25 \%$ \\
\hline DETA $(100 \%)$, cyclen $(25 \%)^{h}$ & - & $+25 \%$ \\
\hline Cyclam & - & $-20 \%$, stoichiometric, $+20 \%$ \\
\hline \multicolumn{3}{|c|}{$\begin{array}{l}\text { a The coatings in bold were surface-functionalised, and the coatings in red were determined to be strongly Cu( } \\
\text { chelating films based on colour intensity. } \\
\text { b Stoichiometric amount of Ancamine }{ }^{\circledR} 2459 \text { but overcured with cyclen. } \\
\text { c Stoichiometric amount of Ancamine }{ }^{\circledR} 2459 \text { but overcured with DETA. } \\
\text { d Enough DETA and cyclen added as hardeners to react with } 75 \% \text { and } 25 \% \text { of the epoxy groups, respectively. } \\
\text { e Enough DETA and cyclen added as hardeners to each react with half of the epoxy groups. } \\
{ }^{f} \text { Enough DETA and cyclen added as hardeners to react with } 25 \% \text { and } 75 \% \text { of the epoxy groups, respectively. } \\
\text { g Stoichiometric amount of cyclen but overcured with DETA. } \\
\text { h Stoichiometric amount of DETA but overcured with cyclen. }\end{array}$} \\
\hline
\end{tabular}




\subsubsection{Preparation of Epoxy Coatings}

A two-pack epoxy was prepared by manually mixing a commercial hardener (Ancamine ${ }^{\circledR} 2459$ Curing Agent; Air Products and Chemicals, Inc.) and a commercial epoxy resin (Epikote ${ }^{\mathrm{TM}}$ 235; Resolution Performance Products) stoichiometrically, in accordance with the manufacturers' specifications: per hundred resin $(\mathrm{phr})=56.3 \mathrm{~g}^{\text {Ancamine }}{ }^{\circledR} 2459$ per $100 \mathrm{~g} \mathrm{Epikote}^{\mathrm{TM}} 235$ (example calculation in the Appendix, Scheme 8.1). Ancamine ${ }^{\circledR} 2459$ is slow-curing, requiring moisture for deprotection of the ketimine-protected amine group before reacting with an epoxy group (Scheme 6.7). Alternative and/or additional polyamine hardeners that could also serve as $\mathrm{Cu}(\mathrm{II})$ ligands (e.g. cyclen, DETA, and cyclam) were mixed manually with Epikote ${ }^{\mathrm{TM}} 235$. These hardeners and combinations are displayed in Table 6.6, and the phr values are listed in Table 6.5, showing the amount of each hardener required to mix stoichiometrically with $100 \mathrm{~g}$ of Epikote ${ }^{\mathrm{TM}} 235$. For the coatings using cyclen as a hardener, cyclen was dissolved in the minimum amount of methanol $(0.8-2.2 \mathrm{mg} / \mu \mathrm{L})$ before mixing with Epikote $^{\mathrm{TM}} 235$, and, for the coatings using cyclam as a hardener, cyclam was dissolved in the minimum amount of 2:1 v/v chloroform:methanol $(\sim 0.3 \mathrm{mg} / \mu \mathrm{L})$ before mixing with Epikote ${ }^{\mathrm{TM}} 235$. DETA was used neat. In the particular cases tabulated, the amounts of hardener were varied up to $\pm 50 \% \mathrm{w} / \mathrm{w}$ and mixed manually with Epikote ${ }^{\mathrm{TM}}$ 235. All of the coatings were applied to a Leneta Form 5DX Brushout card using the standard draw-down method with a bar film applicator (1120/25/100, S232228, Sheen), demonstrated in Figure 6.2, and they were cured overnight at room temperature.

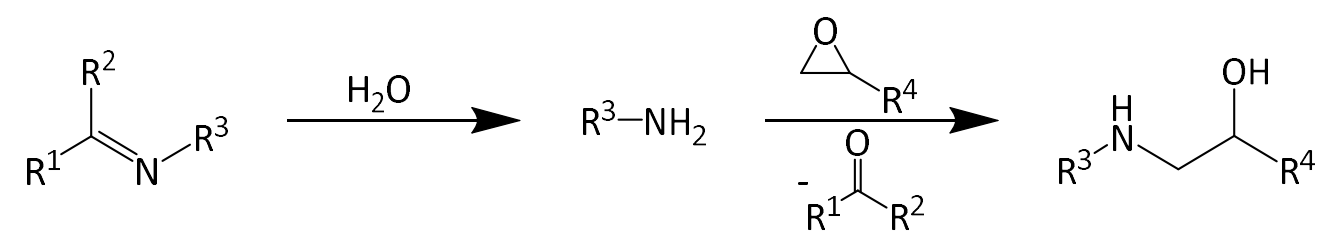

Scheme 6.7. Curing process for Ancamine ${ }^{\circledR}$ 2459: ketimine deprotection of an amine, which then reacts with an epoxide. A side product of the deprotection is a volatile ketone.

\subsubsection{Surface-Functionalisation of Epoxy Coatings}

As in Table 6.6, the surfaces of the epoxy coatings undercured with Ancamine ${ }^{\circledR} 2459$ were functionalised with cyclam, cyclen, DETA, and PEI:

Cyclam (51 mg, $0.25 \mathrm{mmol})$ was dissolved in 2:1 v/v water:methanol $(750 \mu \mathrm{L})$, and then an aliquot $(125 \mu \mathrm{L})$ of the solution was added to a defined surface area of each of the following 
coatings: $-50 \%,-40 \%,-30 \%$, and $-20 \% \mathrm{w} / \mathrm{w}$ epoxy coatings (Ancamine ${ }^{\circledR} 2459$ and Epikote ${ }^{\mathrm{TM}} 235$ ). The aliquots were left on the coatings overnight ( $\sim 16 \mathrm{~h})$, and then the coatings were washed with water.

Cyclen (60 mg, $0.35 \mathrm{mmol}$ ) was dissolved in water $(300 \mu \mathrm{L})$ and added to cover a defined surface area of the $-40 \%$ w/w epoxy coating (Ancamine ${ }^{\circledR} 2459$ and Epikote ${ }^{\mathrm{TM}} 235$ ). After $24 \mathrm{~h}$, the aliquot was removed via pipetting, and the coating was washed with water.

DETA was added neat to the $-40 \%$ and $-30 \%$ w/w epoxy coatings (Ancamine ${ }^{\circledR} 2459$ and Epikote $^{\mathrm{TM}} 235$ ), covering a defined surface area for $24 \mathrm{~h}$ before removal via pipetting. Then, the coatings were washed with water.

PEI (branched, average $M_{w} \sim 800$, average $M_{n} \sim 600$; Sigma-Aldrich) was added neat to the $-40 \% w / w$ epoxy coating (Ancamine ${ }^{\circledR} 2459$ and Epikote ${ }^{\mathrm{TM}}$ 235), covering a defined surface area for $24 \mathrm{~h}$ before removal via pipetting. Then, the coating was washed with water.

\subsubsection{Application of Cu(II) to Epoxy Coatings}

To each prepared epoxy coating, including the surface-functionalised areas, a drop of aqueous copper(II) nitrate (50 mM; $20 \mu \mathrm{L}$ ) was added with an Eppendorf pipette for 2-6.5 h. Then, the coatings were washed with water.

\subsubsection{Quantification of Cu(II)-Loading}

The epoxy coatings assessed for $\mathrm{Cu}(\mathrm{II})$-loading were prepared as in Section 6.3.3.1 and were as follows: Ancamine ${ }^{\circledR} 2459$ and Epikote ${ }^{\mathrm{TM}} 235$ (stoichiometric), cyclen and Epikote ${ }^{\mathrm{TM}} 235$ ( $\pm 20 \% \mathrm{w} / \mathrm{w}$, $+10 \% \mathrm{w} / \mathrm{w}$, stoichiometric), Ancamine ${ }^{\circledR} 2459 /$ cyclen and Epikote $^{\mathrm{TM}} 235$ (+20\% w/w, $\left.+10 \% \mathrm{w} / \mathrm{w}\right)$, DETA $(25 \%) /$ cyclen (100\%) and Epikote ${ }^{\mathrm{TM}} 235(+25 \% \mathrm{w} / \mathrm{w})$, and DETA (100\%)/cyclen (25\%) and Epikote ${ }^{\mathrm{TM}} 235$ $(+25 \% \mathrm{w} / \mathrm{w})$. With the exception of stoichiometric Ancamine ${ }^{\circledR} 2459 /$ Epikote $^{\mathrm{TM}}$ 235, these are the supposed strongly $\mathrm{Cu}(\mathrm{II})$-chelating films highlighted in red in Table 6.6. Glass vials were washed with water $(3 \times)$ and air-dried. Each prepared epoxy coating $(150 \pm 10 \mathrm{mg})$ was delivered to the bottom of a vial and cured uncapped at $50{ }^{\circ} \mathrm{C}$ and $50 \%$ humidity for $24 \mathrm{~h}$. Once cured, the coatings were washed with water ( $3 \times)$ and air-dried, and the mass and exposed surface area (Appendix, Figure 8.38) of each coating were recorded. Aqueous copper(II) nitrate (50 mM; $500 \mu \mathrm{L} /$ vial) was added to the vials, which sat capped at room temperature. Water $(500 \mu \mathrm{L} /$ vial) was added to the vials serving as blanks. After $24 \mathrm{~h}$, the coatings were washed with water $(3 \mathrm{x})$ and air-dried. In preparation for ICP-MS (Cu) analysis, 
concentrated nitric acid (70\% v/v, aq; $500 \mu \mathrm{L})$ was added to each vial, and the vials were heated at $70^{\circ} \mathrm{C}$ for $2 \mathrm{~h}$ to digest the resin and release the copper into solution. The digested solutions were diluted $1,000-10,000 \times$ with $3 \% \mathrm{v} / \mathrm{v}$ aq nitric acid, bringing them into the range of the ICP-MS standards. If any particles were observed in a dilution, the sample was centrifuged for $10 \mathrm{~min}$ at 1788 RCF. The copper retained by each coating was quantified via ICP-MS analysis. 


\subsection{CHAPTER 4}

\subsubsection{Silica Functionalisation}

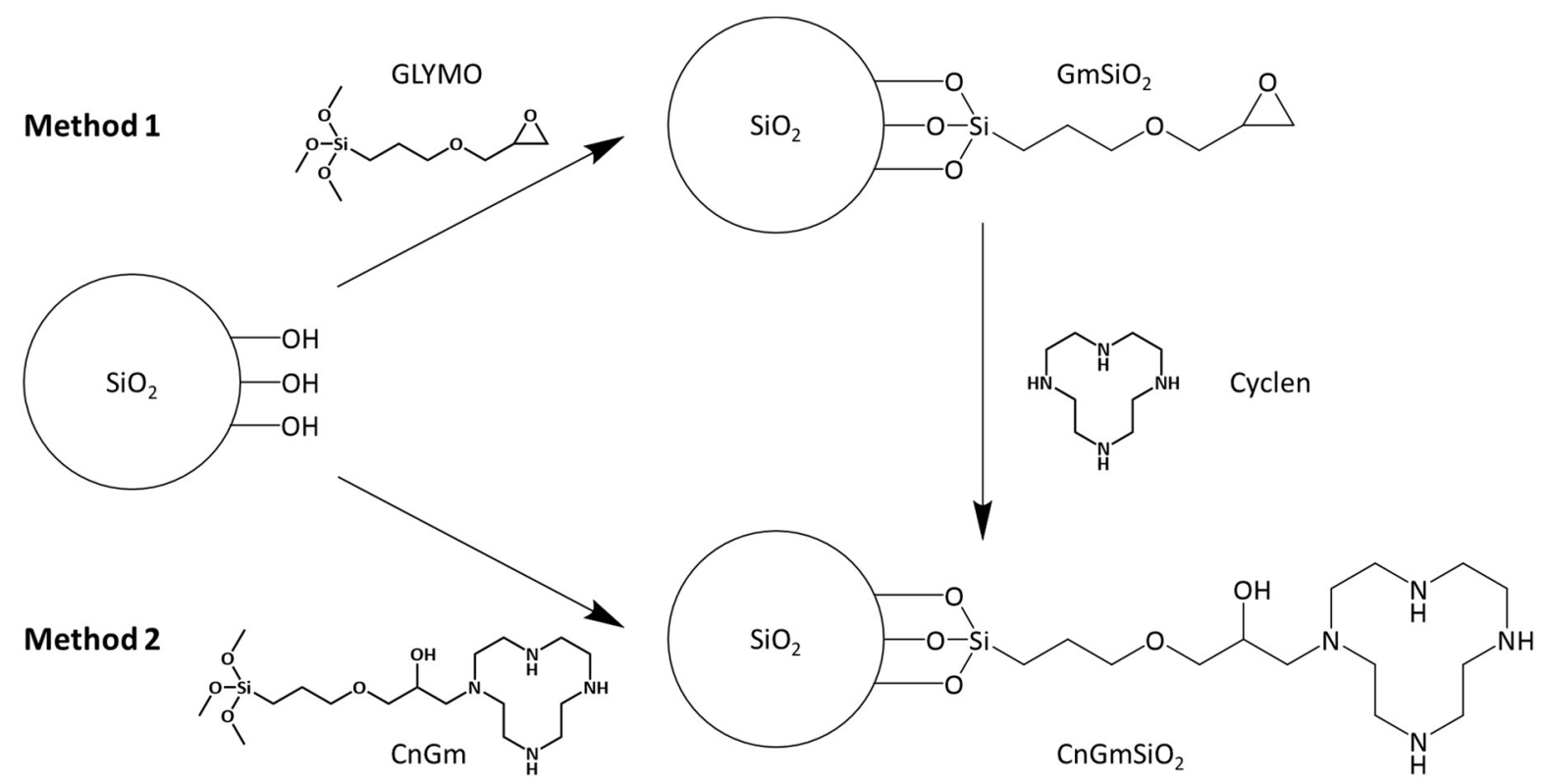

Scheme 6.8. Two silica functionalisation methods. Method 1: Reaction between GLYMO and the silanol groups of silica $\left(\mathrm{SiO}_{2}\right)$ produces $\mathrm{GLYMO}$-functionalised silica $\left(\mathrm{GmSiO}_{2}\right)$, and then the reaction between $\mathrm{GmSiO}_{2}$ and cyclen produces cyclen-GLYMO-silica $\left(\mathrm{CnGmSiO}_{2}\right)$. Method 2: GLYMO and cyclen react first to produce cyclen-GLYMO $(\mathrm{CnGm})$, and then $\mathrm{CnGm}$ reacts with silica to produce $\mathrm{CnGmSiO}_{2}$.

\subsubsection{Method 1}

\subsection{Synthesis of $\mathrm{GmSiO}_{2}$}

Reaction conditions in the procedures for silica-functionalisation with GLYMO (Scheme 6.9) are summarised in Table 6.7.
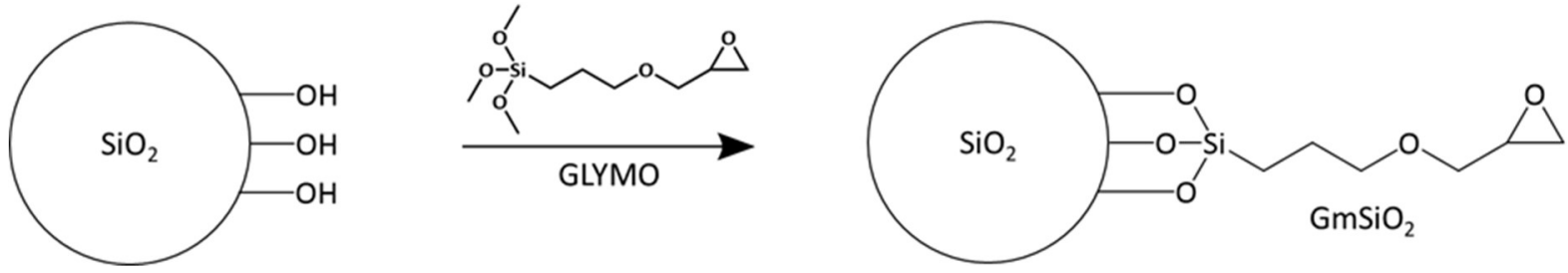

Scheme 6.9. Synthesis of $\mathrm{GmSiO}_{2}$. 
Table 6.7. Reaction conditions in Procedures 1-4 for $\mathrm{GmSiO}_{2}$ synthesis.

\begin{tabular}{lcccc}
\hline Conditions & Procedure 1 & Procedure 2 & Procedure 3 & Procedure 4 \\
\hline $\mathrm{SiO}_{2}(\mathrm{~g})$ & $0.91(\mathrm{dry})$ & 1.02 (dry) & 1.00 (wet) & 2.50 (dry) \\
$\mathrm{GLYMO}(\mathrm{g}, \mathrm{mmol})$ & $0.27,1.1$ & $2.1,9.1$ & $4.3,18$ & $3.7,16$ \\
Anhydrous toluene $(\mathrm{mL})$ & 5 & 4 & - & 35 \\
Triethylamine $(\mu \mathrm{L})$ & - & - & - & 75 \\
Temperature $\left({ }^{\circ} \mathrm{C}\right)$ & $20(\mathrm{RT})$ & 80 & 100 & 110.6 (reflux) \\
Time $(\mathrm{h})$ & 24 & 72 & 69 & 42 \\
\hline
\end{tabular}

\section{Procedure 1}

GLYMO (0.25 mL, $1.1 \mathrm{mmol})$ was added to silica $(0.91 \mathrm{~g})$ in anhydrous toluene $(5 \mathrm{~mL})$ under argon, and the reaction mixture was stirred $24 \mathrm{~h}$ at room temperature. The white silica product $(0.97 \mathrm{~g})$ was filtered, washed with anhydrous toluene $(6 \times 10 \mathrm{~mL})$, and dried by rotary evaporation for $30 \mathrm{~min}$.

IR: Silica $-v_{\max } / \mathrm{cm}^{-1} 3351 \mathrm{w}$ (br, O-H stretching), 1063s (br, Si-O-Si asymmetric stretching), 964m (br, $\mathrm{Si}-\mathrm{OH}$ stretching), $794 \mathrm{~m}$ (br, Si-O-Si symmetric stretching); ${ }^{262,333,334} \mathrm{GLYMO}-\mathrm{v}_{\max } / \mathrm{cm}^{-1} 2943 \mathrm{~m}$ and $2841 \mathrm{~m}\left(\mathrm{C}-\mathrm{H}\right.$ stretching in $\mathrm{Si}-\mathrm{O}-\mathrm{CH}_{3}$ and $\left.\mathrm{CH}_{2}\right), 1467 \mathrm{vw}\left(\mathrm{C}-\mathrm{H}\right.$ in-plane rocking in $\mathrm{Si}-\mathrm{O}-\mathrm{CH}_{3}$ and $\left.\mathrm{CH}_{2}\right)$, 1254vw (Si-C bending), 1191m (C-O stretching of aliphatic ether), 1076vs (Si-O-C stretching), 909w (epoxy ring $\mathrm{C}-\mathrm{O}$ stretching), 816s (Si-C stretching); ${ }^{335}$ Procedure $1 \mathrm{GmSiO}_{2}-\mathrm{v}_{\max } / \mathrm{cm}^{-1} 1060 \mathrm{~s}(\mathrm{br}$, Si-O-Si asymmetric stretching), 953m (br, Si-OH stretching), $801 \mathrm{~m}$ (br, Si-O-Si symmetric stretching). ${ }^{262,333,334}$

\section{Procedure 2}

GLYMO (2.0 $\mathrm{mL}, 9.1 \mathrm{mmol})$ was added to silica $(1.02 \mathrm{~g})$ in anhydrous toluene $(4 \mathrm{~mL})$ under argon, and the reaction mixture was stirred $72 \mathrm{~h}$ at $80^{\circ} \mathrm{C}$. The white silica product $(1.18 \mathrm{~g})$ was filtered, washed with anhydrous toluene $(6 \times 10 \mathrm{~mL})$, and dried via rotary evaporation.

IR: $v_{\max } / \mathrm{cm}^{-1} 1058 \mathrm{~s}$ (br, Si-O-Si asymmetric stretching), 948vw (br, Si-OH stretching), 908vw (br, epoxy ring $\mathrm{C}-\mathrm{O}$ stretching), 797m (br, Si-O-Si symmetric stretching), 729m, 694w.

\section{Procedure 3}

In this procedure, the silica was used without drying. GLYMO $(4.0 \mathrm{~mL}, 18 \mathrm{mmol})$ was added to silica $(1.00 \mathrm{~g})$, and the reaction mixture was stirred $69 \mathrm{~h}$ at $100{ }^{\circ} \mathrm{C}$. The white silica product $(1.40 \mathrm{~g})$ was filtered, washed with toluene $(80 \mathrm{~mL})$, and dried by rotary evaporation.

IR: $v_{\max } / \mathrm{cm}^{-1} 1049 \mathrm{~s}(\mathrm{br}, \mathrm{Si}-\mathrm{O}-\mathrm{Si}$ asymmetric stretching), 914w (br, epoxy ring C-O stretching), 796m (br, Si-O-Si symmetric stretching). 


\section{Procedure 4}

Protocols by Bagnoud et al. ${ }^{255}$ and Gros et al. ${ }^{241}$ were followed. GLYMO (3.5 mL, $16 \mathrm{mmol}$ ) and triethylamine ( $75 \mu \mathrm{L}, 0.54 \mathrm{mmol}$ ) were added to silica $(2.50 \mathrm{~g})$ in anhydrous toluene $(35 \mathrm{~mL})$ under argon, and the reaction mixture was stirred and heated to reflux temperature for $42 \mathrm{~h}$. The pale-yellow silica product ( $3.17 \mathrm{~g}$ ) was filtered, washed ( $70 \mathrm{~mL}$ anhydrous toluene, $100 \mathrm{~mL}$ acetone, $100 \mathrm{~mL}$ ether), and dried via rotary evaporation.

IR: $v_{\max } / \mathrm{cm}^{-1} 1066 \mathrm{~s}$ (br, Si-O-Si asymmetric stretching), 920vw (br, epoxy ring C-O stretching), 801m (br, Si-O-Si symmetric stretching).

\subsection{End-Capping $\mathrm{GmSiO}_{2}$}

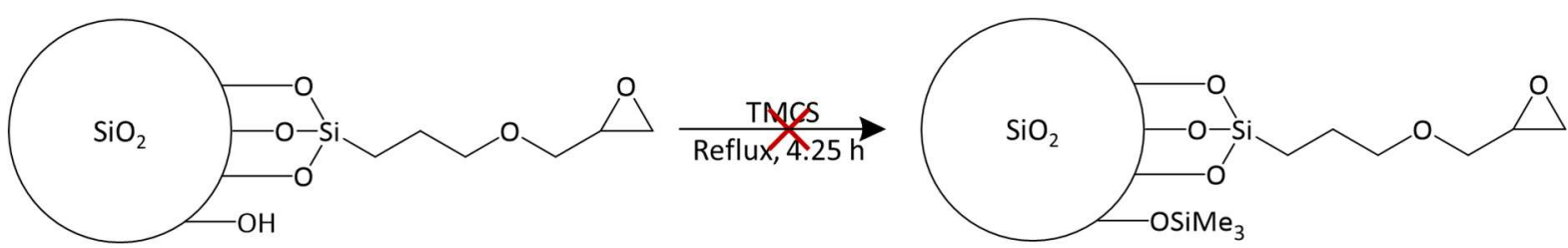

Scheme 6.10. Attempted end-capping of $\mathrm{GmSiO}_{2}$.

Following the protocol by Gros et al. ${ }^{241}$ (Scheme 6.10), $\mathrm{GmSiO}_{2}$ from Procedure 4 ( $2.95 \mathrm{~g}$ ) suspended in trimethylchlorosilane (TMCS; $12 \mathrm{~mL}, 92 \mathrm{mmol}$ ) was heated to reflux temperature for $4.25 \mathrm{~h}$ under argon. The silica product $(2.93 \mathrm{~g})$ was vacuum-filtered to remove excess TMCS, washed with water to neutral $\mathrm{pH}$, and dried for $18 \mathrm{~h}$ under high vacuum $(0.2-1.5 \mathrm{mmHg})$.

IR: $v_{\max } / \mathrm{cm}^{-1} 1059 \mathrm{~s}$ (br, Si-O-Si asymmetric stretching), 793m (br, Si-O-Si symmetric stretching).

\subsection{Synthesis of $\mathrm{CnGmSiO}_{2}$}

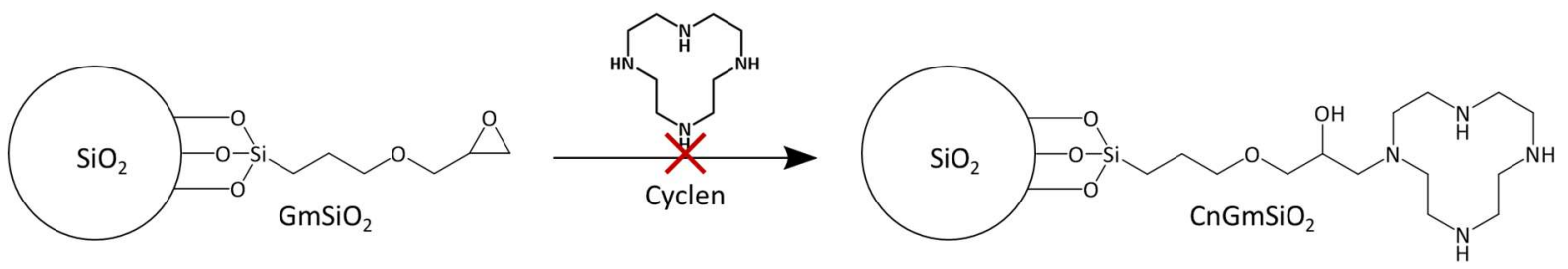

Scheme 6.11. Attempted synthesis of $\mathrm{CnGmSiO}_{2}$. 
$\mathrm{CnGmSiO}_{2}$ was synthesised following the procedure by Gros et al. ${ }^{241}$ (Scheme 6.11). A solution of cyclen $(0.59 \mathrm{~g}, 3.3 \mathrm{mmol})$ in water $(59 \mathrm{~mL})$ was added to the end-capped $\mathrm{GmSiO}_{2}(2.93 \mathrm{~g})$ from Procedure 4 . The suspension was heated at $80^{\circ} \mathrm{C}$ for $48 \mathrm{~h}$ without stirring but was occasionally mixed by gentle swirling, thus preventing damage to the particles. After $48 \mathrm{~h}$, the product $(2.95 \mathrm{~g})$ was filtered to remove excess cyclen and was washed with dilute hydrochloric acid $(0.1 \mathrm{M}$, aq; $2 \times 10 \mathrm{~mL}$ ), water (until the filtrate no longer turned a dark blue colour upon the addition of $50 \mathrm{mM}$ aq copper(II) sulfate), and hot methanol.

IR: $v_{\max } / \mathrm{cm}^{-1} 1055 \mathrm{~s}$ (br, Si-O-Si asymmetric stretching), $950 \mathrm{~m}$ (br, Si-OH stretching), 789m (br, Si-O-Si symmetric stretching).

\subsubsection{Method 2}

\subsection{Synthesis of $\operatorname{Cn}(\mathrm{Gm})_{n}$}

Reaction conditions in the procedures for $\mathrm{Cn}(\mathrm{Gm})_{\mathrm{n}}$ synthesis (Scheme 6.12) are summarised in Table 6.8.

Table 6.8. Reaction conditions in Procedures 1-6 for $\mathrm{Cn}(\mathrm{Gm})_{\mathrm{n}}$ synthesis.

\begin{tabular}{|c|c|c|c|c|c|c|}
\hline Conditions & $\begin{array}{c}\text { Procedure } \\
1\end{array}$ & $\begin{array}{c}\text { Procedure } \\
2\end{array}$ & $\begin{array}{c}\text { Procedure } \\
3\end{array}$ & $\begin{array}{c}\text { Procedure } \\
4\end{array}$ & $\begin{array}{c}\text { Procedure } \\
5\end{array}$ & $\begin{array}{c}\text { Procedure } \\
6\end{array}$ \\
\hline GLYMO (mmol) & 4.4 & 1.1 & 2.3 & 1.1 & 1.1 & 8.9 \\
\hline Cyclen (mmol, eq) & $\begin{array}{l}1.1 \\
0.26\end{array}$ & $\begin{array}{l}4.8, \\
4.3\end{array}$ & $\begin{array}{l}0.56, \\
0.25\end{array}$ & $\begin{array}{l}1.2, \\
1.1\end{array}$ & $\begin{array}{l}1.2 \\
1.1\end{array}$ & $\begin{array}{l}9.7 \\
1.1\end{array}$ \\
\hline $\mathrm{Al}(\mathrm{OTf})_{3}(\mathrm{mmol})$ & - & - & - & 0.1 & 0.1 & - \\
\hline Solvent & $\begin{array}{c}2: 1 \\
\mathrm{MeOH}: \mathrm{H}_{2} \mathrm{O}\end{array}$ & toluene & toluene & $\mathrm{CHCl}_{3}$ & $\mathrm{CDCl}_{3}$ & $\mathrm{CHCl}_{3}$ \\
\hline Volume solvent $(\mathrm{mL})$ & 23.8 & 7.5 & 10 & 2 & 2 & 16 \\
\hline Temperature $\left({ }^{\circ} \mathrm{C}\right)$ & 20 (RT) & $\begin{array}{l}110.6 \\
\text { (reflux) }\end{array}$ & $\begin{array}{l}110.6 \\
\text { (reflux) }\end{array}$ & $20(\mathrm{RT})$ & 20 (RT) & 20 (RT) \\
\hline Time & $5 \mathrm{~h}$ & $24 \mathrm{~h}$ & $97.5 \mathrm{~h}$ & $90 \mathrm{~h}$ & $69 \mathrm{~h}$ & $8-10$ days \\
\hline References & $\begin{array}{c}\text { Bereczki et } \\
\text { al. }^{258}\end{array}$ & $\begin{array}{c}\text { Barreto et } \\
\text { al. }{ }^{259}\end{array}$ & $\begin{array}{c}\text { Barreto et } \\
\text { al. } 259\end{array}$ & $\begin{array}{c}\text { Williams } \\
\text { and } \\
\text { Cullen }^{266}\end{array}$ & $\begin{array}{c}\text { Williams } \\
\text { and } \\
\text { Cullen }^{266}\end{array}$ & \\
\hline
\end{tabular}


<smiles>C1CNCCNCCNCCNC1</smiles><smiles>CO[Si](CCCOCC1CO1)(OC)OC</smiles>

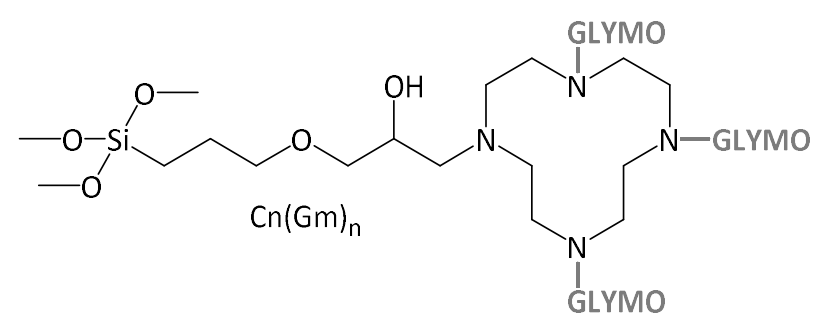

Scheme 6.12. Synthesis of $\mathrm{Cn}(\mathrm{Gm})_{\mathrm{n}}$.

\section{Procedure 1}

Following the procedure by Bereczki et al. ${ }^{258}$, GLYMO $(1.0 \mathrm{~mL}, 4.4 \mathrm{mmol}, 3.9 \mathrm{eq})$ was added to cyclen $(0.20 \mathrm{~g}, 1.1 \mathrm{mmol})$ dissolved in a solution of water $(8 \mathrm{~mL})$ and methanol $(15.8 \mathrm{~mL})$ under argon, and the reaction mixture was stirred at room temperature for $5 \mathrm{~h}$. In order to monitor the reaction by ${ }^{1} \mathrm{H}$ NMR spectroscopy, an aliquot $(1 \mathrm{~mL})$ of the crude reaction was dried by rotary evaporation and dissolved in a deuterated solvent. However, the residue was insoluble in a range of solvents $(2: 1 \mathrm{v} / \mathrm{v}$ $\mathrm{CD}_{3} \mathrm{OD}: \mathrm{D}_{2} \mathrm{O}, \mathrm{CHCl}_{3}, \mathrm{H}_{2} \mathrm{O}$, DMSO, 1:1 v/v DCM:MeOH). Therefore, test reactions in NMR tubes were set up to monitor the reaction. An aliquot $(5 \mathrm{~mL})$ of an aqueous solution of copper(II) acetate $(0.24 \mathrm{M}$; teal solution) was added to the reaction mixture, and immediate complexation was evidenced by a colour change from teal to dark blue.

\section{Test Reaction 1}

GLYMO $(0.040 \mathrm{~mL}, 0.18 \mathrm{mmol}, 3.9 \mathrm{eq})$ was added to cyclen $(0.008 \mathrm{~g}, 0.05 \mathrm{mmol})$ dissolved in a solution of deuterium oxide $(0.322 \mathrm{~mL})$ and methanol- $d_{4}(0.635 \mathrm{~mL})$, and the reaction was monitored by ${ }^{1} \mathrm{H}$ NMR spectroscopy for $2 \mathrm{~h}$. The ${ }^{1} \mathrm{H}$ NMR spectra revealed that the majority of GLYMO remained unreacted in this time period.

\section{Test Reaction 2}

GLYMO $(0.030 \mathrm{~mL}, 0.13 \mathrm{mmol}, 4.6 \mathrm{eq})$ was added to cyclen $(0.005 \mathrm{~g}, 0.03 \mathrm{mmol})$ dissolved in a solution of deuterium oxide $(0.242 \mathrm{~mL})$ and methanol- $d_{4}(0.476 \mathrm{~mL})$, and the reaction was monitored by ${ }^{1} \mathrm{H}$ NMR spectroscopy for $24 \mathrm{~h}$. The ${ }^{1} \mathrm{H}$ NMR spectra revealed that the majority of GLYMO remained unreacted in this time period.

\section{Procedure 2}

Following the procedure by Barreto et al. ${ }^{259}$, GLYMO $(0.26 \mathrm{~mL}, 1.1 \mathrm{mmol})$ in toluene $(5 \mathrm{~mL})$ was added dropwise to a solution of cyclen $(0.84 \mathrm{~g}, 4.8 \mathrm{mmol}, 4.3 \mathrm{eq})$ in hot toluene $(7.5 \mathrm{~mL})$. The reaction mixture 
was heated to reflux temperature for $24 \mathrm{~h}$ and then cooled to room temperature to precipitate the excess cyclen, which was removed by filtration and washed with cold toluene $(25 \mathrm{~mL})$. The toluene filtrate was evaporated to dryness via rotary evaporation, and the residual solid was dissolved in chloroform (10 mL), washed with sodium hydroxide $(0.1 \mathrm{M}$, aq; $2 \times 5 \mathrm{~mL})$, dried over magnesium sulfate, filtered, and concentrated to yield the product as a colourless oil $\left(0.32 \mathrm{~g}, 44 \%\right.$ if $\mathrm{Cn}(\mathrm{Gm})_{2}-70 \%$ if $\mathrm{CnGm}$ ). The reaction was monitored for the disappearance of GLYMO by TLC and ${ }^{1} \mathrm{H}$ NMR spectroscopy.

${ }^{1} \mathrm{H}$ NMR $\left(500 \mathrm{MHz}, \mathrm{CDCl}_{3}\right): \delta 4.31-4.11(\mathrm{~m}, 1 \mathrm{H}), 3.86(\mathrm{dd}, J=10.8,2.9 \mathrm{~Hz}, 1 \mathrm{H}), 3.66-3.59(\mathrm{~m}, 1 \mathrm{H}), 3.57$ (s, 2H), 3.56-3.33 (m, 5H), 3.21 (t, J = 10.4 Hz, 1H), 2.88-2.72 (m, 5H), $2.69(\mathrm{~s}, 4 \mathrm{H}), 2.68-2.39(\mathrm{~m}, 14 \mathrm{H})$, 2.14-1.88 (m, 1H), 1.86-1.75 ( $\mathrm{m}, 1 \mathrm{H}), 1.75-1.58(\mathrm{~m}, 1 \mathrm{H}), 1.19-0.93(\mathrm{~m}, 1 \mathrm{H}), 0.91-0.51(\mathrm{~m}, 2 \mathrm{H})$. ESI-MS m/z (rel intensity): 605.3 (34), 604.3 (66), $603.3\left(82 \text {, [3GLYMO - } \mathrm{CH}_{3}+2 \mathrm{H}\right]^{+}$), 589.3 (21, [3GLYMO $\left.2 \mathrm{CH}_{3}+3 \mathrm{H}\right]^{+}$), $583.3(59), 582.3(95), 581.3(100), 567.3(21), 558.3(29), 557.2(61), 535.3(45), 424.2$ (17), $399.2(18$, [2GLYMO - 2CH 3 + 3H] ]), 378.3 (21), 377.3 (74), 291.3 (82), 284.3 (21), 275.2 (45), 268.2 (39), 212.7 (32), $189.2(59), 173.2\left(20\right.$, [cyclen $\left.+\mathrm{H}^{+}\right)$.

\section{Procedure 3}

Following the procedure by Barreto et al. ${ }^{259}$ with modification, GLYMO $(0.51 \mathrm{~mL}, 2.3 \mathrm{mmol}, 4.0 \mathrm{eq})$ in toluene $(5 \mathrm{~mL})$ was added dropwise to a solution of cyclen $(0.10 \mathrm{~g}, 0.56 \mathrm{mmol})$ in hot toluene $(5 \mathrm{~mL})$. The reaction mixture was heated to reflux temperature for $97.5 \mathrm{~h}$ and then cooled to room temperature. The product was concentrated by rotary evaporation, dried further with a high vacuum pump (0.2-1.5 $\mathrm{mmHg}$ ) at room temperature, and, subsequently, formed a highly insoluble, intractable solid.

\section{Procedure 4}

Based on the procedure by Williams and Cullen ${ }^{266}$, GLYMO $(0.25 \mathrm{~mL}, 1.1 \mathrm{mmol})$ was added dropwise to a mixture of cyclen $(0.22 \mathrm{~g}, 1.2 \mathrm{mmol}, 1.1 \mathrm{eq})$ and $\mathrm{Al}(\mathrm{OTf})_{3}(0.05 \mathrm{~g}, 0.1 \mathrm{mmol})$ in anhydrous chloroform $(2 \mathrm{~mL})$ at room temperature under dry conditions and argon. An aliquot of the reaction mixture $(150 \mu \mathrm{L})$ was sampled $67 \mathrm{~h}$ later and prepared for ${ }^{1} \mathrm{H}$ NMR analysis by the addition of sodium bicarbonate (saturated, aq; $1 \mathrm{~mL}$ ), extraction with dichloromethane $(2 \mathrm{~mL})$, washing of the organic layers with water $(2 \mathrm{~mL})$, drying over magnesium sulfate, filtration, concentration, and dissolution in chloroform- $d$. The reaction mixture was stirred for $90 \mathrm{~h}$ total and then was quenched by the addition of sodium bicarbonate (saturated, aq; $5 \mathrm{~mL}$ ) and extracted with dichloromethane $(3 \times 5 \mathrm{~mL})$. Washing the combined organic layers with water $(2 \times 10 \mathrm{~mL})$ resulted in the precipitation of a white solid. The organic layers were concentrated, yielding a clear, intractable, plastic-like film. 


\section{Procedure 5}

Based on the procedure by Williams and Cullen ${ }^{266}$, GLYMO $(0.25 \mathrm{~mL}, 1.1 \mathrm{mmol})$ was added dropwise to a mixture of cyclen $(0.22 \mathrm{~g}, 1.2 \mathrm{mmol}, 1.1 \mathrm{eq})$ and $\mathrm{Al}(\mathrm{OTf})_{3}(0.06 \mathrm{~g}, 0.1 \mathrm{mmol})$ in anhydrous chloroform- $d(2 \mathrm{~mL})$ at room temperature under dry conditions and argon. The reaction was monitored by ${ }^{1} \mathrm{H}$ NMR spectroscopy by diluting an aliquot of the crude reaction mixture $(40 \mu \mathrm{L})$ with chloroform- $d(650 \mu \mathrm{L})$. The reaction mixture was stirred for $69 \mathrm{~h}$ until the reaction was complete, as determined by ${ }^{1} \mathrm{H}$ NMR spectroscopy. An aliquot of the completed reaction mixture $(100 \mu \mathrm{L})$ was prepared for ${ }^{1} \mathrm{H}$ NMR analysis by the addition of chloroform-d $(700 \mu \mathrm{L})$ and sodium carbonate $(10 \% \mathrm{w} / \mathrm{w}$, aq; $5 \mathrm{~mL})$, drying of the organic layer over magnesium sulfate, and filtration. Attempts to concentrate the reaction mixture produced a clear, intractable, plastic-like film.

${ }^{1} \mathrm{H}$ NMR $\left(500 \mathrm{MHz}, \mathrm{CDCl}_{3}\right): \delta 3.89(\mathrm{~s}, 1 \mathrm{H}), 3.56(\mathrm{~s}, 6 \mathrm{H}), 3.43(\mathrm{~s}, 6 \mathrm{H}), 3.00-2.71(\mathrm{~m}, 3 \mathrm{H}), 2.71-2.61(\mathrm{~m}$, $4 \mathrm{H}), 2.61-2.10(\mathrm{~m}, 5 \mathrm{H}), 1.88-1.47(\mathrm{~m}, 2 \mathrm{H}), 0.84-0.39(\mathrm{~m}, 2 \mathrm{H})$. ESI-MS $m / z$ (rel intensity): $904.5(26)$, 903.5 (48), 889.5 (19), 858.4 (22), 857.4 (41), 843.4 (28), 688.4 (46), 687.4 (93), 645.4 (100, [Cn(Gm) $\left.\left.+\mathrm{H}]^{+}\right), 631.4(57), 621.3(63), 599.3(74), 585.3(46), 571.3(22), 441.4\left(19,\left[\mathrm{Cn}(\mathrm{Gm})_{3}+2 \mathrm{H}\right]^{+2}\right]\right), 409.3$ $\left(56,\left[\mathrm{CnGm}+\mathrm{H}^{+}\right), 395.3(81), 381.2\left(67,\left[\mathrm{CnGm}-2 \mathrm{CH}_{3}+3 \mathrm{H}\right]^{+}\right), 367.2\left(70,\left[\mathrm{CnGm}-3 \mathrm{CH}_{3}+4 \mathrm{H}\right]^{+}\right), 323.3\right.$ (65, $\left.\left.\left[\mathrm{Cn}(\mathrm{Gm})_{2}+2 \mathrm{H}\right]^{+2}\right]\right), 316.3(43), 284.3(24), 173.2\left(19,[\text { cyclen }+\mathrm{H}]^{+}\right), 166.2(15)$.

\section{Procedure 6}

GLYMO (2.0 mL, $8.9 \mathrm{mmol})$ was added dropwise to a solution of cyclen $(1.73 \mathrm{~g}, 9.74 \mathrm{mmol}, 1.12 \mathrm{eq})$ in anhydrous chloroform ( $16 \mathrm{~mL}$ ) at room temperature under dry conditions and an argon atmosphere. The reaction was monitored by ${ }^{1} \mathrm{H}$ NMR spectroscopy by diluting an aliquot of the crude reaction mixture $(40 \mu \mathrm{L})$ with chloroform-d $(650 \mu \mathrm{L})$, and the reaction mixture, which was clear with a faint yellow tinge, was stirred for 8-10 days until the closed epoxide of GLYMO was undetectable by ${ }^{1} \mathrm{H}$ NMR spectroscopy. The crude material was used without purification.

${ }^{1} \mathrm{H}$ NMR $\left(500 \mathrm{MHz}, \mathrm{CDCl}_{3}\right): \delta$ 3.91-3.72 (m, 1H), 3.58-3.51 (m, 3H), 3.51-3.46 (m, 2H), 3.46-3.24 (m, 7H), 2.91-2.66 (m, 4H), $2.64(\mathrm{~s}, 5 \mathrm{H}), 2.60(\mathrm{~s}, 4 \mathrm{H}), 2.56-2.15(\mathrm{~m}, 6 \mathrm{H}), 1.82-1.42(\mathrm{~m}, 2 \mathrm{H}), 0.78-0.42(\mathrm{~m}$, 2H); $\left.{ }^{13} \mathrm{C} \mathrm{NMR} \mathrm{(126} \mathrm{MHz,} \mathrm{CDCl}_{3}\right): \delta$ 73.5, 73.3, 68.6, 58.1, 52.8, 50.6, 50.4, 47.3, 46.6, 46.1, 45.7, 24.1, 22.9, 22.8, 7.3, 6.8, 5.4, 5.3. ESI-HRMS: calcd for $\mathrm{C}_{17} \mathrm{H}_{41} \mathrm{~N}_{4} \mathrm{O}_{5} \mathrm{Si}[\mathrm{M}+\mathrm{H}]^{+} 409.2846$; found 409.2843; ESI-MS $m / z$ (rel intensity): $645.4\left(4,\left[\mathrm{Cn}(\mathrm{Gm})_{2}+\mathrm{H}^{+}{ }^{+}\right), 613.4\right.$ (9), 599.4 (18), 585.3 (5), 488.3 (3), 411.3 (3), $410.3(16), 409.3\left(100,[\mathrm{CnGm}+\mathrm{H}]^{+}\right), 377.3(51), 367.2\left(6,\left[\mathrm{CnGm}-3 \mathrm{CH}_{3}+4 \mathrm{H}\right]^{+}\right), 279.1(12), 252.1$ (3), $225.8(6), 173.2\left(51,[\text { cyclen }+\mathrm{H}]^{+}\right), 142.0(31)$. 


\subsection{Synthesis of $\pm \mathrm{Cu}(\mathrm{II})-\mathrm{CnGmSiO}_{2}$}

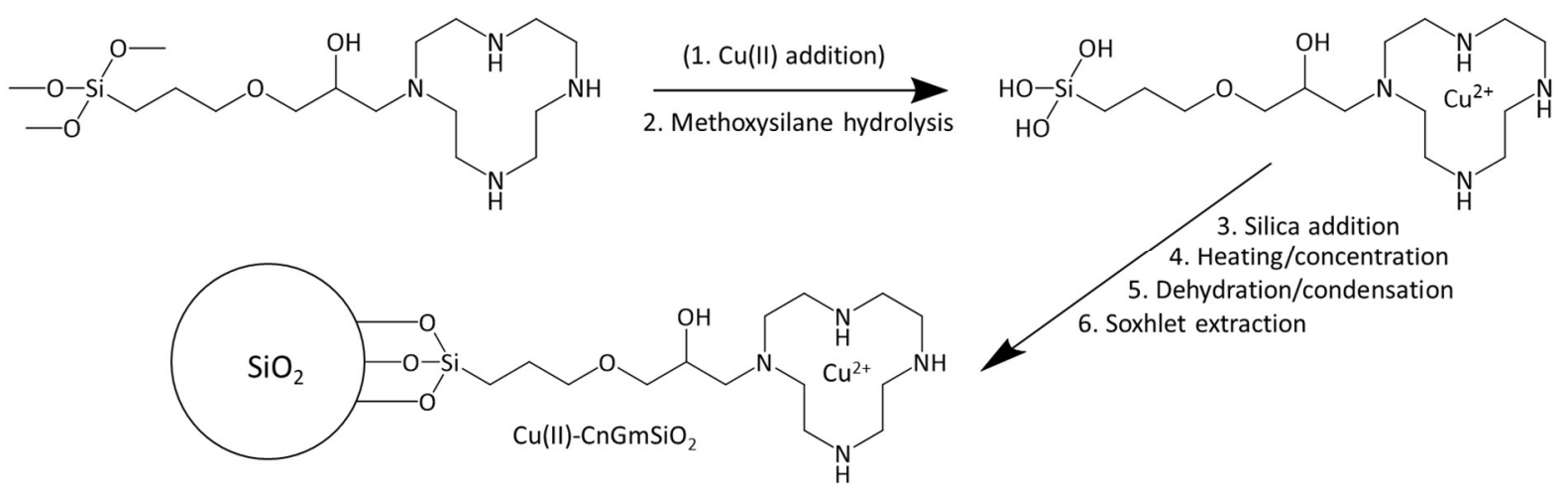

Scheme 6.13. Synthesis of $\pm \mathrm{Cu}(\mathrm{II})-\mathrm{CnGmSiO}{ }_{2}$.

In order to synthesise $\mathrm{Cu}(\mathrm{II})-\mathrm{CnGmSiO}_{2}$ (Scheme 6.13), $\mathrm{Cu}(\mathrm{II})$ may be added first to produce the $\mathrm{Cu}(\mathrm{II})-\mathrm{CnGm}$ complex (1). Then, hydrolysis of the methoxysilane groups (2) is followed by the addition of silica (3) and heating and concentration (4) to drive the dehydration/condensation reaction (5). Finally, the $\pm \mathrm{Cu}(\mathrm{II})-\mathrm{CnGmSiO} 2$ product is washed via Soxhlet extraction (6).

\section{Batch 1: $\mathrm{Cu}(\mathrm{II})-\mathrm{CnGmSiO}{ }_{2}$}

Aqueous copper(II) nitrate $(0.3 \mathrm{M} ; 20 \mathrm{~mL})$ was added to the reaction mixture (approximately $1.8 \mathrm{~g}$ or $4.3 \mathrm{mmol} \mathrm{CnGm}$ in $7.8 \mathrm{~mL} \mathrm{CHCl}$ ) from implementation of scaled-down Procedure 6 for $\mathrm{CnGm}$ synthesis. The organic layer was extracted further with aqueous copper(II) nitrate $(0.3 \mathrm{M} ; 2 \times 10 \mathrm{~mL})$ and washed with water until it was no longer a blue colour $(5 \times 5 \mathrm{~mL})$. The organic layer was analysed by ${ }^{1} \mathrm{H}$ NMR spectroscopy $\left(1: 1 \mathrm{v} / \mathrm{v} \mathrm{CDCl} 3: \mathrm{CD}_{3} \mathrm{OD}\right)$. Aqueous ammonium hydroxide ( $1 \% \mathrm{v} / \mathrm{v} ; 3.55 \mathrm{~mL}$ ) was added to the isolated, dark blue aqueous layer and vortexed, and a sample was analysed by MS. Then, silica (14.60 g) was added, and the mixture was vortexed and concentrated under vacuum at $70^{\circ} \mathrm{C}$ for $1 \mathrm{~h}$. The royal blue $\mathrm{Cu}(\mathrm{II})-\mathrm{CnGmSiO}_{2}$ product $(16.687 \mathrm{~g}$ ) was washed via Soxhlet extraction with water for $24 \mathrm{~h}$, dried at $120^{\circ} \mathrm{C}$ for $2 \mathrm{~h}$, and submitted for CHN elemental and IPC-MS (Cu) analyses.

ESI-HRMS: calcd for $\mathrm{C}_{14} \mathrm{H}_{31} \mathrm{CuN}_{4} \mathrm{O}_{4} \mathrm{Si}\left[\mathrm{M}-\mathrm{H}_{2} \mathrm{O}\right]^{+}$410.1411; found 410.1364; ESI-MS m/z (rel intensity): 607.2 (3), 606.2 (8), 604.2 (14), 431.2 (4), 430.1 (16), 428.1 (30, [Cu(II)-CnGm - 3CH $+2 \mathrm{H}]^{+}$), 410.1 (24, [Cu(II)- $\left.\mathrm{CnGm}-3 \mathrm{CH}_{3}-\mathrm{H}_{2} \mathrm{O}+3 \mathrm{H}\right]^{+}$), $311.6(3), 304.1$ (6), 303.6 (18), 302.6 (30), 293.6 (5), 233.1 (2), 216.1 (10), 215.6 (54), $\left.214.6(100 \text {, [Cu(II)-CnGm - 3CH }+3 \mathrm{H}]^{2+}\right), 205.6$ (38), 190.0 (27), 117.5 (16, [Cu(II)cyclen] ${ }^{2+}$ ). CHN: Silica - found C, <0.30\%; H, 0.96\%; N, <0.30\%; Batch $1 \mathrm{Cu}(I I)-C n G m S i O_{2}-$ found C, 
1.80\%; H, 0.77\%; N, 0.47\%. ICP-MS: Silica - < 0.0001\% w/w Cu; Batch $1 \mathrm{Cu(II)-CnGmSiO}{ }_{2}-0.48 \%$ w/w $\mathrm{Cu}$.

\section{Batch 2: $\mathrm{Cu}(\mathrm{II})-\mathrm{CnGmSiO}{ }_{2}$}

Copper(II) nitrate in methanol $(0.25 \mathrm{M} ; 50 \mathrm{~mL}$ ) was added to the reaction mixture (approximately $3.6 \mathrm{~g}$ or $8.8 \mathrm{mmol} \mathrm{CnGm}$ in $15.8 \mathrm{~mL} \mathrm{CHCl}$ ) from Procedure 6 for $\mathrm{CnGm}$ synthesis. A sample of the dark blue solution of $\mathrm{Cu}(\mathrm{II})-\mathrm{CnGm}$ was analysed by MS. Methoxysilane hydrolysis followed the procedure by $\mathrm{Lu}^{269}$. Methanol $(67.57 \mathrm{~mL})$, aqueous ammonium hydroxide $(28 \% \mathrm{v} / \mathrm{v} ; 5 \mathrm{~mL})$, and silica $(26.68 \mathrm{~g})$ were added to the reaction mixture, which was stirred and heated for $1 \mathrm{~h}$ at $60{ }^{\circ} \mathrm{C}$. The mixture was concentrated under vacuum at $70^{\circ} \mathrm{C}$ for $1 \mathrm{~h}$, and the royal blue $\mathrm{Cu}(\mathrm{II})-\mathrm{CnGmSiO}{ }_{2}$ product $(28.57 \mathrm{~g})$ was washed by Soxhlet extraction with water for $24 \mathrm{~h}$, dried, and submitted for CHN elemental and ICP-MS (Cu) analyses.

The samples were prepared for ICP-MS analysis as follows: the mass of the silica sample ( $\sim 50 \mathrm{mg}$ ) in a pre-cleaned Teflon beaker was recorded to five decimal-points, as were all other masses, and a few drops of concentrated nitric acid $(67-69 \% \mathrm{v} / \mathrm{v})$ were added to wet the sample. Concentrated hydrofluoric acid (48\% v/v; $2 \mathrm{~mL}$ ) was added, and the sample was capped and heated at reflux temperature for 3 days. Then, the sample was evaporated to dryness, nitric acid ( $9 M ; 2 \mathrm{~mL}$ ) was added, and the sample was capped and heated at reflux temperature overnight. Again, the sample was evaporated to dryness, nitric acid $(9 \mathrm{M} ; 2 \mathrm{~mL})$ was added, and the sample was capped and heated at reflux temperature until complete dissolution. The solution was transferred to a pre-cleaned polypropylene bottle, and water was added to the bottle until the recorded mass was approximately $50 \mathrm{~g}$. If required, the sample was diluted further for analysis by diluting an aliquot ( $100 \mathrm{mg}$ ) of the solution in a centrifuge tube with nitric acid ( $3 \% \mathrm{v} / \mathrm{v} ; ~ 10 \mathrm{~mL}$ final volume).

ESI-HRMS: calcd for $\mathrm{C}_{17} \mathrm{H}_{39} \mathrm{CuN}_{4} \mathrm{O}_{5} \mathrm{Si}[\mathrm{M}-\mathrm{H}]^{+}$470.1986; found 470.1978; ESI-MS $\mathbf{m} / \mathbf{z}$ (rel intensity): 927.3 (2), 895.3 (3), 835.4 (6), 833.4 (8), 819.4 (2), 709.2 (1), 662.3 (3), 648.2 (7), 646.2 (8), 618.2 (4), $529.2(2), 473.2(3), 471.2(6), 470.2\left(3,[\mathrm{Cu}(\mathrm{II})-\mathrm{CnGm}-\mathrm{H}]^{+}\right), 458.2(9), 456.2\left(16,\left[\mathrm{Cu}(\mathrm{II})-\mathrm{CnGm}-\mathrm{CH}_{3}\right]^{+}\right)$, $442.2\left(9,\left[\mathrm{Cu}(\mathrm{II})-\mathrm{CnGm}-2 \mathrm{CH}_{3}+\mathrm{H}\right]^{+}\right), 428.2\left(6,\left[\mathrm{Cu}(\mathrm{II})-\mathrm{CnGm}-3 \mathrm{CH}_{3}+2 \mathrm{H}\right]^{+}\right), 424.2$ (10), 330.6 (4), 324.6 (7), 316.6 (11), 307.6 (7), 234.1 (9), 214.6 (12), 212.6 (15), 192.0 (25), 190.0 (55), 145.0 (100). CHN: found C, 4.17\%; H, 0.92\%; N, 1.18\%. ICP-MS: $2.60 \%$ w/w Cu.

\section{Batch 3: $\mathrm{CnGmSiO}$}

The Batch 2 procedure was followed without the addition of copper(II) nitrate, and methoxysilane hydrolysis followed the procedure by $\mathrm{Lu}^{269}$. Methanol $(57.30 \mathrm{~mL})$, aqueous ammonium hydroxide $(28 \% \mathrm{v} / \mathrm{v} ; 2.5 \mathrm{~mL})$, and silica $(13.00 \mathrm{~g})$ were added to the reaction mixture (approximately $1.7 \mathrm{~g}$ or 
$4.3 \mathrm{mmol} \mathrm{CnGm}$ in $7.7 \mathrm{~mL} \mathrm{CHCl}$ ) from implementation of scaled-down Procedure 6 for $\mathrm{CnGm}$ synthesis. The mixture was heated while stirring for $1 \mathrm{~h}$ at $60^{\circ} \mathrm{C}$ and then dried via rotary evaporation for $1 \mathrm{~h}$. The white $\mathrm{CnGmSiO}_{2}$ product (16.03 g) was washed by Soxhlet extraction with water for $24 \mathrm{~h}$, dried, and submitted for $\mathrm{CHN}$ elemental analysis.

CHN: found C, $4.52 \% ; H, 1.22 \% ; \mathrm{N}, 1.24 \%$.

\section{Batch 4: $\mathrm{CnGmSiO}_{2}$}

The procedure from Batch 3 was repeated exactly, and the white $\mathrm{CnGmSiO}_{2}$ product ( $16.28 \mathrm{~g}$ ) was submitted for $\mathrm{CHN}$ elemental analysis.

CHN: found C, 4.81\%; H, 0.94\%; N, 1.46\%.

\subsection{Study of GLYMO Hydrolysis}

The hydrolytic stability of GLYMO (Scheme 6.14) was tested under basic conditions, with and without heating, and in various solvents and solvent combinations.

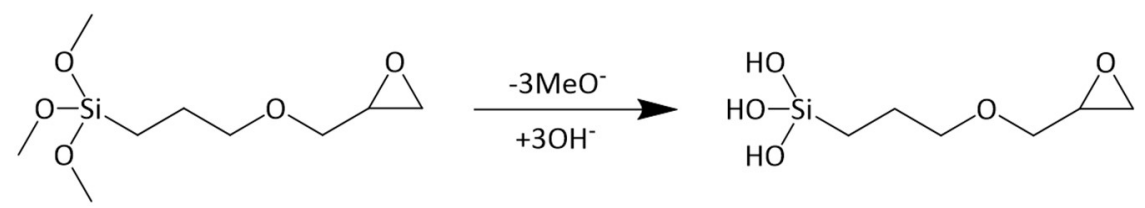

Scheme 6.14. Hydrolysis of GLYMO methoxysilane groups.

$\mathrm{CDCl}_{3}, \mathrm{RT}$

GLYMO (20 mg, $0.085 \mathrm{mmol}$ ) was added to chloroform- $d(700 \mu \mathrm{L})$, and the ${ }^{1} \mathrm{H}$ NMR spectrum was acquired immediately, as well as after 6 days sitting at room temperature.

$C D_{3} \mathrm{OD}, 60^{\circ} \mathrm{C}$

GLYMO (27 mg, $0.11 \mathrm{mmol})$ was added to methanol- $d_{4}(800 \mu \mathrm{L})$, and the solution was heated for 2 days at $60{ }^{\circ} \mathrm{C}$. The ${ }^{1} \mathrm{H}$ NMR spectrum was acquired at $19 \mathrm{~h}$ and $40 \mathrm{~h}$.

Batch 1 Conditions: $0.05 \% \mathrm{v} / \mathrm{v} \mathrm{NH} \mathrm{H}_{4} \mathrm{OH}, \mathrm{D}_{2} \mathrm{O}, \mathrm{RT}$

GLYMO (11 $\mathrm{mg}, 0.045 \mathrm{mmol}$ ) was added to a solution of ammonium hydroxide $(0.05 \% \mathrm{v} / \mathrm{v})$ in deuterium oxide (total solution volume $685.5 \mu \mathrm{L}$ ), and the ${ }^{1} \mathrm{H}$ NMR spectrum was acquired at $0.2 \mathrm{~h}$ and $4.7 \mathrm{~h}$. 


\section{Lu ${ }^{269}$ Conditions: $0.04 \% \mathrm{v} / \mathrm{v} \mathrm{NH} \mathrm{NH}_{4} \mathrm{OH} \mathrm{CD}_{3} \mathrm{OD}, 1.6 \% \mathrm{v} / \mathrm{v} \mathrm{H}_{2} \mathrm{O}, 60^{\circ} \mathrm{C}$}

GLYMO (10 mg, $0.044 \mathrm{mmol})$ was added to a solution of ammonium hydroxide $(0.04 \% \mathrm{v} / \mathrm{v})$ in water $(1.6 \% \mathrm{v} / \mathrm{v})$ and methanol- $d_{4}$ (total solution volume $691 \mu \mathrm{L}$ ), and the solution was heated at $60^{\circ} \mathrm{C}$. The ${ }^{1} \mathrm{H}$ NMR spectrum was acquired at $1.1 \mathrm{~h}$ and $2.3 \mathrm{~h}$.

Lu ${ }^{269}$ Conditions: $1 \% \mathrm{v} / \mathrm{v} \mathrm{NH}{ }_{4} \mathrm{OH}, \mathrm{CD}_{3} \mathrm{OD}, 3.6 \% \mathrm{v} / \mathrm{v} \mathrm{H}_{2} \mathrm{O}, 60^{\circ} \mathrm{C}$

GLYMO (10 $\mathrm{mg}, 0.044 \mathrm{mmol})$ was added to a solution of ammonium hydroxide $(1 \% \mathrm{v} / \mathrm{v})$ in water $(3.6 \% \mathrm{v} / \mathrm{v})$ and methanol- $d_{4}$ (total solution volume $691 \mu \mathrm{L}$ ), and the solution was heated for $1 \mathrm{~h}$ at $60{ }^{\circ} \mathrm{C}$. Then, the ${ }^{1} \mathrm{H}$ NMR spectrum was acquired.

Batches 2-4 Conditions: $1 \% \mathrm{v} / \mathrm{v} \mathrm{NH} \mathrm{NH}_{4} \mathrm{OH}, \mathrm{CD}_{3} \mathrm{OD}, 3.7 \% \mathrm{v} / \mathrm{v} \mathrm{H}_{2} \mathrm{O}, 11 \% \mathrm{v} / \mathrm{v} \mathrm{CHCl}{ }_{3}, 60^{\circ} \mathrm{C}$

GLYMO (10 mg, $0.044 \mathrm{mmol}$ ) was added to a solution of ammonium hydroxide $(1 \% \mathrm{v} / \mathrm{v})$ in water $(3.7 \% \mathrm{v} / \mathrm{v})$, chloroform $(11 \% \mathrm{v} / \mathrm{v})$, and methanol- $d_{4}$ (total solution volume $\left.675.2 \mu \mathrm{L}\right)$, and the solution was heated for $1 \mathrm{~h}$ at $60^{\circ} \mathrm{C}$. Then, the ${ }^{1} \mathrm{H}$ NMR spectrum was acquired.

\subsubsection{4 $\mathrm{Cu}(\mathrm{II})$-Loading by $\mathrm{CnGmSiO}_{2}$ (Batches 3 and 4)}

$\mathrm{CnGmSiO}_{2}(0.10 \mathrm{~g})$ from Batches 3 and 4 was added to an aqueous solution of copper(II) nitrate ( $50 \mathrm{mM} ; 3 \mathrm{~mL}$ ), and the mixture was stirred for $24 \mathrm{~h}$ and filtered. The now-blue silica was washed with water $(5 \times 5 \mathrm{~mL})$ and acetone $(1 \times 3 \mathrm{~mL})$, dried, and submitted for ICP-MS (Cu) analysis, following the sample preparation outlined for Batch $2 \mathrm{Cu}(\mathrm{II})-\mathrm{CnGmSiO}_{2}$ in Section 6.4.1.2.2.

ICP-MS: Batch 3-0.96\% w/w Cu; Batch $4-1.02 \%$ w/w Cu.

\subsection{Adsorption of Cu(II)-Cyclen on Silica}

Copper(II) nitrate trihydrate ( $91 \mathrm{mg}, 0.38 \mathrm{mmol}$ ) and aqueous ammonium hydroxide ( $1 \% \mathrm{v} / \mathrm{v} ; 0.24 \mathrm{~mL}$ ) were added to cyclen $(59 \mathrm{mg}, 0.34 \mathrm{mmol})$ in water $(7.58 \mathrm{~mL})$. Then, silica $(1.00 \mathrm{~g})$ was added, and the mixture was concentrated via rotary evaporation for $1 \mathrm{~h}$ at $70^{\circ} \mathrm{C}$. The resulting light blue silica was washed by Soxhlet extraction with water for $25.3 \mathrm{~h}$, dried, and submitted for $\mathrm{CHN}$ elemental and ICP-MS (Cu) analyses.

CHN: found C, 0.53\%; H, 0.87\%; N, < 0.30\%. ICP-MS: 0.28\% w/w Cu. 


\subsection{Cu(II)-Retention by Unfunctionalised Silica}

Silica (0.10 g) was added to an aqueous solution of copper(II) nitrate (50 mM; $3 \mathrm{~mL})$, and the mixture was stirred for $24 \mathrm{~h}$ and filtered. The silica was washed with water $(5 \times 5 \mathrm{~mL})$ and acetone $(1 \times 3 \mathrm{~mL})$, dried, and submitted for ICP-MS ( $\mathrm{Cu}$ ) analysis, following the sample preparation outlined for Batch 2 $\mathrm{Cu}(\mathrm{II})-\mathrm{CnGmSiO}_{2}$ in Section 6.4.1.2.2. A blank was prepared by repeating the procedure with silica in water.

ICP-MS: $0.06 \% \mathrm{w} / \mathrm{w} \mathrm{Cu}$.

\subsubsection{Incorporation of Silica in a Coating}

A two-pack epoxy was prepared by mixing a commercial hardener (Ancamine ${ }^{\circledR} 2459$ Curing Agent; Air Products and Chemicals, Inc.) and a commercial epoxy resin (Epikote ${ }^{\mathrm{TM}}$ 235; Resolution Performance Products) in accordance with the manufacturers' specifications: 0.563 g Ancamine ${ }^{\circledR} 2459 / \mathrm{g}$ Epikote ${ }^{\mathrm{TM}}$ 235. Immediately following manual mixing of the two components, one coat of the viscous, dark yellow epoxy resin was applied using the standard draw-down method (bar film applicator: 1120/25/100, S232228, Sheen; Figure 6.2) to a single side of a black vinyl chloride/acetate copolymer (black scrub test panel, Leneta). An inverted funnel was then used to add silica to a defined surface area of the still-uncured resin so that, at minimum, one layer of silica covered the resin. The coating was allowed to cure at room temperature overnight, and, the following day, the procedure was repeated for the second side. After allowing the second side to cure at room temperature overnight, the silica/epoxy $\left(\mathrm{SiO}_{2} / \mathrm{epx}\right)$-type samples were brushed, washed with water, and dried with compressed air, and squares $(23 \mathrm{~mm} \times 23 \mathrm{~mm})$ coated on both sides were cut from the material. These squares were tested immediately for bacterial adherence. The four silica samples prepared for testing were as follows: 1) Batch $4 \mathrm{CnGmSiO}_{2}$ (free ligand), 2) Batch $4 \mathrm{CnGmSiO}_{2}+\mathrm{Cu}$ (II) (post-loaded), 3) Batch $2 \mathrm{Cu}(\mathrm{II})-\mathrm{CnGmSiO}_{2}$ (pre-loaded), and 4) unfunctionalised $\mathrm{SiO}_{2}$. To post-load the Batch 4 $\mathrm{CnGmSiO}_{2}$, the squares coated on both sides with $\mathrm{CnGmSiO}_{2}$ were submerged in an aqueous solution of an excess of copper(II) nitrate $(50 \mathrm{mM} ; 16.5 \mathrm{~mL})$ for $24 \mathrm{~h}$, washed with water, dried with compressed air, and tested immediately for bacterial adherence. Images and the elemental composition of the $\mathrm{SiO}_{2} /$ epx-type coatings were obtained by SEM-EDS (Appendix, Figures 8.45-51). 


\subsection{CHAPTER 5}

\subsubsection{Method Development with E. coli NZRM 3647}

In Figure 6.3, the bacterial adherence assay is illustrated and the areas to be addressed for method development are highlighted in red.

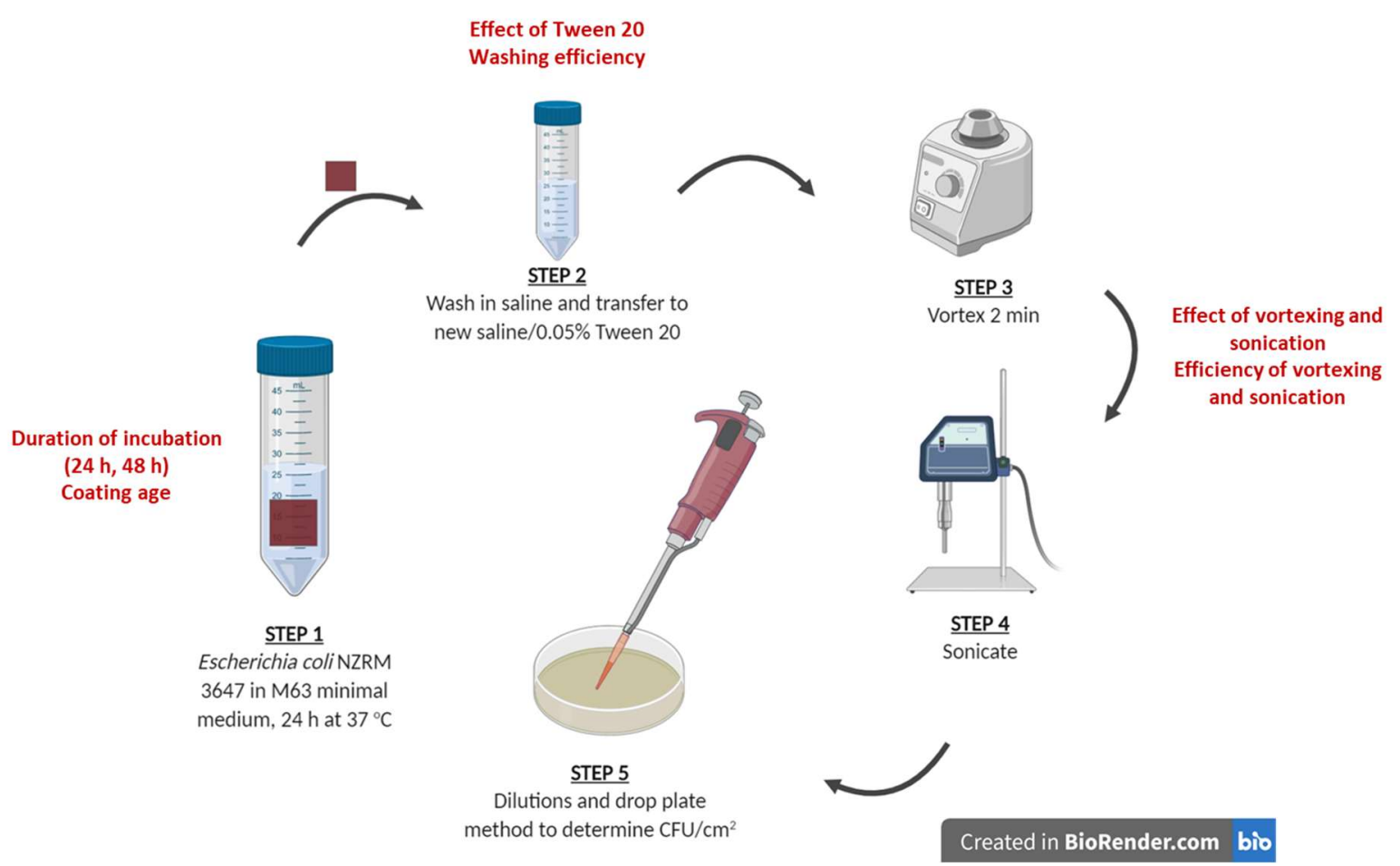

Figure 6.3. Steps of the bacterial adherence assay and, in red, areas to be addressed for method development (created with Biorender.com).

\subsubsection{Duration of Incubation}

An overnight culture was prepared by inoculating nutrient broth $(3 \mathrm{~mL})$ with a colony of E. coli NZRM 3647 from a nutrient agar plate and incubating for $16 \mathrm{~h}$ at $37{ }^{\circ} \mathrm{C}$ with shaking. Two squares $(25 \mathrm{~mm} \times 25 \mathrm{~mm}$ ) cut from a vinyl chloride/acetate copolymer (black scrub test panel, Leneta) and sterilised with $70 \% \mathrm{v} / \mathrm{v}$ aq ethanol were submerged in overnight culture diluted 50× with M63 minimal medium (final volume $16.5 \mathrm{~mL}$ ) in two tubes (one square/tube; Figure 6.4). At $37^{\circ} \mathrm{C}$ without shaking, one tube was incubated $24 \mathrm{~h}$, and the other tube was incubated $48 \mathrm{~h}$. Following incubation, the $\mathrm{OD}_{600}$ of each culture was measured, and each square was transferred to sterile saline $(20 \mathrm{~mL})$ for $5 \mathrm{~min}$ to 
remove planktonic bacteria. After the saline wash, the squares were transferred to tubes containing sterile solutions of $0.05 \% \mathrm{v} / \mathrm{v}$ Tween 20 in saline (final volume $20.040 \mathrm{~mL}$ ), and then the solutions were vortexed $2 \mathrm{~min}$ at maximum speed and sonicated $8 \mathrm{~s}$ at $40 \%$ amplitude to detach bacteria from the squares. Serial dilutions $(10 x)$ of these solutions were performed to count colonies by the drop plate method.

Sterilised, uncoated squares $(\times 2)$

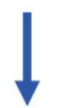

Diluted cultures $(\times 2)$ of $E$. coli NZRM

3647 in M63 minimal medium

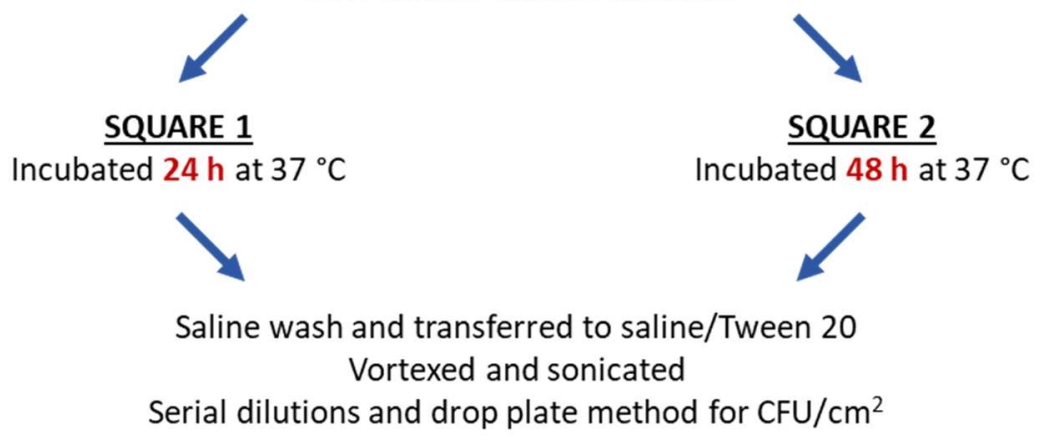

Figure 6.4. Testing the effect of the duration of incubation on bacterial adherence ( $24 \mathrm{~h}$ vs $48 \mathrm{~h}$ ).

\subsubsection{Coating Age}

A single coat of paint or resin was applied to one side of a black plastic sheet (vinyl chloride/acetate copolymer, black scrub test panel, Leneta) by the standard draw-down method (Figure 6.2, bar film applicator: $1120 / 25 / 100$, S232228, Sheen) and was cured at room temperature overnight. The following day, the procedure was repeated for the other side of the plastic sheet. After overnight curing of the second side, squares $(23 \mathrm{~mm} \times 23 \mathrm{~mm})$ were cut from the coated sheets for immediate testing of bacterial adherence to "fresh" coatings, or the coated sheets were allowed to sit for two weeks at room temperature before cutting out the squares for bacterial adherence testing. Four sample square types were prepared: 1) uncoated plastic squares, 2) plastic squares coated with marine paint (Altex Yacht and Boat Paint, Aurora Red, Ablative Antifouling No. 5) containing high amounts of copper $\left(40-50 \% \mathrm{Cu}_{2} \mathrm{O}\right), 3$ ) plastic squares coated with a commercial, two-pot epoxy resin (Epikote $^{\mathrm{TM}} 235$ and Ancamine ${ }^{\circledR}$ 2459; prepared as in Section 6.4.2), and 4) plastic squares coated with a commercial acrylic paint (LR200, Resene Paints Ltd.). The samples were sterilised with UV light for 20 min per side in a laminar flow hood. 
An overnight culture was prepared by inoculating nutrient broth $(3 \mathrm{~mL})$ with a colony of $E$. coli NZRM 3647 from a nutrient agar plate and incubating for $16 \mathrm{~h}$ at $37{ }^{\circ} \mathrm{C}$ with shaking. Each of the sterilised sample squares were submerged in overnight culture diluted with M63 minimal medium to $\mathrm{OD}_{600} 0.05$ (final volume $16.5 \mathrm{~mL} ; 5 \pm 2\left(\times 10^{7}\right) \mathrm{CFU} / \mathrm{mL}$ ), and the diluted cultures were incubated $24 \mathrm{~h}$ at $37^{\circ} \mathrm{C}$ without shaking (Figure 6.5). Then, the $\mathrm{OD}_{600}$ of each culture was measured. The squares were transferred to sterile saline $(20 \mathrm{~mL})$ for $5 \mathrm{~min}$ to remove planktonic bacteria, and a pipet was used to rinse both sides of the squares with sterile saline ( $500 \mu \mathrm{L} / \mathrm{side})$. The squares were transferred to tubes containing sterile solutions of $0.05 \% \mathrm{v} / \mathrm{v}$ Tween 20 in saline (final volume $20.040 \mathrm{~mL}$ ), and the solutions were vortexed $2 \mathrm{~min}$ at maximum speed and sonicated $8 \mathrm{~s}$ at $40 \%$ amplitude to detach bacteria from the squares. Serial dilutions $(10 x)$ of these solutions were performed to count colonies by the drop plate method. The experiment was independently repeated three times.

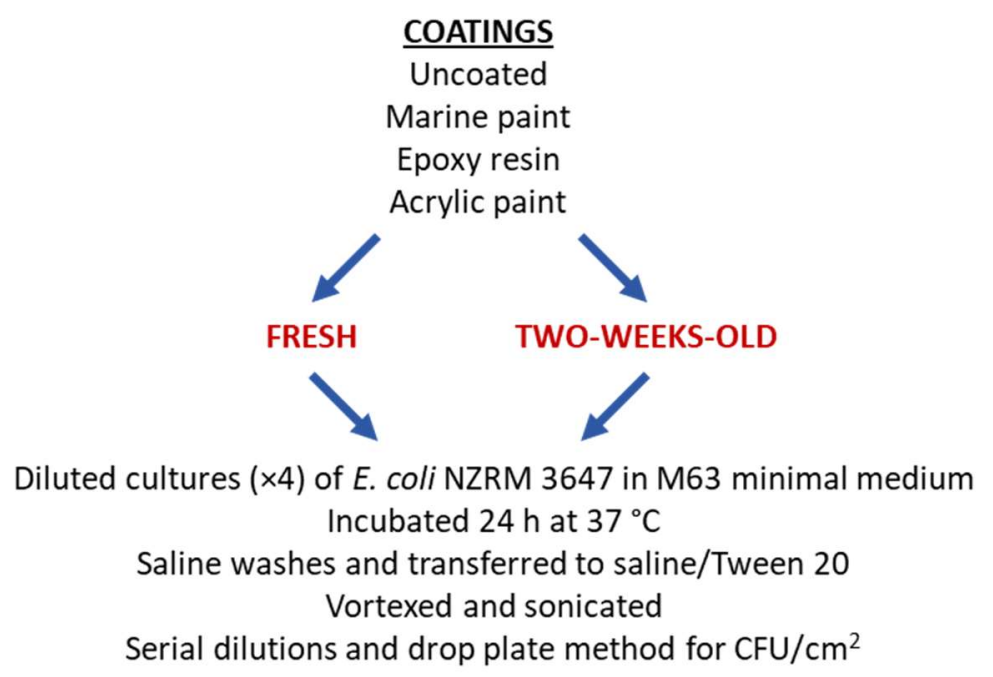

Figure 6.5. Testing the effect of coating age on bacterial adherence.

\subsubsection{Effect of Tween 20}

An overnight culture was prepared by inoculating nutrient broth $(3 \mathrm{~mL})$ with a colony of E. coli NZRM 3647 from a nutrient agar plate and incubating for $16 \mathrm{~h}$ at $37{ }^{\circ} \mathrm{C}$ with shaking. Two squares $(25 \mathrm{~mm} \times 25 \mathrm{~mm}$ ) cut from a vinyl chloride/acetate copolymer (black scrub test panel, Leneta) and sterilised with $70 \% \mathrm{v} / \mathrm{v}$ aq ethanol were submerged in overnight culture diluted 50× with M63 minimal medium (final volume $20 \mathrm{~mL}$ ) in two tubes (one square/tube), and the diluted cultures were incubated $24 \mathrm{~h}$ at $37^{\circ} \mathrm{C}$ without shaking (Figure 6.6). Following incubation, the $\mathrm{OD}_{600}$ of each culture was measured, and each square was transferred to sterile saline $(20 \mathrm{~mL})$ for $5 \mathrm{~min}$ to remove planktonic 
bacteria. Then, one square was transferred to another tube of sterile saline $(20 \mathrm{~mL})$, and the other square was transferred to a tube containing a sterile solution of $0.05 \% \mathrm{v} / \mathrm{v}$ Tween 20 in saline (final volume $20.0401 \mathrm{~mL}$ ). Both tubes were vortexed $2 \mathrm{~min}$ at maximum speed to detach bacteria from the squares. Serial dilutions $(10 \times)$ of the vortexed solutions were performed to count colonies by the drop plate method.

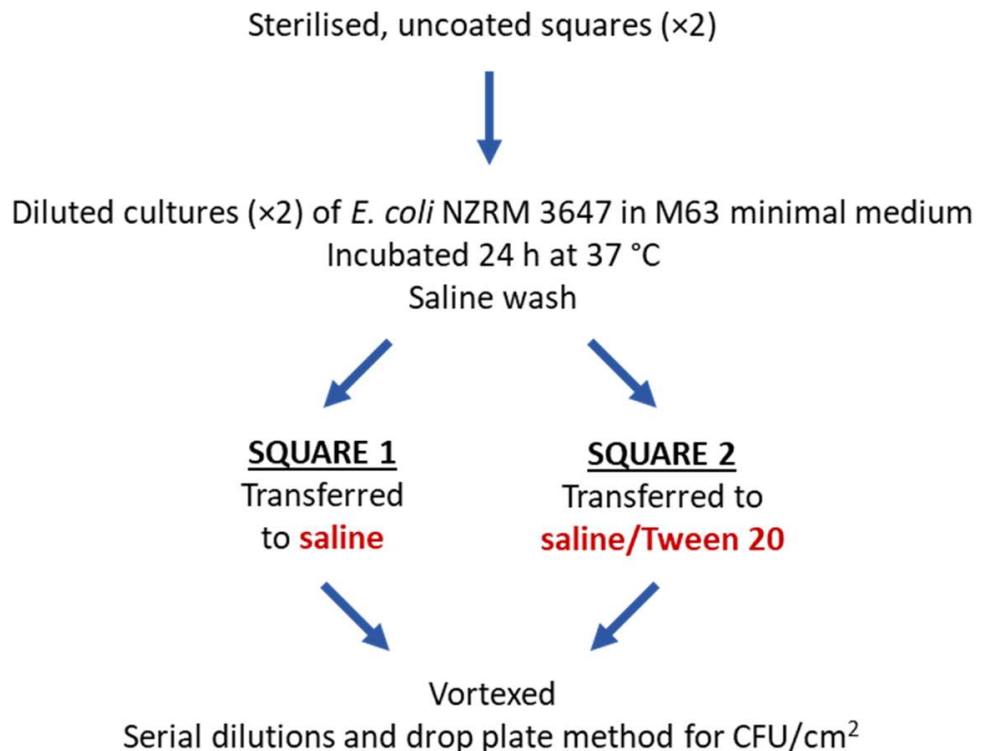

Figure 6.6. Testing the effect of Tween 20 in the bacterial adherence assay.

\subsubsection{Washing Efficiency}

An overnight culture was prepared by inoculating nutrient broth $(3 \mathrm{~mL})$ with a colony of E. coli NZRM 3647 from a nutrient agar plate and incubating for $16 \mathrm{~h}$ at $37^{\circ} \mathrm{C}$ with shaking. One square (23 mm $\times 23 \mathrm{~mm}$ squares) was cut from a vinyl chloride/acetate copolymer (black scrub test panel, Leneta) and sterilised with UV light for $20 \mathrm{~min}$ per side in a laminar flow hood (Figure 6.7). The sterilised square was submerged in overnight culture diluted with M63 minimal medium to $\mathrm{OD}_{600} 0.05$ (final volume $16.5 \mathrm{~mL} ; 5 \pm 2\left(\times 10^{7}\right) \mathrm{CFU} / \mathrm{mL}$ ), and the diluted culture was incubated $24 \mathrm{~h}$ at $37^{\circ} \mathrm{C}$ without shaking. Following incubation, the $\mathrm{OD}_{600}$ of each culture was measured. For the first wash, the square was transferred to sterile saline $(20 \mathrm{~mL})$ for $5 \mathrm{~min}$ to remove planktonic bacteria, and a pipet was used to rinse both sides of the square with sterile saline $(500 \mu \mathrm{L} / \mathrm{side})$. For the second wash, the square was transferred to sterile saline $(20 \mathrm{~mL})$ for $5 \mathrm{~min}$. Then, the square was transferred to a tube containing sterile saline/0.05\% v/v Tween 20 (final volume $20.0401 \mathrm{~mL}$ ), vortexed 2 min at maximum speed, and sonicated $8 \mathrm{~s}$ at $40 \%$ amplitude. Tween 20 (25\% v/v, aq; $40.1 \mu \mathrm{L})$ was added to both wash solutions to bring the final concentration of Tween 20 to $0.05 \% \mathrm{v} / \mathrm{v}$. Serial dilutions (10x) of the first 
and second wash solutions and the detachment solution containing the square were performed to count colonies by the drop plate method. This experiment was repeated for a $\mathrm{Cu}(\mathrm{II})-\mathrm{CnGmSiO} / \mathrm{epx}-$ coated square (Section 6.4.2).

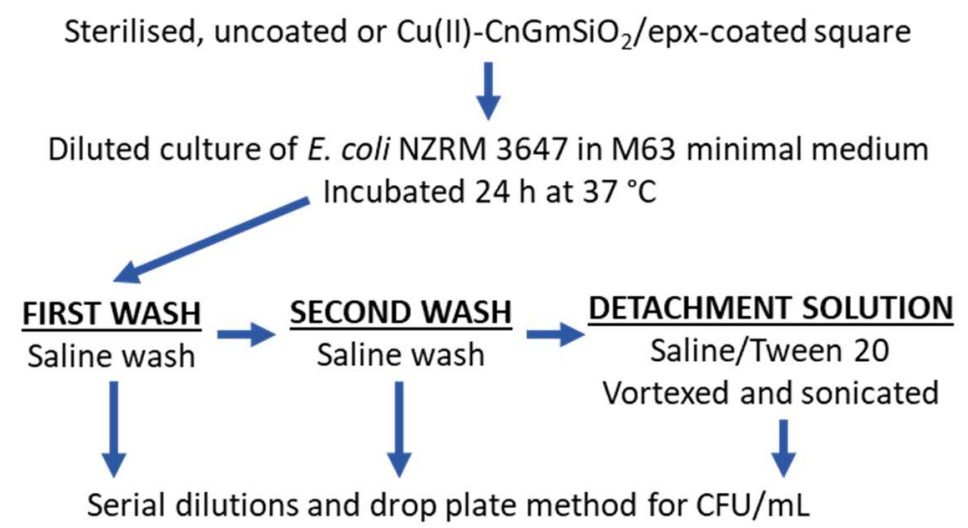

Figure 6.7. Enumeration of bacterial counts (E. coli) in washes.

\subsubsection{Effect of Vortexing and Sonication}

An overnight culture was prepared by inoculating nutrient broth $(3 \mathrm{~mL})$ with a colony of E. coli NZRM 3647 from a nutrient agar plate and incubating for $16 \mathrm{~h}$ at $37^{\circ} \mathrm{C}$ with shaking. A tube containing overnight culture diluted 50x with M63 minimal medium (final volume $25 \mathrm{~mL}$ ) was incubated $24 \mathrm{~h}$ at $37^{\circ} \mathrm{C}$ without shaking (Figure 6.8). Then, the $\mathrm{OD}_{600}$ of the culture was measured, and an aliquot of the culture ( $5 \mathrm{~mL}$ ) was transferred to another tube. The first tube (now containing $20 \mathrm{~mL}$ ) was vortexed for 2 min at maximum speed and sonicated $8 \mathrm{~s}$ at $40 \%$ amplitude. Serial dilutions (10x) of the cultures in both tubes were performed to count colonies by the drop plate method.

\section{Diluted culture of E. coli NZRM 3647 in M63 minimal medium (TUBE 1)} Incubated $24 \mathrm{~h}$ at $37^{\circ} \mathrm{C}$

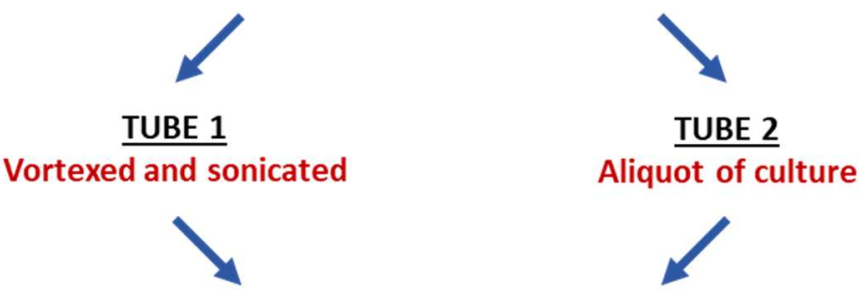

Serial dilutions and drop plate method for $\mathrm{CFU} / \mathrm{mL}$

Figure 6.8. Testing the combined effect of vortexing and sonication on planktonic bacteria. 


\subsubsection{Efficiency of Vortexing and Sonication ( \pm Tween 20)}

\subsection{Uncoated Squares: Tween $20(+)$}

An overnight culture was prepared by inoculating nutrient broth $(3 \mathrm{~mL})$ with a colony of $E$. coli NZRM 3647 from a nutrient agar plate and incubating for $16 \mathrm{~h}$ at $37{ }^{\circ} \mathrm{C}$ with shaking. Three squares $(25 \mathrm{~mm} \times 25 \mathrm{~mm}$ ) cut from a vinyl chloride/acetate copolymer (black scrub test panel, Leneta) and sterilised with $70 \% \mathrm{v} / \mathrm{v}$ aq ethanol were submerged in overnight culture diluted 50× with M63 minimal medium (final volume $16.5 \mathrm{~mL}$ ) in three tubes (one square/tube), and the diluted cultures were incubated $24 \mathrm{~h}$ at $37^{\circ} \mathrm{C}$ without shaking (Figure 6.9). Following incubation, the $\mathrm{OD}_{600}$ of each culture was measured, and each square was transferred to sterile saline $(20 \mathrm{~mL})$ for $5 \mathrm{~min}$ to remove planktonic bacteria. After the saline wash, the squares were transferred to tubes containing sterile saline/0.05\% v/v Tween 20 (final volume $20.040 \mathrm{~mL}$ ). The three tubes received different treatments: 1) the first tube was gently inverted $3 \times$ for the homogeneous distribution of cells ("Control"), 2) the second tube ("Vortex $1 x^{\prime \prime}$ ) was vortexed 2 min at maximum speed, the square was transferred to another tube containing sterile saline/0.05\% v/v Tween 20 (final volume $20.040 \mathrm{~mL}$ ), and then that tube ("Vortex 2x") was vortexed 2 min at maximum speed, and 3) the third tube ("Vortex \& Sonicate") was vortexed $2 \mathrm{~min}$ at maximum speed and sonicated $8 \mathrm{~s}$ at $40 \%$ amplitude. Serial dilutions (10x) of the "Control", "Vortex 1x", "Vortex 2x", and "Vortex \& Sonicate" solutions were performed to count colonies by the drop plate method.

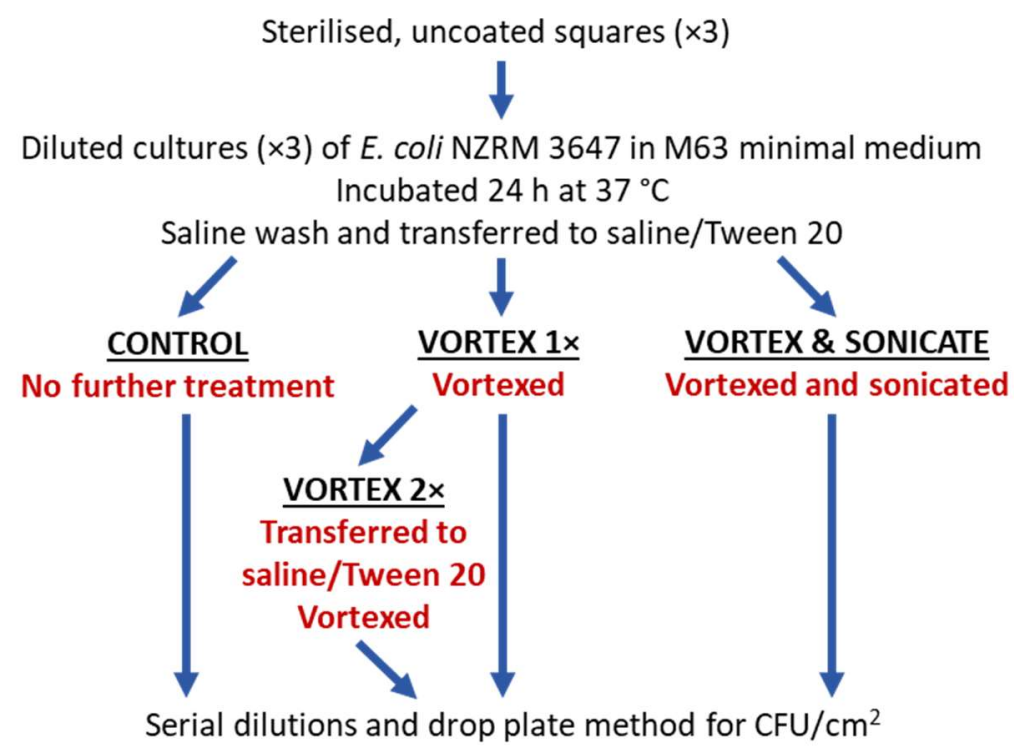

Figure 6.9. Testing the efficiency of vortexing and sonication with Tween 20 in the removal of adherent bacteria from uncoated squares. 


\subsection{Uncoated Squares: Tween $20( \pm)$}

An overnight culture was prepared by inoculating nutrient broth $(3 \mathrm{~mL})$ with a colony of E. coli NZRM 3647 from a nutrient agar plate and incubating for $16 \mathrm{~h}$ at $37{ }^{\circ} \mathrm{C}$ with shaking. Three squares $(23 \mathrm{~mm} \times 23 \mathrm{~mm}$ ) were cut from a vinyl chloride/acetate copolymer (black scrub test panel, Leneta) and sterilised with UV light for 20 min per side in a laminar flow hood (Figure 6.10). Then, each sterilised square was submerged in overnight culture diluted with M63 minimal medium to $\mathrm{OD}_{600} 0.05$ (final volume $16.5 \mathrm{~mL} ; 5 \pm 2\left(\times 10^{7}\right) \mathrm{CFU} / \mathrm{mL}$ ), and the diluted cultures were incubated $24 \mathrm{~h}$ at $37^{\circ} \mathrm{C}$ without shaking. Following incubation, the $\mathrm{OD}_{600}$ of each culture was measured. The squares were transferred to sterile saline $(20 \mathrm{~mL})$ for $5 \mathrm{~min}$ to remove planktonic bacteria, and a pipet was used to rinse both sides of the squares with sterile saline $(500 \mu \mathrm{L} / \mathrm{side})$. Two of the squares were transferred to tubes (one square/tube) containing sterile saline/0.05\% v/v Tween 20 (final volume $20.0401 \mathrm{~mL}$ ), and the last square was transferred to a tube containing sterile saline $(20 \mathrm{~mL})$. One of the Tween 20 solutions was vortexed 2 min at maximum speed and sonicated $8 \mathrm{~s}$ at $40 \%$ amplitude. The other two detachment solutions were gently inverted $3 \times$ for the homogeneous distribution of cells in each solution. Serial dilutions (10x) of all square-containing solutions were performed to count colonies by the drop plate method. The experiment was independently repeated three times.

Sterilised, uncoated squares $(\times 3)$

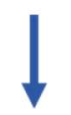

Diluted cultures ( $\times 3$ ) of E. coli NZRM 3647 in M63 minimal medium Incubated $24 \mathrm{~h}$ at $37^{\circ} \mathrm{C}$

Saline washes
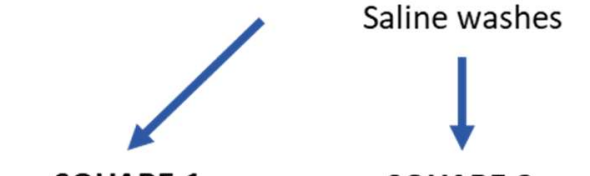

SQUARE 2

$\frac{\text { SQUARE } 1}{\text { Transferred to }}$

saline/Tween 20

Transferred to

saline/Tween 20

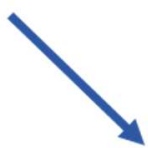

Vortexed and

sonicated

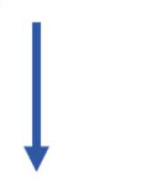

SQUARE 3

Transferred

to saline
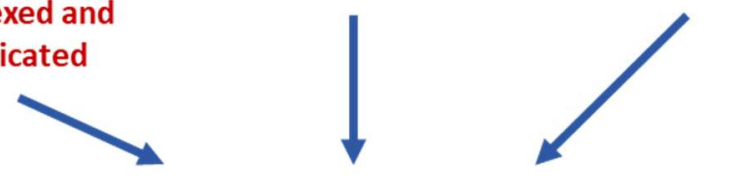

Serial dilutions and drop plate method for $\mathrm{CFU} / \mathrm{cm}^{2}$

Figure 6.10. Testing the efficiency of vortexing and sonication with or without Tween 20 in the removal of adherent bacteria from uncoated squares. 


\subsubsection{3 $\mathrm{SiO}_{2} /$ epx-Coated Squares}

Overnight cultures $(\times 2)$ were prepared by inoculating nutrient broth $(3 \mathrm{~mL})$ with a colony of $E$. coli NZRM 3647 from a nutrient agar plate and incubating for $16 \mathrm{~h}$ at $37{ }^{\circ} \mathrm{C}$ with shaking. Two $\mathrm{SiO}_{2} / \mathrm{epx}-$ coated squares $(23 \mathrm{~mm} \times 23 \mathrm{~mm}$ ) were prepared (Section 6.4.2) and sterilised with UV light for $20 \mathrm{~min}$ per side in a laminar flow hood (Figure 6.11). Then, each sterilised square was submerged in overnight culture diluted with $\mathrm{M} 63$ minimal medium to $\mathrm{OD}_{600} 0.05$ (final volume $16.5 \mathrm{~mL} ; 5 \pm 2\left(\times 10^{7}\right) \mathrm{CFU} / \mathrm{mL}$ ), and the diluted cultures were incubated $24 \mathrm{~h}$ at $37^{\circ} \mathrm{C}$ without shaking. Following incubation, the $\mathrm{OD}_{600}$ of each culture was measured. The squares were transferred to sterile saline $(20 \mathrm{~mL})$ for $5 \mathrm{~min}$ to remove planktonic bacteria, and a pipet was used to rinse both sides of the squares with sterile saline ( $500 \mu \mathrm{L} / \mathrm{side}$ ). One square was transferred to a tube containing sterile saline $/ 0.05 \% \mathrm{v} / \mathrm{v}$ Tween 20 (final volume $20.0401 \mathrm{~mL}$ ), vortexed $2 \mathrm{~min}$ at maximum speed, and sonicated $8 \mathrm{~s}$ at $40 \%$ amplitude. The other square was transferred to a tube containing sterile saline $(20 \mathrm{~mL})$ and gently inverted $3 \times$ for the homogeneous distribution of cells in solution. Serial dilutions (10x) of all square-containing solutions were performed to count colonies by the drop plate method. The experiment was independently repeated three times.

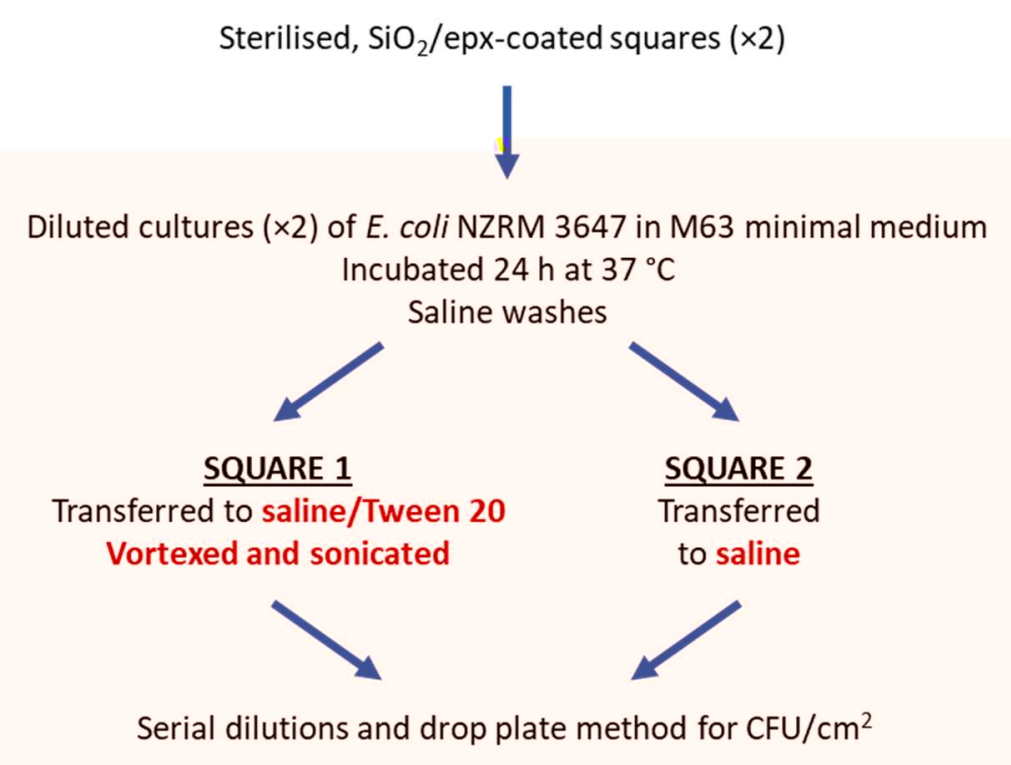

Figure 6.11. Testing the efficiency of vortexing and sonication with or without Tween 20 in the removal of adherent bacteria from $\mathrm{SiO}_{2} /$ epx-coated squares. 


\subsection{Crystal Violet Staining}

The procedure by O'Toole et $a l^{302}$ was utilised for this experiment. An overnight culture was prepared by inoculating nutrient broth $(3 \mathrm{~mL})$ with a colony of $E$. coli NZRM 3647 from a nutrient agar plate and incubating for $16 \mathrm{~h}$ at $37{ }^{\circ} \mathrm{C}$ with shaking. Two squares $(25 \mathrm{~mm} \times 25 \mathrm{~mm})$ cut from a vinyl chloride/acetate copolymer (black scrub test panel, Leneta) and sterilised with $70 \% \mathrm{v} / \mathrm{v}$ aq ethanol were submerged in overnight culture diluted 50x with M63 minimal medium (final volume $16.5 \mathrm{~mL}$ ) in two tubes (one square/tube), and the diluted cultures were incubated $24 \mathrm{~h}$ at $37^{\circ} \mathrm{C}$ without shaking (Figure 6.12). Following incubation, the $\mathrm{OD}_{600}$ of each culture was measured, and each square was transferred to sterile saline $(20 \mathrm{~mL})$ for $5 \mathrm{~min}$ to remove planktonic bacteria. Then, the squares were transferred to tubes containing sterile saline/0.05\% v/v Tween 20 (final volume $20.0401 \mathrm{~mL}$ ). One tube was vortexed $2 \mathrm{~min}$ at maximum speed to detach bacteria from the square. Then, both squares were transferred to $0.005 \% \mathrm{v} / \mathrm{v}$ aq crystal violet $(20 \mathrm{~mL})$ for $15 \mathrm{~min}$, rinsed in sterile water, and transferred to $4: 1 \mathrm{v} / \mathrm{v}$ ethanol:acetone $(20 \mathrm{~mL})$ for $10 \mathrm{~min}$ to de-stain the squares. As a blank, following previous staining procedures, a plastic square unexposed to bacteria was dyed, rinsed, and de-stained. The absorbance of the dye in each de-staining solution was measured at $570 \mathrm{~nm}$.

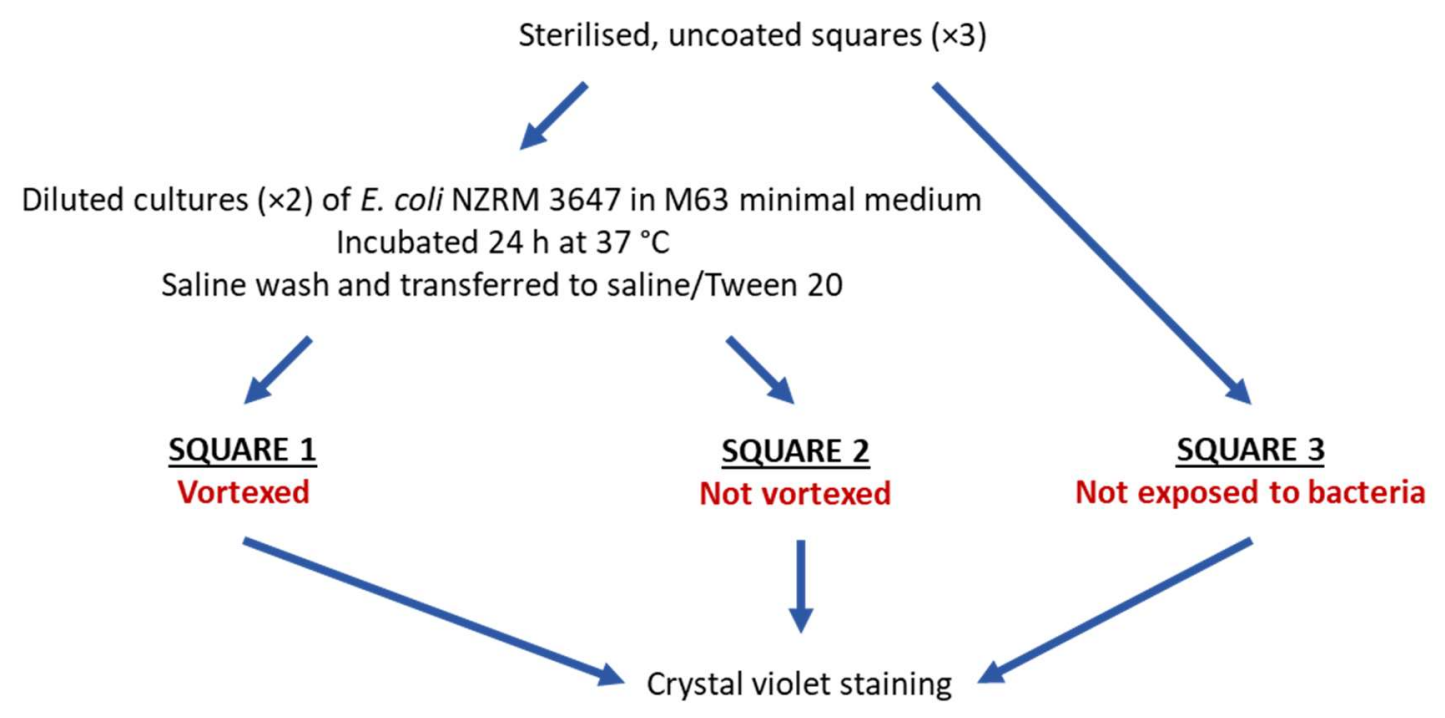

Figure 6.12. Testing the efficiency of vortexing in the removal of adherent bacteria via crystal violet staining. 


\subsubsection{Adherence of $E$. coli NZRM 3647 to $\mathrm{SiO}_{2} / \mathrm{epx}$-Type Coatings}

\subsubsection{Adherence Assay}

Refer to Figure 6.3. The adherence of E. coli NZRM 3647 to the $\mathrm{SiO}_{2} /$ epx-type samples from Section 6.4.2 was tested. An uncoated sample square and sample squares coated with the commercial epoxy resin and marine paint, as in Section 6.5.1.2, were also tested. An overnight culture was prepared by inoculating nutrient broth $(3 \mathrm{~mL})$ with a colony of $E$. coli NZRM 3647 from a nutrient agar plate and incubating for $16 \mathrm{~h}$ at $37^{\circ} \mathrm{C}$ with shaking. The squares were sterilised with UV light for $20 \mathrm{~min}$ per side in a laminar flow hood, and then each of the sterilised squares were submerged in overnight culture diluted with $\mathrm{M} 63$ minimal medium to $\mathrm{OD}_{600} 0.05$ (final volume $16.5 \mathrm{~mL} ; 5 \pm 2\left(\times 10^{7}\right) \mathrm{CFU} / \mathrm{mL}$ ). The diluted cultures were incubated $24 \mathrm{~h}$ at $37^{\circ} \mathrm{C}$ without shaking. Following incubation, the $\mathrm{OD}_{600}$ of each culture was measured, and the squares were transferred to sterile saline $(20 \mathrm{~mL})$ for $5 \mathrm{~min}$ to remove planktonic bacteria. A pipet was used to rinse both sides of the squares with sterile saline ( $500 \mu \mathrm{L} / \mathrm{side}$ ) before transferring the squares to tubes containing sterile solutions of $0.05 \% \mathrm{v} / \mathrm{v}$ Tween 20 in saline (final volume $20.0401 \mathrm{~mL}$ ). These solutions were vortexed 2 min at maximum speed and sonicated $8 \mathrm{~s}$ at $40 \%$ amplitude to detach bacteria from the squares. Serial dilutions $(10 \times)$ of the solutions were performed to count colonies by the drop plate method. The experiment was independently repeated three times.

\subsubsection{Copper-Leaching Experiments}

The amount of copper leaching out of the copper coatings into the liquid medium during the $24 \mathrm{~h}$ incubation period was measured both in the absence and presence of E. coli NZRM 3647. Squares were coated with the commercial marine paint (Altex Yacht and Boat Paint, Aurora Red, Ablative Antifouling No. 5), as in Section 6.5.1.2, and with Batch $2 \mathrm{Cu}(\mathrm{II})-\mathrm{CnGmSiO}_{2} / \mathrm{epx}$ and Batch $4 \mathrm{CnGmSiO}_{2}$ $+\mathrm{Cu}(I I) / \mathrm{epx}$, as in Section 6.4.2.

\subsection{E. coli Absent}

Coated squares $(23 \mathrm{~mm} \times 23 \mathrm{~mm}$ ) were sterilised by exposure of each side to UV light for $20 \mathrm{~min}$ in a laminar flow hood and were submerged in $\mathrm{M} 63$ minimal medium $(16.5 \mathrm{~mL})$ at $37^{\circ} \mathrm{C}$ without shaking. After $24 \mathrm{~h}$, the squares were removed. In order to quantify the copper in the liquid medium via ICP-MS, 
the solutions were diluted $10,000-25,000 \times$ with $3 \% \mathrm{v} / \mathrm{v}$ nitric acid (aq) to bring the copper concentration within the range of the standards. M63 minimal medium served as a blank. The experiment was independently repeated three times.

ICP-MS: Marine Paint - $3.3 \pm 0.5$ ppm Cu; Batch $2 \mathrm{Cu}(I I)-C n G m S i O_{2} / e p x-3.6 \pm 0.3$ ppm Cu; Batch 4 $\mathrm{CnGmSiO}+\mathrm{Cu}(\mathrm{II}) / \mathrm{epx}-2.3 \pm 0.1 \mathrm{ppm} \mathrm{Cu}$.

\subsection{E. coli Present}

An overnight culture was prepared by inoculating nutrient broth $(3 \mathrm{~mL})$ with a colony of $E$. coli NZRM 3647 from a nutrient agar plate and incubating for $16 \mathrm{~h}$ at $37^{\circ} \mathrm{C}$ with shaking. Coated squares (23 $\mathrm{mm} \times 23 \mathrm{~mm}$ ) were submerged in overnight culture diluted with M63 minimal medium to $\mathrm{OD}_{600} 0.05$ (final volume $16.5 \mathrm{~mL} ; 5 \pm 2\left(\times 10^{7}\right) \mathrm{CFU} / \mathrm{mL}$ ) for $24 \mathrm{~h}$ at $37^{\circ} \mathrm{C}$ without shaking. Following incubation, the cultures were filter-sterilised $\left(0.2 \mu \mathrm{m}\right.$ pore size, Sartorius Minisart $\left.{ }^{\circledR}\right)$ and centrifuged (10 min, 3000 RCF). The supernatant was submitted for copper analysis via ICP-MS and diluted $1,000-50,000 \times$ with $3 \% \mathrm{v} / \mathrm{v}$ nitric acid (aq) to bring the copper concentration within the range of the standards. M63 minimal medium served as a blank. The experiment was independently repeated three times.

ICP-MS: Marine Paint - 7.3 \pm 0.4 ppm Cu; Batch $2 \mathrm{Cu}(I I)-C n G m S i O_{2} / e p x-6.0 \pm 0.4$ ppm Cu; Batch 4 $\mathrm{CnGmSiO}+\mathrm{Cu}(\mathrm{II}) / \mathrm{epx}-12 \pm 2 \mathrm{ppm} \mathrm{Cu}$.

\subsubsection{Growth of E. coli \pm Shaking}

An overnight culture was prepared by inoculating nutrient broth $(3 \mathrm{~mL})$ with a colony of E. coli NZRM 3647 from a nutrient agar plate and incubating for $16 \mathrm{~h}$ at $37{ }^{\circ} \mathrm{C}$ with shaking. Two squares $(23 \mathrm{~mm} \times 23 \mathrm{~mm}$ ) were cut from a vinyl chloride/acetate copolymer (black scrub test panel, Leneta) and sterilised with UV light for 20 min per side in a laminar flow hood (Figure 6.13). Then, each sterilised square was submerged in overnight culture diluted with $\mathrm{M} 63$ minimal medium to $\mathrm{OD}_{600} 0.05$ (final volume $16.5 \mathrm{~mL} ; 5 \pm 2\left(\times 10^{7}\right) \mathrm{CFU} / \mathrm{mL}$ ), and the diluted cultures were incubated $24 \mathrm{~h}$ at $37^{\circ} \mathrm{C}-$ one without shaking and the other with shaking. To prepare two other cultures without squares, the overnight culture was diluted with M63 minimal medium to $\mathrm{OD}_{600} 0.05$ (final volume $16.5 \mathrm{~mL}$; $\left.5 \pm 2\left(\times 10^{7}\right) \mathrm{CFU} / \mathrm{mL}\right)$, and the diluted cultures were incubated $24 \mathrm{~h}$ at $37^{\circ} \mathrm{C}$ - one without shaking and the other with shaking. Following incubation, the $\mathrm{OD}_{600}$ of each culture was measured. 


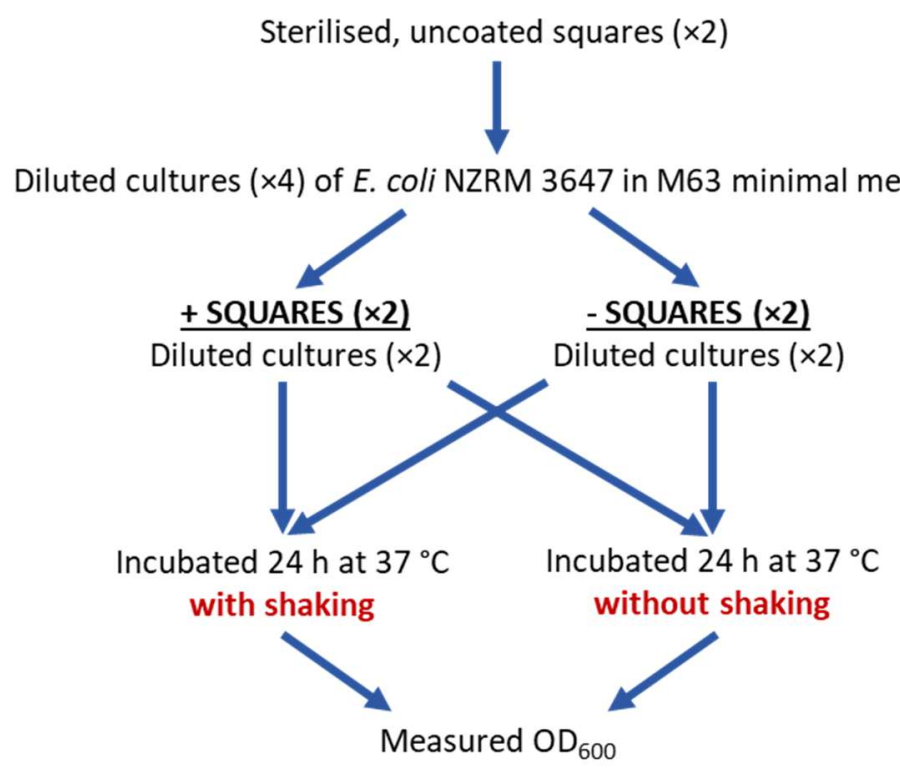

Figure 6.13. Testing the growth of E. coli NZRM 3647 with and without shaking and in the presence or absence of an uncoated square.

\subsubsection{Adherence Assay: V. harveyi NZRM 2698}

\subsubsection{Effects of Vortexing, Sonication, and Tween 20 on Planktonic Bacteria}

An overnight culture was prepared by inoculating Luria marine broth $(10 \mathrm{~mL})$ with a colony of V. harveyi NZRM 2698 from a Luria marine agar plate and incubating for $16 \mathrm{~h}$ at $30{ }^{\circ} \mathrm{C}$ with shaking. Then, in a sterile flask, the overnight culture was diluted to $\mathrm{OD}_{600} 0.05$ with Luria marine broth (final volume $100 \mathrm{~mL} ; 1.4 \pm 0.3\left(\times 10^{8}\right) \mathrm{CFU} / \mathrm{mL}$ ) and incubated $24 \mathrm{~h}$ at $30^{\circ} \mathrm{C}$ with shaking (Figure 6.14). After $24 \mathrm{~h}$, the $O \mathrm{DD}_{600}$ of the culture was measured, and five aliquots $(20 \mathrm{~mL})$ were transferred to five different tubes:

1) The first tube was vortexed for 2 min at maximum speed.

2) The second tube was sonicated for $8 \mathrm{~s}$ at $40 \%$ amplitude $(\sim 0.2 \mathrm{~kJ})$.

3) Tween 20 ( $25 \% \mathrm{v} / \mathrm{v}$, aq; $40.1 \mathrm{uL}$ ) was added to the third tube and gently mixed by inversion, yielding a final concentration of $0.05 \% \mathrm{v} / \mathrm{v}$ Tween 20 .

4) Tween $20(25 \% \mathrm{v} / \mathrm{v}$, aq; $40.1 \mathrm{uL})$ was added to the fourth tube, yielding a final concentration of $0.05 \% \mathrm{v} / \mathrm{v}$ Tween 20 . This tube was then vortexed for $2 \mathrm{~min}$ at maximum speed and sonicated for $8 \mathrm{~s}$ at $40 \%$ amplitude ( $0.2 \mathrm{~kJ})$. 
5) The fifth tube served as a control and was only gently mixed by inversion prior to serial dilutions.

Serial dilutions $(10 x)$ of the cultures in all five tubes were performed to count colonies by the drop plate method.

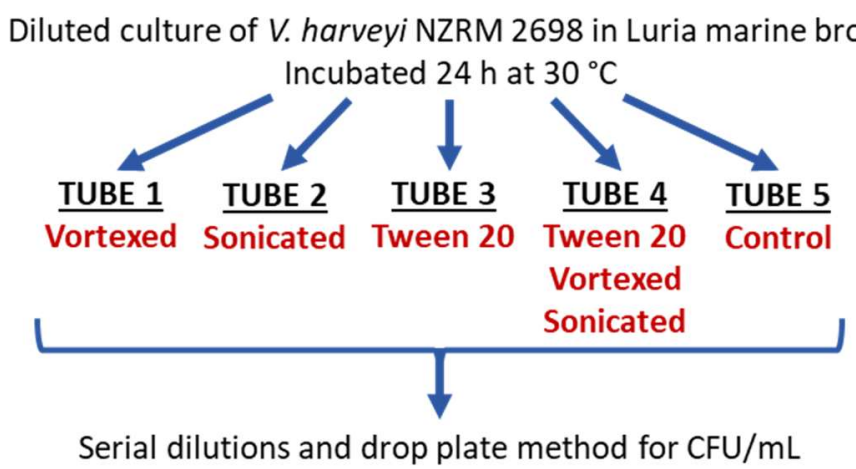

Figure 6.14. Testing the effects of vortexing, sonication, and Tween 20 on planktonic bacteria.

\subsubsection{Checking for Cells Clumping}

An overnight culture was prepared by inoculating Luria marine broth $(3 \mathrm{~mL})$ with a colony of $V$. harveyi NZRM 2698 from a Luria marine agar plate and incubating for $16 \mathrm{~h}$ at $30^{\circ} \mathrm{C}$ with shaking. The overnight culture was checked for clumping cells via Gram staining, as in Section 6.1.8. Then, Tween 20 (25\% v/v, aq) was added to the culture and mixed, yielding a final concentration of $0.05 \% \mathrm{v} / \mathrm{v}$ Tween 20 , and this culture was also checked for clumping cells via Gram staining.

\subsubsection{Effect of Tween 20 in Adherence Assay (2\% vs 0.85\% w/w NaCl)}

An overnight culture was prepared by inoculating Luria marine broth $(3 \mathrm{~mL})$ with a colony of $V$. harveyi NZRM 2698 from a Luria marine agar plate and incubating for $16 \mathrm{~h}$ at $30{ }^{\circ} \mathrm{C}$ with shaking. Four $\mathrm{CnGmSiO}_{2}$ /epx-coated squares ( $23 \mathrm{~mm} \times 23 \mathrm{~mm}$ ) were prepared, as in Section 6.4.2, and sterilised with UV light for 20 min per side in a laminar flow hood (Figure 6.15). Two of the sterilised squares ("-Tween 20") were submerged in overnight culture diluted with Luria marine broth to $\mathrm{OD}_{600} 0.05$ (final volume $\left.16.5 \mathrm{~mL} ; 1.4 \pm 0.3\left(\times 10^{8}\right) \mathrm{CFU} / \mathrm{mL}\right)$. Tween $20(25 \% \mathrm{v} / \mathrm{v}$, aq) was added to the remaining overnight culture and mixed, yielding a final concentration of $0.05 \% \mathrm{v} / \mathrm{v}$ Tween 20 . The other two sterilised squares ("+Tween 20") were submerged in $0.05 \% \mathrm{v} / \mathrm{v}$ Tween $20 /$ overnight culture diluted 
with Luria marine broth to $\mathrm{OD}_{600} 0.05$ (final volume $16.5 \mathrm{~mL}$ ). All of the diluted cultures were incubated $24 \mathrm{~h}$ at $30^{\circ} \mathrm{C}$ with shaking. Following incubation, the $\mathrm{OD}_{600}$ of each culture was measured. Two squares (“0.85\% $\mathrm{NaCl}$ ") - one "+Tween 20 " and one "-Tween 20" - were transferred to sterile saline $(0.85 \% \mathrm{w} / \mathrm{w} \mathrm{NaCl}$, aq; $20 \mathrm{~mL})$ for $5 \mathrm{~min}$ to remove planktonic bacteria, and a pipet was used to rinse both sides of the squares with sterile saline $(500 \mu \mathrm{L} /$ side). The other two squares (" $2 \% \mathrm{NaCl}$ ") were transferred to sterile $2 \% \mathrm{w} / \mathrm{w}$ aq sodium chloride $(20 \mathrm{~mL})$ for $5 \mathrm{~min}$ to remove planktonic bacteria, and a pipet was used to rinse both sides of the squares with sterile $2 \% \mathrm{w} / \mathrm{w}$ aq sodium chloride $(500 \mu \mathrm{L} / \mathrm{side})$. Then, the four squares were transferred to the following solutions for the detachment of adherent bacteria:

1) “ $0.85 \% \mathrm{NaCl}$, + Tween 20 ”: sterile saline/0.05\% Tween $20(20 \mathrm{~mL})$,

2) “ $0.85 \% \mathrm{NaCl}$, -Tween 20 ”: sterile saline $(20 \mathrm{~mL})$,

3) “ $2 \% \mathrm{NaCl}$, + Tween 20 ": sterile $2 \% \mathrm{w} / \mathrm{w}$ aq sodium chloride/0.05\% v/v Tween $20(20 \mathrm{~mL})$, and

4) “ $2 \% \mathrm{NaCl}$, -Tween 20 ": sterile $2 \% \mathrm{w} / \mathrm{w}$ aq sodium chloride $(20 \mathrm{~mL})$.

The detachment solutions were vortexed 2 min at maximum speed. Serial dilutions (10x) of the detachment solutions were performed using the appropriate diluent (as above) to count colonies by the drop plate method.

Sterilised, $\mathrm{CnGmSiO}_{2}$ /epx-coated squares ( $\left.\times 4\right)$

$\underline{0.85 \% ~ N a C l},+$ Tween 20

Transferred to diluted culture of $V$. harveyi NZRM 2698 in Luria marine broth/Tween 20 Incubated $24 \mathrm{~h}$ at $30^{\circ} \mathrm{C}$

$0.85 \% \mathrm{NaCl}$ washes

Transferred to $0.85 \% \mathrm{NaCl} /$ Tween 20

$0.85 \% \mathrm{NaCl}$, -Tween 20

Transferred to diluted culture of $V$. harveyi

NZRM 2698 in Luria marine broth Incubated $24 \mathrm{~h}$ at $30^{\circ} \mathrm{C}$

$0.85 \% \mathrm{NaCl}$ washes Transferred to $0.85 \% \mathrm{NaCl}$

\section{$2 \% \mathrm{NaCl}$, +Tween 20}

Transferred to diluted culture of $\mathrm{V}$. harveyi NZRM 2698 in Luria marine broth/Tween 20 Incubated $24 \mathrm{~h}$ at $30^{\circ} \mathrm{C}$ $2 \% \mathrm{NaCl}$ washes Transferred to $2 \% \mathrm{NaCl} /$ Tween 20

$\underline{2 \%} \mathrm{NaCl}$, -Tween 20

Transferred to diluted culture of $V$. harveyi NZRM 2698 in Luria marine broth Incubated $24 \mathrm{~h}$ at $30^{\circ} \mathrm{C}$ $2 \% \mathrm{NaCl}$ washes Transferred to $2 \% \mathrm{NaCl}$

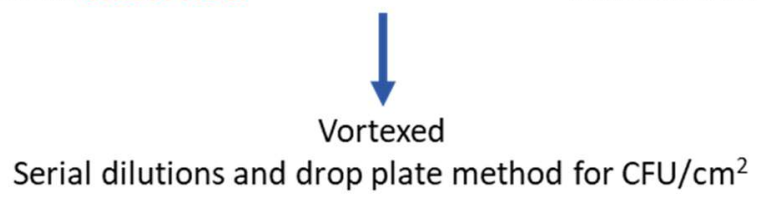

Figure 6.15. Testing the effect of Tween 20 in the adherence assay and comparing the use of saline $v s$ $2 \% \mathrm{w} / \mathrm{w}$ aq $\mathrm{NaCl}$ for the washes, detachment solution, and the diluent. 


\subsubsection{Duration of Incubation and Efficiency of Vortexing and}

\section{Sonication}

An overnight culture was prepared by inoculating Luria marine broth $(3 \mathrm{~mL})$ with a colony of $V$. harveyi NZRM 2698 from a Luria marine agar plate and incubating for $16 \mathrm{~h}$ at $30^{\circ} \mathrm{C}$ with shaking. Four $\mathrm{SiO}_{2} / \mathrm{epx}-$ coated samples (11.5 $\mathrm{mm} \times 23 \mathrm{~mm}$ ) were prepared, as in Section 6.4.2, and sterilised with UV light for 20 min per side in a laminar flow hood (Figure 6.16). The sterilised samples were submerged in overnight culture diluted to $\mathrm{OD}_{600} 0.05$ with Luria marine broth (final volume $16.5 \mathrm{~mL} ; 1.4 \pm 0.3\left(\times 10^{8}\right)$ $\mathrm{CFU} / \mathrm{mL}$ ) in four tubes (one square/tube), and the diluted cultures were incubated at $30{ }^{\circ} \mathrm{C}$ with shaking. The different samples were treated as follows:

1) Sample 1 was incubated for $24 \mathrm{~h}$ at $30{ }^{\circ} \mathrm{C}$ with shaking. After incubation, the $O D_{600}$ of the culture was measured, and the sample was transferred to $2 \% \mathrm{w} / \mathrm{w}$ aq sodium chloride $(20 \mathrm{~mL})$ for $5 \mathrm{~min}$ to remove planktonic bacteria. A pipet was used to rinse both sides of the sample with sterile $2 \% \mathrm{w} / \mathrm{w}$ aq sodium chloride before transferring it to a tube containing sterile $2 \% \mathrm{w} / \mathrm{w}$ sodium chloride $(20 \mathrm{~mL})$. This tube was vortexed 2 min at maximum speed and sonicated $8 \mathrm{~s}$ at $40 \%$ amplitude $(\sim 0.2 \mathrm{~kJ}$ ) to detach bacteria from the sample.

2) Sample 2 was incubated for $48 \mathrm{~h}$ at $30{ }^{\circ} \mathrm{C}$ with shaking. After incubation, the $\mathrm{OD}_{600}$ of the culture was measured, and the sample was transferred to sterile $2 \% \mathrm{w} / \mathrm{w}$ aq sodium chloride $(20 \mathrm{~mL})$ for $5 \mathrm{~min}$ to remove planktonic bacteria. A pipet was used to rinse both sides of the sample with sterile $2 \% \mathrm{w} / \mathrm{w}$ aq sodium chloride before transferring it to a tube containing sterile $2 \% \mathrm{w} / \mathrm{w}$ aq sodium chloride $(20 \mathrm{~mL})$. This tube was vortexed 2 min at maximum speed and sonicated $8 \mathrm{~s}$ at $40 \%$ amplitude $(\sim 0.2 \mathrm{~kJ})$ to detach bacteria from the sample.

3) Sample 3 was incubated for $48 \mathrm{~h}$ at $30{ }^{\circ} \mathrm{C}$ with shaking. After incubation, the $\mathrm{OD}_{600}$ of the culture was measured, and the sample was transferred to sterile $2 \% \mathrm{w} / \mathrm{w}$ aq sodium chloride $(20 \mathrm{~mL})$ for $5 \mathrm{~min}$ to remove planktonic bacteria. A pipet was used to rinse both sides of the sample with sterile $2 \% \mathrm{w} / \mathrm{w}$ aq sodium chloride before transferring it to a tube containing sterile $2 \% \mathrm{w} / \mathrm{w}$ aq sodium chloride $(20 \mathrm{~mL})$. The contents of the tube were gently mixed by inversion prior to serial dilutions.

4) Sample 4 was incubated for $48 \mathrm{~h}$ at $30{ }^{\circ} \mathrm{C}$ with shaking. After incubation, the $O D_{600}$ of the culture was measured, and the sample was transferred to sterile $2 \% \mathrm{w} / \mathrm{w}$ aq sodium chloride $(20 \mathrm{~mL})$ for $5 \mathrm{~min}$ to remove planktonic bacteria. A pipet was used to rinse both sides of the sample with sterile $2 \% \mathrm{w} / \mathrm{w}$ aq sodium chloride before transferring it to a tube containing sterile $2 \% \mathrm{w} / \mathrm{w}$ aq sodium chloride $(20 \mathrm{~mL})$. This tube was vortexed 2 min at maximum speed ("Sample $4, \times 1$ "). Then, the sample was transferred to another tube containing sterile $2 \% \mathrm{w} / \mathrm{w}$ 
aq sodium chloride $(20 \mathrm{~mL})$, and this tube was also vortexed 2 min at maximum speed ("Sample 4, ×2").

Serial dilutions (10x) of the "Sample 1", "Sample 2", "Sample 3", "Sample 4, $\times 1$ ", and "Sample 4, ×2" detachment solutions were performed to count colonies by the drop plate method.

Sterilised, $\mathrm{SiO}_{2} /$ epx-coated samples ( $\times 4$ )

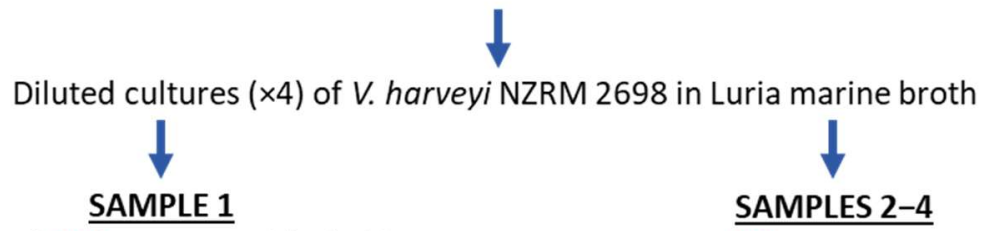

Incubated $24 \mathrm{~h}$ at $30^{\circ} \mathrm{C}$ with shaking

Incubated $48 \mathrm{~h}$ at $30^{\circ} \mathrm{C}$ with shaking

$2 \% \mathrm{NaCl}$ washes and transferred to $2 \% \mathrm{NaCl} 2 \% \mathrm{NaCl}$ washes and transferred to $2 \% \mathrm{NaCl}$

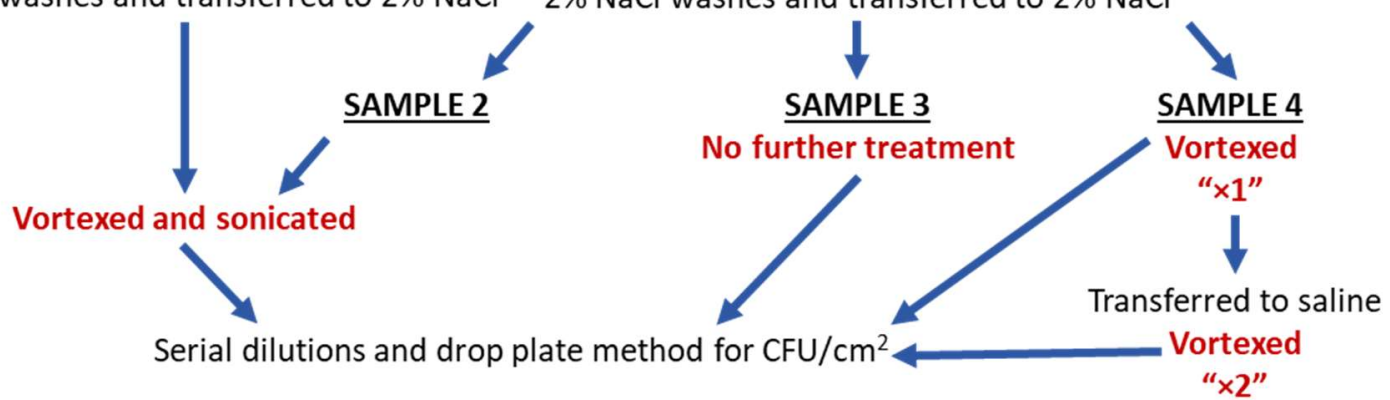

Figure 6.16. Testing the duration of incubation and the efficiency of vortexing and sonication in the removal of adherent bacteria.

\subsubsection{Enumeration of Cells in Washes}

An overnight culture was prepared by inoculating Luria marine broth $(3 \mathrm{~mL})$ with a colony of $V$. harveyi NZRM 2698 from a Luria marine agar plate and incubating for $16 \mathrm{~h}$ at $30^{\circ} \mathrm{C}$ with shaking. One $\mathrm{CnGmSiO}_{2} /$ epx-coated square $(23 \mathrm{~mm} \times 23 \mathrm{~mm})$ was prepared, as in Section 6.4.2, and sterilised with UV light for 20 min per side in a laminar flow hood (Figure 6.17). The sterilised square was submerged in overnight culture diluted with Luria marine broth to $\mathrm{OD}_{600} 0.05$ (final volume $16.5 \mathrm{~mL} ; 1.4 \pm 0.3$ $\left.\left(\times 10^{8}\right) \mathrm{CFU} / \mathrm{mL}\right)$, and the diluted culture was incubated $24 \mathrm{~h}$ at $30{ }^{\circ} \mathrm{C}$ with shaking. Following incubation, the $\mathrm{OD}_{600}$ of the culture was measured. For the first wash, the square was transferred to sterile $2 \% \mathrm{w} / \mathrm{w}$ aq sodium chloride $(20 \mathrm{~mL}$ ) for $5 \mathrm{~min}$ to remove planktonic bacteria, and a pipet was used to rinse both sides of the square with sterile $2 \% \mathrm{w} / \mathrm{w}$ aq sodium chloride ( $500 \mu \mathrm{L} / \mathrm{side}$ ). For the second wash, the square was transferred to sterile $2 \% \mathrm{w} / \mathrm{w}$ aq sodium chloride $(20 \mathrm{~mL})$ for $5 \mathrm{~min}$. Then, the square was transferred to another tube containing sterile $2 \% \mathrm{w} / \mathrm{w}$ aq sodium chloride 
$(20 \mathrm{~mL})$ and vortexed $2 \mathrm{~min}$ at maximum speed. Both wash solutions were vortexed, and serial dilutions (10x) of the first and second wash solutions and the detachment solution containing the square were performed to count colonies by the drop plate method.

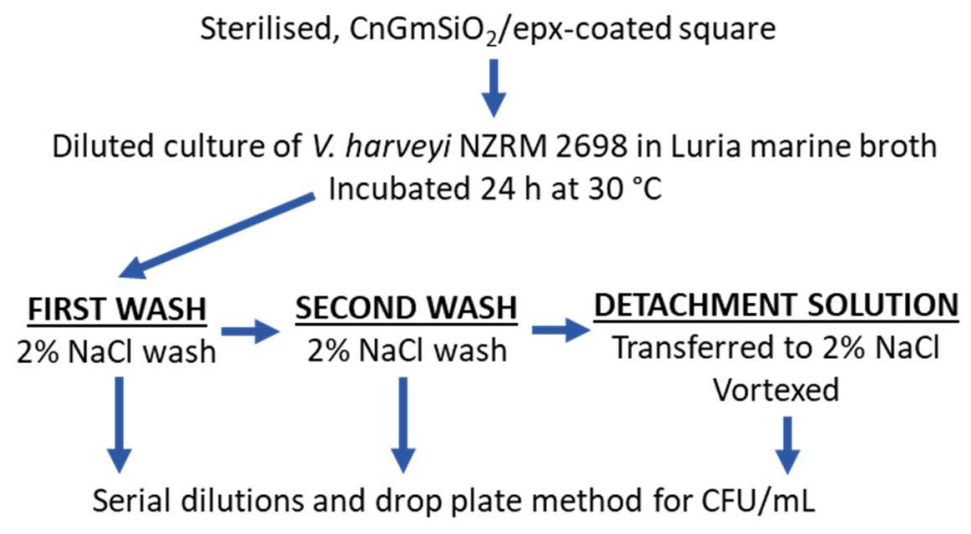

Figure 6.17. Enumeration of bacterial counts (V. harveyi) in washes.

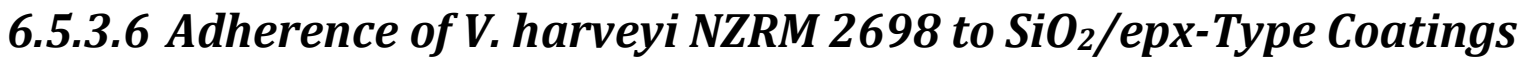

The adherence of $V$. harveyi NZRM 2698 to the $\mathrm{SiO}_{2}$ /epx-type coatings from Section 6.4.2 was tested. An uncoated sample square and sample squares coated with the commercial epoxy resin and marine paint, as in Section 6.5.1.2, were also tested. An overnight culture was prepared by inoculating Luria marine broth $(3 \mathrm{~mL})$ with a colony of $V$. harveyi NZRM 2698 from a Luria marine agar plate and incubating for $16 \mathrm{~h}$ at $30^{\circ} \mathrm{C}$ with shaking. The squares were sterilised with UV light for $20 \mathrm{~min}$ per side in a laminar flow hood, and then each of the sterilised squares were submerged in overnight culture diluted with Luria marine broth to $\mathrm{OD}_{600} 0.05$ (final volume $16.5 \mathrm{~mL} ; 1.4 \pm 0.3\left(\times 10^{8}\right) \mathrm{CFU} / \mathrm{mL}$ ). The diluted cultures were incubated $24 \mathrm{~h}$ at $30^{\circ} \mathrm{C}$ with shaking. Following incubation, the $\mathrm{OD}_{600}$ of each culture was measured, and the squares were transferred to sterile $2 \% \mathrm{w} / \mathrm{w}$ aq sodium chloride $(20 \mathrm{~mL})$ for $5 \mathrm{~min}$ to remove planktonic bacteria. A pipet was used to rinse both sides of the squares with sterile $2 \% \mathrm{w} / \mathrm{w}$ aq sodium chloride $(500 \mu \mathrm{L} / \mathrm{side})$ before transferring the squares to new tubes containing sterile $2 \% \mathrm{w} / \mathrm{w}$ aq sodium chloride $(20 \mathrm{~mL})$. These solutions were vortexed $2 \mathrm{~min}$ at maximum speed and serially-diluted $(10 x)$ to count colonies by the drop plate method. The experiment was independently repeated three times. 


\section{REFERENCES}

1. Bighiu, M. A., Eriksson-Wiklund, A.-K. \& Eklund, B. Biofouling of leisure boats as a source of metal pollution. Environ. Sci. Pollut. Res. 24, 997-1006 (2017).

2. Gittens, J. E., Smith, T. J., Suleiman, R. \& Akid, R. Current and emerging environmentallyfriendly systems for fouling control in the marine environment. Biotechnol. Adv. 31, 1738-1753 (2013).

3. Almeida, E., Diamantino, T. C. \& de Sousa, O. Marine paints: the particular case of antifouling paints. Prog. Org. Coatings 59, 2-20 (2007).

4. Callow, M. E. \& Callow, J. A. Marine biofouling: a sticky problem. Biologist 49, 1-5 (2002).

5. Davidson, I. et al. Mini-review: assessing the drivers of ship biofouling management - aligning industry and biosecurity goals. Biofouling 32, 411-428 (2016).

6. Townsin, R. L. The ship hull fouling penalty. Biofouling 19, 9-15 (2003).

7. Martín-Rodríguez, A. J. et al. From broad-spectrum biocides to quorum sensing disruptors and mussel repellents: antifouling profile of alkyl triphenylphosphonium salts. PLoS One 10, 1-30 (2015).

8. Amara, I., Miled, W., Slama, R. B. \& Ladhari, N. Antifouling processes and toxicity effects of antifouling paints on marine environment. A review. Environ. Toxicol. Pharmacol. 57, 115-130 (2018).

9. Qian, P. Y., Lau, S. C. K., Dahms, H. U., Dobretsov, S. \& Harder, T. Marine biofilms as mediators of colonization by marine macroorganisms: implications for antifouling and aquaculture. Mar. Biotechnol. 9, 399-410 (2007).

10. Flemming, H.-C. Why microorganisms live in biofilms and the problem of biofouling. in Marine and Industrial Biofouling (eds. Flemming, H.-C., Sriyutha Murthy, P., Venkatesan, R. \& Cooksey, K. E.) 3-12 (Spring-Verlag Berlin Heidelberg, 2009). doi:10.1007/978-3-540-69796-1

11. Flemming, H.-C. Biofouling and me: my Stockholm syndrome with biofilms. Water Res. 173, 115 (2020).

12. Dang, H. \& Lovell, C. R. Microbial surface colonization and biofilm development in marine environments. Microbiol. Mol. Biol. Rev. 80, 91-138 (2016).

13. Fusetani, N. Biofouling and antifouling. Nat. Prod. Rep. 21, 94-104 (2004).

14. Burgess, J. G. et al. The development of a marine natural product-based antifouling paint. Biofouling 19 Suppl, 197-205 (2003).

15. Callow, J. A. \& Callow, M. E. Trends in the development of environmentally friendly foulingresistant marine coatings. Nat. Commun. 2, 244 (2011). 
16. Lindholdt, A., Dam-Johansen, K., Olsen, S. M., Yebra, D. M. \& Kiil, S. Effects of biofouling development on drag forces of hull coatings for ocean-going ships: a review. J. Coatings Technol. Res. 12, 415-444 (2015).

17. Al-Naamani, L., Dobretsov, S., Dutta, J. \& Burgess, J. G. Chitosan-zinc oxide nanocomposite coatings for the prevention of marine biofouling. Chemosphere 168, 408-417 (2017).

18. Schultz, M. P. Effects of coating roughness and biofouling on ship resistance and powering. Biofouling 23, 331-41 (2007).

19. Schultz, M. P., Bendick, J. A., Holm, E. R. \& Hertel, W. M. Economic impact of biofouling on a naval surface ship. Biofouling 27, 87-98 (2011).

20. Carve, M., Scardino, A. \& Shimeta, J. Effects of surface texture and interrelated properties on marine biofouling: a systematic review. Biofouling 35, 597-617 (2019).

21. Boren, T. Eco-efficiency: a new tool to support better decisions on hull coating investments. (2010).

22. Buskens, P., Wouters, M., Rentrop, C. \& Vroon, Z. A brief review of environmentally benign antifouling and foul-release coatings for marine applications. J. Coat. Technol. Res. 10, 29-36 (2013).

23. Dafforn, K. A., Lewis, J. A. \& Johnston, E. L. Antifouling strategies: history and regulation, ecological impacts and mitigation. Mar. Pollut. Bull. 62, 453-465 (2011).

24. Hopkins, G. A. \& Forrest, B. M. A preliminary assessment of biofouling and non-indigenous marine species associated with commercial slow-moving vessels arriving in New Zealand. Biofouling 26, 613-621 (2010).

25. Coutts, A. D. M. \& Taylor, M. D. A preliminary investigation of biosecurity risks associated with biofouling on merchant vessels in New Zealand. New Zeal. J. Mar. Freshw. Res. 38, 215-229 (2004).

26. Coutts, A. D. M. \& Forrest, B. M. Development and application of tools for incursion response: lessons learned from the management of the fouling pest Didemnum vexillum. J. Exp. Mar. Bio. Ecol. 342, 154-162 (2007).

27. Hole, W. Marine Fouling and Its Prevention, Contribution No. 580 from the Woods Hole Oceanographic Institution. (United States Naval Institute, 1952). doi:10.1575/1912/191

28. Yebra, D. M., Kiil, S. \& Dam-Johansen, K. Antifouling technology-past, present and future steps towards efficient and environmentally friendly antifouling coatings. Prog. Org. Coatings 50, $75-$ 104 (2004).

29. Bentley, J. \& Turner, G. P. A. Introduction to Paint Chemistry and Principles of Paint Technology. (Chapman \& Hall, 1998). 
30. Paint and Surface Coatings: Theory and Practice. (Woodhead Publishing Limited, 1999).

31. Yang, W. J., Neoh, K.-G., Kang, E.-T., Teo, S. L.-M. \& Rittschof, D. Polymer brush coatings for combating marine biofouling. Prog. Polym. Sci. 39, 1017-1042 (2014).

32. Evans, S. M., Leksono, T. \& McKinnell, P. D. Tributyltin pollution: a diminishing problem following legislation limiting the use of TBT-based anti-fouling paints. Mar. Pollut. Bull. 30, 1421 (1995).

33. Tribou, M. \& Swain, G. The effects of grooming on a copper ablative coating: a six year study. Biofouling 33, 494-504 (2017).

34. Schiff, K., Diehl, D. \& Valkirs, A. Copper emissions from antifouling paint on recreational vessels. Mar. Pollut. Bull. 48, 371-377 (2004).

35. Voulvoulis, N., Scrimshaw, M. D. \& Lester, J. N. Comparative environmental assessment of biocides used in antifouling paints. Chemosphere 47, 789-795 (2002).

36. Lagerström, M., Norling, M. \& Eklund, B. Metal contamination at recreational boatyards linked to the use of antifouling paints - investigation of soil and sediment with a field portable XRF. Environ. Sci. Pollut. Res. 23, 10146-10157 (2016).

37. Karlsson, J., Ytreberg, E. \& Eklund, B. Toxicity of anti-fouling paints for use on ships and leisure boats to non-target organisms representing three trophic levels. Environ. Pollut. 158, 681-687 (2010).

38. Andersson, S. \& Kautsky, L. Copper effects on reproductive stages of Baltic Sea Fucus vesiculosus. Mar. Biol. 125, 171-176 (1996).

39. Debourg, C., Johnson, A., Lye, C., Törnqvist, L. \& Unger, C. Antifouling Products: Pleasure Boats, Commercial Vessels, Nets, Fish Cages and Other Underwater Equipment. (The Swedish National Chemicals Inspectorate, 1993).

40. Ytreberg, E., Karlsson, J. \& Eklund, B. Comparison of toxicity and release rates of $\mathrm{Cu}$ and $\mathrm{Zn}$ from anti-fouling paints leached in natural and artificial brackish seawater. Sci. Total Environ. 408, 2459-2466 (2010).

41. Lindén, J. B., Larsson, M., Coad, B. R., Skinner, W. M. \& Nydén, M. Polyethyleneimine for copper absorption: kinetics, selectivity and efficiency in artificial seawater. RSC Adv. 4, 25063 (2014).

42. Kennish, M. J. Practical Handbook of Marine Science. (CRC Press LLC, 2001).

43. Banerjee, I., Pangule, R. C. \& Kane, R. S. Antifouling coatings: recent developments in the design of surfaces that prevent fouling by proteins, bacteria, and marine organisms. Adv. Mater. 23, 690-718 (2011).

44. Francolini, I., Vuotto, C., Piozzi, A. \& Donelli, G. Antifouling and antimicrobial biomaterials: an overview. Apmis 125, 392-417 (2017). 
45. Roosjen, A., van der Mei, H. C., Busscher, H. J. \& Norde, W. Microbial adhesion to poly(ethylene oxide) brushes: influence of polymer chain length and temperature. Langmuir 20, 1094910955 (2004).

46. Brady, R. F. \& Singer, I. L. Mechanical factors favoring release from fouling release coatings. Biofouling 15, 73-81 (2000).

47. Jones, D. R. H. \& Ashby, M. F. Engineering Materials 1: An Introduction to Properties, Applications and Design. (Elsevier Ltd., 2019).

48. Brady, R. F. Properties which influence marine fouling resistance in polymers containing silicon and fluorine. Prog. Org. Coatings 35, 31-35 (1999).

49. Damodaran, V. B. \& Murthy, N. S. Bio-inspired strategies for designing antifouling biomaterials. Biomater. Res. 20, 1-11 (2016).

50. Curtis, A. S. G. \& Varde, M. Control of cell behavior: topological factors. J. Natl. Cancer Inst. 33, 15-26 (1964).

51. Recum, A. F. et al. Surface roughness, porosity, and texture as modifiers of cellular adhesion. Tissue Eng 2, 241-253 (1996).

52. Curtis, A. \& Wilkinson, C. Topographical control of cells. Biomaterials 18, 1573-1583 (1997).

53. Bettinger, C. J., Langer, R. \& Borenstein, J. T. Engineering substrate topography at the microand nanoscale to control cell function. Angew. Chemie - Int. Ed. 48, 5406-5415 (2009).

54. Schumacher, J. F. et al. Engineered antifouling microtopographies - effect of feature size, geometry, and roughness on settlement of zoospores of the green alga Ulva. Biofouling 23, 5562 (2007).

55. Carman, M. L. et al. Engineered antifouling microtopographies - correlating wettability with cell attachment. Biofouling 22, 11-21 (2006).

56. Cao, X. et al. Interaction of zoospores of the green alga Ulva with bioinspired micro- and nanostructured surfaces prepared by polyelectrolyte layer-by-layer self-assembly. Adv. Funct. Mater. 20, 1984-1993 (2010).

57. Fu, J. et al. Combat biofouling with microscopic ridge-like surface morphology: a bioinspired study. J. R. Soc. Interface 15, 1-8 (2018).

58. Zhang, P., Lin, L., Zang, D., Guo, X. \& Liu, M. Designing bioinspired anti-biofouling surfaces based on a superwettability strategy. Small 13, 1-9 (2017).

59. Zorba, V. et al. Biomimetic artificial surfaces quantitatively reproduce the water repellency of a lotus leaf. Adv. Mater. 20, 4049-4054 (2008).

60. Sanchez-Cano, C. \& Carril, M. Recent developments in the design of non-biofouling coatings for nanoparticles and surfaces. Int. J. Mol. Sci. 21, 1-24 (2020). 
61. Xie, Q., Pan, J., Ma, C. \& Zhang, G. Dynamic surface antifouling: mechanism and systems. Soft Matter 15, 1087-1107 (2019).

62. Zhang, Z. et al. Polysulfobetaine-grafted surfaces as environmentally benign ultralow fouling marine coatings. Langmuir 25, 13516-13521 (2009).

63. Gudipati, C. S., Greenlief, C. M., Johnson, J. A., Prayongpan, P. \& Wooley, K. L. Hyperbranched fluoropolymer and linear poly(ethylene glycol) based amphiphilic crosslinked networks as efficient antifouling coatings: an insight into the surface compositions, topographies, and morphologies. J. Polym. Sci. Part A Polym. Chem. 42, 6193-6208 (2004).

64. Gudipati, C. S., Finlay, J. A., Callow, J. A., Callow, M. E. \& Wooley, K. L. The antifouling and fouling-release performance of hyperbranched fluoropolymer (HBFP)-poly(ethylene glycol) (PEG) composite coatings evaluated by adsorption of biomacromolecules and the green fouling alga Ulva. Langmuir 21, 3044-3053 (2005).

65. Olsen, S. M., Pedersen, L. T., Laursen, M. H., Kiil, S. \& Dam-Johansen, K. Enzyme-based antifouling coatings: a review. Biofouling 23, 369-383 (2007).

66. de Souza Santos, M., Salomon, D., Li, P., Krachler, A.-M. \& Orth, K. Vibrio parahaemolyticus virulence determinants. in The Comprehensive Sourcebook of Bacterial Protein Toxins (eds. Alouf, J., Ladant, D. \& Popoff, M. R.) 230-260 (Elsevier Ltd., 2015).

67. Aykin, E., Omuzbuken, B. \& Kacar, A. Microfouling bacteria and the use of enzymes in ecofriendly antifouling technology. J. Coatings Technol. Res. 16, 847-856 (2019).

68. Tasso, M. et al. Antifouling potential of Subtilisin A immobilized onto maleic anhydride copolymer thin films. Biofouling 25, 505-516 (2009).

69. Dobretsov, S. et al. Inhibition of marine biofouling by bacterial quorum sensing inhibitors. Biofouling 27, 893-905 (2011).

70. Dobretsov, S., Teplitski, M. \& Paul, V. Mini-review: quorum sensing in the marine environment and its relationship to biofouling. Biofouling 25, 413-427 (2009).

71. Maximilien, R. et al. Chemical mediation of bacterial surface colonisation by secondary metabolites from the red alga Delisea pulchra. Aquat. Microb. Ecol. 15, 233-246 (1998).

72. Manefield, M. et al. Evidence that halogenated furanones from Delisea pulchra inhibit acylated homoserine lactone (AHL)-mediated gene expression by displacing the AHL signal from its receptor protein. Microbiology 145, 283-291 (1999).

73. Ozcelik, B. et al. Poly(ethylene glycol)-based coatings combining low-biofouling and quorumsensing inhibiting properties to reduce bacterial colonization. ACS Biomater. Sci. Eng. 3, 78-87 (2017).

74. Egan, S., James, S., Holmström, C. \& Kjelleberg, S. Inhibition of algal spore germination by the 
marine bacterium Pseudoalteromonas tunicata. FEMS Microbiol. Ecol. 35, 67-73 (2001).

75. Satheesh, S., Ba-akdah, M. A. \& Al-Sofyani, A. A. Natural antifouling compound production by microbes associated with marine macroorganisms - a review. Electron. J. Biotechnol. 21, 2635 (2016).

76. Pallavicini, P., Dacarro, G., Diaz-Fernandez, Y. A. \& Taglietti, A. Coordination chemistry of surface-grafted ligands for antibacterial materials. Coord. Chem. Rev. 275, 37-53 (2014).

77. Lindén, J. B. et al. Polyethyleneimine for copper absorption II: kinetics, selectivity and efficiency from seawater. RSC Adv. 5, 51883-51890 (2015).

78. Kaur, S., Kempon, I. M., Lindén, J. B., Larsson, M. \& Nydén, M. Unhindered copper uptake by glutaraldehyde-polyethyleneimine coatings in an artificial seawater model system with adsorbed swollen polysaccharides and competing ligand EDTA. Biofouling 33, 184-194 (2017).

79. Movahedi, A., Lundin, A., Kann, N., Nydén, M. \& Moth-Poulsen, K. Cu(I) stabilizing crosslinked polyethyleneimine. Phys. Chem. Chem. Phys. 17, 18327-18336 (2015).

80. Hirose, K. Conditional stability constants of metal complexes of organic ligands in sea water: past and present, and a simple coordination chemistry model. Anal. Chim. Acta 284, 621-634 (1994).

81. Nydén, B. M. et al. Metal ion binding polymers and uses thereof. (2014).

82. Elmas, S. et al. Porous PEI coating for copper ion storage and its controlled electrochemical release. Adv. Sustain. Syst. 1900123, 1-9 (2020).

83. Ligand Design in Medicinal Inorganic Chemistry. (John Wiley \& Sons, Ltd, 2014). doi:10.1002/9781118697191.ch2

84. Lawrance, G. A. Introduction to Coordination Chemistry. (John Wiley \& Sons, Inc., 2009).

85. Hancock, R. D. \& Martell, A. E. Ligand design for selective complexation of metal ions in aqueous solution. Chem. Rev. 89, 1875-1914 (1989).

86. Delangle, P. \& Mintz, E. Chelation therapy in Wilson's disease: from D-penicillamine to the design of selective bioinspired intracellular $\mathrm{Cu}(\mathrm{I})$ chelators. Dalt. Trans. 41, 6359-6370 (2012).

87. Andersson Trojer, M., Movahedi, A., Blanck, H. \& Nydén, M. Imidazole and triazole coordination chemistry for antifouling coatings. J. Chem. 2013, 1-23 (2013).

88. Lu, J. et al. Copper(II)-selective chelation improves function and antioxidant defences in cardiovascular tissues of rats as a model of diabetes: comparisons between triethylenetetramine and three less copper-selective transition-metal-targeted treatments. Diabetologia 53, 1217-1226 (2010).

89. Amorim, M. T. S., Chaves, S., Delgado, R. \& Frausto da Silva, J. J. R. Oxatriaza macrocyclic ligands: studies of protonation and metal complexation. J. Chem. Soc. Dalt. Trans. 3065-3072 
(1991).

90. Cooper, G. J. S. Therapeutic potential of copper chelation with triethylenetetramine in managing diabetes mellitus and Alzheimer's disease. Drugs 71, 1281-1320 (2011).

91. Roberts, E. A. \& Schilsky, M. L. Diagnosis and treatment of Wilson disease: an update. Hepatology 47, 2089-2111 (2008).

92. Granholm, K., Harju, L. \& Ivaska, A. Desorption of metal ions from kraft pulps. Part 1. Chelation of hardwood and softwood kraft pulp with EDTA. BioResources 5, 206-226 (2010).

93. Jones, C. J. Bonding in transition metal complexes. in $d$ - and f-Block Chemistry (ed. Abel, E. W.) 97-129 (Royal Society of Chemistry, 2001). doi:10.1021/ic50006a055

94. Hosmane, N. S. Review of bonding theories for d-block metal complexes. in Advanced Inorganic Chemistry 89-114 (Elsevier Inc., 2017). doi:10.1016/b978-0-12-801982-5.00006-0

95. Persson, I., Persson, P., Sandström, M. \& Ullström, A.-S. Structure of Jahn-Teller distorted solvated copper(II) ions in solution, and in solids with apparently regular octahedral coordination geometry. J. Chem. Soc. Dalt. Trans. 1256-1265 (2002). doi:10.1039/b200698g

96. Halcrow, M. A. Jahn-Teller distortions in transition metal compounds, and their importance in functional molecular and inorganic materials. Chem. Soc. Rev. 42, 1784-1795 (2013).

97. Jones, C. J. The thermodynamics of complex formation. in $d$-and f-Block Chemistry (ed. Abel, E. W.) 71-96 (John Wiley \& Sons, Ltd, 2001). doi:10.1039/9781847550682-00071

98. Fraústo da Silva, J. J. R. The chelate effect redefined. J. Chem. Educ. 60, 390-392 (1983).

99. Jones, C. J. Coordination compounds. in d-and f-Block Chemistry (ed. Abel, E. W.) 54-70 (Royal Society of Chemistry, 2001). doi:https://doi.org/10.1039/9781847550682

100. Hutton, A. T. \& Linder, P. W. Stability constants \& their determination. in Encyclopedia of Inorganic Chemistry 1-9 (John Wiley \& Sons, Ltd., 2006). doi:10.1002/0470862106.ia227

101. Gans, P., Sabatini, A. \& Vacca, A. Investigation of equilibria in solution. Determination of equilibrium constants with the HYPERQUAD suite of programs. Talanta 43, 1739-1753 (1996).

102. Hill, Z. D. \& MacCarthy, P. Novel approach to Job's method: an undergraduate experiment. J. Chem. Educ. 63, 162-167 (1986).

103. Bruneau, E., Lavabre, D., Levy, G. \& Micheau, J. C. Quantitative analysis of continuous-variation plots with a comparison of several methods: spectrophotometric study of organic and inorganic 1:1 stoichiometry complexes. J. Chem. Educ. 69, 833-837 (1992).

104. de Araújo, E. L., Barbosa, H. F. G., Dockal, E. R. \& Cavalheiro, É. T. G. Synthesis, characterization and biological activity of $\mathrm{Cu}(\mathrm{II}), \mathrm{Ni}(\mathrm{II})$ and $\mathrm{Zn}(\mathrm{II})$ complexes of biopolymeric Schiff bases of salicylaldehydes and chitosan. Int. J. Biol. Macromol. 95, 168-176 (2017).

105. Kumar, S., Pandey, P. K., Sinha, N., Chaudhari, S. \& Sharma, S. Spectroscopic characterisation 
of metal complexes with tetradentate ligand. J. Phys. Sci. 29, 1-11 (2018).

106. Alkaya, Z. A. et al. Synthesis and characterization of $\mathrm{Cu}(\mathrm{II})$ complexes of 2-amino-6sulfamoylbenzothiazole and their inhibition studies on carbonic anhydrase isoenzymes. Polyhedron 151, 199-205 (2018).

107. Okagu, O. D. et al. Synthesis and characterization of $\mathrm{Cu}(\mathrm{II}), \mathrm{Co}(\mathrm{II})$ and $\mathrm{Ni}(\mathrm{II})$ complexes of a benzohydrazone derivative: spectroscopic, DFT, antipathogenic and DNA binding studies. J. Mol. Struct. 1183, 107-117 (2019).

108. Ramadan, S., Hambley, T. W., Kennedy, B. J. \& Lay, P. A. NMR spectroscopic characterization of copper(II) and zinc(II) complexes of indomethacin. Inorg. Chem. 43, 2943-2946 (2004).

109. Parodi, F. Physics and chemistry of microwave processing. in Comprehensive Polymer Science and Supplements (eds. Allen, G. \& Bevington, J. C.) 669-728 (Elsevier Ltd., 1989).

110. Hwang, S. \& Meyerhoff, M. E. Polyurethane with tethered copper(II)-cyclen complex: preparation, characterization and catalytic generation of nitric oxide from S-nitrosothiols. Biomaterials 29, 2443-2452 (2008).

111. Kurnoskin, A. V. Metalliferous epoxy chelate polymers: 1 . synthesis and properties. Polymer (Guildf). 34, 1060-1067 (1993).

112. Abd El-Wahab, H., Abd El-Fattah, M., Ahmed, A. H., Elhenawy, A. A. \& Alian, N. A. Synthesis and characterization of some arylhydrazone ligand and its metal complexes and their potential application as flame retardant and antimicrobial additives in polyurethane for surface coating. J. Organomet. Chem. 791, 99-106 (2015).

113. González-Rodríguez, V. et al. Improving titanium dioxide dispersion in water through surface functionalization by a dicarboxylic acid. J. Dispers. Sci. Technol. 40, 1039-1045 (2019).

114. Vincent, M., Duval, R. E., Hartemann, P. \& Engels-Deutsch, M. Contact killing and antimicrobial properties of copper. J. Appl. Microbiol. 124, 1032-1046 (2018).

115. Grass, G., Rensing, C. \& Solioz, M. Metallic copper as an antimicrobial surface. Appl. Environ. Microbiol. 77, 1541-1547 (2011).

116. Solioz, M. Copper disposition in bacteria. in Clinical and Translational Perspectives on WILSON DISEASE (eds. Kerkar, N. \& Roberts, E. A.) 101-113 (Elsevier Inc., 2019). doi:10.1016/b978-0$12-810532-0.00011-2$

117. Hans, M., Mathews, S., Mücklich, F. \& Solioz, M. Physicochemical properties of copper important for its antibacterial activity and development of a unified model. Biointerphases 11, $1-8$ (2016).

118. Sato, T. et al. Assessment of the anti-biofouling potentials of a copper iodide-doped nylon mesh. Appl. Microbiol. Biotechnol. 95, 1043-1050 (2012). 
119. Luo, J., Hein, C., Mücklich, F. \& Solioz, M. Killing of bacteria by copper, cadmium, and silver surfaces reveals relevant physicochemical parameters. Biointerphases 12, 1-6 (2017).

120. Dupont, C. L., Grass, G. \& Rensing, C. Copper toxicity and the origin of bacterial resistance new insights and applications. Metallomics 3, 1109-1118 (2011).

121. Stevenson, J., Barwinska-Sendra, A., Tarrant, E. \& Waldron, K. J. Mechanism of action and applications of the antimicrobial properties of copper. in Microbial Pathogens and Strategies for Combating Them: Science, Technology and Education 468-479 (2013).

122. McElroy, D. J. et al. Effect of copper on multiple successional stages of a marine fouling assemblage. Biofouling 33, 904-916 (2017).

123. Cuenot, F., Meyer, M., Bucaille, A. \& Guilard, R. A molecular approach to remove lead from drinking water. J. Mol. Liq. 118, 89-99 (2005).

124. Chester, R. \& Jickells, T. Marine Geochemistry. (John Wiley \& Sons, Ltd, 2012). doi:10.1007/97894-010-9488-7

125. Gattuso, J.-P. \& Hansson, L. Ocean Acidification. (Oxford University Press, Inc., 2011).

126. Chouyyok, W. et al. Selective removal of copper(II) from natural waters by nanoporous sorbents functionalized with chelating diamines. Environ. Sci. Technol. 44, 6390-6395 (2010).

127. Daines, A. M., Robinson, H., Glenny, M., Williams, D. B. G. \& Hinkley, S. F. R. Linear and macrocyclic water soluble polyacylhydrazones and their utilisation in coatings. Prog. Org. Coatings 121, 38-44 (2018).

128. Daines, A. Personal communication.

129. Harris, D. C. \& Bertolucci, M. D. Symmetry and Spectroscopy: An Introduction to Vibrational and Electronical Spectroscopy. (Dover Publications, Inc., 1978).

130. Conry, R. R. Copper: inorganic \& coordination chemistry. in Encyclopedia of Inorganic Chemistry 1-19 (John Wiley \& Sons, Ltd., 2006). doi:10.1002/0470862106.ia052

131. El-Wahab, H. A. The synthesis and characterization of the hydrazone ligand and its metal complexes and their performance in epoxy formulation surface coatings. Prog. Org. Coatings 89, 106-113 (2015).

132. Rodríguez-Argüelles, M. C. et al. Antibacterial and antifungal activity of metal(II) complexes of acylhydrazones of 3-isatin and 3-(N-methyl)isatin. Polyhedron 28, 2187-2195 (2009).

133. Zha, D. \& You, L. Multiresponsive dynamic covalent assemblies for the selective sensing of both $\mathrm{Cu}^{2+}$ and $\mathrm{CN}^{-}$in water. ACS Appl. Mater. Interfaces (2016). doi:10.1021/acsami.5b11552

134. Sayer, J. M., Pinsky, B., Schonbrunn, A. \& Washtien, W. Mechanism of carbinolamine formation. J. Am. Chem. Soc. 96, 7998-8009 (1974).

135. Brokaite, K., Mickevicius, V. \& Mikulskiene, G. Synthesis and structural investigation of some 
1,4-disubstituted-2-pyrrolidinones. ARKIVOC 61-67 (2006).

136. Ershov, A. Y. et al. Tautomerism and conformational isomerism of mercaptoacetylhydrazones of aliphatic and aromatic aldehydes. Russ. J. Org. Chem. 45, 660-666 (2009).

137. Gu, W. et al. Synthesis and antibacterial evaluation of new $\mathrm{N}$-acylhydrazone derivatives from dehydroabietic acid. Molecules 17, 4634-4650 (2012).

138. Wyrzykiewicz, E. \& Prukała, D. New isomeric $\mathrm{N}$-substituted hydrazones of 2-, 3- and 4pyridinecarboxaldehydes. J. Heterocycl. Chem. 35, 381-387 (1998).

139. Palla, G., Predieri, G., Domiano, P., Vignali, C. \& Turner, W. Conformational behaviour and E/Z isomerization of $\mathrm{N}$-acyl and $\mathrm{N}$-aroylhydrazones. Tetrahedron 42, 3649-3654 (1986).

140. Ferraresi-Curotto, V., Echeverría, G. A., Piro, O. E., Pis-Diez, R. \& González-Baró, A. C. Synthesis and characterization of a series of isoniazid hydrazones. Spectroscopic and theoretical study. J. Mol. Struct. 1133, 436-447 (2017).

141. Cordier, C. et al. Salicylaldehyde benzoyl hydrazone: isomerization due to water. A structural analysis using a combination of NMR, IR, and theoretical investigations. Struct. Chem. 15, 295307 (2004).

142. Koh, L. L. et al. Complexes of salicylaldehyde acylhydrazones: cytotoxicity, QSAR and crystal structure of the sterically hindered $t$-butyl dimer. J. Inorg. Biochem. 72, 155-162 (1998).

143. Friestad, G. K. Chiral $\mathrm{N}$-acylhydrazones: versatile imino acceptors for asymmetric amine synthesis. European J. Org. Chem. 2005, 3157-3172 (2005).

144. Van Dijken, D. J., Kovaříček, P., Ihrig, S. P. \& Hecht, S. Acylhydrazones as widely tunable photoswitches. J. Am. Chem. Soc. 137, 14982-14991 (2015).

145. Chaur, M. N., Collado, D. \& Lehn, J.-M. Configurational and constitutional information storage: multiple dynamics in systems based on pyridyl and acyl hydrazones. Chem. - A Eur. J. 17, 248258 (2011).

146. Popov, L. D., Askalepova, O. I., Levchenkov, S. I. \& Kogan, V. A. Protolitic complexing properties of acylhydrazones of substituted salicylaldehydes and stability constants of their copper(II) complexes. Russ. J. Inorg. Chem. 52, 626-629 (2007).

147. Renuka, M. \& Hussain Reddy, K. Non-extractive spectrophotometric determination of copper(II) in leafy vegetable samples using salicylaldehyde acetoylhydrazone (SAAH). Int. J. Curr. Multidiscip. Stud. 1, 32-36 (2013).

148. Yousef Ebrahimipour, S., Sheikhshoaie, I., Crochet, A., Khaleghi, M. \& Fromm, K. M. A new mixed-ligand copper(II) complex of (E)-N'-(2-hydroxybenzylidene) acetohydrazide: synthesis, characterization, NLO behavior, DFT calculation and biological activities. J. Mol. Struct. 1072, 267-276 (2014). 
149. Abd El Wahed, M. G., Bayoumi, H. A. \& Mohammed, M. I. Physical properties of some acetylbenzaldehydehydrazone metal complexes. Bull. Korean Chem. Soc. 24, 1313-1318 (2003).

150. Sanjeev, R., Jagannadham, V. \& Vrath, R. V. Implications of a novel interpretation of the isosbestic point. Chem. New Zeal. 76, 133-135 (2012).

151. Nowicka-Jankowska, T. Some properties of isosbestic points. J. Inorg. Nucl. Chem. 33, 20432050 (1971).

152. Gans, P. Personal communication.

153. Hinkley, S. F. R. Personal communication.

154. Lehn, J.-M. Dynamers: dynamic molecular and supramolecular polymers. Prog. Polym. Sci. 30, 814-831 (2005).

155. Roy, N., Bruchmann, B. \& Lehn, J.-M. DYNAMERS: dynamic polymers as self-healing materials. Chem. Soc. Rev. 44, 3786-3807 (2015).

156. Zhang, Y. \& Barboiu, M. Constitutional dynamic materials - toward natural selection of function. Chem. Rev. 116, 809-834 (2016).

157. Buchs, B. et al. Release of bioactive volatiles from supramolecular hydrogels: influence of reversible acylhydrazone formation on gel stability and volatile compound evaporation. Org. Biomol. Chem. 9, 2906-2919 (2011).

158. Butler, A. Acquisition and utilization of transition metal ions by marine organisms. Science 281, 207-210 (1998).

159. Singh, V. P. \& Gupta, P. Synthesis, physico-chemical characterization and antimicrobial activity of cobalt(II), nickel(II), copper(II), zinc(II) and cadmium(II) complexes with some acyldihydrazones. J. Enzyme Inhib. Med. Chem. 23, 797-805 (2008).

160. Ozay, H., Baran, Y. \& Miyamaez, H. The stabilities and formation kinetics of some macrocycles with copper(II): crystal structures of some pendant arm macrocycles. J. Coord. Chem. 64, 14691480 (2011).

161. Lee, S. C. et al. Highly selective copper(II) ion receptors: tetraazacrown ethers bearing two 8hydroxyquinoline side arms. Inorganica Chim. Acta 317, 174-180 (2001).

162. Esteves, C. V. et al. Remarkable inertness of copper(II) chelates of cyclen-based macrobicycles with two trans-N-acetate arms. Inorg. Chem. 52, 5138-5153 (2013).

163. Füzerová, S. et al. Cyclam (1,4,8,11-tetraazacyclotetradecane) with one methylphosphonate pendant arm: a new ligand for selective copper(II) binding. Dalt. Trans. 2908-2915 (2005). doi:10.1039/b507062g

164. Lima, L. M. P., Esteban-Gómez, D., Delgado, R., Platas-Iglesias, C. \& Tripier, R. Monopicolinate 
cyclen and cyclam derivatives for stable copper(II) complexation. Inorg. Chem. 51, 6916-6927 (2012).

165. Thom, V. J., Hosken, G. D. \& Hancock, R. D. Anomalous metal ion size selectivity of tetraaza macrocycles. Inorg. Chem. 24, 3378-3381 (1985).

166. Kodama, M. \& Kimura, E. A thermodynamic and kinetic interpretation of the macrocyclic effect. Polarographic studies on copper(II) 1,4,7,10-tetraazacyclododecane complexation. J. Chem. Soc., Chem. Commun. 326-327 (1975).

167. Martell, A. E. \& Hancock, R. D. The chelate, macrocyclic, and cryptate effects. in Coordination Chemistry (ed. Kauffman, G. B.) 240-254 (American Chemical Society, 1994). doi:10.1021/bk1994-0565.ch020

168. Hancock, R. D. \& McDougall, G. J. The macrocyclic effect in tetraaza-macrocyclic ligands. Adv. Mol. Relax. Interact. Process. 18, 99-108 (1980).

169. Firdaus, F., Fatma, K., Azam, M. \& Shakir, M. Synthesis, spectroscopic, thermal, and antimicrobial studies of tetradentate 12 and 14 member Schiff bases and their complexes with Fe(III), Co(II), and Cu(II). J. Coord. Chem. 63, 3956-3968 (2010).

170. Li, S. et al. The synthesis and activities of novel mononuclear or dinuclear cyclen complexes bearing azole pendants as antibacterial and antifungal agents. Eur. J. Med. Chem. 84, 677-686 (2014).

171. Shakir, M., Azim, Y., Chishti, H. T. N. \& Parveen, S. Synthesis, characterization of complexes of $\mathrm{Co}(\mathrm{II}), \mathrm{Ni}(\mathrm{II}), \mathrm{Cu}(\mathrm{II})$ and $\mathrm{Zn}(\mathrm{II})$ with 12-membered Schiff base tetraazamacrocyclic ligand and the study of their antimicrobial and reducing power. Spectrochim. Acta - Part A Mol. Biomol. Spectrosc. 65, 490-496 (2006).

172. Hirose, K., Dokiya, Y. \& Sugimura, Y. Determination of conditional stability constants of organic copper and zinc complexes dissolved in seawater using ligand exchange method with EDTA. Mar. Chem. 11, 343-354 (1982).

173. Hirose, K. Metal-organic matter interaction: ecological roles of ligands in oceanic DOM. Appl. Geochemistry 22, 1636-1645 (2007).

174. Felix, V. et al. Electron spin resonance studies and crystal structures of copper(II) complexes of some 12-, 13- and 14-membered oxatriaza macrocycles. J. Chem. Soc. Dalt. Trans. 3099-3106 (1994).

175. Kodama, M. \& Kimura, E. Reaction of cobalt(II) macrocyclic tetra-amine complexes with dioxygen. J. Chem. Soc. Dalt. Trans. 327-333 (1980).

176. Thom, V. J. \& Hancock, R. D. The stability of nickel(II) complexes of tetra-aza macrocycles. J. Chem. Soc. Dalt. Trans. 1877-1880 (1985). doi:10.1039/DT9850001877 
177. Kodama, B. M. \& Kimura, E. Equilibria and kinetics of complex formation between zinc(II), lead(II), and cadmium(II), and 12-, 13-, 14-, and 15-membered macrocyclic tetra-amines. J. Chem. Soc. Dalt. Trans. 2269-2276 (1977).

178. Hancock, R. D., Wade, P. W., Ngwenya, M. P., de Sousa, A. S. \& Damu, K. V. Ligand design for complexation in aqueous solution. 2. Chelate ring size as a basis for control of size-based selectivity for metal ions. Inorg. Chem. 29, 1968-1974 (1990).

179. Hinz, F. P. \& Margerum, D. W. Ligand solvation and the macrocyclic effect. Nickel(II)-tetramine complexes. Inorg. Chem. 13, 2941-2949 (1974).

180. Nakani, B. S. \& Hancock, R. D. The chelate, cryptate and macroyclic effects. S. Afr. J. Chem. 36, 117 (1983).

181. Wadas, T. J., Wong, E. H., Weisman, G. R. \& Anderson, C. J. Coordinating radiometals of copper, gallium, indium, yttrium and zirconium for PET and SPECT imaging of disease. Chem Rev. 110, 2858-2902 (2010).

182. Hancock, R. D. Macrocycles and their selectivity for metal ions on the basis of size. Pure Appl. Chem. 58, 1445-1452 (1986).

183. Paul, S. Crosslinking: chemistry of surface coatings. in Comprehensive Polymer Science and Supplements (eds. Allen, G. \& Bevington, J. C.) 149-192 (Elsevier Ltd., 1996). doi:10.1016/b9780-08-096701-1.00186-5

184. Bossion, A. et al. Opportunities for organocatalysis in polymer synthesis via step-growth methods. Prog. Polym. Sci. 90, 164-210 (2019).

185. Armelin, E. et al. Marine paint fomulations: conducting polymers as anticorrosive additives. Prog. Org. Coatings 59, 46-52 (2007).

186. Prisacariu, C. Polyurethane Elastomers: From Morphology to Mechanical Aspects. (SpringerVerlag/Wien, 2011). doi:10.1007/978-3-7091-0514-6

187. Puiu, S. C. et al. Metal ion-mediated nitric oxide generation from polyurethanes via covalently linked copper(II)-cyclen moieties. J. Biomed. Mater. Res. - Part B Appl. Biomater. 91B, 203-212 (2009).

188. Xie, Q., Ma, C., Liu, C., Ma, J. \& Zhang, G. Poly(dimethylsiloxane)-based polyurethane with chemically attached antifoulants for durable marine antibiofouling. ACS Appl. Mater. Interfaces 7, 21030-21037 (2015).

189. Hamerton, I., Howlin, B. J. \& Jepson, P. Metals and coordination compounds as modifiers for epoxy resins. Coord. Chem. Rev. 224, 67-85 (2002).

190. Kurnoskin, A. V. Metalliferous epoxy chelate polymers: 2 . influence of structural fragments on properties. Polymer (Guildf). 34, 1068-1076 (1993). 
191. Kurnoskin, A. V. Metalliferous epoxy chelate polymers: 3. influence of structural fragments on the properties of similar matrices. Polymer (Guildf). 34, 1077-1088 (1993).

192. Kurnoskin, A. V. Water resistance of metalliferous epoxy chelate polymers. J. Appl. Polym. Sci. 45, 1557-1567 (1992).

193. Döring, M. \& Arnold, U. Polymerization of epoxy resins initiated by metal complexes. Polym. Int. 58, 976-988 (2009).

194. Lakshmi, B., Shivananda, K. N., Prakash, G. A., Rama, K. R. K. \& Mahendra, K. N. Synthesis of $\mathrm{Co}(\mathrm{II}), \mathrm{Ni}(\mathrm{II})$ and $\mathrm{Cu}(\mathrm{II})$ complexes from Schiff base ligand and reactivity studies with thermosetting epoxy resin. Bull. Korean Chem. Soc. 32, 1613-1619 (2011).

195. Thomas, R. Practical Guide to ICP-MS: A Tutorial for Beginners. (CRC Press LLC, 2013).

196. Ojeil, M., Jermann, C., Holah, J., Denyer, S. P. \& Maillard, J. Y. Evaluation of new in vitro efficacy test for antimicrobial surface activity reflecting UK hospital conditions. J. Hosp. Infect. 85, 274281 (2013).

197. Wiegand, C. et al. Critical physiological factors influencing the outcome of antimicrobial testing according to ISO 22196 / JIS Z 2801. PLoS One 13, 1-15 (2018).

198. Campos, M. D., Zucchi, P. C., Phung, A., Leonard, S. N. \& Hirsch, E. B. The activity of antimicrobial surfaces varies by testing protocol utilized. PLoS One 11, 1-11 (2016).

199. Standard, I. ISO 22196:2007 - Plastics - Measurement of antibacterial activity on plastics surfaces. 2007, (2007).

200. Burr, D. Personal communication.

201. Mytilinaios, I., Salih, M., Schofield, H. K. \& Lambert, R. J. W. Growth curve prediction from optical density data. Int. J. Food Microbiol. 154, 169-176 (2012).

202. Monod, J. The growth of bacterial cultures. Annu. Rev. Microbiol. 3, 371-394 (1949).

203. Ferry, J. D. \& Carritt, D. E. Action of antifouling paints. Ind. Eng. Chem. 38, 612-617 (1946).

204. Rascio, V. J. D. Antifouling coatings: where do we go from here. Corros. Rev. 18, 133-154 (2000).

205. Woods Hole Oceanographic Institute. The physical chemistry of compounds of copper and mercury and their interactions with sea water. in Marine Fouling and Its Prevention 264-276 (George Banta Publishing Co., 1952).

206. Macomber, L. \& Imlay, J. A. The iron-sulfur clusters of dehydratases are primary intracellular targets of copper toxicity. Proc. Natl. Acad. Sci. U. S. A. 106, 8344-8349 (2009).

207. Glenny, M. Personal communication.

208. Dorsey, J. G., Dorsey, G. F., Rutenberg, A. C. \& Green, L. A. Determination of the epoxide equivalent weight of glycidyl ethers by proton magnetic resonance spectrometry. Anal. Chem. 
49, 1144-1145 (1977).

209. González Garcia, F., Montedo da Silva, P., Guenther Soares, B. \& Rieumont Briones, J. Combined analytical techniques for the determination of the amine hydrogen equivalent weight in aliphatic amine epoxide hardeners. Polym. Test. 26, 95-101 (2007).

210. Product data sheet Epikote ${ }^{\mathrm{TM}}$ Resin 235. (2004).

211. Epoxy curing agents and modifiers: Ancamine ${ }^{\circledR} 2459$ Curing Agent.

212. Burton, B. L. Amine-blushing problems? No sweat! in Epoxy Resin Formulators' Meeting of the Society of the Plastics Industry 1-17 (2001).

213. Patel, R. N. et al. Characterization and biological activities of two copper(II) complexes with diethylenetriamine and 2,2'-bipyridine or 1,10-phenanthroline as ligands. Spectrochim. Acta Part A Mol. Biomol. Spectrosc. 62, 261-268 (2005).

214. Villis, P. C. M. et al. Diethylenetriamine ion-imprinted silica gel for copper determination in tap water. J. Appl. Electrochem. 48, 867-883 (2018).

215. Fang, Z., Yan, J., Yu, W., Zhang, N. \& Zhang, S. Three Schiff base complexes based on diethylenetriamine: synthesis, structure, DNA binding and cleavage, and in vitro cytotoxicity. Transit. Met. Chem. 44, 463-474 (2019).

216. Dacarro, G. et al. Monolayers of polyethilenimine on flat glass: a versatile platform for cations coordination and nanoparticles grafting in the preparation of antibacterial surfaces. Dalt. Trans. 41, 2456-2463 (2012).

217. Shaw, R. A. \& Mantsch, H. H. Near-IR spectrometers. in Encyclopedia of Spectroscopy and Spectrometry (ed. Lindon, J. C.) 1451-1461 (Elsevier Ltd., 2000).

218. Cholake, S. T. et al. Quantitative analysis of curing mechanisms of epoxy resin by mid- and nearFourier transform infrared spectroscopy. Def. Sci. J. 64, 341-321 (2014).

219. Beć, K. B. \& Huck, C. W. Breakthrough potential in near-infrared spectroscopy: spectra simulation. A review of recent developments. Front. Chem. 7, 1-22 (2019).

220. Cañavate, J., Colom, X., Pagès, P. \& Carrasco, F. Study of the curing process of an epoxy resin by FTIR spectroscopy. Polym. Plast. Technol. Eng. 39, 937-943 (2000).

221. González González, M., Cabanelas, J. C. \& Baselga, J. Applications of FTIR on epoxy resins identification, monitoring the curing process, phase separation and water uptake. in Infrared Spectroscopy - Materials Science, Engineering and Technology (ed. Theophile, T.) 261-284 (IntechOpen, 2012).

222. Poisson, N., Lachenal, G. \& Sautereau, H. Near- and mid-infrared spectroscopy studies of an epoxy reactive system. Vib. Spectrosc. 12, 237-247 (1996).

223. Morgan, R. J. The effect of thermal history and strain rate on the mechanical properties of 
diethylenetriamine-cured bisphenol-A-diglycidyl ether epoxies. J. Appl. Polym. Sci. 23, 27112717 (1979).

224. Matějka, L. Amine cured epoxide networks: formation, structure, and properties. Macromolecules 33, 3611-3619 (2000).

225. Saucy, D. A., Simko, S. J. \& Linton, R. W. Comparison of photoacoustic and attentuated total reflectance sampling depths in the infrared region. Anal. Chem. 57, 871-875 (1985).

226. Hobbs, A. L. \& Almirall, J. R. Trace elemental analysis of automotive paints by laser ablationinductively coupled plasma-mass spectrometry (LA-ICP-MS). Anal. Bioanal. Chem. 376, 12651271 (2003).

227. Hebbar, R. S., Isloor, A. M. \& Ismail, A. F. Contact angle measurements. in Membrane Characterization 219-255 (Elsevier B.V, 2017).

228. Al-Kattan, A. et al. Characterization of materials released into water from paint containing nano-SiO 2 . Chemosphere 119, 1314-1321 (2015).

229. Zhou, S., Wu, L., Sun, J. \& Shen, W. The change of the properties of acrylic-based polyurethane via addition of nano-silica. Prog. Org. Coatings 45, 33-42 (2002).

230. Manoudis, P. N., Tsakalof, A., Karapanagiotis, I., Zuburtikudis, I. \& Panayiotou, C. Fabrication of super-hydrophobic surfaces for enhanced stone protection. Surf. Coatings Technol. 203, 13221328 (2009).

231. Wallström, E., Jespersen, H. T. \& Schaumburg, K. A new concept for anti-fouling paint for yachts. Prog. Org. Coatings 72, 109-114 (2011).

232. Masato Kishihara, O., Akira Saito, F., Hiroshi Yamashita, H., Toshimitsu Muramatsu, Y. \& Yoichi Yonehara, C. Antifouling coating composition. (1999).

233. Simendinger, W. H. Antifouling coating composition. (2003).

234. Michailidis, M. et al. Modified mesoporous silica nanoparticles with a dual synergetic antibacterial effect. ACS Appl. Mater. Interfaces 9, 38364-38372 (2017).

235. Brandès, S., David, G., Suspène, C., Corriu, R. J. P. \& Guilard, R. Exceptional affinity of nanostructured organic-inorganic hybrid materials towards dioxygen: confinement effect of copper complexes. Chem. - A Eur. J. 13, 3480-3490 (2007).

236. Barbette, F. et al. Extraction of uranyl ions from aqueous solutions using silica-gel-bound macrocycles for alpha contaminated waste water treatment. Anal. Chim. Acta 502, 179-187 (2004).

237. Dubois, G. et al. Coordination chemistry in the solid: study of the incorporation of $\mathrm{Cu}(\mathrm{II})$ into cyclam-containing hybrid materials. Angew. Chemie - Int. Ed. 40, 1087-1090 (2001).

238. Dubois, G., Tripier, R., Brandès, S., Denat, F. \& Guilard, R. Cyclam complexes containing silica 
gels for dioxygen adsorption. J. Mater. Chem. 12, 2255-2261 (2002).

239. Dubois, G. et al. First organic-inorganic hybrid materials with controlled porosity incorporating cyclam units. Chem. Commun. 2283-2284 (1999). doi:10.1039/a907217i

240. Corriu, R. J. P., Embert, F., Guari, Y., Reyé, C. \& Guilard, R. Coordination chemistry in the solid: evidence for coordination modes within hybrid materials different from those in solution. Chem. - A Eur. J. 8, 5732-5741 (2002).

241. Gros, C. et al. New silica-gel-bound polyazacycloalkanes and characterization of their copper(II) complexes using electron spin resonance spectroscopy. J. Chem. Soc. - Dalt. Trans. 1209-1214 (1996). doi:10.1039/DT9960001209

242. Corriu, R. J. P. et al. Preparation of ordered SBA-15 mesoporous silica containing chelating groups. Study of the complexation of Eu(II) inside the pore channels of the materials. New J. Chem. 28, 156-160 (2004).

243. Corriu, R. J. P., Mehdi, A., Reyé, C. \& Thieuleux, C. Direct synthesis of functionalized mesoporous silica by non-ionic assembly routes. Quantitative chemical transformations within the materials leading to strongly chelated transition metal ions. Chem. Mater. 16, 159-166 (2004).

244. Kassiba, A. et al. EPR investigations of mesoporous silica doped with metal transitions ions. J. Phys. Chem. Solids 67, 875-881 (2006).

245. Corriu, R. J. P., Mehdi, A., Reyé, C. \& Thieuleux, C. Control of coordination chemistry in both the framework and the pore channels of mesoporous hybrid materials. New J. Chem. 27, 905908 (2003).

246. Gong, Y. \& Lee, H. K. Application of cyclam-capped $\beta$-cyclodextrin-bonded silica particles as a chiral stationary phase in capillary electrochromatography for enantiomeric separations. Anal. Chem. 75, 1348-1354 (2003).

247. Sujandi, Han, S.-C., Han, D.-S., Jin, M.-J. \& Park, S.-E. Catalytic oxidation of cycloolefins over Co(cyclam)-functionalized SBA-15 material with $\mathrm{H}_{2} \mathrm{O}_{2}$. J. Catal. 243, 410-419 (2006).

248. Puranik, D. B. et al. Copper removal from fuel by solid-supported polyamine chelating agents. Energy and Fuels 12, 792-797 (1998).

249. Veuthey, J. L., Bagnoud, M. A. \& Haerdi, W. Enrichment of amino and carboxylic acids using copper-loaded silica pre-columns coupled on-line with HPLC. Int. J. Environ. Anal. Chem. 26, 157-166 (1986).

250. Bagnoud, M. A. \& Haerdi, W. Metal-loaded silicas for on-line coupling of enrichment precolumn and analytical columns. Int. J. Environ. Anal. Chem. 38, 97-104 (1990).

251. Bradshaw, J. S. et al. New nitrogen-containing macrocyclic ligands covalently attached to silica 
gel and their use in separating metal cations. Solvent Extr. Ion Exch. 7, 855-864 (1989).

252. Goubert-Renaudin, S. et al. Factors affecting copper(II) binding to multiarmed cyclam-grafted mesoporous silica in aqueous solution. Langmuir 25, 9804-9813 (2009).

253. Da'na, E. Adsorption of heavy metals on functionalized-mesoporous silica: a review. Microporous Mesoporous Mater. 247, 145-157 (2017).

254. Díaz-García, D. et al. Preparation and study of the antibacterial applications and oxidative stress induction of copper maleamate-functionalized mesoporous silica nanoparticles. Pharmaceutics 11, 1-18 (2019).

255. Bagnoud, M. A., Haerdi, W. \& Veuthey, J. L. Outer-sphere ligand-exchange chromatography with copper-loaded macrocyclic-bonded silica column. Chromatographia 29, 495-499 (1990).

256. Plueddemann, E. P. Silane Coupling Agents. (Springer Science + Business Media, LLC, 1982).

257. Jal, P. K., Patel, S. \& Mishra, B. K. Chemical modification of silica surface by immobilization of functional groups for extractive concentration of metal ions. Talanta 62, 1005-1028 (2004).

258. Bereczki, H. F., Daróczi, L., Fábián, I. \& Lázár, I. Sol-gel synthesis, characterization and catalytic activity of silica aerogels functionalized with copper(II) complexes of cyclen and cyclam. Microporous Mesoporous Mater. 234, 392-400 (2016).

259. Barreto, J. A., Matterna, M., Graham, B., Stephan, H. \& Spiccia, L. Synthesis, colloidal stability and ${ }^{64} \mathrm{Cu}$ labeling of iron oxide nanoparticles bearing different macrocyclic ligands. New J. Chem. 35, 2705-2712 (2011).

260. Ortega, F., Velez, E. \& Somanathan, R. Synthesis and use of reverse-phase silica gel for HPLC in undergraduate chemistry. J. Chem. Educ. 73, A26 (1996).

261. Fulmer, G. R. et al. NMR chemical shifts of trace impurities: common laboratory solvents, organics, and gases in deuterated solvents relevant to the organometallic chemist. Organometallics 29, 2176-2179 (2010).

262. Capeletti, L. B. \& Zimnoch, J. H. Fourier Transform Infrared and Raman characterization of silicabased materials. in Applications of Molecular Spectroscopy to Current Research in the Chemical and Biological Sciences (ed. Stauffer, M. T.) 3-22 (IntechOpen, 2016). doi:10.5772/64477

263. Tripp, C. P. \& Hair, M. L. Reaction of chloromethylsilanes with silica: a low-frequency infrared study. Langmuir 7, 923-927 (1991).

264. Wan, F., Li, C., Jiang, L. \& Li, Y. Synthesis and characterization of binuclear Zn(II)-cyclen complexes bridged by $\alpha, \omega$-bis(4-methylphenoxy) alkanes. Res. Chem. Intermed. 38, 2085-2096 (2012).

265. Parker, R. E. \& Isaacs, N. S. Mechanisms of epoxide reactions. Chem. Rev. 59, 737-799 (1959).

266. Williams, D. B. G. \& Cullen, A. Al(OTf) ${ }_{3}$-mediated epoxide ring-opening reactions: toward 
piperazine-derived physiologically active products. J. Org. Chem. 74, 9509-9512 (2009).

267. Williams, D. B. G. \& Lawton, M. Aluminium triflate: a remarkable Lewis acid catalyst for the ring opening of epoxides by alcohols. Org. Biomol. Chem. 3, 3269-3272 (2005).

268. Sepeur, S. Nanotechnology: Technical Basics and Applications. (Vincentz Network, 2008).

269. Lu, H.-T. Synthesis and characterization of amino-functionalized silica nanoparticles. Colloid J. 75, 311-318 (2013).

270. Teughels, W., Van Assche, N., Sliepen, I. \& Quirynen, M. Effect of material characteristics and/or surface topography on biofilm development. Clin. Oral Implants Res. 17, 68-81 (2006).

271. Kozlovsky, A., Artzi, Z., Moses, O., Kamin-Belsky, N. \& Greenstein, R. B.-N. Interaction of chlorhexidine with smooth and rough types of titanium surfaces. J. Periodontol. 77, 1194-1200 (2006).

272. Loesche, W. J. Role of Streptococcus mutans in human dental decay. Microbiol. Rev. 50, 353380 (1986).

273. Miyoshi, K. Surface Characterization Techniques: An Overview. (Glenn Research Center, 2002).

274. Scimeca, M., Bischetti, S., Lamsira, H. K., Bonfiglio, R. \& Bonanno, E. Energy dispersive X-ray (EDX) microanalysis: a powerful tool in biomedical research and diagnosis. Eur. J. Histochem. 62, 1-10 (2018).

275. Goldstein, J. I. et al. Scanning Electron Microscopy and X-ray Microanalysis. (Springer-Verlag New York, 2018). doi:10.1007/978-1-4939-6676-9

276. Stojilovic, N. Why can't we see hydrogen in X-ray photoelectron spectroscopy? J. Chem. Educ. 89, 1331-1332 (2012).

277. Konopka, J. Options for quantitative analysis of light elements by SEM/EDS. (Thermo Fisher Scientific Inc., 2013).

278. Inkson, B. J. 2 - Scanning electron microscopy (SEM) and transmission electron microscopy (TEM) for materials characterization. in Materials Characterization Using Nondestructive Evaluation (NDE) Methods (eds. Hübschen, G., Altpeter, I., Tschuncky, R. \& Herrmann, H.-G.) 17-43 (Woodhead Publishing, 2016).

279. Ni, C. Scanning electron microscopy (SEM). in Encyclopedia of Tribology (eds. Wang, Q. J. \& Chung, Y.-W.) 150, 2977-2982 (Springer US, 2013).

280. Newbury, D. E. \& Ritchie, N. W. M. Is scanning electron microscopy/energy dispersive X-ray spectrometry (SEM/EDS) quantitative? Scanning 35, 141-168 (2013).

281. Newbury, D. E. \& Ritchie, N. W. M. Performing elemental microanalysis with high accuracy and high precision by scanning electron microscopy/silicon drift detector energy-dispersive X-ray spectrometry (SEM/SDD-EDS). J. Mater. Sci. 50, 493-518 (2015). 
282. Briand, J. F. Marine antifouling laboratory bioassays: an overview of their diversity. Biofouling 25, 297-311 (2009).

283. Garrett, T. R., Bhakoo, M. \& Zhang, Z. Bacterial adhesion and biofilms on surfaces. Prog. Nat. Sci. 18, 1049-1056 (2008).

284. Wang, Y., Lee, S. M. \& Dykes, G. The physicochemical process of bacterial attachment to abiotic surfaces: challenges for mechanistic studies, predictability and the development of control strategies. Crit. Rev. Microbiol. 41, 452-464 (2015).

285. Hermansson, M. The DLVO theory in microbial adhesion. Colloids Surfaces B Biointerfaces 14, 105-119 (1999).

286. Harder, T. \& Yee, L. H. Bacterial adhesion and marine fouling. in Advances in Marine Antifouling Coatings and Technologies (eds. Hellio, C. \& Yebra, D.) 113-131 (Elsevier Science \& Technology, 2009). doi:10.1533/9781845696313.1.113

287. Donlan, R. M. Biofilms: microbial life on surfaces. Emerg. Infect. Dis. 8, 881-890 (2002).

288. Rijnaarts, H. H. M., Norde, W., Bouwer, E. J., Lyklema, J. \& Zehnder, A. J. B. Bacterial adhesion under static and dynamic conditions. Appl. Environ. Microbiol. 59, 3255-3265 (1993).

289. Dewanti, R. \& Wong, A. C. L. Influence of culture conditions on biofilm formation by Escherichia coli 0157:H7. Int. J. Food Microbiol. 26, 147-164 (1995).

290. Moodie, L. W. K. et al. Probing the structure-activity relationship of the natural antifouling agent polygodial against both micro- and macrofoulers by semisynthetic modification. J. Nat. Prod. 80, 515-525 (2017).

291. Cuadrado-Silva, C. T., Castellanos, L., Arévalo-Ferro, C. \& Osorno, O. E. Detection of quorum sensing systems of bacteria isolated from fouled marine organisms. Biochem. Syst. Ecol. 46, 101-107 (2013).

292. Nandhini, S. \& Revathi, K. Study on biofouling organisms present on the surface of boats in Royapuram, Chennai. Nat. Environ. Pollut. Technol. 15, 257-261 (2016).

293. Mittelman, M. W. et al. Test systems for determining antifouling coating efficacy using on-line detection of bioluminescence and fluorescence in a laminar-flow environment. J. Microbiol. Methods 18, 51-60 (1993).

294. Angell, P., Arrage, A. A., Mittelman, M. W. \& White, D. C. On line, non-destructive biomass determination of bacterial biofilms by fluorometry. J. Microbiol. Methods 18, 317-327 (1993).

295. Karunasagar, I., Otta, S. K. \& Karunasagar, I. Biofilm formation by Vibrio harveyi on surfaces. Aquaculture 140, 241-245 (1996).

296. Armstrong, E., Boyd, K. G., Pisacane, A., Peppiatt, C. J. \& Burgess, J. G. Marine microbial natural products in antifouling coatings. Biofouling 16, 215-224 (2000). 
297. Xu, Q., Barrios, C. A., Cutright, T. \& Newby, B. M. Z. Assessment of antifouling effectiveness of two natural product antifoulants by attachment study with freshwater bacteria. Environ. Sci. Pollut. Res. 12, 278-284 (2005).

298. Barrios, C. A., Xu, Q., Cutright, T. \& Newby, B. M. Z. Incorporating zosteric acid into silicone coatings to achieve its slow release while reducing fresh water bacterial attachment. Colloids Surfaces B Biointerfaces 41, 83-93 (2005).

299. Cassé, F. \& Swain, G. W. The development of microfouling on four commercial antifouling coatings under static and dynamic immersion. Int. Biodeterior. Biodegrad. 57, 179-185 (2006).

300. Santos Mendonça, R. C., Morelli, A. M. F., Pereira, J. A. M., de Carvalho, M. M. \& de Souza, N. L. Prediction of Escherichia coli 0157:H7 adhesion and potential to form biofilm under experimental conditions. Food Control 23, 389-396 (2012).

301. Danese, P. N., Pratt, L. A., Dove, S. L. \& Kolter, R. The outer membrane protein, Antigen 43, mediates cell-to-cell interactions within Escherichia coli biofilms. Mol. Microbiol. 37, 424-432 (2000).

302. O'Toole, G. A. et al. Genetic approaches to study of biofilms. Methods Enzymol. 310, 91-109 (1999).

303. Naves, P. et al. Measurement of biofilm formation by clinical isolates of Escherichia coli is method-dependent. J. Appl. Microbiol. 105, 585-590 (2008).

304. Mire, C. E., Tourjee, J. A., O'Brien, W. F., Ramanujachary, K. V. \& Hecht, G. B. Lead precipitation by Vibrio harveyi: evidence for novel quorum-sensing interactions. Appl. Environ. Microbiol. 70, 855-864 (2004).

305. Muazu, A. et al. Differential biofilm formation and chemical disinfection resistance of Escherichia coli on stainless steel and polystyrene tissue culture plate. Int. J. Res. Med. Sci. 3, 3300-3307 (2015).

306. Merritt, J. H., Kadouri, D. E. \& O’Toole, G. A. Growing and analyzing static biofilms. Curr. Protoc. Microbiol. Chapter 1, 1-29 (2005).

307. Epstein, A. K., Hochbaum, A. I., Kim, P. \& Aizenberg, J. Control of bacterial biofilm growth on surfaces by nanostructural mechanics and geometry. Nanotechnology 22, 1-18 (2011).

308. Herigstad, B., Hamilton, M. \& Heersink, J. How to optimize the drop plate method for enumerating bacteria. J. Microbiol. Methods 44, 121-129 (2001).

309. Wu, C., Lim, J. Y., Fuller, G. G. \& Cegelski, L. Disruption of E. coli amyloid-integrated biofilm formation at the air-liquid interface by a polysorbate surfactant. Langmuir 29, 920-926 (2013).

310. Pankey, G. A. \& Sabath, L. D. Clinical relevance of bacteriostatic versus bactericidal mechanisms of action in the treatment of Gram-positive bacterial infections. Clin. Infect. Dis. 38, 864-870 
(2004).

311. Day, D. Personal communication.

312. Santo, C. E., Taudte, N., Nies, D. H. \& Grass, G. Contribution of copper ion resistance to survival of Escherichia coli on metallic copper surfaces. Appl. Environ. Microbiol. 74, 977-986 (2008).

313. Pallavicini, P. et al. A monolayer of a $\mathrm{Cu}^{2+}$-tetraazamacrocyclic complex on glass as the adhesive layer for silver nanoparticles grafting, in the preparation of surface-active antibacterial materials. New J. Chem. 35, 1198-1201 (2011).

314. Warnes, S. L., Caves, V. \& Keevil, C. W. Mechanism of copper surface toxicity in Escherichia coli 0157:H7 and Salmonella involves immediate membrane depolarization followed by slower rate of DNA destruction which differs from that observed for Gram-positive bacteria. Environ. Microbiol. 14, 1730-1743 (2012).

315. Singh, A., Yeager, R. \& McFeters, G. A. Assessment of in vivo revival, growth, and pathogenicity of Escherichia coli strains after copper- and chlorine-induced injury. Appl. Environ. Microbiol. 52, 832-837 (1986).

316. Grey, B. \& Steck, T. R. Concentrations of copper thought to be toxic to Escherichia coli can induce the viable but nonculturable condition. Appl. Environ. Microbiol. 67, 5325-5327 (2001).

317. Lindgren, J. F. et al. Copper release rate needed to inhibit fouling on the west coast of Sweden and control of copper release using zinc oxide. Biofouling 34, 453-463 (2018).

318. He, T. et al. Polydopamine assisted immobilisation of copper(II) on titanium for antibacterial applications. Mater. Technol. 30, B68-B72 (2015).

319. Perrin, C. et al. Nickel promotes biofilm formation by Escherichia coli K-12 strains that produce curli. Appl. Environ. Microbiol. 75, 1723-1733 (2009).

320. Long, T. E. Repurposing thiram and disulfiram as antibacterial agents for multidrug-resistant Staphylococcus aureus infections. Antimicrob. Agents Chemother. 61, 1-8 (2017).

321. Trueba, F. J. \& Woldringh, C. L. Changes in cell diameter during the division cycle of Escherichia coli. J. Bacteriol. 142, 869-878 (1980).

322. Law, B. Pharmaceuticals/basic drugs: liquid chromatography. in Encyclopedia of Separation Science (ed. Wilson, I. D.) 3701-3708 (Elsevier Science Ltd., 2000).

323. Arrage, A. A. et al. On-line monitoring of antifouling and fouling-release surfaces using bioluminescence and fluorescence measurements during laminar flow. J. Ind. Microbiol. 15, 277-282 (1995).

324. Sarkheil, M., Sourinejad, I., Mirbakhsh, M., Kordestani, D. \& Johari, S. A. Antibacterial activity of immobilized silver nanoparticles on TEPA-Den-SiO ${ }_{2}$ against shrimp pathogen, Vibrio sp. Persian1. Aquac. Res. 48, 2120-2132 (2017). 
325. Brandt, S. \& Podivinsky, E. Resuscitation of putative viable but non-culturable (VNC) foodborne bacteria of significance to New Zealand. 1-44 (2008).

326. Wang, L. et al. Use of bacteriophages to control Escherichia coli 0157:H7 in domestic ruminants, meat products, and fruits and vegetables. Foodborne Pathog. Dis. 14, 483-493 (2017).

327. M63 Medium Broth. 1-3 (2015).

328. Smith, A. C. \& Hussey, M. A. Gram stain protocols. 1-9 (2005).

329. Sadek, B. et al. Non-imidazole histamine H3 receptor ligands incorporating antiepileptic moieties. Eur. J. Med. Chem. 77, 269-279 (2014).

330. Yang, Z. D., Song, Z. W., Ren, J., Yang, M. J. \& Li, S. Improved thin-layer chromatography bioautographic assay for the detection of actylcholinesterase inhibitors in plants. Phytochem. Anal. 22, 509-515 (2011).

331. Gao, W., Li, Q., Chen, J., Wang, Z. \& Hua, C. Total synthesis of six 3,4-unsubstituted coumarins. Molecules 18, 15613-15623 (2013).

332. Kozlov, N. G. \& Basalaeva, L. I. Vanillin esters in reactions with indan-1,3-dione. Chem. Heterocycl. Compd. 42, 1223-1228 (2006).

333. Bois, L. et al. Functionalized silica for heavy metal ions adsorption. Colloids Surfaces $A$ Physicochem. Eng. Asp. 221, 221-230 (2003).

334. Cui, Y. et al. A dual solvent evaporation route for preserving carbon nanoparticle fluorescence in silica gel and producing white light-emitting diodes. Mater. Chem. Front. 1, 387-393 (2017).

335. Li, J. et al. Liquid oxygen compatibility and cryogenic mechanical properties of a novel phosphorous/silicon containing epoxy-based hybrid. RSC Adv. 6, 91012-91023 (2016). 


\section{APPENDIX}

\subsection{CHAPTER 2}
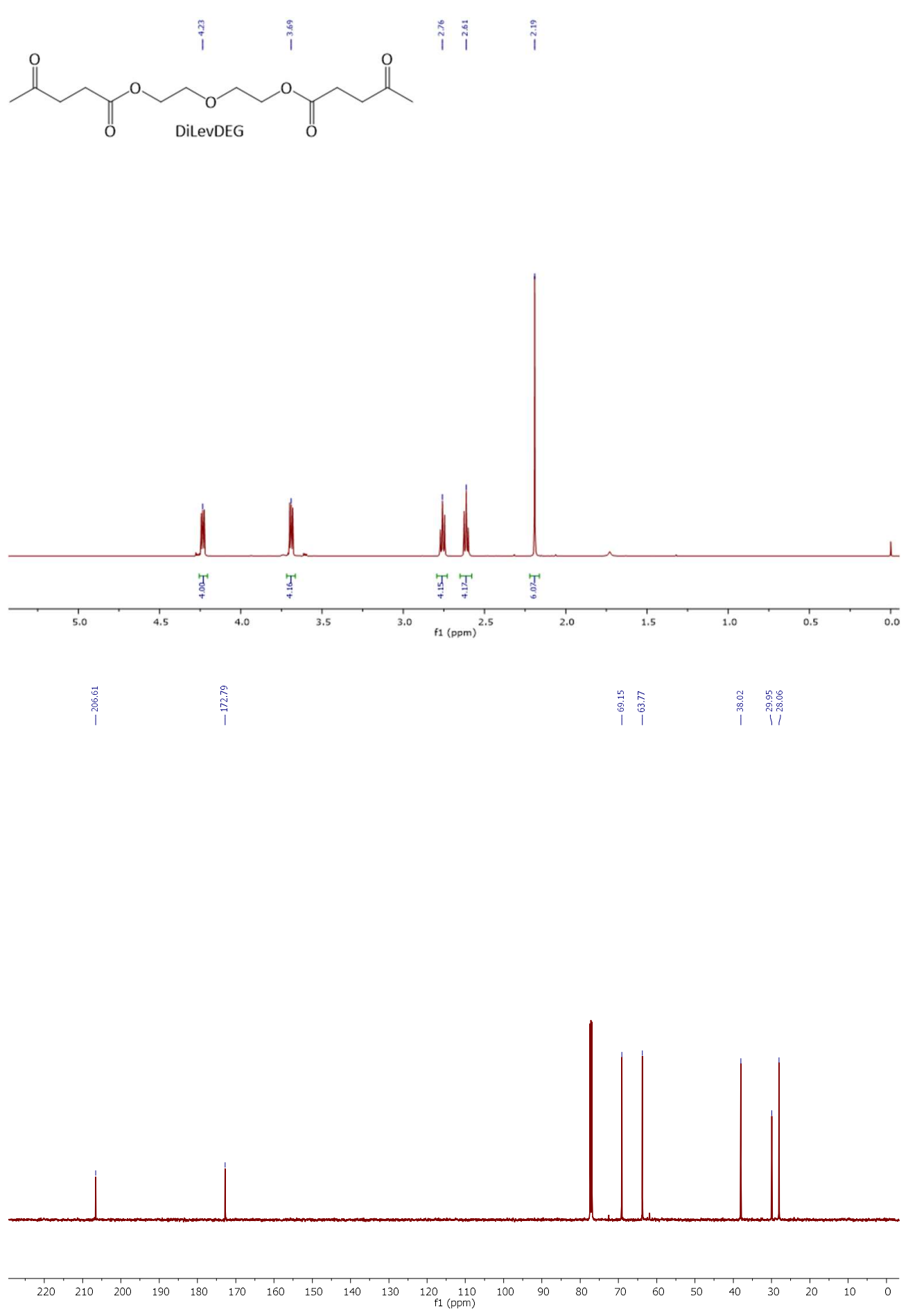

Figure 8.1. ${ }^{1} \mathrm{H}$ and ${ }^{13} \mathrm{C}$ NMR spectra $\left(\mathrm{CDCl}_{3}\right)$ of DiLevDEG $\left(\delta_{\mathrm{H}} 1.73 \mathrm{ppm}=\right.$ water; $\delta_{\mathrm{H}} 4.26 \& 3.60 \mathrm{ppm}=$ DEG). 

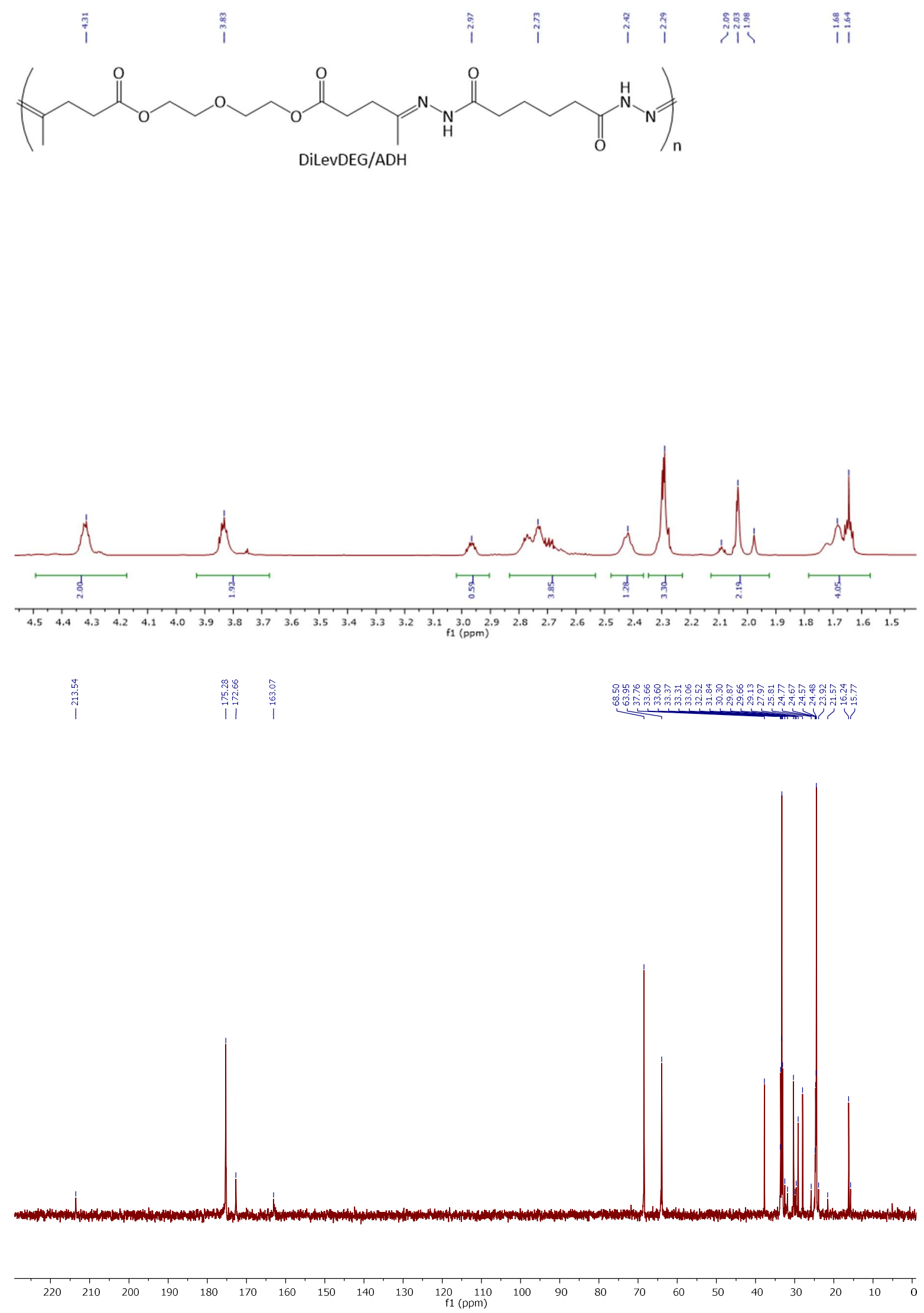

Figure 8.2. ${ }^{1} \mathrm{H}$ and ${ }^{13} \mathrm{C}$ NMR spectra (crude; $\mathrm{D}_{2} \mathrm{O}$ ) of DiLevDEG/ADH polyacylhydrazone. 

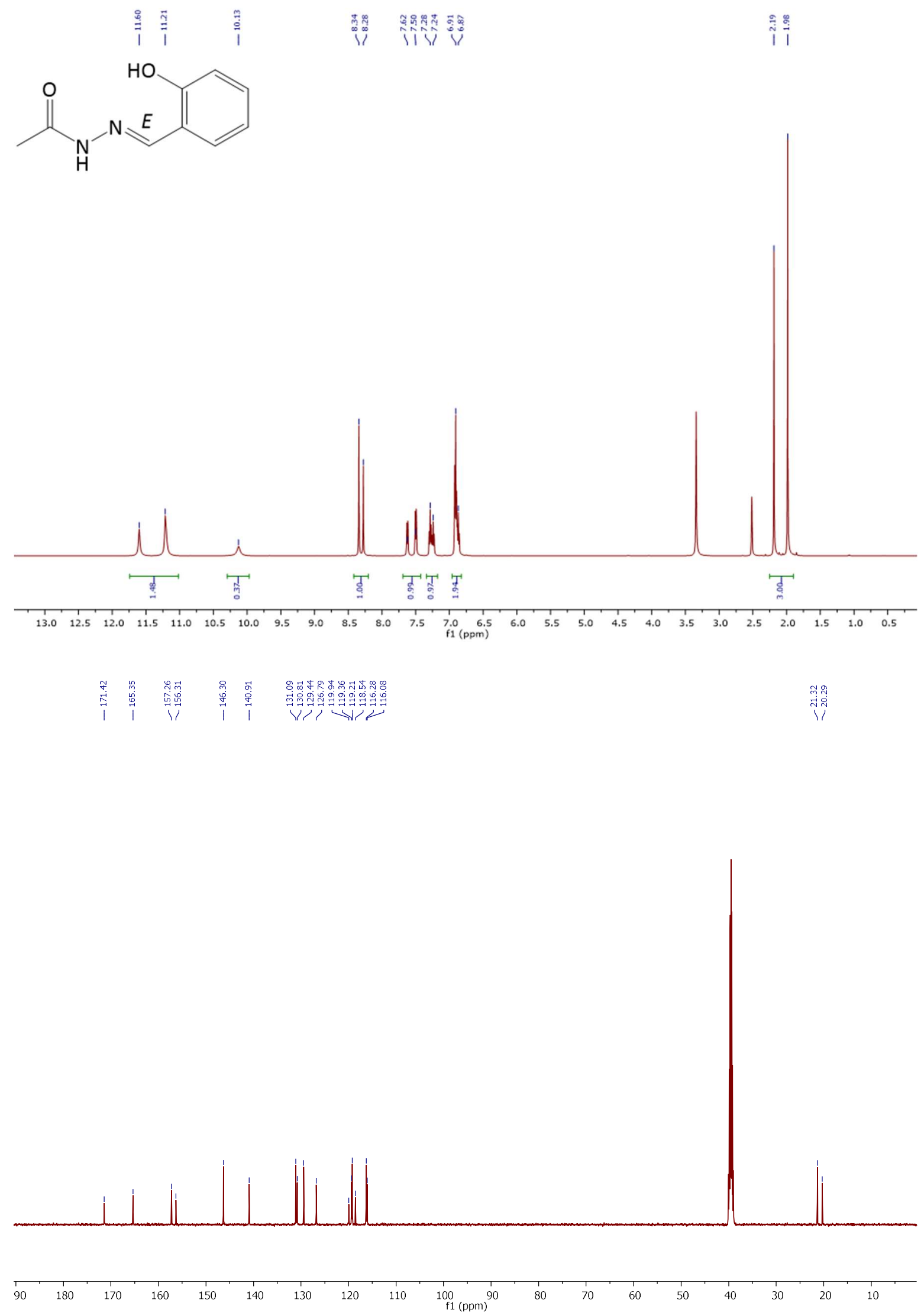

Figure 8.3. ${ }^{1} \mathrm{H}$ and ${ }^{13} \mathrm{C}$ NMR spectra (DMSO- $\left.d_{6}\right)$ of $1 \mathrm{a}\left(\delta_{\mathrm{H}} 3.33 \mathrm{ppm}=\right.$ water). 
<smiles>[Z]C(=O)N/N=C/c1ccccc1O</smiles>

Table 8.1. Peak assignments in ${ }^{1} \mathrm{H}$ and ${ }^{13} \mathrm{C}$ NMR spectra of 1a.

\begin{tabular}{lll}
\hline Position & $\delta_{c^{a}, \mathrm{~b}}$, Type & $\delta_{\mathrm{H}^{\mathrm{a}, \mathrm{c}}}(\mathrm{J}$ in Hz$)$ \\
\hline 1 & $146.3,140.9, \mathrm{CH}$ & $8.34, \mathrm{~s} ; 8.27, \mathrm{~s}$ \\
\hline 2 & $119.9,118.5, \mathrm{C}$ & - \\
\hline 3 & $157.3,156.3, \mathrm{C}$ & - \\
\hline 4 & $116.3,116.1, \mathrm{CH}$ & $6.94-6.83, \mathrm{~m}$ \\
\hline 5 & $131.1,130.8, \mathrm{CH}$ & $7.28, \mathrm{td}(7.6,1.6) ; 7.24, \mathrm{td}(7.5,1.6)$ \\
\hline 6 & $119.4,119.2, \mathrm{CH}$ & $6.94-6.83, \mathrm{~m}$ \\
\hline 7 & $129.4,126.8, \mathrm{CH}$ & $7.62, \mathrm{dd}(7.8,1.7) ; 7.49, \mathrm{dd}(7.5,1.7)$ \\
\hline $1^{\prime}$ & $171.4,165.4, \mathrm{C}$ & - \\
\hline $2^{\prime}$ & $21.3,20.3, \mathrm{CH}$ & $2.18, \mathrm{~s} ; 1.98, \mathrm{~s}$ \\
\hline $\mathrm{OH}$ & - & $10.13, \mathrm{~s}$ \\
$\mathrm{NH}$ & - & $11.60, \mathrm{~s} ; 11.21, \mathrm{~s}$ \\
\hline
\end{tabular}

${ }^{a}$ Where multiple values are provided, rotamers were observed in solution.

b $126 \mathrm{MHz}$, Referenced to DMSO- $d_{6}\left(\delta_{c} 39.52 \mathrm{ppm}\right){ }^{261}$ Elucidated/confirmed by DEPT, HSQC, and HMBC.

c $500 \mathrm{MHz}$, Referenced to TMS $\left(\delta_{\mathrm{H}} 0.00 \mathrm{ppm}\right)$. Elucidated/confirmed by HSQC, HMBC, and COSY. 

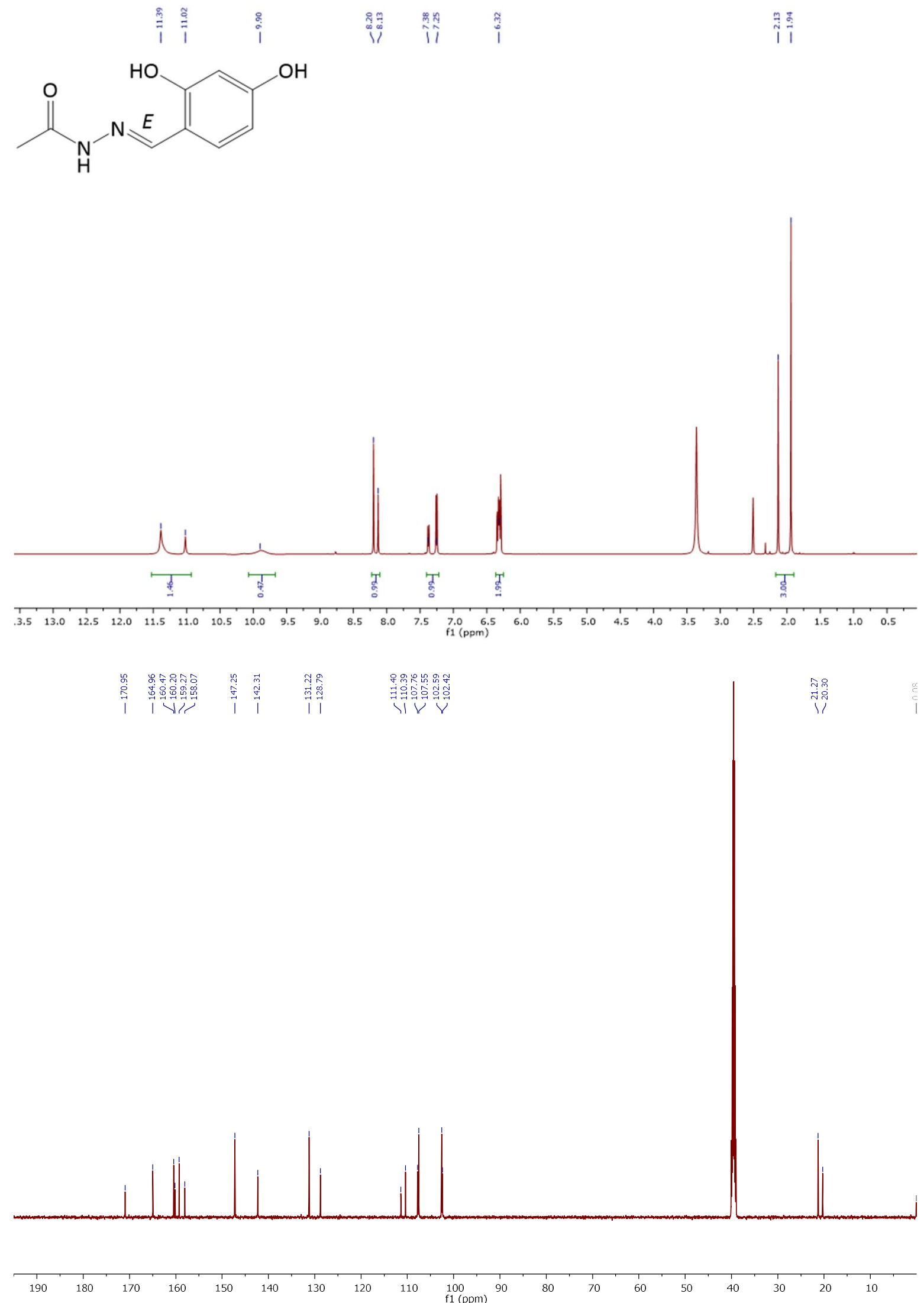

Figure 8.4. ${ }^{1} \mathrm{H}$ and ${ }^{13} \mathrm{C}$ NMR spectra (DMSO- $\left.d_{6}\right)$ of $\mathbf{1 b}\left(\delta_{\mathrm{H}} 3.36 \mathrm{ppm}=\right.$ water; $\delta_{\mathrm{H}} 2.32 \mathrm{ppm}=$ contaminant). 


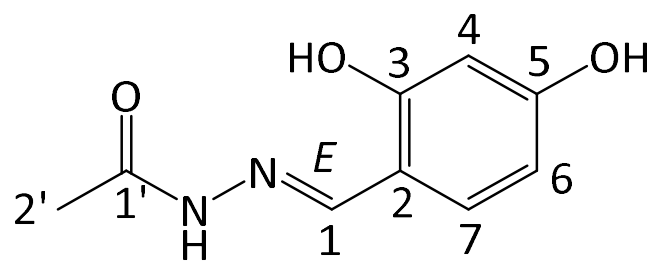

Table 8.2. Peak assignments in ${ }^{1} \mathrm{H}$ and ${ }^{13} \mathrm{C}$ NMR spectra of $\mathbf{1 b}$.

\begin{tabular}{lll}
\hline Position & $\delta_{c^{a, b}, \text { Type }}$ & $\delta_{H^{a, c}}(J$ in Hz $)$ \\
\hline 1 & $147.3,142.3, \mathrm{CH}$ & $8.20, \mathrm{~s} ; 8.13, \mathrm{~s}$ \\
\hline 2 & $111.4,110.4, \mathrm{C}$ & - \\
\hline 3 or 5 & $160.5,160.2, \mathrm{C}$ & - \\
\hline 4 & $102.6,102.4, \mathrm{CH}$ & $6.37-6.27, \mathrm{~m}$ \\
\hline 5 or 3 & $159.3,158.1, \mathrm{C}$ & - \\
\hline 6 & $131.2,128.8, \mathrm{CH}$ & $7.38, \mathrm{~d}(9.1) ; 7.25, \mathrm{~d}(8.4)$ \\
\hline 7 & $107.8,107.6, \mathrm{CH}$ & $6.37-6.27, \mathrm{~m}$ \\
\hline $1^{\prime}$ & $171.0,165.0, \mathrm{C}$ & - \\
\hline $2^{\prime}$ & $21.3,20.3, \mathrm{CH}_{3}$ & $2.13, \mathrm{~s} ; 1.94, \mathrm{~s}$ \\
\hline 3 or $5 \mathrm{OH}$ & - & $9.89, \mathrm{~s}$ \\
\hline NH & - & $11.39, \mathrm{~s} ; 11.02, \mathrm{~s}$ \\
\hline
\end{tabular}

${ }^{a}$ Where multiple values are provided, rotamers were observed in solution.

b $126 \mathrm{MHz}$, Referenced to DMSO- $d_{6}(\delta 39.52 \mathrm{ppm}) .^{261}$ Elucidated/confirmed by DEPT, HSQC, and HMBC.

c $500 \mathrm{MHz}$, Referenced to TMS ( $\delta 0.00$ ppm). Elucidated/confirmed by HSQC, HMBC, and COSY. 
<smiles>COC(=O)CC/C(C)=N/NC(C)=O</smiles>
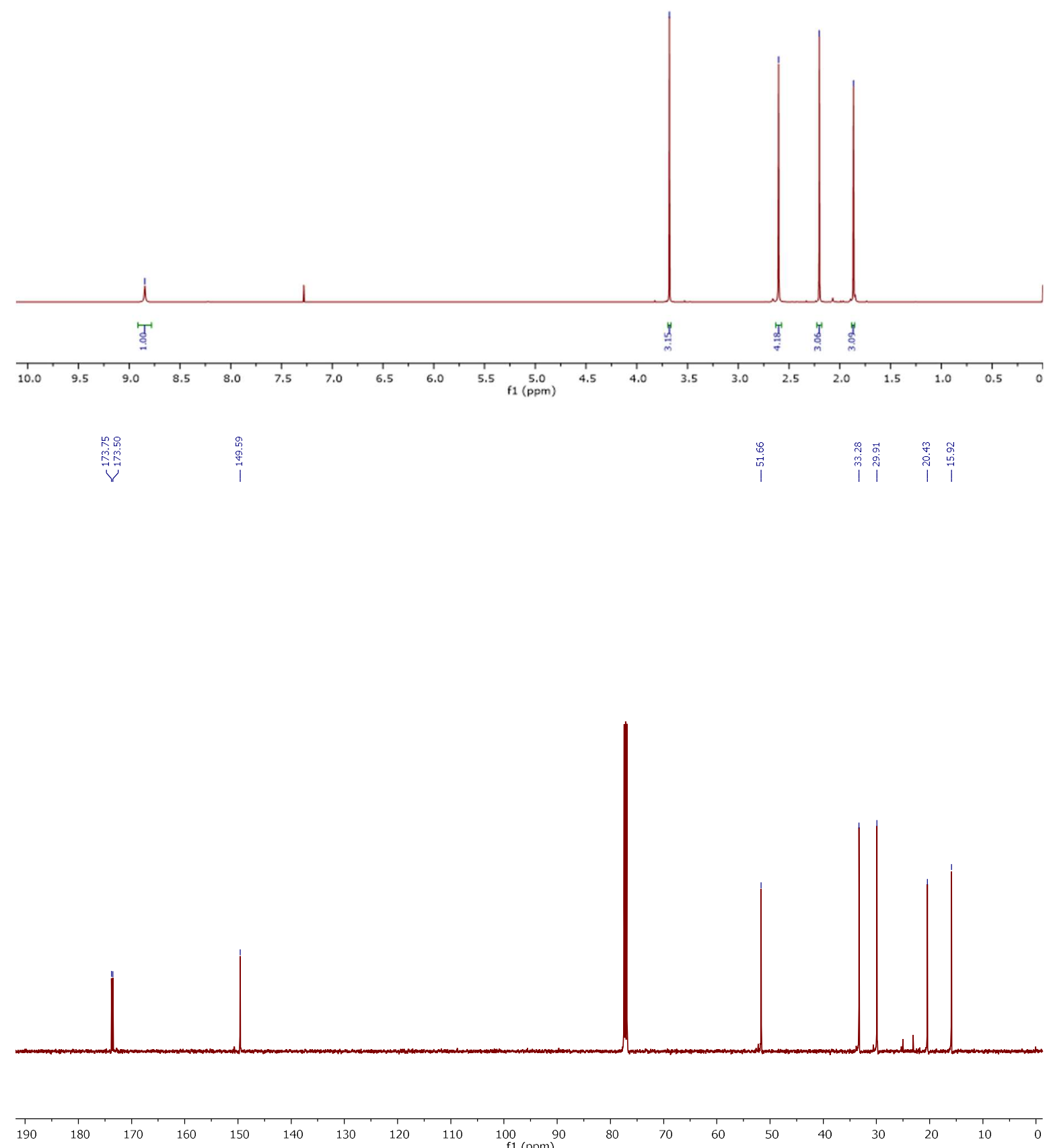

Figure 8.5. ${ }^{1} \mathrm{H}$ and ${ }^{13} \mathrm{C}$ NMR spectra $\left(\mathrm{CDCl}_{3}\right)$ of $\mathbf{1 i}$. 
<smiles>[Z]C(=O)NN=C(I)CCC(=O)OC</smiles>

Table 8.3. Peak assignments in ${ }^{1} \mathrm{H}$ and ${ }^{13} \mathrm{C}$ NMR spectra of $\mathbf{1 i}$.

\begin{tabular}{|c|c|c|}
\hline Position & $\delta_{c}{ }^{a}$, Type & $\delta_{H}^{b}(J$ in $\mathrm{Hz})$ \\
\hline 1 & $15.9, \mathrm{CH}_{3}$ & $1.87, \mathrm{~s}$ \\
\hline 2 & 149.6, C & - \\
\hline 3 & $33.3, \mathrm{CH}_{2}$ & $2.63-2.57, \mathrm{~m}$ \\
\hline 4 & $29.9, \mathrm{CH}_{2}$ & $2.63-2.57, \mathrm{~m}$ \\
\hline 5 & $173.5, \mathrm{C}$ & - \\
\hline 6 & $51.7, \mathrm{CH}_{3}$ & $3.68, \mathrm{~s}$ \\
\hline $1^{\prime}$ & $173.8, C$ & - \\
\hline $2^{\prime}$ & $20.4, \mathrm{CH}_{3}$ & $2.20, \mathrm{~s}$ \\
\hline $\mathrm{NH}$ & - & $8.84, s$ \\
\hline
\end{tabular}


<smiles>CCOC(=O)CCC(C)=NNC(C)=O</smiles>
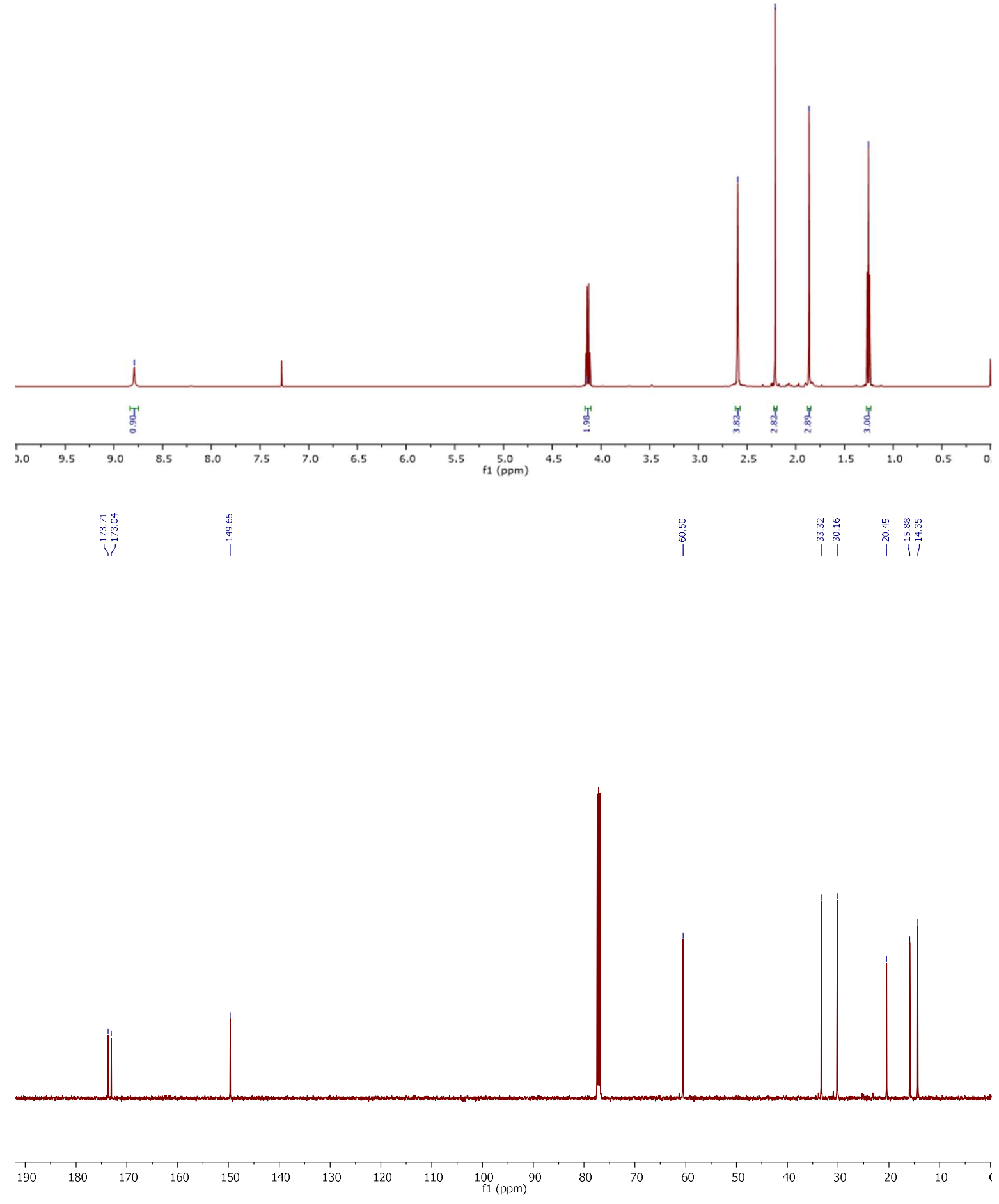

Figure 8.6. ${ }^{1} \mathrm{H}$ and ${ }^{13} \mathrm{C}$ NMR spectra $\left(\mathrm{CDCl}_{3}\right)$ of $\mathbf{1 j}$. 


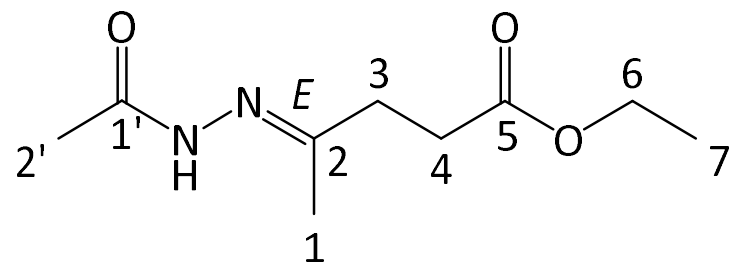

Table 8.4. Peak assignments in ${ }^{1} \mathrm{H}$ and ${ }^{13} \mathrm{C}$ NMR spectra of $\mathbf{1 j}$.

\begin{tabular}{lll}
\hline Position & $\delta_{c^{a}}{ }^{2}$, Type & $\delta_{\mathrm{H}^{\mathrm{b}}}(\mathrm{J}$ in Hz$)$ \\
\hline 1 & $15.9, \mathrm{CH}_{3}$ & $1.86, \mathrm{~s}$ \\
\hline 2 & $149.7, \mathrm{C}$ & - \\
\hline 3 & $33.3, \mathrm{CH}_{2}$ & $2.62-2.58, \mathrm{~m}$ \\
\hline 4 & $30.2, \mathrm{CH}_{2}$ & $2.62-2.58, \mathrm{~m}$ \\
\hline 5 & $173.0, \mathrm{C}$ & - \\
\hline 6 & $60.5, \mathrm{CH}_{2}$ & $4.13, \mathrm{q}(7.1)$ \\
\hline 7 & $14.4, \mathrm{CH}_{3}$ & $1.25, \mathrm{t}(7.1)$ \\
$1^{\prime}$ & $173.7, \mathrm{C}$ & - \\
$2^{\prime}$ & $20.5, \mathrm{CH}_{3}$ & $2.21, \mathrm{~s}$ \\
$\mathrm{NH}$ & - & $8.79, \mathrm{~s}$ \\
\hline
\end{tabular}

a $126 \mathrm{MHz}$, Referenced to $\mathrm{CDCl}_{3}(\delta 77.16 \mathrm{ppm}){ }^{261}$ Elucidated/confirmed by DEPT, HSQC, and HMBC.

b $500 \mathrm{MHz}$, Referenced to TMS ( $\delta 0.00 \mathrm{ppm})$. Elucidated/confirmed by HSQC, HMBC, and COSY. 

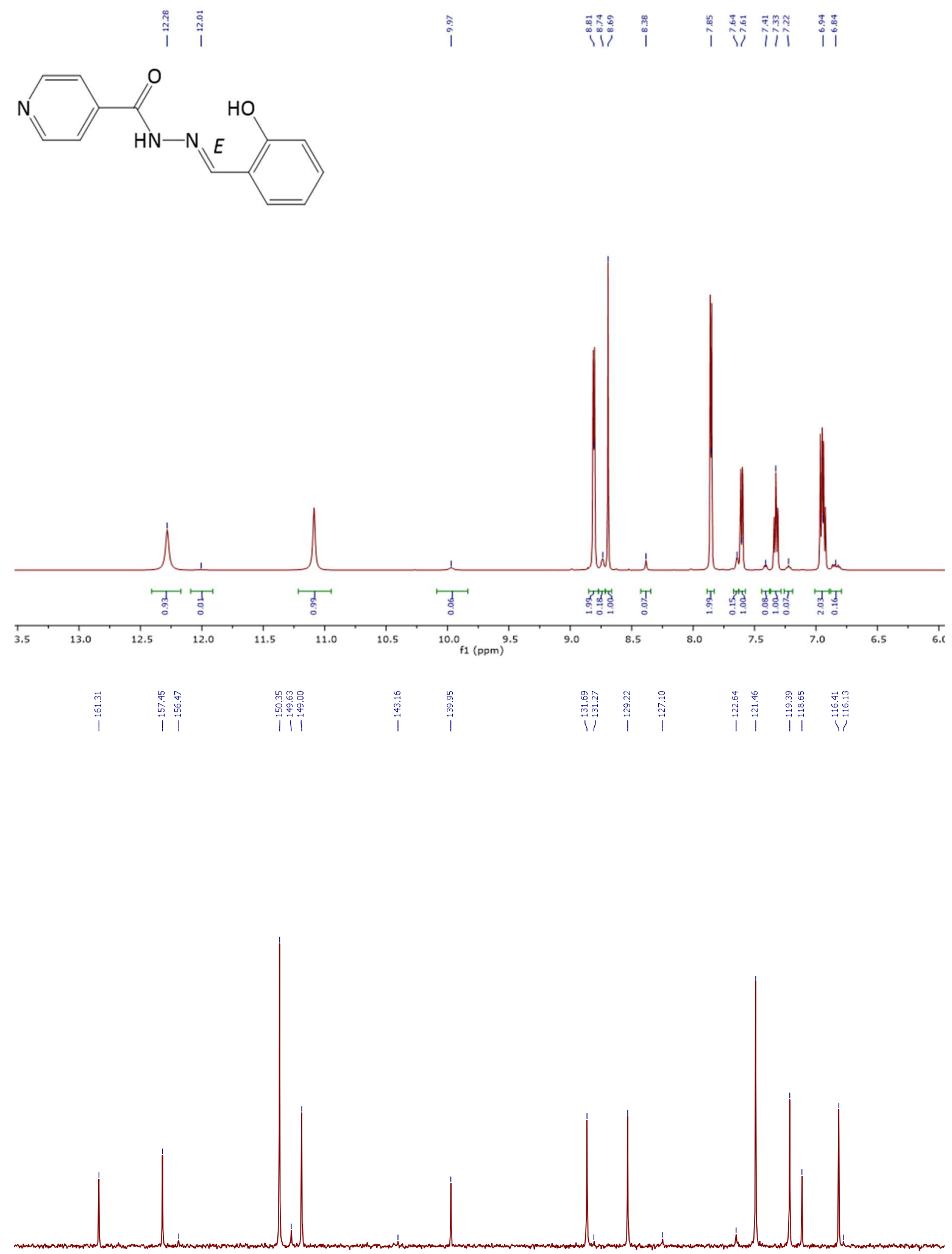

$\begin{array}{lllllllllllllllllllllllllllllllllll}166 & 164 & 162 & 160 & 158 & 156 & 154 & 152 & 150 & 148 & 146 & 144 & 142 & 140 & 138 & 136 & 134 & 132 & 130 & 128 & 126 & 124 & 122 & 120 & 118 & 116 & 114 & 112 & 1\end{array}$

Figure 8.7. ${ }^{1} \mathrm{H}$ and ${ }^{13} \mathrm{C}$ NMR spectra (DMSO- $d_{6}$ ) of $2 \mathrm{a}$. 


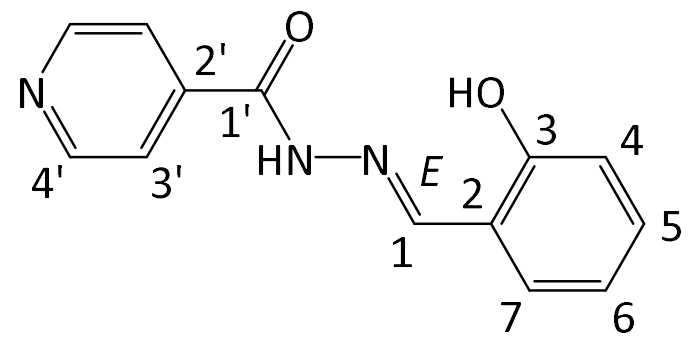

Table 8.5. Peak assignments in ${ }^{1} \mathrm{H}$ and ${ }^{13} \mathrm{C}$ NMR spectra of $2 \mathrm{a}$.

\begin{tabular}{lll}
\hline Position & $\boldsymbol{\delta}_{\mathrm{C}^{\mathrm{a}, \mathrm{b}}, \text { Type }}$ & $\boldsymbol{\delta}_{\mathrm{H}^{\mathrm{acc}}}(\boldsymbol{J}$ in Hz$)$ \\
\hline 1 & $149.0,143.2, \mathrm{CH}$ & $8.69, \mathrm{~s} ; 8.38, \mathrm{~s}$ \\
\hline 2 & $118.7, \mathrm{C}$ & - \\
\hline 3 & $157.5,156.5, \mathrm{C}$ & - \\
\hline 4 & $116.4,116.1, \mathrm{CH}$ & $6.98-6.90, \mathrm{~m} ; 6.88-6.79, \mathrm{~m}$ \\
\hline 5 & $131.7,131.1, \mathrm{CH}$ & $7.33, \mathrm{td}(7.4,1.6) ; 7.22, \mathrm{t}(7.3)$ \\
\hline 6 & $119.4, \mathrm{CH}$ & $6.98-6.90, \mathrm{~m} ; 6.88-6.79, \mathrm{~m}$ \\
\hline 7 & $129.2,127.1, \mathrm{CH}$ & $7.61, \mathrm{dd}(7.7,1.8) ; 7.41, \mathrm{~d}(7.5)$ \\
\hline $1^{\prime}$ & $161.3, \mathrm{C}$ & - \\
\hline $2^{\prime}$ & $140.0, \mathrm{C}$ & - \\
\hline $3^{\prime}$ & $122.6,121.5, \mathrm{CH}$ & $7.85, \mathrm{dd}(4.4,1.7) ; 7.64, \mathrm{~d}(4.6)$ \\
\hline $4^{\prime}$ & $150.4,149.6, \mathrm{CH}$ & $8.81, \mathrm{dd}(4.5,1.6) ; 8.74, \mathrm{~d}(4.3)$ \\
\hline $\mathrm{OH}$ & - & $11.09, \mathrm{~s} ; 9.97, \mathrm{~s}$ \\
\hline $\mathrm{NH}$ & - & $12.28, \mathrm{~s} ; 12.01, \mathrm{~s}$ \\
\hline
\end{tabular}

a Where multiple values are provided, rotamers were observed in solution.

b $126 \mathrm{MHz}$, Referenced to DMSO- $d_{6}(\delta 39.52 \mathrm{ppm}) .{ }^{261}$ Elucidated/confirmed by HSQC.

c $500 \mathrm{MHz}$, Referenced to TMS ( $\delta 0.00 \mathrm{ppm}$ ). Elucidated/confirmed by HSQC and COSY. 


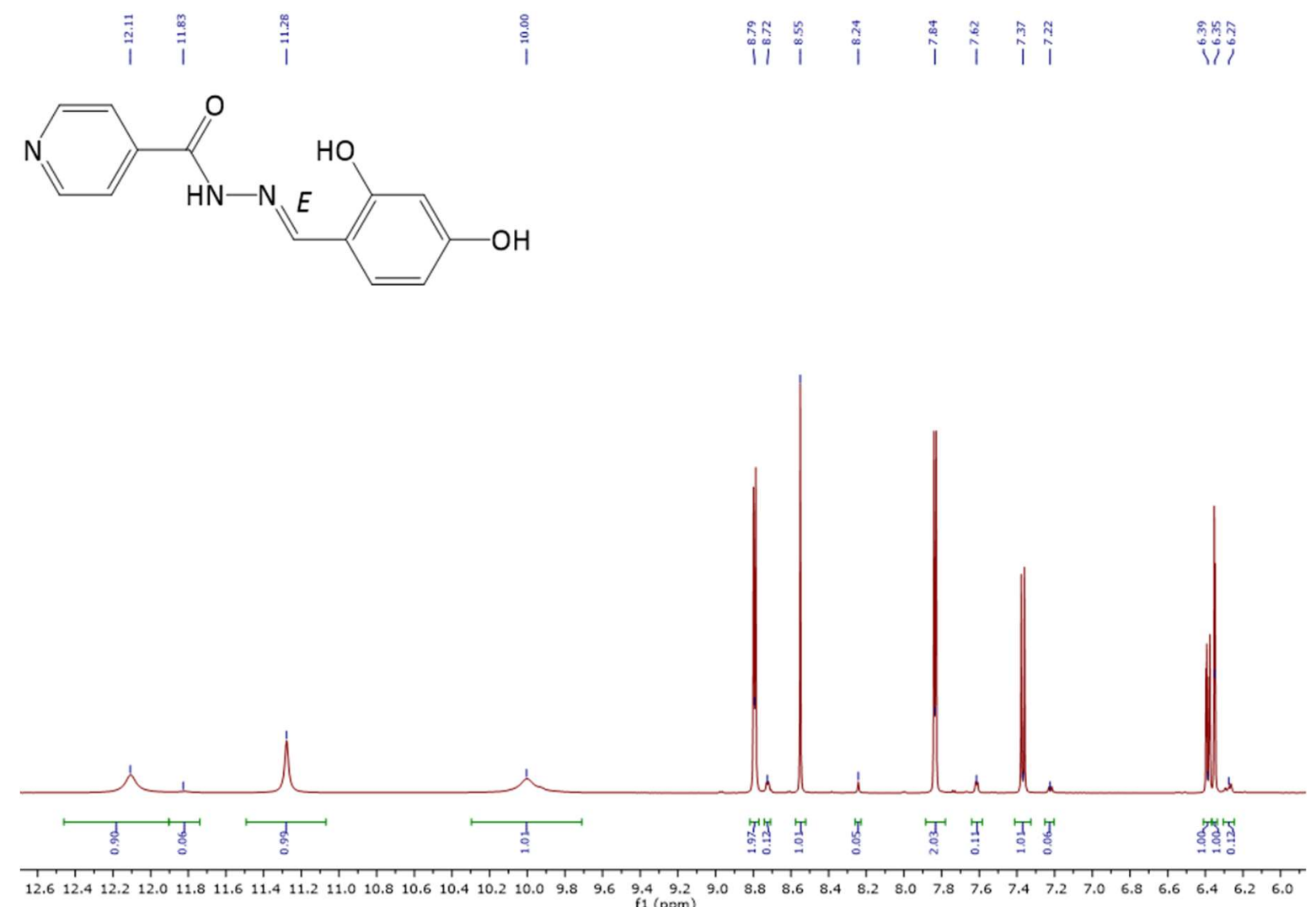
$\begin{array}{llllllllllllllllllllllllllllllllllllllllllllllll}12.6 & 12.4 & 12.2 & 12.0 & 11.8 & 11.6 & 11.4 & 11.2 & 11.0 & 10.8 & 10.6 & 10.4 & 10.2 & 10.0 & 9.8 & 9.6 & 9.4 & 9.2 & 9.0 & 8.8 & 8.6 & 8.4 & 8.2 & 8.0 & 7.8 & 7.6 & 7.4 & 7.2 & 7.0 & 6.8 & 6.6 & 6.4 & 6.2 & 6.0\end{array}$

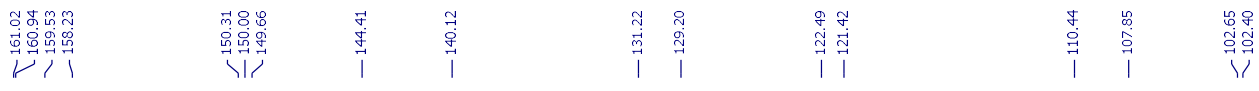
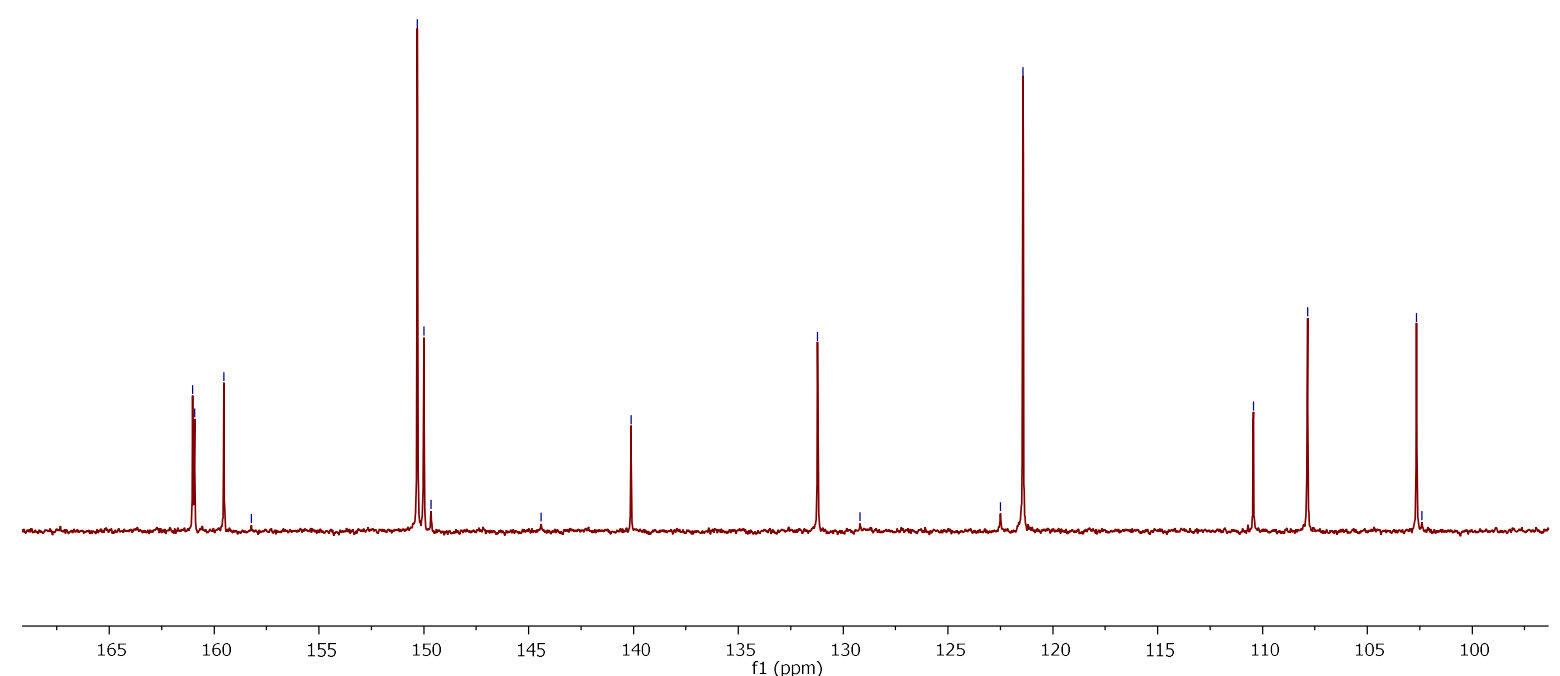

Figure 8.8. ${ }^{1} \mathrm{H}$ and ${ }^{13} \mathrm{C}$ NMR spectra (DMSO- $d_{6}$ ) of $\mathbf{2} \mathbf{b}$. 


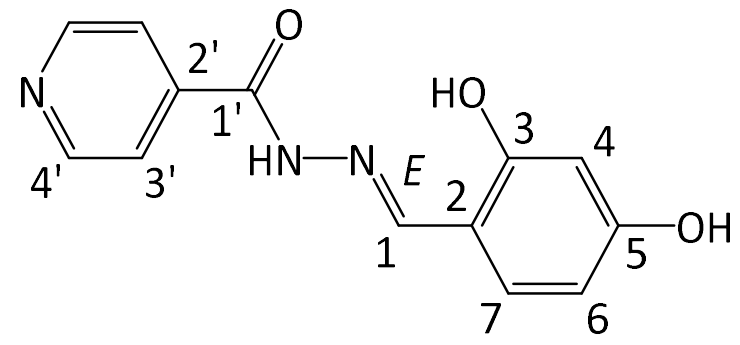

Table 8.6. Peak assignments in ${ }^{1} \mathrm{H}$ and ${ }^{13} \mathrm{C}$ NMR spectra of $\mathbf{2} \mathbf{b}$.

\begin{tabular}{|c|c|c|}
\hline Position & $\delta_{c}^{a, b}$, Type & $\boldsymbol{\delta}_{\mathrm{H}}^{\mathrm{a}, \mathrm{c}}(\boldsymbol{J}$ in $\mathrm{Hz})$ \\
\hline 1 & $150.0,144.4, \mathrm{CH}$ & $8.55, \mathrm{~s} ; 8.24, \mathrm{~s}$ \\
\hline 2 & $110.4, \mathrm{C}$ & - \\
\hline 3 & $161.0,160.9,159.5$, or $158.2, C$ & - \\
\hline 4 & $102.7,102.4, \mathrm{CH}$ & $6.35, \mathrm{~d}(2.4) ; 6.30-6.25, \mathrm{~m}$ \\
\hline 5 & $161.0,160.9,159.5$, or $158.2, \mathrm{C}$ & - \\
\hline 6 & $107.9, \mathrm{CH}$ & $6.39, \mathrm{dd}(8.4,2.3) ; 6.30-6.25, \mathrm{~m}$ \\
\hline 7 & $131.2,129.2, \mathrm{CH}$ & $7.37, \mathrm{~d}(8.4) ; 7.22, \mathrm{~d}(8.3)$ \\
\hline $1^{\prime}$ & $161.0,160.9,159.5$, or $158.2, \mathrm{C}$ & - \\
\hline $2^{\prime}$ & $140.1, \mathrm{C}$ & - \\
\hline $3^{\prime}$ & $122.5,121.4, \mathrm{CH}$ & $7.84, \mathrm{dd}(4.5,1.5) ; 7.61, \mathrm{~d}(5.1)$ \\
\hline $4^{\prime}$ & $150.3,149.7, \mathrm{CH}$ & $8.79, \mathrm{dd}(4.4,1.6) ; 8.72, \mathrm{~d}(5.3)$ \\
\hline $3 \mathrm{OH}$ & - & $12.11, \mathrm{~s} ; 11.82, \mathrm{~s}$ \\
\hline $5 \mathrm{OH}$ & - & $10.00, \mathrm{~s}$ \\
\hline $\mathrm{NH}$ & - & $11.28, \mathrm{~s}$ \\
\hline
\end{tabular}

${ }^{a}$ Where multiple values are provided, rotamers were observed in solution.

b $126 \mathrm{MHz}$, Referenced to DMSO- $d_{6}(\delta 39.52 \mathrm{ppm}) .^{261}$ Elucidated/confirmed by HSQC.

c $500 \mathrm{MHz}$, Referenced to TMS ( $\delta 0.00 \mathrm{ppm})$. Elucidated/confirmed by HSQC and COSY. 

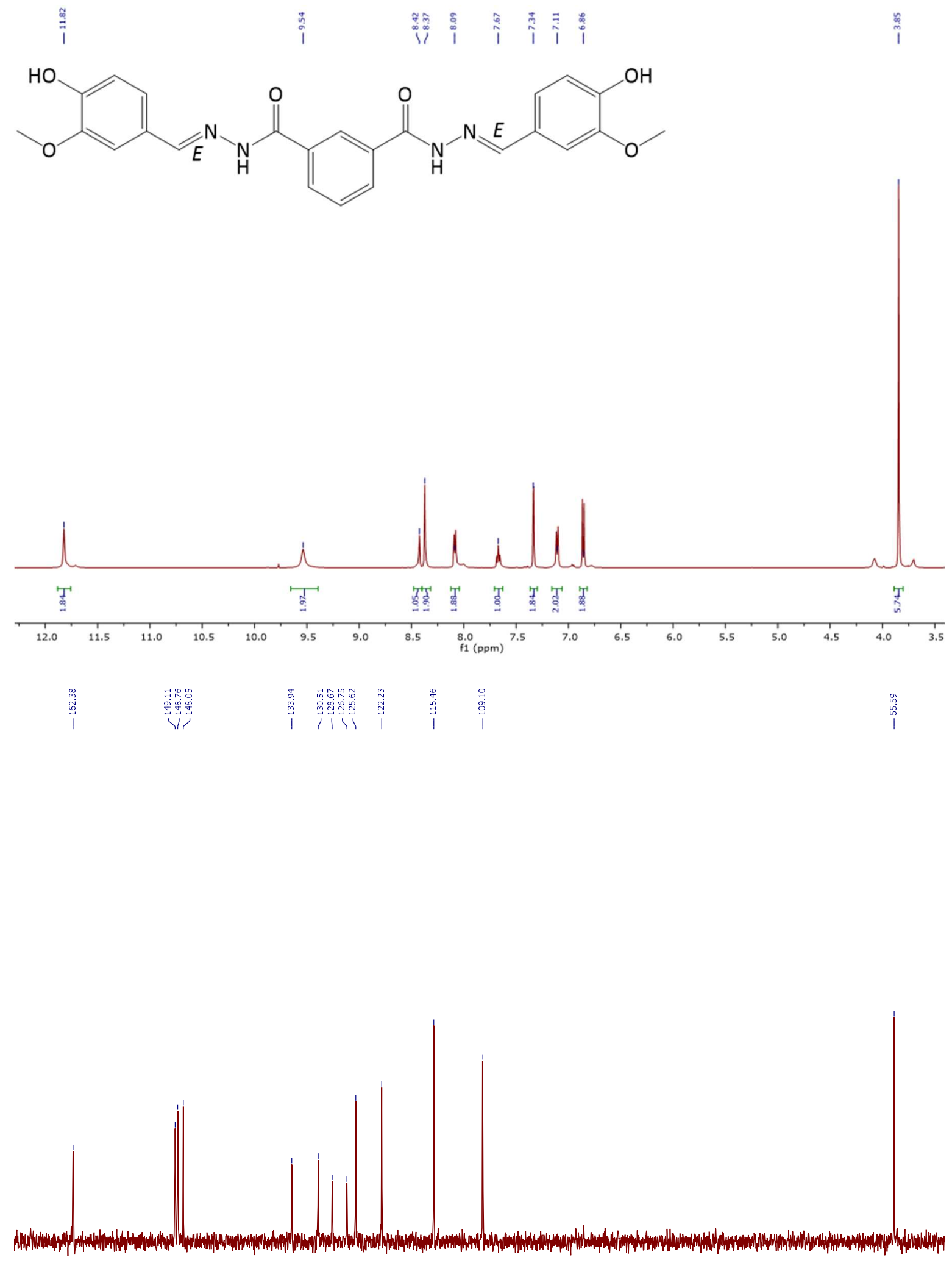

$\begin{array}{lllllllllllllllllllllllllllll}70 & 165 & 160 & 155 & 150 & 145 & 140 & 135 & 130 & 125 & 120 & 115 & \underset{f 1}{110} & 105 & 100 & 95 & 90 & 85 & 80 & 75 & 70 & 65 & 60 & 55 & 50\end{array}$

Figure 8.9. ${ }^{1} \mathrm{H}$ and ${ }^{13} \mathrm{C}$ NMR spectra (DMSO- $d_{6}$ ) of $3 \mathrm{c}$. 
<smiles>COc1cc(/C=N/NC(=O)c2cccc(C(=O)N/N=C/c3ccc(O)c(OS)c3)c2)ccc1O</smiles>

Table 8.7. Peak assignments in ${ }^{1} \mathrm{H}$ and ${ }^{13} \mathrm{C}$ NMR spectra of $\mathbf{3 c}$.

\begin{tabular}{lll}
\hline Position & $\delta_{\mathrm{C}^{\mathrm{a}}}$, Type & $\boldsymbol{\delta}_{\mathrm{H}^{\mathrm{b}}}(\mathrm{J}$ in Hz$)$ \\
\hline 1 & $148.8, \mathrm{CH}$ & $8.37, \mathrm{~s}$ \\
\hline 2 & $125.6, \mathrm{C}$ & - \\
\hline 3 & $109.1, \mathrm{CH}$ & $7.34, \mathrm{~s}$ \\
\hline 4 & $148.1, \mathrm{C}$ & - \\
\hline 5 & $55.6, \mathrm{CH}_{3}$ & $3.85, \mathrm{~s}$ \\
\hline 6 & $149.1, \mathrm{C}$ & - \\
\hline 7 & $115.5, \mathrm{CH}$ & $6.86, \mathrm{~d}(8.1)$ \\
\hline 8 & $122.2, \mathrm{CH}$ & $7.11, \mathrm{~d}(8.0)$ \\
\hline $1^{\prime}$ & $162.4, \mathrm{C}$ & - \\
\hline $2^{\prime}$ & $133.9, \mathrm{C}$ & - \\
\hline $3^{\prime}$ & $126.8, \mathrm{CH}$ & $8.42, \mathrm{~s}$ \\
\hline $4^{\prime}$ & $130.5, \mathrm{CH}$ & $8.09, \mathrm{~d}(7.7)$ \\
\hline $5^{\prime}$ & $128.7, \mathrm{CH}$ & $7.67, \mathrm{t}(7.7)$ \\
\hline $\mathrm{OH}^{\prime}$ & - & $9.54, \mathrm{~s}$ \\
\hline $\mathrm{NH}^{\prime}$ & - & $11.82, \mathrm{~s}$ \\
\hline $126 \mathrm{MHz}$ & & \\
\hline
\end{tabular}

a $126 \mathrm{MHz}$, Referenced to DMSO- $d_{6}(\delta 39.52 \mathrm{ppm}){ }^{261}$ Elucidated/confirmed by DEPT.

b $500 \mathrm{MHz}$, Referenced to TMS ( $\delta 0.00 \mathrm{ppm}$ ). Elucidated/confirmed by HSQC. 


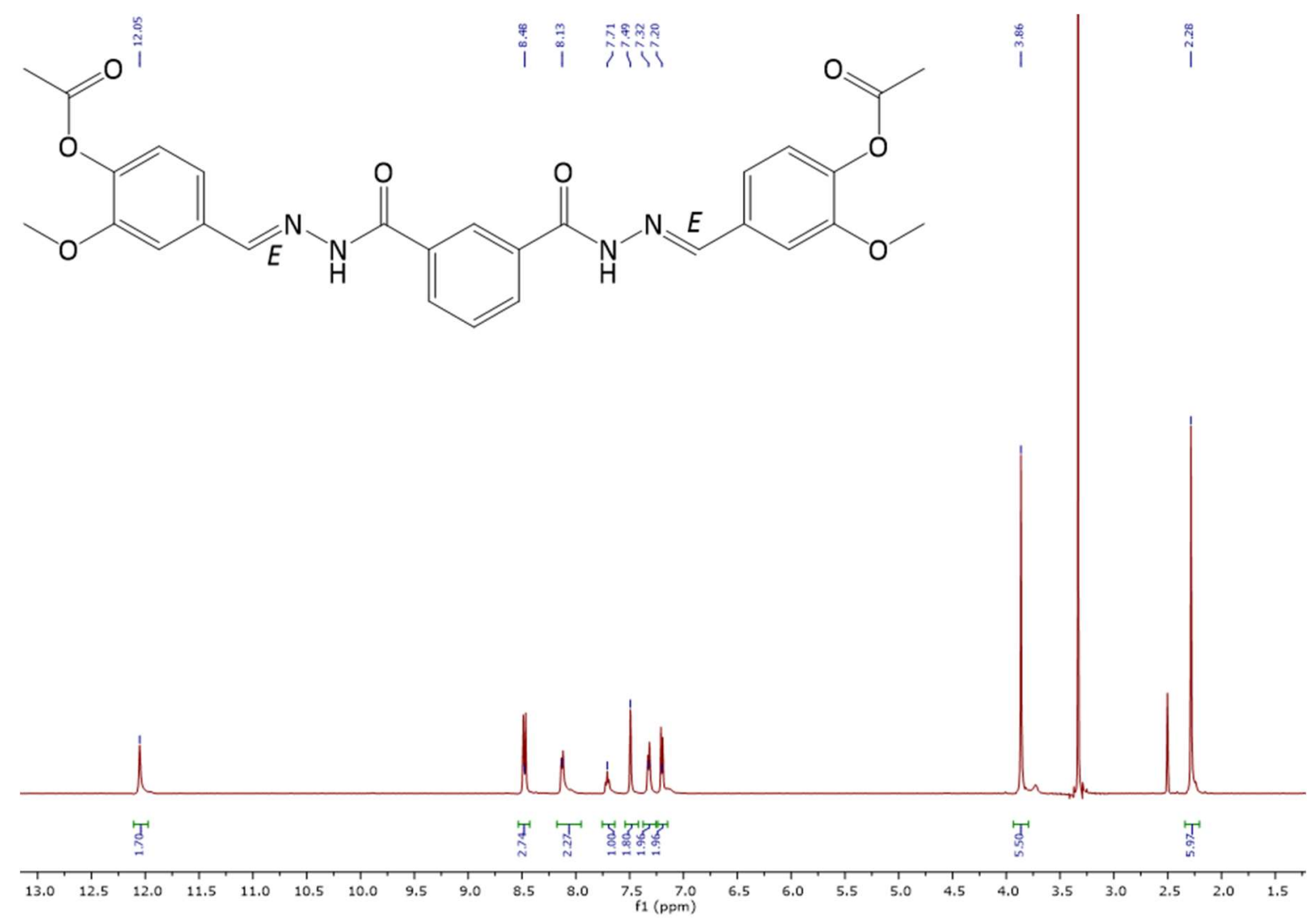

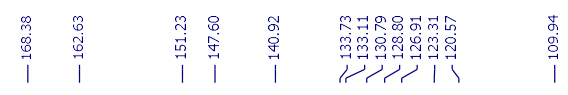
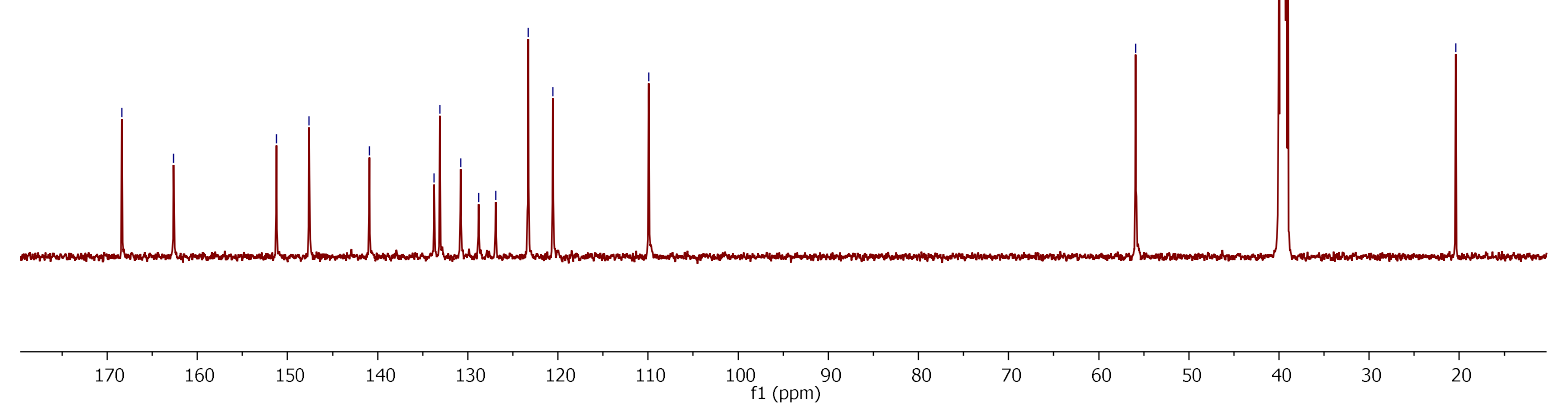

Figure 8.10. ${ }^{1} \mathrm{H}$ and ${ }^{13} \mathrm{C}$ NMR spectra (DMSO- $\left.d_{6}\right)$ of $\mathbf{3 d}\left(\delta_{H} 3.33 \mathrm{ppm}=\right.$ water). 
<smiles>COc1cc(/C=N/NC(=O)c2cccc(C(=O)N/N=C/c3ccc(OC(C)=O)c(OC)c3)c2)ccc1OC(C)=O</smiles>

Table 8.8. Peak assignments in ${ }^{1} \mathrm{H}$ and ${ }^{13} \mathrm{C}$ NMR spectra of $\mathbf{3 d}$.

\begin{tabular}{lll}
\hline Position & $\delta_{c^{a},}$, Type & $\delta_{H^{b}}(\boldsymbol{J i n ~ H z})$ \\
\hline 1 & $147.6, \mathrm{CH}$ & $8.49, \mathrm{~s}$ \\
\hline 2 & $133.7, \mathrm{C}$ & - \\
\hline 3 & $109.9, \mathrm{CH}$ & $7.49, \mathrm{~s}$ \\
\hline 4 & $151.2, \mathrm{C}$ & - \\
\hline 5 & $55.9, \mathrm{CH}_{3}$ & $3.86, \mathrm{~s}$ \\
\hline 6 & $140.9, \mathrm{C}$ & - \\
\hline 7 & $168.4, \mathrm{C}$ & - \\
\hline 8 & $20.4, \mathrm{CH}$ & $2.28, \mathrm{~s}$ \\
\hline 9 & $123.3, \mathrm{CH}$ & $7.20, \mathrm{~d}(8.1)$ \\
\hline 10 & $120.6, \mathrm{CH}$ & $7.32, \mathrm{~d}(7.9)$ \\
\hline $1^{\prime}$ & $162.6, \mathrm{C}$ & - \\
\hline $2^{\prime}$ & $133.1, \mathrm{C}$ & - \\
\hline $3^{\prime}$ & $126.9, \mathrm{CH}$ & $8.47, \mathrm{~s}$ \\
\hline $4^{\prime}$ & $130.8, \mathrm{CH}$ & $8.13, \mathrm{~d}(7.5)$ \\
\hline $5^{\prime}$ & $128.8, \mathrm{CH}$ & $7.71, \mathrm{t}(7.6)$ \\
\hline $\mathrm{NH}^{\prime}$ & - & $12.05, \mathrm{~s}$ \\
\hline $126 \mathrm{MHz}$ & & \\
\hline
\end{tabular}

a $126 \mathrm{MHz}$, Referenced to DMSO- $d_{6}(\delta 39.52 \mathrm{ppm}){ }^{261}$ Elucidated/confirmed by DEPT.

b $500 \mathrm{MHz}$, Referenced to TMS ( $\delta 0.00 \mathrm{ppm}$ ). Elucidated/confirmed by HSQC. 


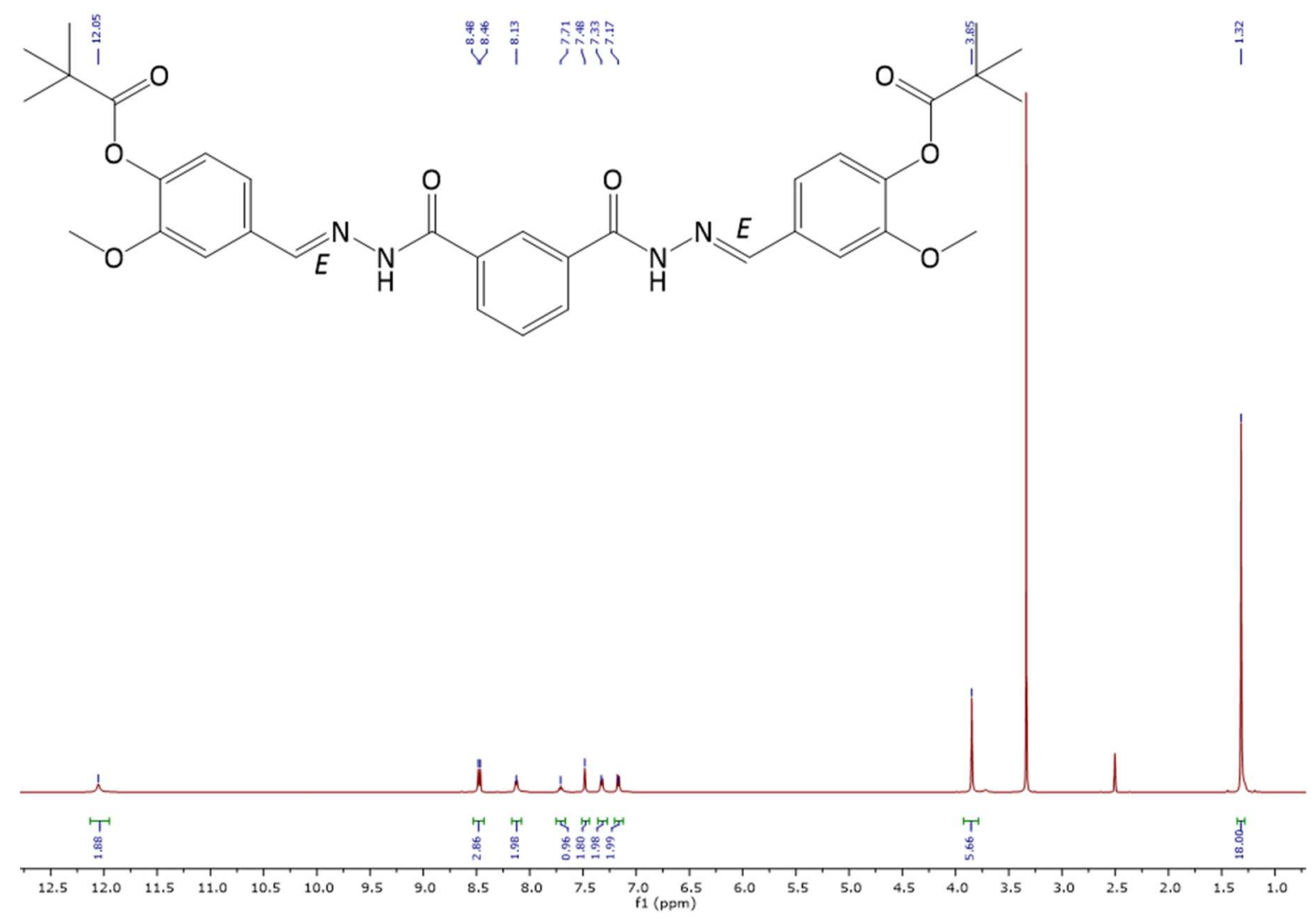

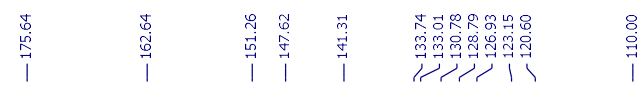
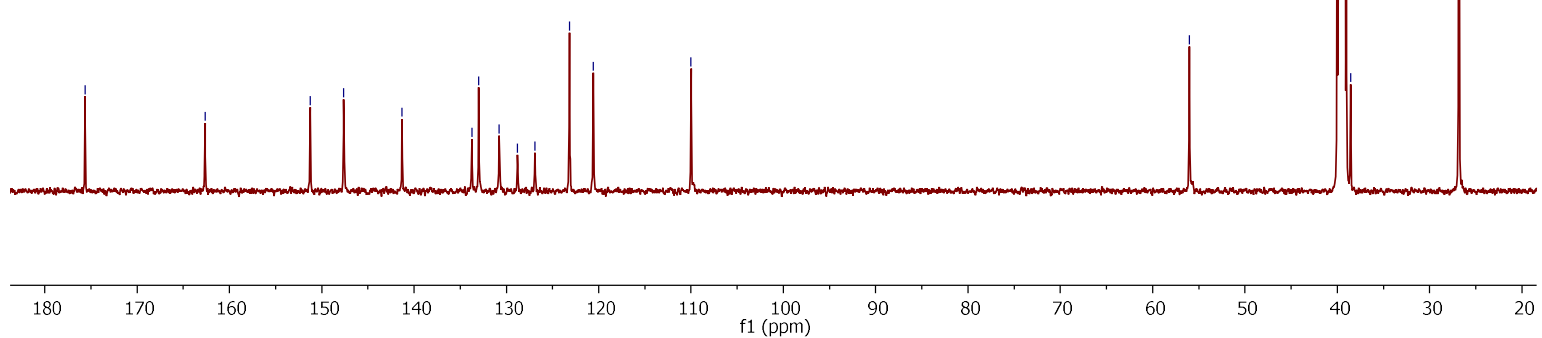

Figure 8.11. ${ }^{1} \mathrm{H}$ and ${ }^{13} \mathrm{C}$ NMR spectra (DMSO- $\left.d_{6}\right)$ of $3 e\left(\delta_{\mathrm{H}} 3.34 \mathrm{ppm}=\right.$ water). 
<smiles>COc1cc(/C=N/NC(=O)c2cccc(C(=O)N/N=C/c3ccc(OC(=O)C(C)(C)C)c(OC)c3)c2)ccc1OC(=O)C(C)(C)C</smiles>

Table 8.9. Peak assignments in ${ }^{1} \mathrm{H}$ and ${ }^{13} \mathrm{C}$ NMR spectra of $3 \mathbf{e}$.

\begin{tabular}{lll}
\hline Position & $\delta_{c^{a}}{ }^{\mathrm{a}}$ Type & $\delta_{\mathrm{H}^{\mathrm{b}}(\mathrm{J} \text { in Hz})}$ \\
\hline 1 & $147.6, \mathrm{CH}$ & $8.48, \mathrm{~s}$ \\
\hline 2 & $133.7, \mathrm{C}$ & - \\
\hline 3 & $110.0, \mathrm{CH}$ & $7.48, \mathrm{~s}$ \\
\hline 4 & $151.3, \mathrm{C}$ & - \\
\hline 5 & $56.0, \mathrm{CH}_{3}$ & $3.85, \mathrm{~s}$ \\
\hline 6 & $141.3, \mathrm{C}$ & - \\
\hline 7 & $175.6, \mathrm{C}$ & - \\
\hline 8 & $38.6, \mathrm{C}$ & - \\
\hline 9 & $26.8, \mathrm{CH}$ & $1.32, \mathrm{~s}$ \\
\hline 10 & $123.2, \mathrm{CH}$ & $7.17, \mathrm{~d}(8.1)$ \\
\hline 11 & $120.6, \mathrm{CH}$ & $7.32, \mathrm{~d}(7.9)$ \\
\hline $1^{\prime}$ & $162.6, \mathrm{C}$ & - \\
\hline $2^{\prime}$ & $133.0, \mathrm{C}$ & - \\
\hline $3^{\prime}$ & $126.9, \mathrm{CH}$ & $8.46, \mathrm{~s}$ \\
\hline $4^{\prime}$ & $130.8, \mathrm{CH}$ & $8.13, \mathrm{~d}(7.5)$ \\
\hline $5^{\prime}$ & $128.8, \mathrm{CH}$ & $7.71, \mathrm{t}(7.6)$ \\
\hline $\mathrm{NH}^{\prime}$ & - & $12.05, \mathrm{~s}$ \\
\hline
\end{tabular}

a $126 \mathrm{MHz}$, Referenced to DMSO- $d_{6}(\delta 39.52 \mathrm{ppm}) .{ }^{261}$ Elucidated/confirmed by DEPT.

${ }^{\mathrm{b}} 500 \mathrm{MHz}$, Referenced to TMS ( $\left.\delta 0.00 \mathrm{ppm}\right)$. Elucidated/confirmed by HSQC. 

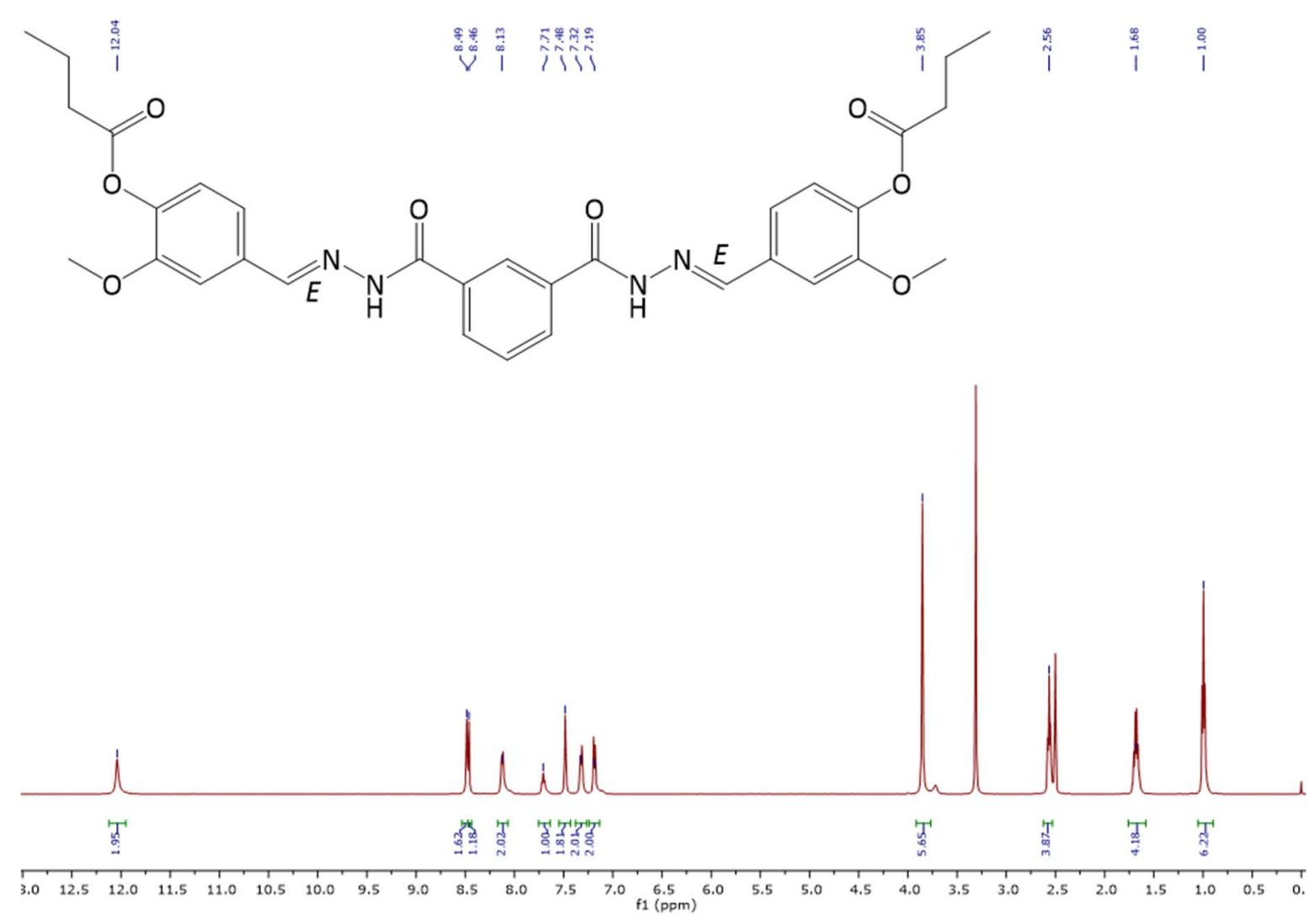

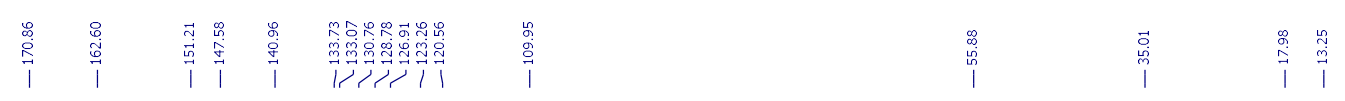

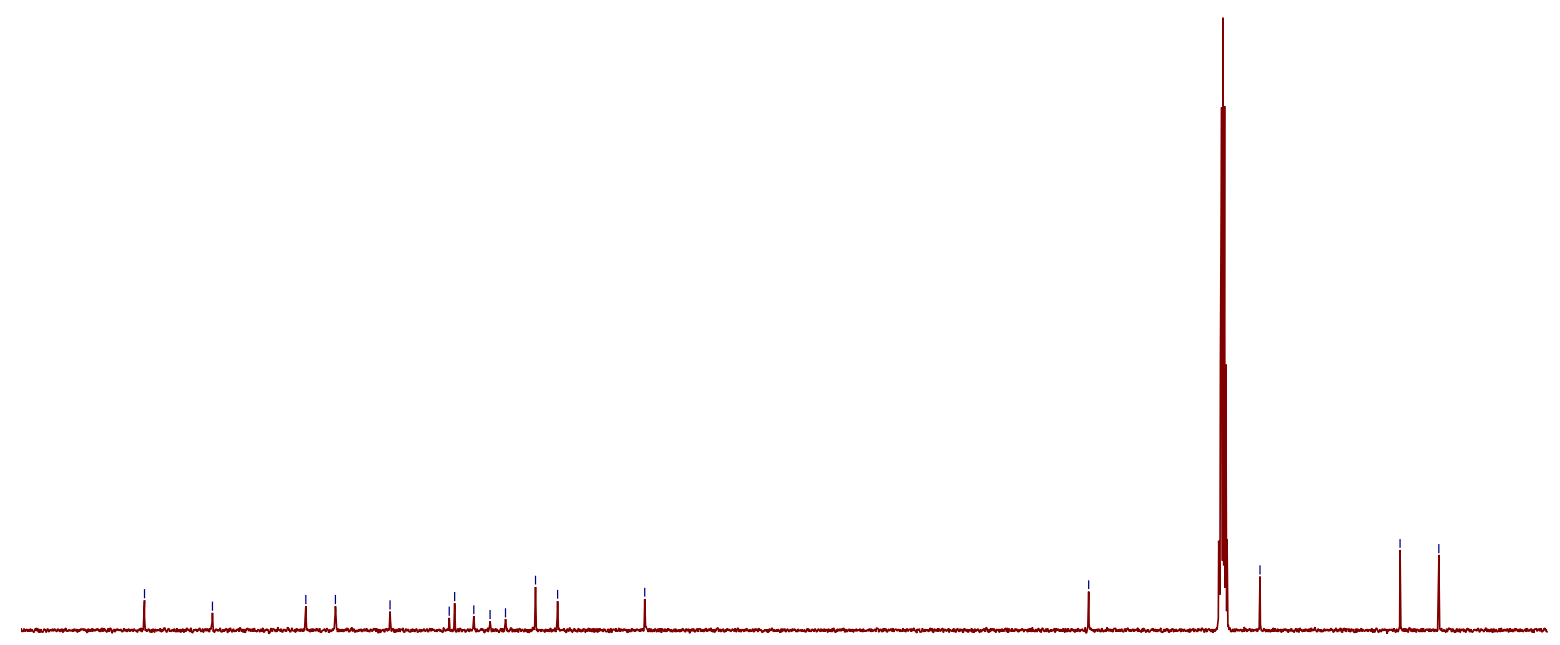

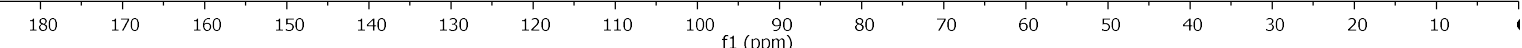

Figure 8.12. ${ }^{1} \mathrm{H}$ and ${ }^{13} \mathrm{C}$ NMR spectra (DMSO- $\left.d_{6}\right)$ of $3 \mathbf{f}\left(\delta_{\mathrm{H}} 3.31 \mathrm{ppm}=\mathrm{HDO}\right)$. 


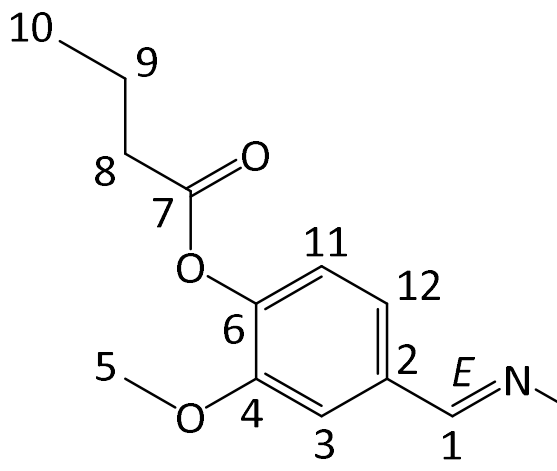<smiles>CCCC(=O)Oc1ccc(/C=N/NC(=O)c2cccc([14C](=O)NC)c2)cc1OC</smiles>

Table 8.10. Peak assignments in ${ }^{1} \mathrm{H}$ and ${ }^{13} \mathrm{C}$ NMR spectra of $\mathbf{3 f}$.

\begin{tabular}{|c|c|c|}
\hline Position & $\delta_{c}{ }^{a}$, Type & $\delta_{H}{ }^{b}(J$ in $\mathrm{Hz})$ \\
\hline 1 & $147.6, \mathrm{CH}$ & $8.49, \mathrm{~s}$ \\
\hline 2 & 133.7, C & - \\
\hline 3 & $110.0, \mathrm{CH}$ & $7.48, \mathrm{~s}$ \\
\hline 4 & $151.2, \mathrm{C}$ & - \\
\hline 5 & $55.9, \mathrm{CH}_{3}$ & $3.85, \mathrm{~s}$ \\
\hline 6 & 141.0, C & - \\
\hline 7 & $170.9, \mathrm{C}$ & - \\
\hline 8 & $35.0, \mathrm{CH}_{2}$ & $2.56, \mathrm{t}(7.1)$ \\
\hline 9 & $18.0, \mathrm{CH}_{2}$ & $1.68, \operatorname{sxt}(7.1)$ \\
\hline 10 & $13.3, \mathrm{CH}_{3}$ & $1.00, \mathrm{t}(7.3)$ \\
\hline 11 & $123.3, \mathrm{CH}$ & $7.19, \mathrm{~d}(8.0)$ \\
\hline 12 & $120.6, \mathrm{CH}$ & $7.32, d(7.7)$ \\
\hline $1^{\prime}$ & 162.6, C & - \\
\hline $2^{\prime}$ & 133.1, C & - \\
\hline $3^{\prime}$ & $126.9, \mathrm{CH}$ & $8.46, \mathrm{~s}$ \\
\hline $4^{\prime}$ & $130.8, \mathrm{CH}$ & $8.13, d(7.3)$ \\
\hline $5^{\prime}$ & $128.8, \mathrm{CH}$ & $7.71, \mathrm{t}(7.4)$ \\
\hline $\mathrm{NH}$ & - & $12.04, \mathrm{~s}$ \\
\hline
\end{tabular}

a $126 \mathrm{MHz}$, Referenced to DMSO- $d_{6}(\delta 39.52 \mathrm{ppm}) .{ }^{261}$ Elucidated/confirmed by DEPT.

${ }^{\mathrm{b}} 500 \mathrm{MHz}$, Referenced to TMS ( $\delta 0.00 \mathrm{ppm}$ ). Elucidated/confirmed by HSQC. 


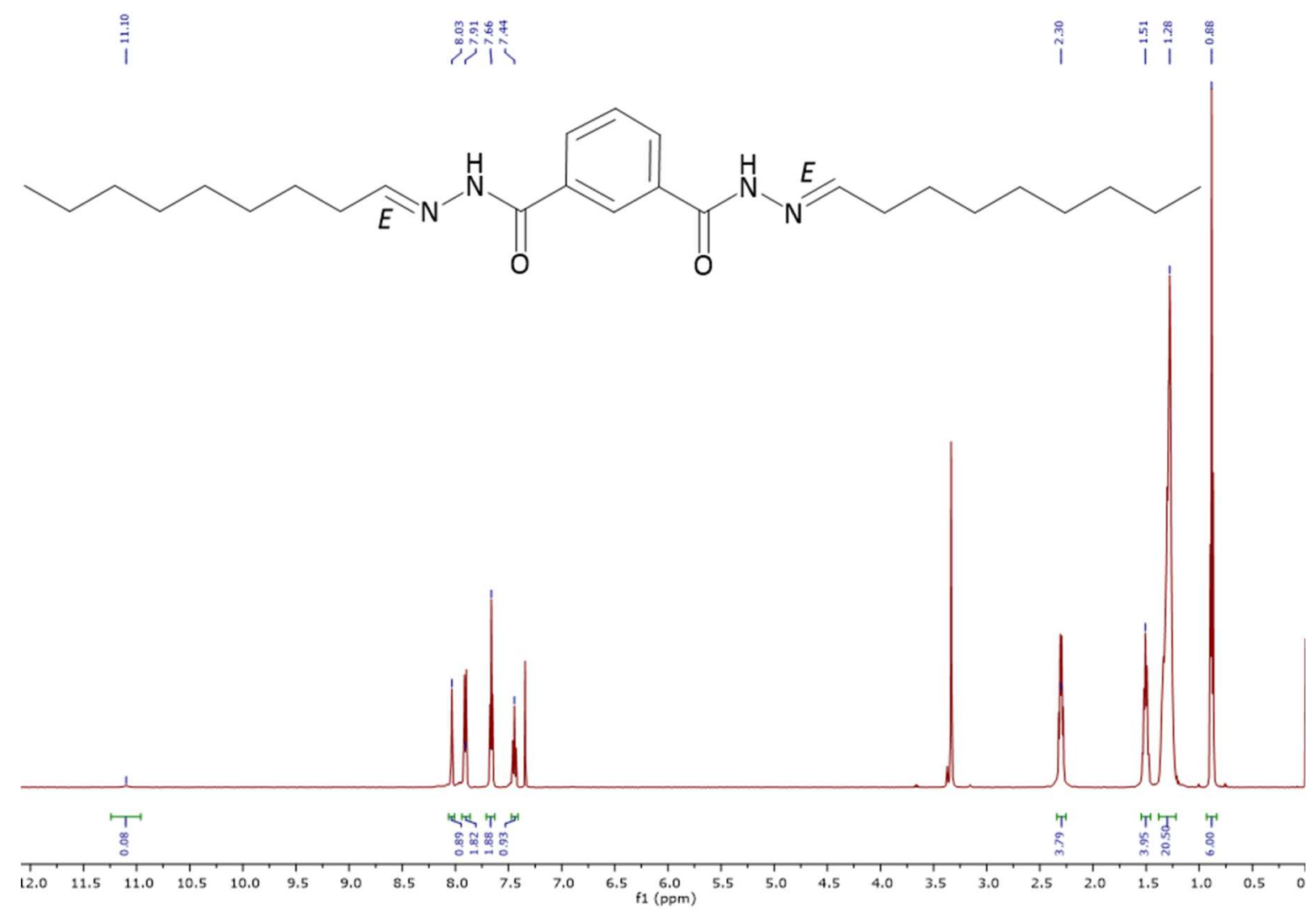

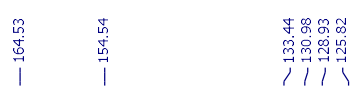

|
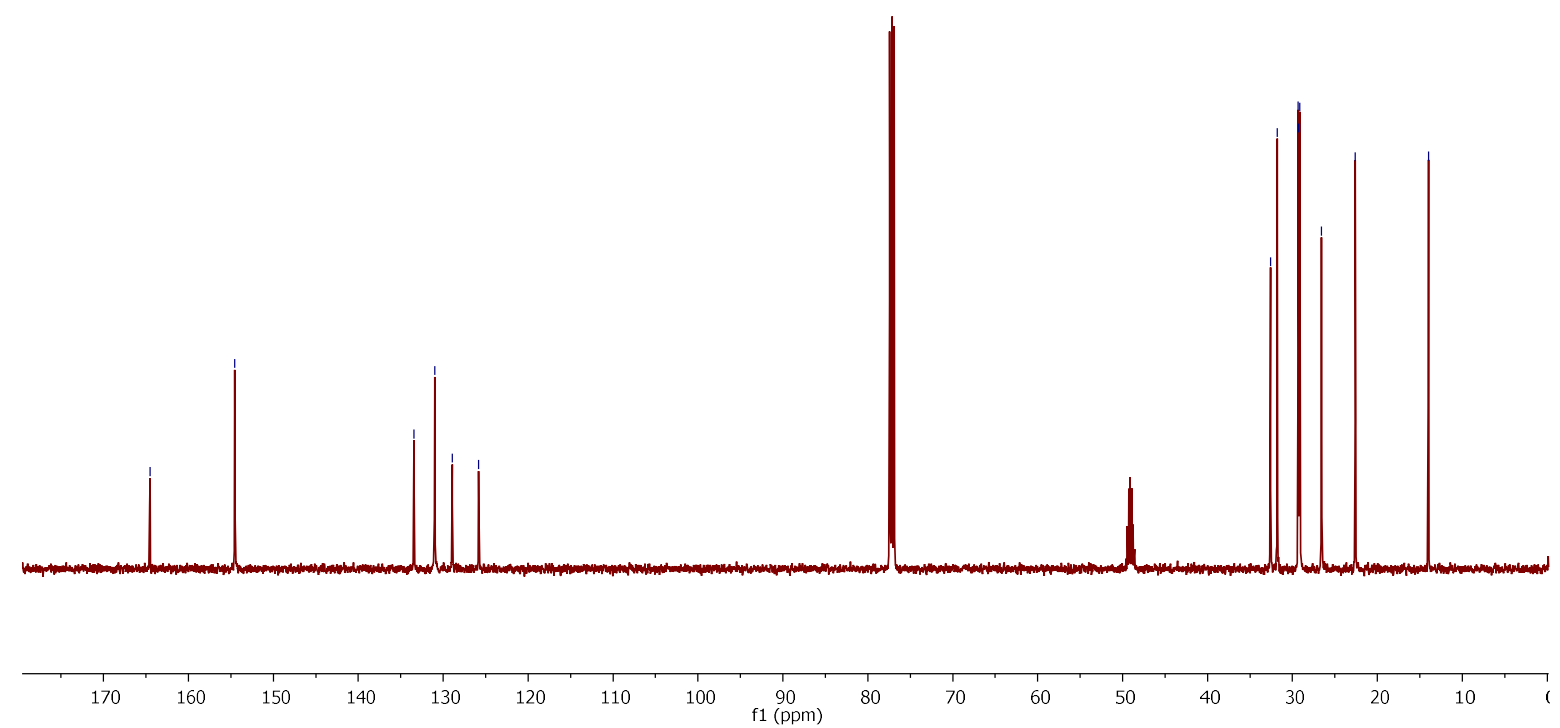

Figure 8.13. ${ }^{1} \mathrm{H}$ and ${ }^{13} \mathrm{C} \mathrm{NMR} \mathrm{spectra}\left(\mathrm{CDCl}_{3} / 10 \% \mathrm{v} / \mathrm{v} \mathrm{CD}_{3} \mathrm{OD}\right)$ of $3 \mathrm{~h}\left(\delta_{\mathrm{H}} 3.35 \& \delta_{\mathrm{c}} 49.1 \mathrm{ppm}=\right.$ methanol). 
<smiles>[2H]C(CC)CCC(=O)CC/C=N/NC(=O)c1cccc(C(=O)N/N=C/CCCCCCCC)c1</smiles>

Table 8.11. Peak assignments in ${ }^{1} \mathrm{H}$ and ${ }^{13} \mathrm{C}$ NMR spectra of $\mathbf{3 h}$.

\begin{tabular}{lll}
\hline Position & $\delta_{c^{a}}{ }^{\mathrm{a}}$ Type & $\delta_{\mathrm{H}^{\mathrm{b}}}(\mathrm{J}$ in Hz) \\
\hline 1 & $154.5, \mathrm{CH}$ & $7.66, \mathrm{t}(5.7)$ \\
\hline 2 & $32.6, \mathrm{CH}_{2}$ & $2.37-2.24, \mathrm{~m}$ \\
\hline 3 & $26.6, \mathrm{CH}_{2}$ & $1.51, \mathrm{p}(7.6)$ \\
\hline 4 & 29.3 or $29.2, \mathrm{CH}_{2}$ & $1.38-1.21, \mathrm{br} \mathrm{m}$ \\
\hline 5 & 29.3 or $29.2, \mathrm{CH}_{2}$ & $1.38-1.21, \mathrm{br} \mathrm{m}$ \\
\hline 6 & 29.3 or $29.2, \mathrm{CH}_{2}$ & $1.38-1.21, \mathrm{br} \mathrm{m}$ \\
\hline 7 & $31.8, \mathrm{CH}_{2}$ & $1.38-1.21, \mathrm{br} \mathrm{m}$ \\
\hline 8 & $22.6, \mathrm{CH}_{2}$ & $1.38-1.21, \mathrm{br} \mathrm{m}$ \\
\hline 9 & $14.0, \mathrm{CH}_{3}$ & $0.88, \mathrm{t}(6.9)$ \\
\hline $1^{\prime}$ & $164.5, \mathrm{C}$ & - \\
\hline $2^{\prime}$ & $133.4, \mathrm{C}$ & - \\
\hline $3^{\prime}$ & $125.8, \mathrm{CH}$ & $8.03, \mathrm{~s}$ \\
\hline $4^{\prime}$ & $131.0, \mathrm{CH}$ & $7.91, \mathrm{~d}(7.7)$ \\
\hline $5^{\prime}$ & $128.9, \mathrm{CH}$ & $7.44, \mathrm{t}(7.7)$ \\
\hline $\mathrm{NH}^{\prime}$ & - & $11.10, \mathrm{~s}$ \\
\hline
\end{tabular}

a $126 \mathrm{MHz}$, Referenced to $\mathrm{CDCl}_{3}(\delta 77.16 \mathrm{ppm}) .{ }^{261}$ Elucidated/confirmed by DEPT.

${ }^{\mathrm{b}} 500 \mathrm{MHz}$, Referenced to TMS ( $\delta 0.00 \mathrm{ppm}$ ). Elucidated/confirmed by HSQC and COSY. 

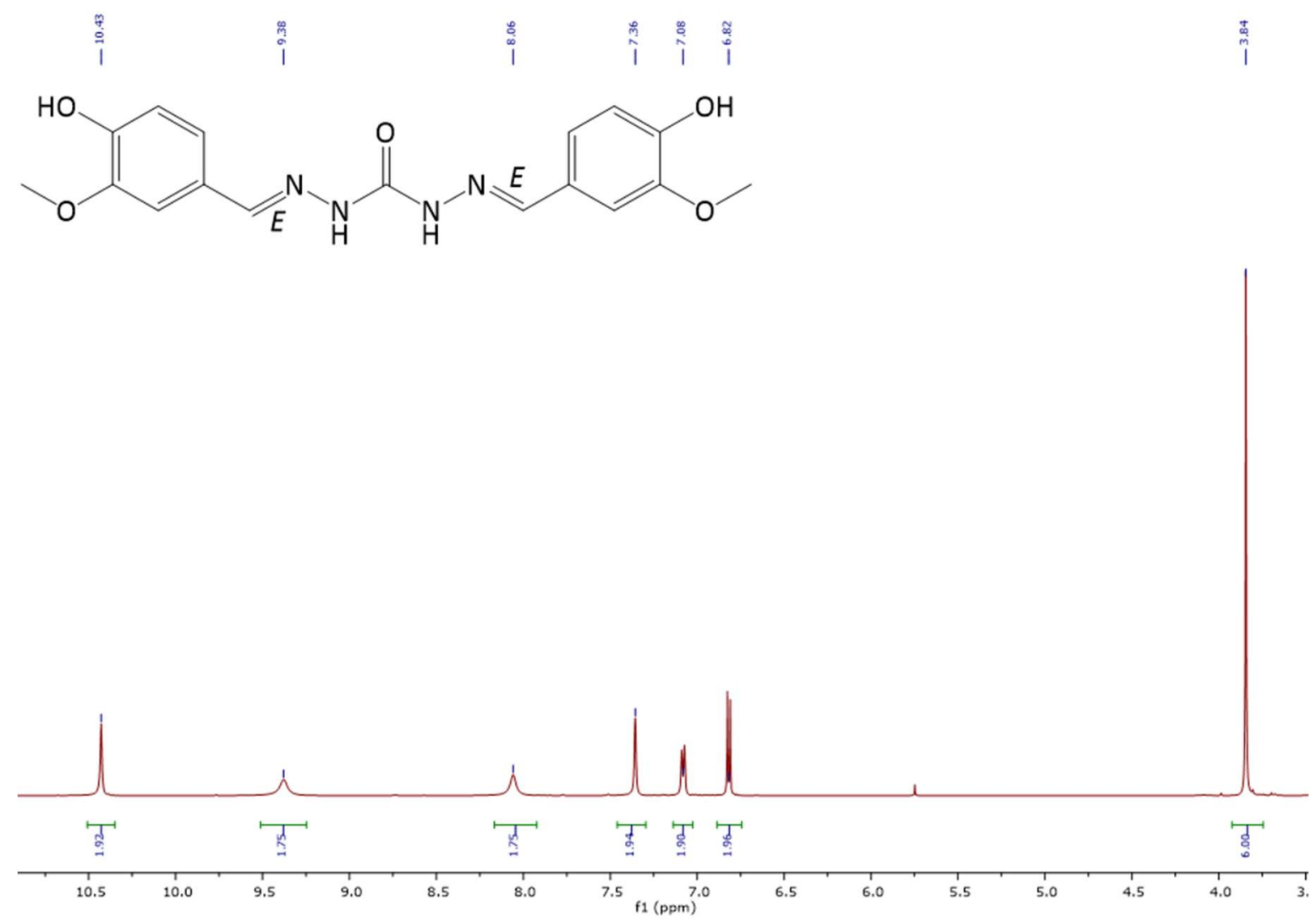

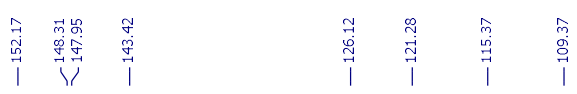
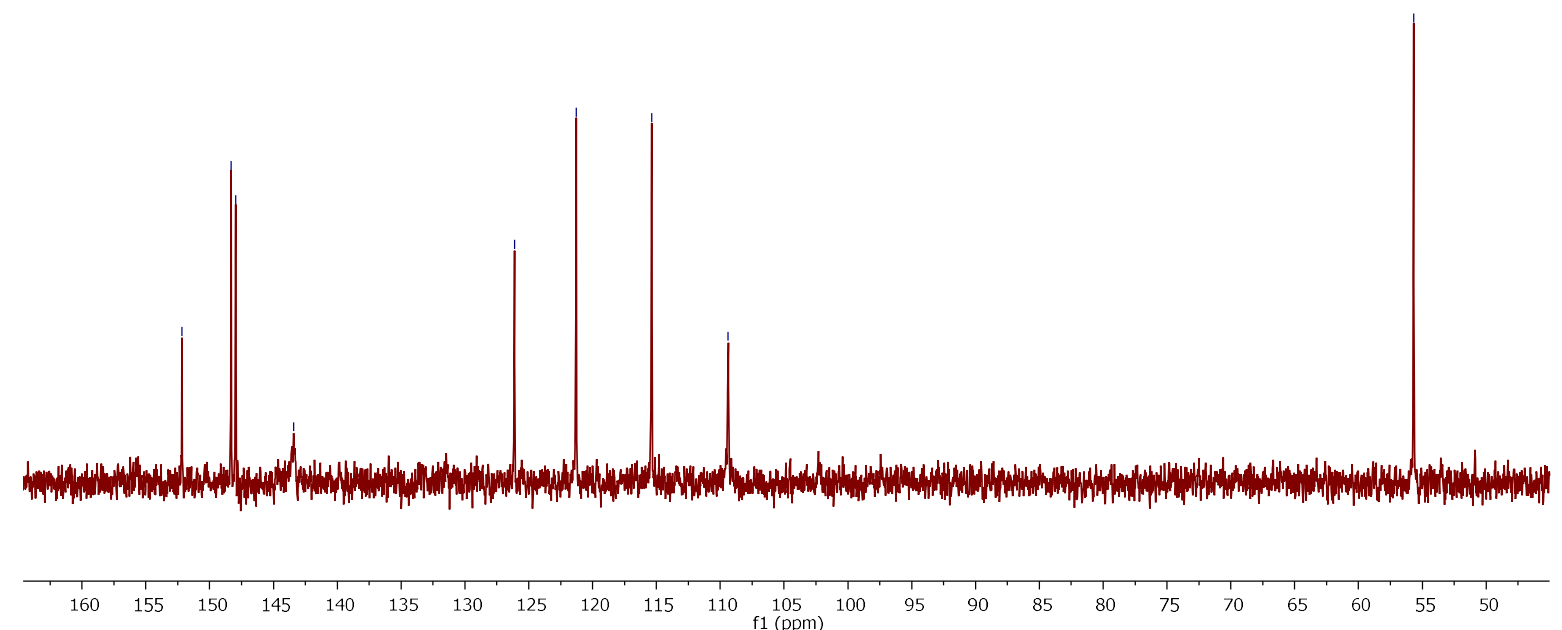

Figure 8.14. ${ }^{1} \mathrm{H}$ and ${ }^{13} \mathrm{C}$ NMR spectra (DMSO- $\left.d_{6}\right)$ of $4 \mathrm{c}\left(\delta_{\mathrm{H}} 5.75 \mathrm{ppm}=\mathrm{DCM}\right)$. 
<smiles>COc1cc(/C=N/NC(=O)N/N=C/c2ccc(O)c(OS)c2)ccc1O</smiles>

Table 8.12. Peak assignments in ${ }^{1} \mathrm{H}$ and ${ }^{13} \mathrm{C}$ NMR spectra of 4c.

\begin{tabular}{lll}
\hline Position & $\delta_{\mathrm{c}^{\mathrm{a}}}$, Type & $\boldsymbol{\delta}_{\mathrm{H}^{\mathrm{b}}}(\mathrm{J}$ in Hz$)$ \\
\hline 1 & $143.4, \mathrm{CH}$ & $8.06, \mathrm{~s}$ \\
\hline 2 & $126.1, \mathrm{C}$ & - \\
\hline 3 & $109.4, \mathrm{CH}$ & $7.36, \mathrm{~s}$ \\
\hline 4 & $148.0, \mathrm{C}$ & - \\
\hline 5 & $55.7, \mathrm{CH}$ & $3.84, \mathrm{~s}$ \\
\hline 6 & $148.3, \mathrm{C}$ & - \\
\hline 7 & $115.4, \mathrm{CH}$ & $6.82, \mathrm{~d}(8.1)$ \\
8 & $121.3, \mathrm{CH}$ & $7.08, \mathrm{~d}(8.0)$ \\
\hline${ }^{\prime}$ & $152.2, \mathrm{C}$ & - \\
\hline $\mathrm{OH}$ & - & $9.38, \mathrm{~s}$ \\
$\mathrm{NH}$ & - & $10.43, \mathrm{~s}$ \\
\hline
\end{tabular}

a $126 \mathrm{MHz}$, Referenced to DMSO- $d_{6}(\delta 39.52 \mathrm{ppm}){ }^{261}$ Elucidated/confirmed by DEPT.

b $500 \mathrm{MHz}$, Referenced to TMS ( $\delta 0.00 \mathrm{ppm}$ ). Elucidated/confirmed by HSQC. 

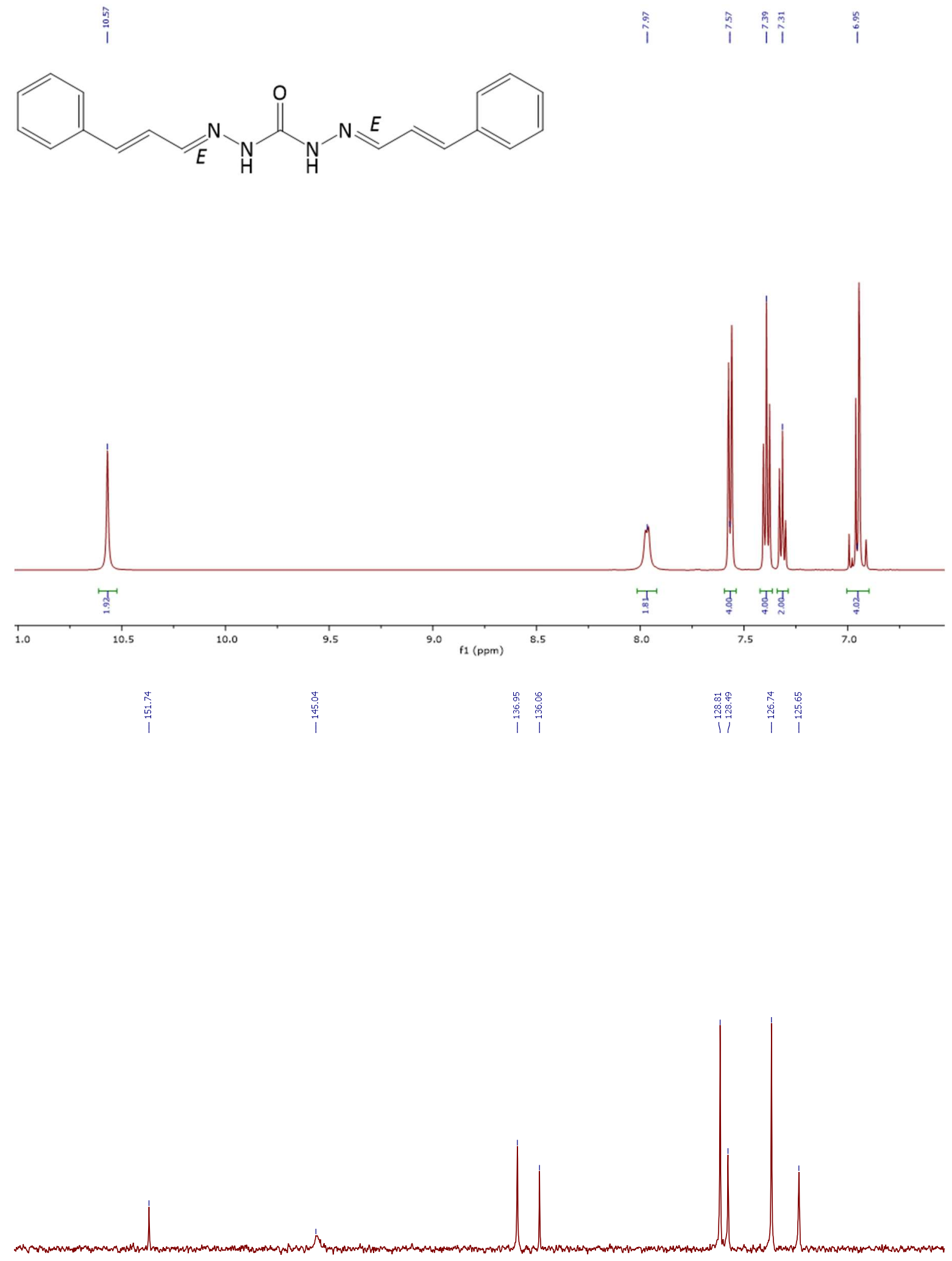

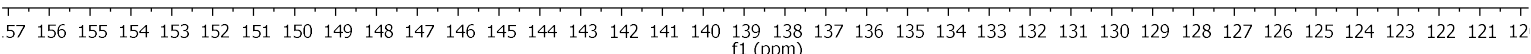

Figure 8.15. ${ }^{1} \mathrm{H}$ and ${ }^{13} \mathrm{C}$ NMR spectra (DMSO- $d_{6}$ ) of $\mathbf{4 g}$. 
<smiles>O=C(N/N=C/C=C/c1ccccc1)N/N=C/c1ccccc1</smiles>

Table 8.13. Peak assignments in ${ }^{1} \mathrm{H}$ and ${ }^{13} \mathrm{C}$ NMR spectra of $\mathbf{4 g}$.

\begin{tabular}{lll}
\hline Position & $\delta_{c^{a}}{ }^{a}$, Type & $\delta_{H}{ }^{b}(J$ in $\mathrm{Hz})$ \\
\hline 1 & $145.0, \mathrm{CH}$ & $7.97, \mathrm{~d}(6.4)$ \\
\hline 2 & $137.0, \mathrm{CH}$ & $7.02-6.89, \mathrm{~m}$ \\
\hline 3 & $125.7, \mathrm{CH}$ & $7.02-6.89, \mathrm{~m}$ \\
\hline 4 & $136.1, \mathrm{C}$ & - \\
\hline 5 & $126.7, \mathrm{CH}$ & $7.57, \mathrm{~d}(7.3)$ \\
\hline 6 & $128.8, \mathrm{CH}$ & $7.39, \mathrm{t}(7.5)$ \\
\hline 7 & $128.5, \mathrm{CH}$ & $7.31, \mathrm{t}(7.3)$ \\
\hline $1 '$ & $151.7, \mathrm{C}$ & - \\
\hline $\mathrm{NH}$ & - & $10.57, \mathrm{~s}$ \\
\hline a $126 \mathrm{MHz}$, Referenced to DMSO- $d_{6}(\delta 39.52 \text { ppm })^{261}$ Elucidated/confirmed by DEPT. \\
${ }^{\mathrm{b}} 500 \mathrm{MHz}$, Referenced to TMS $(\delta 0.00 \mathrm{ppm})$. Elucidated/confirmed by HSOC.
\end{tabular}



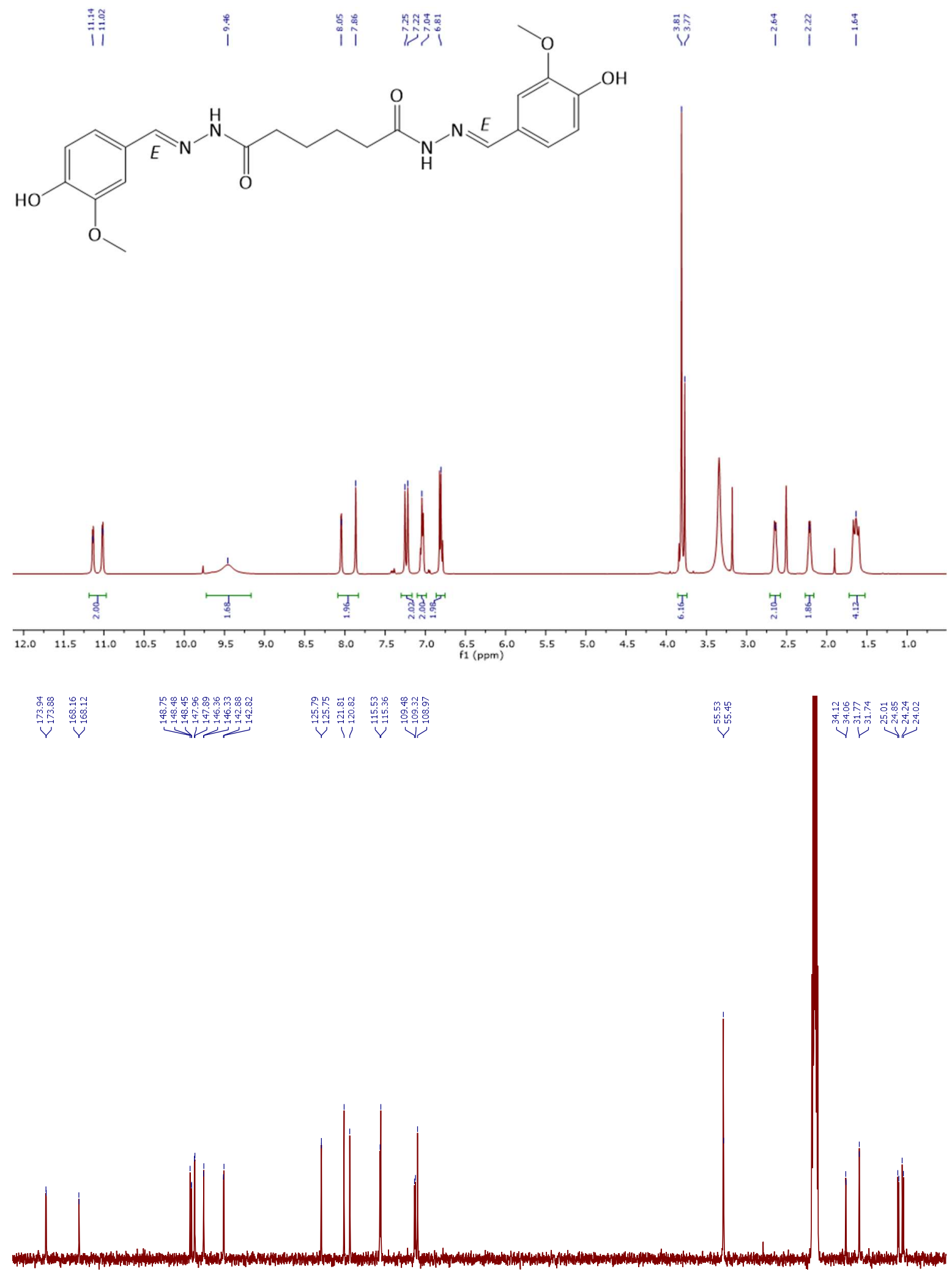

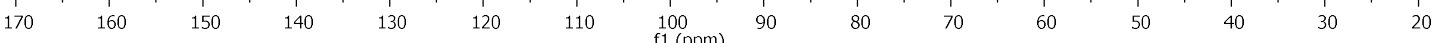

Figure 8.16. ${ }^{1} \mathrm{H}$ and ${ }^{13} \mathrm{C}$ NMR spectra (crude; DMSO- $\left.d_{6}\right)$ of $5 \mathrm{c}\left(\delta_{\mathrm{H}} 9.77 \mathrm{ppm}=\right.$ contaminant, $\delta_{\mathrm{H}} 3.34 \mathrm{ppm}$ = water, $\delta_{H} 3.18 \mathrm{ppm}=$ methanol, $\delta_{H} 1.90 \mathrm{ppm}=$ acetic acid). 
<smiles>COc1cc(/C=N/NC(=O)C[C]CCC(=O)N/N=C/c2ccc(O)c(OC)c2)ccc1O</smiles>

Table 8.14. Peak assignments in ${ }^{1} \mathrm{H}$ and ${ }^{13} \mathrm{C}$ NMR spectra of $\mathbf{5 c}$.

\begin{tabular}{lll}
\hline Position & $\delta_{\mathrm{c}^{\mathrm{a}, \mathrm{b}}, \text { Type }}$ & $\delta_{\mathrm{H}^{\mathrm{a}, \mathrm{c}}}(\boldsymbol{J}$ in Hz) \\
\hline 1 & $146.4,146.3,142.9,142.8, \mathrm{CH}$ & $8.05, \mathrm{~s} ; 7.86, \mathrm{~s}$ \\
\hline 2 & $125.8,125.8, \mathrm{C}$ & - \\
\hline 3 & $109.5,109.3,109.0, \mathrm{CH}$ & $7.25, \mathrm{~s} ; 7.22, \mathrm{~s}$ \\
\hline 4 & $148.0,147.9, \mathrm{C}$ & - \\
\hline 5 & $55.5,55.5, \mathrm{CH} 3$ & $3.81, \mathrm{~s} ; 3.77, \mathrm{~s}$ \\
\hline 6 & $148.8,148.5,148.5, \mathrm{C}$ & - \\
\hline 7 & $115.5,115.4, \mathrm{CH}$ & $6.84-6.76, \mathrm{~m}$ \\
\hline 8 & $121.8,120.8, \mathrm{CH}$ & $7.08-7.00, \mathrm{~m}$ \\
\hline $1^{\prime}$ & $173.9,173.9,168.2,168.1, \mathrm{C}$ & - \\
\hline $2^{\prime}$ & $34.1,34.1,31.8,31.7, \mathrm{CH}_{2}$ & $2.69-2.60, \mathrm{~m} ; 2.26-2.17, \mathrm{~m}$ \\
$3^{\prime}$ & $25.0,24.9,24.2,24.0, \mathrm{CH}_{2}$ & $1.70-1.57, \mathrm{~m}$ \\
\hline $\mathrm{OH}$ & - & $9.45, \mathrm{~s}$ \\
\hline $\mathrm{NH}$ & - & $11.14, \mathrm{~d}(5.3) ; 11.02, \mathrm{~d}(5.0)$ \\
\hline
\end{tabular}

a Where multiple values are provided, rotamers were observed in solution.

${ }^{\mathrm{b}} 126 \mathrm{MHz}$, Referenced to DMSO- $d_{6}(\delta 39.52 \mathrm{ppm}) .{ }^{261}$ Elucidated/confirmed by DEPT.

c $500 \mathrm{MHz}$, Referenced to TMS ( $\delta 0.00 \mathrm{ppm})$. Elucidated/confirmed by HSQC. 

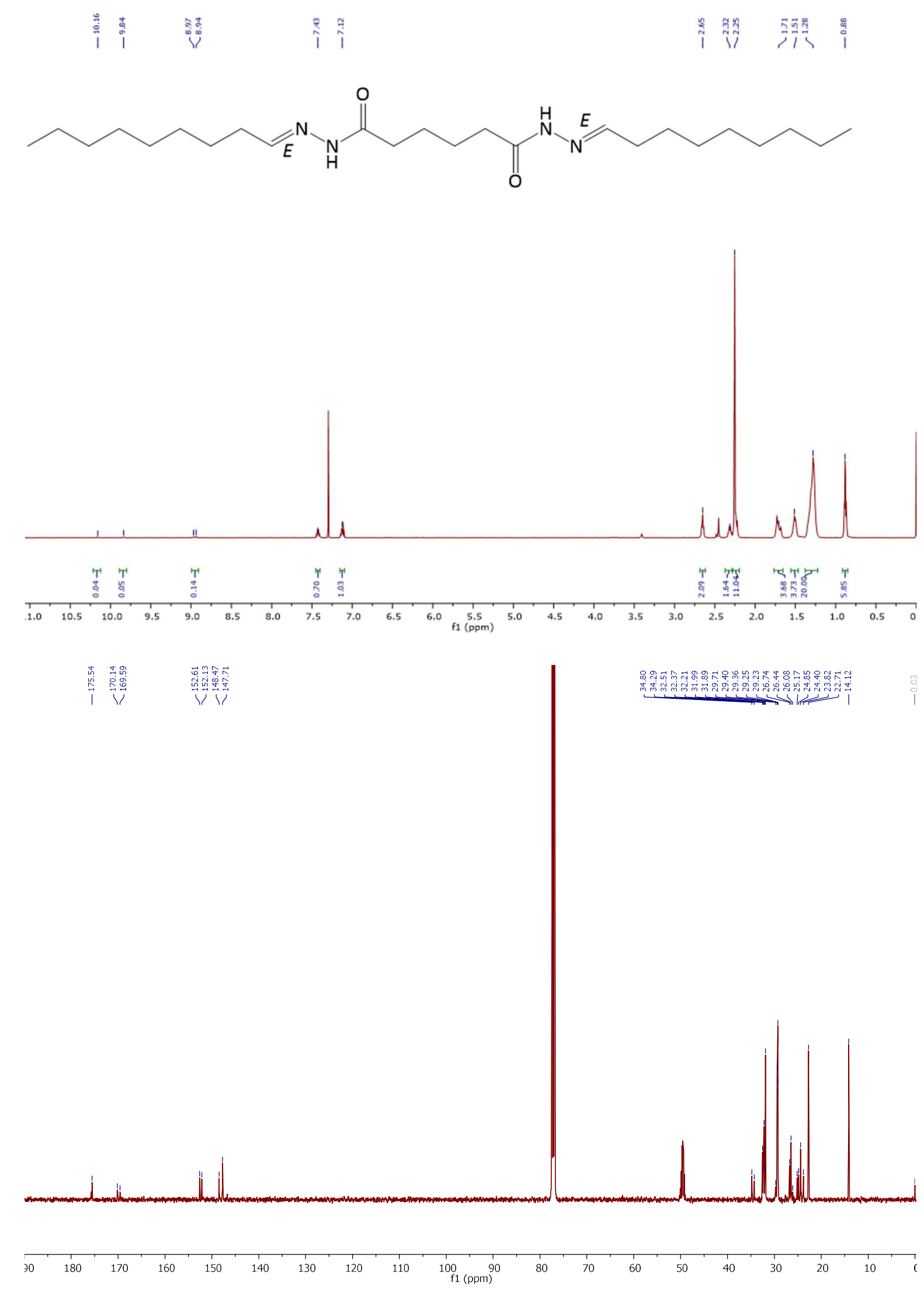

Figure 8.17. ${ }^{1} \mathrm{H}$ and ${ }^{13} \mathrm{C}$ NMR spectra (crude; $\left.\mathrm{CDCl}_{3} / 5 \% \mathrm{v} / \mathrm{v} \mathrm{CD}_{3} \mathrm{OD}\right)$ of $5 \mathrm{~h}\left(\delta_{\mathrm{H}} 2.45 \& 2.42 \mathrm{ppm}=\right.$ contaminant, $\delta_{H} 3.37 \& \delta c 49.5 \mathrm{ppm}=$ methanol). 


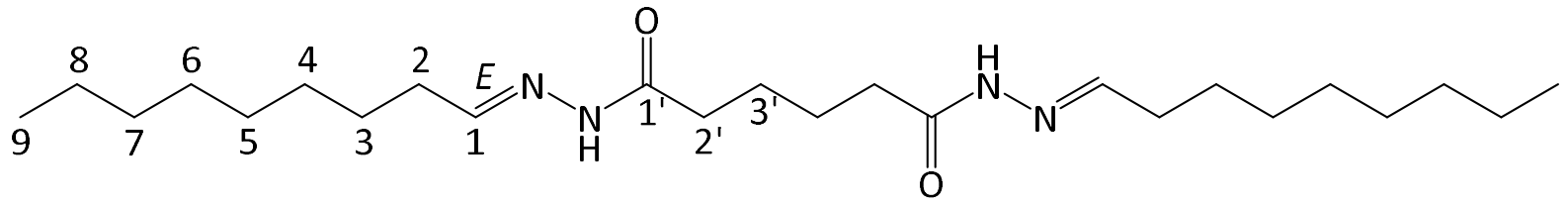

Table 8.15. Peak assignments in ${ }^{1} \mathrm{H}$ NMR spectra of $\mathbf{5 h}$.

\begin{tabular}{lll}
\hline Position & $\delta_{c^{\mathrm{a}, \mathrm{b}}, \text { Type }}$ & $\delta_{\mathrm{H}^{\mathrm{a}, \mathrm{c}}}(\mathrm{J}$ in $\mathrm{Hz})$ \\
\hline 1 & $152.6,152.1,148.5,147.7, \mathrm{CH}$ & $7.46-7.39, \mathrm{~m} ; 7.16-7.07, \mathrm{~m}$ \\
\hline 2 & 32.5 (possibly 34.8 or 34.3), $\mathrm{CH}_{2}$ & $2.36-2.28, \mathrm{~m} ; 2.28-2.20^{\mathrm{d}}, \mathrm{m}$ \\
\hline 3 & $26.7,26.4,26.1, \mathrm{CH}_{2}$ & $1.57-1.43, \mathrm{~m}$ \\
\hline $4-8$ & $32.0,31.9,29.7,29.4,29.4,29.3,29.2$, & $1.38-1.19, \mathrm{~m}$ \\
\hline 9 & $22.7, \mathrm{CH}_{2}$ & $0.93-0.81, \mathrm{~m}$ \\
\hline $1^{\prime}$ & $14.1, \mathrm{CH}_{3}$ & - \\
$2^{\prime}$ & $175.5,170.1,169.6, \mathrm{C}$ & $2.70-2.60, \mathrm{~m} ; 2.28-2.20^{\mathrm{d}}, \mathrm{m}$ \\
$3^{\prime}$ & $32.4,32.2$ (possibly 34.8 or 34.3), $\mathrm{CH}_{2}$ & $1.79-1.64, \mathrm{~m}$ \\
$\mathrm{NH}^{\mathrm{e}}$ & $25.2,24.9,24.4,23.8, \mathrm{CH}_{2}$ & $10.16, \mathrm{~s} ; 9.84, \mathrm{~s} ; 8.97, \mathrm{~s} ; 8.94, \mathrm{~s}$ \\
\hline
\end{tabular}

a Where multiple values are provided, rotamers were observed in solution.

b $126 \mathrm{MHz}$, Referenced to $\mathrm{CDCl}_{3}(\delta 77.16 \mathrm{ppm}) \cdot{ }^{261}$ Elucidated/confirmed by DEPT and HSQC.

c $500 \mathrm{MHz}$, Referenced to TMS ( $\delta 0.00 \mathrm{ppm})$. Elucidated/confirmed by COSY and HSQC.

d Overlapping HDO peak.

exchangeable. 

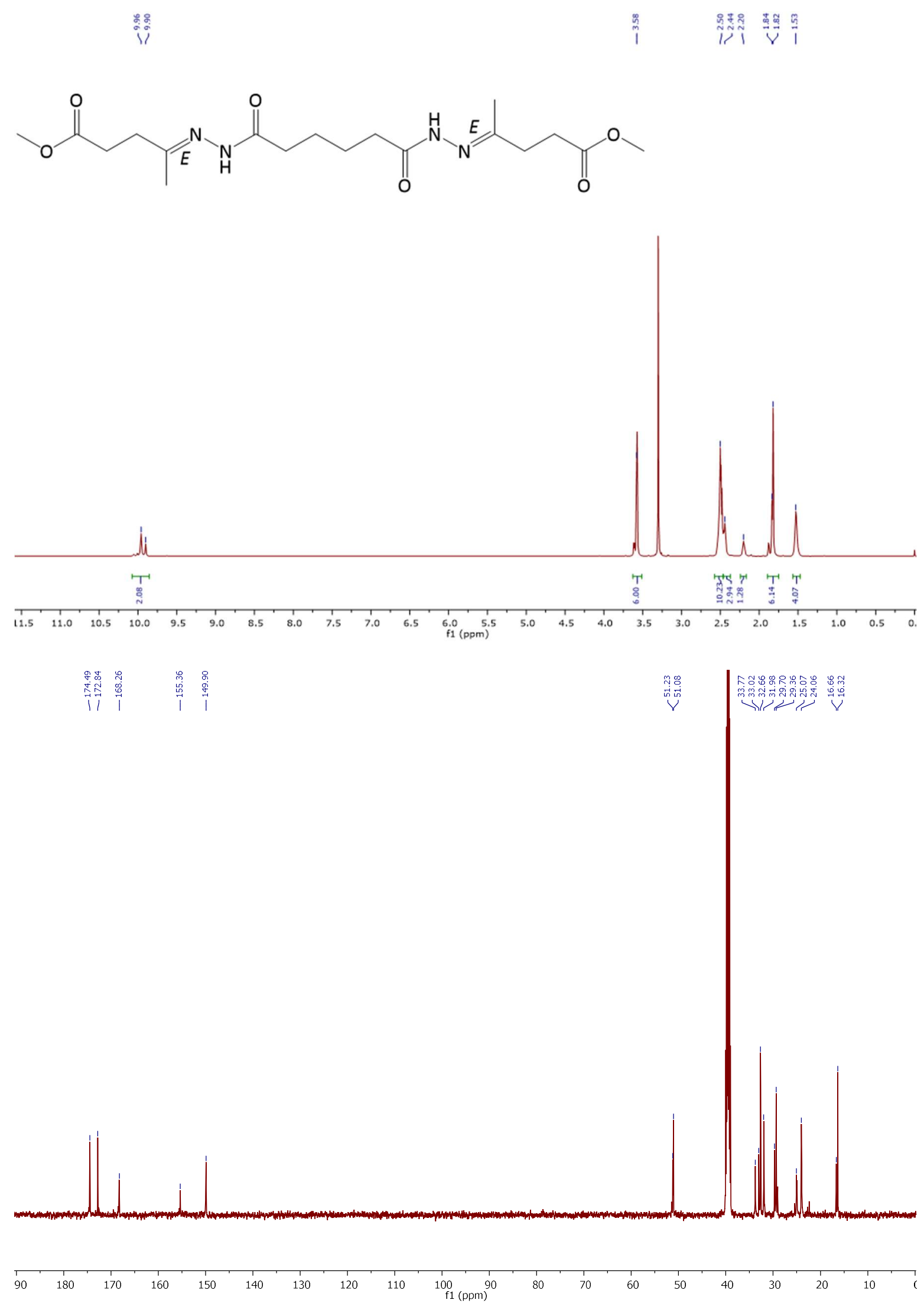

Figure 8.18. ${ }^{1} \mathrm{H}$ and ${ }^{13} \mathrm{C}$ NMR spectra $\left(\mathrm{DMSO}-d_{6}\right)$ of $5 \mathrm{i}\left(\delta_{\mathrm{H}} 3.30 \mathrm{ppm}=\mathrm{HDO}\right)$. 


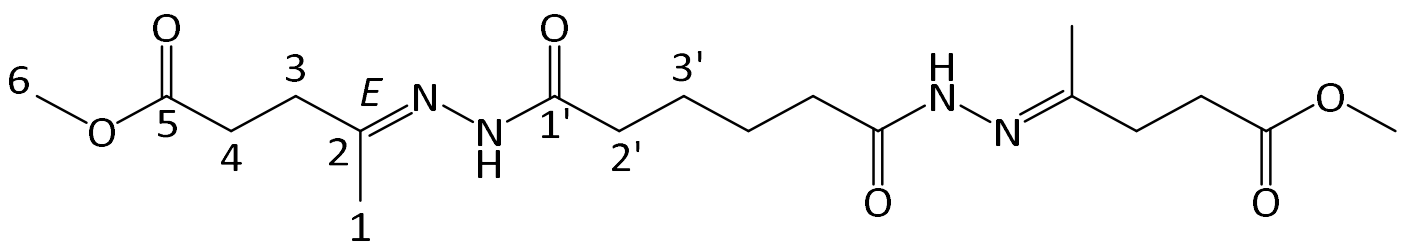

Table 8.16. Peak assignments in ${ }^{1} \mathrm{H}$ and ${ }^{13} \mathrm{C}$ NMR spectra of $\mathbf{5 i}$.

\begin{tabular}{lll}
\hline Position & $\delta_{c^{a, b}, \text { Type }}$ & $\delta_{H^{a, c}}(\boldsymbol{J}$ in Hz) \\
\hline 1 & $16.7,16.3, \mathrm{CH}_{3}$ & $1.84, \mathrm{~s} ; 1.82, \mathrm{~s}$ \\
\hline 2 & $155.4,149.9, \mathrm{C}$ & - \\
\hline 3 & $33.0,32.7, \mathrm{CH}_{2}$ & $2.57-2.46^{\mathrm{d}}, \mathrm{m}$ \\
\hline 4 & $29.7,29.4, \mathrm{CH}_{2}$ & $2.57-2.46^{\mathrm{d}}, \mathrm{m}$ \\
\hline 5 & $172.8, \mathrm{C}$ & - \\
\hline 6 & $51.2,51.1, \mathrm{CH}_{3}$ & $3.58, \mathrm{~s}$ \\
\hline $1^{\prime}$ & $174.5,168.3, \mathrm{C}$ & - \\
\hline $2^{\prime}$ & $33.8,32.0, \mathrm{CH}_{2}$ & $2.46-2.37, \mathrm{~m} ; 2.24-2.17, \mathrm{~m}$ \\
\hline $3^{\prime}$ & $25.1,24.1, \mathrm{CH}_{2}$ & $1.60-1.45, \mathrm{~m}$ \\
\hline $\mathrm{NH}$ & - & $9.96, \mathrm{~s} ; 9.90, \mathrm{~s}$ \\
\hline
\end{tabular}

a Where multiple values are provided, rotamers were observed in solution.

${ }^{\mathrm{b}} 126 \mathrm{MHz}$, Referenced to DMSO- $d_{6}(\delta 39.52 \mathrm{ppm}) .{ }^{261}$ Elucidated/confirmed by DEPT, HSQC, and HMBC.

c $500 \mathrm{MHz}$, Referenced to TMS ( $\delta 0.00 \mathrm{ppm}$ ). Elucidated/confirmed by HSQC, HMBC, and COSY.

${ }^{\mathrm{d}}$ Overlapping DMSO- $d_{6}$ peak. 

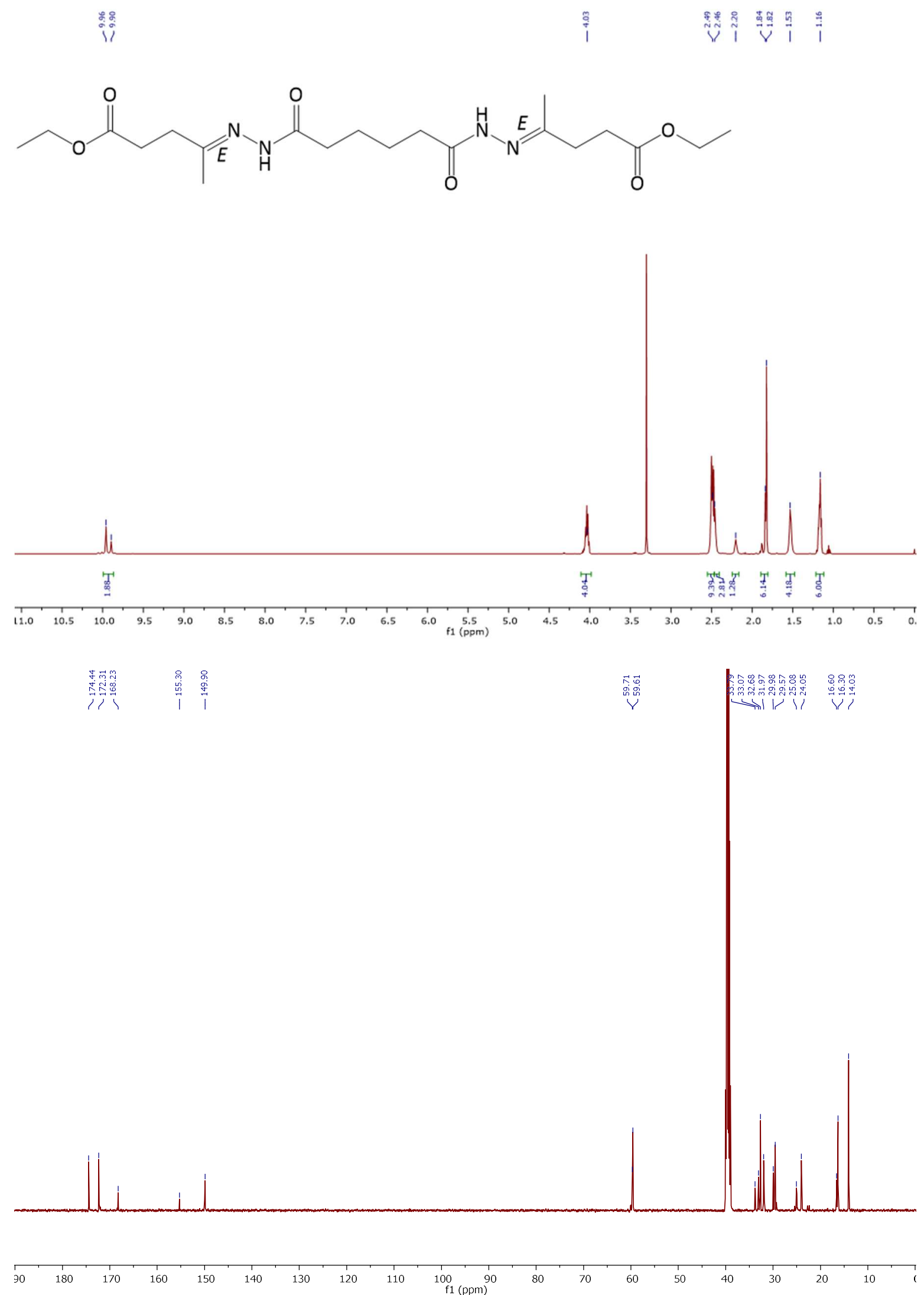

Figure 8.19. ${ }^{1} \mathrm{H}$ and ${ }^{13} \mathrm{C}$ NMR spectra (DMSO- $\left.d_{6}\right)$ of $5 \mathbf{j}\left(\delta_{H} 3.30 \mathrm{ppm}=\mathrm{HDO}, \delta_{H} 1.06 \mathrm{ppm}=\right.$ ethanol). 
<smiles>[H][Z2](CCCC(=O)NN=C(C)CCC(=O)OCC)C(=O)NN=C([3H])CC(C)C(=O)OC[3H]</smiles>

Table 8.17. Peak assignments in ${ }^{1} \mathrm{H}$ and ${ }^{13} \mathrm{C}$ NMR spectra of $\mathbf{5 j}$.

\begin{tabular}{lll}
\hline Position & $\delta_{c^{a, b}, \text { Type }}$ & $\delta_{\mathrm{H}^{a, c}}(\mathrm{~J}$ in Hz$)$ \\
\hline 1 & $16.6,16.3, \mathrm{CH}_{3}$ & $1.84, \mathrm{~s} ; 1.82, \mathrm{~s}$ \\
\hline 2 & $155.3,149.9, \mathrm{C}$ & - \\
\hline 3 & $33.1,32.7, \mathrm{CH}_{2}$ & $2.55-2.47^{\mathrm{d}}, \mathrm{m}$ \\
\hline 4 & $30.0,29.6, \mathrm{CH}_{2}$ & $2.55-2.47^{\mathrm{d}}, \mathrm{m}$ \\
\hline 5 & $172.3, \mathrm{C}$ & - \\
\hline 6 & $59.7,59.6, \mathrm{CH}_{2}$ & $4.09-3.99, \mathrm{~m}$ \\
\hline 7 & $14.0, \mathrm{CH}_{3}$ & $1.22-1.13, \mathrm{~m}$ \\
\hline $1^{\prime}$ & $174.4,168.2, \mathrm{C}$ & - \\
\hline $2^{\prime}$ & $33.8,32.0, \mathrm{CH}_{2}$ & $2.47-2.40, \mathrm{~m}, 2.22-2.18, \mathrm{~m}$ \\
\hline $3^{\prime}$ & $25.1,24.1, \mathrm{CH}_{2}$ & $1.59-1.46, \mathrm{~m}$ \\
\hline $\mathrm{NH}$ & - & $9.96, \mathrm{~s} ; 9.90, \mathrm{~s}$ \\
\hline
\end{tabular}

a Where multiple values are provided, rotamers were observed in solution.

b $126 \mathrm{MHz}$, Referenced to DMSO- $d_{6}(\delta 39.52 \mathrm{ppm}) .{ }^{261}$ Elucidated/confirmed by DEPT, HSQC, and HMBC.

c $500 \mathrm{MHz}$, Referenced to TMS ( $\delta 0.00 \mathrm{ppm}$ ). Elucidated/confirmed by HSQC, HMBC, and COSY.

${ }^{\mathrm{d}}$ Overlapping DMSO- $d_{6}$ peak. 


\subsection{CHAPTER 3}

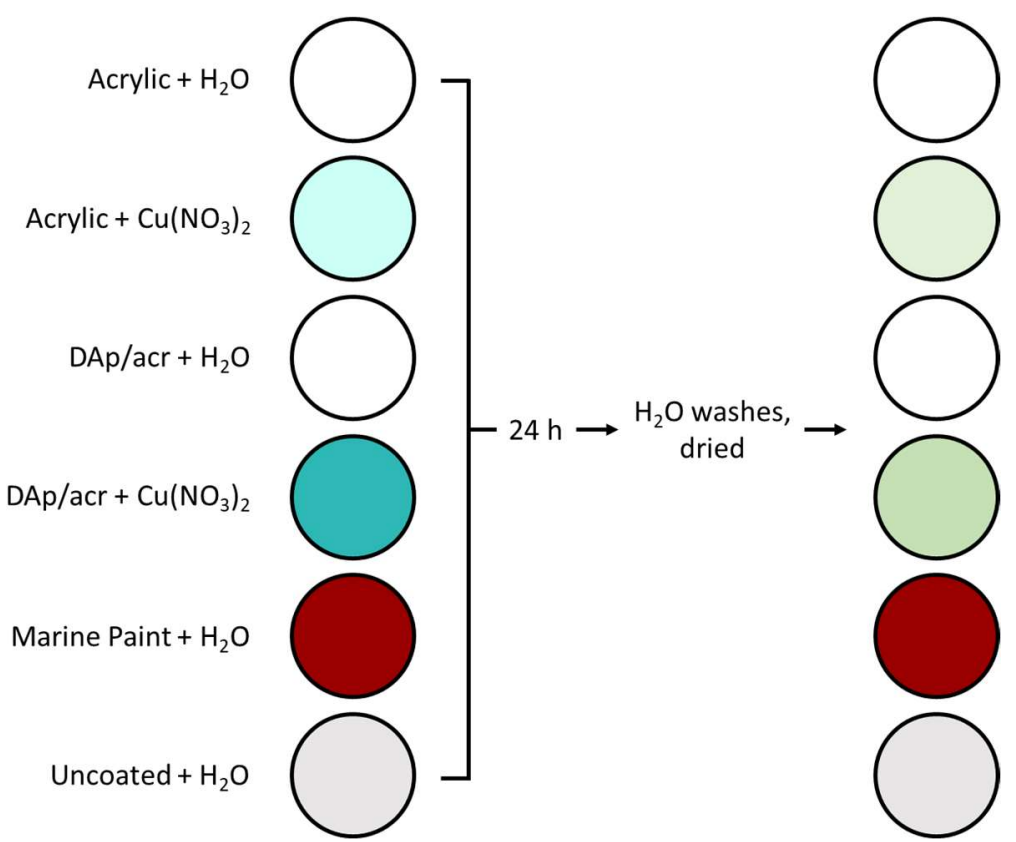

Figure 8.20. Preparation of the coated, 24-well plate(s) in Chapters 3 (Sections 3.2.2 and 3.2.3) and 6 (Sections 6.3.1.2 and 6.3.1.3).

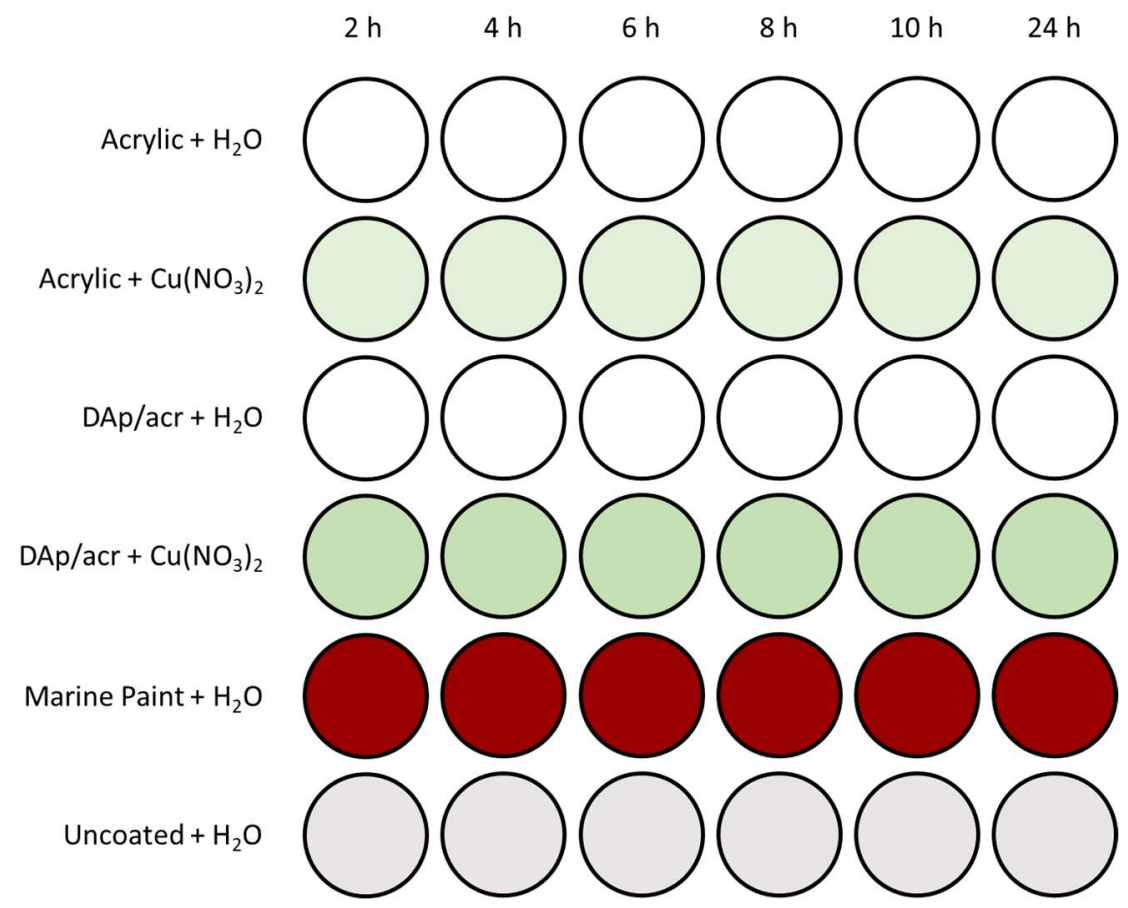

Figure 8.21. Plate setup for growth assay (Chapters 3 and 6, Sections 3.2.2 and 6.3.1.2). 


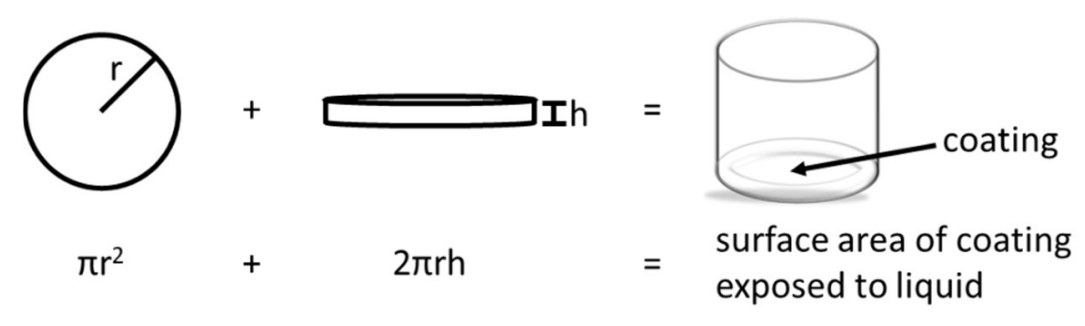

Figure 8.22. Formula for calculating the coating's surface area (cylindrical shape) exposed to liquid (Chapters 3 and 6, Sections 3.2.4 and 6.3.1.5).

Table 8.18. Results of combinations of amine and/or alcohol components with Desmodur ${ }^{\circledR}$ W.

\begin{tabular}{|c|c|c|}
\hline $\begin{array}{l}\text { Amine/Alcohol } \\
\text { Component }\end{array}$ & $\begin{array}{c}\text { mol Amine and/or Alcohol } \\
\text { Component per } 1 \text { mol Desmodur }{ }^{\circledR} \mathrm{W}\end{array}$ & Results \\
\hline $\operatorname{DETA}\left(20^{\circ} \mathrm{C}, \mathrm{RT}\right)$ & 0.67 & $\begin{array}{c}\text { exothermic; immediate } \\
\text { solidification; soft polyurea }\end{array}$ \\
\hline $\operatorname{DETA}\left(0^{\circ} \mathrm{C}\right)$ & 0.67 & $\begin{array}{l}\text { exothermic; immediate } \\
\text { solidification; soft polyurea }\end{array}$ \\
\hline TEG & 0.99 & clear, patchy polyurethane coating \\
\hline TEG, DETA & 0.90 (TEG); 0.071 (DTA) & polyurethane/polyurea gel \\
\hline 1,2-Dianilinoethane & 0.99 & $\begin{array}{l}\text { no observable reaction with the } \\
\text { hindered amine }\end{array}$ \\
\hline
\end{tabular}

Epikote $^{\mathrm{TM}}$ 235: Epoxy Resin

$\mathrm{WPE}=177-182 \mathrm{~g} / \mathrm{eq}$ epoxy group $($ mean $=179.5 \mathrm{~g} / \mathrm{eq}$ epoxy group $)$

$\underline{\text { Ancamine }^{\circledR} \text { 2459: Hardener }}$

AHEW $=101 \mathrm{~g} /$ eq amine hydrogen

$$
p h r=\frac{A H E W}{W P E} \times 100=\frac{101 \frac{\mathrm{g}}{\mathrm{eq}}}{179.5 \frac{\mathrm{g}}{\mathrm{eq}}} \times 100=56.3 \mathrm{~g} \text { hardener } \text { per } 100 \mathrm{~g} \text { resin }
$$

Scheme 8.1. Example calculation of the weight of hardener required per $100 \mathrm{~g}$ resin (phr). 
Table 8.19. Properties of epoxy coatings prepared with epoxy resin Epikote ${ }^{\mathrm{TM}} 235$ and hardeners Ancamine ${ }^{\circledR} 2459$, cyclen, DETA, and cyclam, as well as surface-functionalised with cyclam, cyclen, DETA, and PEI (bold red = strongly Cu(II)-chelating films, based on colour intensity).

\begin{tabular}{|c|c|c|c|c|c|c|c|c|}
\hline Hardener & $\begin{array}{c}\text { Surface } \\
\text { Functionalisation }\end{array}$ & $\begin{array}{l}\text { \% Over (+)/ } \\
\text { Undercure (-) }\end{array}$ & Appearance & $\begin{array}{l}\text { Softness/ } \\
\text { Hardness }\end{array}$ & $\begin{array}{c}\text { Ease of } \\
\text { Draw-Down }\end{array}$ & $\begin{array}{l}\text { Colour Change } \\
\text { Upon } \mathrm{Cu}(\mathrm{II})- \\
\text { Addition (Y/N) }\end{array}$ & Colour Intensity & $\begin{array}{l}\text { Free Epoxy } \\
\text { by IR (Y/N) }\end{array}$ \\
\hline $\begin{array}{l}\text { Ancamine }^{\circledR} \\
2459\end{array}$ & - & $-50 \%$ & $\begin{array}{l}\text { yellow, clear, } \\
\text { splotchy }\end{array}$ & soft & splotchy & - & - & $\mathrm{Y}$ \\
\hline $\begin{array}{l}\text { Ancamine }^{\circledR} \\
2459\end{array}$ & cyclam & $-50 \%$ & $\begin{array}{l}\text { yellow, clear, } \\
\text { uneven surface }\end{array}$ & $\begin{array}{l}\text { hard, } \\
\text { flexible }\end{array}$ & - & $Y$ - purple & medium & $Y$ \\
\hline $\begin{array}{l}\text { Ancamine }^{\circledR} \\
2459\end{array}$ & - & $-40 \%$ & yellow, clear & $\begin{array}{l}\text { hard, } \\
\text { flexible }\end{array}$ & slightly splotchy & $\begin{array}{l}\mathrm{N} \text { - retention but no } \\
\text { obvious change }\end{array}$ & weak & $Y$ \\
\hline $\begin{array}{l}\text { Ancamine }^{\circledR} \\
2459\end{array}$ & cyclen & $-40 \%$ & $\begin{array}{c}\text { yellow, blushing, } \\
\text { matte }\end{array}$ & $\begin{array}{c}\text { hard, } \\
\text { flexible }\end{array}$ & easy, uniform & Y-dark blue & medium & $\mathrm{Y}$ \\
\hline $\begin{array}{l}\text { Ancamine }^{\circledR} \\
2459\end{array}$ & cyclam & $-40 \%$ & yellow, clear & $\begin{array}{l}\text { hard, } \\
\text { flexible }\end{array}$ & - & $\begin{array}{l}\mathrm{N} \text { - retention but no } \\
\text { obvious change }\end{array}$ & weak & $\mathrm{Y}$ \\
\hline $\begin{array}{l}\text { Ancamine }^{\circledR} \\
2459\end{array}$ & DETA & $-40 \%$ & yellow, matte & soft & easy, uniform & $\mathrm{Y}$-dark blue & v. strong & $\mathbf{N}$ \\
\hline $\begin{array}{l}\text { Ancamine }^{\circledR} \\
2459\end{array}$ & PEI & $-40 \%$ & $\begin{array}{l}\text { yellow, opaque, } \\
\text { blushing, matte }\end{array}$ & flexible & easy, uniform & Y - dark blue & strong & $\mathbf{N}$ \\
\hline $\begin{array}{l}\text { Ancamine }^{\circledR} \\
2459\end{array}$ & - & $-30 \%$ & yellow, clear & $\begin{array}{l}\text { hard, } \\
\text { flexible }\end{array}$ & slightly splotchy & $\begin{array}{l}\mathrm{N} \text { - retention but no } \\
\text { obvious change }\end{array}$ & medium & $\mathrm{Y}$ \\
\hline $\begin{array}{l}\text { Ancamine }^{\circledR} \\
2459\end{array}$ & cyclam & $-30 \%$ & yellow, clear & $\begin{array}{l}\text { hard, } \\
\text { flexible }\end{array}$ & - & $\begin{array}{l}\mathrm{N} \text { - retention but no } \\
\text { obvious change }\end{array}$ & weak & $\mathrm{Y}$ \\
\hline $\begin{array}{l}\text { Ancamine }^{\circledR} \\
2459\end{array}$ & DETA & $-30 \%$ & yellow, matte & soft & - & Y-dark blue & v. strong & $\mathbf{N}$ \\
\hline $\begin{array}{l}\text { Ancamine }^{\circledR} \\
2459\end{array}$ & - & $-20 \%$ & yellow, clear & $\begin{array}{l}\text { hard, } \\
\text { flexible }\end{array}$ & easy, uniform & $\begin{array}{l}\mathrm{N} \text { - retention but no } \\
\text { obvious change }\end{array}$ & medium & $Y$ \\
\hline $\begin{array}{l}\text { Ancamine }^{\circledR} \\
2459\end{array}$ & cyclam & $-20 \%$ & yellow, clear & $\begin{array}{l}\text { hard, } \\
\text { brittle }\end{array}$ & - & $\begin{array}{l}\mathrm{N} \text { - retention but no } \\
\text { obvious change }\end{array}$ & weak & $Y$ \\
\hline $\begin{array}{l}\text { Ancamine }^{\circledR} \\
2459\end{array}$ & - & $-10 \%$ & yellow, clear & $\begin{array}{l}\text { hard, } \\
\text { flexible }\end{array}$ & easy, uniform & $\begin{array}{l}\mathrm{N} \text { - retention but no } \\
\text { obvious change }\end{array}$ & medium & $\mathrm{Y}$ \\
\hline $\begin{array}{l}\text { Ancamine }^{\circledR} \\
2459\end{array}$ & cyclam & $-10 \%$ & yellow, clear & $\begin{array}{l}\text { hard, } \\
\text { brittle }\end{array}$ & - & $\begin{array}{l}\mathrm{N} \text { - retention but no } \\
\text { obvious change }\end{array}$ & weak & $\mathrm{Y}$ \\
\hline
\end{tabular}




\begin{tabular}{|c|c|c|c|c|c|c|c|c|}
\hline $\begin{array}{l}\text { Ancamine }^{\circledR} \\
2459\end{array}$ & - & stoichiometric & yellow, clear & $\begin{array}{l}\text { hard, } \\
\text { flexible }\end{array}$ & easy, uniform & $\begin{array}{l}\mathrm{N} \text { - retention but no } \\
\text { obvious change }\end{array}$ & medium & $Y$ \\
\hline $\begin{array}{l}\text { Ancamine }^{\circledR} \\
2459\end{array}$ & - & $+10 \%$ & yellow, clear & $\begin{array}{l}\text { hard, } \\
\text { flexible }\end{array}$ & easy, uniform & $\begin{array}{l}\mathrm{N} \text { - retention but no } \\
\text { obvious change }\end{array}$ & medium & $Y$ \\
\hline $\begin{array}{l}\text { Ancamine }^{\circledast} \\
2459\end{array}$ & - & $+20 \%$ & yellow, clear & $\begin{array}{l}\text { hard, } \\
\text { flexible }\end{array}$ & easy, uniform & $\begin{array}{l}\mathrm{N} \text { - retention but no } \\
\text { obvious change }\end{array}$ & medium & Y \\
\hline $\begin{array}{l}\text { Ancamine }^{\circledR} \\
2459\end{array}$ & - & $+50 \%$ & yellow, clear & $\begin{array}{l}\text { hard, } \\
\text { brittle }\end{array}$ & easy, uniform & $\begin{array}{l}\mathrm{N} \text { - retention but no } \\
\text { obvious change }\end{array}$ & medium & $\mathrm{N}$ \\
\hline $\begin{array}{l}\text { Ancamine }^{\circledR} \\
2459, \text { cyclen }^{a}\end{array}$ & - & $+10 \%$ (cyclen) & yellow, clear & $\begin{array}{l}\text { hard, } \\
\text { brittle }\end{array}$ & easy, uniform & Y - dark blue & strong & $\mathbf{N}$ \\
\hline $\begin{array}{l}\text { Ancamine }^{\circledR} \\
2459, \text { cyclen }^{\text {a }}\end{array}$ & - & $+20 \%$ (cyclen) & yellow, clear & $\begin{array}{l}\text { hard, } \\
\text { brittle }\end{array}$ & easy, uniform & Y - dark blue & strong & $\mathbf{N}$ \\
\hline $\begin{array}{l}\text { Ancamine }^{\circledR} \\
2459, \text { DETA }^{\text {b }}\end{array}$ & - & $+10 \%$ (DETA) & yellow, clear & $\begin{array}{l}\text { hard, } \\
\text { brittle }\end{array}$ & easy, uniform & Y-dark blue & medium & $\mathrm{N}$ \\
\hline $\begin{array}{l}\text { Ancamine }^{\circledR} \\
2459, \text { DETA }^{\text {b }}\end{array}$ & - & $+20 \%$ (DETA) & yellow, clear & $\begin{array}{l}\text { hard, } \\
\text { brittle }\end{array}$ & easy, uniform & $\mathrm{Y}$-dark blue & medium & $\mathrm{N}$ \\
\hline Cyclen & - & $-20 \%$ & milky, clear & $\begin{array}{l}\text { hard, } \\
\text { brittle }\end{array}$ & poor, uneven & $\mathrm{Y}$-dark blue & medium & Y \\
\hline Cyclen & - & $-10 \%$ & milky, clear & $\begin{array}{l}\text { hard, } \\
\text { brittle }\end{array}$ & poor, uneven & Y-dark blue & medium & $Y$ \\
\hline Cyclen & - & stoichiometric & milky, clear & $\begin{array}{l}\text { hard, } \\
\text { brittle }\end{array}$ & slightly uneven & Y-dark blue & medium & $\mathrm{Y}$ \\
\hline Cyclen & - & $+10 \%$ & milky, clear & $\begin{array}{l}\text { hard, } \\
\text { brittle }\end{array}$ & slightly uneven & Y-dark blue & medium-strong & $\mathrm{Y}$ \\
\hline Cyclen & - & $+20 \%$ & milky, clear & $\begin{array}{l}\text { hard, } \\
\text { brittle }\end{array}$ & poor, uneven & Y - dark blue & v. strong & $\mathbf{Y}$ \\
\hline DETA & - & $-20 \%$ & $\begin{array}{l}\text { splotchy, white } \\
\text { blushing }\end{array}$ & $\begin{array}{l}\text { soft, } \\
\text { sticky }\end{array}$ & $\begin{array}{l}\text { v. poor, uneven, } \\
\text { splotchy }\end{array}$ & Y-dark blue & medium, uneven & $\mathrm{Y}$ \\
\hline DETA & - & $-10 \%$ & $\begin{array}{l}\text { splotchy, white } \\
\text { blushing }\end{array}$ & $\begin{array}{l}\text { soft, } \\
\text { sticky }\end{array}$ & $\begin{array}{l}\text { v. poor, uneven, } \\
\text { splotchy }\end{array}$ & Y-dark blue & medium, uneven & $\mathrm{Y}$ \\
\hline DETA & - & stoichiometric & $\begin{array}{l}\text { splotchy, white } \\
\text { blushing }\end{array}$ & $\begin{array}{l}\text { soft, } \\
\text { sticky }\end{array}$ & $\begin{array}{l}\text { v. poor, uneven, } \\
\text { splotchy }\end{array}$ & $\mathrm{Y}$-dark blue & medium, uneven & $Y$ \\
\hline DETA & - & $+10 \%$ & $\begin{array}{l}\text { splotchy, white } \\
\text { blushing }\end{array}$ & $\begin{array}{l}\text { soft, } \\
\text { sticky }\end{array}$ & $\begin{array}{l}\text { v. poor, uneven, } \\
\text { splotchy }\end{array}$ & Y-dark blue & medium, uneven & $\mathrm{Y}$ \\
\hline
\end{tabular}




\begin{tabular}{|c|c|c|c|c|c|c|c|c|}
\hline DETA & - & $+20 \%$ & $\begin{array}{c}\text { splotchy, white } \\
\text { blushing }\end{array}$ & $\begin{array}{l}\text { soft, } \\
\text { sticky }\end{array}$ & $\begin{array}{c}\text { v. poor, uneven, } \\
\text { splotchy }\end{array}$ & $\mathrm{Y}$ - dark blue & strong, uneven & $\mathbf{Y}$ \\
\hline $\begin{array}{l}\text { DETA }(75 \%), \\
\text { cyclen }(25 \%)^{c}\end{array}$ & - & stoichiometric & $\begin{array}{l}\text { splotchy, milky } \\
\text { white blushing }\end{array}$ & $\begin{array}{l}\text { sticky, } \\
\text { brittle }\end{array}$ & $\begin{array}{l}\text { v. poor, uneven, } \\
\text { splotchy }\end{array}$ & $\mathrm{Y}$-dark blue & medium & $\mathrm{Y}$ \\
\hline $\begin{array}{l}\text { DETA }(50 \%), \\
\text { cyclen }(50 \%)^{d}\end{array}$ & - & stoichiometric & $\begin{array}{l}\text { milky white } \\
\text { blushing }\end{array}$ & $\begin{array}{l}\text { hard, } \\
\text { brittle }\end{array}$ & slightly splotchy & $\mathrm{Y}$-dark blue & medium & $\mathrm{Y}$ \\
\hline $\begin{array}{l}\text { DETA }(25 \%) \text {, } \\
\text { cyclen }(75 \%)^{\mathrm{e}}\end{array}$ & - & stoichiometric & $\begin{array}{l}\text { milky white } \\
\text { blushing }\end{array}$ & $\begin{array}{l}\text { hard, } \\
\text { brittle }\end{array}$ & $\begin{array}{c}\text { slightly } \\
\text { splotchy/uneven }\end{array}$ & Y-dark blue & medium & $\mathrm{Y}$ \\
\hline $\begin{array}{l}\text { DETA }(25 \%) \text {, } \\
\text { cyclen }(100 \%)^{f}\end{array}$ & - & $+25 \%$ & $\begin{array}{l}\text { milky white } \\
\text { blushing }\end{array}$ & $\begin{array}{l}\text { hard, } \\
\text { brittle }\end{array}$ & $\begin{array}{l}\text { slightly splotchy, } \\
\text { mostly uniform }\end{array}$ & $\mathrm{Y}$ - dark blue & strong & $\mathbf{Y}$ \\
\hline $\begin{array}{l}\text { DETA }(100 \%) \text {, } \\
\text { cyclen }(25 \%)^{\mathrm{g}}\end{array}$ & - & $+25 \%$ & $\begin{array}{l}\text { splotchy, milky } \\
\text { white blushing }\end{array}$ & $\begin{array}{l}\text { sticky, } \\
\text { brittle }\end{array}$ & $\begin{array}{c}\text { v. poor, uneven, } \\
\text { splotchy }\end{array}$ & Y - dark blue & strong & $\mathbf{Y}$ \\
\hline Cyclam & - & $-20 \%$ & clear, colourless & $\begin{array}{l}\text { hard, } \\
\text { brittle }\end{array}$ & easy, uniform & Y-purple & $\begin{array}{c}\text { v. weak (aq), } \\
\text { medium (MeOH) }\end{array}$ & $Y$ \\
\hline Cyclam & - & stoichiometric & clear, colourless & $\begin{array}{l}\text { hard, } \\
\text { brittle }\end{array}$ & easy, uniform & Y-purple & $\begin{array}{c}\text { v. weak (aq), } \\
\text { medium (MeOH) }\end{array}$ & $\mathrm{Y}$ \\
\hline Cyclam & - & $+20 \%$ & clear, colourless & $\begin{array}{l}\text { hard, slight } \\
\text { flexibility }\end{array}$ & easy, uniform & $Y$ - purple & $\begin{array}{c}\text { v. weak }(\mathrm{aq}) \\
\text { medium }(\mathrm{MeOH})\end{array}$ & $Y$ \\
\hline
\end{tabular}

a Stoichiometric amount of Ancamine ${ }^{\circledR} 2459$ but overcured with cyclen.

${ }^{\mathrm{b}}$ Stoichiometric amount of Ancamine ${ }^{\circledR} 2459$ but overcured with DETA.

c Enough DETA and cyclen added as hardeners to react with $75 \%$ and $25 \%$ of the epoxy groups, respectively.

d Enough DETA and cyclen added as hardeners to each react with half of the epoxy groups.

${ }^{e}$ Enough DETA and cyclen added as hardeners to react with $25 \%$ and $75 \%$ of the epoxy groups, respectively.

f Stoichiometric amount of cyclen but overcured with DETA.

${ }^{\mathrm{g}}$ Stoichiometric amount of DETA but overcured with cyclen. 
(a)

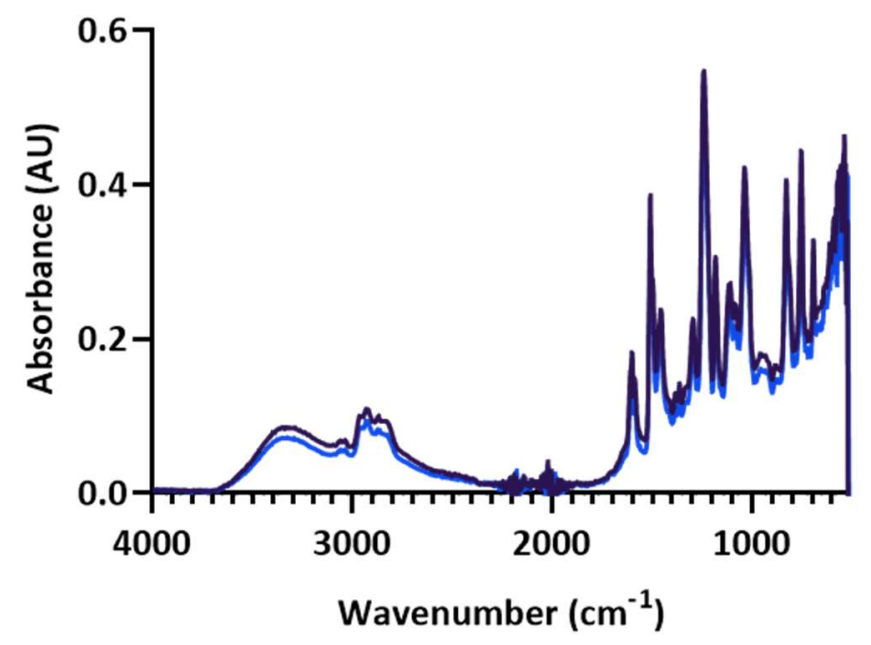

- $+20 \% \mathrm{w} / \mathrm{w}$ cyclen

$-+10 \% \mathrm{w} / \mathrm{w}$ cyclen

(b)

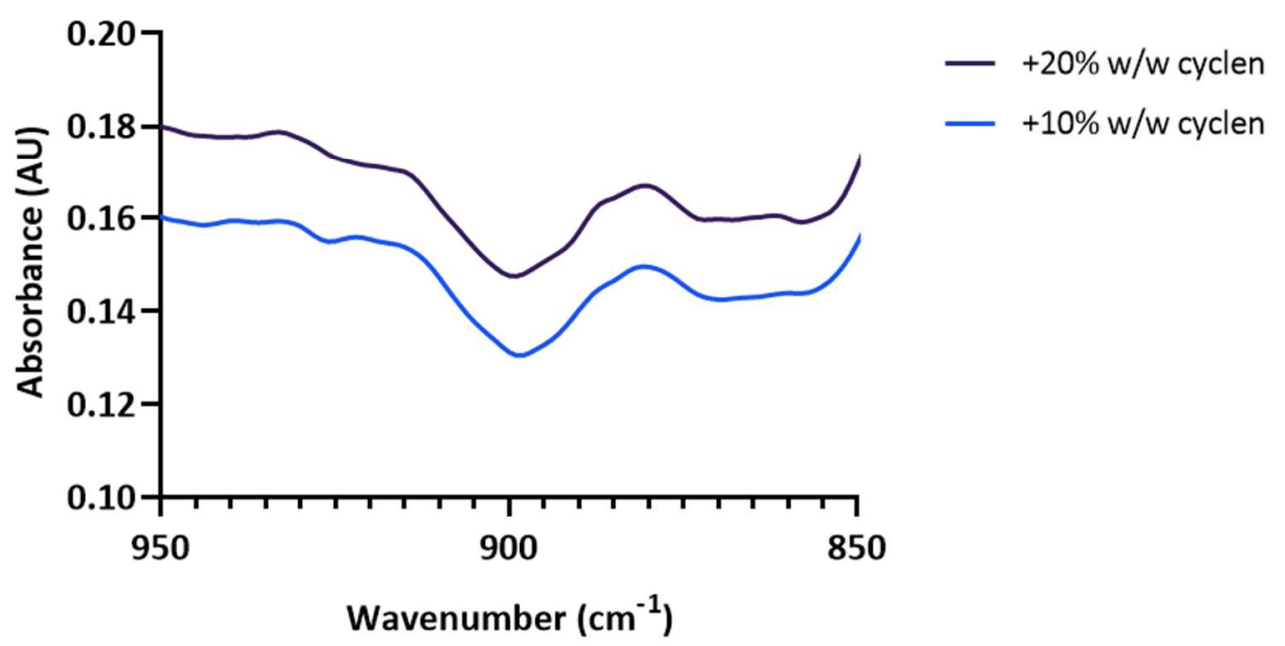

Figure 8.23. FT-IR spectra of coatings prepared from Epikote ${ }^{\mathrm{TM}} 235$ and Ancamine ${ }^{\circledR} 2459$ in stoichiometric proportions and overcured $(+10 \% \mathrm{w} / \mathrm{w},+20 \% \mathrm{w} / \mathrm{w})$ with cyclen: $(a)$ is the full spectrum, and (b) shows an expanded view of the bands due to the epoxy group ( $915 \mathrm{~cm}^{-1}$ ). 
(a)

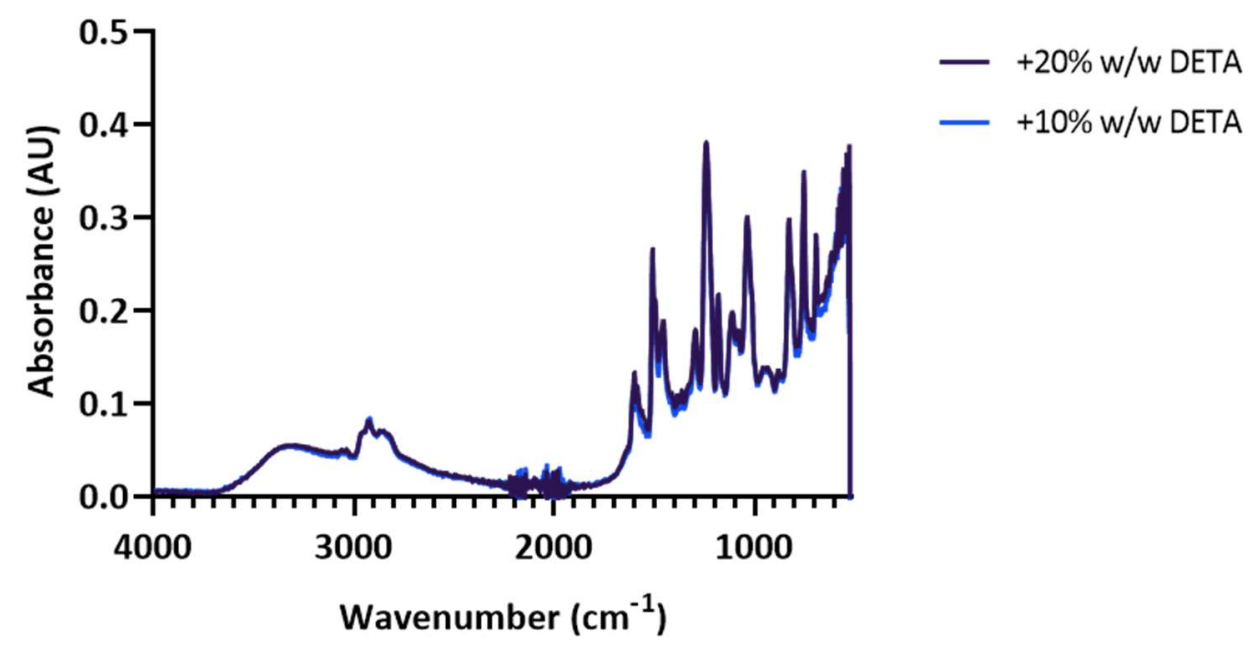

(b)

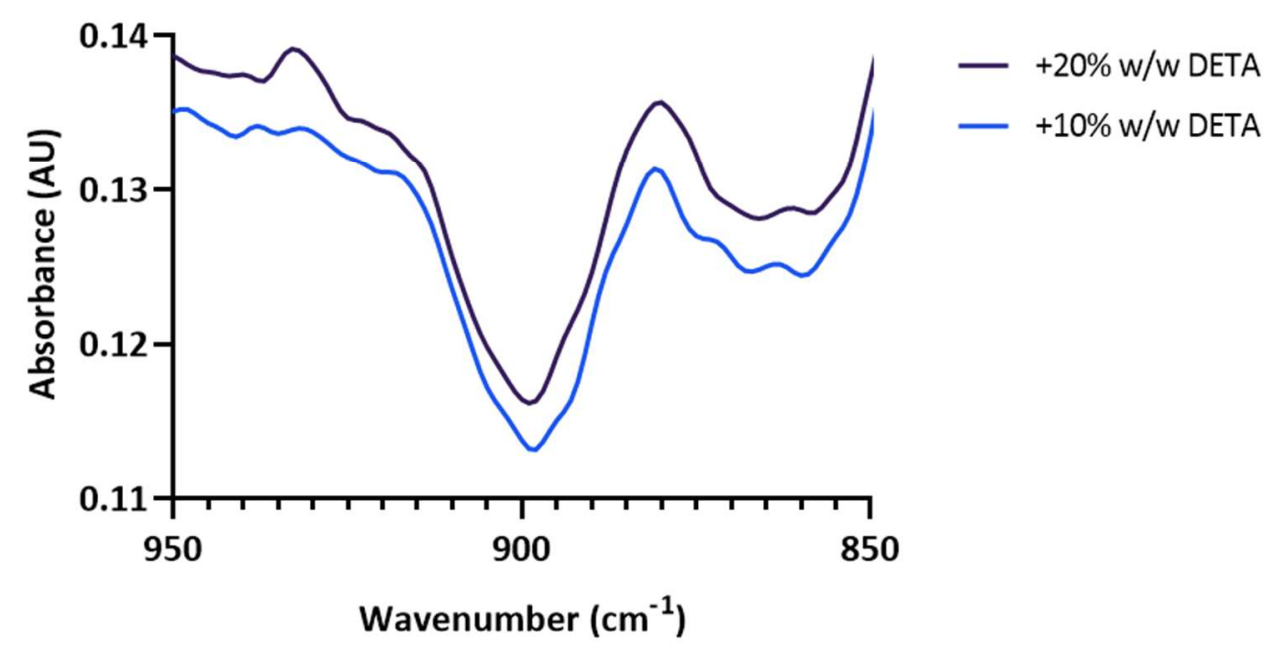

Figure 8.24. FT-IR spectra of coatings prepared from Epikote ${ }^{\mathrm{TM}} 235$ and Ancamine ${ }^{\circledR} 2459$ in stoichiometric proportions and overcured $(+10 \% \mathrm{w} / \mathrm{w},+20 \% \mathrm{w} / \mathrm{w})$ with DETA: $(a)$ is the full spectrum, and (b) shows an expanded view of the bands due to the epoxy group ( $\left.915 \mathrm{~cm}^{-1}\right)$. 
(a)

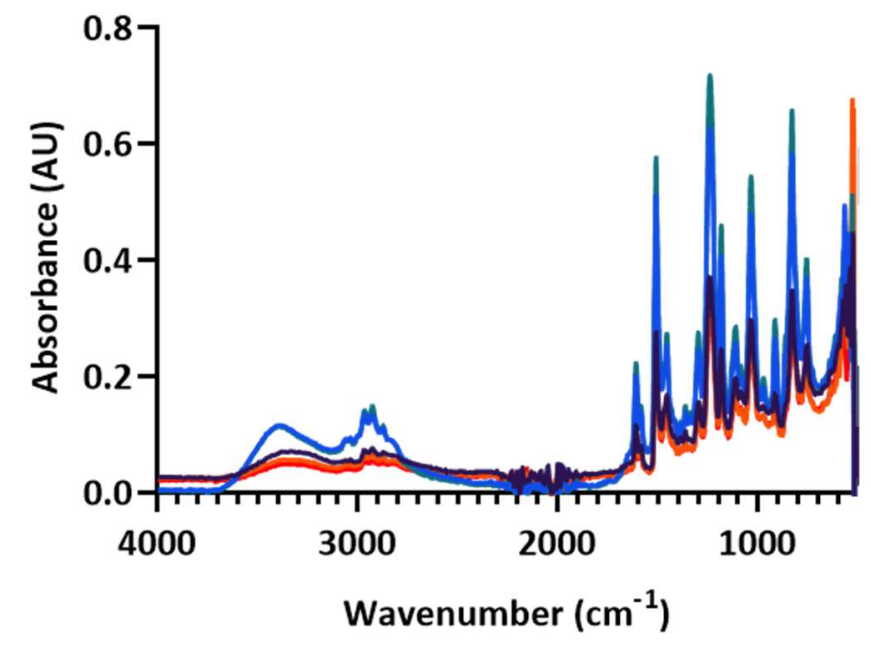

— $+25 \%$ w/w DETA

$+25 \% \mathrm{w} / \mathrm{w}$ cyclen Stoichiometric (DETA/cyclen 75/25)

Stoichiometric (DETA/cyclen 50/50)

Stoichiometric (DETA/cyclen 25/75)

(b)

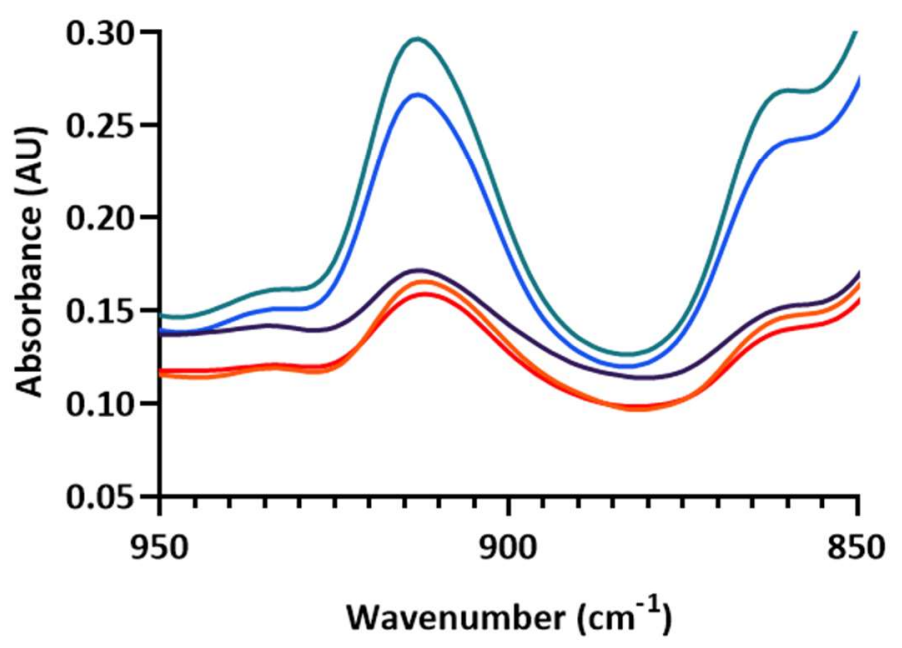

— $+25 \%$ w/w DETA

- $+25 \% \mathrm{w} / \mathrm{w}$ cyclen Stoichiometric (DETA/cyclen 75/25) Stoichiometric (DETA/cyclen 50/50) Stoichiometric (DETA/cyclen 25/75)

Figure 8.25. FT-IR spectra of coatings prepared from Epikote ${ }^{\mathrm{TM}} 235$ and DETA and/or cyclen in stoichiometric proportions or overcured $(+25 \% \mathrm{w} / \mathrm{w})$ with DETA or cyclen: $(a)$ is the full spectrum, and (b) shows an expanded view of the bands due to the epoxy group ( $915 \mathrm{~cm}^{-1}$; adjusted baseline). 
(a)

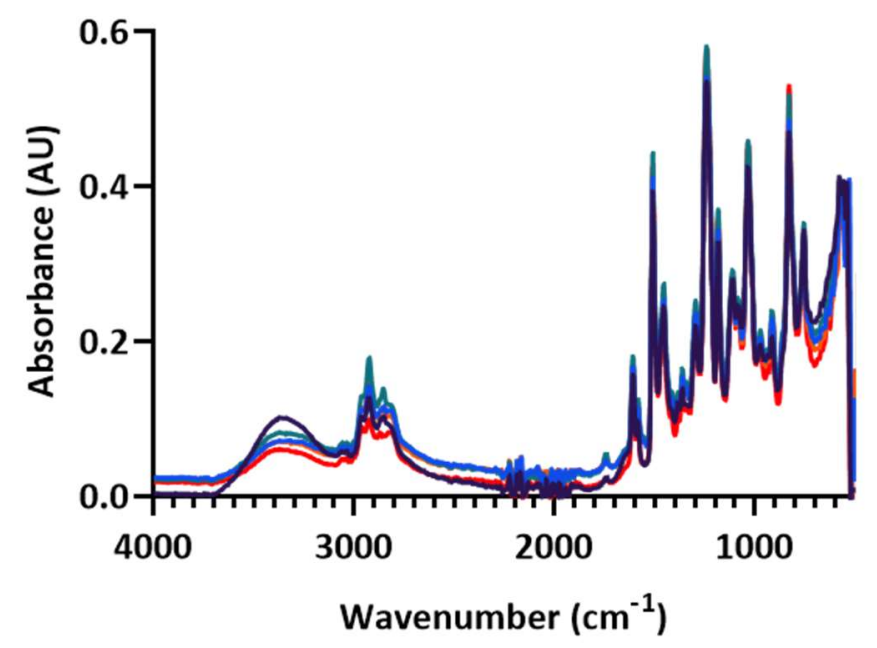

- $+20 \% \mathrm{w} / \mathrm{w}$ cyclen

- $+10 \% \mathrm{w} / \mathrm{w}$ cyclen

- Stoichiometric

$-10 \% \mathrm{w} / \mathrm{w}$ cyclen

$-20 \% \mathrm{w} / \mathrm{w}$ cyclen

(b)

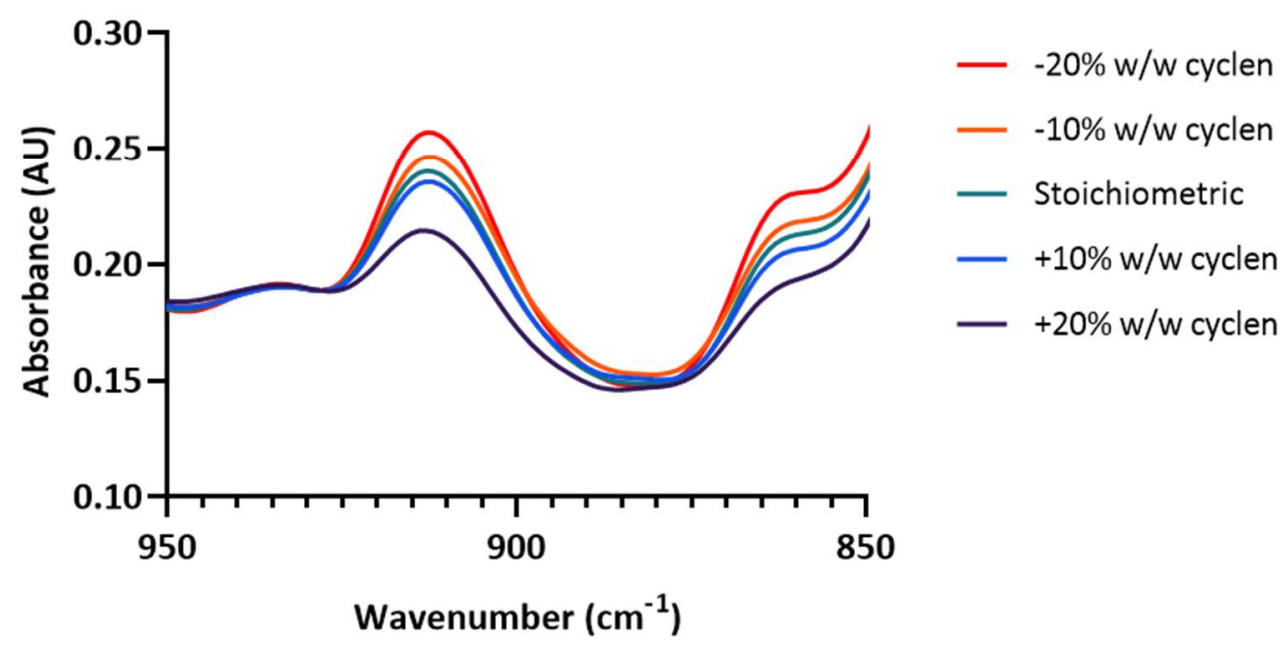

Figure 8.26. FT-IR spectra of coatings prepared from Epikote ${ }^{\mathrm{TM}} 235$ and cyclen in stoichiometric proportions or under/overcured $(\mathbf{2 0 \%} \mathrm{w} / \mathrm{w})$ with cyclen: $(\mathrm{a})$ is the full spectrum, and (b) shows an expanded view of the bands due to the epoxy group ( $915 \mathrm{~cm}^{-1}$; adjusted baseline). 
(a)

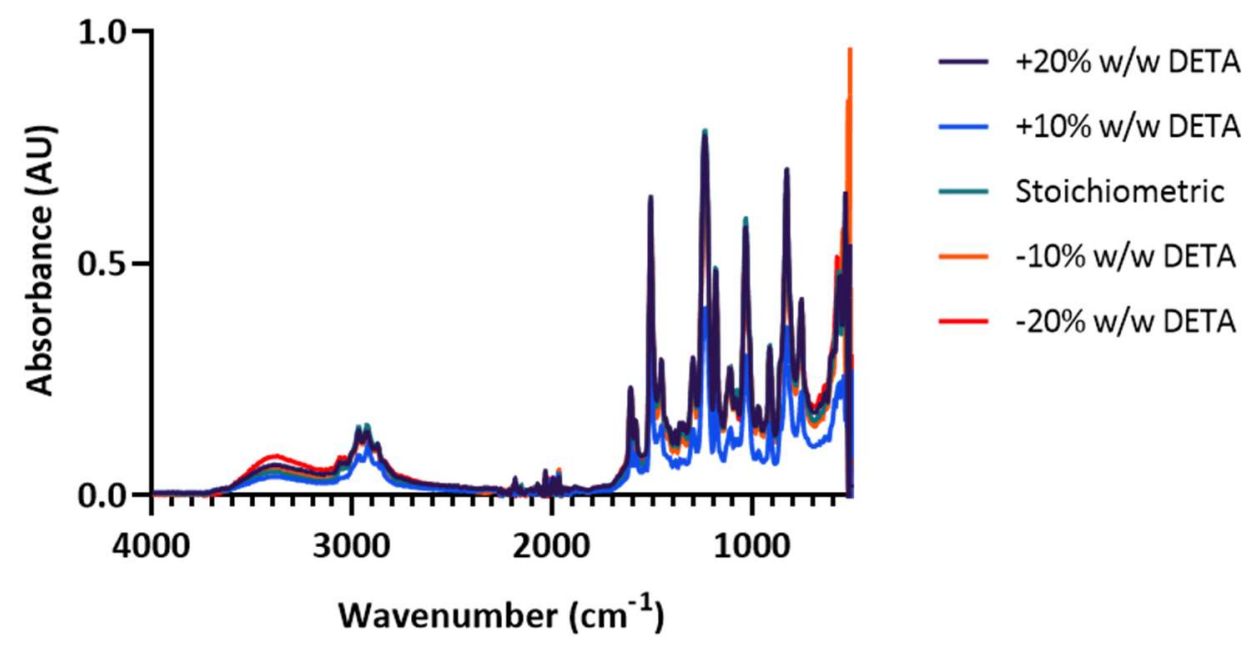

(b)

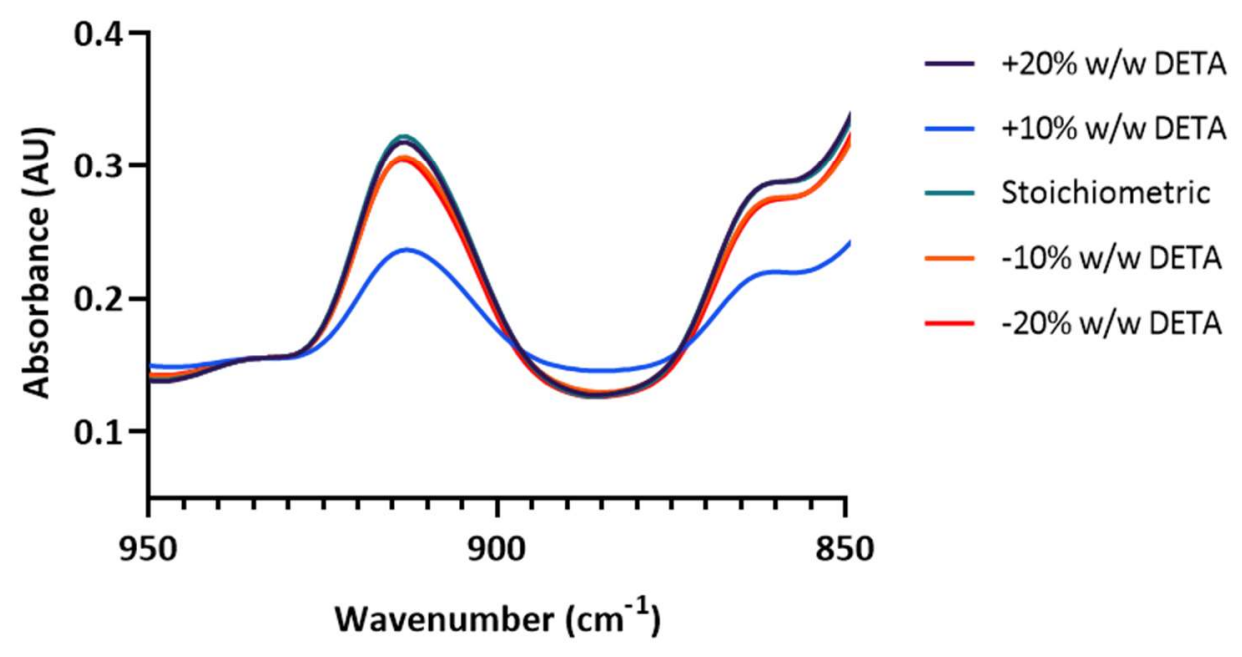

Figure 8.27. FT-IR spectra of coatings prepared from Epikote $^{\mathrm{TM}} 235$ and DETA in stoichiometric proportions or under/overcured $(\mathbf{2 0 \%} \mathbf{w} / \mathbf{w})$ with DETA: $(a)$ is the full spectrum, and (b) shows an expanded view of the bands due to the epoxy group ( $915 \mathrm{~cm}^{-1}$; adjusted baseline). 
(a)

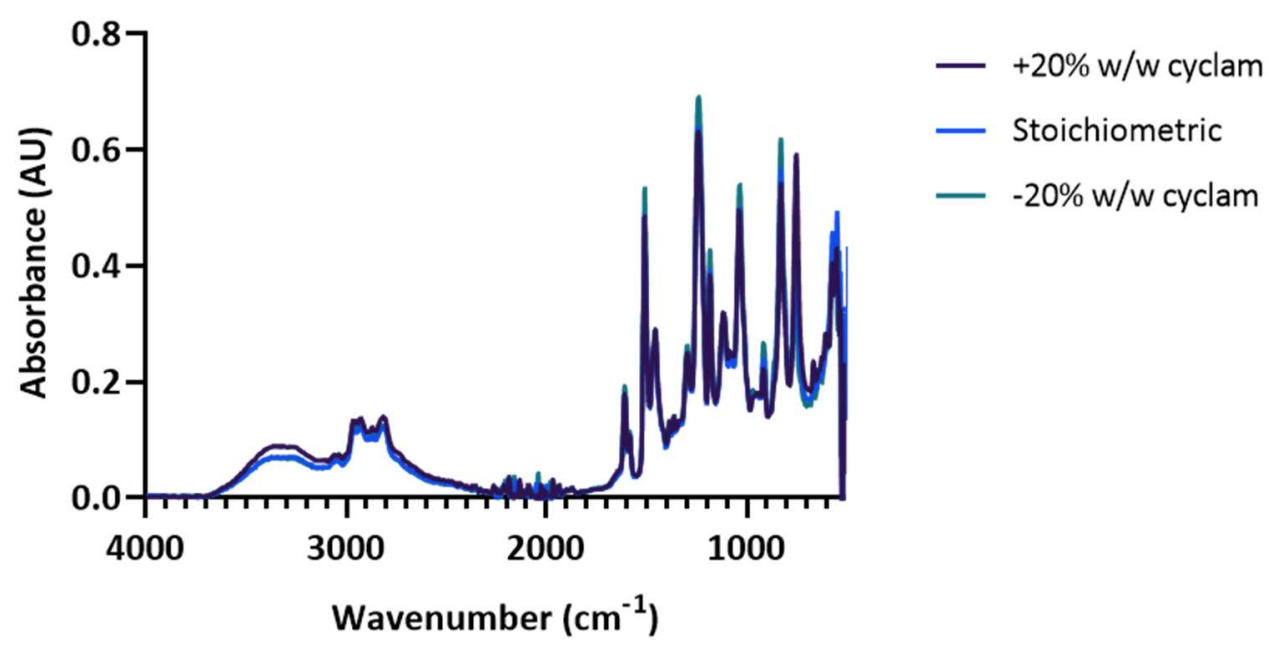

(b)

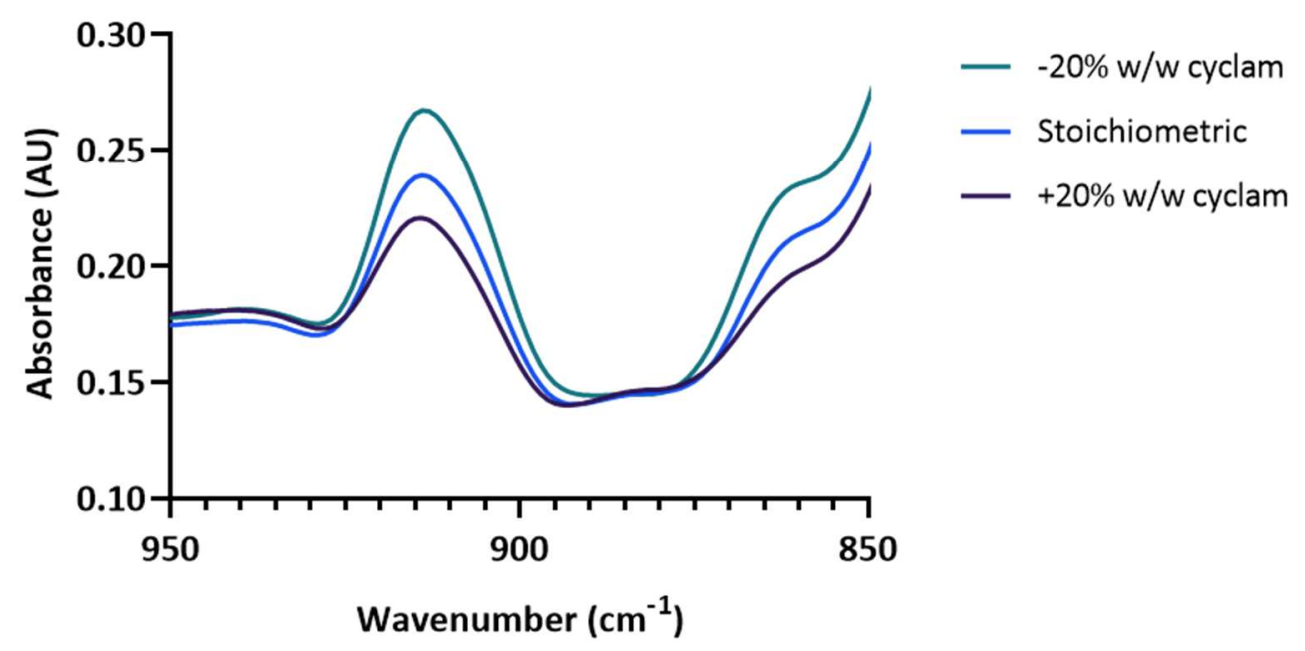

Figure 8.28. FT-IR spectra of coatings prepared from Epikote ${ }^{\mathrm{TM}} 235$ and cyclam in stoichiometric proportions or under/overcured $(\mathbf{2 0 \%} \mathrm{w} / \mathrm{w})$ with cyclam: $(\mathrm{a})$ is the full spectrum, and (b) shows an expanded view of the bands due to the epoxy group $\left(\sim 915 \mathrm{~cm}^{-1}\right)$. 


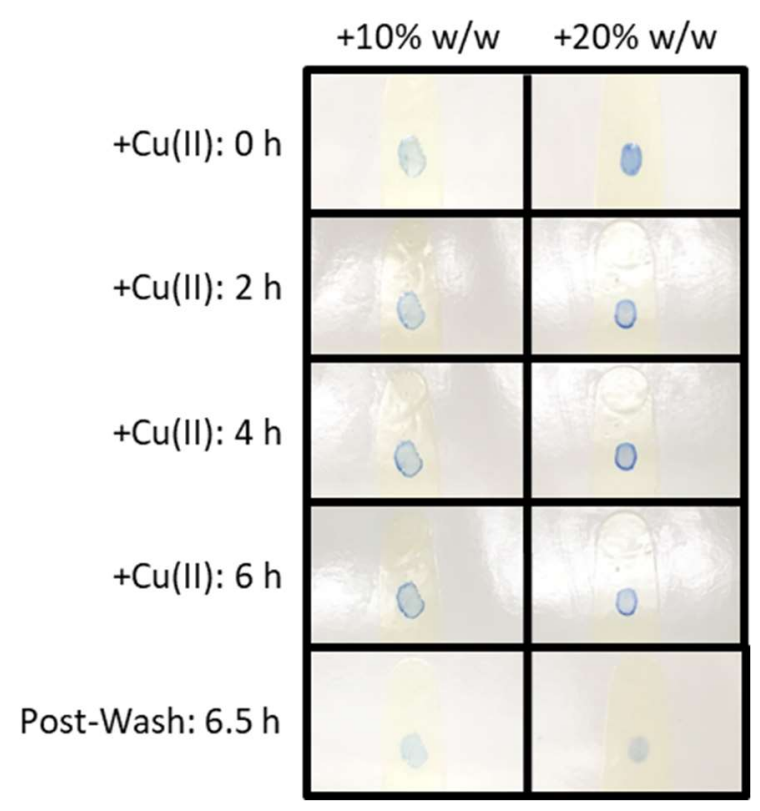

Figure 8.29. Monitoring the $\mathrm{Cu}(\mathrm{II})$-addition to coatings prepared from Epikote ${ }^{\mathrm{TM}} 235$ and Ancamine ${ }^{\circledR}$ 2459 in stoichiometric proportions and overcured $(+10 \% \mathrm{w} / \mathrm{w},+20 \% \mathrm{w} / \mathrm{w})$ with cyclen.

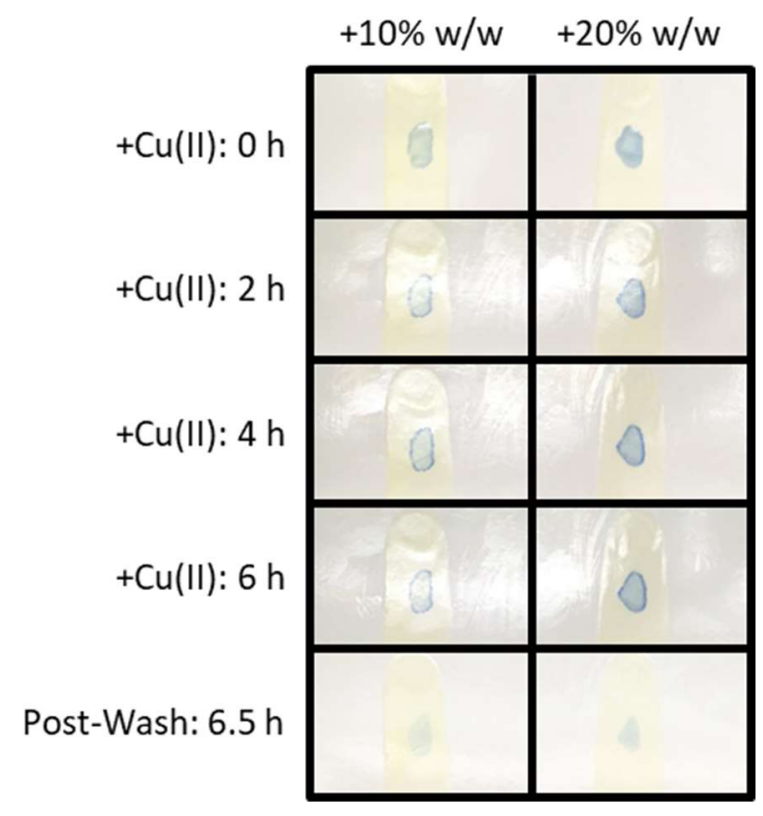

Figure 8.30. Monitoring the $\mathrm{Cu}(\mathrm{II})$-addition to coatings prepared from Epikote ${ }^{\mathrm{TM}} 235$ and Ancamine ${ }^{\circledR}$ 2459 in stoichiometric proportions and overcured $(+10 \% \mathrm{w} / \mathrm{w},+20 \% \mathrm{w} / \mathrm{w})$ with DETA. 


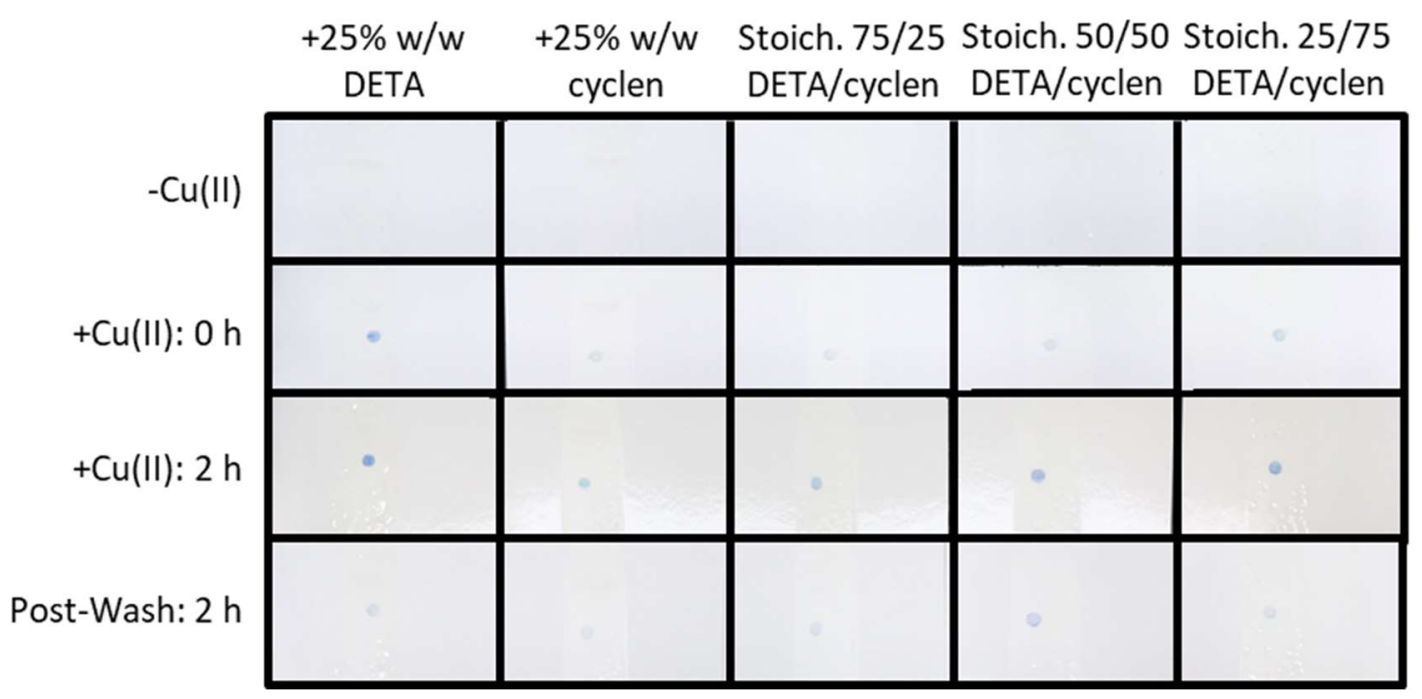

Figure 8.31. Monitoring the $\mathrm{Cu}(\mathrm{II})$-addition to coatings prepared from Epikote ${ }^{\mathrm{TM}} 235$ and DETA and/or cyclen in stoichiometric proportions or overcured $(+25 \% \mathrm{w} / \mathrm{w})$ with DETA or cyclen.

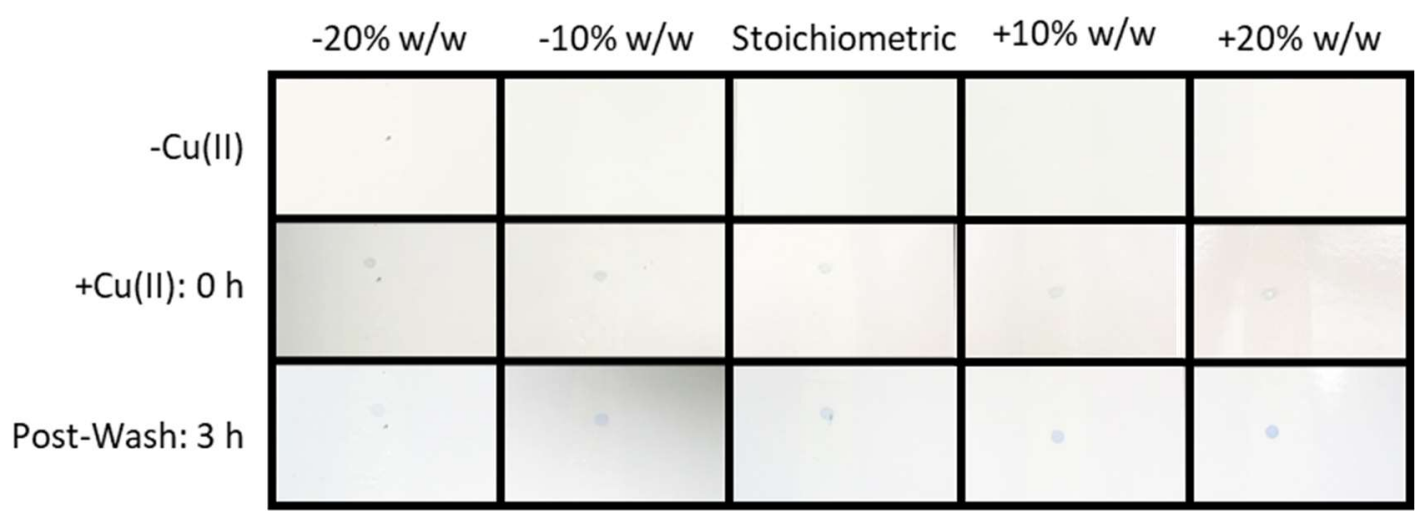

Figure 8.32. Monitoring the $\mathrm{Cu}(\mathrm{II})$-addition to coatings prepared from Epikote ${ }^{\mathrm{TM}} 235$ and cyclen in stoichiometric proportions or under/overcured $( \pm 20 \% \mathrm{w} / \mathrm{w})$ with cyclen. 


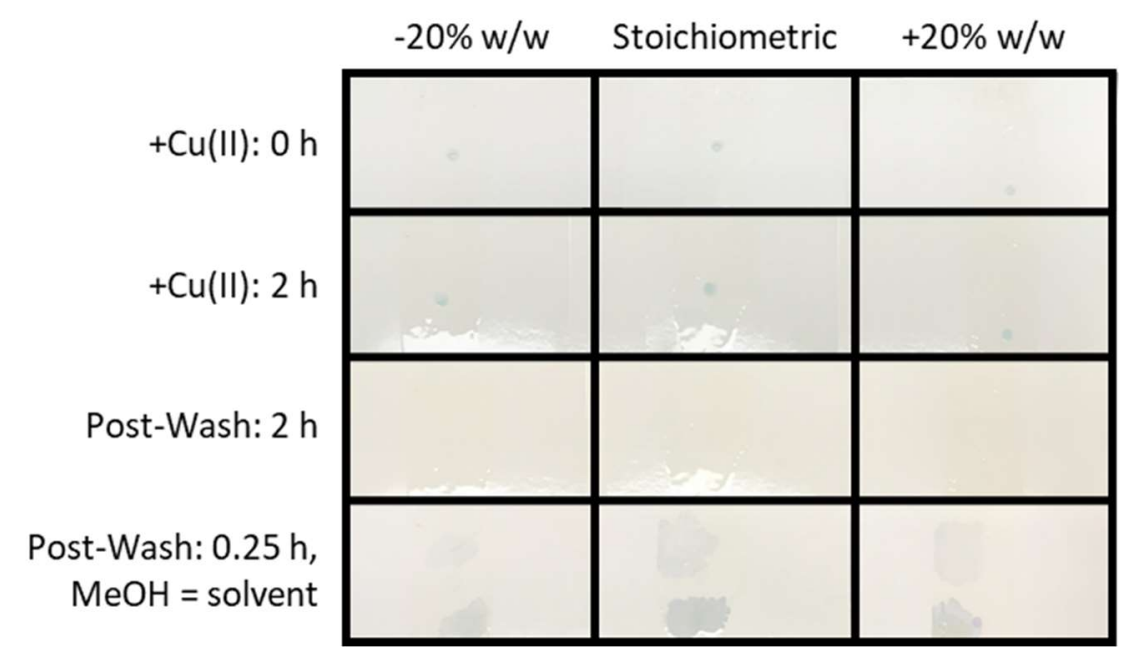

Figure 8.33. Monitoring the $\mathrm{Cu}(\mathrm{II})$-addition to coatings prepared from Epikote ${ }^{\mathrm{TM}} 235$ and cyclam in stoichiometric proportions or under/overcured $( \pm 20 \% \mathrm{w} / \mathrm{w})$ with cyclam. 
(a)

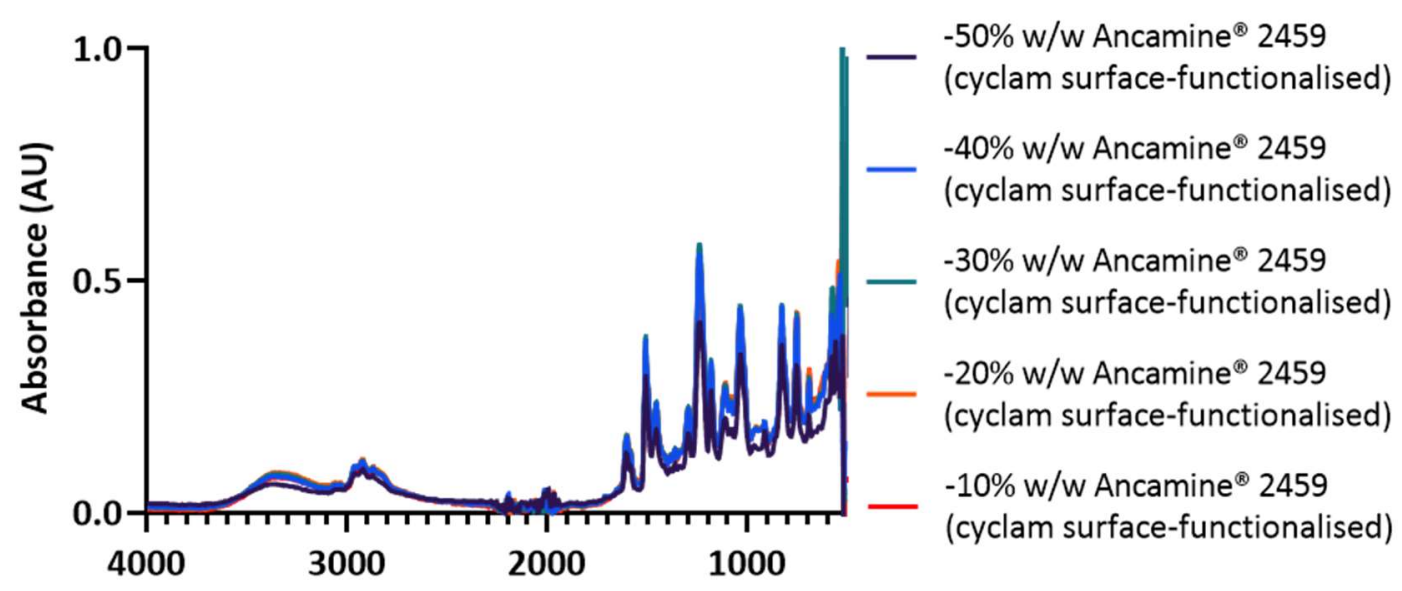

Wavenumber $\left(\mathrm{cm}^{-1}\right)$

(b)

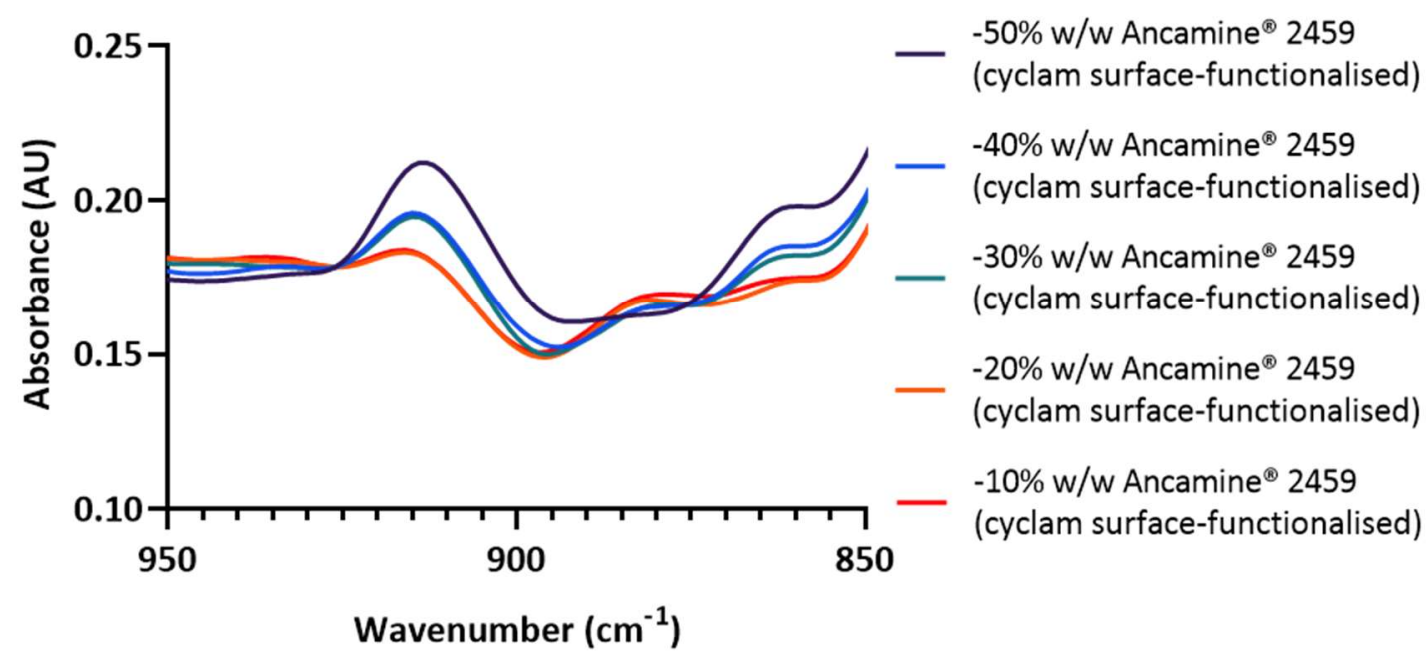

Figure 8.34. FT-IR spectra of coatings prepared from Epikote ${ }^{\mathrm{TM}} 235$ undercured $(10-50 \% \mathrm{w} / \mathrm{w})$ with Ancamine ${ }^{\circledR} 2459$ and surface-functionalised with cyclam: (a) is the full spectrum, and (b) shows an expanded view of the bands due to the epoxy group ( $915 \mathrm{~cm}^{-1}$; adjusted baseline). 
(a)

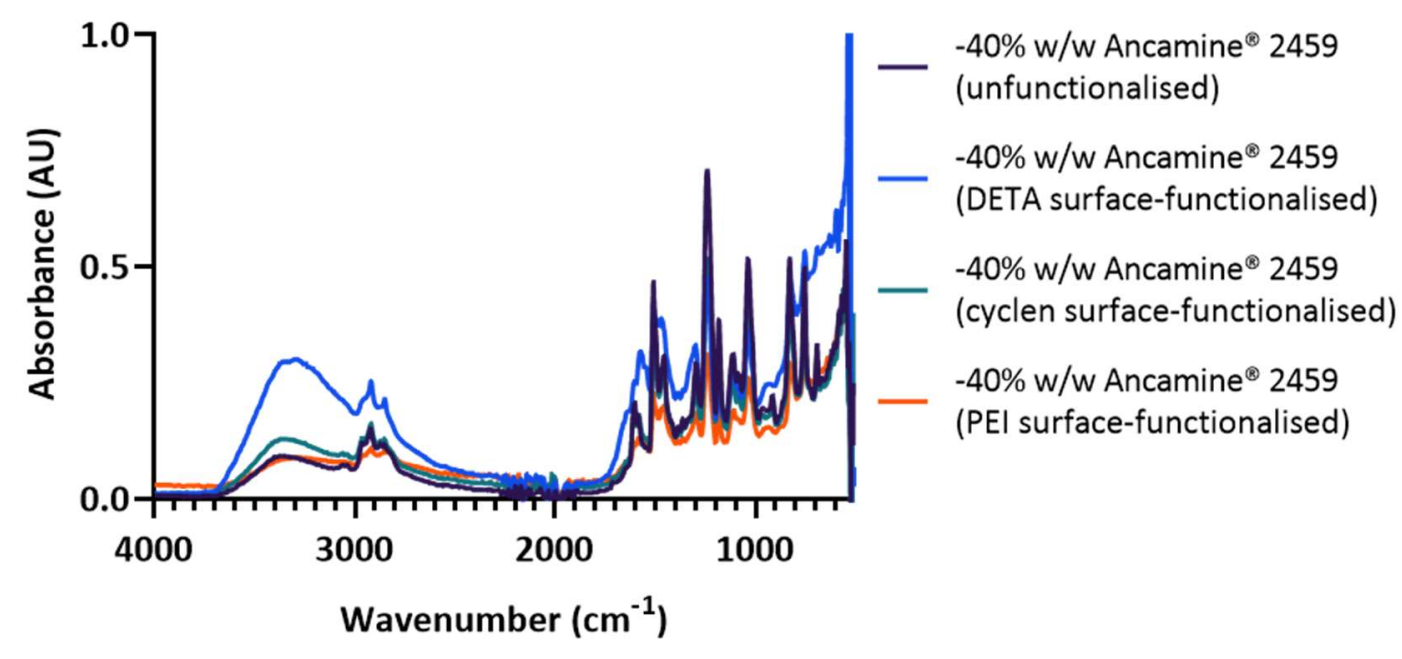

(b)

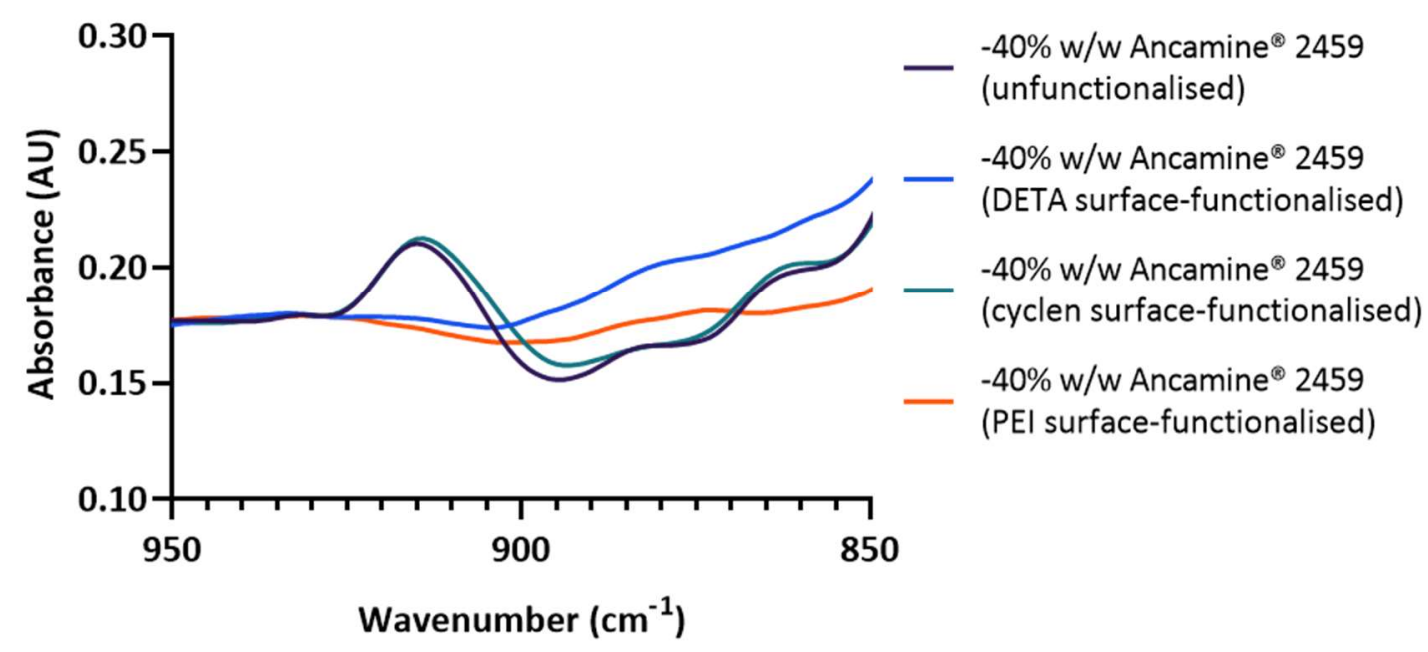

Figure 8.35. FT-IR spectra of coatings prepared from Epikote ${ }^{\mathrm{TM}} 235$ undercured $(40 \% \mathrm{w} / \mathrm{w})$ with Ancamine ${ }^{\circledR} 2459$ and surface-functionalised with DETA, cyclen, and PEI: (a) is the full spectrum, and (b) shows an expanded view of the bands due to the epoxy group ( $\sim 15 \mathrm{~cm}^{-1}$; adjusted baseline). 


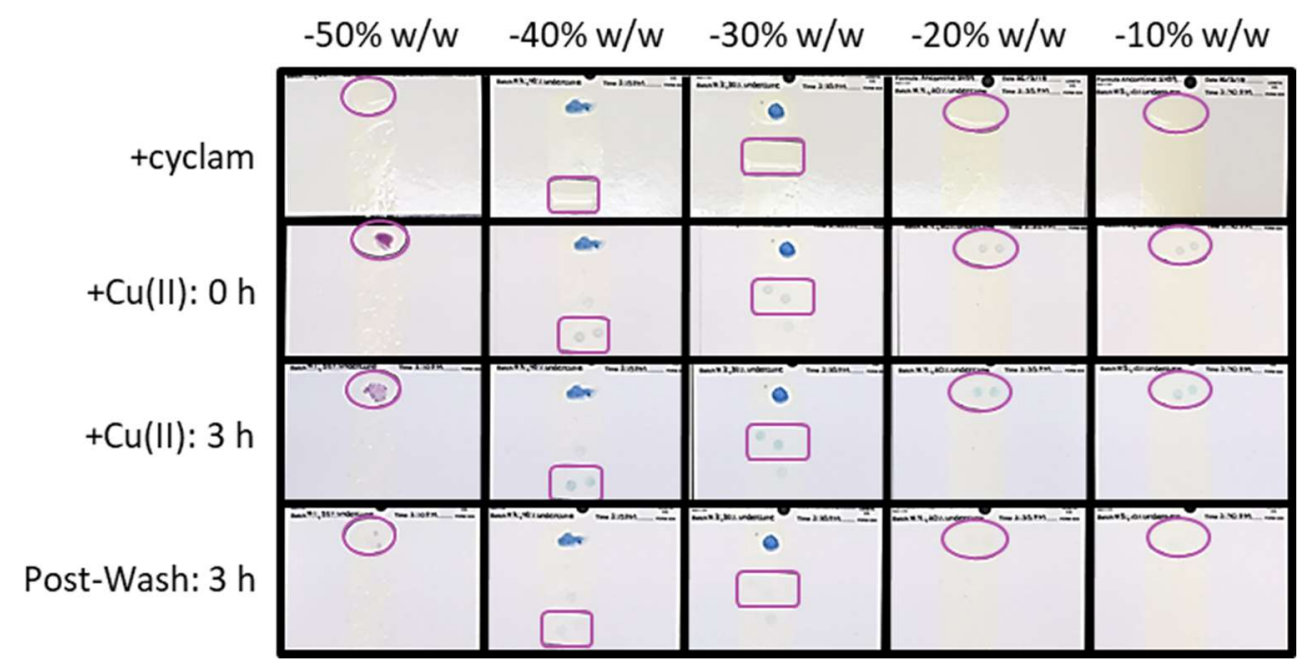

Figure 8.36. Monitoring the $\mathrm{Cu}(\mathrm{II})$-addition to coatings prepared from Epikote ${ }^{\mathrm{TM}} 235$ undercured $(10-50 \% \mathrm{w} / \mathrm{w})$ with Ancamine ${ }^{\circledR} 2459$ and surface-functionalised with cyclam in the purple-outlined area.

+ligands

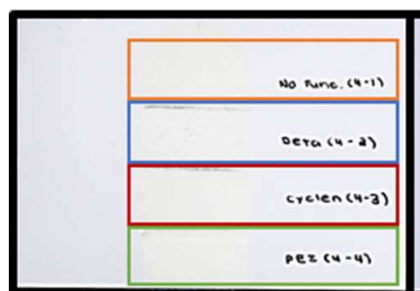

$+\mathrm{Cu}(\mathrm{II}): \mathrm{Oh}$

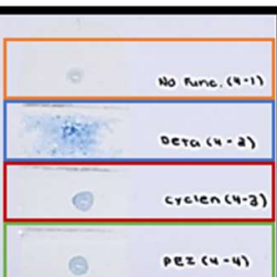

$+\mathrm{Cu}(\mathrm{II}): 2 \mathrm{~h}$

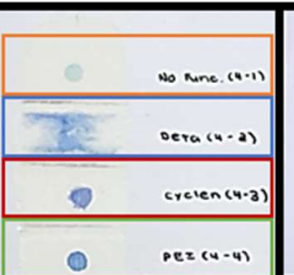

Post-Wash: $2 \mathrm{~h}$

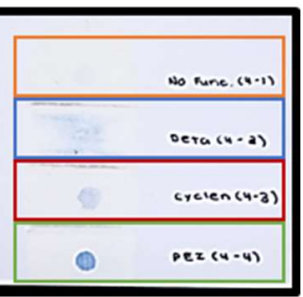

Figure 8.37. Monitoring the $\mathrm{Cu}(\mathrm{II})$-addition to coatings prepared from Epikote ${ }^{\mathrm{TM}} 235$ undercured (40\% w/w) with Ancamine ${ }^{\circledR} 2459$ and surface-functionalised with DETA (blue), cyclen (red), and PEI (green). The area outlined in orange was unfunctionalised. 


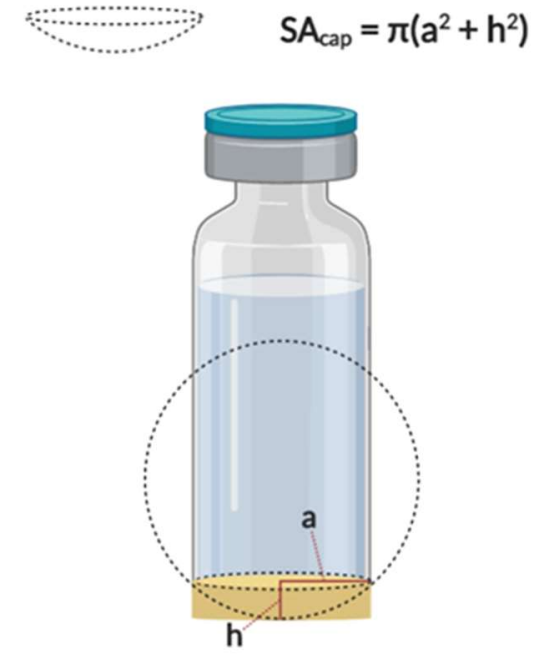

Created in BioRender.com bio

Figure 8.38. Formula for calculating the coating's surface area (spherical shape) exposed to liquid (created with Biorender.com; Chapters 3 and 6, Sections 3.3.3 and 6.3.3.4). 


\subsection{CHAPTER 4}

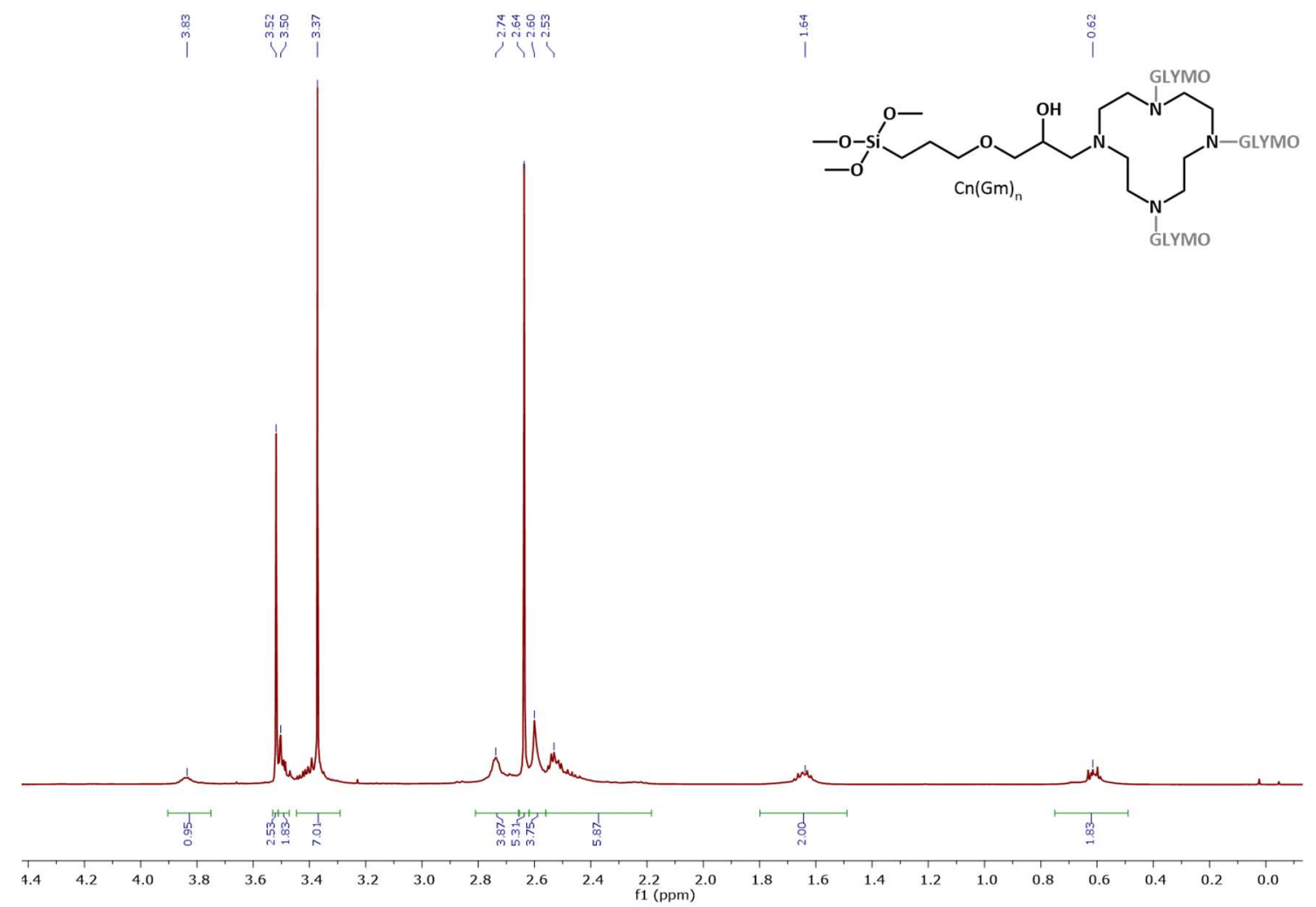

Figure 8.39. ${ }^{1} \mathrm{H}$ NMR spectrum (crude; $\mathrm{CDCl}_{3}$ ) of $\mathrm{Cn}(\mathrm{Gm})_{n}$ from Procedure 6 .

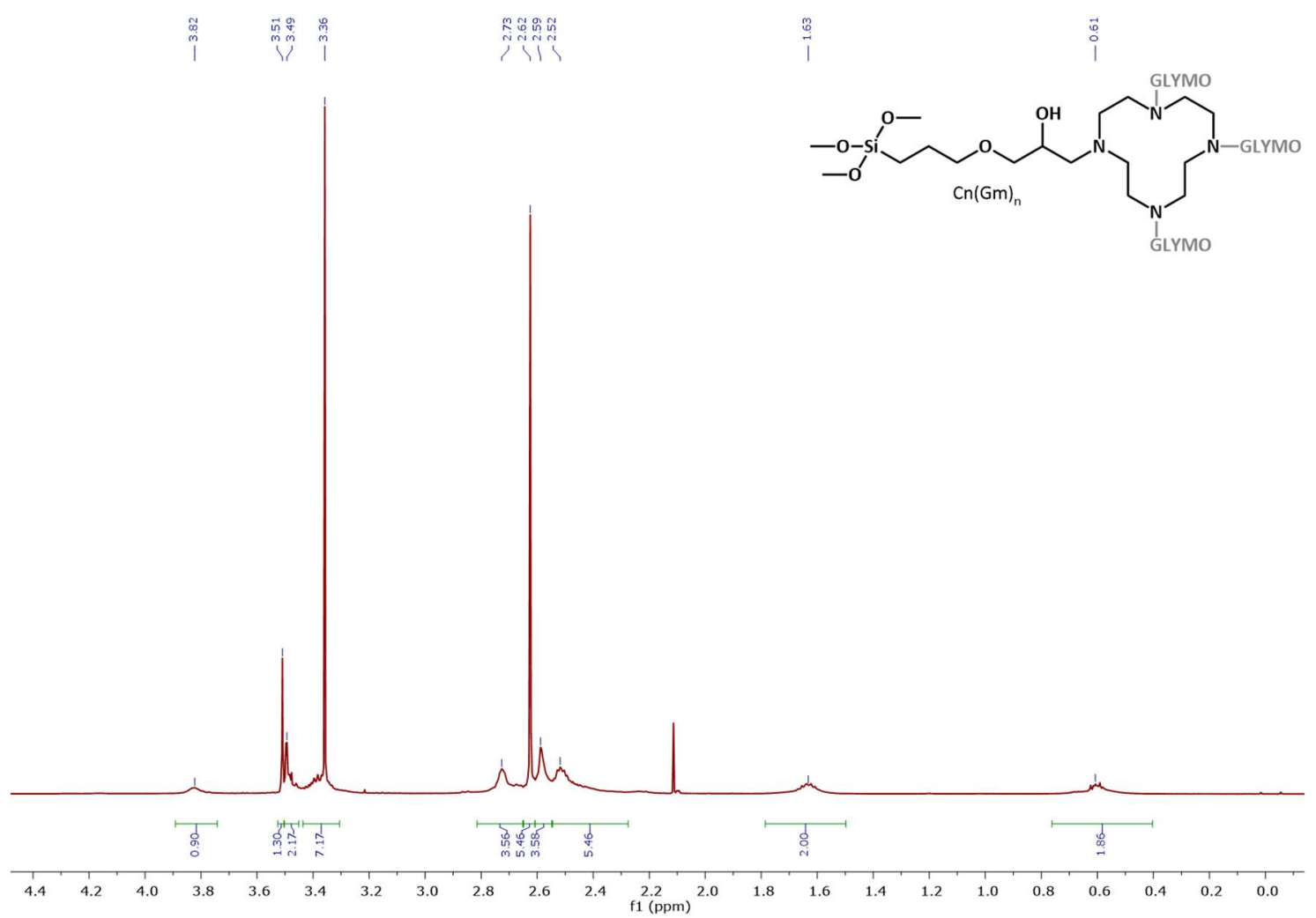

Figure 8.40. ${ }^{1} \mathrm{H}$ NMR spectrum (crude; $\mathrm{CDCl}_{3}$ ) of $\mathrm{Cn}(\mathrm{Gm})_{\mathrm{n}}$ from Procedure $6+$ pyridine- $d_{5}$. 

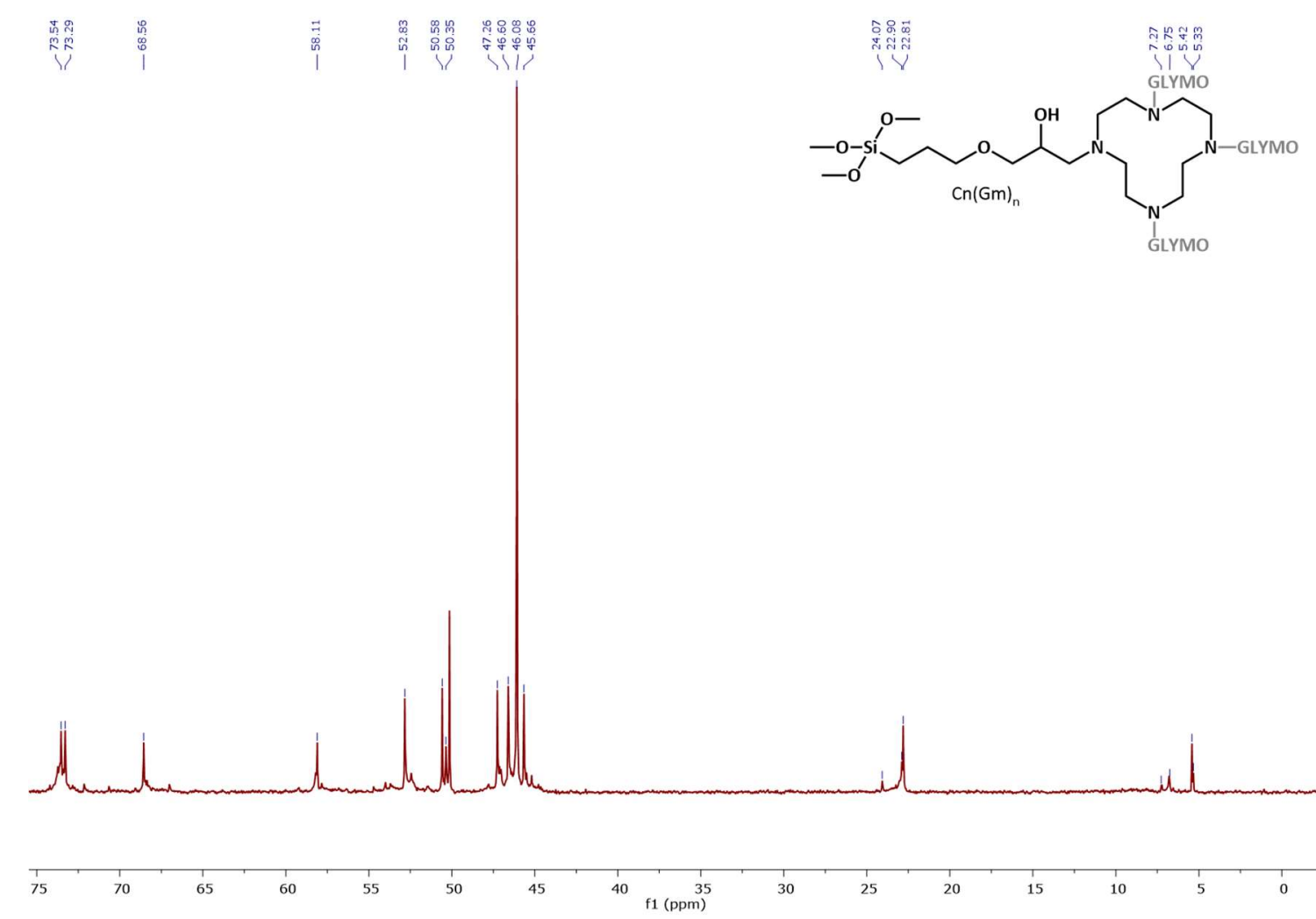

Figure 8.41. ${ }^{13} \mathrm{C}$ NMR spectrum (crude; $\mathrm{CDCl}_{3}$ ) of $\mathrm{Cn}(\mathrm{Gm})_{n}$ from Procedure 6.

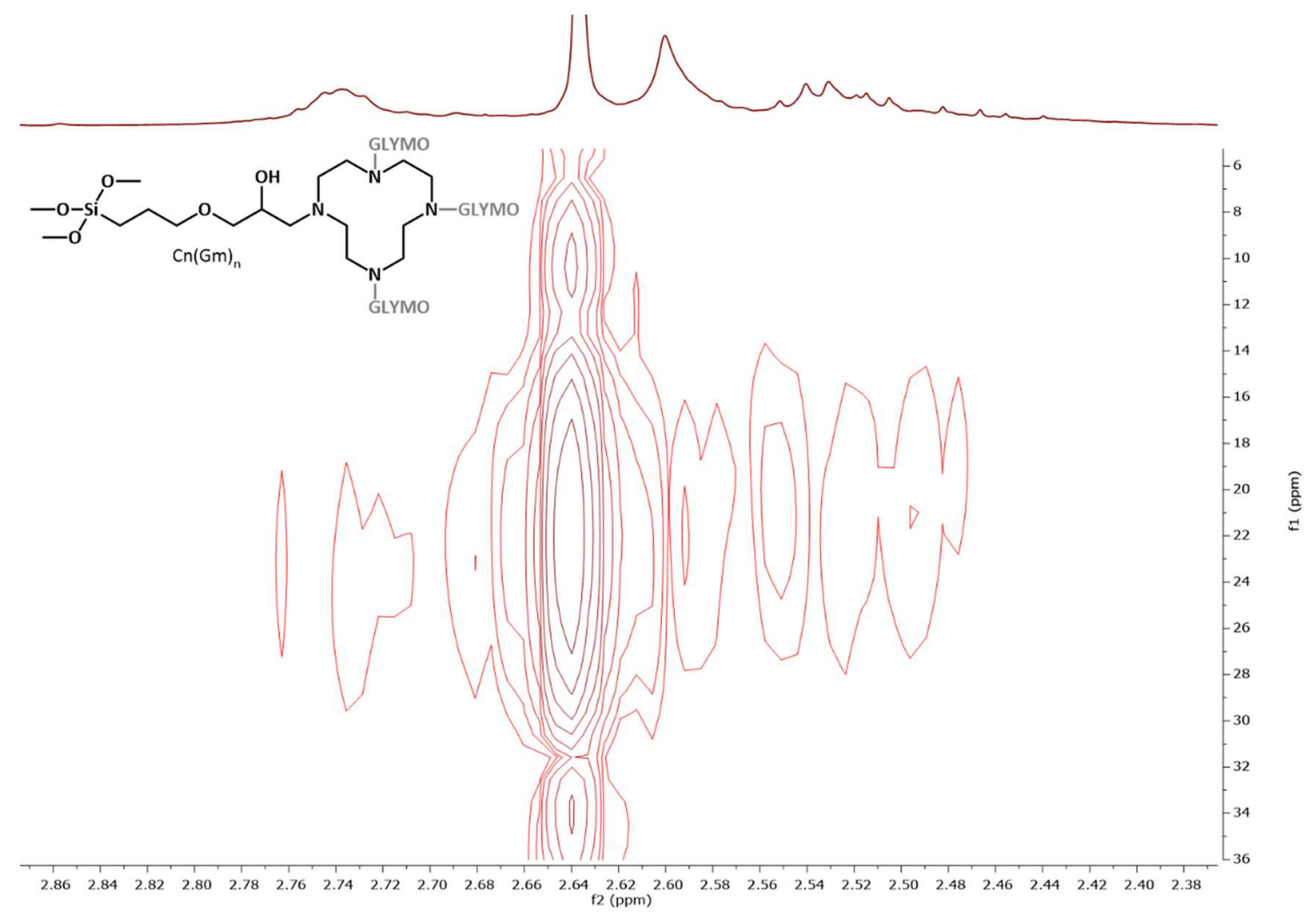

Figure 8.42. $2 \mathrm{D}\left({ }^{1} \mathrm{H},{ }^{15} \mathrm{~N}\right)-\mathrm{HMBC} \mathrm{NMR}$ spectrum (crude; $\left.\mathrm{CDCl}_{3}\right)$ of $\mathrm{Cn}(\mathrm{Gm})_{\mathrm{n}}$ from Procedure 6. 


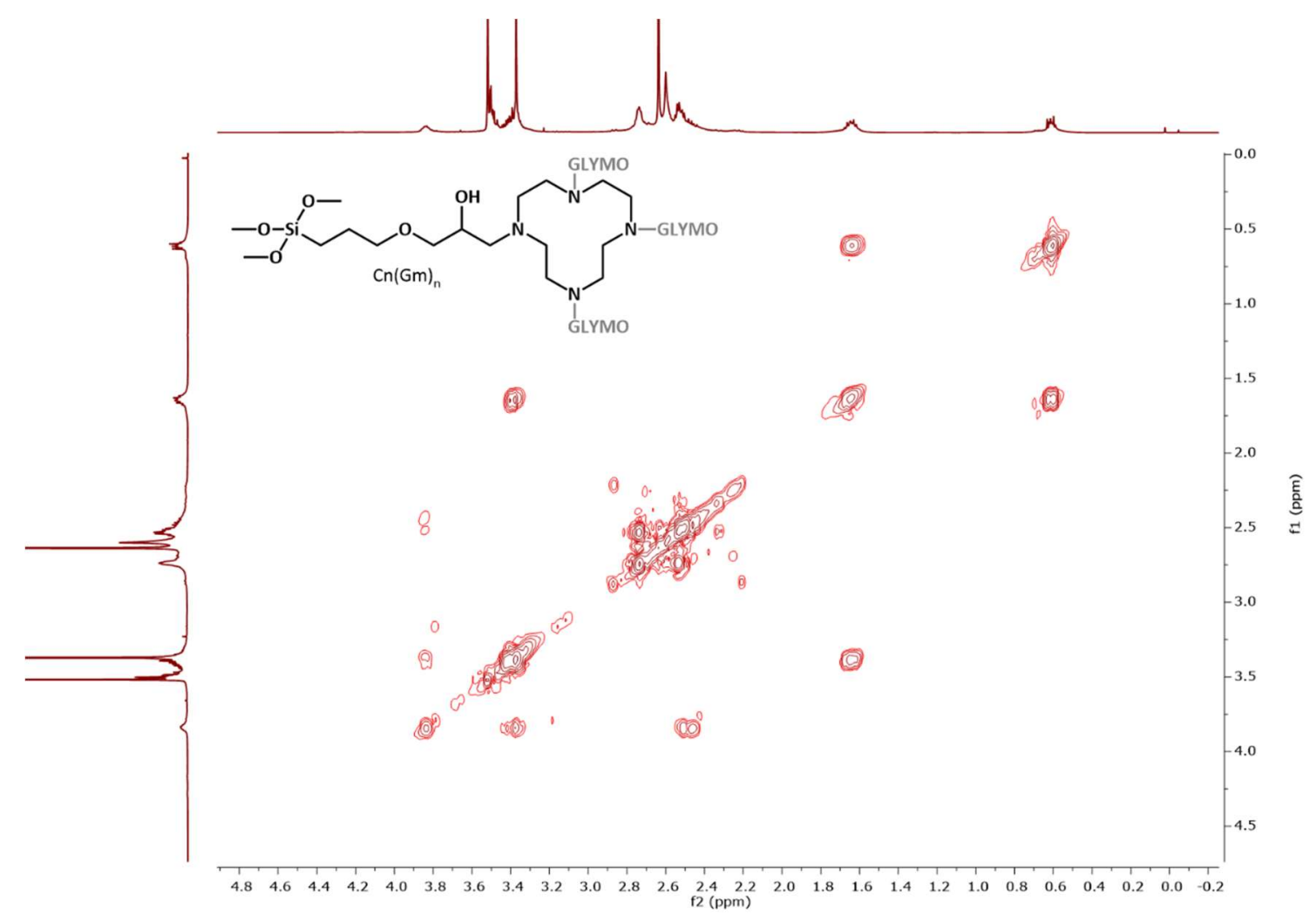

Figure 8.43. $2 \mathrm{D}\left({ }^{1} \mathrm{H},{ }^{1} \mathrm{H}\right)$-COSY NMR spectrum (crude; $\left.\mathrm{CDCl}_{3}\right)$ of $\mathrm{Cn}(\mathrm{Gm})_{\mathrm{n}}$ from Procedure 6.
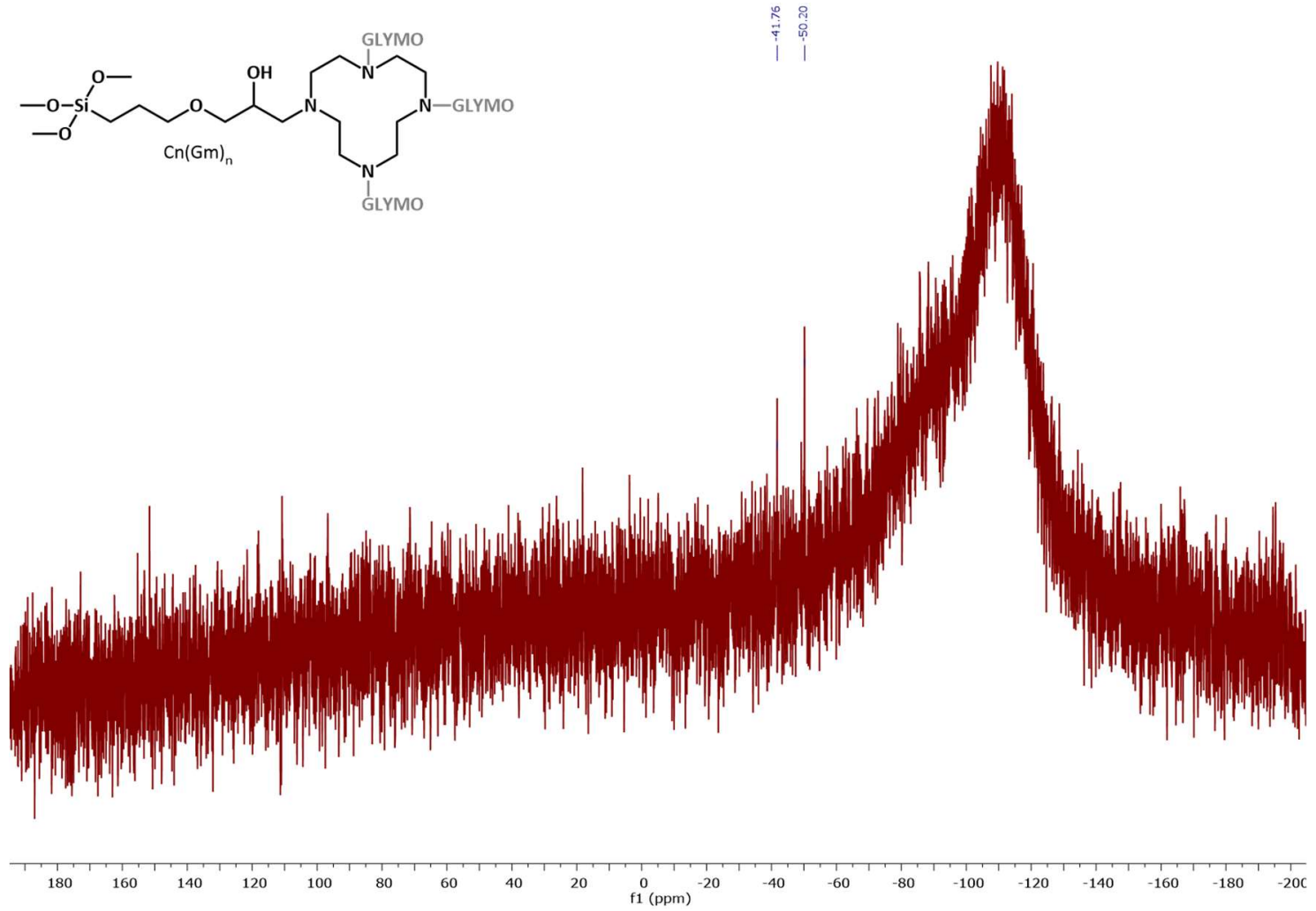

Figure 8.44. ${ }^{29} \mathrm{Si} N M R$ spectrum (crude; $\left.\mathrm{CDCl}_{3}\right)$ of $\mathrm{Cn}(\mathrm{Gm})_{\mathrm{n}}$ from Procedure $6\left(\delta_{\mathrm{Si}}-109.7 \mathrm{ppm}=\mathrm{NMR}\right.$ tube). 


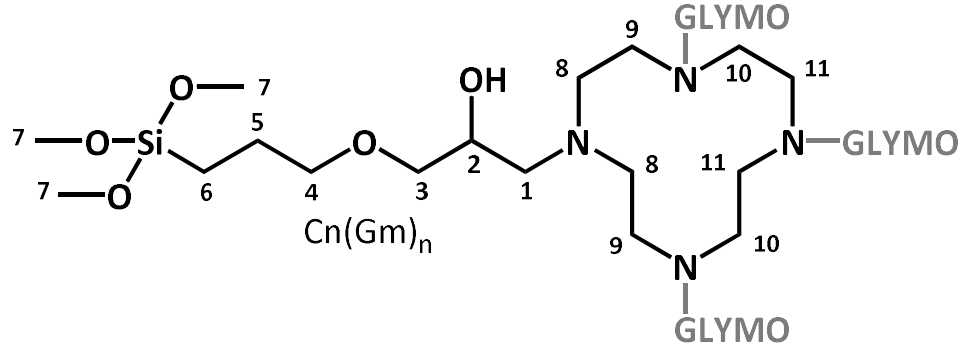

Table 8.20. Peak assignments in ${ }^{1} \mathrm{H}$ and ${ }^{13} \mathrm{C}$ NMR spectra of Procedure $6 \mathrm{Cn}(\mathrm{Gm})_{n}$.

\begin{tabular}{|c|c|c|}
\hline Position & $\delta_{c}$, Type $^{a, c}$ & $\delta_{H}(\# H)^{b, c}$ \\
\hline 1 & $58.1, \mathrm{CH}_{2}$ & $2.56-2.15, \mathrm{~m}(6 \mathrm{H})$ \\
\hline 2 & $68.6, \mathrm{CH}$ & $3.91-3.72, \mathrm{~m}(1 \mathrm{H})$ \\
\hline 3 & $73.3, \mathrm{CH}_{2}$ & $3.46-3.24, \mathrm{~m}(7 \mathrm{H})$ \\
\hline 4 & $73.5, \mathrm{CH}_{2}$ & $3.46-3.24, \mathrm{~m}(7 \mathrm{H})$ \\
\hline 5 & $24.1,22.9,22.8, \mathrm{CH}_{2}$ & $1.82-1.42, \mathrm{~m}(2 \mathrm{H})$ \\
\hline 6 & $7.3,6.8,5.4,5.3, \mathrm{CH}_{2}$ & $0.78-0.42, \mathrm{~m}(2 \mathrm{H})$ \\
\hline 7 & $50.6,50.4, \mathrm{CH}_{3}$ & $3.58-3.51, \mathrm{~m}(3 \mathrm{H}) ; 3.51-3.46, \mathrm{~m}(2 \mathrm{H})$ \\
\hline 8 & $52.8, \mathrm{CH}_{2}$ & $2.60, \mathrm{~s}(4 \mathrm{H})$ \\
\hline Cyclen $\mathrm{CH}_{2} \mathrm{~S}$ & $47.3,46.6,46.1,45.7, \mathrm{CH}_{2}$ & $2.91-2.66, \mathrm{~m}(4 \mathrm{H}) ; 2.64, \mathrm{~s}(5 \mathrm{H}) ; 2.60, \mathrm{~s}(4 \mathrm{H}) ; 2.56-2.15, \mathrm{~m}(6 \mathrm{H})$ \\
\hline
\end{tabular}




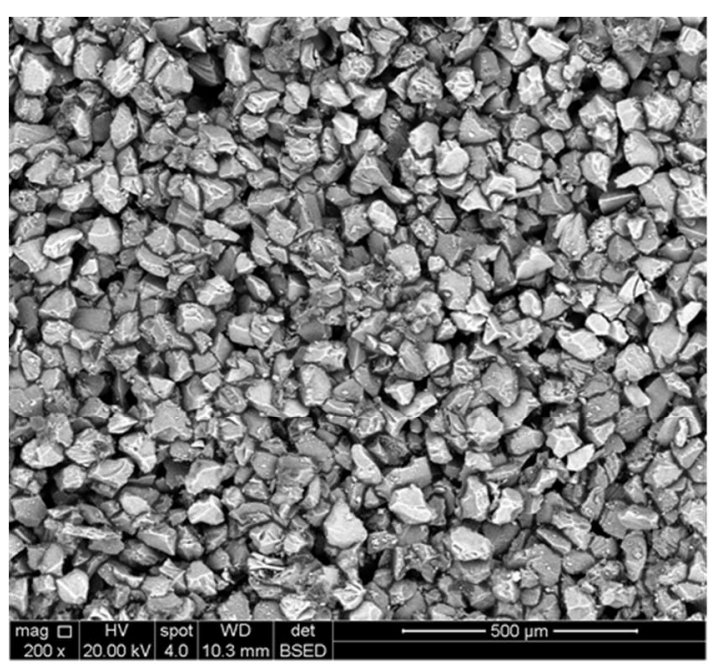

$\mathrm{SiO}_{2}$

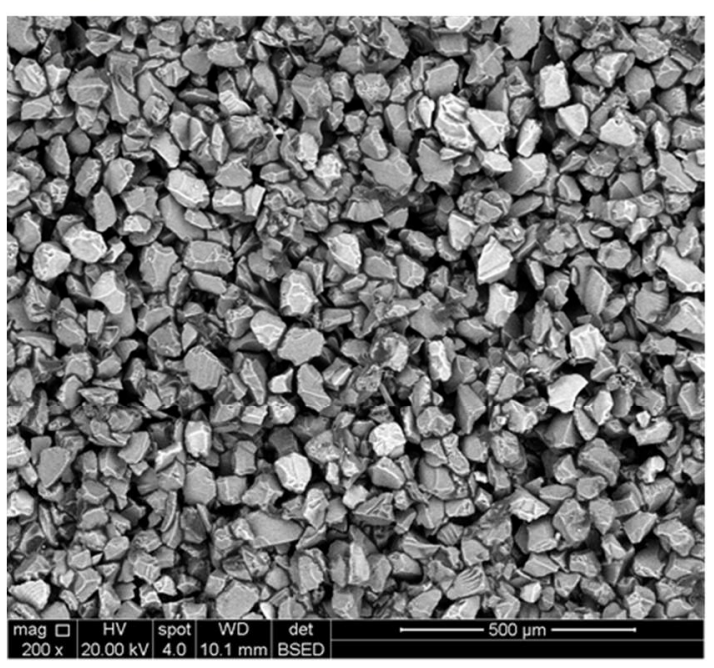

$\mathrm{CnGmSiO}_{2}$
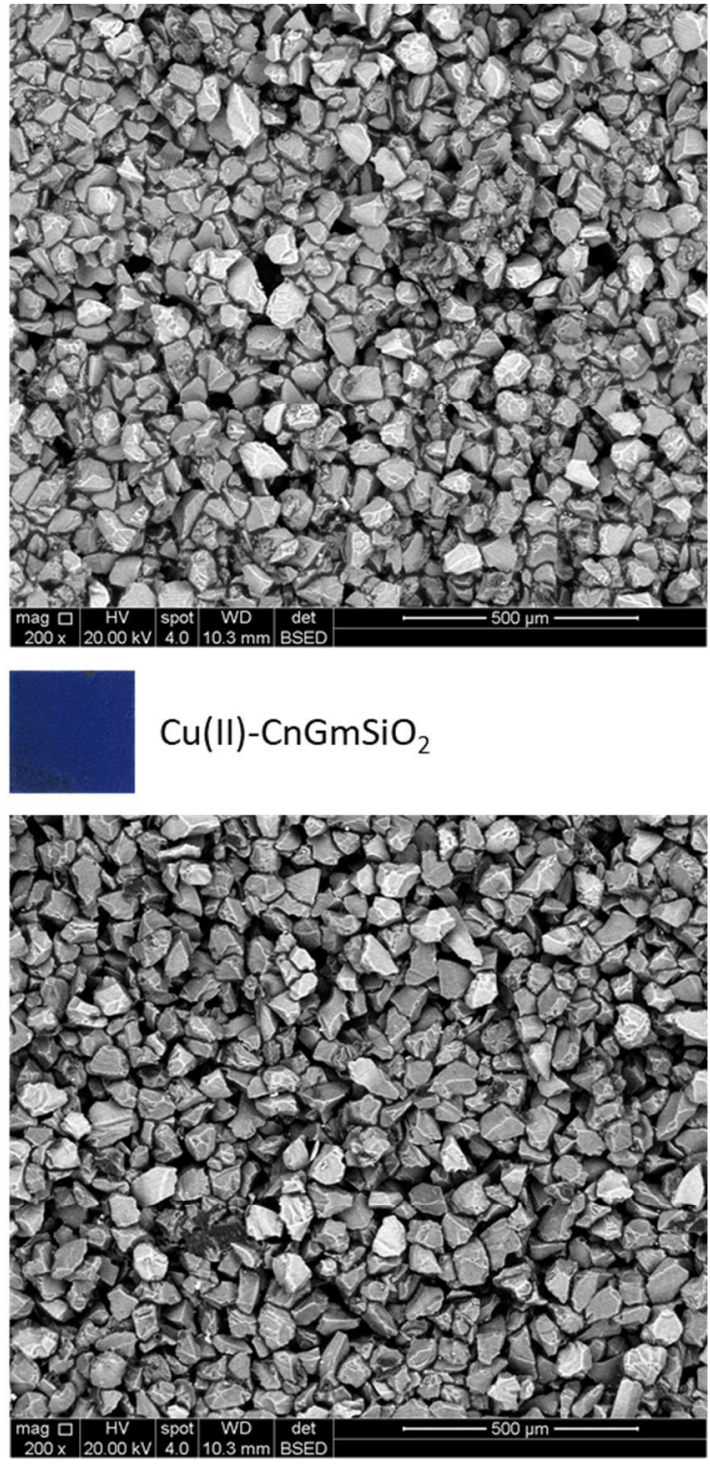

$\mathrm{CnGmSiO}_{2}+\mathrm{Cu}(\mathrm{II})$

Figure 8.45. SEM backscatter images at 200 $\times$ magnification of the $\mathrm{SiO}_{2} /$ epx-type squares to be tested for bacterial adherence (HV $20 \mathrm{kV}$, spot size 4, WD $10 \mathrm{~mm}$, BSED). The coatings include unfunctionalised $\mathrm{SiO}_{2} /$ epx, Batch $2 \mathrm{Cu}(\mathrm{II})-\mathrm{CnGmSiO}_{2} /$ epx (pre-loaded), Batch $4 \mathrm{CnGmSiO}$ /epx (free ligand), and Batch $4 \mathrm{CnGmSiO}+\mathrm{Cu}(I I) / e p x$ (post-loaded). 

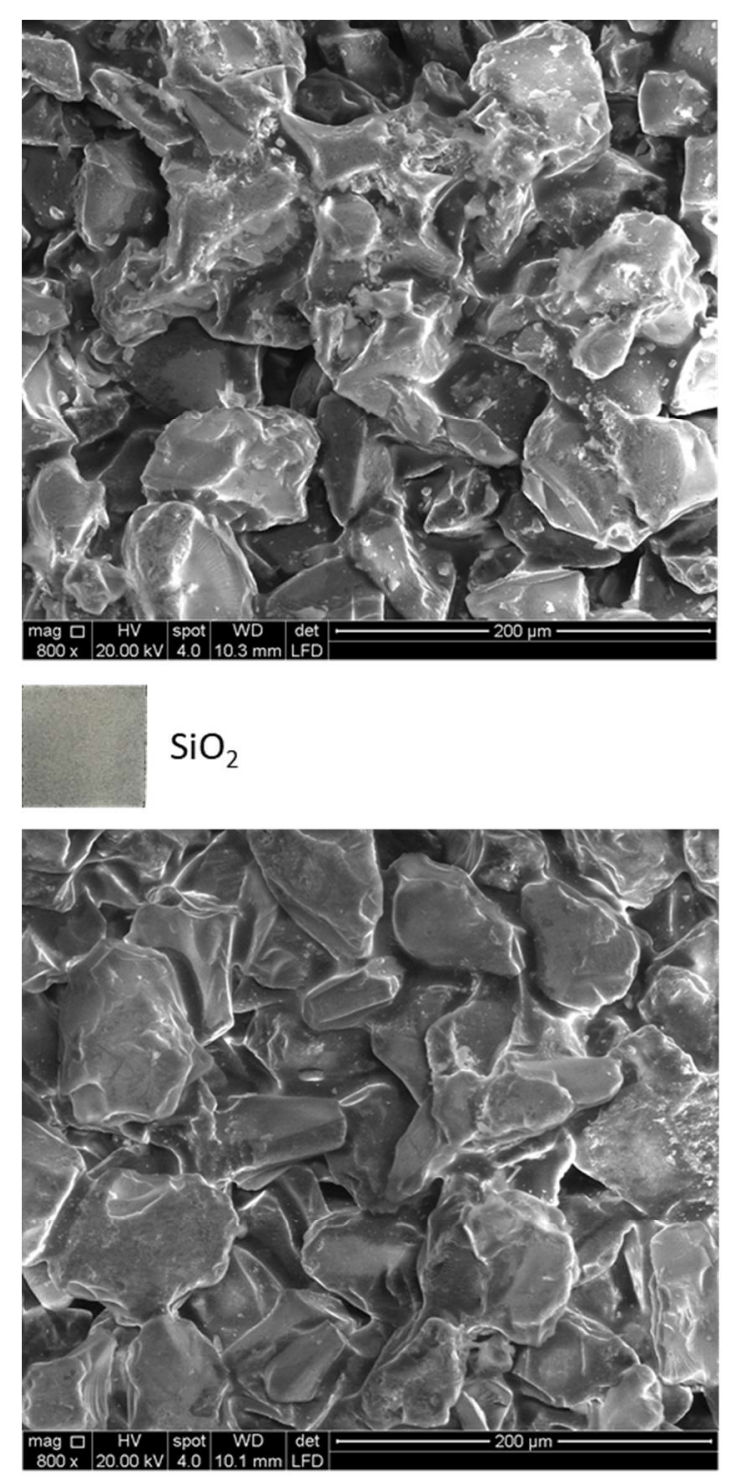

$\mathrm{CnGmSiO}_{2}$
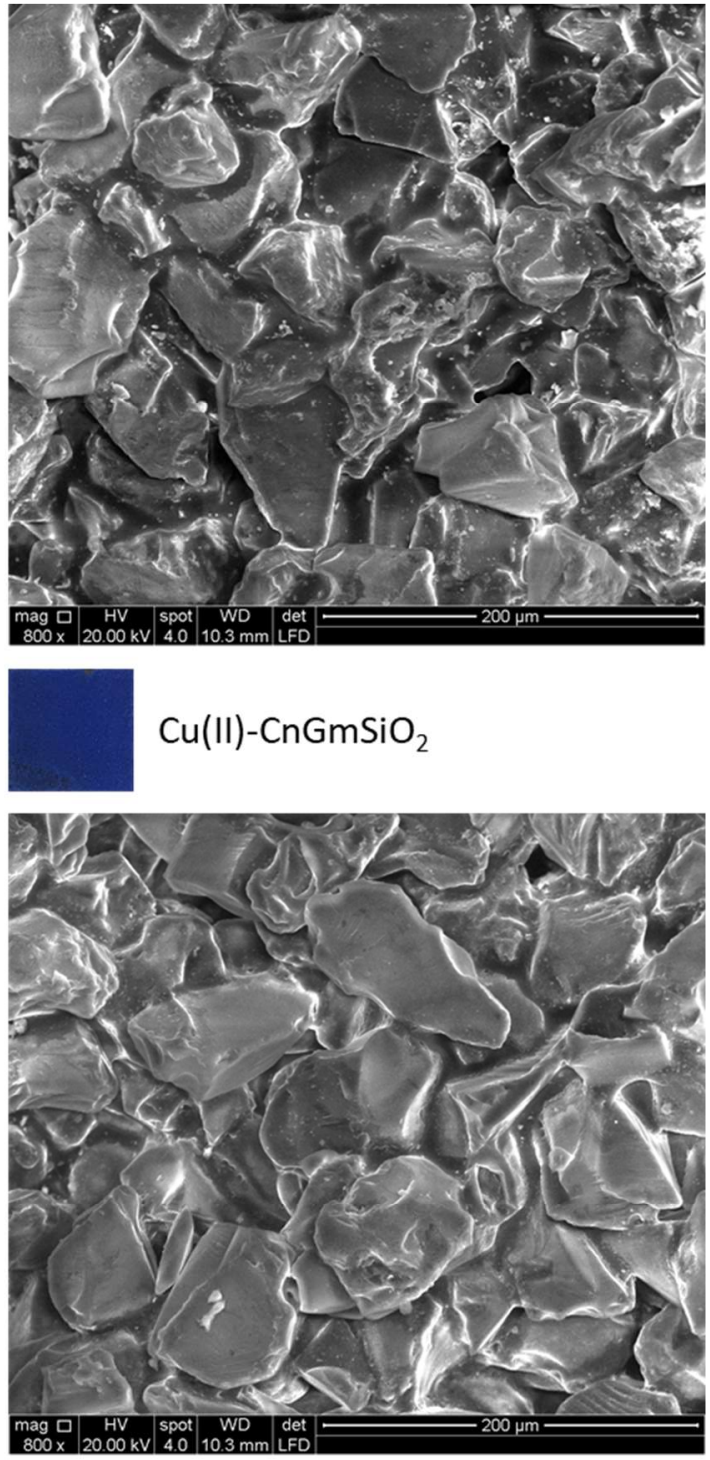

$\mathrm{CnGmSiO}_{2}+\mathrm{Cu}(\mathrm{II})$

Figure 8.46. SEM secondary electron images at $800 \times$ magnification of the $\mathrm{SiO}_{2} / \mathrm{epx}$-type squares to be tested for bacterial adherence (HV $20 \mathrm{kV}$, spot size 4, WD $10 \mathrm{~mm}$, LFD). The coatings include unfunctionalised $\mathrm{SiO}_{2} /$ epx, Batch $2 \mathrm{Cu}(\mathrm{II})-\mathrm{CnGmSiO}_{2} /$ epx (pre-loaded), Batch $4 \mathrm{CnGmSiO} /$ epx (free ligand), and Batch $4 \mathrm{CnGmSiO}+\mathrm{Cu}(I I) / e p x$ (post-loaded). 

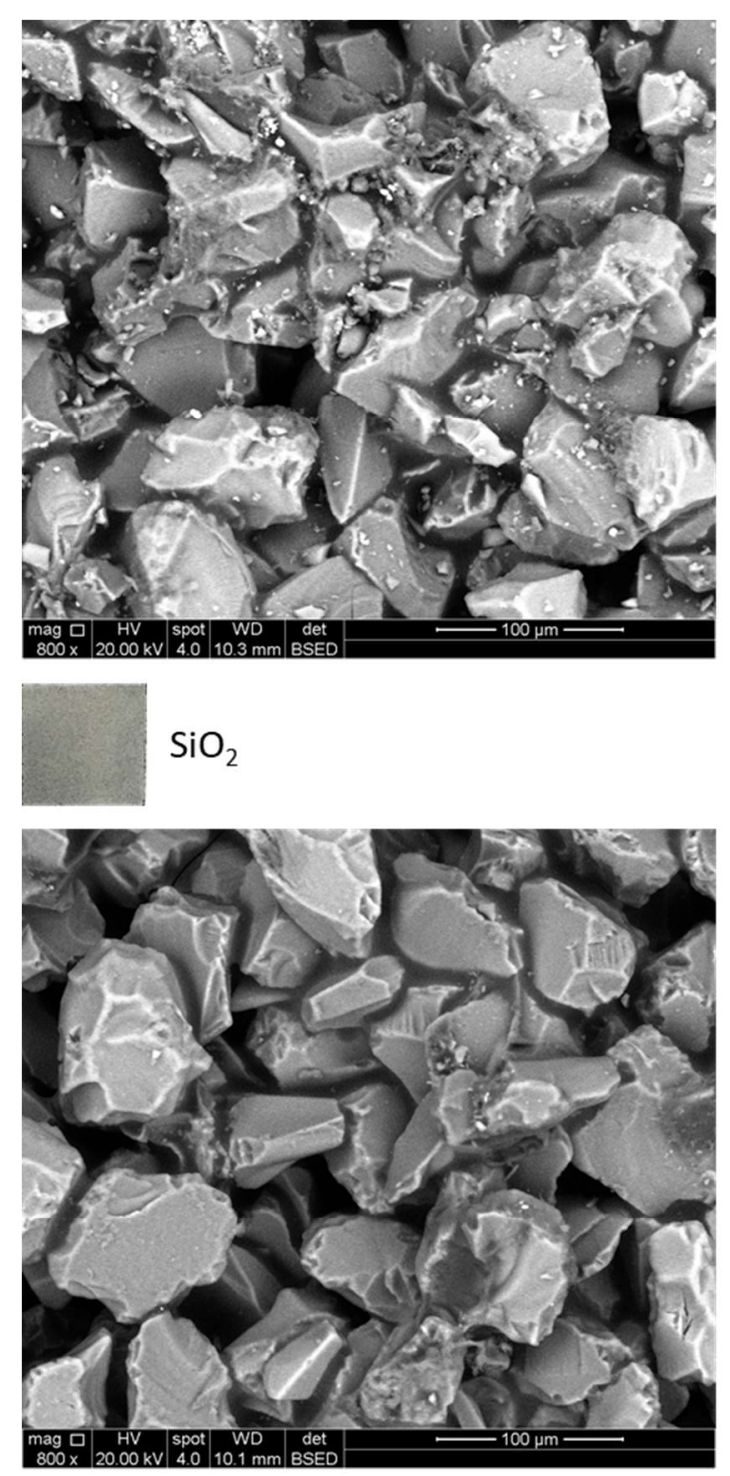

$\mathrm{CnGmSiO}_{2}$
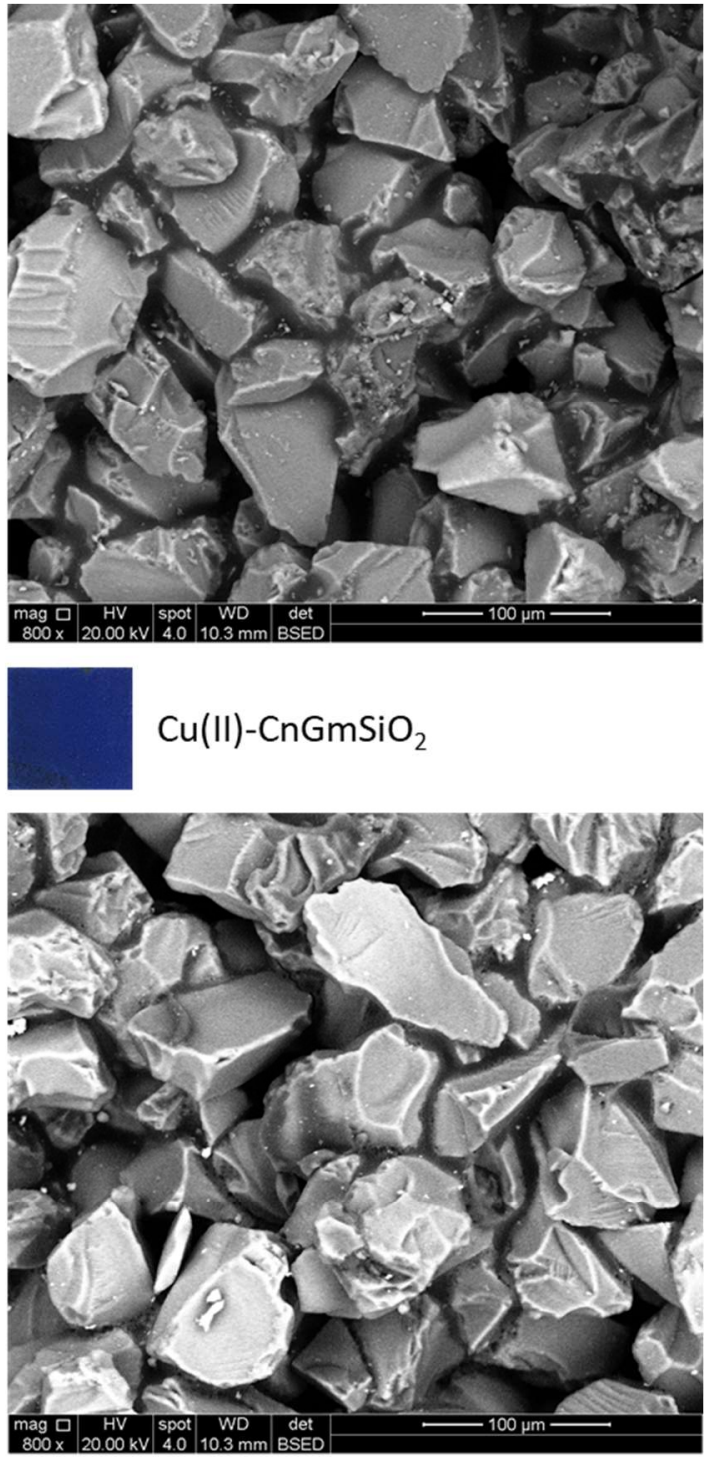

$\mathrm{CnGmSiO}_{2}+\mathrm{Cu}(\mathrm{II})$

Figure 8.47. SEM backscatter images at $800 \times$ magnification of the $\mathrm{SiO}_{2} /$ epx-type squares to be tested for bacterial adherence (HV $20 \mathrm{kV}$, spot size 4, WD $10 \mathrm{~mm}$, BSED). The coatings include unfunctionalised $\mathrm{SiO}_{2} /$ epx, Batch $2 \mathrm{Cu}(\mathrm{II})-\mathrm{CnGmSiO}_{2} /$ epx (pre-loaded), Batch $4 \mathrm{CnGmSiO} /$ epx (free ligand), and Batch $4 \mathrm{CnGmSiO}_{2}+\mathrm{Cu}(\mathrm{II}) / \mathrm{epx}$ (post-loaded). 


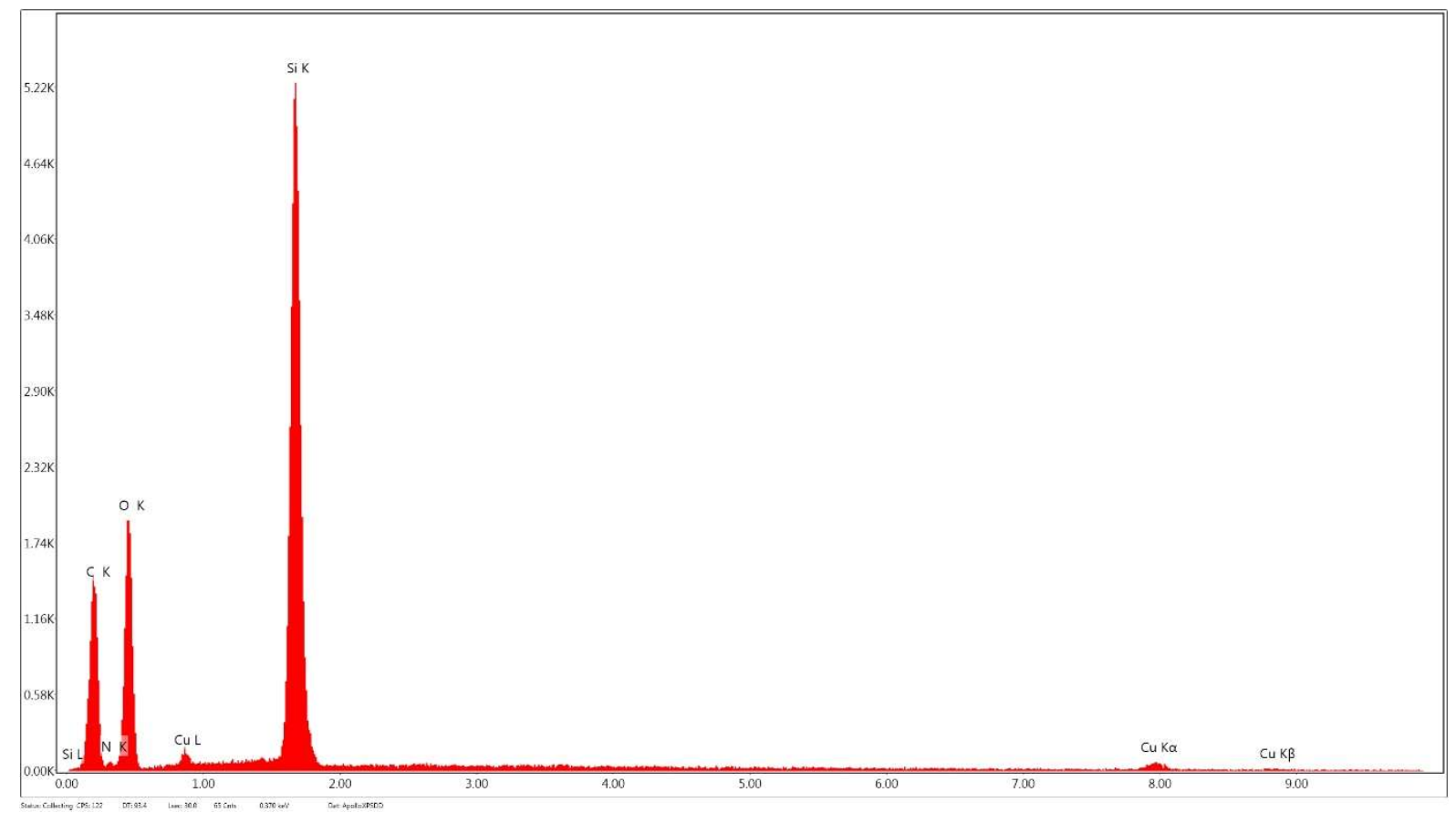

Figure 8.48. EDS spectrum (counts vs keV) of Batch $2 \mathrm{Cu}(\mathrm{II})-\mathrm{CnGmSiO}_{2} / \mathrm{epx}$ (pre-loaded) coating (200x magnification, HV $20 \mathrm{kV}$, spot size 6, WD $10 \mathrm{~mm}$, 30 s live time, $1.6 \mu$ s amplitude time, $35.3^{\circ}$ take-off angle, $129 \mathrm{eV}$ resolution).

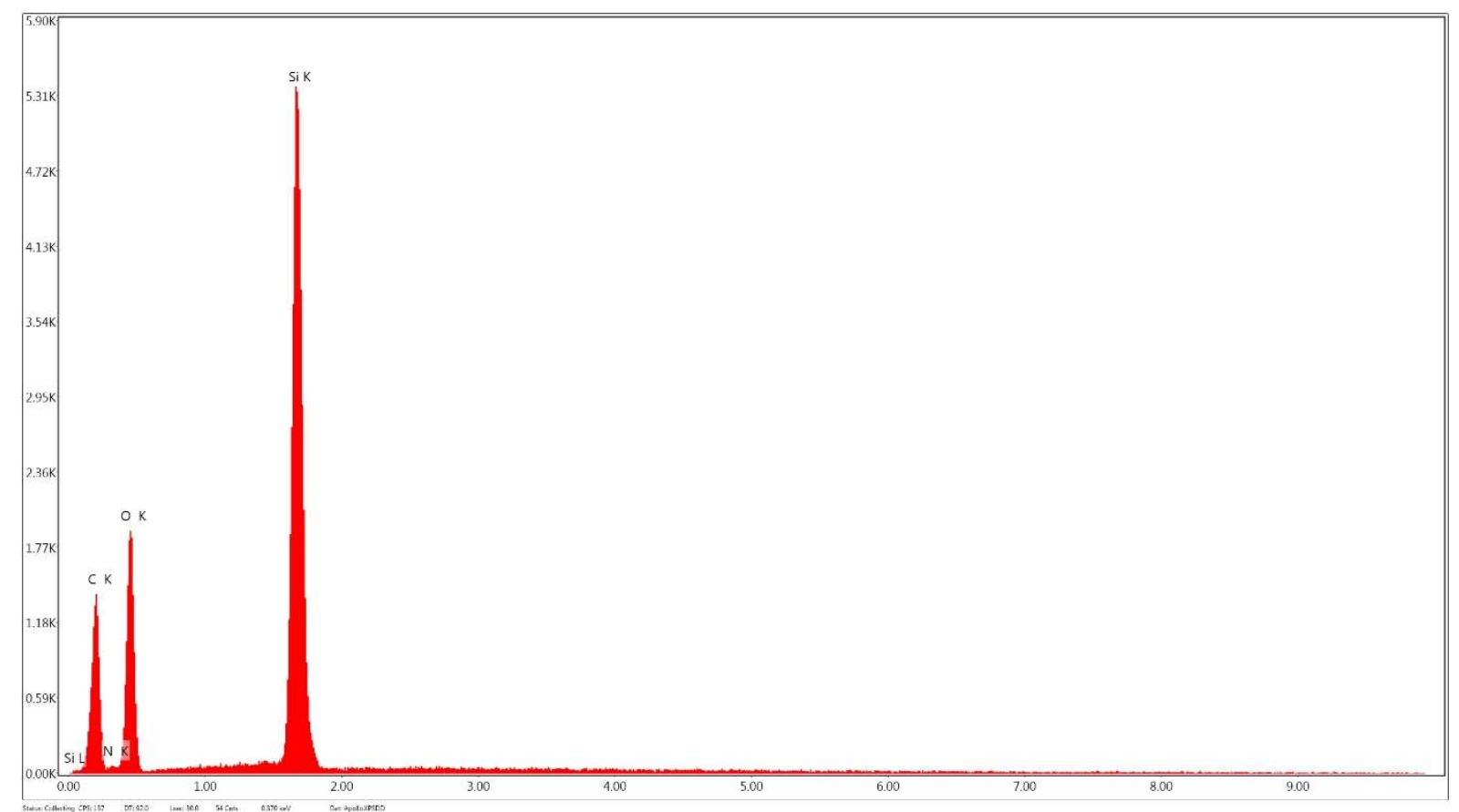

Figure 8.49. EDS spectrum (counts vs keV) of Batch $4 \mathrm{CnGmSiO}_{2} / \mathrm{epx}$ (free ligand) coating (200x magnification, HV $20 \mathrm{kV}$, spot size 6, WD $10 \mathrm{~mm}$, $30 \mathrm{~s}$ live time, $1.6 \mu \mathrm{s}$ amplitude time, $35.1^{\circ}$ take-off angle, $129 \mathrm{eV}$ resolution). 


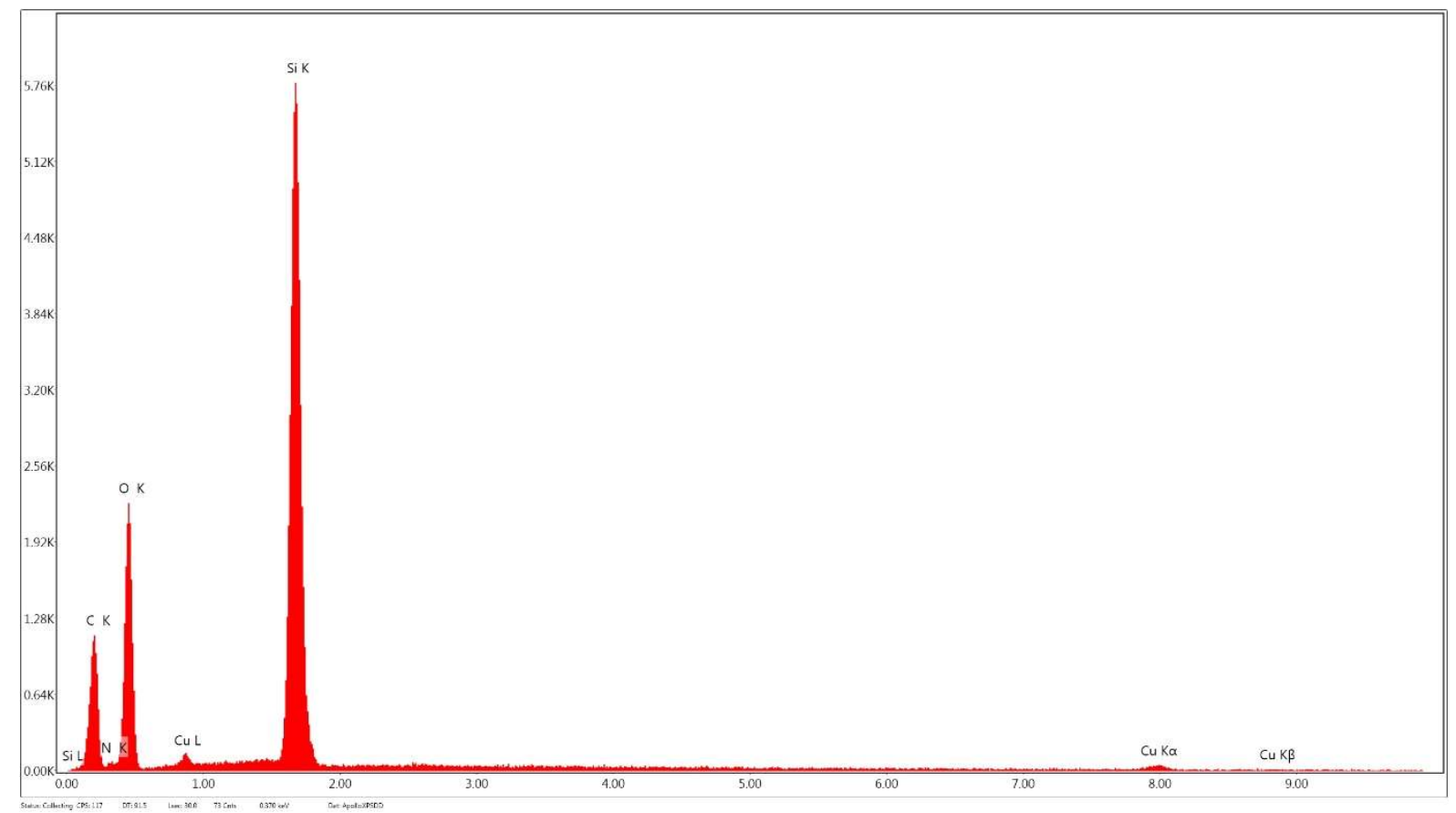

Figure 8.50. EDS spectrum (counts vs keV) of Batch $4 \mathrm{CnGmSiO}_{2}+\mathrm{Cu}(\mathrm{II}) / \mathrm{epx}$ (post-loaded) coating

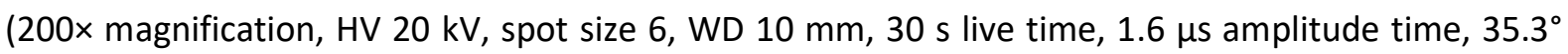
take-off angle, $129 \mathrm{eV}$ resolution).

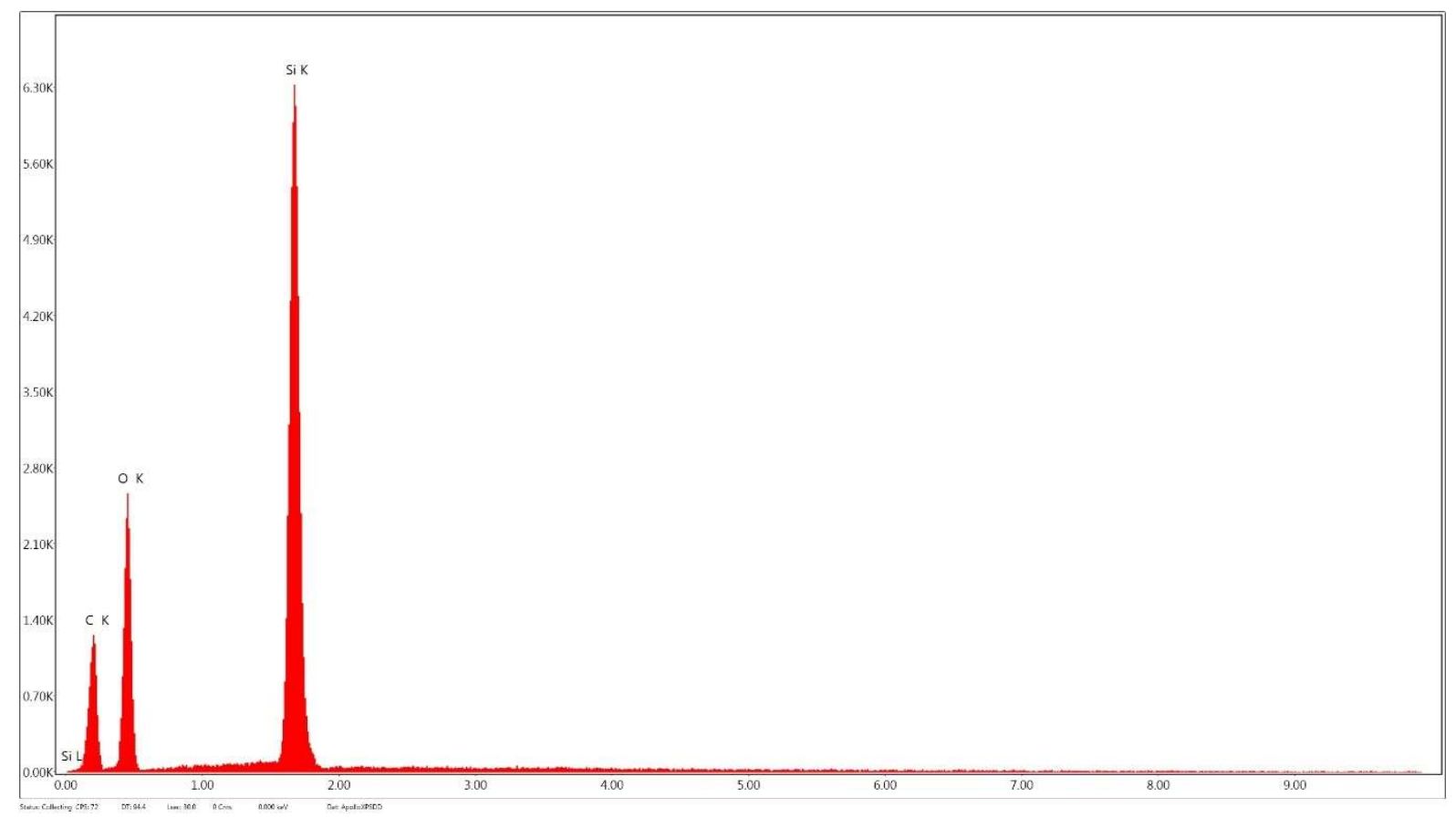

Figure 8.51. EDS spectrum (counts vs keV) of unfunctionalised $\mathrm{SiO}_{2} / \mathrm{epx}$ coating (200x magnification, HV 20 kV, spot size 6, WD 10 mm, 30 s live time, $1.6 \mu \mathrm{s}$ amplitude time, 35.3 take-off angle, $129 \mathrm{eV}$ resolution). 


\subsection{CHAPTER 5}

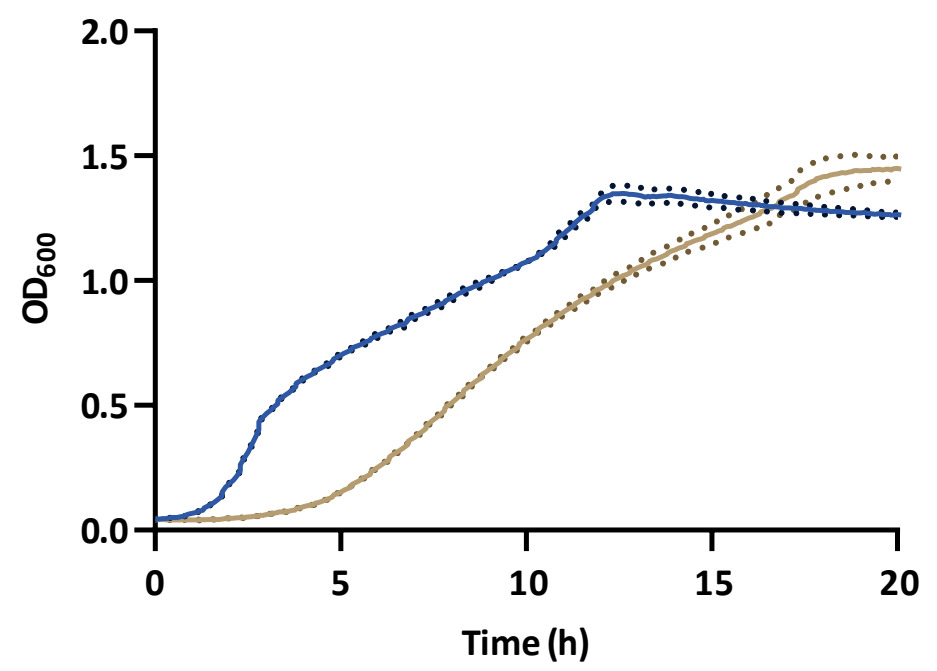

— Nutrient Broth $\quad$ - M63 Minimal Medium

Figure 8.52. Growth curves of E. coli NZRM 3647 cultured in nutrient broth or M63 minimal medium. The solid line represents the mean $(b r=3)$, and the dotted line represents the SD.

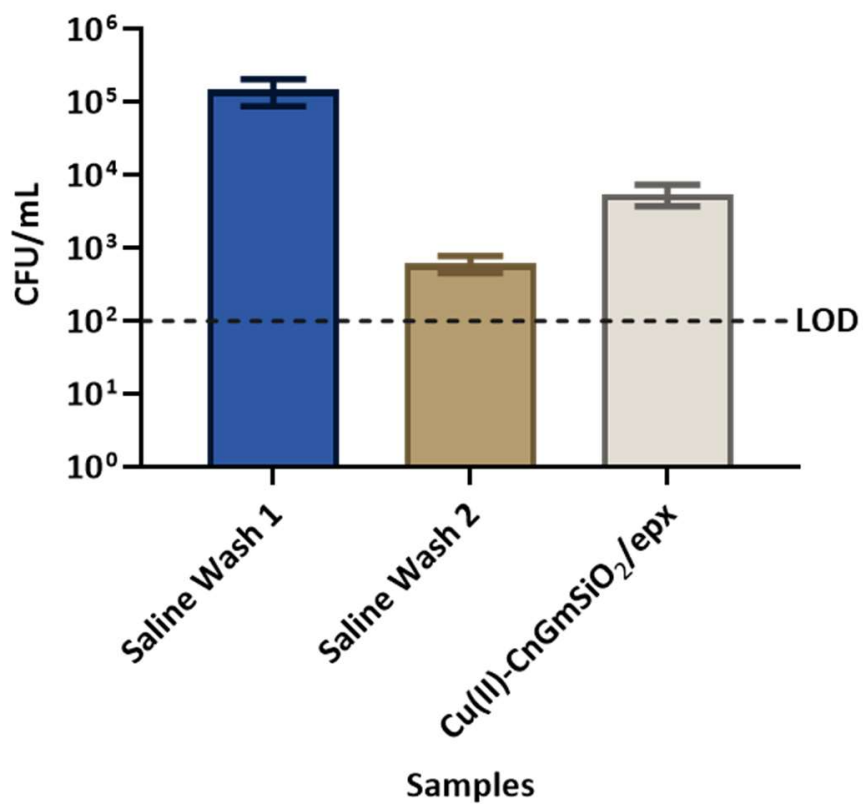

Figure 8.53. Enumeration of bacterial counts in the saline washes: results of CFU/mL in saline washes and the detachment solution containing a $\mathrm{Cu}(\mathrm{II})-\mathrm{CnGmSiO}_{2} / \mathrm{epx}$-coated square. The bars represent the mean $(\operatorname{tr}=5) \pm S D(L O D=100 \mathrm{CFU} / \mathrm{mL})$. 
Diameter $\mathrm{SiO}_{2}$ particles: $35-75 \mu \mathrm{m}$ (treat particles as spheres)

Dimensions of sample square: $23 \mathrm{~mm} \times 23 \mathrm{~mm}$

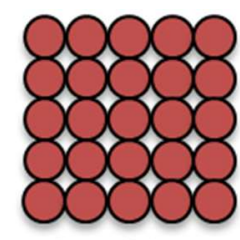

$2 \times S A_{\text {red }}$

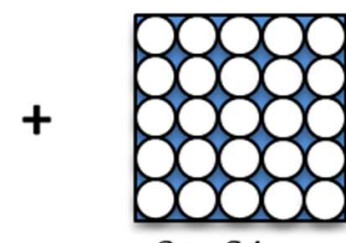

$2 \times S A_{\text {blue }}$

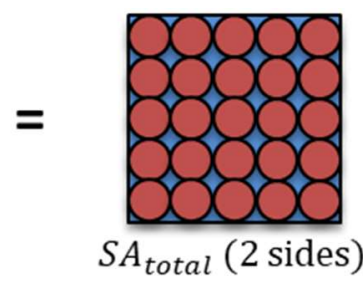

$S A_{\text {total }}$ (2 sides)

$S A_{\text {red }}=\frac{1}{2} \times 4 \pi r^{2} \times(\#$ spheres in row $) \times(\#$ spheres in column $)$

$$
S A_{\text {red }}=2 \pi r^{2} \times\left(\frac{23}{d}\right)^{2}=264.5 \pi=831 \mathrm{~mm}^{2}=8.31 \mathrm{~cm}^{2}
$$

$$
S A_{\text {blue }}=(23 \times 23)-\left(\pi r^{2} \times \text { total \#circles }\right)
$$

$S A_{\text {blue }}=529-\left(\pi r^{2} \times\left(\frac{23}{d}\right)^{2}\right)=529-\frac{529 \pi}{4}=114 \mathrm{~mm}^{2}=1.14 \mathrm{~cm}^{2}$

$$
S A_{\text {total }}=(2 \times 8.31)+(2 \times 1.14)=18.9 \mathrm{~cm}^{2}
$$

Scheme 8.2. Surface area calculation for $\mathrm{SiO}_{2} /$ epx-type coatings.

Table 8.21. Results of $\mathrm{BET}$ surface area analysis of $\mathrm{SiO}_{2}$, Batch $4 \mathrm{CnGmSiO}_{2}$, and Batch 2 $\mathrm{Cu}(\mathrm{II})-\mathrm{CnGmSiO}$.

\section{Sample}

$\mathrm{SiO}_{2}$ (Sigma-Aldrich) $^{\mathrm{b}}$

$\mathrm{SiO}_{2}$ (measured) ${ }^{c}$

Batch $4 \mathrm{CnGmSiO}_{2}$

Batch $2 \mathrm{Cu}(\mathrm{II})-\mathrm{CnGmSiO}_{2}$

a $-5 \%$ error.

${ }^{\mathrm{b}}$ Value reported by Sigma-Aldrich.

${ }^{c}$ Value measured in this work.

\section{Specific Surface Area $\left(\mathrm{m}^{2} / \mathrm{g}\right)^{\mathrm{a}}$}

500

411

271

255 
(a)

Diluted cultures ( $\times 2$ ) of $E$. coli NZRM 3647 in M63 minimal medium Incubated $24 \mathrm{~h}$ at $37^{\circ} \mathrm{C}$

Saline wash and transferred to saline/Tween 20
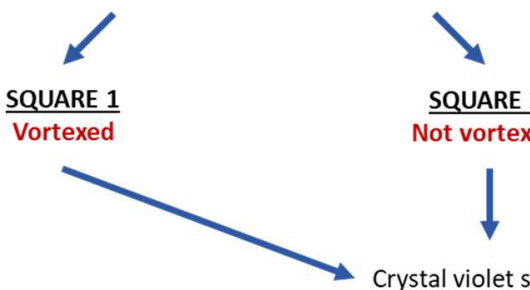

Not vortexed

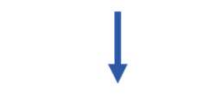

Crystal violet staining (b)

\begin{tabular}{|c|c|}
\hline Treatment & Absorbance $570 \mathrm{~nm}^{2}$ \\
\hline - Vortexing & 0.008 \\
\hline + Vortexing & 0.010 \\
\hline
\end{tabular}

+ Vortexing experiment. Blank subtracted.

Figure 8.54. Testing the efficiency of vortexing in the removal of adherent bacteria via crystal violet staining: (a) procedure and (b) tabulated results of crystal violet staining of vortexed (+) and not vortexed (-) samples.

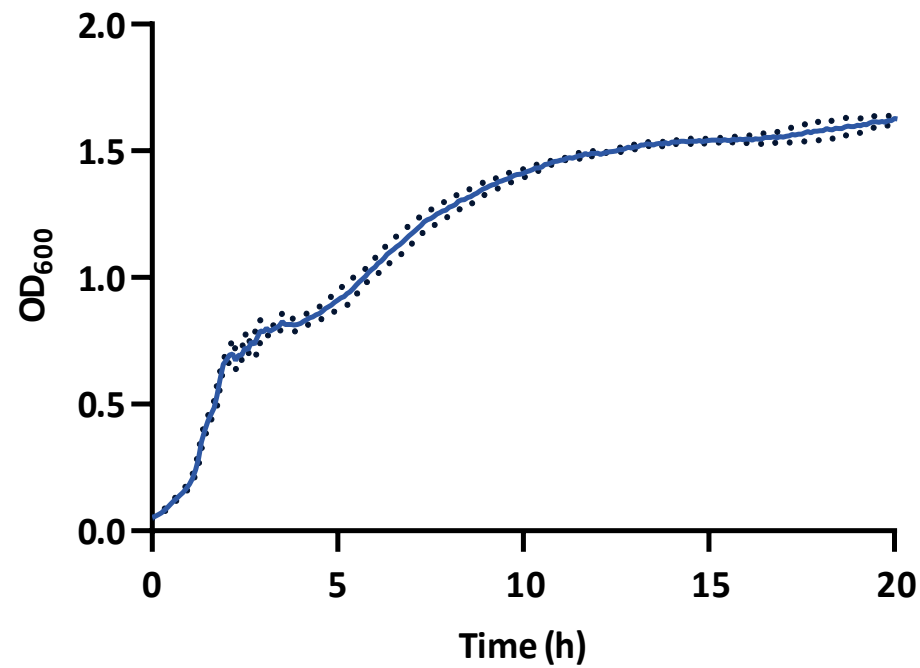

Figure 8.55. Growth curve of $V$. harveyi NZRM 2698 cultured in Luria marine broth. The solid line represents the mean $(b r=3)$, and the dotted line represents the SD. 